\title{
Model Validation Status Review
}

Prepared for:

U.S. Department of Energy

Yucca Mountain Site Characterization Office

P.O. Box 30307

North Las Vegas, Nevada 89036-0307

Prepared by:

Bechtel SAIC Company LLC

1180 Town Center Drive

Las Vegas, Nevada 89144

Under Contract Number:

DE-AC08-01RW12101 


\section{DISCLAIMER}

This report was prepared as an account of work sponsored by an agency of the United States Government. Neither the United States Government nor any agency thereof, nor any of their employees, nor any of their contractors, subcontractors or their employees, makes any warranty, express or implied, or assumes any legal liability or responsibility for the accuracy, completeness, or any third party's use or the results of such use of any information, apparatus, product, or process disclosed, or represents that its use would not infringe privately owned rights. Reference herein to any specific commercial product, process, or service by trade name, trademark, manufacturer, or otherwise, does not necessarily constitute or imply its endorsement, recommendation, or favoring by the United States Government or any agency thereof or its contractors or subcontractors. The views and opinions of authors expressed herein do not necessarily state or reflect those of the United States Government or any agency thereof. 
Model Validation Status Review

TDR-WIS-MD-000005 REV 00

November 2001

Prepared by:

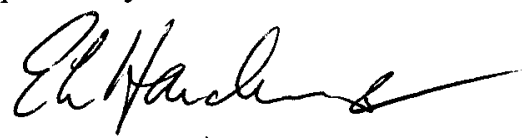

Ernest L. Hardin

$11 / 28 / 01$

Date

Technical Issues Lead, BSC Chief Science Office

Technical Check by:
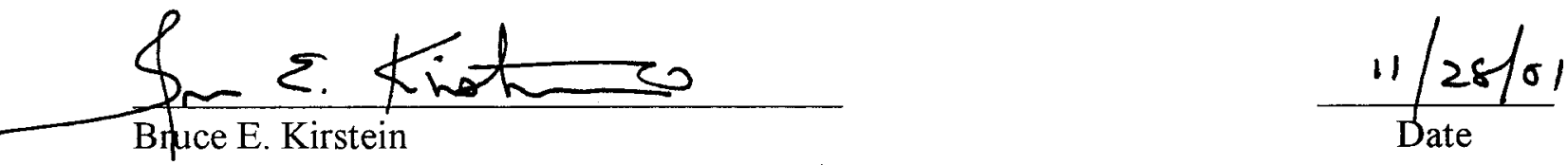

Principal Engineer, BSC Chief Science Office

Compliance Check by:
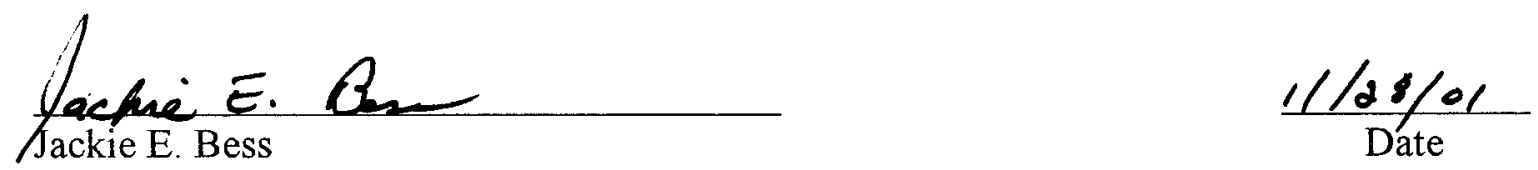

Licensing Engineer, Engineering and Preclosure

Safety Analysis Team

Approved by:

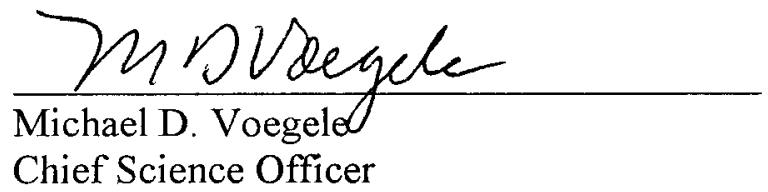

$\frac{1 /-28-01}{\text { Date }}$ 


\title{
CHANGE HISTORY
}

\author{
Revision Interim \\ Number Change Notice Description of Change \\ $0 \quad 0 \quad$ Initial Issue (no documents superseded)
}


INTENTIONALLY LEFT BLANK 


\section{EXECUTIVE SUMMARY}

This Model Validation Status Review (MVSR) technical report was prepared as part of the (amended) response to Corrective Action Request BSC-01-D-001 (Clark 2001, Krisha 2001). This report documents a systematic, one-time review of all the Analysis/Model Reports (AMRs) that support the Total System Performance Assessment - Site Recommendation (TSPA-SR), by an independent team under the direction of the Bechtel SAIC Company Chief Science Officer.

A total of 125 AMRs plus other documents and data were reviewed. The review team first identified the models documented in the AMRs. Then they determined how the models were used in development of the TSPA-SR. Finally, the models were assigned to three categories ("bins") according to the extent to which model validation was achieved in compliance with Administrative Procedure AP-3.10Q, Analyses and Models. Review-team recommendations are provided for improvement of documentation, and in some cases, for further model development, testing, or data collection to improve the models in support of licensing activities.

A total of 128 models were identified, of which 17 were assigned to Bin 1 (compliant validation), 77 were assigned to Bin 2 (information readily available for validation but documentation not in compliance), and 34 were assigned to Bin 3 (not in compliance and not readily validated). For the 34 Bin-3 models, impact reviews were conducted and the resulting impact review documentation is summarized in Section 6, and included in Appendix IV to this technical report. For all 34 Bin-3 models, the impact reviews found no significant impact from the model validation review findings, on the conclusions of the TSPA-SR (i.e., the calculated annual dose is negligible).

\section{Impact Review Summary for Bin-3 Models}

Of the 34 Bin-3 models, the impact reviews for 24 can be summarized as follows:

- Sixteen were found not to support TSPA-SR, i.e., the output from these models was not used as input to the system model. All 16 of these models are developmental, and some have been superseded by other products. Of these 16 models, only six were used for screening out of features, events, and processes (FEPs) from the TSPA-SR.

- Five additional Bin-3 models (not among the 16 discussed above) were originally intended by the authors to be analyses or calculations (i.e., not requiring validation). At the time this work was documented, the authors determined that model validation was not required based on consideration of details such as the analysis methods used and the contribution to TSPA-SR. The review team identified these as models requiring validation, using the criteria from Section 3.4.2 of this report.

- Three additional Bin-3 models in the Biosphere area were embedded within the GENII-S dose-assessment code and were not previously recognized as discrete models. (The GENII-S code is a widely used and accepted approach, but application to a potential Yucca Mountain geologic repository is not yet fully documented.) 
The remaining 10 of the Bin-3 models were used for TSPA-SR, and were originally intended as models requiring validation. Some examples of these are the WAPDEG Analysis of Waste Package and Drip Shield Degradation, the DRKBA Rockfall Model, and the TSPA-SR Model. These are discussed in detail in Sections 6 and 7 of this technical report.

Impact reviews for all 34 Bin-3 models are provided in Appendix IV to this report. A frequent explanation for no impact is that these models are bounding, or conservative with respect to the effect on the calculated annual dose in TSPA-SR. (Conservatism is not a compliant grounds for model validation according to AP-3.10Q.) Another type of explanation is based on minimal impact of the model on TSPA-SR dose calculations. For example, impact reviews for the Bin-3 cladding models (Section 6.11) state that a sensitivity study was performed for TSPA-SR, in which the performance of cladding was minimized, and the impact on calculated annual dose was minor.

Yet another type of explanation is based on differing technical opinions. For example, for the TSPA-SR Model (Section 6.21.1) and WAPDEG Model (Section 6.10.8) there are disagreements between reviewers and the responsible Project staff as to whether the available validating information provides sufficient confidence for the intended uses of the models (i.e., Bin 2 or Bin 3). The impact reviews for these models reiterate how the available information does support sufficient confidence in the model results. It is anticipated that the potential for such disagreements in the future will decrease through revision and enhancement of the documentation.

In summary, for all 34 models assigned to Bin 3, the impact reviews provide the basis for a determination that the conclusions of TSPA-SR (i.e., calculated annual dose as noted above) are not significantly impacted by the findings of this model validation review.

\section{Discussion of Binning Results}

The review findings combined assessments of procedural compliance, with expert opinions concerning the suitability of the validation arguments and the availability of additional supporting information. Procedural compliance was relied upon to discern Bin-1 models from noncompliant ones. The reviewers often expressed the need for better documentation of models for which they were in basic agreement, and for which validating information is available. This helps to explain the relatively large number of models in Bin 2 . The need for additional documentation is attributable to the need for better communication to an external audience, and limitations on the technical information available and known to the authors when the reports were prepared.

The basis for assignment to Bins 2 and 3 varied somewhat among the reviewers according to their individual technical understanding of the models and the intended uses. Neither Bin 2 or Bin 3 is, by definition, in compliance with AP-3.10Q so these binning decisions were based primarily on expert opinion as to the suitability of available information from all sources, to support the models for their intended uses.

In the process of identifying models, this MVSR identified 33 analyses and calculations defined according to AP-3.10Q and AP-3.12Q, Calculations, respectively, which were not documented 
as models but should have been. Documenting these as models would ensure that validation is performed, commensurate with the intended use for TSPA-SR and/or FEP screening. The criteria used by the MVSR reviewers to judge when model documentation is required are discussed in Section 3.4. In addition, 13 AMRs were identified which had been designated as models, but which were used in such a way that validation is not required. For example, the three reports that support the Integrated Site Model (Table 1, Model Area T) were designated by the authors as models, but considered to be analyses by this review. The MVSR reviewers recommend that these products be reconsidered as analyses or calculations which have no need for model validation.

\section{Report Organization}

Section 1 describes the Objective and Scope, and Section 2 the Quality Assurance requirements for the MVSR activity. Section 3 describes the review methodology, including the 21 technical model areas used to organize this review. Section 4 lists the documents that were reviewed. Section 5 presents biographical information for the 32 reviewers, and how they were assigned. Section 6 describes the identification of models, how the models were used in support of TSPA$\mathrm{SR}$, the basis for categorization ("binning"), and recommendations. Section 6 also provides a summary of the impact reviews performed for Bin-3 models. The detailed information in Section 6 is organized according to the technical model areas. Section 7 gives a detailed summary of the binning results, and summarizes the conclusions of the impact reviews. Section 8 lists the references used. Appendices I, II, and III contain additional review comments and recommendations for improvement of selected models and documentation. Appendix IV contains the impact review documents developed for the 34 Bin-3 models. 
INTENTIONALLY LEFT BLANK 


\section{CONTENTS}

Page

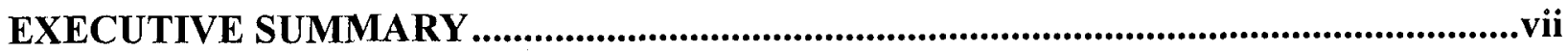

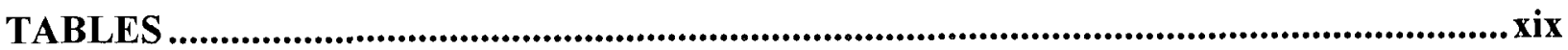

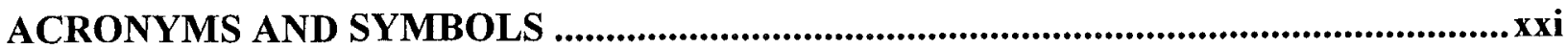

1. Objective and Scope...........................................................................................1

1.1 Background on Model Validation for Site Characterization at Yucca Mountain ....... 1

1.1.1 Model Validation - NUREG-1636 Definition ....................................... 1

1.1.2 Model Validation - AP-3.10Q Procedural Definition ................................. 2

1.1.3 Previous Identification of Model Validation Deficiencies ........................... 3

1.2 Relationship of this Review to a Potential Future Site Recommendation ...................3

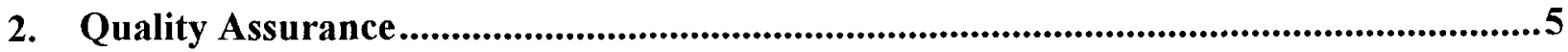

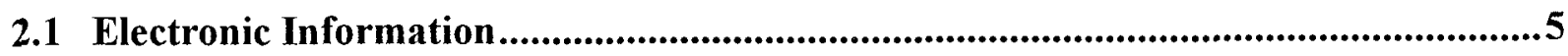

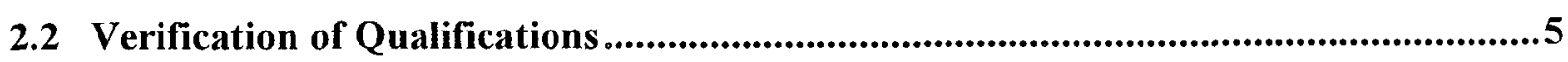

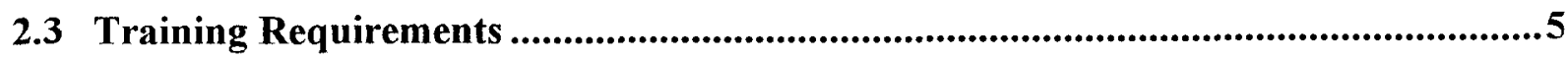

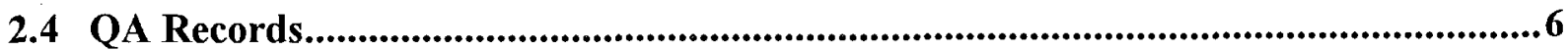

3. Review Methodology and Criteria...............................................................................

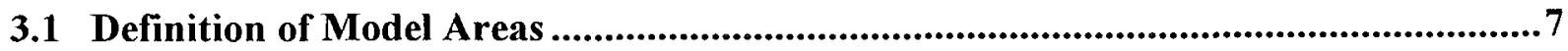

3.2 Roles of Technical Specialists and Model Area Leads ..........................................7

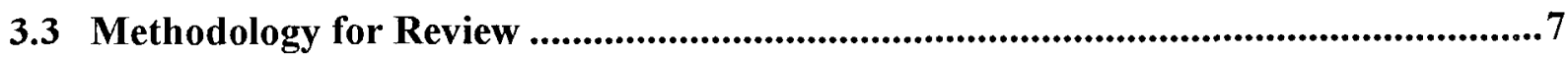

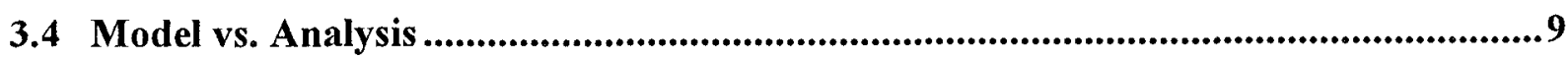

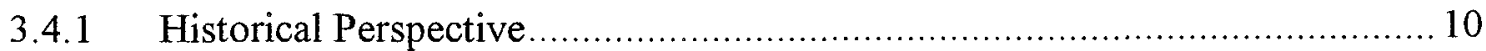

3.4.2 Criteria for Discerning Models vs. Analyses .................................. 10

4. Documents Included in Review ............................................................................... 13

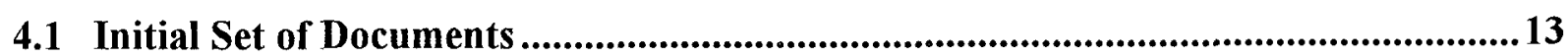

4.2 Justification for Additional Documentation ...........................................................13

5. Selection of Technical Specialists and Model Area Leads ...........................................15

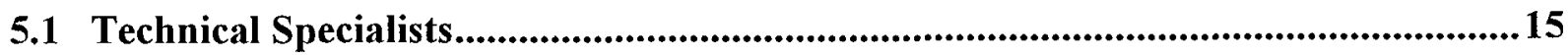

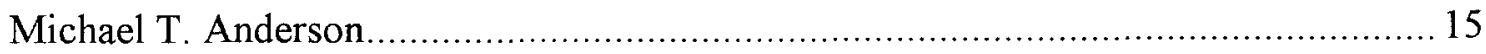

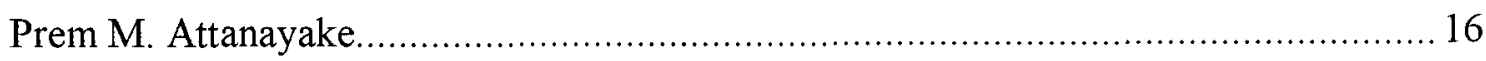

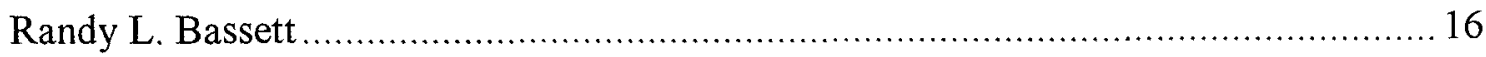

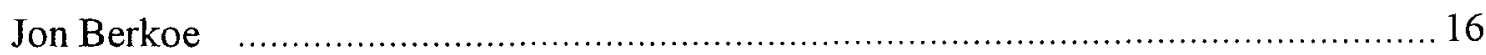

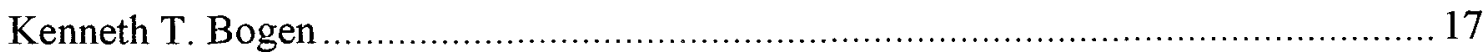

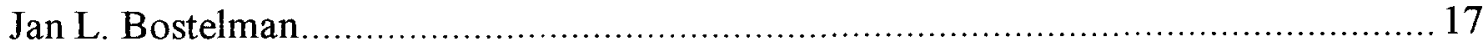

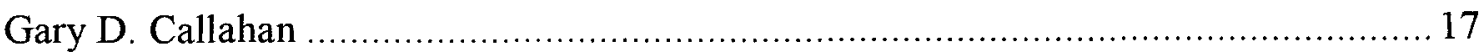


Kerry L. DeVries …………… 18

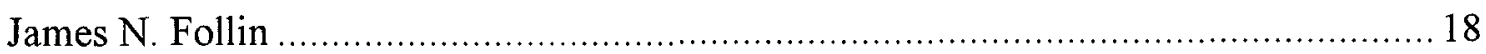

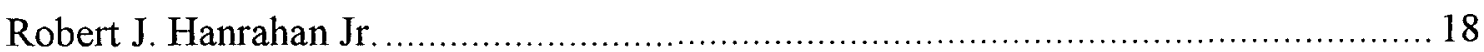

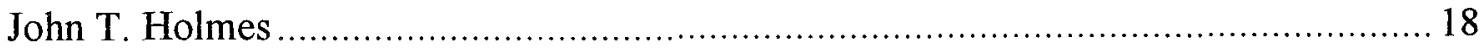

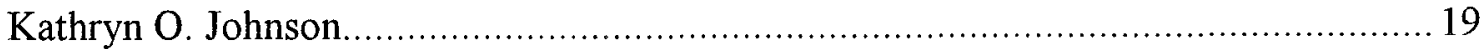

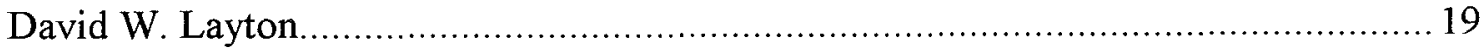

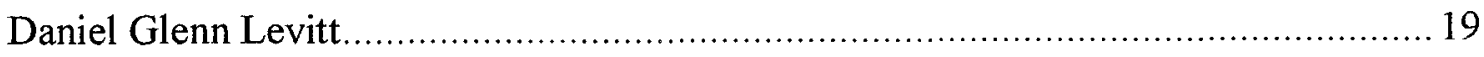

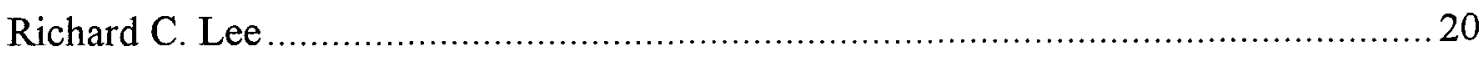

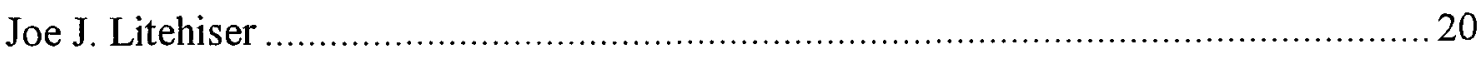

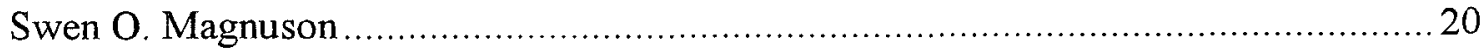

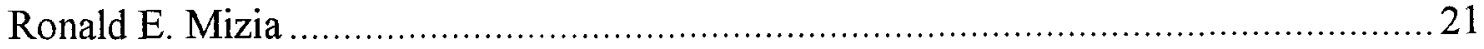

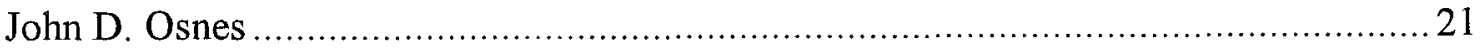

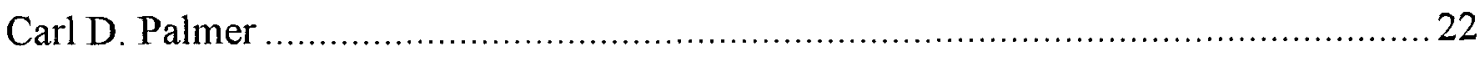

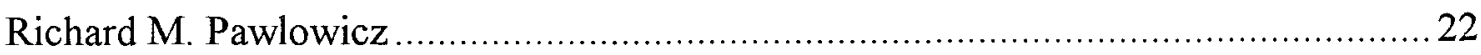

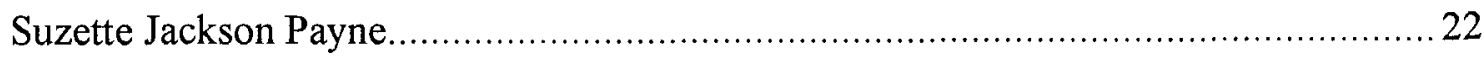

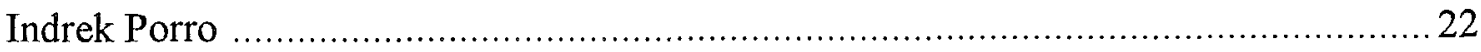

Stuart E. Rawlinson

George D. Redden

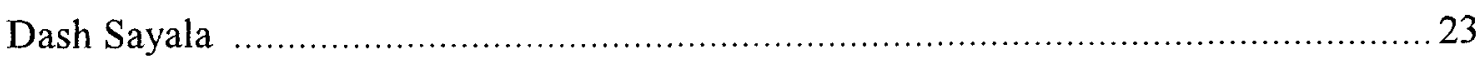

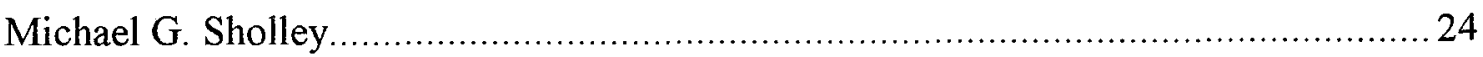

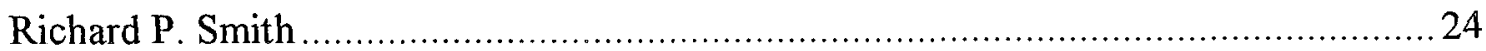

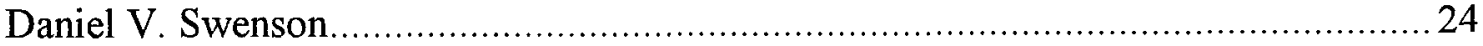

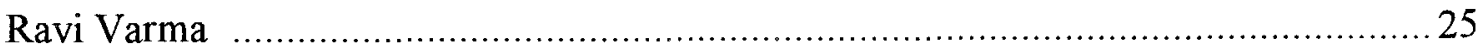

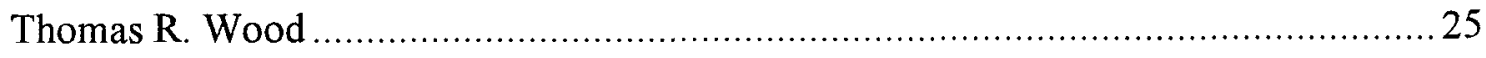

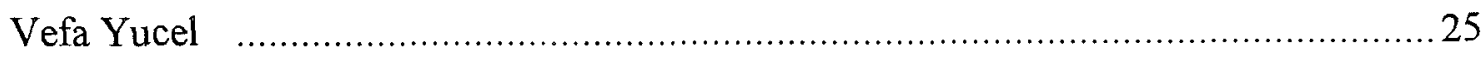

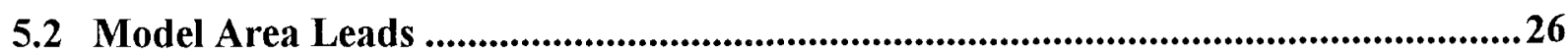

6. Review Findings and Recommendations.........................................................................2

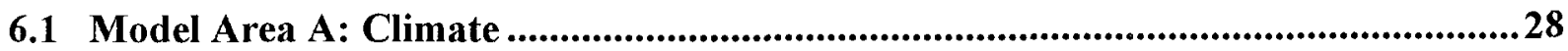

6.1.1 Precession-Based Orbital Clock Model (A.1) ........................................ 28

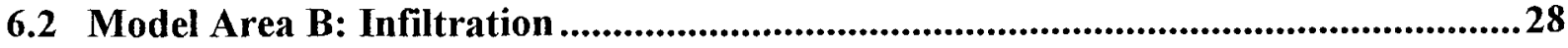

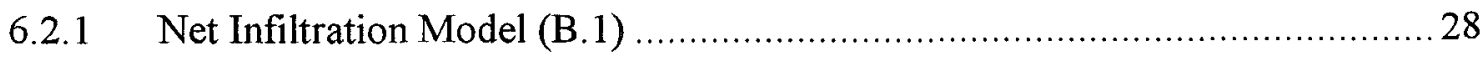

6.3 Model Area C: Unsaturated Zone Flow .................................................................30

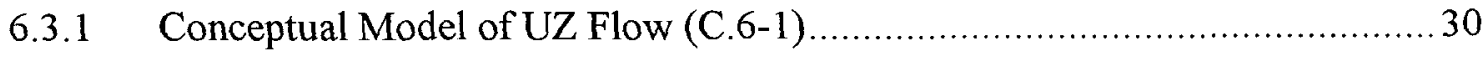

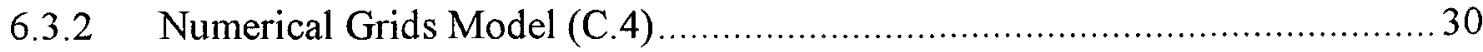

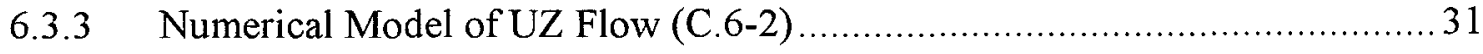

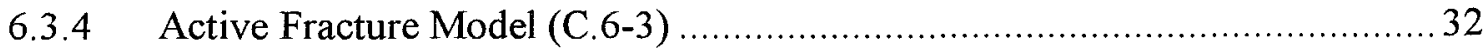


6.3.5 Calibrated Properties Model (C.2) .................................................. 33

6.3.6 UZ Flow Model (C.1-1) .......................................................... 33

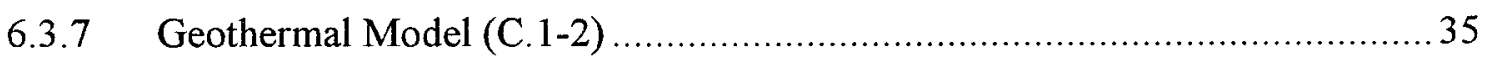

6.3.8 Conceptual Model of Perched Water (C.1-3) ...................................... 35

6.3.9 3-D Perched Water Calibration Model (C. 1-4) ..................................... 36

6.4 Model Area D: Mountain-Scale Thermal-Hydrology ................................................37

6.4.1 Mountain-Scale Coupled Processes (Thermal-Hydrology)

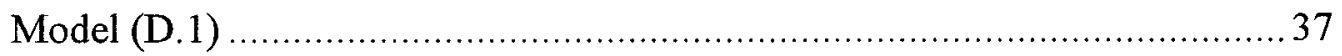

6.5 Model Area E: Ambient/Thermal Drift Seepage .............................................39

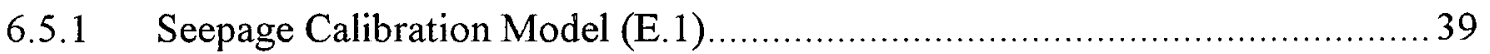

6.5.2 Seepage Model for Performance Assessment Including Drift

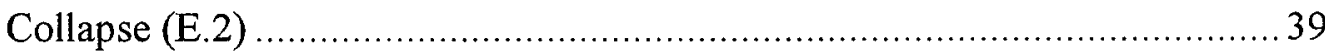

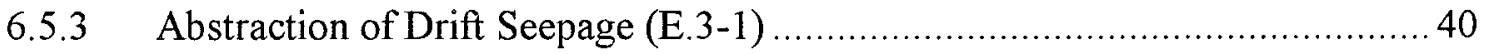

6.5.4 Abstraction of Thermal Seepage Effects (E. 3-2) .............................. 41

6.6 Model Area F: Mountain-Scale/Near-Field Thermal-Hydrologic-Chemical Model ...........................................................................................................42

6.6.1 Drift-Scale Test Thermal-Hydrologic-Chemical Calibration Model (F.1-1) .................................................................. 42

6.6.2 Thermal-Hydrologic-Chemical Seepage Model (F.1-2) .......................... 43

6.7 Model Area G: Mountain-Scale/Near-Field Thermal-Hydrologic-Mechanical

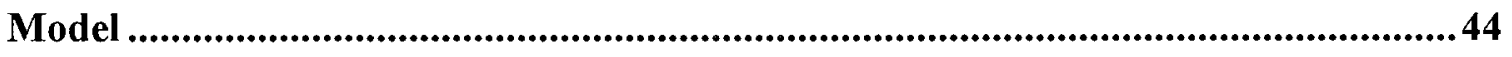

6.7.1 Thermal-Hydrologic-Mechanical (THM) Model (G.1) ........................... 44

6.8 Model Area H: In-Drift Chemistry ...............................................................46

6.8.1 High-Relative Humidity Salts Model (H.1) ..................................... 46

6.8.2 In-Drift Microbial Communities (H.6) ............................................... 49

6.8.3 Gas Flux and Fugacity Model (H.3-1) ....................................... 50

6.8.4 In-Drift Gas Flux and Composition Model (H.5) ............................... 51

6.8.5 Cement Grout Model (H.3-2) ............................................... 51

6.8.6 Seepage/Cement Interaction Model (H.7) ..................................... 52

6.8.7 Corrosion of Steel Used in the Ex-Container EBS (H.3-3) ......................... 53

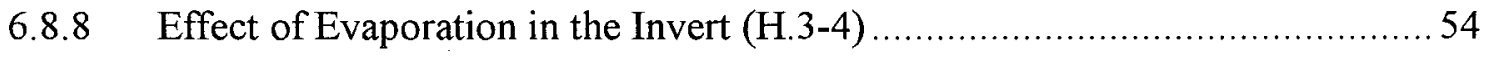

6.8.9 EBS Colloids Model (H.3-5) ................................................ 54

6.8.10 Normative Precipitates/Salts Model (H.3-6) ................................. 55

6.9 Model Area I: EBS Moisture Distribution and Thermal-Hydrology ........................56

6.9.1 Multiscale TH Model (I.1) ......................................................... 56

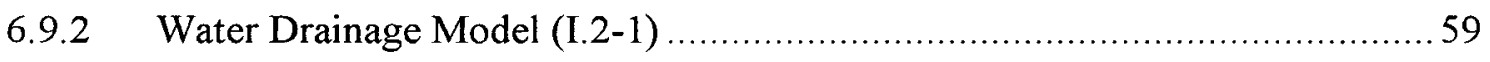




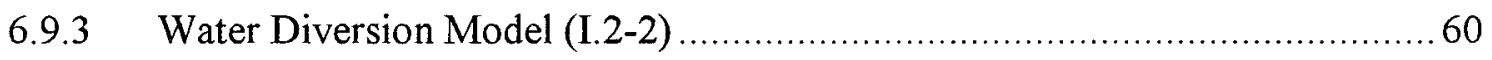

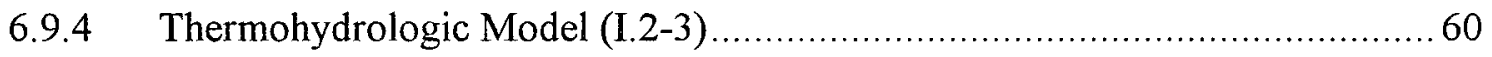

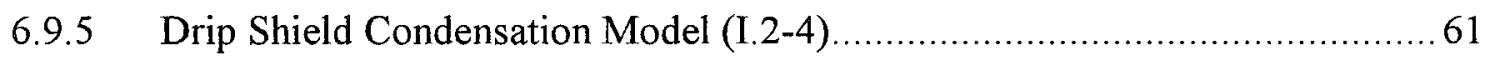

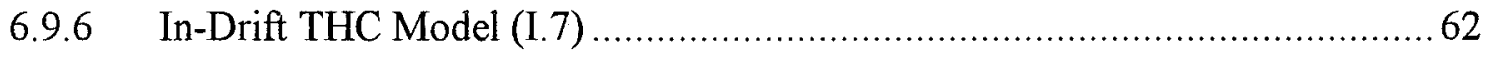

6.9.7 Effective Thermal Conductivity Model (I.8) ..............................................63

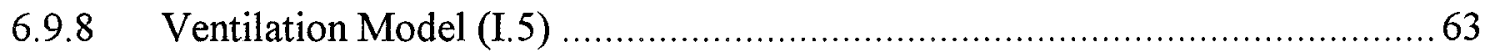

6.10 Model Area J: Waste Package/Drip Shield Degradation................................................64

6.10.1 General and Localized Corrosion of the Waste Package Outer

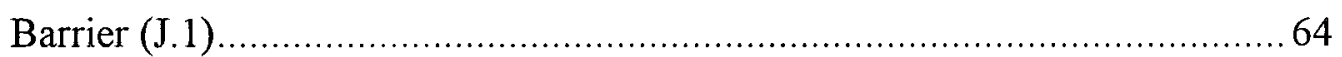

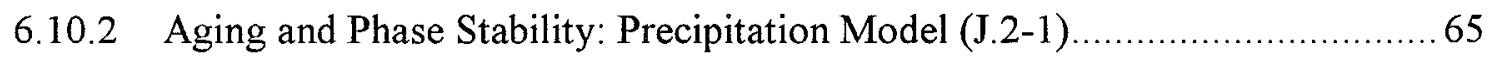

6.10.3 Aging and Phase Stability: Long Range Ordering Model (J.2-2)................. 66

6.10.4 General and Localized Corrosion of the Drip Shield (J.3) ............................66

6.10.5 Degradation of Stainless Steel Structural Materials (J.4) .............................67

6.10.6 Alloy-22 Potential-Based Localized Corrosion Initiation Threshold

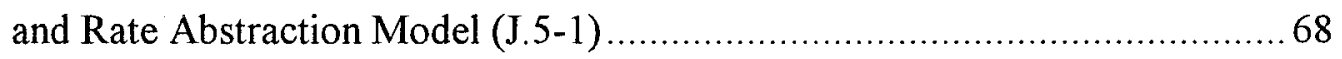

6.10.7 Drip Shield Ti-7 Potential-Based Localized Corrosion Initiation

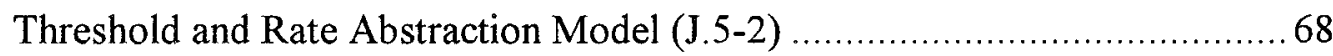

6.10.8 WAPDEG Analysis of Waste Package and Drip Shield Degradation (J.6).

6.10.9 Calculation of the General Corrosion Rate for the Drip Shield and Waste Package Outer Barrier to Support WAPDEG Analysis (J.7)............... 71

6.10.10 Incorporation of Uncertainty and Variability of Drip Shield and Waste Package Degradation in WAPDEG (J.8) …...................................... 73

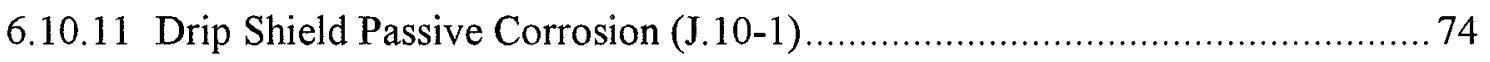

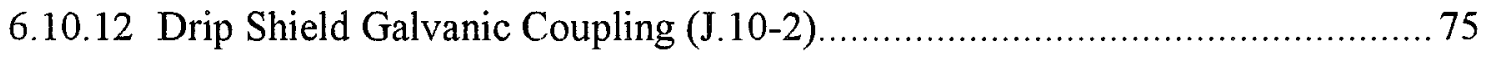

6.10.13 Stress Corrosion Cracking Threshold Model (J.11-1) …............................. 76

6.10.14 Stress Corrosion Cracking Slip Dissolution/Film Rupture

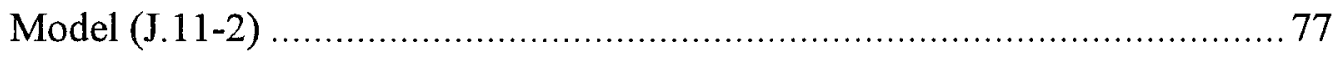

6.10.15 Manufacturing Defects Abstraction Model (J.12-1) ...................................78

6.10.16 Stress and Stress Intensity Factor Profile Abstraction Model (J.12-2) ........... 78

6.10.17 Slip Dissolution Abstraction Model (J.12-3) …....................................... 78

6.10.18 Threshold Stress Intensity Factor Abstraction Model (J.12-4)_.................... 79

6.11 Model Area K: Waste Form Degradation .....................................................................79

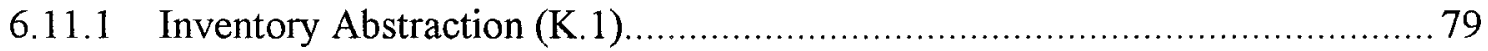

6.11.2 CSNF Waste Form Summary Degradation Abstraction (K.2) …................... 80

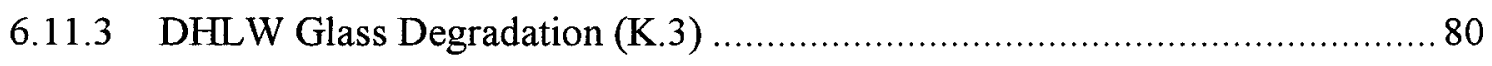


6.11.4 Waste Form Degradation Abstraction - Upper Limit Model (K.4-1) ............ 81

6.11.5 Waste Form Degradation Abstraction - Conservative Model (K.4-2) ........... 81

6.11.6 Waste Form Degradation Abstraction - Best Estimate Model (K.4-3) ...........82

6.11.7 Waste Form Degradation Abstraction - Immobilized Pu Model (K.4-4)...... 82

6.11.8 In-Package Source Term Abstraction (K.5) ........................................ 83

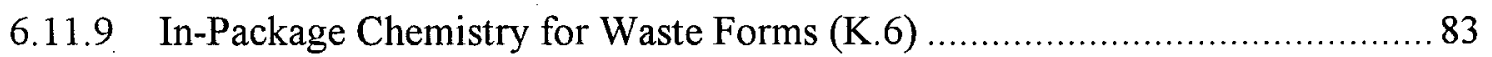

6.11.10 In-Package Chemistry Abstraction (K.7) .................................... 84

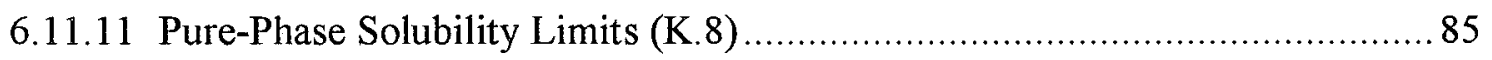

6.11.12 Dissolved Concentration Limits (K.9) ................................................. 85

6.11.13 Secondary Uranium-Phase Paragenesis and Incorporation of

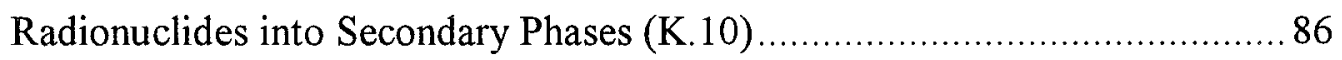

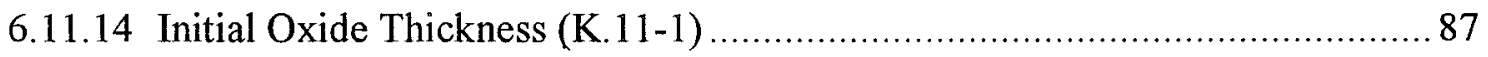

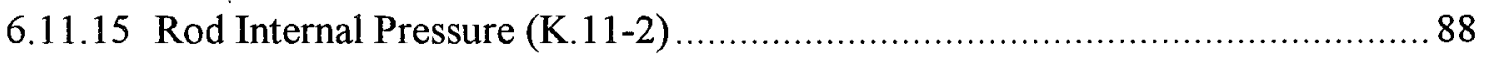

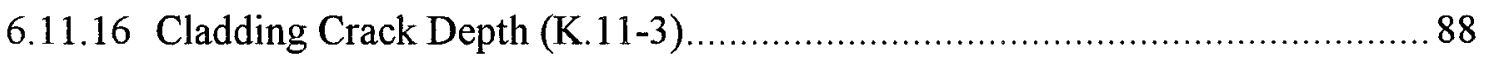

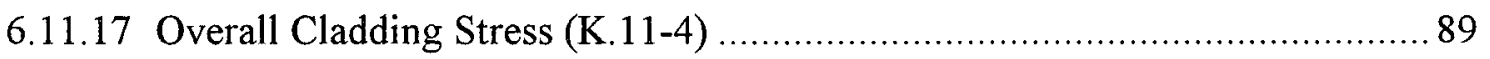

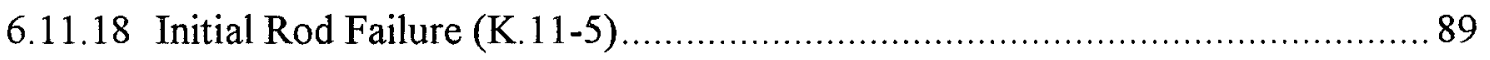

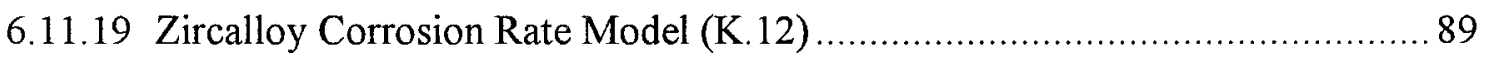

6.11.20 Residual Stress in CSNF Cladding Material (K.13) ............................. 90

6.11.21 Alternative Wet Clad Unzipping Model (K.14-1) ................................. 91

6.11.22 Bounding Model for Clad Unzipping Velocity (K.14-2) .........................92

6.11.23 Clad Dry Unzipping Model (K.15) ............................................... 93

6.11.24 Summary and Abstraction - Clad Unzipping and Fuel

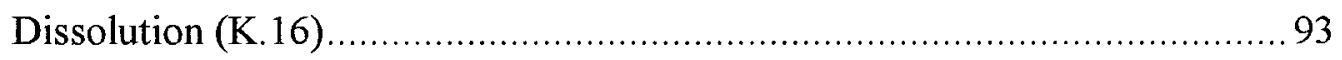

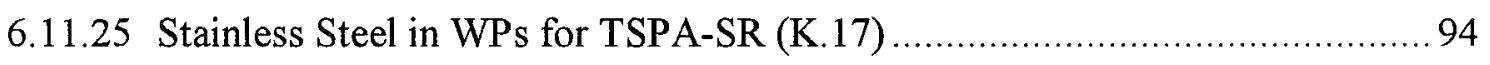

6.11.26 Thermal Evaluation of Breached 21-PWR Waste Packages (K.18) .............. 95

6.11.27 Breakage of CSNF Clad by Seismic Loading (K.19-1) .......................... 96

6.11.28 Breakage of CSNF Clad by Static Loading (K.19-2) .............................. 96

6.11.29 Colloid-Associated Radionuclide Concentration Limits (K.21)................... 97

6.11.30 Waste Form Colloid-Associated Concentration Limits: Abstraction

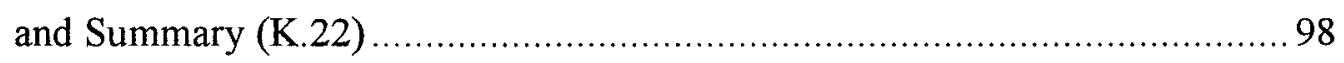

6.12 Model Area L: EBS Degradation........................................................................99

6.12.1 DRKBA Rockfall Model (L.1) ............................................... 99

6.12.2 Rockfall on Drip Shield Model (L.3) ........................................ 100

6.12.3 Flow into Waste Packages Through Small Lid Openings (FLO)

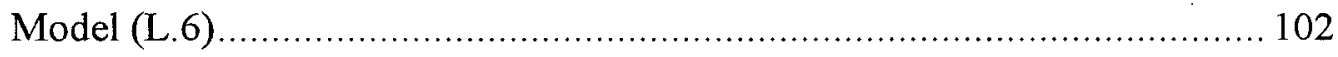


6.13 Model Area M: EBS Radionuclide Transport................................................... 102

6.13.1 In-Drift Colloids and Concentrations Model (M.3) ............................. 102

6.13.2 Invert Diffusion Properties Model (M.1) ............................................ 105

6.13.3 In-Drift Transport of Radionuclides Model (M.2) ............................... 106

6.13.4 EBS Radionuclide Transport Abstraction Model (M.5) .......................... 107

6.13.5 Seepage/Invert Interactions Model (M.4)......................................... 109

6.14 Model Area N: Unsaturated Zone Transport.......................................................... 110

6.14.1 Equilibrium Matrix Sorption Basis (N.1-1) ..................................... 110

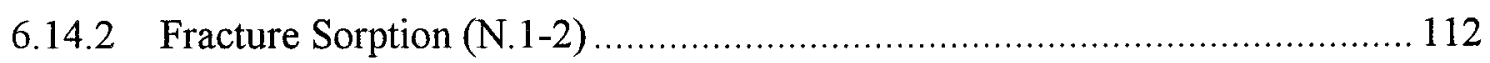

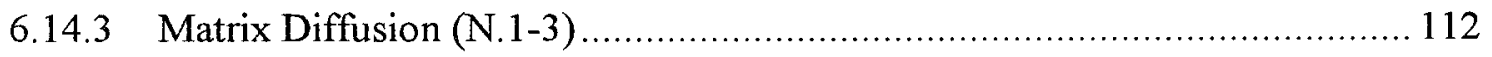

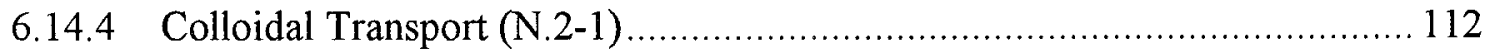

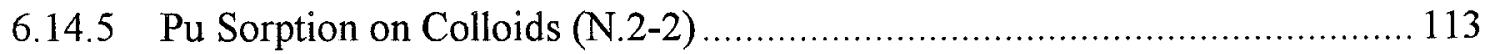

6.14.6 FRACL Calibration to Borehole Chloride (N.3-1) ........................... 114

6.14.7 EOS9nT Calibration to ESF Chloride Profile (N.3-2) ......................... 115

6.14.8 Fracture-to-Matrix Colloid Filtration (N.4) ................................... 116

6.15 Model Area O: Saturated Zone Flow ...............................................................116

6.15.1 Site-Scale Saturated Zone Flow Model (0.5) .................................. 116

6.16 Model Area P: Saturated Zone Transport ......................................................116

6.16.1 Sub-Gridblock Scale Dispersion in 3-D Heterogeneous Fractured

Media (P.6) ......................................................................... 116

6.16.2 Saturated Zone Colloid Facilitated Transport (P.2) ............................ 117

6.16.3 Pipe Model for Daughter Radionuclides (P.4-1) ................................ 118

6.16.4 Abstraction of FEHM and Coupling with UZ Mass Flux (P.4-2) .............. 119

6.16.5 Transport Parameters from C-Wells and Laboratory Studies (P.4-3)......... 120

6.17 Model Area Q: Biosphere................................................................................. 121

6.17.1 Crop Interception Fraction Submodel (Q.2-1).............................. 121

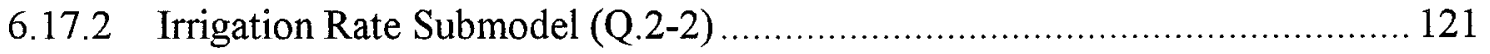

6.17.3 Dose Conversion for Ingestion (Q.4-1) ...................................... 121

6.17.4 Dose Conversion for Inhalation (Q.4-2) .................................... 122

6.17.5 Dose Conversion for External Exposure (Q.4-3) .............................. 122

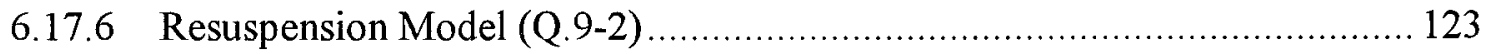

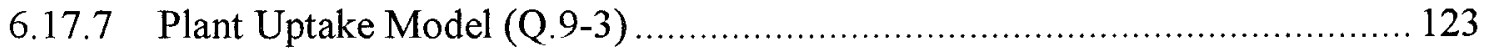

6.17.8 Surface Soil Model in GENII-S (Q.9-1) ...................................... 124

6.17.9 Radionuclide Transfer to Animals (Q.9-4) ................................... 125

6.17.10 Radionuclide Transfer to Aquatic Food (Q.9-5) .............................. 126 
6.18 Model Area R: Disruptive Events/Igneous Disruption Consequences

6.18.1 Conditional Distribution for Number of Eruptive Centers Model (R.1)

6.18.2 Geometry of Volcanic Feeder System Model (R.2) ............................ 127

6.18.3 Volcanic Eruption Release Model (R.3-1) ............................................ 129

6.18.4 In-drift Damage Due to Dike Intersection Model (R.3-2)......................... 130

6.18.5 Mass Loading Decay Model Following Deposition of Volcanic

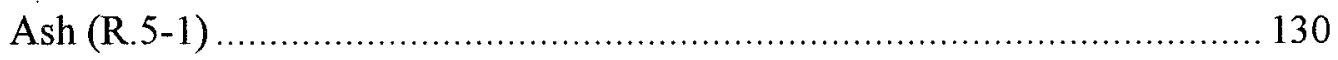

6.18.6 Dose Conversion Factor Model for Inhalation - Igneous Disruption (R.5-2)...

6.19 Model Area S: Seismic Hazards......................................................................... 131

6.19.1 Vibratory Ground Motion Hazard Model (S.1-1).................................. 131

6.19.2 Fault Rupture Hazard Model (S. 1 -2) ....................................... 132

6.20 Model Area T: Integrated Site Model........................................................... 133

6.21 Model Area U: Performance Assessment Model..................................................... 133

6.21.1 Total System Performance Assessment-Site Recommendation

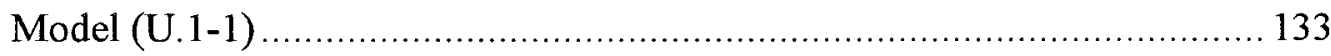

6.21.2 Soil Removal Model for Volcanic Disruption (U.1-2) ......................... 136

6.21.3 Pu-Ceramic Degradation Model for TSPA-SR (U.4) ............................. 137

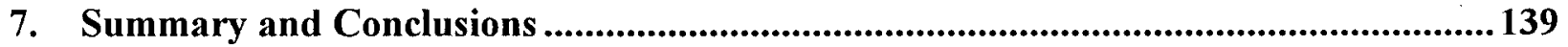

7.1 Summary of Review Findings by Model Area ....................................................... 139

7.2 Impact Reviews for Bin-3 Models.............................................................151

7.3 Summary of Binning Results ............................................................................... 152

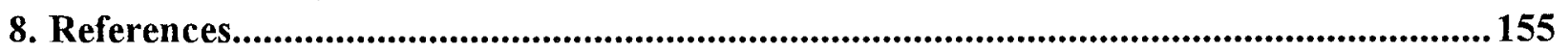

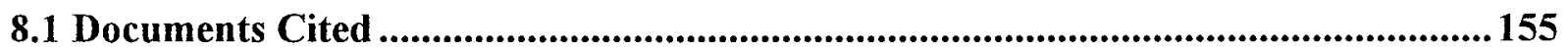

8.2 Codes, Standards, Regulations, and Procedures............................................... 170

8.3 Source Data, Listed by Data Tracking Numbers ................................................ 171

Appendix I - Additional Comments and Recommendations on the Drift-Scale Test ThermalHydrologic-Chemical Calibration Model (F.1-1) and the Thermal- Hydrologic-Chemical Seepage Model (F.1-2).

Appendix II - Additional Comments and Recommendations on the Total System Performance Assessment - Site Recommendation Model (U.1): Basis for Assessment of Validation for the TSPA-SR Model

Appendix III - Additional Comments on Document Content Reorganization for the TSPA Model Report, and the TSPA Technical Report...................................................... III-1

Appendix IV - Impact Reviews for Bin-3 Models as Submitted by Responsible Project Staff. 
Thermal-Hydrologic-Mechanical (THM) Model (G.1) .............................IV-2

Cement Model (H.3-2) ......................................................................

Corrosion of Steel Used in the Ex-Container EBS (H.3-3) ................................... IV-7

Effect of Evaporation in the Invert (H.3-4) ..................................................

EBS Colloids Model (H.3-5) ..............................................................

Seepage/Cement Interaction Model (H.7) ..................................................

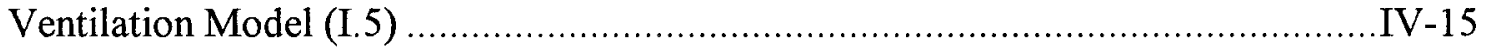

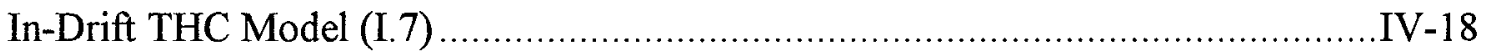

Effective Thermal Conductivity Model (I.8) ................................................. IV-19

WAPDEG Analysis of Waste Package and Drip Shield Degradation (J.6).............IV-21

Incorporation of Uncertainty and Variability of Drip Shield and Waste Package Degradation in WAPDEG (J.8) .......................................................

Waste Form Degradation Abstraction - Best Estimate Model (K.4-3) .................IV-29

Waste Form Degradation Abstraction - Immobilized Pu Model (K.4-4) ................IV-30

Alternate Wet Clad Unzipping Model (K.14-1) .............................................IV-31

Summary and Abstraction - Clad Unzipping and Fuel Dissolution (K.16) .............IV-32

Thermal Evaluation of Breached 21-PWR Waste Packages (K.18) .......................IV-34

Breakage of CSNF Clad by Seismic Loading (K.19-1) ..................................IV-35

Breakage of CSNF Clad by Static Loading (K.19-2) .....................................

DRKBA Rockfall Model (L. 1) .................................................................

Flow into Waste Packages Through Small Lid Openings (FLO) Model (L.6) .........IV-40

In-Drift Transport of Radionuclides Model (M.2) .........................................

In-Drift Colloids and Concentrations Model (M.3) .........................................IV-43

Seepage/Invert Interactions Model (M.4) .................................................... IV-44

EBS Radionuclide Transport Abstraction Model (M.5) ....................................IV-45

FRACL Calibration to Borehole Chloride (N.3-1) .....................................IV-46

Abstraction of FEHM and Coupling with UZ Mass Flux (P.4-2) .........................IV-49

Transport Parameters from C-Wells and Laboratory Studies (P.4-3) ...................IV-52

Surface Soil Model in GENII-S (Q.9-1) ..........................................

Radionuclide Transfer to Animals (Q.9-4) .............................................

Radionuclide Transfer to Aquatic Food (Q.9-5) .............................................

Geometry of Volcanic Feeder System Model (R.2) ........................................IV-60

Total System Performance Assessment-Site Recommendation Model (U.1-1) .......IV-62

Soil Removal Model for Volcanic Disruption (U.1-2) ................................... IV-69

Pu-Ceramic Degradation Model for TSPA-SR (U.4) .......................................... 


\section{TABLES}

Page

1. List of Model Areas and Associated AMRs and Other Reports for Status Review 173

2. Original Planned Schedule of Tasks Performed for Model Validation Status Review 182

3. List of Supplementary Review Materials Distributed to the Technical Specialists. 183

4. Assignment of Technical Specialists to Model Areas (X denotes supporting role) 185

5. Model Area Leads, Representing Responsible Project Staff. 186

6. Summary of Binning Results from Model Validation Status Review for Each Model Area

7. Summary of Bin-3 Models, Showing How They are Used, and the Model Designation in the Principal Supporting Document.

8. Models which are Documented as Analyses or Calculations. 198

9. Recommended Analyses Identified in This Review which are Documented as Models 200 


\section{INTENTIONALLY LEFT BLANK}




\section{ACRONYMS and SYMBOLS}

A

AMR

ANL

ANSYS

APC

apmf

B

BDCF

BSC

C

Calc.

CAR

CDF

CD-ROM

CFR

CLST

CODATA

CRWMS M\&O

CSNF

CSO

CTD

$\mathrm{CTD}^{*}$

$\mathrm{CTD}^{* *}$

$\overline{\mathrm{CTD}}$

CTD *

c

D

$\overline{\mathrm{D}}$

DCF

DDT

DHLW

DIRS

DLL

DOE

DPC

DRKBA

DSNF

DS

DST
Stochastic variate used in an example of Monte Carlo analysis

Analysis/Model Report

Argonne National Laboratory

Finite element modeling software; not specified as an acronym

Aqueous Phase Corrosion

Two-point approximating probability mass function

Stochastic variate used in an example of Monte Carlo analysis

Biosphere Dose Conversion Factors

Bechtel SAIC Company, LLC

Stochastic variate used in an example of Monte Carlo analysis

Calculation

Corrective Action Request

Cumulative Distribution Function

Compact Disc Read Only Memory

Code of Federal Regulations

Container Life and Source Term KTI

Committee on Data for Science and Technology

Civilian Radioactive Waste Management System Management and

Operating Contractor

Commercial Spent Nuclear Fuel

Chief Science Officer

Critical-target dose

Reasonable upper confidence bound on simulated CTD

Alternative estimator: expected value of CTD conditioned on exceedance probability

Estimator of E(CTD)

A reasonable upper bound on $\mathrm{E}(\mathrm{CTD})$ incorporating uncertainty

Random variate with chi-square distribution

Stochastic variate used in an example of Monte Carlo analysis

Mean of D

Dose Conversion Factor

Discrete-heat-source, Drift-scale, Thermal-conduction

Defense High-Level Waste

Document Input Reference System

Dynamically linked library

U.S. Department of Energy

Discrete Probability Calculus

Discrete Region Key-Block Analysis

Defense Spent Nuclear Fuel

Drip Shield

Drift Scale Test 
DTN

E

EBS

ECRB

ECM

EDA-II

EPA

$\mathrm{EQ} 3 / 6$

ESF

E(CTD)

$\mathrm{E}(G(L(\mathbf{x})))$

$\mathrm{E}(\mathbf{x})$

FEPs

FEHM

FGR

FLO

FR

FRACL

$f\left(x_{i}\right)$

G

GE

GENII-S

GSD

HAC

HIC

HLW

HMW

IAEA

ICSU

INEEL

KTI

$L(\mathbf{x})$

LA

LDTH

LLC

LLNL
Data Tracking Number

Stochastic variate used in an example of Monte Carlo analysis

Engineered Barrier System

Enhanced Characterization of Repository Block

Effective-Continuum Model (also equivalent-continuum model)

Enhanced Design Alternative II

U.S. Environmental Protection Agency

Geochemical modeling software

Exploratory Studies Facility

Expected value for the critical-target dose

Expected value for the approximation to the output from TSPA

Expected value(s) for the reduced set of quantities representing important inputs to the TSPA model

Features, Events, and Processes

Finite Element Heat and Mass Transfer

Federal Guidance Report

Flow into waste packages through small Lid Openings

Federal Register

Geochemical transport simulator; not specified as an acronym

Approximation to the TSPA model representing major monotonic

dependence on inputs

Analytic and/or interpolated function representing output of TSPA

General Electric Co.

Biosphere modeling code

Geometric standard deviation

Humid-Air Corrosion

Hydrogen-Induced Cracking

High-Level Waste

Harvie, Moeller, and Weare database for EQ3/6

International Atomic Energy Agency

International Council of Science Unions

Idaho National Engineering and Environmental Laboratory

Key Technical Issue

Linear function representing simplified functionality of the TSPA model

License Application

Line-averaged-heat-source, Drift-scale, Thermohydrologic

Limited Liability Corporation

Lawrence Livermore National Laboratory 


\begin{tabular}{|c|c|}
\hline $\begin{array}{l}\text { LANL } \\
\text { LRO } \\
\text { LWR }\end{array}$ & $\begin{array}{l}\text { Los Alamos National Laboratory } \\
\text { Long Range Ordering } \\
\text { Light Water Reactor }\end{array}$ \\
\hline MIC & Microbially Influenced Corrosion \\
\hline MING & Nutrient and energy balance software for microbial studies \\
\hline MOP & Member Of Public \\
\hline MSTHM & Multi-Scale Thermohydrology Model \\
\hline MT & Metric Ton \\
\hline MVSR & Model Validation Status Review \\
\hline MW & MegaWatt \\
\hline $\mathrm{M}$ & Mean of a distribution representing model input $\mathrm{X}$ \\
\hline $\mathrm{m}$ & Sample mean for $n$-samples of model input $\mathrm{X}$ \\
\hline NEA & $\begin{array}{l}\text { Nuclear Energy Agency (Organisation for Economic Co-operation and } \\
\text { Development) }\end{array}$ \\
\hline NFE & Near-Field Environment \\
\hline NIST & National Institute of Standards and Technology \\
\hline No. & Number \\
\hline NRC & U.S. Nuclear Regulatory Commission \\
\hline NTS & Nevada Test Site \\
\hline NUFT & Nonisothermal Unsaturated Flow and Transport simulator \\
\hline $\mathrm{n}$ & Number of samples \\
\hline $\mathrm{n}_{\mathrm{sim}}$ & Number of Monte Carlo realizations \\
\hline OQA & Office of Quality Assurance \\
\hline$p$ & Probability \\
\hline pdf & Probability distribution function \\
\hline PIT & Pitzer database for EQ3/6 \\
\hline PMR & Process Model Report \\
\hline PNL & Pacific Northwest Laboratory \\
\hline PVHA & Probabilistic Volcanic Hazards Assessment \\
\hline PWR & Pressurized Water Reactor \\
\hline QA & Quality Assurance \\
\hline $\mathrm{RC}$ & Relative univariate Contribution \\
\hline RELAP & Geochemical dual-porosity transport code; not specified as an acronym \\
\hline RETRAN & Geochemical dual-porosity transport code; not specified as an acronym \\
\hline $\mathrm{RH}$ & Relative Humidity \\
\hline RT & Radionuclide Transport \\
\hline RTA & Reactive Transport Application (software) \\
\hline SCC & Stress Corrosion Cracking \\
\hline SCFT & Solid-Centered Flow-Through mode for EQ3/6 \\
\hline
\end{tabular}




\begin{tabular}{|c|c|}
\hline SDT & Smeared-heat-source, Drift-scale, Thermal-conduction \\
\hline SEM & Scanning Electron Microscopy \\
\hline SHT & Single Heater Test \\
\hline SMT & Smeared-heat-source, Mountain-scale, Thermal-conduction \\
\hline SNF & Spent Nuclear Fuel \\
\hline SR & Site Recommendation \\
\hline $\mathrm{SZ}$ & Saturated Zone \\
\hline 2-D & Two-Dimensional \\
\hline $3-\mathrm{D}$ & Three-Dimensional \\
\hline T2R3D & Unsaturated zone transport software; not specified as an acronym \\
\hline TEM & Transmission Electron Microscopy \\
\hline $\mathrm{TH}$ & Thermal Hydrology \\
\hline THC & Thermal-Hydrologic-Chemical \\
\hline THM & Thermal-Hydrologic-Mechanical \\
\hline TM & Thermal-Mechanical \\
\hline TRACRN & Groundwater transport simulation software; not specified as an acronym \\
\hline TSPA-SR & Total-System Performance Assessment for Site Recommendation \\
\hline $\mathrm{t}$ & Random variate with Student-t distribution \\
\hline UDEC & Distinct-element geomechanical modeling software \\
\hline UFA & Ultra-centrifuge Flow Apparatus \\
\hline USA & United States of America \\
\hline USGS & United States Geologic Survey \\
\hline $\mathrm{UZ}$ & Unsaturated Zone \\
\hline $\mathrm{V}$ & Variance of a distribution representing model input $\mathrm{X}$ \\
\hline $\mathrm{v}$ & Sample variance for $n$-samples of model input X \\
\hline vs. & versus \\
\hline WAPDEG & Waste Package Degradation Model \\
\hline WF & Waste Form \\
\hline WIPP & Waste Isolation Pilot Plant \\
\hline WPOB & Waste Package Outer Barrier \\
\hline WP & Waste Package \\
\hline $\mathrm{X}$ & A variable representing one model input \\
\hline $\mathbf{x}$ & A reduced set representing important inputs to the TSPA model \\
\hline $\mathrm{X}_{\mathrm{i}}$ & $\begin{array}{l}\text { Vector of inputs to an approximate function } \mathrm{f}\left(\mathrm{x}_{\mathrm{i}}\right) \text { representing major } \\
\text { dependencies for the TSPA model }\end{array}$ \\
\hline$x_{i 1}, x_{i 2}$ & $\begin{array}{l}\text { Output values for a 2-point approximating probability mass function } \\
\text { (examples } x_{12} \text { and } x_{22} \text { are used) }\end{array}$ \\
\hline YM & Yucca Mountain \\
\hline YMP & $\begin{array}{l}\text { Yucca Mountain Site Characerization Project } \\
\text { years }\end{array}$ \\
\hline
\end{tabular}




\section{Objective and Scope}

The primary objective for the Model Validation Status Review was to perform a one-time evaluation of model validation associated with the analysis/model reports (AMRs) containing model input to total-system performance assessment (TSPA) for the Yucca Mountain site recommendation (SR). This review was performed in response to Corrective Action Request BSC-01-C-01 (Clark 2001, Krisha 2001) pursuant to Quality Assurance review findings of an adverse trend in model validation deficiency. The review findings in this report provide the following information which defines the extent of model validation deficiency and the corrective action needed:

- AMRs that contain or support models are identified, and conversely, for each model the supporting documentation is identified.

- The use for each model is determined based on whether the output is used directly for TSPA-SR, or for screening (exclusion) of features, events, and processes (FEPs), and the nature of the model output.

- Two approaches are used to evaluate the extent to which the validation for each model is compliant with AP-3.10Q (Analyses and Models). The approaches differ in regard to whether model validation is achieved within individual AMRs as originally intended, or whether model validation could be readily achieved by incorporating information from other sources.

- Recommendations are presented for changes to the AMRs, and additional model development activities or data collection, that will remedy model validation review findings, in support of licensing activities.

The Model Validation Status Review emphasized those AMRs that support TSPA-SR (CRWMS M\&O 2000bl and 2000bm). A series of workshops and teleconferences was held to discuss and integrate the review findings. The review encompassed 125 AMRs (Table 1) plus certain other supporting documents and data needed to assess model validity. The AMRs were grouped in 21 model areas representing the modeling of processes affecting the natural and engineered barriers, plus the TSPA model itself. Description of the model areas is provided in Section 3, and the documents reviewed are described in Section 4. The responsible manager for the Model Validation Status Review was the Chief Science Officer (CSO) for Bechtel-SAIC Co. (BSC). The team lead was assigned by the CSO. A total of 32 technical specialists were engaged to evaluate model validation status in the 21 model areas. The technical specialists were generally independent of the work reviewed, meeting technical qualifications as discussed in Section 5.

\subsection{Background on Model Validation for Site Characterization at Yucca Mountain}

\subsubsection{Model Validation - NUREG-1636 Definition}

The following information summarizes the approach to model validation that is taken in NUREG-1636 (Regulatory Perspectives on Model Validation in High-Level Radioactive Waste Management Programs: A Joint NRC/SKI White Paper; Eisenberg et al. 1999). This NUREG provided guidance for the authors of the AP-3.10Q Administrative Procedure. It is not binding on the YMP but is considered to be reliable guidance for how acceptable model validation can be achieved. The definition for model validation in NUREG-1636 is consistent with, but less 
prescriptive than the definition from AP-3.10Q discussed below. As stated in NUREG-1636 (Eisenberg et al. 1999; p. 25):

"The goal of validation is to obtain sufficient confidence, commensurate with the model's intended use, that the models are able to describe the behavior of interest in the real system. Confidence is gained in two ways: i) by examining the theoretical or scientific basis for the model to assure it is sufficient for the application of the model; and ii) by evaluating the application of scientific principles in the model to assure the application is appropriate, which can be accomplished by reviewing application of the principles in similar [analogous] circumstances."

To paraphrase, NUREG-1636 defines model validation to consist of confidence-building activities or information which show that performance assessment (and its constituent models) demonstrates a reasonable expectation that the respository system will comply with regulatory standards (applying the change from the definition of "reasonable assurance" as implemented in 66 FR 55732). A regulatory approach to validation requires an adequate description of the physical behavior of interest, for a given purpose. Thus the intended use is a key feature of model validation.

One approach to model validation involves comparison of model predictions to empirical information such as test data, or scientific data from other sources such as peer-reviewed literature. This approach is likely to be more effective when the predictions are generated prior to collecting the data. If a single data set is available for both model development and validation, then confidence-building may be achieved by using one part of the data set to model another. Theoretical analysis may be used in place of site-specific test data, if the approach is applicable and supported by empirical data from other sites or sources. Other avenues for confidencebuilding are available such as publication of model results in peer reviewed journals, presentation in public meetings attended by scientific peers, and formal peer review. Conservative bounding models may be used, but requirements for validation are similar because of the need for confidence in the bounds.

NUREG-1636 recommends that a model validation strategy should be established prior to model development if possible, and consist of a description of the confidence-building activities or information. Quantitative goals should be established so that model validation can be interpreted unequivocally. The overall objective of model validation documentation is to "...facilitate acceptance or rejection of models used, based on transparent and logical reasoning..." (Eisenberg et al. 1999, p.29).

\subsubsection{Model Validation - AP-3.10Q Procedural Definition}

The controlling procedure for AMRs (AP-3.10Q, Analyses and Models) requires that models be validated to an extent that depends on the intended use as stated in Section 5.3(a):

"The appropriate level of confidence for a model shall be determined based on the intended use of the model and the importance of the model for assessing postclosure system performance..."

Validation is then defined in Section 5.3.b: 
"...model validation shall consist of comparing analysis results against data acquired from the laboratory, field experiments, natural and man-made analog studies, or other relevant observations. The criteria used to evaluate the appropriateness and adequacy of the model for its intended use may be qualitative or quantitative but must be justified in the model documentation."

For validation of models for which no data from laboratory or field experiments, natural and man-made analog studies, or other relevant observations are available, the following additional methods for validation are provided in Section 5.3.c:

“1) Peer review (see AP-2.12Q, Peer Review) or review by international collaborations

2) Technical review through publication in the open literature

3) Review of model calibration parameters for reasonableness, or consistency in explanation of all relevant data

4) Comparison of analysis results with the results from alternative conceptual models including supporting information to establish basis for confidence in selected model

5) Calibration and corroboration within experimental data sets

6) Comparison of analysis results with data attained during Performance Confirmation studies."

This procedural definition is consistent with the Quality Assurance Requirements and Description (DOE 2000; Supplement III, Section III.2.6). It is based on NUREG-1636, and applicable to all Yucca Mountain Site Characterization Project model development activities. Accordingly, the procedural definition is used for implementing the Model Validation Status Review. The procedural definition still requires application of expert judgment in the determination that sufficient confidence has been obtained (or could be readily obtained) commensurate with the model's intended use. It is the need for expert judgment (by independent technical specialists) that has prompted this review.

\subsubsection{Previous Identification of Model Validation Deficiencies}

Review by the Office of Quality Assurance (OQA) for the Yucca Mountain Site Characterization Project determined that a significant fraction (more than half) of the models documented in AMRs supporting TSPA-SR failed to achieve validation using any of the approaches given above. In addition, OQA determined that some AMRs were designated as analyses but actually were models for which no validation was documented (Clark 2001). The Model Validation Status Review was performed to further define the extent of these conditions and to identify the needed corrective actions.

\subsection{Relationship of this Review to a Potential Future Site Recommendation}

The Model Validation Status Review was intended to support preparation of the Yucca Mountain Site Recommendation in these ways:

- Address a Corrective Action Request (Clark 2001). 
- Provide guidance for corrective actions to improve the models and model documentation.

The results for these two objectives are documented in this report. A team of technical specialists was convened to evaluate which of the SR-supporting technical work products contain models, and whether the work (as documented) complies with procedural requirements for model validation (AP-3.10Q). The recommendations describe further documentation, and in some cases additional testing, data collection, and model development, which would bring models into compliance with AP-3.10Q, in support of licensing activities. These recommendations are separate from, and have not been compared or integrated with, the current baseline plan for future technical work by the YMP. Because the impact reviews in this report document that the results of TSPA-SR are not significantly impacted by the recommendations for additional work, this additional work is not necessary to support a potential site suitability determination. 


\section{Quality Assurance}

This document has been prepared in accordance with AP-3.11Q, Technical Reports, and the Technical Work Plan for Model Validation Status Review (BSC 2001j). The technical work plan was developed in accordance with AP-2.21Q, Quality Determinations and Planning for Scientific, Engineering, and Regulatory Compliance Activities. Applicability of the QA program is documented in an Activity Evaluation per AP-2.21Q. The Activity Evaluation (BSC 2001j, Addendum B) has concluded that this document is quality-affecting and subject to the QA controls of the Quality Assurance Requirements and Description (DOE 2000).

\subsection{Electronic Information}

Results of an evaluation required by AP-SV.1Q (Control of the Electronic Management of Information) indicated that specific controls were required for data transfer (BSC 2001j, Addendum C). Accordingly, the following controls were implemented in the preparation of this report. Access to all computers used was password-protected. Access to data residing on network servers was controlled by assigned access privileges. All volumes on which data were stored were subject to periodic backup, and the backup media were labeled and stored. Backups were maintained until the information was accepted by the Records Processing Center.

All documents reviewed by this activity were distributed to the technical specialists on CD-ROM (Compact Disc Read Only Memory) media. Mastering and duplication of CD-ROMs were performed by BSC Document Control. The properties inherent to the CD-ROM media provided adequate control to protect information integrity and to ensure that the information was readily retrievable.

Transfer of model reviews from the technical specialists to the team lead for incorporation in the final report was by signed hard copy. Electronic files were also submitted, and the electronic files were verified against the hard copies (Hardin 2001c).

\subsection{Verification of Qualifications}

All the technical specialists who participated in the Model Validation Status Review met the requirements of a Senior Engineering Specialist/Senior Science Specialist (Grade 27 or higher) in the BSC organization (Hardin 2001b). The position description (BSC Code \#175A/117A) includes specific requirements for academic attainment in a related discipline, and years of relevant employment experience. These qualifications were verified in accordance with AP-2.2Q (Establishment and Verification of Required Education and Experience of Personnel). Evidence of verification is available for inspection.

\subsection{Training Requirements}

A job function category was created for Technical Specialist/Reviewer, and assigned specific training requirements in accordance with AP-2.1Q (Indoctrination and Training of Personnel). A training program suitable for presentation to personnel who would be working at home-office locations, was developed by the BSC Training Department. This consisted of a standard training course: Introduction to Quality Assurance (LPGET00-013 Rev. 01, BSC 2001l), and an orientation briefing developed for this activity entitled: Technical Specialist/Reviewer Briefing (BRETEC01-030 Rev. 00, BSC 2001m). A total of 32 technical specialists received this training. 


\subsection{QA Records}

The QA-records produced by this activity include the following:

- Training summary memo - Description of the selection, training, qualification verification, and instruction for the technical specialists (Hardin 2001b).

- Final workshop memo - Summary of the 3-day final workshop in Las Vegas (Hardin 2001c).

- Team review draft report and technical specialist comments - The team review draft of the report was circulated for informal review and comment by the technical specialists (Hardin 2001c)

- Informal comments on the team review draft, received electronically from the technical specialists (Hardin 2001c)

In addition, training and qualification verification records are the responsibility of the Training and Human Resources Departments:

- Training assignment matrix

- Training attendance records

- Experience and education verification records.

Finally, records generated during checking, review, and approval of the final report are submitted as a records package when those activities are complete:

- Check copy of final report (consolidated first draft with reviewer's comments)

- Backcheck copy draft of report

- Originator's and checker's checklists

- Formal review draft of report

- Review record and concurrence

- Concurrence draft/final checking draft of report. 


\section{Review Methodology and Criteria}

The review methodology and criteria were documented in work plan: Technical Work Plan for Model Validation Status Review (BSC 2001j) prepared in accordance with AP-2.21Q (Quality Determinations and Planning for Scientific, Engineering, and Regulatory Compliance Activities). The following sections describe the 21 model areas selected to group the 125 AMRs and other documents that were evaluated. The sections then describe the roles of the technical specialists and model-area leads who constituted the review team, and describes the methodology for the review. There is also a discussion of the terms "model" and "analysis," with additional guidance that was used in the review activity to discern when a validated model is appropriate.

\subsection{Definition of Model Areas}

Model areas were selected to group the AMRs and other documents in a manner that is aligned with the technical specialties available. The approach followed that used in a previous report (YMP 2001) to organize models for a review of uncertainties that impact TSPA dose assessment. A list of model areas was extracted from Figures 1-1, 2-1, 3-1, 4-1, 5-1, 6-1, 7-1, 8-1 through 8$5,9-1,10-1,11-1,12-1,13-1,14-1,15-1,16-1$, and 17-1 of that report, with modifications. The resulting list is shown in Table 1 (List of Model Areas and Associated AMRs and Other Reports for Status Review). This approach encompasses all the AMRs, while providing enough individual categories to limit the number of reports in each model area. Also, the model areas are defined so as to permit small groups consisting of one to four specialists to adequately cover the technical material in each area. In general the results of the model validation review, particularly the identification of models, did not prove sensitive to the approach taken for categorizing the AMRs. This is because the same AMRs were reviewed in multiple model areas, by different technical specialists, where appropriate.

\subsection{Roles of Technical Specialists and Model Area Leads}

The team lead selected 32 technical specialists, as described in Section 5, Selection of Technical Specialists and Model Area Leads, to evaluate model validation status. The role of the technical specialists was to identify and describe the models represented by the documentation, and to evaluate their validation status in accordance with AP-3.10Q, using a binning process. One technical specialist was assigned as the lead for each of the 21 model areas, and multiple specialists were generally involved in review of each model area. This organization promoted integration between model areas because specialists had overlapping assignments.

In addition, another group of participants called model-area leads were involved in the activity. These were individuals from Project staff who were responsible for producing AMRs in the model areas, and who knew the models. The role of the model-area leads was to interact with the technical specialists at the final workshop, and to assist the team lead in formulating recommendations in response to the review comments.

\subsection{Methodology for Review}

The Model Validation Status Review emphasized those AMRs and supporting documentation that supported TSPA-SR (CRWMS M\&O 2000bl and 2000bm). A total of 125 AMRs were reviewed, plus other supporting data, calculation reports, and technical reports. The selection of 
documents for review and the assignment of the documents to model areas are discussed in Section 4, Documents Included in Review.

A 1-day kickoff workshop was held for QA training, and briefings on TSPA and model validation. The relevant Corrective Action Request (Clark 2001) was discussed. This training was repeated three times to accommodate all technical specialists (see Table 2).

A review period was scheduled during which the technical specialists received the documents in electronic form, and performed the reviews while working at their home-office locations. The team lead conducted teleconferences during this period to monitor progress, and the lead specialist also coordinated activities among the assigned reviewers, for each model area.

The following series of steps was used to review each model area:

A. AMRs in each model area, including analyses, that contain models were identified. Also, for each model any other documentation that supported validation (i.e. entire AMRs, parts of AMRs, calculation reports, technical reports, or other documents) was also identified.

B. The intended use for each model was determined as a starting point for evaluating model validation. Assessments included whether each model supports a Principal Factor, and whether it was used directly in TSPA-SR. The Repository Safety Strategy (CRWMS M\&O 2001o, Volume 1, Sections 1 through 4) was used to define Principal Factors. Sources used to determine whether a model was used for TSPA-SR, included the TSPA$S R$ model report (see model area $U$ in Table 1), and direct contact with the AMR originators.

C. A unique, short text description was developed for each model. A short text description of the intended use, including a description of the model output, and identification of the other models that use this as input, was also developed.

D. Each model was evaluated to determine whether validation (as defined in AP-3.10Q) was achieved in a single, principal AMR. The entire content of the AMR was considered, not just the model validation subsections. Where model validation was deemed by this criterion to be adequate for its intended use, the model was assigned to "Bin 1."

E. Each model not assigned to "Bin 1" was evaluated to determine whether existing documentation (possibly including other AMRs, other reports, data, publications, etc.) provided adequate confidence to support model validation for its intended use as defined in AP-3.10Q. Where model validation was deemed by this criterion to be adequate for its intended use, the model was assigned to "Bin 2."

F. Models that were not assigned to "Bin 1" or "Bin 2" were assigned to "Bin 3."

In addition, the technical specialists identified some models for which improvements to the documentation (e.g., revisions to one or more AMRs) are needed for clarity of model validation arguments. These comments are provided in Section 6, Review Findings and Recommendations.

A final workshop was conducted at the conclusion of the review period, for presentations by the lead technical specialist for each model area. Each lead specialist presented the models that were identified and described in Steps A through $\mathrm{C}$ above, and the binning described in Steps D through F. At the conclusion of the workshop the technical specialists submitted their findings in writing, on signed forms. 
It is noted that revised findings for Model J.8 (see Section 6.10.10) was received from the technical specialist reviewers assigned to this model area after the model-binning and impact reviews were completed. This revised findings recommend that Model J.8 be considered an analysis instead of a model, in which case validation would not be required. However, this input was not received in time to be incorporated in the findings of the Model Validation Status Review (MVSR) as represented in this report.

After the final workshop, the model-area leads formulated written assessments of the impact of Bin-3 findings on the results of TSPA-SR, and recommendations for additional work needed to prepare these models for a potential License Application (LA). These submittals by the technical specialists and the model-area leads formed the basis for Section 6, Review Findings and Recommendations, and Section 7, Summary and Conclusions, of this report.

A draft report was then prepared by the team lead and distributed electronically to the technical specialists and model-area leads for informal review and comment. Comments were incorporated in the text of the report, or appended to the report, as appropriate. The resulting version was Rev. 00A, submitted to checking in accordance with AP-3.11Q (Technical Reports). The check copy was revised to produce Rev. 00B, which was distributed for Project-wide review in accordance with AP-2.14Q (Review of Technical Products and Data). After reviewer-concurrence the final version was approved by the BSC Chief Science Officer.

\subsection{Model vs. Analysis}

Procedure AP-3.10Q gives overlapping definitions for the terms "model" and "analysis," and further clarification was developed for uniform and accurate identification of models and analyses. This is important because models require validation per the procedure, whereas analyses may not (depending on whether they contain models). The goal was clear definition for when validation is required (model) and when it is not (simple analysis). The terms are defined in the procedure (AP-3.10Q) as follows:

Model - A representation of a process, system, or phenomenon, along with any hypotheses required to describe the process or system or explain the phenomenon, often mathematically. A model may be used as an input to an analysis or calculation. Various qualifiers may be added to the term model to indicate the intended use of the model. For example, a process model is a model of a particular process, an abstraction model is a simplified representation of a process model generally created to facilitate its incorporation in a system model, and a system model combines several process models and/or abstraction models to represent an integrated system.

Analysis - 1) The process of translating design inputs into the design of systems, structures, and components; 2) the process of defining, investigating, validating, reviewing, and documenting the study or evaluation of the performance of a natural or engineered system or a component of that system; or 3) a scientific investigation that quantitatively or qualitatively describes, interprets, explains, or models a natural system, process, or phenomenon. An analysis can include one or more models and/or one or more calculations, as well as interpretation of the results of models and calculations. 
Difficulty arises in determining whether a report contains a model or analysis, for example, whether it is a "representation... along with hypotheses required to describe the process..." or a "scientific investigation that....describes, interprets, [or] explains..." Accordingly, the following additional information and criteria were developed.

\subsubsection{Historical Perspective}

Procedure AP-3.10Q was developed initially in 1999 as part of a campaign to re-engineer the processes used for technical work on the Yucca Mountain Project. One goal of that campaign was to reduce the total number of procedures, so a procedure for engineering design analysis was modified to also control model development by the science program.

Design analyses are used to develop configurations and operating controls for engineered systems, structures, or components, and to show that they meet design requirements. Modeling is used to predict performance of the natural and engineered barriers in support of the Total System Performance Assessment (TSPA). Validation is required for models but not for simple analyses that do not contain models. However, differences between models and analyses were not clearly defined, so the applicability of model validation was not clearly defined, and model validation was not achieved uniformly.

\subsubsection{Criteria for Discerning Models vs. Analyses}

Identifying models means discriminating models vs. analyses. In addition to the procedure definitions quoted above, the following additional considerations were developed for discriminating models vs. analyses, and distributed to the technical specialists (Hardin 2001a):

- Sensitivity to waste isolation performance - If the TSPA results are relatively sensitive to the output of an analysis (compared with other inputs), then justification for the methodology, assumptions, etc., used is especially important, and the analysis may be more appropriately documented as a model.

- Need for consideration of alternatives - Where there is a clear choice among available methods a simple analysis may suffice. As examples consider the use of standard engineering methods for structural analysis, or the use of standard statistical methods for analyzing site characterization data. However, where alternative analysis methods exist and selection has the potential to affect TSPA, documentation as a model may provide needed validation, i.e. appropriate confidence in the output commensurate with its use for TSPA (see Hardin 2001a).

- Complexity of mathematical operation - Complex or extensive mathematical operations typically involve assumptions or hypotheses. If the output has the potential to affect TSPA, the appropriate degree of justification, i.e. validation, may be achieved by documenting the operations as a model.

- Performance modeling vs. design analysis - Simple analyses may suffice to compare calculated results with design requirements. By contrast, models are typically used to predict the long-term performance of natural or engineered components as they degrade and no longer serve their designed functions.

These aspects may occur separately or in combination, and to the extent that they apply, validated models will generally provide more confidence than simple analyses. For each model area consisting of analysis/model reports (AMRs), the review team identified the models that 
require validation and reached this finding independently of the original designation by the report originator (as a model or analysis, or both).

Any decision to document technical work as an analysis or a model is ultimately based on judgment as to the confidence needed for the intended use. Model documentation (with validation) may provide additional confidence relative to analysis documentation. No lack of validity or quality is necessarily implied where this model validation status review recommends that existing models be documented as analyses instead. These are recommendations only, and not final determinations as to the future status of such documentation. 


\section{INTENTIONALLY LEFT BLANK}




\section{Documents Included in Review}

The most recent versions of the AMRs (available electronically from Document Control as of June 11, 2001) were evaluated. This means that for a few AMRs, a more recent version was reviewed than was cited in the TSPA-SR documentation (CRWMS M\&O 2000bl and 2000bm). The document version numbers constituting the initial set for this review are described in Section 4.1, Initial Set of Documents, of this report. Additional documents and data items were added at the request of the review team as discussed in Section 4.2, Justification for Additional Documentation, of this report.

\subsection{Initial Set of Documents}

The AMRs selected for review are listed in Table 1, where they are grouped by model area as defined in Section 3.1. This review list of documents includes the AMRs available from BSC Document Control on June 11, 2001. The review list includes all of the AMRs that were reviewed by QA prior to issuance of the Corrective Action Request discussed previously (Clark 2001).

The most recent versions of the AMRs (as of June 11, 2001) were reviewed (listed in Table 1). For some AMRs this meant that a more recent version was reviewed than was cited in the TSPASR documentation (CRWMS M\&O 2000bl and 2000bm) or reviewed by QA (Clark 2001).

The review set included some AMRs, or parts of them, that were not used for TSPA-SR but were generally developed for screening features, events, and processes (FEPs), i.e., determination as to whether FEPs are included or excluded from explicit representation in the TSPA-SR (see CRWMS M\&O 2000bm, Appendix B for a discussion of FEPs and FEP screening). The validity of FEPs screening arguments was not considered in the scope of the MVSR. The FEPs screening AMRs were included among the documents for review only to support assessment of the intended use for models documented in other AMRs.

The initial review set of documents was issued by Document Control on a CD-ROM, which was submitted to the Records Information System (Hardin 2001b).

\subsection{Justification for Additional Documentation}

Documents and data requested by the technical specialists as supplementary review material are listed in Table 3 (List of Supplemental Review Materials Requested by, and Distributed to, the Technical Specialists). These represent additional information used to evaluate the intended use of models or the basis for validation. These documents and data were obtained from the BSC Technical Data Management System (for DTNs) or from the Records Information System (for records assigned accession numbers).

The supplementary review material was issued by Document Control on a CD-ROM, which was submitted to the Records Processing Center (Hardin 2001b). 
INTENTIONALLY LEFT BLANK 


\section{Selection of Team Lead, Technical Specialists, and Model Area Leads}

The team lead was selected by Michael D. Voegele, Chief Science Officer for BSC, to be Ernest L. Hardin (Voegele 2001). Dr. Hardin is a geoscientist with over $16 \mathrm{yr}$ technical and managerial work experience for the Yucca Mountain Site Characterization Project, and international waste disposal projects. He holds a Ph.D. in Hydrology from the University of Arizona, a Master's in Earth Science from the Massachusetts Institute of Technology, and a B.S. in Applied Geophysics from the University of Utah. He is currently assigned as Lead for Technical Issues in the Chief Science Office. He can be contacted by telephone (702/295-3963) or email (ernest_hardin@ymp.gov).

\subsection{Technical Specialists}

A total of 32 technical specialists were assigned to the 21 model areas as shown in Table 4 (Assignment of Technical Specialists to Model Areas). The table shows the lead, and other specialists assigned to each area.

The technical specialists were independent, having not been involved with originating, checking, reviewing, or approving any of the AMRs or other documents reviewed. As required in the plan for the Model Validation Status Review (MVSR) (BSC 2001j, Addendum A) the technical specialists were selected to emphasize independence, although complete independence was not necessary.

Independence was a potential concern only in the selection of Mr. John Holmes as lead technical specialist for the Waste Form model area, because of his affiliation with Sandia National Laboratories. It was determined that $\mathrm{Mr}$. Holmes was retired from Sandia and would serve as a consultant. Also, his previous involvement with Yucca Mountain pre-dated the development of AMRs in this model area. Further, he is highly qualified, being former manager of waste-form investigations for the Waste Isolation Pilot Plant.

The following biographical information was abstracted from the resumes of the technical specialists. Information on education and work experience was independently verified by BSC Human Resources; other information that may be included has not been independently verified.

\section{Michael T. Anderson}

Mr. Anderson has been with Bechtel-BWXT/INEEL since 1989, and is experienced in the development of nondestructive examination techniques for power generating plants, processing facilities, and specialized applications. In addition, he is the author of numerous scientific publications related to his work. Previous work experience includes: Arizona Nuclear Power Company, Senior Engineer responsible for the development and coordination of programs for 1270 MW-PWR nuclear generating plants; and Northern States Power Company, Materials and Special Processes Engineer responsible for coordination of programs at $540 \mathrm{MW}$-PWR nuclear generating plants, and Principal Project Coordinator for steam generator tubing examinations at Prairie Island Nuclear Generating Plant. Mr. Anderson's academic background includes an M.S. in Metallurgy from the University of Idaho, and a B.S. in Nondestructive Testing Science from Athens State College. In the Model Validation Status Review, he supported review in the Waste Package/Drip Shield Degradation modeling area. He can be contacted by telephone, (208) 5268780 ; or by e-mail, mta2@inel.gov. 


\section{Prem M. Attanayake}

Dr. Attanayake has been Chief Hydrogeologist with Bechtel Corporation in San Franciso, California, since 1994. His previous Bechtel experience includes: Manager, Engineering Geology Group; Supervising Geologist/Hydrogeologist; and Senior Geologist/Hydrogeologist. He has worked at Lawrence Berkeley Laboratory as a Research Scientist; University of Windsor and Technical University of Nova Scotia as a Research Assistant; and Geoscience Consultants, Ibadan, Nigeria, as a Consulting Geologist. He has authored numerous scientific publications on geohydrology topics. Dr. Attanayake's academic background includes: a Ph.D. in Civil Engineering, Technical University of Nova Scotia; a Master of Applied Science in Geological Engineering, University of Windsor, and an M.S. in Petroleum Geology/Reservoir Engineering, Friendship University, Moscow. Additional course work was taken at University of California, Berkeley; University of California, Davis; Princeton University; and University of Waterloo. In the Model Validation Status Review, Dr. Attanayake was assigned as the lead technical specialist in the Saturated Zone (SZ) Flow modeling area, and also supported review in the Unsaturated Zone (UZ) Flow model area. He can be contacted by telephone, (415) 768-0454; or by e-mail, pmattana@bechtel.com.

\section{Randy L. Bassett}

Dr. Bassett currently serves as President of Geochemical Technologies Corporation, Adjunct Professor of the Department of Hydrology and Water Resources at the University of Arizona, and Faculty Director of the Isotope Laboratory in that department. His areas of expertise include brine geochemistry, contaminant chemistry, isotope geochemistry, chemical basis for radioactive waste disposal, and flow and transport modeling. Dr. Bassett holds a Ph.D. in Environmental Geochemistry from Stanford University, and also a B.S in Geology, and an M.S. in Geochemistry. He has authored numerous scientific publications on geochemistry topics, in addition to participating on advisory committees and serving as a lecturer. He is currently an associate editor for Ground Water and Applied Geochemistry. In the Model Validation Status Review, Dr. Basset supported reviews in the Mountain-Scale/Near-Field THC model area and the In-Drift Chemistry modeling area. He can be contacted by telephone, (303) 423-8187; or by e-mail, bassett@geochemtech.com.

\section{Jon Berkoe}

Mr. Berkoe has $17 \mathrm{yr}$ of experience as a specialist in fluid dynamics and heat transfer for the petroleum and chemical, aerospace, environmental, metallurgical, fossil and nuclear power, and transportation industries. $\mathrm{He}$ is currently the Principal Engineer and Manager of the Computational Fluid Dynamics and Advanced Simulation Group, Bechtel Corporation, San Francisco, California. He has contributed to more than 40 Bechtel projects since 1992 including: solvent extraction settler design, electro-winning cell ventilation system, copper smelter emissions control, and building wind effects and structural force predictions. He holds M.S. and B.S. degrees in Mechanical Engineering. Mr. Berkoe is also recognized for his contributions to conference proceedings, journal writings, and a patent concerning ventilation for electrolytic cell. In the Model Validation Status Review, he supported review in the Mountain-Scale TH and the EBS Moisture Distribution and TH modeling areas. He can be contacted by telephone, (415) 768-2149; or by e-mail, jberkoe@bechtel.com. 


\section{Kenneth T.Bogen}

Dr. Bogen has worked as an environmental health scientist in Health \& Ecological Assessment Division, at Lawrence Livermore National Laboratory, University of California, Livermore, California, since 1986. Previous experience included being a Science Policy Analyst, U.S. Library of Congress, Congressional Research Service, Science Policy Research Division. His academic background includes: A.B. in Biology, Princeton University; M.A., Science, Technology, and Public Policy, George Washington University; and an M.P.H. and a Dr.P.H., Environmental Health Science, University of California, Berkeley. His research focus includes cancer risk assessment, regulatory toxicology, biological modeling of carcinogenesis, and physiologically based pharmacokinetic models for volatile organic compounds. Dr. Bogen served in 1995 as President of the Northern California Chapter of the Society for Risk Analysis; served in 1992-1994 on the National Research Council committee that issued the NRC report, Science and Judgment in Risk Assessment (1994); and in 2000-2001 chaired the U.S. CPSC Chronic Hazards Advisory Panel on Diisononyl Pthalate (DINP). Dr. Bogen also has participated in other scientific peer review panels, holds a number of patents, and has authored many scientific publications. In the Model Validation Status Review, he was assigned as the lead technical specialist in the Biosphere model area, and also supported review in the Performance Assessment modeling area. He can be contacted by telephone, (925) 422-0902; or by e-mail, bogen@LLNL.gov.

\section{Jan L. Bostelman}

Ms. Bostelman's technical background includes more than $21 \mathrm{yr}$ of experience in safety and material assessments, their application to nuclear reactor systems and non-nuclear process facilities, and mathematical and computer modeling. She has been employed by Innovative Technology Solutions Corporation since 1996, with responsibilities that include commercial nuclear safety analyses and fuel management optimization. She was previously with Omaha Public Power District Fort Calhoun Nuclear Station as a supervisor in the Reactor Performance area. She holds a B.S. in Chemical Engineering, and has finished an M.S. in Mechanical Engineering. She has done additional graduate work in scanning electron microscopy, high-level radioactive waste processing and vitrification, and bioremediation/biochemical engineering. She is a licensed Professional Metallurgical Engineer. In addition, she has authored numerous publications and presentations. In the Model Validation Status Review, Ms. Bostelman supported reviews in the Waste Package/Drip Shield Degradation model area. She can be contacted by telephone, (402) 488-0991; or by e-mail, jan_bostelman@ymp.gov or rosa@ITSC.com.

\section{Gary D. Callahan}

Dr. Callahan has more than $25 \mathrm{yr}$ experience in the development and application of techniques used to evaluate unique geomechanics problems, much of this time with RE/SPEC, Inc. in Rapid City, South Dakota. He has directed numerous projects that included material properties testing and numerical analyses for commercial clients and in support of the DOE Office of Civilian Radioactive Waste Management, and the Waste Isolation Pilot Plant. Dr. Callahan's areas of technical expertise include constitutive model and computer code development; mechanical, thermal, and structural analyses; waste management programs and environmental site characterization; materials testing; and rock mechanics. His academic background includes a B.S. and an M.S. in Mechanical Engineering, and a Ph.D. in Geo-Engineering. In 1999, he received the Applied Research Award for significant and original research from the U.S. 
National Committee for Rock Mechanics. He has authored numerous publications related to these projects. In the Model Validation Status Review, Dr. Callahan supported review in the PA Modeling and Disruptive Events-Igneous Disruption modeling areas. He can be contacted by telephone, (605) 394-6400; or by e-mail, gdcalla@respec.com.

\section{Kerry L. DeVries}

Mr. DeVries is currently a Project Engineer for Geomechanics, RE/SPEC, Inc., Rapid City, South Dakota. His primary areas of technical expertise are in thermomechanical finite element modeling, in situ rock mechanics tests, constitutive model development, and rock mechanics particularly in salt formations. Since $1987 \mathrm{Mr}$. DeVries has performed many statistical and numerical analyses to support prediction of the structural response of mines and underground storage facilities. Mr. DeVries is the author of numerous publications and reports related to this work. His academic background includes a B.S. in Mechanical Engineering from South Dakota State University, and an M.S. in Mechanical Engineering from the South Dakota School of Mines and Technology. In the Model Validation Status Review, Mr. DeVries was assigned as the lead technical specialist in the Mountain-Scale/Near-Field THM model area, and also supported review in the EBS Degradation modeling area. He can be contacted by telephone, (605) 3946400; or by e-mail, kldevries@respec.com.

\section{James N. Follin}

Dr. Follin has been with Bechtel-Bettis Laboratory since 1993, working in submarine propulsion systems, environmental affairs, and quality assurance. He is currently on special assignment for repository topics, including thermal, criticality, spent fuel transportation, and fuel performance analyses. Dr. Follin has a B.A. in Physics from Johns Hopkins University, an M.S. in Physics from Texas A\&M University, and a Ph.D. in Engineering and Public Policy from CarnegieMellon University. In the Model Validation Status Review, Dr. Follin supported review in the Waste Form Degradation modeling area. He can be contacted by telephone, (412) 476-5934; or by e-mail, follinjn@bettis.gov or follin@adelphia.net.

\section{Robert J. Hanrahan Jr.}

Dr. Hanrahan is currently with Los Alamos National Laboratory as the Team Leader in Electrochemistry and Corrosion, in the Metallurgy Group. His research interests include high temperature oxidation and corrosion of metals and ceramics; corrosion of actinides; beryllium alloy development; and synthesis, processing and fabrication of nuclear fuels. Dr Hanrahan has a B.S. and an M.S. in Nuclear Engineering, and a Ph.D. in Materials Science and Engineering, from the University of Florida. He has authored numerous publications related to his work at Los Alamos. In the model Validation Status Review, Dr. Hanrahan supported review in the Waste Package/Drip Shield Degradation and Waste Form Degradation modeling areas. He can be contacted by telephone, (505) 667-9560; or by e-mail, hanrahan@lanl.gov.

\section{John T. Holmes}

Mr. Holmes recently retired from Sandia National Laboratories, where he worked since 1976. The last $10 \mathrm{yr}$ of this period, he managed the technical staff who developed chemistry and transport technology required to certify the safety of the Waste Isolation Pilot Plant. His 39-year career also encompasses work in solar thermal energy technology and environment, safety and health. The first $15 \mathrm{yr}$ of Mr. Holmes' professional career were spent at Argonne National Laboratory where his responsibilities spanned a broad range of nuclear power-reactor 
technology. He has been the recipient of numerous awards, including R\&D-100 Awards for a hydrogen detection system that alarms water-to-sodium leaks in sodium-heated steam generators for liquid-metal-cooled nuclear power reactors, and solar detoxification of hazardous organic materials in ground water. Mr. Holmes has a B.S. in Chemical Engineering from the University of Wisconsin, and an M.S. in Chemical Engineering from the University of California at Berkeley. He has completed extensive additional training in management, and environment, safety and health programs. Mr. Holmes is the author of approximately 70 publications in the open literature, government reports, and published abstracts. He holds six chemical process and process-monitoring patents. In the Model Validation Status Review, Mr. Holmes was assigned as the lead technical specialist in the Waste Form Degradation model area. He can be contacted by telephone, (505) 292-0898; or by e-mail, holmesjm@lobo.net.

\section{Kathryn O. Johnson}

Dr. Johnson has more than $20 \mathrm{yr}$ experience in environmental consulting, specializing in geochemistry, geohydrology, and contaminant fate and transport. Her experience includes geochemical modeling, data collection and analysis, compliance activities, negotiation of investigative and remediation strategies with the EPA and State Agencies, and expert testimony. She is currently the Owner/Principal of MATRIX Consulting Group, started in 1998. From 1990 to 1998, Dr. Johnson was the Owner/Principal of Johnson Environmental Concepts, and previously spent $4 \mathrm{yr}$ with Morrison Knudsen Environmental Services. She has a B.S. in Chemistry and Mathematics from Black Hills State College, an M.S. in Chemistry from Iowa State University, and a Ph.D. in Geology from the South Dakota School of Mines and Technology. Dr. Johnson has also held several appointments, among them a gubernatorial appointment to the South Dakota Board of Minerals and Environment; and an appointment by Senator Daschle to the Congressional Commission on the Advancement of Women and Minorities in Science, Engineering and Technology. She is the author of numerous publications and presentations. In the Model Validation Status Review Dr. Johnson was assigned as the lead technical specialist in the SZ Transport modeling area, and also supported review in the In-Drift Chemistry model area. She can be contacted by telephone, (605) 343-3534, ext. 212; or by email, kjohnson@matrixcgi.com.

\section{David W. Layton}

Dr. Layton has been the Division Leader, Health and Ecological Assessment division, Lawrence Livermore National Laboratory since 1996, although he has been with the group in various positions since 1975. He has a B.A. in Earth Science and a Ph.D. in Water Resources Administration. His professional activities include: Chairman of the Working Group on Exposure Assessment, International Commission for Protection Against Environmental Mutagens and Carcinogens, 1990-1993; member of the U.S. Department of Energy Peer-Review Panel on Low-Level Waste Disposal Sites, 1988-1997; and member of the Editorial Advisory Board, Journal of Soil Contamination, Association for the Environmental Health of Soils, 1996present. In the Model Validation Status Review, Dr. Layton supported review in the Biosphere modeling area. He can be contacted by telephone, (925) 422-0918; or by e-mail, laytonl@1lnl.gov.

\section{Daniel Glenn Levitt}

Dr. Levitt has been a Principal Hydrologist with Bechtel Nevada since 1996. Previously, he was a Senior Hydrologist for Reynolds Electrical \& Engineering Co. (REECo) Inc., technical lead for 
environmental monitoring of radioactive waste management sites at the Nevada Test Site, and technical lead for the Nevada Test Site groundwater monitoring program. He has been responsible for groundwater, vadose zone, meteorology, air, radiation exposure, and biota monitoring programs for Area 3 and Area 5 Radioactive Waste Management Sites. Dr. Levitt has a B.A. in Geology from Colorado College, and an M.S. and Ph.D. in Soil Science from the University of Arizona. He is the author of numerous publications and presentations. In the Model Validation Status Review, Dr. Levitt was assigned as the lead technical specialist in the Infiltration model area, and also supported review in the Ambient/Thermal Drift Seepage model area. He can be contacted by telephone, (702) 295-7343; or by e-mail, levittdg@nv.doe.gov.

\section{Richard C. Lee}

Dr. Lee has conducted a broad range of geophysical and seismological investigations during the last $20 \mathrm{yr}$, including seismic structure, earthquake source characterization, and hazard assessments for a variety of tectonic environments. He has field and analytical experience with geophysical site characterization and seismic site response for critical facilities; and regulatory and technical management experience involving large geotechnical databases and geographic information systems. Dr. Lee has been with the Savannah River Site since 1991. His academic background includes a B.S. in Physics from California State University/Fresno, and an M.A. and Ph.D. in Geophysics from the University of California at Berkeley. In addition to many projectspecific reports and technical abstracts, he has written numerous publications. In the Model Validation Status Review, Dr. Lee supported review in the Seismic Hazard model area. He can be contacted by telephone, (803) 952-6538; or by e-mail, rxlee@bechtel.com.

\section{Joe J. Litehiser}

Dr. Litehiser is Chief Seismologist for Geological Services, Bechtel Corporation, San Francisco, California. Much of his professional career has been conducting site characterization investigations for earthquake hazard evaluation for low-level and high-level nuclear waste disposal, nuclear power plants, and a variety of civil projects including dams, pipelines, transportation corridors, mining projects, petroleum projects, industrial facilities, and fossilfueled power plants. His work currently includes oversight of geophysical investigation for foundation studies, remote sensing, and blast monitoring. Since 1974 he has supervised all seismological investigations and analyses for Bechtel throughout the United States and overseas. Dr. Litehiser is Secretary of the Seismological Society of America and a member of the Earthquake Engineering Research Institute. He is Chairman of ANS 2.7, a committee charged with maintaining the nuclear industry standard for characterization of neotectonic features. Dr. Litehiser has written numerous reports for Bechtel projects, and has authored or co-authored a number of papers in the open scientific literature. He has an A.B. in Geology from Indiana University, and an M.A. in Geophysics and Ph.D. in Seismology from the University of California at Berkeley. In the Model Validation Status Review, Dr. Litehiser was assigned as the lead specialist in the Seismic Hazard model area. He can be contacted by telephone, (415) 768-7145; or by e-mail, jjlitehi@bechtel.com.

\section{Swen O. Magnuson}

Mr. Magnuson is an Advisory Scientist in Geosciences with Bechtel BWXT Idaho. His responsibilities include development of models for use in simulation of buried hazardous and radioactive wastes; interaction with regulatory agency personnel; contributing to field characterization studies; and direction of supporting personnel assisting in modeling studies. 
Previously, he was Staff Scientist with Integrated Earth Science/Geotechnologies, Lockheed Idaho Technologies, and Senior Scientist with the Subsurface and Environmental Modeling Unit, EG\&G Idaho. Mr. Magnuson has a B.S. in Geological Engineering from the Montana College of Mineral Science and Technology, and an M.S. in Hydrology from the New Mexico Institute of Mining and Technology. He received the Best Paper Award, American Nuclear Society, in 1989 at the Annual Meeting in Atlanta, Georgia: "Modeling Contaminant Migration from a MixedWaste disposal Site: Studies of Controlling Factors and Processes." Additionally, he is the author of numerous papers, reports, and presentations. In the Model Validation Status Review, Mr. Magnuson was assigned as the lead technical specialist in the UZ Transport modeling area, and also supported review in the UZ Flow modeling area. He can be contacted by telephone, (208) 526-8618; or by e-mail,smm@inel.gov.

\section{Ronald E. Mizia}

Mr. Mizia is an Engineering Fellow in the Nuclear Energy Systems Engineering Division at the Idaho National Engineering and Environmental Laboratory (INEEL), currently working with Bechtel BWXT Idaho. He has more than $20 \mathrm{yr}$ experience in materials, metallurgical, and corrosion engineering, in the areas of stainless and nickel-based alloy development and testing, corrosion testing of metallic and nonmetallic materials, welding, facility and component design, structural integrity, failure analysis, lifetime prediction, and nondestructive examination. Much of this work has been directed to handling and disposal of acidic, high-level radioactive wastes. His most recent work involves characterization and lifetime prediction for materials used in storage canisters for DOE spent fuel, and development of gadolinium-containing stainless steel and nickel-based alloys to be used as fixed neutron absorbers in these canisters. Mr. Mizia has a B.S. and an M.S. in Mechanical Engineering from the Michigan Technological University. He has authored numerous publications and is co-inventor on several patents. In the Model Validation Status Review, Mr. Mizia was assigned as lead specialist in the Waste Package/Drip Shield Degradation model area. He can be contacted by telephone, (208) 526-3352; or by e-mail, rma@inel.gov.

\section{John D. Osnes}

Dr. Osnes is currently Manager of Geomechanics and Resident Consultant for RE/SPEC, Inc. in Rapid City, South Dakota. He has more than 20 yr of experience in geotechnical engineering, primarily in the areas of rock mechanics and subsurface flow. His primary expertise is in geotechnical engineering, environmental compliance, engineering software development, and probabilistic and statistical methods. Since 1977 he has developed and used analytical and numerical models to analyze problems involving fluid flow, heat transfer, and mechanical behavior in geological materials. His project experience includes surface subsidence and slope stability analyses, groundwater transport modeling, and thermal and structural analysis of underground excavations for mining, waste disposal, and for hydrocarbon storage. Dr. Osnes' academic background includes a B.S. in Mechanical Engineering from the South Dakota School of Mines and Technology, and an M.S. and Ph.D. in Civil Engineering from the University of California at Berkeley. He has authored numerous publications and reports throughout his career. In the Model Validation Status Review Dr. Osnes was assigned as the lead specialist in the EBS Degradation modeling area, and also supported review of the Integrated Site Model and EBS Radionuclide Transport modeling areas. He can be contacted by telephone, (605) 394-6400; or by e-mail, jdosnes@respec.com. 


\section{Carl D. Palmer}

Dr. Palmer is employed with Bechtel-BWXT/INEEL as an Advisory Scientist, and has $20 \mathrm{yr}$ experience conducting research on aqueous geochemistry, solute transport, and hydrogeology. This includes identification of phases affecting solute transport, measurement of thermodynamic properties of chromium-enriched phases at waste sites, rates of redox transformations, installation and monitoring of wells in clay tills, heat transport in porous media, and bioavailability of cesium in the rhizosphere. In addition, he has $15 \mathrm{yr}$ experience teaching aqueous geochemistry, subsurface hydrology, and groundwater modeling. Dr. Palmer's academic background includes a B.S. in Mathematics, a B.S. in Geology, an M.S. in Geology, all from the Pennsylvania State University. In addition he holds a Ph.D. in Hydrogeology from the University of Waterloo. He is the author of numerous publications, reports, and presentations. In the Model Validation Status Review, Dr. Palmer was assigned as the lead technical specialist in the In-Drift Chemistry model area, and also supported review in the EBS Degradation model area. He can be contacted by telephone, (208) 526-4478; or by e-mail, palmcd@inel.gov.

\section{Richard M. Pawlowicz}

Dr. Pawlowicz, an employee with Bechtel Jacobs Company, LLC, is currently a Technical Lead and Subcontract Technical Representative for the ORNL Project, Melton Valley Hydrofracture Well Plugging and Abandonment project. The project scope includes closure of deep wells associated with low-level waste disposal in the subsurface on the Oak Ridge National Laboratory site. His academic background includes a B.S. and M.S. in Geology from the University of Toledo, and a Ph.D. in Geology from the New Mexico Institute of Mining and Technology. In the Model Validation Status Review, Dr. Pawlowicz supported reviews in the Saturated Zone Flow and Saturated Zone Transport modeling areas. He can be contacted by telephone, (865) 241-8031; or by e-mail, pawlowiczrm@bechteljacobs.org.

\section{Suzette Jackson Payne}

Ms. Payne is an Advisory Scientist at the Idaho National Engineering and Environmental Laboratory (INEEL) and has been the Program Manager for the INEEL Seismic Monitoring Program since 1984. Her work has included the design, maintenance, and operation of 26 seismograph stations and 25 strong-motion accelerographs, and analyzing and interpreting seismograms and accelerograms for annual documentation of eastern Idaho earthquake activity. She was lead for the INEEL seismic hazards assessments and development of probabilistic design basis earthquake parameters for INEEL facilities, and providing technical input to and review of INEEL safety analysis reports, environmental impact statements, and facility siting studies pertaining to seismic hazards and design. She has provided technical input to and review of INEEL documents pertaining to seismic and volcanic hazards. She has a B.S. from the University of Utah, and an M.S. in Geophysics from Boise State University. In the Model Validation Status Review, Ms. Payne supported review in the Disruptive Events - Igneous Disruption modeling area. She can be contacted by telephone, (208) 526-4293; or by e-mail, msj1@inel.gov.

\section{Indrek Porro}

Dr. Porro is currently with Bechtel BWXT Idaho as an Advisory Engineer/Scientist. He has spent the last $11 \mathrm{yr}$ as a soil physicist/vadose zone hydrologist investigating soil water and contaminant transport issues related to waste management and environmental restoration. He is 
principal scientist on a multi-year project for field-testing and evaluation of the hydrologic behavior of engineered soil covers. His background includes research on soil-plant-atmosphere interactions. Dr. Porro also spent $5 \mathrm{yr}$ as an electrical engineer in the electrical power industry designing electrical systems for nuclear power plants. He has a B.S. in Electrical Engineering from Rutgers University, a B.S. and M.S. in Soil Science from North Carolina State University, and a Ph.D. in Agronomy from New Mexico State University. In addition, he has authored numerous publications, reports, and presentations. In the Model Validation Status Review, Dr. Porro was assigned as the lead technical specialist in the UZ Flow modeling area, and also supported review in the UZ Transport model area. He can be contacted by telephone, (208) 5260906; or by e-mail, ixp@inel.gov.

\section{Stuart E. Rawlinson}

Dr. Rawlinson is a Principal Scientist with Bechtel-Nevada. Currently he is manager of the Geological and Hydrological Services Group (15 geologists/hydrologists). Previously he managed the Environmental Assessment and Closure Section, and the Waste Management Department, both within the Environmental Restoration and Waste Management Division of Raytheon Services Nevada. In this capacity he was responsible for developing a site characterization program to meet regulatory requirements for operating two radioactive waste management sites at the Nevada Test Site. Dr. Rawlinson has authored approximately 70 technical publications through the State of Alaska, professional journals, books, and the Department of Energy. He has a B.S. in Geology from California State University/Long Beach, and M.S. and Ph.D. degrees in Geology from the University of Alaska. In the Model Validation Status Review, Dr. Rawlinson was assigned as the lead specialist in the Climate modeling area, and also supported review in the Ambient/Thermal Drift Seepage modeling area. He can be contacted by telephone, (702) 295-1185; or by e-mail, rawlinse@nv.doe.gov.

\section{George D. Redden}

Dr. Redden is currently an Advisory Scientist with Bechtel-BWXT Idaho. From 1992 to 1998 he was associated with the Department of Civil and Environmental Engineering at Stanford University. His principal interests include environmental chemistry, geochemistry, and biochemistry of aquatic systems; fate of trace elements and radionuclides, and heavy metal interactions with mineral surfaces and organic matter (natural and contaminant) in natural environments. He also worked in coordination chemistry; surface chemistry of solute-solid interactions (redox processes and adsorption); global elemental cycles; and global environmental issues from both scientific and societal aspects. Dr. Redden's academic background includes a B.S. in Chemistry from Stanford University, an M.S. in Oceanography from Oregon State University, and a Ph.D. in Civil Engineering from Stanford University. He has authored numerous publications and been involved in many research projects in his area of expertise. In the Model Validation Status Review, Dr. Redden was assigned as the lead technical specialist in the EBS Radionuclide Transport model area, and also supported review in the UZ Transport model area. He can be contacted by telephone, (208) 526-0765; or by e-mail, reddgd@inel.gov.

\section{Dash Sayala}

Dr. Sayala has more than 28 yr of professional experience in geochemistry, hydrogeology, geologic disposal, environmental assessments, and program management. He has provided technical support to U.S. and international agency programs for disposal of high-level, low-level, and transuranic radioactive wastes, and hazardous wastes. He is a consultant with Management 
Solutions, Inc. and previously worked with other companies including Kathpal Technologies, Science Applications International Corporation (SAIC), and Mitre Corporation. He contributed to the U.S. Agency for International Development (USAID) Privatization Environmental Project and USAID Global Environmental Center Project. He was employed by SAIC from 1991 to 1996, where he provided technical support to Department of Energy environmental waste management projects, Waste Isolation Pilot Plant program, and to activities of the DOE Office of Nuclear Safety Policies and Standards. Dr. Sayala holds B.S. and M.S. degrees in Geology from the Osmania University, India. In addition he has an M.S. in Geochemistry from the University of New Mexico, and a Ph.D. in Geochemistry from The George Washington University. He has authored numerous publications and reports during his career. In the Model Validation Status Review, Dr. Sayala was assigned as lead technical specialist in the Mountain-Scale/Near-field THC model area, and also supported review in the In-Drift Chemistry model area. He can be contacted by telephone, (703) 938-1081; or by e-mail, dsayala@aol.com.

\section{Michael G. Sholley}

Mr. Sholley has provided engineering geology support for $24 \mathrm{yr}$ on civil engineering projects (site investigation and design for power plants, dams, bridges, tunnels, pipelines, water supply, and roadways), mining engineering projects (geotechnical investigation and analysis), and environmental engineering projects at the Savannah River Site, Hanford, and elsewhere. He has conducted hydrogeologic investigations, engineering geology investigations, geologic reconnaissance and mapping. In addition, he has performed and supervised rock mechanics analyses, software development, and groundwater modeling. Mr. Sholley has been employed with Bechtel Environmental, Inc., Geotechnical and Engineering Technologies Group as an Engineering Geology/Hydrogeology Specialist since 1995. Mr. Sholley holds a B.S. in Geological Engineering from the University of Idaho, and an M.S. in Geotechnical Engineering from the University of California at Berkeley. In the Model Validation Status Review, Mr. Sholley was assigned as the lead technical specialist in the Integrated Site Model area, and also supported review in the SZ Flow model area. He can be contacted by telephone, (415) 768-6092; or by e-mail, mgsholle@bechtel.com.

\section{Richard P. Smith}

Dr. Smith has worked at the Idaho National Engineering and Environmental Laboratory (INEEL) since 1986. His experience includes Principal Investigator for neotectonic studies of the Snake River Plain and northern Basin-and-Range for seismic and volcanic hazards assessment; Principal Investigator for design and implementation of geotechnical studies for nuclear reactor and hazardous waste facilities siting, determination of volume, mass, and configuration of molten material in the core of the Three Mile Island reactor for post-mortem accident investigation, and evaluation of innovative mining systems for energy development. Dr. Smith holds a B.S. in Geology from Marshall University, and M.S. and Ph.D. degrees in Geology from the University of Colorado. He has authored numerous publications, presentations, and abstracts. In the Model Validation Status Review, Dr. Smith was assigned as lead technical specialist in the Disruptive Events-Igneous Disruption modeling area. He can be contacted by telephone, (208) 526-9896; or by e-mail, rps3@inel.gov.

\section{Daniel V. Swenson}

Dr. Swenson is currently on sabbatical from a faculty appointment at Kansas State University, and is President of Thunderhead Engineering. His engineering expertise includes stress analysis, 
finite element modeling, fracture mechanics, computer graphics, and software development. His interactive pre- and post-processors for the TOUGH2, TETRAD, and STAR codes are currently under development. Dr. Swenson has a B.S. in Mechanical Engineering from Kansas State University, an M.S. in Mechanical Engineering from Carnegie-Mellon University, and a Ph.D. in Civil Engineering from Cornell University. He has authored numerous publications, reports, and presentations. In the Model Validation Status Review, Dr. Swenson was assigned as the lead technical specialist in the Mountain-Scale TH and the EBS Moisture Distribution and TH model areas, and also supported review in the Mountain-Scale/Near-Field THC model area. He can be contacted by telephone, (785) 770-8511; or by e-mail, swenson@thunderheadeng.com.

\section{Ravi Varma}

Dr. Varma is currently a Technical Staff Member and supervisor for Los Alamos National Laboratory, Materials Science and Technology Division. He has been involved with many projects, including nuclear materials $(\mathrm{Pu} / \mathrm{U})$ disposition; microwave-assisted processing for immobilization of $\mathrm{Pu} / \mathrm{U}$ waste in glass/ceramics; and beryllium testing (understanding fracture concerns for various vintages of $\mathrm{Be}$ and $\mathrm{Be}$-welds). He has tested and evaluated a number of critical non-nuclear components (including the $\mathrm{Be}-\mathrm{Al} / \mathrm{Si}-\mathrm{Be}$ and the $21 \mathrm{Cr}-6 \mathrm{Ni}-9 \mathrm{Mn}$ welds) for the Stockpile Stewardship programs. His academic background includes a B.Sc. and an M.S. in Chemistry, and a Ph.D. in Chemical Physics from the University of Pennsylvania. In the Model Validation Status, Review Dr. Varma supported review of the Waste Form Degradation and Waste Package/Drip Shield Degradation modeling areas. He can be contacted by telephone, (303) 871 7464 or (303) 644-5396; or by e-mail, rvarma@lanl.gov or rvarma@du.edu

\section{Thomas R. Wood}

Mr. Wood has been an Advisory Scientist with Bechtel-BWXT Idaho since 1999. He has extensive experience in site characterization, basic and applied research, management, and environmental remediation during $21 \mathrm{yr}$ of professional practice. He has expertise in groundwater and vadose zone hydrology, geophysics, and geology. His current research interests include application of deterministic-chaos theory to the fluid flow through variably saturated fractured basalt. Mr. Wood has a B.S. in Geophysics from Western Washington University, and an M.S. in Geological Engineering from Washington State University. He has authored numerous publications and presentations. In the Model Validation Status Review, Mr. Wood was assigned as lead technical specialist in the Ambient/Thermal Drift Seepage modeling area, and supported review of the SZ Transport area. He can be contacted by telephone, (208) 526-1293; or by e-mail, tqw@inel.gov.

\section{Vefa Yucel}

Mr. Yucel has been a Principal Hydrologist with Bechtel-Nevada since 1996, working to support the waste management, engineering, environmental restoration, monitoring, and environmental remediation projects on the Nevada Test Site. He has developed the performance assessment and composite analysis documents for securing the Disposal Authorizations for two LLW disposal facilities at the Nevada Test Site. He participated in the International Atomic Energy Agency working group on Improving Long Term Safety Assessments Methodologies for Near Surface Radioactive Waste Disposal Facilities (ISAM). He also contributed to the South Africa Borehole Safety Case for African sealed source radionuclide inventory. Mr. Yucel's academic background includes a B.S. and an M.S. in Civil Engineering from Iowa State University, and graduate classes at Stanford University. In the Model Validation Status Review, Mr. Yucel was assigned 
as lead technical specialist in the PA Modeling area. He can be contacted by telephone, (702) 295-1158; or by e-mail, yucelv@nv.doe.gov.

\subsection{Model Area Leads}

Assigned model area leads are shown in Table 5 (Model Area Leads, Representing Responsible Project Staff). These individuals were selected from among supervisory staff in the Science and Analysis Project area of the BSC organization. Their participation was documented in accordance with the plan for the MVSR (BSC 2001j; Hardin 2001c). They participated in the final workshop (Task 6 in Table 2) and contributed text describing recommended additional work on model validation, for inclusion in this report. 


\section{Review Findings and Recommendations}

This section presents information developed by the technical specialists who reviewed the 125 AMRs plus other documents. The review information is organized around the 21 model areas, and the models (not necessarily AMRs) identified within each area. For binning purposes (see Section 3.3) it was necessary to identify the principal AMR which contains the documentation of each model. Many models are fully described by a single AMR, however, some are not and the distinction between models and AMRs encouraged the reviewers to consider how the technical content is organized.

All of the AMRs in each model area (Table 1) were reviewed, however some did not contribute to the models and are not discussed in this section. It is the judgment of the technical specialists that these AMRs contain supporting analyses, or sensitivity analyses, and are not models (see Section 3.4 for definitions). Conversely, a number of AMRs that were designated by the authors as analyses, were found to constitute the principal documentation for models in this review (see Table 7). In addition, a number of calculation reports were also found to document models (Table 7).

For each model the text provides a short description, a statement of the use of the model, and explanation of the basis for binning. The results of the model identification and binning are summarized in Table 6 . Information on use is primarily limited to whether substantive output of the model is used for TSPA-SR, and whether the model is used in screening (excluding) FEPs from consideration in TSPA-SR.

For Bin-2 and Bin-3 models, recommendations are provided as to further documentation, and additional testing, data collection, and model development as appropriate, which could bring the models into compliance with AP-3.10Q. Recommendations for improvement of Bin-1 models are also provided in some cases. These recommendations may not have been incorporated into the baseline work plan, schedule, and budget for the Yucca Mountain Project.

For some Bin-2 models, supplementary information from Project staff is provided in Section 6 to clarify certain aspects of model validation, and to describe future investigations that can be used to increase confidence. In accordance with the Technical Work Plan for this activity (BSC 2001j) supplementary information is included in Section 6 where provided by Project staff.

For Bin-3 models, impact reviews are provided in Appendix IV as signed reviews prepared by responsible Project staff. These reviews address the impact of model validation review findings on the conclusions of TSPA-SR, i.e. the calculated annual dose histories. In addition, where applicable the impact reviews also list the existing Key Technical Issue (KTI) agreements that previously addressed those technical questions and concerns represented by the model validation review findings. The impact reviews also describe the path forward, i.e. further documentation, development, or data support that is planned for models that are intended to support a potential License Application.

Some of the path forward description pertains to ongoing work, but much of it pertains to future work. Discussion of future work should be considered as recommendations from the review team (including Project staff), and such work may not have been incorporated into the baseline work plan, schedule, or budget for the Yucca Mountain Project. 
Where appropriate, the impact reviews address the review findings on a point-by-point basis, but this is not done if a simpler or more compelling basis for the impact review is available (e.g., the model is not used or it is bounding).

\subsection{Model Area A: Climate}

\subsubsection{Precession-Based Orbital Clock Model (A.1)}

Description: This model provides the timing for future changes in climate, based on earthorbital parameters and the Devil's Hole oxygen isotope record as captured in precipitated vein calcite. Vein-calcite samples were analyzed for oxygen isotopes, and dated using radiometric methods. Assuming cyclicity of climate change, this "clock" is used to forecast the beginning and end of future major climatic events in the Yucca Mountain region. The model provides information on the timing sequence, but not the magnitude and nature of climate change. The magnitude and nature of climate changes are predicted using corollary records from other sites that are natural analogs to Yucca Mountain, e.g., the microfossil record from Owens Lake. This model is documented in the AMR entitled Future Climate Analysis (USGS 2000).

Use of the Model: The sequence of climate changes is used in the infiltration model (Model Area B), which is a boundary condition on the UZ flow and transport model, seepage models, and thermal-hydrology models used for TSPA-SR. Output from the model consists of the timing for a series of forecasted future climate changes.

Review Findings: The Precession-Based Orbital Clock Model was assigned to Bin 1, meaning that compliant validation was achieved in the principal AMR. As the basis for this finding the reviewers determined that the earth-orbital parameter data, the correlation of oxygen isotope ratios with global temperature, and the Devil's Hole oxygen-isotopic record and radiometric chronology, are all published in peer-reviewed literature. All of these data are described as accepted or qualified.

Other aspects of this AMR, such as the use of climate information from analog sites, were considered by the reviewer to constitute an analysis as defined by AP-3.10Q.

Supplementary Response from Project Staff: External reviewers have pointed out in past reviews that it is difficult to validate the model for future climates. For example, one of the DOE-NRC agreements arising from the Key Technical Issue for Total System Performance Assessment Integration (TSPAI) (Cornell 2001) involves justification for the use of the analog site temperature data (TSPAI 3.19). In addition, there are concerns about the approach of using historical data and neglecting anthropogenic effects, especially given the current global warming situation. Work to improve the million-year climate model is ongoing, but this work is not expected to significantly change the conclusions of the TSPA-SR. This is because output from the climate model constrains the infiltration model, and the impact of changes in the infiltration model would be insignificant as discussed in Section 6.2.

\subsection{Model Area B: Infiltration}

\subsubsection{Net Infiltration Model (B.1)}

Description: This model estimates the net infiltration used as an upper boundary condition for the site-scale UZ Flow Model (Section 6.3.6 of this report). The model is documented in the AMR entitled: Simulation of Net Infiltration for Modern and Potential Future Climates (USGS 
2001a). Estimates of net infiltration are provided as raster-based, 2-D grids of spatially distributed, time-averaged infiltration rates for various present and future climate conditions. The climate conditions used for TSPA-SR consist of three cases: the lower-bound, mean, and upperbound cases which represent a range of uncertainty on net infiltration. For each of these cases the climate evolves through time, in stages (modern, monsoonal, and glacial-transition) representing the next $10,000 \mathrm{yr}$. In summary, for each such case and stage a separate infiltration map is developed; the result is a set of nine maps that represent uncertainty in present as well as future conditions.

Use of the Model: The set of nine raster grid maps ( 3 stages multiplied by 3 cases) provides spatially detailed representation of the magnitude and distribution of net-infiltration rates, and is used to define the upper flux boundary conditions for UZ flow and transport simulations.

Review Findings: The Net Infiltration Model is assigned to Bin 2, meaning that compliant validation was not achieved because additional documentation is needed to justify certain aspects, specifically:

- Rooting depths - The model assumes 6-meter rooting depths if the soil is $6 \mathrm{~m}$ deep. This assumption may be non-conservative and warrants additional justification. Literature on rooting depths at the Nevada Test Site suggests that 6-meter rooting depth would be rare.

- Neutron log data - Model output should be compared to neutron logging data that were used to calibrate the 1996 version of the Net Infiltration Model. Instead, the 1999 version (reviewed here) is calibrated using 2-yr of stream-flow data. The neutron logging data may have uncertainties associated with interpretation of volumetric moisture content, but it is an extensive record and should be assessed as part of this model.

Both these aspects can be addressed without further model development or data collection, and would support the degree of validation appropriate for the intended use of this model.

The other AMR reviewed in this model area, entitled: Analysis of Infiltration Uncertainty (CRWMS M\&O 2000e) was determined by the reviewer to constitute an analysis as defined by AP-3.10Q.

Supplementary Response from Project Staff: This model has been the subject of DOE-NRC agreements arising from the Key Technical Issues for Unsaturated and Saturated Flow Under Isothermal Conditions (USFIC) and Total System Performance Assessment Integration (TSPAI) (Cornell 2001; Reamer 2001). Resolution of these agreements will involve: 1) justification of Monte Carlo parameter distributions (USFIC 3.1 and 3.2 ); 2) comparison of the existing "bucket" or plug-flow model with the Richards' equation (TSPAI 3.18); and 3) update of infiltration model parameters and validation (TSPAI 3.19 through 3.21).

Work to address these issues is ongoing, but the results are not expected to significantly impact the conclusions of the TSPA-SR because a wide range of infiltration rates is already incorporated in the UZ flow and transports models used for TSPA-SR, and recent studies have shown (BSC 2001d, Sections 3.2 and 3.3) that the presence of nonwelded tuff units above the repository horizon tends to re-distribute percolation flux and make it more uniform (even with spatial variation of infiltration at the surface). 


\subsection{Model Area C: Unsaturated Zone Flow}

\subsubsection{Conceptual Model of UZ Flow (C.6-1)}

Description: This is a conceptual model that describes flow issues and processes considered to be relevant for modeling flow and transport within the unsaturated zone (UZ) at Yucca Mountain. These issues and processes are integrally related to the hydrogeologic features of the UZ, which consists of heterogeneous volcanic rocks that have been welded and fractured to varying degrees, and are divided into hydrogeologic units based roughly on the degree of welding. Water moving downward through these units is considered to be partitioned between fractures and the rock matrix. The relevant issues and processes are: infiltration, fracture and matrix flow components, fracture-matrix interaction, perched water, effects of major faults, transient flow, focusing flow and fast flow paths, and the gas flow process. The model is documented in the AMR: Conceptual and Numerical Models for UZ Flow and Transport (CRWMS M\&O 2000q).

Use of the Model: This model develops the framework that is used for UZ flow and transport, and seepage modeling, used in TSPA-SR.

Review Findings: This conceptual model has no output to which validation methods in AP3.10Q Section 5.3.b or 5.3.c can be applied. However, this model does serve as the basis for the UZ Flow Model, and validation of the UZ Flow Model could effectively serve as validation of the Conceptual Model of UZ Flow. Accordingly, revision of the documentation (e.g., inclusion of this model with the UZ Flow Model described in Section 6.3.6 of this report) can be used to provide validation. The Conceptual Model of UZ Flow is therefore assigned to Bin 2.

\subsubsection{Numerical Grids Model (C.4)}

Description: This model is used to develop numerical grids that represent the unsaturated hydrogeologic system of Yucca Mountain. These grids are integral to the numerical implementation of the UZ Flow Model. The model comprises four submodels: 1) a 1-D Vertical Column Grid Submodel (for hydrogeologic property set inversion), 2) a 2-D Vertical Crosssections Grid Submodel (for fault hydrogeologic property calibrations), 3) a 3-D Calibration Grid Submodel, and 4) a 3-D Grid Submodel (for generating flow fields for performance assessment). The 1-D Vertical Column Grid Submodel consists of columns centered at borehole locations. The 2-D Vertical Cross-sections Grid Submodel consists of an east-west, crosssectional grid through borehole UZ-7a located within the Ghost Dance fault zone.

There are two 3-D grids developed: 1) the 3-D Calibration Grid Submodel, and 2) the 3-D Grid Submodel. The 3-D Calibration Grid Submodel consists of a plan-view grid with additional discretization along major faults, the Exploratory Studies Facility (ESF), and the Enhanced Characterization of Repository Block (ECRB) drift that crosses the repository block. The grid is structured such that it contains columns of gridblocks centered at borehole locations. The 3-D Grid Submodel consists of a plan-view grid with finer resolution at the repository horizon and within the Calico Hills nonwelded hydrogeologic unit, but without the additional discretization at the ESF or ECRB drift. The numerical grids are based on data extracted from the Geologic Framework Model and the Integrated Site Model (see Section 6.20), and the definitions of hydrogeologic units. The Numerical Grids Model is documented in the AMR entitled: Development of Numerical Grids for UZ Flow and Transport Modeling (CRWMS M\&O 2000t). 
Use of the Model: The output of this model consists of numerical grids developed to represent the UZ at Yucca Mountain. These grids include a 1-D vertical column grid used for hydrogeologic property set inversion, a 2-D vertical cross-sectional grid used for fault property calibrations, a 3-D grid is used for 3-D model calibrations, and a different 3-D grid used for generating flow fields for the TSPA-SR.

Review Findings: Verification of grid parameters is documented in the subject AMR (CRWMS $\mathrm{M} \& \mathrm{O}$ 2000t). Gridblock material names and elevations are verified by comparison with stratigraphic information from the Geologic Framework Model Analysis Model Report (BSC 2001e). Spot checks involving hand calculations of gridblock volumes, connection lengths, interface areas between gridblocks, and direction of absolute permeability were consistent with model results.

Sensitivity studies examining the effect of grid resolution on flow and transport simulations were conducted using grids documented in previous reports. These studies indicated that current resolution is adequate. However, these results should be only corroborative material for this model. Although the verification activities cited above provide reasonable assurance that the model works as intended, additional sensitivity studies using this model are needed to provide confidence in the validity of this model and, also, some measure of the predictive uncertainty involved. Accordingly, the Numerical Grids Model is assigned to Bin 2.

\subsubsection{Numerical Model of UZ Flow (C.6-2)}

Description: This is a conceptual model that describes the numerical approach used to implement the Unsaturated Zone (UZ) Flow Model. Unsaturated zone flow at Yucca Mountain is described using a dual-continuum approach, whereby fractures and matrix are treated as two separate, but interacting continua. Each gridblock is subdivided into one fracture block and one matrix block. Global flow is thought to occur within both fracture and matrix continua, which is referred to as the dual-permeability approach. A geologic-based, deterministic approach, in which an entire model layer is assigned uniform properties, is used for characterizing mountainscale flow and transport in the unsaturated zone of Yucca Mountain. Porous-medium equivalence is used to describe water flow in the fracture continuum in the dual-permeability approach. Specifically, Darcy's law is used to determine water flux within the fracture continuum, and constitutive relations between relative permeability, capillary pressure, and saturation are employed for modeling water flow. The model is documented in the AMR: Conceptual and Numerical Models for UZ Flow and Transport (CRWMS M\&O 2000q).

Use of the Model: This model develops the mathematical representation for UZ flow and transport, used in TSPA-SR.

Review Findings: This model has no output to which validation methods in AP-3.10Q Section 5.3.b or 5.3.c can be applied. However, this model does serve as the basis for the UZ Flow Model. Validation of the UZ Flow Model could effectively serve as validation of the Numerical Model of UZ Flow. Accordingly, revision of the documentation (e.g., inclusion of this model with the UZ Flow Model described in Section 6.3.6 of this report) can be used to provide validation. Accordingly, the Numerical Model of UZ Flow is assigned to Bin 2.

Supplementary Response from Project Staff: The dual-continuum model is a simple representation of unsaturated, fractured rock (fractures and matrix are both represented by continua). Discrete-fracture effects may be important at the field scale that controls seepage into 
the drifts. There has been little investigation of discrete-fracture models, and their importance cannot be readily evaluated. This concern has been the subject of a DOE-NRC agreement arising from the Key Technical Issue for Unsaturated and Saturated Flow Under Isothermal Conditions (USFIC) (Reamer 2001). This agreement (USFIC 4.06) calls for comparison of continuum and discrete fracture network models, or justification of the continuum approach at the scale of the seepage model grid. The issue will be investigated using data from the Alcove $8 /$ Niche 3 field test and the 1-m3 block multifracture lab test.

\subsubsection{Active Fracture Model (C.6-3)}

Description: This is a conceptual model that describes the fracture-matrix interaction in the unsaturated zone at Yucca Mountain. The water-flow pattern in the unsaturated fractured rock at Yucca Mountain is expected to be characterized by significant preferential (fingering) flow at a fracture-network scale. The active fracture model (AFM) concept is based on the reasoning that as a result of fingering flow, only a portion of fractures in a connected, unsaturated fracture network contributes to liquid water flow, while others are simply by-passed. The portions of the connected fractures that actively conduct water are called active fractures. It is hypothesized that the number of active fractures in the UZ of Yucca Mountain is small compared to the total number of connected fractures so that active fractures, rather than total fractures, must be used in numerical models. It is also hypothesized that the number of active fractures within a grid block is large, such that a continuum approach is still valid for describing fracture flow. Active fractures are treated as a portion of the "homogeneous" fracture continuum for a given gridblock using a "fraction of active fractures" parameter. The model is documented in the AMR: Conceptual and Numerical Models for UZ Flow and Transport (CRWMS M\&O 2000q).

Use of the Model: The AFM is used to represent the hydrologic characteristics of rock fractures for the UZ Flow Model, Mountain-Scale/Near-Field THC Models, and the Multiscale Thermohydrology Model, which provide output that is abstracted for use in TSPA-SR.

Review Findings: This model has no output to which validation methods in AP-3.10Q Section 5.3.b or 5.3.c can be applied. However, this model does serve as the basis for the UZ Flow Model, and validation of the UZ Flow Model could effectively serve as validation of the AFM. Accordingly, revision of the documentation (e.g., inclusion of this model with the UZ Flow Model described in Section 6.3.6 of this report) can be used to provide the needed validation, and the Active Fracture Model is assigned to Bin 2.

Supplementary Response from Project Staff: The AFM is used for all UZ flow, seepage, and transport models. Validation of the AFM will be based on field and lab evidence, in addition to numerical considerations. Validation of the AFM has been the subject of a DOE-NRC agreement arising from the Key Technical Issue for Total System Performance Assessment Integration (TSPAI) (Cornell 2001). This agreement involves independent lines of evidence to provide additional confidence in the use of the active fracture continuum concept in the UZ transport model (TSPAI 3.28). Recent preliminary results from the ongoing IAEA peer review of the TSPA-SR also indicate that the AFM needs further validation. Ongoing seepage and transport tests at Alcove $8 / \mathrm{Niche} 3$, and laboratory testing using a $1-\mathrm{m}^{3}$ block of fractured, welded tuff will provide data for validating this key conceptual model. 


\subsubsection{Calibrated Properties Model (C.2)}

Description: This is a process model that implements an inverse-modeling parameter estimation procedure to provide calibrated hydrologic property sets for use in other process models to simulate unsaturated zone (UZ) flow and transport, and thermally driven coupled hydrologic processes. The model comprises three submodels: 1) 1-D Mountain-scale Calibration Submodel, 2) 1-D Drift-scale Calibration Submodel, and 3) 2-D Fault Calibration Submodel. Model parameters that are calibrated include: fracture and matrix permeability, fracture and matrix van Genuchten parameters, and a fracture activity parameter. These parameters are estimated for 31 model layers. Parameter estimation is performed for the mean, upper-bound, and lower-bound infiltration cases. Saturation, water potential, and pneumatic data from 11 surface-based boreholes are used as constraint data for estimating unit parameters in the 1-D submodels. Saturation, water potential, and pneumatic pressure data from one borehole within the Ghost Dance fault zone are used as constraint data for estimating unit parameters in the 2-D submodel. The Calibrated Properties Model is documented in AMR: Calibrated Properties Model (CRWMS M\&O 2000i).

Use of the Model: Model output consists of mountain-scale, drift-scale, and fault property sets, which are used in the UZ Flow Model, mountain-scale/near-field coupled process models, and the Multiscale Thermohydrology Model. The hydrologic properties comprise matrix and fracture permeability, matrix and fracture van Genuchten parameters, and a fracture activity parameter.

Review Findings: Validation of the Calibrated Properties Model is accomplished using a combination of several approaches, each of which is based on AP-3.10Q. First, the calibrated property sets are developed using a calibration methodology within experimental data sets ("cross-validation"). Saturation data, in situ water potential data, pneumatic pressure data, and prior property information are inverted for the calibrations. Second, the calibrated parameters are reviewed for reasonableness and consistency in explanation of relevant data. Most of the calibrated parameters are consistent with the prior information (parameters determined by other means) and for those that are not, the change is reasonably explained in light of other data. Third, previous UZ Flow Model calibration efforts using essentially the same methodology as used here have undergone technical review through publication in the open literature (Bandurraga and Bodvarsson 1999; Ahlers et al. 1999). This can be considered as corroborative because those models are not documented in the subject AMR (CRWMS M\&O 2000i). Fourth and finally, UZ Flow Model predictions using the calibrated property sets were compared to data not used in the calibration process. The first three approaches are found in the subject AMR, but lack the strength on their own to constitute adequate validation. The comparison of model predictions using the calibrated properties to data not used in the calibration process is found in the supporting AMR UZ Flow Models and Submodels (CRWMS M\&O 2000bq) and constitutes a much stronger validation approach. Revision of the documentation is likely to produce a compliant validation argument, so the Calibrated Properties Model is assigned to Bin 2.

\subsubsection{UZ Flow Model (C.1-1)}

Description: This is a 3-D process model of flow used to generate flow fields for various applications in support of TSPA-SR. Eighteen flow fields are generated for the TSPA-SR based on nine infiltration maps and two conceptual models of perched water. Nine infiltration maps are used to account for the present-day, monsoon, and glacial transition climate stages, with the mean, lower-bound, and upper-bound infiltration cases (see Section 6.2.1 of this report). The 
future monsoonal and glacial-transition climate periods are used to account for possible greater precipitation and infiltration. The infiltration bounds account for uncertainty in the Net Infiltration Model (see Section 6.2 of this report). This model incorporates flow issues and processes identified in the Conceptual Model of UZ Flow that are considered to be relevant for modeling flow and transport within the unsaturated zone (UZ) at Yucca Mountain (Section 6.3.1). The 3-D grid used for this model includes a refined mesh in the vicinity of the potential repository, but no refinement along the Enhanced Characterization of Repository Block (ECRB) cross-drift and the Exploratory Studies Facility (ESF). This model is documented in the AMR: Unsaturated Zone Flow Model and Submodels (CRWMS M\&O 2000bq).

Use of the Model: 3-D flow fields are used for UZ transport modeling in support of TSPA-SR.

Review Findings: The UZ Flow Model has been validated against four different sets of data acquired at Yucca Mountain: 1) Alcove 1 Test data, 2) ECRB observation data, 3) SD-6 and WT-24 data, and 4) 3-D gas flow data.

The Alcove 1 Test is an infiltration and tracer transport test that was performed in the ESF Alcove 1 located in the Tiva Canyon Tuff unit. The infiltration test involved applying water at the ground surface directly over the end of Alcove 1. A non-sorbing bromide tracer was introduced into the infiltrating water during the later stages of the test. Seepage into the alcove and the tracer arrival time were recorded. The comparisons between predicted and observed seepage rates and tracer transport were reasonably good compared to results typically reported in the technical literature for similar model-data comparisons.

The ECRB data consist of water-potential data collected from heat dissipation probes installed in the tunnel wall along the ECRB tunnel inside ESF. UZ Flow Model water potential predictions were compared to field observation data. Although the comparisons are limited, the UZ Flow Model generally predicts the range of water-potential from in-situ measurements and the model predictions are within the range of the in-situ measurements.

Data from boreholes SD-6 and WT-24 consist of liquid saturation measured on samples of the tuff matrix. Model predictions are generally consistent with field measurements for both boreholes (comparing the extent of model-data differences with the magnitude of trends in the field measurements). The model accurately predicted the location of perched water observed in borehole WT-24, and no perched water at borehole SD-6. The model did not accurately predict the matrix saturation in the CHn unit at the location of borehole SD-6, nor did it accurately predict a low-saturation layer within the $\mathrm{CHn}$ unit at borehole WT-24. Data gaps at these particular units for these two boreholes lead to poorly constrained estimates of model parameters.

Pneumatic data from six boreholes are compared to a fully 3-D pneumatic simulation, and good agreement between the predictions and observed data are obtained (also comparing model-data differences with trends in the field measurements).

The UZ Flow Model validation studies have demonstrated that the model can reasonably match different types of data acquired from field experiments and observations (e.g., water potentials, liquid saturation, seepage rate, breakthrough concentrations, and pneumatic pressures). The model apparently captures the important features of the UZ flow regime at Yucca Mountain. By proxy, this serves as validation for the contributing models identified in this report. Recognizing the extent of model-data comparison used for model validation, the UZ Flow Model is assigned to Bin 1. However, it is recommended that more specific criteria for model-data comparisons be 
developed, based on the sensitivity of model output. Also, the documentation of contributing models is distributed among several reports that could be consolidated to improve model transparency, and to achieve validation of the contributing models in accordance with AP-3.10Q.

\subsubsection{Geothermal Model (C.1-2)}

Description: This is a 3-D process model of flow and heat transfer used to calibrate the geothermal conditions of the Unsaturated Zone (UZ) Flow Model and provide ambient temperature distributions for determining boundary and initial conditions for thermal-hydrologic models. This model uses the 3-D calibration grid, which includes refined gridding along the Enhanced Characterization of Repository Block (ECRB) and the Exploratory Studies Facility (ESF). In addition to calculating thermal boundary and initial conditions, the Geothermal Model also establishes that the mass and energy balance aspects of UZ hydrology are represented consistently by the UZ Flow Model. This model is documented in the AMR: Unsaturated Zone Flow Model and Submodels (CRWMS M\&O 2000bq).

Use of the Model: Ambient temperature distributions for determining boundary and initial conditions for thermal hydrologic models.

Review Findings: Validation of the Geothermal Model is accomplished through calibration and corroboration within a field measurement data set (similar to "cross-validation"). Temperature profiles in six boreholes are used to calibrate the thermal property values for each rock unit (accounting for the effects of infiltration and saturation-dependent thermal conductivity). Comparison of calculated temperature profiles with measured temperature data shows close agreement for every borehole, except near the ground surface where there are seasonal temperature fluctuations. The criteria for model-data comparison is similar to the approach described above (Section 6.3.6). Seasonal changes in surface temperature are expected to have little impact on steady-state heat flow in the deeper UZ (more than 20 meters).

Although the calibration was conducted using an effective-continuum model (ECM) formulation with the 3-D Calibration Grid Submodel (Section 6.3.2), it is applicable to other model formulations such as the dual-permeability approach used for the UZ Flow Model, because the ambient heat flow is dominated by steady-state heat and mass transfer processes. For steady-state processes the ECM model predicts similar results to those from the dual-permeability model. Accordingly, the Geothermal Model is assigned to Bin 1. The validation argument could be strengthened with use of the dual-permeability formulation instead of the ECM formulation.

\subsubsection{Conceptual Model of Perched Water (C.1-3)}

Description: This is a conceptual model that describes features and processes considered to be relevant for the formation of perched water that has been observed within the unsaturated zone (UZ) at Yucca Mountain. Two versions of the model are considered:

- The first conceptual model incorporates these three features: 1) no large-scale vertically connected potentially fluid-conducting fractures transect the low-permeability units underlying the perched water; 2) both vertical and horizontal permeabilities within and below the perched water zone are small compared with permeabilities outside perching zones; and 3) the percolation flux exceeds the lower matrix hydraulic conductivity. 
- The second conceptual model is derived from the first, whereby the perching mechanism is controlled only by the low-permeability zeolitic matrix of the CHn unit, and it is assumed that fractures are not present in perching layers.

This model is documented in the AMR: Unsaturated Zone Flow Model and Submodels (CRWMS M\&O 2000bq).

Use of the Model: This model develops the framework that is used for the 3-D Perched Water Calibration Model, which contributes to the UZ Flow Model used for TSPA-SR.

Review Findings: The Conceptual Model of Perched Water has no output to which validation methods in AP-3.10Q Section 5.3.b or 5.3.c can be applied. However, the Conceptual Model of Perched Water serves as the basis for the 3-D Perched Water Calibration Model. Accordingly, revision of the documentation (e.g., inclusion of this model with the 3-D Perched Water Calibration Model described in Section 6.3.9 of this report) can be used to provide the needed validation, and the Conceptual Model of Perched Water is assigned to Bin 1.

\subsubsection{3-D Perched Water Calibration Model (C.1-4)}

Description: This 3-D process model of unsaturated zone (UZ) flow is used to calibrate and analyze two perched water conceptual models, and thereby contribute to understanding of the occurrence of perched water and the properties of the hydrostratigraphic units where perched water has been observed. The two conceptual models are documented in the Conceptual Model for Perched Water (see Section 6.3.8 of this report). A third scenario that cannot predict perched water in the UZ is also examined; this scenario provides an extreme case in which maximum flow through zeolites occurs. The third scenario is used primarily for sensitivity analysis and comparison with the two water-perching models. The 3-D Perched Water Calibration Model uses the 3-D Calibration Grid Submodel (Section 6.3.2), which includes refined gridding along the Enhanced Characterization of Repository Block (ECRB) and Exploratory Studies Facility (ESF). The conceptual models are implemented by locally modifying rock properties in several grid layers near the observed perched zones. This model is documented in the AMR: Unsaturated Zone Flow Model and Submodels (CRWMS M\&O 2000bq).

Use of the Model: Fracture and matrix permeability, fracture and matrix van Genuchten parameters, and the active fracture parameter for model layers associated with perched water occurrence, are used for the UZ Flow Model.

Review Findings: Validation of the 3-D Perched Water Calibration Model is accomplished, in part, through calibration and corroboration within experimental data sets (similar to a "crossvalidation" approach). The model is calibrated against matrix liquid saturations, matrix water potentials, and perched water elevations as observed from boreholes and the ECRB, and good agreement with the measured data is obtained. This approach builds confidence because the model-data agreement is obtained for different types of observational data, at different locations in the site area.

Validation of the 3-D Perched Water Calibration Model is also addressed through comparison of analysis results from alternative conceptual models to establish confidence in the selected model. Two conceptual models for perched water are analyzed, and one is chosen based on a closer match to observations of perched water occurrence. 
Validation also includes the comparison of model simulation results using calibrated parameters against measured data not used in the calibrations. Simulated matrix saturation results were compared to field measurements from two boreholes, one with perched water and the other without. Model predictions of the presence or absence of perched water were consistent with field observations. The model also predicts greater saturations than observed in parts of the $\mathrm{CHn}$ unit. These discrepancies were attributed to data gaps in the Geologic Framework Model (BSC 2001e) and the assumptions made to cover these gaps. The data gaps are expected to be resolved with revision of the Geologic Framework Model AMR.

The combination of validation approaches used establishes the appropriateness and adequacy of the model for its intended use. The validation approaches cited above are documented in the AMR. Accordingly, the 3-D Perched Water Calibration Model is assigned to Bin 1.

Supplementary Response from Project Staff: Although this model is calibrated to field observations, questions remain concerning transport pathways through or around occurrences of perched water, as indicated by the greater predicted saturations in the $\mathrm{CHn}$ unit. However, uncertainty associated with this model does not significantly impact the conclusions of TSPASR, because similar results have been obtained using different conceptual models for perched water as discussed above.

\subsection{Model Area D: Mountain-Scale Thermal-Hydrology}

\subsubsection{Mountain-Scale Coupled Processes (Thermal-Hydrology) Model (D.1)}

Description: This model consists of several mountain-scale simulations of the repository used to evaluate coupled thermal-hydrologic processes. This model is documented in the AMR entitled: Mountain-Scale Coupled Processes (TH) Models (CRWMS M\&O 2000av). The model uses 3-D and 2-D simulations with the TOUGH2 code, to calculate temperatures, fluid saturation, and fluid flow rates for $100,000 \mathrm{yr}$. All models represent the repository from an elevation of approximately $750 \mathrm{~m}$ to $1500 \mathrm{~m}$ above mean sea level. The 3-D model bounds the potential repository while the 2-D models represent vertical, N-S cross-sections through the potential repository.

TOUGH2 implements the Integral Finite Difference Method to simulate multi-phase, multicomponent flow of mass (liquid, vapor, and air) and heat. A dual-permeability approach is used to represent the fractures and the rock matrix as different continua. Matrix and fracture flow properties are assumed to be temporally invariant in the simulations. Thermal-hydrologicchemical coupling effects, such as blocking of flow due to silica precipitation, and thermalmechanical coupling effects, such as change in fracture aperture and flow due to thermal stress, are not included.

The top boundary condition is a fixed temperature with infiltration prescribed in the same manner as the UZ Flow Model. The bottom boundary condition is handled in different ways for the different simulations. Heat loads are $72.7 \mathrm{~kW} / \mathrm{acre}$, neglecting ventilation during the 50 -yr preclosure period except for Case 6. Perched water effects are ignored. Infiltration is specified as modern-stage, mean-case except for Cases 5 and 6 , which also use the transitions to monsoonal and glacial-transition conditions identified in Section 6.1 of this report.

Use of the Model: Results from the Mountain-Scale Coupled Processes (TH) Model are not used in TSPA-SR. Rather, the results are used to support the conceptual basis for modeling drift 
seepage and radionuclide transport in the UZ. The model is cited in the AMR entitled: Features, Events, and Processes in Unsaturated Zone Flow and Transport (BSC 2001b).

Review Findings: The Mountain-Scale Coupled Processes (TH) Model is assigned Bin 2, meaning that compliant validation was not achieved because additional documentation is needed, specifically:

- Model validation arguments should be updated to include the Single Heater Test (SHT) and the Drift Scale Test (DST) comparisons.

- The AMR does not confirm that the initialization run of TOUGH2 provides a reasonable match to ambient conditions predicted by the UZ Flow Model. In addition, the model does not replicate known perched water data.

- Coupled thermal-hydrologic-chemical and thermal-hydrologic-mechanical processes are neglected in the model. While this may be appropriate for a model of limited scope, it should be addressed more fully in the discussion of the applicability of the model results. This documentation should include assessment of the published literature on this subject, including mechanical and chemical effects on permeability of geothermal reservoirs (e.g., Dixie Valley, Nevada as investigated by the U.S. Geological Survey), and alternative THC models of repository evolution (e.g., Lawrence Livermore National Laboratory).

- The AMR does not address the heterogeneity of permeability from fractures on these different scales. There may be a few large scale heterogeneities that act as rapid transit channels for flow.

- Justification for mesh design in the TOUGH2 analysis. In the vertical direction, both the 3-D and 2-D meshes appear to violate the Voronoï condition, which might affect convergence or accuracy of the numerical solution. Also, the coarse mesh used around the repository openings may introduce artifacts in the results. Provide justification for the mesh designs that address these questions.

- Inconsistent use of different approaches to ventilation, different infiltration rates, and different thermal loads for Cases 1 through 6.

- Missing specification of complete material properties, such as permeability and porosity of the rock matrix and the fractures.

- Incomplete evaluation of the results from different cases. For example, there is no explanation for the difference in results at $10 \mathrm{yr}$ for Case 4 (p. 67) and for Case 5 (p. 7475) reviewed in the AMR.

It is believed that the additional documentation can be provided without further model development or data collection, and would support the degree of validation that is appropriate for the intended use of this model.

Supplementary Response from Project Staff: It is difficult to validate mountain-scale coupled processes models such as the Mountain-Scale Coupled Processes TH Model, based on relatively short-term, drift-scale tests such as the SHT and DST. Coupled THC and THM processes are simulated by separate models (Sections 6.6 and 6.7 of this report; see also BSC 2001d, Sections 3.3 and 3.4). 
It is noted that grid blocks in the vertical direction are horizontal (90 degrees to gravity), so the Voronoï condition of having the nodal connection perpendicular to nodal interface is always honored. Convergence of these models is not a problem. Gridding always affects the accuracy of modeled results and can introduce artifacts, but grid refinement is used in these models where needed. Ideally one wants to maximize grid refinement, e.g., in comparison of numerical results to an analytical solution, the finer the grid, the better the agreement. However, in solving real problems other factors must be considered and grid refinement is constrained by feasibility.

Results of testing and modeling studies so far indicate that the impacts of thermally-induced coupled processes are not significant for performance (BSC 2001d, Sections 3, 4, and 11). However, some processes have not been directly investigated, such as the effects of TH and THC on radionuclide transport. Work is ongoing to reduce uncertainties in the mountain-scale coupled processes models. Data collected from the final heating phase and the cooling phase of the DST will be analyzed. Additional data may be collected from future thermal testing. In addition, validation of these models is being investigated using data from geothermal analog sites.

\subsection{Model Area E: Ambient/Thermal Drift Seepage}

\subsubsection{Seepage Calibration Model (E.1)}

Description: This model provides the general framework for modeling seepage that is used for TSPA-SR. It also provides estimates of the seepage-relevant van Genuchten capillary-strength parameter $(1 / \alpha)$ from data from liquid-release experiments performed in the Exploratory Studies Facility at Yucca Mountain. The model is documented in the AMR entitled: Seepage Calibration Model and Seepage Testing Data (CRWMS M\&O 2001p).

Use of the Model: Estimates of the seepage-relevant van Genuchten capillary-strength parameter (1/ $\alpha$ ), which are used in Seepage Model for Performance Assessment (PA) Including Drift Collapse for TSPA-SR (CRWMS M\&O 2000be).

Review Findings: The Seepage Calibration Model is assigned to Bin 2 because additional support is needed in these areas:

- The model validation argument needs to incorporate available seepage test data from the lower lithophysal zone of Topopah Spring welded unit, because this is the principal host rock unit for the TSPA-SR repository conceptual design.

- The assumption that evaporation is negligible in the interpretation of seepage test data needs to be justified. Also, the argument that a non-conservative assumption in an inverse model is actually conservative (p. 28 of the AMR) is not explained.

\subsubsection{Seepage Model for Performance Assessment Including Drift Collapse (E.2)}

Description: This model includes seepage predictions for repository conditions, for a nominal case and for a submodel incorporating the effects of rockfall on the drift opening profile, for the middle nonlithophysal and lower lithophysal Topopah Spring Tuff units. These results are used to develop the probability distribution of water seepage into waste-emplacement drifts. The model is documented in the AMR entitled: Seepage Model for PA Including Drift Collapse (CRWMS M\&O 2000be).

The AMR documents numerical simulations that were performed using stochastic representation of hydrologic properties. The effects of alternative drift opening geometry are represented using 
opening profiles generated by the DRKBA Rockfall Model (Section 6.12.1 of this report) and other approaches. Seepage of water into waste-emplacement drifts is considered to have relatively strong impact on long-term safety of the repository system.

Use of the Model: Ranges of seepage fraction and seepage percentage values for different values of average bulk permeability, heterogeneity of the permeability field, van Genuchten $1 / \alpha$ parameter, spatial correlation length, and percolation flux. This information, and the effects on seepage from drift collapse, are input to the Abstraction of Drift Seepage (CRWMS M\&O $2001 \mathrm{~b}$ ). In addition, the distribution or rock block sizes is used to develop the design basis for resistance of the drip shield to rockfall, and supports the drip shield structural calculation (CRWMS M\&O 2000bw).

Review Findings: The Seepage Model for Performance Assessment Including Drift Collapse was assigned to Bin 2, because of the need for additional documentation as described below. Model validation is claimed in accordance with Section 5.3.b in AP-3.10Q based on model-data comparison documented for the Seepage Calibration Model (Section 6.5.1 above). However, the modeling approach for TSPA-SR is not exactly the same as used for model calibration with field test data (Section 6.5.1). The model for TSPA-SR used 0.5-m grid spacing while 0.1-m grid spacing was used for model-data comparison. The AMR states that seepage increased by zero to $3 \%$ when the grid size decreased from $0.5 \mathrm{~m}$ to $0.25 \mathrm{~m}$ (pp. 51-52), however comparison with the calibration model approach was not performed.

In addition, the model output should include surface-needle effect (i.e. enhancement of seepage because of rock bolts) because it could increase seepage significantly (p. 53). This effect is included in the AMR entitled: Abstraction of Drift Seepage (CRWMS M\&O 2001b) and consistency of the documentation is needed for validation.

Finally, the assumption that evaporation is negligible in the interpretation of seepage test data needs to be justified, and the argument that a non-conservative assumption in an inverse model is actually conservative is not adequately explained (p. 24 of the AMR).

\subsubsection{Abstraction of Drift Seepage (E.3-1)}

Description: This model determines the distributions of seep spacing and flow rate in the emplacement drifts. It is documented in the AMR entitled: Abstraction of Drift Seepage (CRWMS M\&O 2001b). Percolation flux and the spacing of active fractures (where there is likely to be flow) are extracted from flow fields generated by the UZ Flow Model (Section 6.3 of this report). This Abstraction of Drift Seepage Model is concerned with the amount of water entering emplacement drifts, but does not address water chemistry. It generates probability distributions that represent the inherent uncertainty and estimated spatial variability of seepage. Seepage is then treated as a stochastic quantity in TSPA simulations by sampling values from the distributions. In defining the probability distributions, the dependence of seepage on key input parameters (including percolation flux, fracture permeability, and fracture capillary strength) is taken into account and the influence of other processes including drift degradation, elevated temperature of the host rock, and flow focusing are addressed.

Use of the Model: Estimates for spacing between seeps, and seep flow rate, and the approach to representing thermal seepage, are used directly in TSPA-SR.

Review Findings: The seepage probability distributions that were performed by the abstraction of drift seepage model were based on the results of the Seepage Calibration Model and the 
Seepage Model for Performance Assessment Including Drift Collapse. The Abstraction of Drift Seepage Model results are consistent with these source models. Spot checks were performed on the weep spacing calculated by the Abstraction of Drift Seepage Model in the output files to ensure that the routine was performing correctly for the range of input parameters used. This is appropriate validation for an abstraction, however, the assumption that THM effects can be neglected needs to be confirmed. Accordingly, the Abstraction of Drift Seepage Model is assigned to Bin 2. Technical issues with the thermal seepage abstraction are discussed in Section 6.5.4 of this report.

Supplementary Response from Project Staff: Significant uncertainties remain in the understanding of coupled processes in the near-field environment. This concern has been the subject of DOE-NRC agreements arising from the Key Technical Issues for Thermal Effects on Flow (TEF) (Reamer and Williams 2001), Evolution of the Near-Field Environment (ENFE) (Williams 2001), and Total System Performance Assessment Integration (TSPAI) (Cornell 2001). Activities for resolution of these agreements will include investigation of sources of water observed in the underground openings at Yucca Mountain, the effects of rock bolts and drift degradation, and the effects of film flow (KTE0201, KEN0107, KEN02016, KEN0402, KEN0105, and TSPAI 3.25). Uncertainties associated with the effects of coupled processes on seepage do not impact the conclusions of TSPA-SR because of conservatism built into the seepage abstraction model.

\subsubsection{Abstraction of Thermal Seepage Effects (E.3-2)}

Description: This model was also referred to by the reviewers as the "CSNF Percolation Flux 5 Meters Above Drift Model." The thermal seepage abstraction is documented in the AMR entitled: Abstraction of Drift Seepage (CRWMS M\&O 2001b) and the abstraction of percolation flux for use to estimate thermal seepage is described in Abstraction of NFE Drift Thermodynamic Environment and Percolation Flux (CRWMS M\&O 2001c). The bin-averaged percolation flux 5 $\mathrm{m}$ above the drift is taken as the percolation flux input into the Abstraction of Drift Seepage Model which is based on ambient seepage testing and analysis. No thermal or other effects are considered to be important in the intervening rock between the drift opening and the prediction horizon $5 \mathrm{~m}$ above.

Use of the Model: Seepage during the thermal period is calculated for use in TSPA-SR, using the method described here.

Review Findings: There is no justification supporting the assumption that the bin-averaged percolation flux $5 \mathrm{~m}$ above the drift opening provides a realistic estimate of the flux reaches the drift wall during the thermal period. Thermally driven processes in the near-field environment may have a significant impact on this flux, and thus on the seepage that could occur. The approach is conservative as it applies to the average behavior of thermal flux over many emplacement locations, however conservatism is not a method of model validation under AP3.10Q, Section 5.3.b or 5.3.c. Therefore, further justification is needed. Accordingly, the Abstraction of Thermal Seepage Effects Model is assigned to Bin 2.

Supplementary Response from Project Staff: It is agreed that there is little justification for selecting the bin-averaged percolation flux $5 \mathrm{~m}$ above the drift opening, to represent the percolation flux available for seepage. Thermal effects on seepage have been further explored in recent work (BSC 2001d, Section 4.3). In addition, a systematic review of UZ models and their TSPA abstractions is planned which will lead to more realistic representations of UZ processes, 
including the coupled processes that strongly influence seepage. Such improvements are not expected to impact the conclusions of TSPA-SR because of the conservative treatment of flow focusing in the current seepage abstraction (i.e., the Abstraction of Drift Seepage Model of ambient seepage, which is also used in the Abstraction of Thermal Seepage Effects).

\subsection{Model Area F: Mountain-Scale/Near-Field Thermal-Hydrologic-Chemical Model}

\subsubsection{Drift-Scale Test Thermal-Hydrologic-Chemical Calibration Model (F.1-1)}

Description: The DST THC Calibration Model is designed for comparison to observations of coupled thermal, hydrologic, and chemical processes from the Drift-Scale Test, at temperatures ranging from $25^{\circ} \mathrm{C}$ to $200^{\circ} \mathrm{C}$. The measured data from the DST are used to validate the THC Seepage Model, which is used for repository predictions. Because THC simulations are computationally intensive and the DST model domain is relatively large, 2-D simulations were performed rather than 3-D. The DST THC Model is thus a 2-D representation using homogeneous rock properties of a 3-D experiment in heterogeneous fractured tuff. This model is documented in the AMR entitled: Drift-Scale Coupled Processes (DST and THC Seepage) Models (BSC 2001a).

Hydrologic, thermal, and geochemical boundary and initial conditions are established, and simulation results, using the TOUGHREACT software code, are compared with the measured data for purposes of model validation. Simulations of THC processes include coupling between heat, water and vapor flow; aqueous and gaseous species transport; and aqueous chemical reactions described by kinetics. Feedback from chemical dissolution/precipitation reactions can simulate changes in the porosity, permeability, and capillary properties for the dual-permeability (fracture and rock matrix) medium representing the rock. Input files for simulations of the DST include hydrologic properties, mineralogical composition, and chemical reaction parameters for the rock surrounding the heated drift. To address uncertainty in local percolation conditions, the simulations were repeated for lower, mean, and upper infiltration estimates and the corresponding hydrologic property sets. Note that simulations for the DST THC Calibration Model are for the present day climate.

The DST has produced an extensive data set for the composition and distribution of migrating liquid water in heated, unsaturated tuff. Thermal-hydrologic model-data comparisons are available from the AMR: Thermal Tests Thermal-Hydrological Analyses/Model Report (CRWMS M\&O 2000bk). Modeling of thermal-hydrologic processes is an important part of the foundation for THC modeling of the DST. In some instances the model-data comparisons were consistent, however, in other cases the calculated results differed from the measured values (for example, above-boiling temperature predictions). It should be noted that the field test is ongoing therefore the data set is incomplete. The model-data comparisons in the subject AMR (BSC 2001a) are somewhat limited; they are limited to gas-phase $\mathrm{CO}_{2}$ measurements from approximately 15 sampling points, and liquid water sample collection points that were more sparse.

Although this model is designated as a "calibration model" the subject AMR does not describe an iterative process by which model input parameters were adjusted to improve model-data comparison. It appears that only a single set of model runs was produced using best-estimate input parameters. Adjustments were made using repeat model runs, so there is justification for describing this as a calibration model. 
Use of the Model: The DST THC Calibration Model is used to validate the THC Seepage Model (Section 6.6.2 of this report) which is used for predicting the effects of geotechnical and hydrothermal processes in the rock. This information is used by other models, to predict the environment within the repository emplacement drifts for TSPA-SR.

Review Findings: Definitions of the conceptual models used in the THC models are vague and the subject AMR does not clearly delineate the relationships between these conceptual models and their probable impacts on the water chemistry or changes in hydrologic properties of the host rock. The AMR also does not provide a clear breakdown of the conceptual basis for the models and how the elements of this conceptual basis will be validated.

In addition, model validation criteria are not well established. This could take the form of a justification for the closeness of model-data comparison using the DST THC Calibration Model that is needed to assure validation of the THC Seepage Model. The AMR contains a number of assumptions for which additional justification is needed. If possible, model inputs should be justified such that assumptions are not needed or are greatly reduced.

In validating the models, the methodology and results for other pertinent models such as the Abstraction of Thermal Seepage Effects (Section 6.5.4 of this report), the THM Model (Section 6.7.1), and the In-Drift Chemistry Models (Section 6.8) are not integrated into the discussion. In accordance with this discussion, the DST THC Calibration Model is assigned to Bin 2. Additional comments and recommendations related to this model are compiled in Appendix I, Additional Comments and Recommendations on the Drift Scale Test Thermal-HydrologicChemical Calibration Model (6.6-1) and the Thermal-Hydrologic-Chemical Seepage Model (6.62).

\subsubsection{Thermal-Hydrologic-Chemical Seepage Model (F.1-2)}

Description: This model consists of a set of thermal-hydrologic-chemical (THC) simulations, each representing a 2-D cross-section through a repository drift. The model inputs are based on the DST THC Calibration Model (Section 6.6.1), with changes to represent repository heating, climate change, and other differences. The THC Seepage Model is implemented for different cases:

- Tptpmn unit properties with backfill - Evaluates THC processes for a repository emplacement drift located in the middle nonlithophysal rock unit, with backfill installed in the emplacement drifts at repository closure.

- Tptpmn unit properties without backfill - Evaluates THC processes for the same case but without backfill.

- Tptpmn heterogeneous properties - Evaluates THC processes for a repository emplacement drift located in the middle nonlithophysal rock unit, without backfill, and using a heterogeneous random permeability field to represent the hydrologic characteristics of the fracture network in the host rock.

- Tptpll unit properties without backfill - Evaluates THC processes for a repository emplacement drift located in the lower lithophysal rock unit, without backfill.

Simulations of THC processes include coupling between heat, water and vapor flow; aqueous and gaseous species transport; and aqueous chemical reactions described by kinetics. Feedback from chemical dissolution/precipitation reactions can simulate changes in the porosity, 
permeability, and capillary properties for the dual-permeability (fracture and rock matrix) medium representing the rock. Input files for simulations of the DST THC Model and the THC Seepage Model include hydrologic properties, mineralogical composition, and chemical reaction parameters for the rock surrounding the heated drift. To address uncertainty in local percolation conditions, the simulations are repeated for lower, mean, and upper infiltration estimates using the corresponding hydrologic property sets. Note that simulations for the THC Seepage Model include climate change implemented as changes in the net infiltration boundary condition at fixed time intervals (Section 6.2 of this report).

Simulations of the THC processes include coupling between heat, water and vapor flow, aqueous and gaseous species transport, and kinetic chemical reactions. Feedback from chemical dissolution/precipitation reactions can produce changes in porosity, permeability, and capillary properties for the dual-permeability (fracture and matrix) rock medium. Input files for simulations of the DST coupled processes include hydrologic properties, mineralogical composition (silicate and non-silicate minerals) and chemical reaction parameters for the rock surrounding the heated drift.

Use of the Model: The THC Seepage Model is used as input to the AMR entitled: Abstraction of Drift-Scale Coupled Processes (CRWMS M\&O 2000a) which develops seepage water compositions and gas-phase compositions for use in TSPA-SR.

Review Findings: (These findings are identical to those listed for the DST THC Calibration Model in Section 6.6.1.) Definitions of the conceptual models used in the THC models are vague and the subject AMR does not clearly delineate the relationships between these conceptual models and their probable impacts on the water chemistry or changes in hydrologic properties of the host rock. The AMR also does not provide a clear breakdown of the conceptual basis for the models and how the elements of this conceptual basis will be validated.

In addition, model validation criteria are not well established. This could take the form of a justification for the closeness of model-data comparison using the DST THC Calibration Model to assure validation of the THC Seepage Model (validation criteria are closely linked with validation of the DST THC Model discussed in Section 6.6.1 of this report). The AMR contains a number of assumptions for which additional justification is needed. If possible, model inputs should be justified such that assumptions are not needed or are greatly reduced.

In validating the models, the methodology and results for other pertinent models such as the Abstraction of Thermal Seepage Effects (Section 6.5.4 of this report), the THM Model (Section 6.7.1), and the In-Drift Chemistry Models (Section 6.8) are not integrated into the discussion. In accordance with this discussion, the DST THC Calibration Model is assigned to Bin 2. Additional comments and recommendations to this report are compiled in Appendix I.

\subsection{Model Area G: Mountain-Scale/Near-Field Thermal-Hydrologic-Mechanical Model}

\subsubsection{Thermal-Hydrologic-Mechanical (THM) Model (G.1)}

Description: This model computes a bounding estimate for fracture permeability changes resulting from heating and cooling in the rock surrounding an emplacement drift. The model is documented in the calculation report: Calculation of Permeability Change Due to Coupled THM Effects (CRWMS M\&O 2000g). Normal and shear displacements of fractures are computed for a long horizontal emplacement drift in the Topopah Spring Tuff member at Yucca Mountain using a distinct-element model. Computed fracture opening and shear displacements are related to 
permeability to provide a bounding estimate of how heating and cooling in the rock surrounding an emplacement drift and the resulting mechanical deformations may affect the fracture permeability of the rock.

Use of the Model: The THM Model is not used directly for TSPA-SR, but is cited in the AMR entitled: Features, Events, and Processes in Thermal Hydrology and Coupled Processes (CRWMS M\&O 2001j).

Review Findings: The validation criteria in AP-3.10Q are not addressed because the THM Model is documented as a calculation, which doesn't require validation. Validation activities should be directed toward obtaining confidence in the expected magnitude of fracture permeability changes caused by thermal and mechanical effects.

It appears that the THM Model approach for modeling fracture deformation in the rock around an emplacement drift, caused by excavation and thermal loading, is too simplistic. Also, predicted displacements were related to fracture permeability changes using a constitutive relationship that may not be representative. Accordingly, the THM Model is assigned to Bin 3 (impact review is provided in Appendix IV). Validation of the model using field data may not be possible unless model-data agreement can be obtained, therefore it is recommended that alternative constitutive relationships be considered.

The THM Model is not fully coupled, in that it computes the thermal-mechanical response of the rock and then uses post-processing to determine changes in hydrologic parameters, rather than a fully coupled process to simulate THM response. This approach is predicated on an assumption that hydrology (particularly mass and enthalpy transport) has little effect on the thermal and mechanical responses.

The deformation of the rock surrounding an emplacement drift will be controlled by rock block movement. However, it is possible that plastic deformation and/or failure of the rock mass could occur over the long period of time that the rock is subjected to stress and temperature gradients. Permanent plastic deformation could alter the fracture aperture and joint normal stresses following the cool-down period, which was not considered by the model. Also, a single realization of orthogonal joint sets is insufficient to provide the level of confidence necessary for the intended use of the model. This fracture geometry does not resemble the orientation and density of fracture sets as described in other AMRs, such as: Fracture Geometry Analysis for the Stratigraphic Units of the Repository Host Horizon (CRWMS M\&O 2000ae).

Additional information on THM modeling was found in the more recent FY01 Supplemental Science and Performance Analysis, Volume I: Scientific Basis and Analyses (BSC 2001d; Sections 3.3.7 and 4.3.7). The additional information identified new models that address nearly all the concerns stated here, however, this additional information was judged not to ameliorate the validation status of the original model identified for the review. The new information while representing appropriate steps in model development, is not sufficient in its present form to serve as the basis for a compliant validation strategy.

Impact Review Summary: The need for additional validation of this model has been previously recognized and steps have already been initiated to complete the validation process. This model was not used in TSPA-SR but did serve as a basis for screening out FEPs. Alternative modeling approaches have been implemented and a new AMR was issued in August 2001 (BSC 2001ag). This new AMR includes more extensive information on model validation based primarily on 
measured field data from the Drift Scale, Large Block, and Single Heater Tests. Additional information to be developed to address DOE-NRC agreements on Key Technical Issue related to THM processes will provide additional confidence.

All analyses completed to date indicate that the THM effects on permeability are relatively small (within an order of magnitude change in permeability, based on measurements from both the SHT and DST) compared to the range of permeability (three to four orders of magnitude) arising from natural spatial heterogeneity. The results of these analyses support the "screening-out" of THM effects from the TSPA-SR as both reasonable and defensible, thus further model validation activities are not expected to impact the conclusions of the TSPA-SR (i.e. annual dose calculations; CRWMS M\&O 2000bm, Section 6.1).

Based on the above information and the additional detail provided in Appendix IV, the conclusion is that model validation review findings associated with the THM Model have no impact on the conclusions of TSPA-SR.

\subsection{Model Area H: In-Drift Chemistry}

\subsubsection{High-Relative Humidity Salts Model (H.1)}

Description: The purpose of the model is to provide estimates for the $\mathrm{pH}$, chloride concentration, ionic strength, and the environment required for complete vaporization of water in the drift as well as the composition of the brine during postclosure. The model examines the evaporation of seepage water on the drip shield, using an assumed incoming water composition, humidity and temperature behavior. It excludes consideration of reaction with other substrates such as the drift wall and invert. The calculations are made using EQ3/6 with a newly developed unqualified database (PT4) that uses the ion-interaction aqueous model. This model is document in the AMR entitled: In-Drift Precipitates/Salts Analysis (CRWMS M\&O 2001l).

Use of the Model: The output from this AMR provides estimates of $\mathrm{pH}$, chloride concentration, and ionic strength, which are intended for use in TSPA-SR. The nature of the general and localized corrosion models implemented in the Waste Package Degradation Model (WAPDEG) for TSPA-SR (see Section 6.10.8 of this report) means these measures of the chemical environment are not actually used to model corrosion. Similarly, the nature of the waste form degradation model is such that it does not change according to the composition of water incident on the waste package. The ionic strength computed for the drift invert is used to estimate a bounding colloid concentration for use in modeling radionuclide transport between the waste package and the drift wall.

Review Findings: The documentation does not provide data that are sufficient to support model validation over the range of conditions required for the intended use. As stated in the AMR, the intended use is "..to estimate within an appropriate level of confidence, the $\mathrm{pH}$, chloride concentration, and ionic strength of water on the drip shield or other location within the drift during the post-closure period." The model validation argument was presented for calculated chloride concentration and ionic strength values up to 10 molal.

First, although not explicitly stated, it is assumed that the $\mathrm{pH}, \mathrm{Cl}$ and ionic strength calculations should all three be valid for this range of 0 to $10 \mathrm{~m}$ ionic strength. There are no qualified $\mathrm{pH}$ data used for the validation. Unqualified experimental data are referenced for comparison to $\mathrm{pH}$ predictions, and these are specified as corroborative only. These unqualified data are the only $\mathrm{pH}$ data used and therefore are needed in the validation, and the comparison of predictions to these 
data are available only for the lowest portion of this ionic strength range, predominantly less than 1 molal, with one data point for $\mathrm{pH}$ at of approximately 2 molal ionic strength. Model predictions of $\mathrm{pH}$ are presented, however, for the full range of ionic strength up to 10 molal. Second, limits for the range of validation for all three parameters $(\mathrm{pH}, \mathrm{Cl}$ and ionic strength) should be provided in the documentation.

The High-Relative Humidity Salts Model is assigned to Bin 2, because a compliant validation strategy can probably be constructed, but this will require many changes to the documentation. The intended use criterion should be reviewed for consistency with the actual use in TSPA-SR, which would be less demanding and more likely to achieve compliant validation. The following comments and recommendations should be addressed.

Validate the model $\mathrm{pH}$ predictions by comparing the computed $\mathrm{pH}$ values against available experimental data for appropriate Yucca Mountain waters, or compare them to mixed salt solubility data from the literature for the full range of ionic strength expected for the intended use. If no experimental data are available, then explicitly state in the AMR the upper limit of ionic strength and temperature for which the $\mathrm{pH}$ calculations performed with this model may be used. Extrapolations of $\mathrm{pH}$ predictions to conditions beyond the available data may be permissible if the appropriate methods of validation are used as specified in AP-3.10Q, but will require additional justification in the AMR.

The validation criteria defined in the AMR are: 1) prediction of $\mathrm{pH}$ within 1 unit, 2) prediction of chloride within one order of magnitude, and 3) prediction of ionic strength within one order of magnitude (p. 43 of the AMR). Although the model predictions for chloride and ionic strength easily meet these criteria, the authors should describe in the AMR the justification for these validation criteria. It is the judgement of the reviewer that these criteria are not sufficiently defined to serve as a basis for validation; for example if the experimental values of chloride and ionic strength were 10 molal, then a prediction between 1 and 100 molal would meet either criterion. This is not a reasonable or definitive test because this essentially spans the entire range of possible solution compositions.

The stated use of the model for TSPA-SR is for estimating $\mathrm{pH}$, chloride, and ionic strength, however predictions of other solution species are made in the AMR. The authors should explicitly state that predictions made by the model for other species have not been validated. In addition it should be clarified that the validation for predicting chloride did not consider the prediction of chloride salt solubility in mixed electrolyte systems. For example, the prediction of halite or sylvite solubility in the presence of other electrolytes was not done. Solubility validation was done using only single salt data. In the comparison for J-13 and Topopah Spring pore water data, the calculations demonstrating chloride accuracy are made only for evaporation of water over a range of concentrations below the solubility of any chloride salts; consequently the model was not tested for any process involving chloride other than simple evaporation. This should be a qualifier to the extent of validation.

A discussion of the qualification status of data is needed. For example, it should be clearly stated that the PT4 database is not verified and the experimental data for J-13 and Topopah Spring tuff used in the evaluations are not qualified data sources.

The modeling assumes that brine reactions occur in a "reactor" but the conceptual model for where this reactor is located in the drift and what it represents physically, and the basis for assuming such a reactor would be representative of the process needed for TSPA-SR, are absent. 
A diagram describing the conceptual model and the boundary conditions would also assist in evaluating the process. Similarly, a discussion is needed to put these salt accumulations in context in quantity, effect, and location to other expected accumulations of salts on the drift walls, in the invert, and on the waste package; many of these salts will be impacted by other reactions beyond evaporation. How does the calculation in this AMR fit with other calculations being done for evaporation and salt accumulation in the drift?

It appears that the PT4 database was developed by the authors using the PIT and HMW databases as the basis. Additional coefficients developed by others such as Reardon (1990; see Section 5.3 of the AMR) were added to extend the use of the database to other components. Although Reardon (1990) may have calibrated and tested his coefficients against a limited amount of experimental data, the PT4 database still needs its own verification.

The use of median values for the beta and $\mathrm{C}$ coefficients needs justification in order to be accepted as reasonable. A discussion of how the use of median values affects accuracy would be another topic that should be discussed in the uncertainty section.

On p. 33 of the AMR, the statement is made that "The dissolved solids in the seepage composition are restricted to $\mathrm{Na}, \mathrm{K}, \mathrm{NO}_{3}, \mathrm{Cl}$, and $\mathrm{CO}_{2}$." If this is the case then the detailed discussion about database modifications to accommodate $\mathrm{Al}, \mathrm{F}, \mathrm{Fe}$, etc., may not be needed in the AMR. Now that this version of the AMR is written and it has been determined that a reduced set of species is needed for the TSPA calculations, an existing database such as PIT could be used rather than the newly modified but unverified PT4 database.

It should be noted that Equation 4 in the AMR is only valid if the solution consists of $\mathrm{Na}, \mathrm{K}$, and chloride under equimolar concentrations, and similarly divalent cations and anions in equimolar concentrations.

The ionic strength computed here is not the effective ionic strength, which can only be computed by considering activities and complexes; rather this is the analytical ionic strength and does not represent the true value. Some discussion should be devoted to this point and its relevance to TSPA-SR.

Supplementary Response from Project Staff: The following information is provided by Project staff, as a result of the inter-disciplinary review of this report, as additional perspective on the findings discussed above.

The documented validation range for the High Relative Humidity Salts Model is stated in terms of water activity down to 0.85 (CRWMS M\&O 2001l, p. 66). For evaporated J-13 water this corresponds to a calculated ionic strength of approximately 10 molal, whereas for evaporated Topopah Spring tuff pore water the calculated ionic strength is approximately 6 molal. From evaporation experiments with waters representing Topopah Spring tuff pore water, $\mathrm{pH}$ measurements were obtained for ionic strength up to 2.8 molal. Thus the validation range is based on extrapolation of the measured $\mathrm{pH}$, which is justified because the behavior of this chemical system in the range of 2.8 and 6 molal ionic strength is straightforward, which is evident from measurements other than $\mathrm{pH}$ in the same experiments. Limits for the validation ranges of all three output parameters from this model $(\mathrm{pH}$, chloride concentration, and ionic strength) are more clearly presented in the latest revision of this AMR, which is in review.

The stated validation criteria were met by the model, and for chloride concentration and ionic strength, the criteria were met with considerable margin. Thus it is agreed that the criteria could 
be tightened. The authors maintain that the model predicts all three measures with more than sufficient confidence for the intended use. It is noted that the model-data comparisons used for validation involve independent data, and none of these data were used in development or calibration of the model. It is acknowledged that model predictions for other species have not been validated, and that predictions for chloride concentration did not consider chloride-salt solubility in mixed electrolyte systems. However, none of the chloride salts approach solubility equilibrium in the validation range, and chloride dominates brine chemistry for the Topopah Spring pore water system, so these qualifications have little impact on confidence in the three model outputs over the validation range.

The conceptual model and boundary conditions used to develop the "stirred reactor" modeling approach are addressed in the AMR (CRWMS M\&O 2001l) particularly in reference to the Low Relative Humidity Salts Model. Confidence in application of the the PT4 database, including the assumptions used for estimation of higher order coefficients, is obtained from model-data comparisons used for model validation.

The statement that "dissolved solids in the seepage composition are restricted to $\mathrm{Na}, \mathrm{K}, \mathrm{NO}_{3}, \mathrm{Cl}$, and $\mathrm{CO}_{2}$ " (CRWMS M\&O 2001l, p. 33) applies to the Low Relative Humidity Salts Model which does not involve Pitzer calculations. The PT4 database was developed to include silica and carbonate species, which are important for $\mathrm{pH}$ prediction in the range of interest although the predicted carbonate- and silica-species concentrations are not included in the validated model output. Equation 4 in the AMR is an approximation with justification provided (CRWMS M\&O $2001 l$, p. 45). Finally, as pointed out by the reviewer, the predicted ionic strength is the analytical value, but the colloids model (the "downstream" user of the ionic strength prediction) is calibrated to this definition of the ionic strength (CRWMS M\&O 2001l, p. 45).

\subsubsection{In-Drift Microbial Communities (H.6)}

Description: This model provides total biomass that can be produced from degradation of materials present in the emplacement drifts (e.g., stainless steel, cement grout) and inputs from the natural system (rock and TSw tuff water) as a function of time, using a mass and energy balance approach. The biomass production is based on a) the total energy available through microbially mediated redox reactions, and b) the number of microbes produced based on availability of nutrients. The MING software code implements the model, and calculates biomass as a function of time based on the inputs. From this calculated biomass, inferences can be made concerning microbial effects on $\mathrm{CO}_{2}$ gas generation, generation of colloids, and abundance of biofilms. The model is documented in the AMR: In-Drift Microbial Communities (CRWMS M\&O 2000an).

Use of the Model: None of the outputs are directly used for TSPA-SR.

Review Findings: The Microbial Communities Model is assigned to Bin 2 because additional documentation is needed to achieve compliant validation that is technically defensible. The following comments and recommendations should be addressed in revision of the model documentation.

Develop a diagrammatic conceptual model, outlining the relevant microbial processes and the implications for repository performance. Establish the type of mathematical modeling needed, including comparison with alternatives. Show how the model simulations will actually be used by other models and TSPA. 
The AMR states that no information is available on the degradation lifetimes for materials such as cement, yet median lifetimes are estimated. What is the basis for these estimates? Material lifetimes for the release of $\mathrm{Fe}^{2+}$ from biotite were selected to provide a conservative bound on the rate of oxidation of iron minerals, but neither biotite nor $\mathrm{Fe}^{2+}$ are important labile constituents of the tuff.

In the MING model, it is assumed that gas flux into the drift does not greatly affect the results for calculations. This should be confirmed because the information that is used is based on calculations performed for the Viability Assessment of a Repository at Yucca Mountain (DOE 1998).

It is assumed that calculated biomass is considered to be recycled by heterotrophs, however, this should be justified using analogous microcosm studies reported from the literature, or by direct laboratory testing.

Connections between other modeling and testing investigations on the Yucca Mountain Project, and the assumptions made regarding microbial activity and the chemical environment, should be clarified. For example, page 61 of the AMR states that time dependent flux of gases $\left(\mathrm{CO}_{2}, \mathrm{~N}_{2}, \mathrm{O}_{2}\right.$ and $\mathrm{CH}_{4}$ ) are one of three sources of nutrients. However, there is no evidence that methane will be present in the repository near-field environment and it is not considered in other studies. As another example, laboratory investigation of microbially influenced corrosion of engineered barrier materials is apparently ongoing at Lawrence Livermore National Laboratory (LLNL), but there is no integration as to the types and rates of microbial activity that are likely to be important.

In Tables 11 and 12 of the AMR, the bacteria should be identified as to aerobic or anaerobic. On page 40 of the AMR, the size variations of bacteria are discussed, but there is no discussion of actual sampling and testing that could confirm these size variations.

On page 127 of the AMR it is mentioned that measurements of microbes were made in the exploratory studies facility; however, it is not clear what kind of microbes they measured (whether they are aerobic/thermophiles or anaerobic/nonthermophiles). Similar distinction is also not made in calculating the biomass under variable conditions.

\subsubsection{Gas Flux and Fugacity Model (H.3-1)}

Description: Calculates $\mathrm{CO}_{2}$ and $\mathrm{O}_{2}$ flux through the unsaturated zone under pre-repository conditions using ${ }^{14} \mathrm{CO}_{2}$ measurements and to estimates $\mathrm{CO}_{2}$ and $\mathrm{O}_{2}$ fluxes using air mass-fraction approach based on the thermal-hydrology model. This model is documented in the AMR entitled: EBS Physical and Chemical Environment Model (CRWMS M\&O 2000ab).

Use of the Model: Model output is not used for TSPA-SR, however, it is used for screening; see EBS Features, Events, and Processes (CRWMS M\&O 2001h).

Review Findings: The Gas Flux and Fugacity Model is assigned to Bin 2 because revisions are needed for compliant validation, and the information needed to do this should be readily available. The following comments should be addressed:

For the steady-state isotopic mass balance calculation for $\mathrm{CO}_{2}$ in the unsaturated zone, it is not clear how the ${ }^{14} \mathrm{C}$ associated with the solid phase is included in Equation $6.2-1$ of the AMR. Is it significant or not? Equation $6.2-2$ of the AMR only accounts for the aqueous phase ${ }^{14} \mathrm{C}$. 
The $\mathrm{Kd}$ that is used (Equation 6.2-3 of the AMR) applies only to the partitioning between the gas phase and the aqueous phase, and the solid phase is ignored. This approach is not justified. This $\mathrm{Kd}$ should be consistent with what would be calculated using an equilibrium assumption and established principles of carbonate chemistry. It should depend on the partial pressure of $\mathrm{CO}_{2}$, the $\mathrm{pH}$ of the water, presence of carbonate minerals, and temperature. It will not be constant as indicated in Equation 6.2-9 of the AMR.

The justification for using a constant multiplier of the gas phase diffusion coefficient to account for both the tortuosity of the medium and oscillatory advection is too vague. This assumption needs to be justified through numerical modeling or experimental data.

These models need to be validated against either field or laboratory data to demonstrate that the simplifying assumptions used in the models are reasonable.

The reactive transport model used to develop an estimate of $\mathrm{CO}_{2}$ fugacity in the host rock at the drift wall used a no-flow boundary at the drift wall. This boundary condition appears to be contradictory for computing the $\mathrm{CO}_{2}$ concentration of a through-flux at the drift wall.

\subsubsection{In-Drift Gas Flux and Composition Model (H.5)}

Description: This is a conceptual model of the changes to in-drift gas flux and composition due to thermal perturbation of the geosphere and reactions of the ambient gases with introduced materials of the EBS. The scope of the model is to evaluate the need to include changes to the flux and composition of in-drift gases, particularly carbon dioxide, oxygen, nitrogen and steam in TSPA analysis. Sources described in the TSPA for the Viability Assessment (DOE 1998), as well as potential additional sources and sinks of in-drift gas were identified. Mass balance calculations for oxygen and $\mathrm{CO}_{2}$ were performed to evaluate the effect of repository material on the gases present in the drift over time. The dominant sink modeled for $\mathrm{CO}_{2}$ was carbonation of calcium phases contained in the cement around rock bolts. The dominant sink modeled for oxygen was the oxidation of iron in metal in the repository. This model is documented in the AMR entitled: In-Drift Gas Flux and Composition (CRWMS M\&O 2000am).

Use of the Model: The model output is a conclusion that in-drift gas flux and composition would not be strongly affected by interactions with in-drift and near-drift materials. No information from this model is used directly as input to the TSPA.

Review Findings: The In-Drift Gas Flux and Composition Model is assigned to Bin 2. Validation must address the use of the simplistic model of sinks for $\mathrm{CO}_{2}$ and oxygen in the drift. The analysis of the magnitude of the sink terms compared to the quantities of the ambient gases is straightforward. Validation could be achieved by comparison of the results with conceptual analysis of more complex models to show that the simplistic assumptions are conservative. This AMR is a candidate for combining with EBS Physical and Chemical Environment Model (CRWMS M\&O 2000ab).

\subsubsection{Cement Grout Model (H.3-2)}

Description: This model was used to determine leachate composition for waters coming in contact with cement grout around rock bolts, and to estimate the flux of leachate that could enter the drift opening as seepage. The authors use EQ3/6 to evaluate the leachate composition produced by equilibrium dissolution of an idealized mineral assemblage representing cement. The model uses a geometrical argument to estimate the quantity of leachate that could enter the 
drift opening as seepage through the grout. This model is documented in the AMR entitled: $E B S$ Physical and Chemical Environment Model (CRWMS M\&O 2000ab).

Use of the Model: Model output is not used for TSPA-SR but is used for FEP screening; see EBS Features, Events, and Processes (CRWMS M\&O 2001h).

Review Findings: The Cement Grout Model is assigned to Bin 3 (impact review is provided in Appendix IV) because of the need for extensive revision and enhancement of the modeling approach. The following comments should be addressed.

At $96^{\circ} \mathrm{C}$, ettringite may not be stable but rather sulfate hydrocalumite (monosulfate) may be the stable phase, particularly if sulfate concentrations are low. A method of synthesizing sulfate hydrocalumite is to boil ettringite.

It is not clear how the calcium silicate hydrate phase is handled in the thermodynamic calculations. It behaves like a solid solution and its "solubility product" changes with the leachate composition. In general, a more thorough discussion of the composition of cement and the thermodynamic properties of the constituents needs to be included, and better representation of cement is needed in the chemical model. There should not be a single leachate composition from the cement grout but rather several compositions reflecting multiple invariant points in the composition. The composition will remain relatively constant until the dominant phase controlling the invariant point is removed. The leaching model is not compared to any literature or experimental data.

If the grout is finer-grained than the formation, then it is possible that there is convergence of flow toward the grout when under unsaturated conditions. Flux through the rock bolt may be as much as twice that as through an equal cross section of unsaturated zone due to the higher unsaturated permeability. Clarify the 1.55 factor that is used to account for "an increase in seepage flow from the effects of the rock bolt protruding into the drift."

Impact Review Summary: The Cement Model was not used in TSPA-SR, but was used as the basis for screening out FEPs. The predicted $\mathrm{pH}$ for leachate compositions is considered conservative. In addition, waste package and drip shield corrosion rate models indicate that water compositions similar to the predicted cement leachate compositions have no significant effect on degradation of these components. Additional information developed to address NRC Key Technical Issue agreements related to cement/seepage composition and interactions will provide further confidence in the model. Based on the above information and the additional detail provided in Appendix IV, the model validation review findings associated with this model have no significant impact on the conclusions of TSPA-SR (CRWMS M\&O 2000bm, Section 6.1).

\subsubsection{Seepage/Cement Interaction Model (H.7)}

Description: Analytical model of the rate of carbonation of grout around rock bolts; based on Fick's first law. No engineering or scientific design software programs were used. This model is documented in the AMR entitled: Seepage/Cement Interaction (CRWMS M\&O 2000bf).

Use of the Model: Model output is not used for TSPA-SR and is not used for FEP screening. Output from this model was identified by the reviewer as available for FEP screening, but the AMR is not cited by EBS Features, Events, and Processes (CRWMS M\&O 2001h).

Review Findings: The model is described as an analysis, however, it is a model because it is a representation of a phenomenon (carbonation of grout) that could have implications for the 
chemistry and stability of the drift. The boundary condition at the rock bolt/grout interface is not clearly stated nor justified, and may be inappropriately represented. Modeling is based on literature values of diffusion coefficients that have a wide range of values. There is little or no comparison with measured or reported rates of carbonation. Extensive revision of the model is needed, including justification for the approach. Accordingly, the Seepage/Cement Interaction Model is assigned to Bin 3 (impact review is provided in Appendix IV). This AMR is a candidate for combining with EBS Physical and Chemical Environment Model (CRWMS M\&O 2000ab).

Impact Review Summary: The Seepage/Cement Interaction Model was not used in TSPA-SR and was not used for FEP screening. Consequently, based on this statement and the additional detail provided in Appendix IV, the model validation review findings associated with this model have no impact on TSPA-SR.

\subsubsection{Corrosion of Steel Used in the Ex-Container EBS (H.3-3)}

Description: Calculate the consumption of $\mathrm{O}_{2}$ by steel corrosion and compare with the influx of gas-phase influx of $\mathrm{O}_{2}$ to the emplacement drifts. This model is documented in the AMR entitled: EBS Physical and Chemical Environment Model (CRWMS M\&O 2000ab).

Use of the Model: Model output is not used for TSPA-SR but is used for screening; see $E B S$ Features, Events, and Processes (CRWMS M\&O 2001h).

Review Findings: The Corrosion of Steel Used in the Ex-Container EBS Model is assigned to Bin 3 (impact review is provided in Appendix IV), because extensive revision of the model is needed, including justification for the approach. The following comments should be addressed:

The model is based on Equation 5-1 of the AMR, which is referenced to another source (Farmer et al., 1998). The equation is developed for one value of the $\mathrm{O}_{2}$ fugacity and then extended to other fugacity values (Equations 6.3-13 and 6.3-14 of the AMR). In particular, the use of Equation 6.3-13 is assumed without justification. The penetration rate in the presence of diminished or variable oxygen fugacity may actually involve the $b_{0}$ or $b_{1}$ terms or an additional term in the exponent in Equation 5-1, rather than a simple multiplier of the exponential term. These assumptions are recognized and identified as to-be-verified in the report, but are not justified. Also, discussion of Equation 6.3-14 of the AMR equates fugacities and the fraction of influx, which does not seem to be obvious and needs to be clarified.

The model implicitly assumes that corrosion occurs only in the presence of dissolved $\mathrm{O}_{2}$; however the reaction $\mathrm{Fe}(0)+2 \mathrm{H}_{2} \mathrm{O}=\mathrm{Fe}^{2+}+2 \mathrm{OH}^{-}+\mathrm{H}_{2}$ may also occur. The rationale for neglecting this reaction needs to explained, or the approach revised.

The report mentions potential analogs for $\mathrm{O}_{2}$ consumption by steel corrosion (without references) and suggests that $\mathrm{O}_{2}$ consumption may be rapid. Yet, $\mathrm{Fe}(\mathrm{III})$ is assumed as the corrosion product rather than Fe(II). There may be a potential contradiction in the way it is presented; if the model is a bounding approach, then this needs to be explained and justified.

The microbial factor is assumed to be a simple multiplier of the rate obtained from Equation 5-1 of the AMR. Even if the method for including microbial effects is valid, the magnitude of the factor (a factor of 6) needs to be verified since it is based on preliminary data, as stated. Similar to the assumption for the fugacity, there may be a better representation of microbial effects on the rate of steel corrosion. 
Impact Review Summary: The Corrosion of Steel Used in the Ex-Container EBS Model estimates a range of effects from steel corrosion on the oxygen content of the gas phase in the emplacement drift. The approach used is considered conservative, and the calculated range of oxygen fugacities is less than the range needed to significantly affect the redox potential of the aqueous phase. Further model development, testing, and comparison to natural or man-made analogs would not likely change this conclusion. The model was not used in TSPA-SR but was used for screening out FEPs. Based on this information and the additional detail provided in Appendix IV, the model validation review findings associated with this model have no impact on the conclusions of TSPA-SR (CRWMS M\&O 2000bm, Section 6.1).

\subsubsection{Effect of Evaporation in the Invert (H.3-4)}

Description: Show the effects of evaporation on the porosity and other hydrologic properties of the invert. This model is documented in the AMR entitled: EBS Physical and Chemical Environment Model (CRWMS M\&O 2000ab).

Use of the Model: Model output is not used for TSPA-SR but is used for screening; see $E B S$ Features, Events, and Processes (CRWMS M\&O 2001h).

Review Findings: The Effect of Evaporation in the Invert Model is assigned to Bin 3 (impact review is provided in Appendix IV) because of the need for extensive revision and enhancement of the modeling approach. The following comments should be addressed:

There are no measurements of change in porosity with precipitation of salts against which the model can be validated.

The model assumes a one-dimensional system but the flow may be three-dimensional, in which case the distribution of minerals formed by evaporating waters would not be uniform.

The model is based on post-processing of the output from thermal-hydrologic models, the THC Seepage Model (see 6.6.2 of this report), and the Normative Precipitates and Salts Model (see 6.8.10 of this report), and therefore its validation depends on (but is not guaranteed by) the validation of these models. It is recommended that, as an alternative, simulation of THC processes in the invert should be done entirely within the reactive transport simulator.

Impact Review Summary: The Effect of Evaporation in the Invert Model was not used in TSPA-SR but was cited in the screening of EBS FEPs. The screening argument recognizes that the invert is a minor barrier to flow in comparison to the drip shield, waste package, and unsaturated zone beneath the drift. Changes in porosity of the invert would have little effect on radionuclide transport. The typical depth dimension of the invert, about one meter, is much less than the transport distance through the unsaturated zone, and the effect on radionuclide transport is therefore much less. Based on this argument and the additional information provided in Appendix IV, there is a negligible impact on the conclusions of TSPA-SR (CRWMS M\&O $2000 \mathrm{bm}$, Section 6.1) from the model validation review findings associated with this model.

\subsubsection{EBS Colloids Model (H.3-5)}

Description: This model provides bounds for radionuclide transport in the emplacement drifts by colloids formed from steel corrosion products. This model is documented in the AMR entitled: EBS Physical and Chemical Environment Model (CRWMS M\&O 2000ab). 
Use of the Model: Model output is not used for TSPA-SR and is not used for FEP screening. Although cited in EBS Features, Events, and Processes (CRWMS M\&O 2001h), the FEP is included, not excluded, based on other models as discussed below and in Appendix IV.

Review Findings: The EBS Colloids Model is assigned to Bin 3 (impact review is provided in Appendix IV) because of the need for extensive revision and enhancement of the modeling approach. The following comments should be addressed:

Sorption to iron-oxide particles is estimated based on constant partition coefficient (Kd), although in general, sorption depends on $\mathrm{pH}$, ionic strength, and the aqueous concentrations. Such an assumption requires justification and supporting data. The applicability of the laboratory data used for $\mathrm{Kd}$ values, to repository drift conditions, is not established. If adsorption is nonlinear or $\mathrm{pH}$ and ionic strength are not constant then the enhancement factors for radionuclide transport are functions of aqueous concentration, $\mathrm{pH}$, and ionic strength.

The model assumes the colloids are comprised of hematite, although a more conservative approach would use goethite because it has a larger specific surface area and sorption capacity.

The concentrations and size distributions of colloids derived from the corrosion of the steel may be very different from those derived from natural processes in the aquifer, hence the representativeness of the groundwater colloid data used in this model is questionable. If the natural colloid data are used to bound colloid concentrations and size, further justification is required. Experimental data are needed to provide more reliable estimates of colloid concentrations.

Impact Review Summary: The EBS Colloids Model was not used in TSPA-SR and did not serve as a basis for screening out FEPs. Colloidal transport in the waste package and the excontainer engineered barrier system is included (not excluded) in TSPA-SR based on the In-Drift Colloids and Concentrations Model (M.3; Section 6.13.1) and the Waste Form ColloidAssociated Concentrations Limits: Abstraction and Summary (K.22; Section 6.11.30). The EBS Colloids Model bounds only one aspect of colloid transport, and will not be carried forward to the potential License Application. Based on the above information and the additional detail provided in Appendix IV, there is no significant impact on the conclusions of TSPA-SR (CRWMS M\&O 2000bm, Section 6.1) from the model validation review findings associated with this model.

\subsubsection{Normative Precipitates/Salts Model (H.3-6)}

Description: The model is intended to predict which minerals and salts will form when water that is similar in composition to J-13 well water, or to Topopah Spring welded tuff matrix porewater, is evaporated completely. This model is documented in the AMR entitled: $E B S$ Physical and Chemical Environment Model (CRWMS M\&O 2000ab).

Use of the Model: Model output is not used for TSPA-SR but is used for screening; see $E B S$ Features, Events, and Processes (CRWMS M\&O 2001h).

Review Findings: The Normative Precipitates/Salts Model is assigned to Bin 2 because revisions are needed for compliant validation, and the information needed to do this should be readily available. The following comments should be addressed:

In the normative analysis there are only 11 measured components (e.g., Tables 6.5-1 and 6.5-2 of the AMR) but there are 16 precipitates listed in Table 6.5-15. It appears that the number of 
unknowns (amount of each phase dissolved or precipitated) exceeds the number of known quantities (change in the concentrations of the various aqueous components). This apparent problem needs to be clarified.

The laboratory experimental data were acquired at atmospheric $\mathrm{P}\left(\mathrm{CO}_{2}\right)$. If the $\mathrm{P}\left(\mathrm{CO}_{2}\right)$ in the drift is expected to higher, the results by be different. May be clarified with simple calculations and arguments.

Because smectites were precipitated, it seems that $\mathrm{Al}$ should have been measured in the solutions. The possibility of precipitating gibbsite or some other Al-oxyhydroxide instead of alumino-silicates should be evaluated.

The normative model requires a priori decisions about what phases will be included. If these choices were based on the results of the powder x-ray diffraction results, then the statement that the normative results replicate the experimental results is a circular argument.

\subsection{Model Area I: EBS Moisture Distribution and Thermal-Hydrology}

\subsubsection{Multiscale TH Model (I.1)}

Description: The Multiscale Thermohydrology Model (MSTHM) is used to calculate thermodynamic conditions in the emplacement drifts and near-field environment (in-drift temperature, relative humidity, liquid saturation, etc.) throughout the repository as a function of time. The MSTHM couples several simpler submodels to obtain the final results. The submodels are used to extrapolate from a 3-D heat conduction analysis to a local thermohydrologic result.

The submodels conceptually include: a 3-D mountain-scale heat conduction model (SMT), a planar drift-scale heat conduction model that represents the length of a single drift with different waste packages and extends from below the water table to the surface (DDT), a drift-scale heat conduction model that represents a local column extending from below the water table to the surface (SDT), and a matching drift-scale thermohydrologic model that represents the same local column using a line-averaged heat source and extends from below the water table to the surface (LDTH). The LDTH model includes the flow of water and water vapor through partiallysaturated fractured rock. All submodels use the NUFT simulation code. This model is important in assessing post-closure performance.

The model is documented in the AMR entitled: Multiscale Thermohydrology Model (CRWMS M\&O 2000aw).

Use of the Model: In-drift temperature, relative humidity, liquid saturation, etc., are input to: Abstraction of NFE Drift Thermodynamic Environment and Percolation Flux (CRWMS M\&O 2001c) for use in TSPA-SR.

Review Findings: The model divides the repository area into 31 columns at which detailed calculations are performed. These independent column results are then interpolated using the 3-D model and the planar waste package model. As an example, the interpolation steps used to calculate the repository temperatures are summarized below (from Section 6.6.3.1 of the AMR):

- Step 1: Perform calculations at each of the 31 columns using both the heat conduction (SDT) and thermohydrologic (LDTH) models. This is done for five different thermal loadings. Perform one planar heat conduction calculation of the 
drift with different waste packages (DDT) and one 3-D heat conduction calculation of the mountain (SMT).

- Step 2: Store the mountain-scale results at locations where final results are desired (623 locations). This is the average host rock temperature at each location.

- Step 3: Develop relationships between the column thermal conduction model results and the column thermohydrologic models at each of the 31 column locations for each of the thermal loadings.

- Step 4: Interpolate the relationships developed in Step 3 to the locations identified in Step 2.

- Step 5: Bracket the 3-D peak mountain-scale heat conduction temperature at each location in Step 2 with the peak column heat conduction temperatures for the different thermal loads at the same location and then use this interpolation to obtain the corresponding column thermohydrologic temperature at this location.

- Step 6: Adjust these temperatures using the planar drift model to account for different waste package thermal loads.

Similar interpolations used for other variables. It is important to note that this interpolation/extrapolation procedure is not the same as the product solution procedure used to extend 1-D results to multiple dimensions in classical conduction problems. The product solution procedure is based on separation of linear partial differential equations into two or three ordinary differential equations. The interpolation/extrapolation procedure in this AMR projects from a thermal conduction temperature to a nonlinear thermohydrologic solution and then makes a final adjustment on that temperature.

The Multiscale Thermohydrology Model is assigned to Bin 2 for the following reasons:

1. The interpolation/extrapolation procedure using submodels described in 6.9.1 of this report is not validated:

- It uses temperature obtained from heat conduction models (SMT and SDT) to extrapolate into the thermohydrologic (LDTH) results. A conduction-only adjustment is made to the interpolated thermohydrologic results. Use of this procedure introduces an assumption that conduction is more important than convection at the mountain-scale. However, the results of the Mountain-Scale Coupled Processes (TH) Models show that TH effects dominate (see Section 6.4 .1 of this report). A better alternative would be to use a relatively coarse mountain scale thermohydrologic model, and then use results from that analysis as boundary conditions on a more refined thermohydrologic drift-scale model.

- The procedure assumes that vertical columns do not interact horizontally and that lateral flow above the repository is not significant. These assumptions need to be justified because the contacts of the different stratigraphic units are sloped, which could cause lateral flow. Justification for these assumptions is also needed because of the existence of perched water, which indicates that lateral flow can occur in portions of the unsaturated zone.

- Results are only presented for plan views at the repository depth and time histories at selected locations. These results are obtained after interpolation and extrapolation of 
submodel results. To properly evaluate the model, typical results of submodels should be presented. For example, a vertical section of SMT (Smeared-heat-source, Mountainscale, Thermal-conduction) results should be presented. Similarly, no results are presented for typical SDT (Smeared-heat-source, Drift-scale, Thermal-conduction), LDTH (Line-averaged-heat-source, Drift-scale, Thermohydrologic), or DDT (Discreteheat-source, Drift-scale, Thermal-conduction) submodels. Therefore, the reviewer can not evaluate the magnitude of the interpolations being used between submodels. Finally, the interpolation/extrapolation procedure could be used to provide 3-D data that would allow comparison with the results of the Mountain-Scale Coupled Processes (TH) Models (CRWMS M\&O 2000av).

- The numerical mesh used in an analysis has a significant bearing on the results. No figures are given for review of any of the submodels (except for a local figure showing the mesh around the drift).

- No validation of the interpolation procedure is provided, other than to say the results were checked carefully. A simplified verification problem could be provided that shows that the interpolation procedure gives the same results as a full $3-D$ analysis of the verification problem.

2. Potentially significant effects, such as thermal-mechanical (TM) and thermal-hydrologicchemical (THC) coupling, are not discussed and no justification is given for not including them. TM and THC effects can change flow by causing fracture aperture changes due to thermal stress or boiling and condensation, which can result in precipitation and sealing of flow paths. Transmissivity of flow through a fracture is a cubic function of fracture aperture. Therefore, the flow through a fracture is highly sensitive and nonlinearly sensitive to changes in fracture aperture. Fracture apertures are affected by both mechanical and chemical processes and these must both be seriously considered when modeling flow in fractured rock.

- In the Dixie Valley, Nevada geothermal reservoir, the United States Geological Survey (USGS) has claimed there is a correlation between the orientation and magnitude of insitu stresses, and the orientation and transmissivity of fractures in that reservoir. While geothermal reservoirs are often extensively fractured, relatively few fractures exhibit significant permeability. In the past, this was generally explained as due to mineral precipitation sealing most of the fractures. Now a consensus may emerge that fractures will eventually seal, while only the tectonically active ones remain open. The AMR should discuss the state of stress and the magnitude of the thermally induced stresses at Yucca Mountain before dismissing thermo-mechanical effects.

In 1998, LLNL developed a model of drift-scale thermal-hydrologic-chemical processes in a Yucca Mountain repository (work that is partially described by Hardin 1998, Section 5.6.5) concluding that a mineral cap would form above the waste packages due to boiling and the precipitation of minerals above the waste packages.

- Fractures, joints, and faults occur on many dimensional scales. The AMR does not address the heterogeneity of permeability from fractures on these different scales. There may be a few large scale heterogeneities that act as rapid transit channels for flow. Again, Yucca Mountain is not a geothermal reservoir, however, in some geothermal reservoirs, tracers have shown rapid transport to distant wells while completely bypassing nearby wells. In the documents reviewed, only pneumatic tests are presented, not liquid tracer 
results. Liquid driven by gravity is likely to move in different paths than the gasses used in pneumatic tests around the potential repository. The active fracture concept is a significant step in a realistic direction for modeling flow in fractures, but geostatistical descriptions and significant quantities of field and laboratory verifications are needed before fractured rock can be modeled with a reasonable level of confidence in the precision of the results.

3. Validation does not make full use of existing data as recommended in AP-3.10Q Section 5.3.b. Specifically, the following uses are not developed:

- No calculations are made to show that the model accurately represents the ambient conditions (natural state). The capability to accurately represent the natural state is a powerful demonstration that geologic properties are represented accurately.

- The model does not replicate known perched water data. Perched water provides an opportunity to validate assumed hydrogeology and analysis.

4. The validation argument uses the model applied in a different manner than used in AMR. In the model validation section, documentation of the comparisons of NUFT with Large Block Test data and the Drift Scale Test data are given. However, it should be noted that for these comparisons, NUFT was run as a thermohydrologic analysis, not a conduction-only analysis. Therefore, these results do not directly validate this AMR, since a different modeling approach is used. This reinforces the need to validate the conduction-only models used in the AMR.

\subsubsection{Water Drainage Model (I.2-1)}

Description: The Water Drainage Model is used to quantify the capability of the drift to remove water naturally after drift backfill. The model represents a vertical column near the center of the repository, extending from the water table to the surface. Simplifying assumptions are made to justify use of steady state flow calculations, rather than transient coupled thermal-hydrologic (TH) calculations. Similarly Thermal-Hydrological-Chemical (THC) and Thermal-HydrologicalMechanical (THM) effects are represented by assuming completely blocked fracture flow in selected locations below the waste packages.

Calculations are made using a 2-D NUFT model of the column. The drift is represented in crosssection, with the area under the drip shield represented as an impermeable solid. The backfill and invert are represented, and the area above the backfill is modeled as host rock. Results consist of the saturation at selected cells in the bottom of the drift.

This model is documented in the AMR: Water Distribution and Removal Model (CRWMS M\&O 2001t), supported by model development in the AMR: Water Drainage Model (CRWMS M\&O 2000bv).

Use of the Model: Model output is not used for TSPA-SR but is used for screening; see EBS Features, Events, and Processes (CRWMS M\&O 2001h).

Review Findings: The Water Drainage Model is assigned to Bin 2. Several assumptions are made to simplify the analysis and further justification is required of these assumptions before the model can be considered validated. Revision should consider the following comments:

The use of a column model assumes that lateral flow above the repository is not significant. If such flow would occur, this would make possible additional focusing of infiltration. Neglecting 
lateral flow needs to be justified, especially in light of the observations of natural perched water in the repository host rock formation, which demonstrates the possibility for lateral flow.

Only one column in the center of the repository was selected for analysis, with a mean glacial infiltration rate of $38.66 \mathrm{~mm} / \mathrm{yr}$ (Section 6.1 .6 of the AMR). However, based on data supplied in Table I-2 of the AMR, mean glacial infiltration rates above the repository vary over a range of 0.733 to $65.0 \mathrm{~mm} / \mathrm{yr}$. The justification for selection of the $38.66 \mathrm{~mm} / \mathrm{yr}$ infiltration rate should be given.

Thermal-Hydrologic-Chemical and Thermal-Hydrologic-Mechanical effects are represented by plugging of the fractures below the invert and the engineered barrier segment. However, the permeability of the rock matrix is not changed, so some flow continues. The usual mechanism assumed to cause plugging of fractures is the formation of heat pipes with associated deposition and dissolution in the fractures. However, heat pipes will not form below the drift. Therefore this process can not be used to justify plugging of only fractures. Instead, whatever process (such as rock flour) is assumed to cause plugging of the fractures could at the same time plug the porous matrix. Justification of assuming plugging only the fractures should be given.

Multiple results are presented in the AMR, including a sand drain and the possibility of no backfill. It is not clear how these options will be incorporated into the final design procedure.

\subsubsection{Water Diversion Model (I.2-2)}

Description: The Water Diversion Model is used to quantify the performance of the drift shield to divert water naturally after drift backfill. The model uses the properties of water at $60^{\circ} \mathrm{C}$ and applies physical principles and the Navier-Stokes equations for constant viscosity and incompressible flow to derive equations for liquid drop size, crevice flow and thin film flow rates with condensation. Simplifying assumptions, such as crevice flow behavior as flow between parallel plates, pendant drops fall from fractures no larger than $1 \mathrm{~mm}$, etc., are made, which are generally conservative.

Calculations are made with Mathcad 7 and checked by hand. The analytic solutions assume the drift shield is symmetric and deformation, such as by rock fall, is not considered. Deposition and mineral precipitation in the capillary barriers are also neglected and the author's definition of viscosity is misleading, however, the results are not affected.

This model is documented in the AMR: Water Distribution and Removal Model (CRWMS M\&O 2001t), supported by model development in the AMR: Water Diversion Model (CRWMS M\&O 2000bu).

Use of the Model: Model output is not used for TSPA-SR but is used for screening; see $E B S$ Features, Events, and Processes (CRWMS M\&O 2001h).

Review Findings: The phenomena are mathematically modeled directly from relatively simple equations and physical principles. This treatment is accurate and properly based upon the relevant physics. The validation discussion is arguably compliant because the intended use does not include application to TSPA-SR, and because of the simple nature of the model. Accordingly, the Water Diversion Model is assigned to Bin 1.

\subsubsection{Thermohydrologic Model (I.2-3)}

Description: The Thermohydrologic Model is used to quantify the environmental conditions within and around the EBS as a function of time, in the presence of seepage imposed as a flux of 
heated water into the emplacement drift. This model is documented in the AMR: Water Distribution and Removal Model (CRWMS M\&O 2001t).

The model represents a vertical column in the repository, extending from the water table to the surface, considering a drift near the center, and a drift near the boundary of the repository. Transient coupled thermal-hydrologic (TH) calculations are made and the active fracture concept is applied to a laterally homogeneous dual permeability continuum. Only porous continuum properties are applied to the EBS backfill materials. Heat generation decay curves are used modified for the no-backfill case and the effect of ventilation is included. Thermo-mechanical and thermo-chemical couplings are not considered.

Calculations are made using a 2-D NUFT model considering upper, mean, and lower infiltration cases. The drift is represented in a symmetric cross-section with the drip shield represented as an impermeable solid. Results consist of time histories of temperature, saturation, relative humidity, liquid flux and evaporation rate in the in-drift environment.

Use of the Model: Model output is not used for TSPA-SR but is used for screening; see EBS Features, Events, and Processes (CRWMS M\&O 2001h).

Review Findings: The model correctly postulates that neglecting drift-scale heterogeneities leads to the result of no discrete seeps into the drifts (pp. 107 and 108 of the AMR). However, because fractures in the host rock range in permeability over many orders of magnitude, it seems reasonable to hypothesize that high-permeability fracture channels may lead to discrete seeps within the drifts. Recognizing this possibility, the authors investigate the effect of discrete seeps based on arbitrarily assumed rates. It is also correctly stated in the AMR that to model the effects of drift-scale fracture heterogeneities in a 3-D simulation would be the most appropriate way to predict if discrete seeps are possible. Further analysis of this possibility and justification of homogeneous fracture properties in each hydrogeologic unit are required before the model can be considered validated. Accordingly, the Thermohydrologic Model is assigned to Bin 2.

Thermo-mechanical and thermo-chemical couplings are not considered. Because saturation and humidity in the drift environment may be sensitive to mechanical deformation and mineral sealing of fractures, the effects of these couplings must be addressed, or neglecting these effects must be justified before validation.

\subsubsection{Drip Shield Condensation Model (I.2-4)}

Description: The software, input, assumptions, and parameters used for the Thermohydrologic Model documented in the AMR entitled Water Distribution and Removal Model (CRWMS $\mathrm{M} \& \mathrm{O} 2001 \mathrm{t}$ ) are used to quantify the potential for condensation on the drip shield as a function of time.

The Thermohydrologic Model represented a vertical column in the repository, extending from the water table to the surface. Transient thermal-hydrologic calculations were made for this scale, and applied to calculate the condensation potential on the drip shield.

Calculations are made using a 2-D NUFT model, and a spreadsheet routine was used to postprocess the results, calculate a condensation index, and interpolate values in steam tables.

This model is documented in the AMR: Water Distribution and Removal Model (CRWMS M\&O 2001t), supported by model development in the AMR: In-Drift Thermal-Hydrological-Chemical Model (BSC 2001r). 
Use of the Model: Model output is not used for TSPA-SR but is used for screening but is used for screening; see Engineered Barrier System Features, Events, and Processes (CRWMS M\&O 2001h).

Review Findings: The condensation index is very close to unity throughout the time history, however, the condensation index predicts condensation only after about 50,000 yr. This suggests that the uncertainty in this result should be rigorously analyzed. Also, a comparison to other test results should be conducted before validation. Accordingly, the Drip Shield Condensation Model is assigned to Bin 2.

\subsubsection{In-Drift THC Model (I.7)}

Description: This model is used to analyze thermal-hydrologic processes within the emplacement drifts, with the drip-shield (and backfill) in place. The software, input, assumptions, and parameters are similar to those used for the Thermohydrologic Model (Section 6.9 .4 of this report) with the notable exceptions that a coarse grid, and the equivalent continuum model (ECM) approach, are used with the NUFT code. The focus of this model is on the potential for condensation on the drip shield as a function of time and simulation of quarter-scale drip shield test results. There is no chemistry component of this model, which was to be added in a later revision.

The model domain is a vertical column in the repository, extending from the water table to the surface. Transient thermal-hydrologic calculations are made for this scale and applied to calculate the condensation potential on the drip shield. Calculations are made using a 2-D NUFT model.

This model is documented in the AMR: In-Drift THC Model (BSC 2001r). This model is a candidate for combination with the Drip Shield Condensation Model documented in the AMR Water Distribution and Removal Model (CRWMS M\&O 2001t).

Use of the Model: Model output is not used for TSPA-SR.

Review Findings: The model-data comparison is unsatisfactory for the intended use of the model to represent repository processes, and the In-Drift THC Model is assigned to Bin 3 (impact review is provided in Appendix IV). The following additional comments are offered for consideration:

The quarter-scale experimental testing was very limited in scope and applicability, and provided a marginal contribution to validation. The test was for the "backfill" condition and no model-data comparison was done for the "no-backfill" condition.

Gridding is not consistent with recommended practice; the model is based on rectilinear Cartesian representation of cylindrical geometry (i.e. a flat drip shield) and this is too inaccurate to resolve radiative heat transfer and other processes that will affect condensation on drip shield.

The model applies a porous-medium "Darcy's Law" type formulation to simulate clear-fluid convective processes. Such processes are better described by a full Navier-Stokes representation, which is completely different from the system of equations solved by NUFT.

Impact Review Summary: The In-Drift THC Model was not used in TSPA-SR and did not serve as a basis for screening out FEPs. The model was preliminary and was developed for the backfill case (coupled thermal-hydrologic-chemical processes were to be incorporated in a 
revision). Consequently, the model validation review findings associated with this model have no impact on TSPA-SR.

\subsubsection{Effective Thermal Conductivity Model (I.8)}

Description: The model derives a simplified "thermal conductivity" parameter used to approximate in-drift coupled radiation, convection, and conduction modes of heat transfer for NUFT simulations representing both pre- and postclosure thermal evolution of the repository emplacement drifts.

The model is documented in the calculation report entitled: Effective Thermal Conductivity for Drift-Scale Models Used in TSPA-SR (CRWMS M\&O 2001g).

Use of the Model: The effective thermal conductivity approximation is used in the Multiscale Thermohydrology Model (CRWMS M\&O 2000aw) for thermal-hydrology calculations that are abstracted for use in TSPA-SR.

Review Findings: The model is documented as a calculation, but should be a model so that it is appropriately validated. No validation is documented, and no model-data comparison was attempted. The Effective Thermal Conductivity Model is assigned to Bin 3 (impact review is provided in Appendix IV), because it is an oversimplification and the correct representation of these processes is an explicit approach that is different altogether. The following additional comments are offered for consideration:

The model assumes concentric cylindrical geometry for the drift opening and the waste package/drip shield. This does not represent the geometry of in-drift components and therefore cannot adequately investigate or represent the potential for "hot spots" in the drift. Also, the model approximates thermal radiation and buoyant convection using the Fourier rate law, which is a completely different physical process. Certain input properties used for this model do not correspond to those for other drift-scale models that support TSPA-SR.

Impact Review Summary: The Effective Thermal Conductivity Model is a simplification that was used in TSPA-SR but was not used as a basis for screening out FEPs. Temperatures computed using this approach are spatially smoothed such that local spot variations in temperature along the surfaces of waste packages, drip shields, or drift walls are not represented. The effect of this smoothing on TSPA-SR is small because the evolution of temperature and humidity primarily affect the timing, but not the occurrence, of other processes such as waste package degradation. Furthermore, the TSPA-SR component models do not have the spatial resolution that would be needed to make use of more detailed information on the variability of temperature in the repository. Also, the effects of small differences in temperature or humidity on processes such as corrosion, as represented in the TSPA-SR model, are minor. Based on the above information and the additional detail provided in Appendix IV, the model validation review findings associated with this model have no significant impact on the conclusions of TSPA-SR (i.e. annual dose calculations; CRWMS M\&O 2000bm, Section 6.1)

\subsubsection{Ventilation Model (I.5)}

Description: This model is used to simulate pre-closure forced ventilation in the emplacement drifts. Forced ventilation is an important means by which heat from the waste packages would be removed from the potential repository. The model is documented in the AMR: Ventilation Model (CRWMS M\&O 2000bx). Forced ventilation or convection would act in concert with thermal 
radiation and buoyant convection to transfer heat within the repository (e.g., to the drift wall or from warmer regions of the repository to cooler regions).

The Ventilation Model considers only sensible heat transfer. Moisture removal by ventilation and potential water movement in the rock mass are not included. The model includes simplified representations of conduction, thermal radiation, and buoyant convection, for simplified geometry. The software code ANSYS (version 5.2) is used to support the model.

Use of the Model: Ventilation heat removal rates are used as input for thermal models of the repository and thermal-hydrologic coupled process models used for TSPA-SR.

Review Findings: The Ventilation Model is not validated for its intended use, and is assigned to Bin 3 (impact review is provided in Appendix IV). Although the ventilation system for the repository is not yet at a conceptual design stage, an effective ventilation model is needed to evaluate the effects of pre-closure ventilation on post-closure performance and to include the operant physics in accurate calculations of the waste package temperatures during both pre- and postclosure periods. The following comments summarize review of the Ventilation Model:

Wall temperatures are "averaged" for a lumped radiation calculation; this approach neglects "hot spots" on waste packages due to geometry effects. Buoyancy is not modeled; this is important because warm air rises affecting the temperature distribution in the drift downstream. Using a single "average" air velocity cannot account for "dead zones" caused by invert, WP support, etc., which could have an important effect on the temperature distribution. Local drift wall temperature is "pre-specified" in the model and needs to be fully coupled to the in-drift calculation for accurate simulation. Moisture effects have not been included, which are important because latent heat could be removed from the system by evaporating water thus increasing ventilation efficiency, and because humid air has less heat capacity than dry air thus decreasing ventilation efficiency. The code used (ANSYS) is qualified for structural analysis and not well suited for flow physics of this type. Finally, there is no model-data comparison provided in the AMR, which would be required for complex simulations of this type.

Impact Review Summary: The Ventilation Model was not used directly in TSPA-SR and did not serve as a basis for screening out FEPs. Thermal analyses and thermal-hydrologic models that support TSPA-SR represent pre-closure ventilation as a decrease in the waste heat output by a fixed proportion; the more detailed information produced by the model is not used. The model is used only to demonstrate the feasibility of thermal management using forced ventilation. More detailed calculations of ventilation performance will be conducted in the future, in conjunction with design of the ventilation system. Based on this information and the additional detail provided in Appendix IV, the model validation review findings associated with the Ventilation Model have no impact on the conclusions of TSPA-SR (CRWMS M\&O 2000bm, Section 6.1).

\subsection{Model Area J: Waste Package/Drip Shield Degradation}

\subsubsection{General and Localized Corrosion of the Waste Package Outer Barrier (J.1)}

Description: This model provides a master process-level model, composed of multiple submodels for dry oxidation, humid air corrosion, and aqueous-phase corrosion, addressing both general and localized modes for corrosion of Alloy-22.

If the relative humidity threshold, which is based on the deliquescence point of $\mathrm{NaNO}_{3}$ and is a function of temperature (approximately 50\% relative humidity) is exceeded, dry oxidation will 
not occur, but humid air corrosion will take place and aqueous phase corrosion (APC) will occur in dripping water (the presence of salts may change the threshold relative humidity for APC). The microbial induced corrosion potential remains to be addressed. The model recommends that the effects of aging be represented by a corrosion enhancement factor.

Use of the Model: The model supports the key elements of the approach used to model general and localized corrosion of Alloy-22 for TSPA-SR, implemented in WAPDEG Analysis of Waste Package and Drip Shield Degradation (CRWMS M\&O 2000br).

Review Findings: Model predictions are compared with measurement data to establish confidence. A compliant validation argument can probably be constructed, by addressing the following comments, hence the General and Localized Corrosion of the Waste Package Outer Barrier Model is assigned to Bin 2.

The model uses general corrosion and critical potentials for projecting the occurrence of general and local corrosion. This may not be completely satisfactory and additional justification is needed. The sub-model applicable to localized corrosion needs to be expanded to include pitting and crevice corrosion for both welded and unwelded Alloy-22. The localized corrosion submodel should be revised to incorporate available new experimental electrochemical corrosion data.

Consistent and conceptually valid probabilistic methodology should be established for obtaining subjective probabilities for total system uncertainties and determining stochastic representations of variability in physical data and performance parameters. In other words, more use of expert judgement is recommended to avoid excessive reliance on deterministic modeling of critical components. Identification of causal relationships would be of benefit in developing probabilistic descriptions of barrier performance; for example, stating the influence of environmental factors such as rockfall and the chemical environment.

It would be beneficial to correlate corrosion data (i.e., on model-data comparison plots) as the sum of a general (uniform) term plus local (pitting) terms, where appropriate. Local corrosion would appear to be a much greater risk for failure, thus it is important to characterize local corrosion explicitly. Local corrosion could be characterized using closed form, stochastic functions that can be more readily interpreted by reviewers, thus increasing confidence.

\subsubsection{Aging and Phase Stability: Precipitation Model (J.2-1)}

Description: This report evaluates phase stability of the waste package outer barrier (WPOB) to support modeling of mechanical properties and corrosion. Two models are presented in the AMR: Aging and Phase Stability of Waste Package Outer Barrier (CRWMS M\&O 2000d) both of which are preliminary and are intended for bounding of potential microstructural changes. This review pertains to the model for precipitation of intermetallic compounds.

The rate of precipitation of second phase particles is evaluated using optical and electron microscopy (SEM and TEM) of coupons of Alloy-22 aged for various times between 1 and 16,000 hours at temperatures from 593 to $800^{\circ} \mathrm{C}$. For the purposes of this model, no distinction is made between various types of precipitates. The times at which precipitates begin to cover grain boundaries, twin boundaries, and within grains, are determined in order to develop an initial estimate of the precipitation kinetics. Nucleation and growth are modeled as an exponential process and the rates of precipitation are plotted as $\log$ (time) vs. reciprocal temperature. These data are extrapolated to repository relevant temperatures to determine the time at which precipitation may be expected to begin. Allowing for the fact that this model is 
very conservative, it still does not predict any precipitation in 10,000 $\mathrm{yr}$ at temperatures as high as $300^{\circ} \mathrm{C}$. The model is supported by calculations of diffusion and precipitation rates using comparison of activation energies derived from this data vs. published results and binary diffusion data.

Use of the Model: The model output consists of a bounding value for the precipitation of intermetallic compounds at the repository temperature. No precipitation is expected in $10,000 \mathrm{yr}$, however, a bounding argument was made for TSPA-SR to account for aging effects and to address uncertainty in the data.

Review Findings: The model does not yield quantitative results for the precipitation of secondary phases at repository temperatures, but this is a direct result of the extremely slow (bordering on nonexistent) rates of the processes of interest. Inclusion of results from ongoing testing and more detailed thermodynamic and kinetic modeling, should be sufficient to adequately validate this model. Accordingly, the Aging and Phase Stability: Precipitation Model is assigned to Bin 2.

\subsubsection{Aging and Phase Stability: Long Range Ordering Model (J.2-2)}

Description: This model evaluates phase stability for the Alloy-22 materials of the waste package outer barrier (WPOB) to support modeling of mechanical properties and corrosion. The model is documented in the AMR entitled: Aging and Phase Stability of the Waste Package Outer Barrier (CRWMS M\&O 2000d). This is one of two models that are presented in the AMR, both of which are preliminary and are intended for bounding of potential microstructural changes. This model for long-range ordering (LRO) of the alloy matrix is documented in Section 6.5 of the AMR. A process similar to that used in the model of intermetallic precipitation is used.

The occurrence (and lack) of LRO is evaluated using optical and electron microscopy (SEM and TEM) on coupons of Alloy-22 (both base-metal and welded) which were aged for various times between 1 and 40,000 hours at temperatures from 260 to $800^{\circ} \mathrm{C}$. LRO was observed in only 5 samples. Nucleation and growth are modeled as an exponential process and the rates of precipitation are plotted as $\log ($ time $)$ vs. reciprocal temperature. These data are extrapolated to repository temperatures in order to determine the time at which precipitation may be expected to begin. It is suggested that LRO may begin in less than $10,000 \mathrm{r}$ at $\mathrm{T}<300^{\circ} \mathrm{C}$, however this is based on only 2 data points.

Use of the Model: The model output consists of bounding conditions for initiation of LRO in Alloy-22. This information is not used in TSPA-SR because LRO is not expected to occur for the anticipated range of repository conditions.

Review Findings: The Long-Range Ordering Model is assigned to Bin 2. Although the model does not give explicit quantitative description of conditions for the initiation of LRO in the potential repository, it provides an valid approach for predicting if this will in fact occur at relevant times and temperatures. It is noted that experiments, reportedly in progress, should significantly improve the predictions of this model.

\subsubsection{General and Localized Corrosion of the Drip Shield (J.3)}

Description: This is a process-level model intended to account for the rate of general and localized corrosion of the drip shield. The model is documented in the AMR entitled: General Corrosion and Localized Corrosion of the Drip Shield (CRWMS M\&O 2000af). The data used were acquired from testing of $\mathrm{Ti}$ grade 16 , as it is reported that $\mathrm{Ti}$ grade 7 was not available in 
the necessary timeframe for initial testing. The model described herein includes sub-models for dry oxidation, humid air corrosion, and general and localized corrosion in aqueous media. A process flow model (similar to that used for alloy Alloy-22 and SS 316-L) is used to determine what type of corrosion dominates at a given time and which rate equation to use. This is summarized in Figure 1 of the AMR.

Use of the Model: This model provides rates of corrosion of the drip shield as a function of the temperature and environment, based on available test data. This information is used as the basis for abstraction of drip shield corrosion for TSPA-SR.

Review Findings: The various submodels are supported by a range of different types of data. There is discussion of uncertainty and variability in Section 1.7 of the AMR. The discussion focuses on the uncertainty in the threshold relative humidity $(\mathrm{RH})$ for humid-air corrosion (HAC) and aqueous-phase corrosion (APC), the assumptions regarding the threshold for crevice corrosion $\left(\mathrm{E}_{\text {corr }}=\mathrm{E}_{\text {crit }}\right.$ at $\left.90-120^{\circ} \mathrm{C}\right)$ and the uncertainty in the estimated general corrosion rates (which are centered around zero). These issues are reported to be the subject of ongoing and additional testing at LLNL. In most cases the existing data are qualified or accepted with the notable exception of the crevice corrosion data where the crevice $\mathrm{pH}$ was not measured. In this case (crevice corrosion) the rates of attack are approximated by using data for extremely aggressive solutions (boiling aqua regia; or $19 \% \mathrm{HCl}, 4 \% \mathrm{FeCl}_{3}$ and $4 \% \mathrm{MgCl}_{2}$ at $82^{\circ} \mathrm{C}$ ). This approach cannot be considered validated. The Bin 2 recommendation for this model is based on the combination of several validated submodels with additional work in progress (including test results for Ti grade 7) that will support model validation.

\subsubsection{Degradation of Stainless Steel Structural Materials (J.4)}

Description: This is a process-level model intended to account for the rate of general and localized corrosion of the internal $316 \mathrm{NG}$ "structural support" layer for the waste package wall. The model is documented in the AMR entitled: Degradation of Stainless Steel Structural Material (CRWMS M\&O 2000s). The model includes sub-models for dry oxidation, humid air corrosion (HAC), and general and localized corrosion in aqueous media (APC). A process flow model (similar to that used for Alloy-22 and Ti grade 7) is used to determine what type of corrosion dominates at a given time and which rate equation to use. This is summarized in Figure 1 of the AMR. Stainless steel $316 \mathrm{~L}$ is used for the experiments rather than $316 \mathrm{NG}$. Any corrosion that is described by this model would require that the waste package outer barrier (WPOB) be penetrated first. This means in practice that the components of the model, which are potentially applicable, are humid air corrosion and general and localized corrosion in aqueous media.

Use of the Model: The model output consists of conditions for which pitting and general corrosion of the $316 \mathrm{NG}$ shell are predicted to occur. This information is not used in TSPA-SR, because there is no performance credit taken for the inner barrier.

Review Findings: The Degradation of Stainless Steel Structural Materials Model is assigned to Bin 2. The various submodels are supported by a range of data. There is discussion of uncertainty and variability in section 1.7 of the AMR. The discussion focuses on the uncertainty in the threshold relative humidity for HAC and APC, the assumptions regarding the threshold for crevice corrosion, and the uncertainty in the estimated general corrosion rates (which are centered around zero). These issues are reported to be the subject of ongoing and additional testing at LLNL. In most cases the existing data are qualified or accepted. There is sufficient 
existing data on ambient corrosion of $316 \mathrm{~L}$ and $316 \mathrm{NG}$ to support validation of this model, especially when combined with in-process data (including test results for $316 \mathrm{NG}$ ).

\subsubsection{Alloy-22 Potential-Based Localized Corrosion Initiation Threshold and Rate Abstraction Model (J.5-1)}

Description: An abstraction model is presented wherein the corrosion of both the drip shield and waste package outer barrier are put into a form that can be used as input for TSPA or other types of modeling of these materials. The model is documented in the AMR entitled: Abstraction of Models for Pitting and Crevice Corrosion of Drip Shield and Waste Package Outer Barrier (CRWMS M\&O 2000b). Two submodels are included within the AMR for Ti grade 7 and Alloy22. The Alloy-22 Potential-Based Localized Corrosion Initiation Threshold and Rate Abstraction Model is described in Section 6.3 of the AMR.

Use of the Model: A function is provided that can be used in TSPA to determine conditions under which accelerated local corrosion of Alloy C-22 may initiate. (These conditions may not be encountered during implementation of the TSPA-SR system model.)

Review Findings: The Alloy-22 Potential-Based Localized Corrosion Initiation Threshold and Rate Abstraction Model is assigned to Bin 1. The models incorporated within this AMR are based entirely on relevant data. The data are used to choose a relatively conservative but quantitative measure (in this case the potential difference between $\mathrm{E}_{\mathrm{corr}}$ and $\mathrm{E}_{\text {critl }}$ ) for predicting the conditions under which pitting corrosion may initiate as a function of potential and $\mathrm{pH}$.

\subsubsection{Ti Grade 7 Potential-Based Localized Corrosion Initiation Threshold and Rate Abstraction Model (J.5-2)}

Description: An abstraction model is presented wherein the corrosion of both the drip shield and waste package outer barrier are put into a form that can be used as input for TSPA or other types of models of these materials. The model is documented in the AMR entitled: Abstraction Models for Pitting and Crevice Corrosion of the Drip Shield and Waste Package Outer Barrier (CRWMS M\&O 2000b). Two submodels are included within the AMR for Ti grade7 and Alloy22. The Ti Grade 7 Potential-Based Localized Corrosion Initiation Threshold and Rate Abstraction Model is described in Section 6.3 of the AMR. Qualified data that has been collected on the corrosion of these alloys is used as the basis for a model that predicts pitting initiation. These are incorporated into a corrosion model that chooses a relatively conservative but quantitative measure (in this case the potential difference between $E_{\text {corr }}$ and $E_{\text {critl }}$ ) for predicting the conditions under which pitting corrosion may initiate as a function of potential and $\mathrm{pH}$.

Use of the Model: A function is provided which can be used in TSPA to determine conditions for which accelerated local corrosion of Ti grade 7 may initiate. (These conditions may not be encountered during implementation of the TSPA-SR system model.)

Review Findings: The Ti Grade 7 Potential-Based Localized Corrosion Initiation Threshold and Rate Abstraction Model is assigned to Bin 1. The models incorporated within this AMR are based on relevant data. The data are used to choose a relatively conservative but quantitative measure (in this case the potential difference between $E_{\text {corr }}$ and $E_{\text {crit1 }}$ ) for predicting the conditions under which pitting corrosion may initiate as a function of potential and $\mathrm{pH}$. The results of this model are then compared to a combination of validated and accepted data (in this case the accepted data is taken from peer reviewed publications) to demonstrate the validity of the model. 


\subsubsection{WAPDEG Analysis of Waste Package and Drip Shield Degradation (J.6)}

Description: The Waste Package Degradation Model (WAPDEG) is the integrated, stochastic model used to analyze waste package and drip shield degradation as a function of time when exposed to repository relevant environments (temperature, relative humidity, chemistry on surface, etc). The overall WAPDEG Model is documented in the AMR entitled: WAPDEG Analysis of Waste Package and Drip Shield Degradation (CRWMS M\&O 2000br). This AMR integrates the following models, which are described separately in this report:

- Waste Package Outer Barrier Localized Corrosion Initiation Threshold and Rate Abstraction Model, which is described in Section 6.10.6 of this report and entitled: Alloy22 Potential-Based Localized Corrosion Initiation Threshold and Rate Abstraction Model (J.5-1).

- Drip Shield Localized Corrosion Initiation Threshold and Rate Abstraction Models, described in Section 6.10.7 and entitled: Ti Grade 7 Potential-Based Localized Corrosion Initiation Threshold and Rate Abstraction Model (J.5-2).

- Manufacturing Defects Abstraction Model (J.12-1), described in Section 6.10.15.

- Stress and Stress Intensity Factor Profile Abstraction Model (J.12-2), described in Section 6.10.16.

- Slip Dissolution Abstraction Model (J.12-3), described in Section 6.10.17.

- Threshold Stress Intensity Factor Abstraction Model (J.12-4), described in Section 6.10 .18 .

This AMR also includes the following submodels, which are also supported by other documentation as noted:

- Relative Humidity Threshold Abstraction Model - The threshold of $50 \%$ relative humidity for aqueous phase corrosion is taken from the AMR: Environment on the Surfaces of the Drip Shield and Waste Package Outer Barrier (CRWMS M\&O 2000ac).

- Drip Shield General Corrosion Abstraction Model - Distribution functions for general corrosion are taken from the calculation report: Calculation of General Corrosion Rate of Drip Shield and Waste Package Outer Barrier to Support WAPDEG Analysis (CRWMS $\mathrm{M} \& \mathrm{O} 2000 \mathrm{f}$.

- Waste Package Outer Barrier General Corrosion Abstraction Model - Distribution functions for general corrosion are taken from the calculation report: Calculation of General Corrosion Rate of Drip Shield and Waste Package Outer Barrier to Support WAPDEG Analysis (CRWMS M\&O 2000f).

- Waste Package Outer Barrier Microbial Induced Corrosion Abstraction Model - Also based on the AMR: General Corrosion and Localized Corrosion of Waste Package Outer Barrier (CRWMS M\&O 2000ag).

- Waste Package Outer Barrier Aging and Phase Stability Abstraction Model - Also based on the AMR: Aging and Phase Stability of Waste Package Outer Barrier (CRWMS $\mathrm{M} \& \mathrm{O} 2000 \mathrm{~d}$ ). 
Use of the Model: The output from WAPDEG is a set of profiles for waste package and drip shield failure (defined as initial and subsequent breaches) that cause through-wall thickness penetrations as a function of time. The WAPDEG results are used as input for the waste form degradation component of the TSPA-SR Model.

Review Findings: The WAPDEG Analysis of Waste Package and Drip Shield Degradation Model is assigned to Bin 3 (impact review is provided in Appendix IV) because there is a missing model that should be incorporated. This model will bring together the effects of the weld, weld heat-affected zone, and base plate microstructure/residual stress profile to predict the resistance of the waste package outer barrier (WPOB) to localized corrosion and stress corrosion cracking. The model will incorporate weld process variation within the number of passes needed to fill the joint and the effects of repair welding. The model will also incorporate the effect of chemistry variation in different heats of Alloy 22 weld wire and base plate.

This model will develop the characterization of the microstructure and residual stress for Alloy 22 under the following conditions:

- Welds and weld heat affected zone of the WPOB in the as-welded and as-welded/solution annealed condition (aged and un-aged)

- The weld and weld heat affected zone of the inner lid of the outer barrier in the WPOB in the as-welded and as-welded/laser peened condition (aged and un-aged)

- The weld and weld heat affected zone of the outer lid of the WPOB in the as-welded and as-welded/induction annealed condition (aged and un-aged).

Items that need further definition in this AMR or the models that it is based on include the following:

- The finite element model analysis documented in the AMR entitled: Stress Corrosion Cracking of the Drip Shield, the Waste Package Outer Barrier, and the Stainless Steel Structured Material (CRWMS M\&O 2000bi) is presented but not validated with measurements of residual stress for the as-welded condition before laser peening or induction annealing. There are assumptions made for the inner lid induction annealed case but no validating data are presented. The residual stress data presented for the laser peened WPOB outer lid closure weld are based on other nickel-based materials and weld joint designs that may not reflect accurately the relevant closure weld design. The residual stress measurement data presented do not account for changes due to the welding process (weld fit-up variations, variations in heat input, travel speed) and possible subsequent repair processes. More data are also required on the equivalence of shot peening and laser peening.

- The use of the term "hoop stress" to define residual stress needs clarification.

- The output of the WAPDEG model concludes that localized corrosion is not possible within the 10,000-yr evaluation period as represented in the TSPA-SR. However, it is the strongly held opinion of the reviewers that the materials used in the waste package should not be judged on the low rate of general corrosion but localized corrosion or stress corrosion cracking (SCC). This is not adequately expressed in WAPDEG despite the uncertainty that surrounds the technical issues. An example of this is the lack of expression of uncertainty related to the efficacy of the stress mitigation procedures for the 
WPOB welds. More data are needed (microstructure and residual stress) from representative weld mockups fabricated using the weld process, joint design, and stress mitigation techniques selected for the WPOB.

- The multiple mechanisms for localized corrosion and cracking, as described in the abstraction models, should be combined probabilistically and then included in the WAPDEG abstraction model. This is needed to avoid oversimplification and to satisfy stakeholders.

It is noted that ongoing investigations and associated unpublished results do not fall under the scope of this review. However, it is acknowledged that some potentially important work is currently underway which could address the foregoing comments.

Impact Review Summary: The WAPDEG model is an integration model for waste package degradation analysis and is based on supporting process models and abstractions models. The abstraction models are based entirely on underlying process models, so the abstraction models and the WAPDEG model are considered validated as long as the corresponding process models are validated. The process models are based on Project-generated data relevant to repository conditions, and are considered validated. The technical basis for the process models is the focus of several DOE-NRC agreements on Key Technical Issues related to Alloy-22 and titanium degradation. The additional information developed to address these agreements will provide further validation of the model. This model is used in TSPA-SR but did not serve as a basis for screening out FEPs. Based on the above information and the additional detail provided in Appendix IV, model validation review findings associated with the WAPDEG Analysis of Waste Package and Drip Shield Degradation Model have no significant impact on the conclusions of TSPA-SR (CRWMS M\&O 2000bm, Section 6.1).

\subsubsection{Calculation of the General Corrosion Rate for the Drip Shield and Waste Package Outer Barrier to Support WAPDEG Analysis (J.7)}

Description: This model produces cumulative distribution functions for Alloy-22 corrosion based on 24-month test data; and for Ti grade 7 (0.12 to $0.25 \%$ palladium) based on data from testing of $\mathrm{Ti}$ grade $16(0.04$ to $0.08 \%$ palladium $)$ corrosion. The tests used some plain (unmodified base metal) test coupons and some with crevices. The test conditions were not specified in the AMR. The model is documented in the AMR entitled: Calculation of General Corrosion Rate of Drip Shield and Waste Package Outer Barrier to Support WAPDEG Analysis (CRWMS M\&O 2000f).

The corrosion rates were determined by coupon weight loss and a correction was made for silica deposition. This correction was based on an integral convolution algorithm for subtracting a uniform, random distribution ( 0 to $63.0 \times 10^{-6} \mathrm{~mm} / \mathrm{yr}$ ) for estimated silica deposition, from the experimental data for corroded coupon weight for Alloy-22 to produce a corrected cumulative distribution function. Figure 1 of the AMR shows the results for the 24-month exposure of Alloy-22; Figure 2 for Ti grade 7. In addition, general corrosion rates for Ti grade 16 are considered and are expected to be similar to Ti grade 7 .

Use of the Model: The basis data and tabulated results of the model are provided in the AMR. The cumulative distribution functions are used directly in the implementation of the WAPDEG model for TSPA-SR. 
Review Findings: This model is assigned to Bin 2. It is determined to be a model, not a calculation, because of the magnitude of corrections applied to the measured corrosion rates. Full validation can be achieved through consideration of the following comments and recommendations.

The analysis of the test data appears to be satisfactory, however, the adjustment for silica deposition results in a correction that is approximately three times larger than the unadjusted corrosion rate. Thus the approach used for applying the silica correction is important. Is the assumption of a linear correction from 0 to $63.0 \times 10^{-6} \mathrm{~mm} / \mathrm{yr}$ appropriate? If so, the general method used to apply this correction appears reasonable; however, there was no justification presented for using a linear stochastic correction. The corrected values are predominately estimates of silica deposits.

The results for the two types of coupons were not separately identified, thus the data from smooth coupons is convoluted with the ribbed coupons. The geometry of the coupons was not described.

The cumulative distribution functions for general corrosion rates exhibit a population of approximately $10 \%$ of the total number of data that have significantly higher corrosion rates than the rest. This raises additional questions:

- Did the ribbed coupons exhibit higher corrosion?

- Was corrosion nonuniform (e.g., pitting) on individual coupons?

- How much spatial variation of the corrosion was there on individual coupons? Does this indicate nonuniformity of the metal substrate and variability from coupon to coupon, or a difference between smooth coupons and coupons with crevices, or a difference in corrosion processes at different exposure conditions?

- What was the true nature of the silica deposits and is the assumption of a linear random distribution from 0 to $63.0 \times 10^{-6} \mathrm{~mm} / \mathrm{yr}$ valid or are the silica deposits nearly constant at $63.0 \times 10^{-6} \mathrm{~mm} / \mathrm{yr}$ ?

Documentation of the corrosion testing procedure and experimental design needs to be better integrated with the justification for this model, to provide better support for the use of test data in developing the important stochastic functions which are used to represent corrosion processes in TSPA.

A broader perspective of the needs for a TSPA should be adopted to support the regulatory compliance strategy. Models that support the TSPA should consider the data necessary to evaluate alternative exposure scenarios, such as the particular effects of brine contact at elevated temperature.

It is recommended that an independent review process be established for determining test conditions for waste package/drip shield materials and to determine the applicability of testing for evaluating predictive uncertainty for disturbed as well as expected conditions. This function should address both stochastic and model uncertainty (including subjective confidence in the models). It is further recommended that a Project-level function should be established to define the scope of uncertainty analysis from a broad, system-performance perspective, and to translate this uncertainty analysis into effective input to the testing programs. Such a function should also promote consistent terminology and methods. 


\subsubsection{Incorporation of Uncertainty and Variability of Drip Shield and Waste Package Degradation in WAPDEG (J.8)}

Description: This model describes how uncertainty and variability are incorporated into modeling of waste package and drip shield degradation. The report is conceptual and quantitative analysis of data is included for illustrative purposes only. Although the treatment of this topic resembles an analysis as defined in AP-3.10Q, the implications for waste package performance are very important, so the AMR is designated as a model requiring validation. The model is documented in the AMR entitled: Incorporation of Uncertainty and Variability of Drip shield and Waste Package Degradation in WAPDEG Analysis (CRWMS M\&O 2000ak).

Use of the Model: Contributes to the conceptual basis for abstractions of Alloy-22 and Ti grade 7 corrosion behavior used in TSPA-SR.

Review Findings: This AMR provides a comprehensive set of examples of computational and statistical methods of analysis. The application of probabilistic risk assessment of nuclear power plants was offered as an example of stochastic uncertainty in a complex system. However, the AMR is restricted to consideration of corrosion data collected under limited exposure conditions. Methods to consider low probability and potentially high consequence scenarios for corrosion failures, based on limited available data, were not addressed in the examples provided, therefore this model is assigned to Bin 3 (impact review is provided in Appendix IV).

The methods for treatment of uncertainty as outlined in this model may produce inaccurate estimates of waste package reliability.

The theory and relationships presented in Section 6.2 of the AMR are important for building confidence in performance of the waste package. This is because failures are most likely to result from numerically minor populations in the corrosion data, e.g., localized pitting. It is recommended that this insight be incorporated in the design of corrosion tests to characterize the possibility for extremal behaviors, and in the analysis of test data that includes multiple modes of behaviors, e.g., general and localized corrosion. Definition of the tails of the probability distributions that describe waste performance should begin with specification of the testing program and the methods of test data analysis.

Such analysis seems to have been deferred to the next level, i.e. TSPA. However, this gives rise to criticism that the system model is over-used, and there is insufficient understanding of extremal behavior at the process level.

Impact Review Summary: The model is developmental and is partially complete in its present form. It was originally intended that the AMR would be revised for use as supporting information for TSPA-SR. This model was not used in TSPA-SR and did not serve as a basis for screening out FEPs. Also, revised input from the technical specialist reviewers assigned to this model area indicates that this model should be considered as an analysis instead, in which case there is no need for validation. (This revised input was received recently, after the model-binning and impact reviews were conducted, and could not be incorporated in the findings of the review.) Consequently, based on this information and the additional detail provided in Appendix IV, the model validation review findings associated with this model have no impact on the conclusions of TSPA-SR (CRWMS M\&O 2000bm, Section 6.1).

\subsubsection{Drip Shield Passive Corrosion (J.10-1)}


Description: The Drip Shield Passive Corrosion model is based on the assumption that hydrogen-induced cracking (HIC) failure will not occur until enough hydrogen is absorbed into Ti grade 7 to exceed a measured critical hydrogen content $\left(\mathrm{H}_{\mathrm{c}}\right)$. The model is documented in the AMR entitled: Hydrogen Induced Cracking of Drip Shield (CRWMS M\&O 2000ah). Observations of $\mathrm{H}_{\mathrm{c}}$ for Ti grade 2, Ti grade 12 and Ti grade 16 are available and are documented in the AMR. Critical hydrogen content for Ti grade 7 is not documented because data were not available. Thus, it is assumed that the $\mathrm{H}_{\mathrm{c}}$ data for Ti grade 16 are applicable to Ti grade 7, which is justified based on the similarity between these alloys (CRWMS M\&O 2000ah, Section 5.2). The model is based only on general passive corrosion of Ti; crevice corrosion is neglected based on information from the AMR entitled: General Corrosion and Localized Corrosion of the Drip Shield (CRWMS M\&O 2000af). The model also neglects hydrogen generation from radiolysis because the radiation annual dose at repository conditions would be too small. No discussion is provided pertaining to hydrogen generation from microbial processes. The model is based on hydrogen efficiency absorption values for other types of Ti (bounding values) and the general corrosion rate. This approach is then used to calculate whether enough hydrogen could be absorbed into Ti grade 7 to exceed the critical hydrogen threshold and thus lead to drip shield failure by HIC. The model concludes that the hydrogen concentration in the drip shield material will remain less than critical concentration for 10,000 yr after emplacement.

Use of the Model: This model is used in the abstraction of drip shield corrosion processes for TSPA-SR to eliminate HIC as a significant degradation model for Ti grade 7 in the potential repository environment.

Review Findings: The subject AMR (CRWMS M\&O 2000ah) includes a separate section that addresses model validation (Section 6.2 .5 of the AMR), but the model is assigned to Bin 2 because additional information is needed to achieve appropriate confidence. The AMR states that the model is validated based on laboratory test results, along with published data from the open literature for prediction of HIC behavior in Ti grade 7 . The same section also states that model predictions show excellent agreement with laboratory test results. This assertion needs additional validation. Natural or man-made analogs were not used in the validation approach.

The first premise of this model is that HIC can be predicted based on hydrogen critical threshold. This should be justified using the open literature, which will also show that the threshold may vary with environmental factors, stress in the material, surface finish, and other factors that are not addressed in the AMR. Ultimately, data should be made available for Ti grade 7 in rolled form, to justify this model.

Next, the cited data are for other Ti alloys, and not Ti grade 7 , yet they are assumed to be applied for $\mathrm{Ti}$ grade 7 . The hydrogen critical concentration for $\mathrm{Ti}$ grade 7 may be more favorable than $\mathrm{Ti}$ grade 16, but this is not shown. Also, the model above does not account for hydrogen generation by crevice corrosion. In the AMR this is discounted based on data for other alloys not specific to Ti grade 7. In addition, the potential effects from heavy metals and halogens are not addressed with respect to crevice corrosion; the text only discusses laboratory test environments using SDW, SCW and SAW for investigation of crevice corrosion. Additional justification should be provided for ignoring crevice corrosion as a hydrogen source.

Microbial corrosion was not discussed in relationship to Ti (i.e. hydrogen generation). Although justification for ignoring microbially influenced corrosion of $\mathrm{Ti}$ alloys can probably be found in the open literature, it is lacking from the model validation arguments. 
This model also used a fractional efficiency for hydrogen absorption. The text says that test data have established an efficiency of 0.1 based on direct analysis of hydrogen absorption during crevice corrosion, but discards that value because Ti grade 7 is immune to crevice corrosion at repository conditions. The basis for ignoring crevice corrosion should be strengthened based on actual Ti grade 7 data, otherwise this fractional efficiency should be used in the model.

The validation discussion states that additional data are being collected, which will improve confidence in the model. It is recommended that such data be acquired for Ti grade 7, and include measurements of hydrogen fractional efficiency absorption, general corrosion rate, and hydrogen critical threshold.

\subsubsection{Drip Shield Galvanic Coupling (J.10-2)}

Description: This model is for the no-backfill design and is documented in the AMR entitled: Hydrogen Induced Cracking of the Drip Shield (CRWMS M\&O 2000ah). The impact of hydrogen induced cracking (HIC) was evaluated in a model for situations in which there is galvanic contact of titanium with carbon steel (no intervening backfill). A qualitative assessment first determined that Fe-Ti couples would be limited. Although galvanic corrosion is stated to be unlikely, a simple mathematical model is developed to evaluate absorption/diffusion of hydrogen in the drip shield. The hydrogen concentration for long term galvanic coupling is reduced to an equation that depends on hydrogen diffusion rate, hydrogen absorption rate, and radius of initial contact. The model indicates that the hydrogen critical threshold for cracking $\left(\mathrm{H}_{\mathrm{c}}\right)$ would not be exceeded (based on an assumed $\mathrm{H}_{\mathrm{c}}$ for Ti grade 7) due to galvanic coupling for 10,000 yr. The model conclusions state that the hydrogen concentration in the drip shield will remain less than the critical concentration for $10,000 \mathrm{yr}$ after emplacement, even if galvanic coupling occurs.

Use of the Model: This model is used in the abstraction of drip shield corrosion processes for TSPA-SR to eliminate HIC originating from galvanic coupling with carbon steel as a significant degradation process for Ti grade 7 in the potential repository environment.

Review Findings: The Drip Shield Galvanic Coupling Model is assigned to Bin 2. For this model there is no explicit section for validation in the AMR. Such a section would need to be added to address the proposed model for HIC due to galvanic coupling. The qualitative discussion in Section 6.3.2 of the AMR states that further quantitative studies may be conducted to determine:

- How much hydrogen would be liberated per unit mass of Fe

- Whether the available hydrogen concentrations could cause HIC in the adjacent Ti

- How fast hydrogen diffuses into the surrounding $\mathrm{Ti}$, thus limiting the hydrogen concentration.

These are important questions that should be addressed in the validation strategy. Also, additional information is needed to confirm that $\mathrm{H}_{\mathrm{c}}$ for Ti grade 7 is indeed similar to $\mathrm{H}_{\mathrm{c}}$ for $\mathrm{Ti}$ grade $16(1000 \mu \mathrm{g} / \mathrm{gm})$. A parametric study was used to evaluate the potential for HIC, but no basis was provided for the specific parameter values chosen. No use of field observations, or natural or man-made analogs, was documented for this model. No alternative approaches for validation were documented for this model (i.e. peer review, publication in peer-reviewed literature, model calibration, comparison to alternative models). Various strategies are possible based on published literature for galvanic processes involving Ti. Also, the effect of the cathode- 
anode area ratio on galvanic corrosion of Ti-Fe couples should be investigated; there is relevant information available from the literature.

\subsubsection{Stress Corrosion Cracking Threshold Model (J.11-1)}

Description: This is a conceptual model for determining susceptibility of the Alloy-22 waste package outer barrier and the Ti grade 7 drip shield to stress corrosion cracking (SCC). The model is documented in the AMR entitled: Stress-Corrosion Cracking of the Drip Shield, the Waste Package Outer Barrier and the Stainless Steel Structural Material (CRWMS M\&O 2000bi). The stainless steel inner barrier is excluded from the model because no credit for this barrier is taken in TSPA-SR. The premise of this model is that some threshold value exists for the stress intensity factor $\left(\mathrm{K}_{\mathrm{ISCC}}\right)$ below which initiation of SCC or propagation of pre-existing flaws does not occur. A finite element analysis is used for determining the stress values that will exist in WP closure welds (other WP welds are fully annealed during fabrication). In the model, only rockfall contributes to potentially significant stress in the drip shield; the resulting SCC is not considered to degrade drip shield performance (CRWMS M\&O 2000z, Section 6.5.3). Deterministic calculations are applied to assess $\mathrm{K}_{\mathrm{ISCC}}$. Uncertainties in the finite element analyses due to experimental data scatter and general corrosion are addressed.

Use of the Model: The model results are used in the AMR entitled: Abstraction of Models of Stress Corrosion Cracking of Drip Shield and Waste Package Outer Barrier and Hydrogen Induced Corrosion of the Drip Shield (CRWMS M\&O 2000c). Analysis documented in that AMR eliminates this model from consideration in TSPA-SR.

Review Findings: This model is only partially validated by experimental laboratory results performed at LLNL for Alloy-22 and Ti grade 12. The tests were performed at $90^{\circ} \mathrm{C}$ in deaerated acidic brine solutions on pre-cracked specimens with applied stress ranging from 20 to $39 \mathrm{ksi}$. These tests yielded $\mathrm{K}_{\mathrm{ISCC}}$ data for model input. Other key parameters of the model are consistent with recent data available from open scientific literature.

Several issues relative to the laboratory tests require further development to support model validation:

- It is unclear whether the laboratory-applied stresses are sufficient to simulate actual conditions in the waste package (it is noted that no laboratory crack-growth samples containing welds have been tested). Justification for why the applied stress levels used in the tests bound important waste package residual stress and/or operating stress scenarios, given final design and welding attributes, should be presented.

- Extrapolation of results from tests performed at $90^{\circ} \mathrm{C}$ should be included for higher temperatures, if possible. If not possible, further laboratory tests are should be performed at higher temperatures.

- Provide further justification for why laboratory results performed on Ti grade 12 are directly applicable to the Ti grade 7 material proposed for use on the drip shield.

Recognizing these needs for additional documentation, the Stress Corrosion Cracking Threshold Model is assigned to Bin 2.

\subsubsection{Stress Corrosion Cracking Slip Dissolution/Film Rupture Model (J.11-2)}


Description: This is a process model for determining the susceptibility of the Alloy-22 waste package outer barrier and the Ti grade 7 drip shield to stress corrosion cracking (SCC), using the slip dissolution/film rupture mechanism. The model is documented in the AMR entitled: StressCorrosion Cracking of the Drip Shield, the Waste Package Outer Barrier and the Stainless Steel Structural Material (CRWMS M\&O 2000bi). The stainless steel inner barrier is excluded from the model because no credit for this barrier is taken in TSPA-SR. The concept for this model is an electrochemical process by which the protective layer of material immediately preceding the crack tip is ruptured by corrosion, allowing the crack to propagate. Rapid oxidation of the ruptured film is controlled by activation and/or diffusion kinetics, however, exposed surfaces quickly form a protective layer and crack propagation slows until the new layer of protective film ruptures. This process is repetitive, given certain environmental, metallurgical, and corrosion potential conditions. A crack will advance based on oxidation charge density and frequency of film rupture as controlled by the fracture strain of the film and the strain rate at the crack tip. The model uses these parameters and Faraday's Law to determine the average environmental crack growth rate. A power-law relationship is developed with material constants that can be measured from laboratory experiments.

Use of the Model: This model is used in the AMR entitled: Abstraction of Models of Stress Corrosion Cracking of Drip Shield and Waste Package Outer Barrier and Hydrogen Induced Corrosion of Drip Shield (CRWMS M\&O 2000c). The SCC growth rate is used in the representation of SCC for TSPA-SR.

Review Findings: This model is only partially validated by experimental laboratory results performed at GE Corporate Research \& Development Center for Alloy-22 and Ti grade 7. The tests were performed on solution-annealed specimens, pre-cracked by fatigue. An applied stress of $30 \mathrm{MPa}$ was used and the transition from fatigue to SCC was addressed by continuing cyclic loading $(\mathrm{R}=$ approx. 0.7$)$ at a low frequency $(0.001 \mathrm{~Hz})$. The samples were immersed in water containing concentrated chemical species representing those expected to exist on the barrier surfaces in situ (although not necessarily at the same concentrations) and autoclave tested at $110^{\circ} \mathrm{C}$. These tests yielded estimates of the SCC growth rate data for Alloy-22 and Ti grade 7 .

Other key parameters of the model are consistent with recent data available from open scientific literature, although this literature is primarily focused on austenitic stainless steels. Therefore, the film oxidation rate and crack tip strain have been determined by empirical methods based on the laboratory results of Alloy 22 and $\mathrm{Ti}$ grade 7 . For this reason, it is crucial that laboratory tests closely simulate metallurgical conditions that may be present in the as-built waste package closure welds.

It is unclear whether the applied tensile stress of $30 \mathrm{MPa}$ adequately simulates the magnitude and orientation of residual stresses that may be found in the completed closure welds. Similarly, other metallurgical parameters, e.g., sensitization and grain growth in the heat-affected-zone resulting from field welding processes have not been addressed by the current laboratory tests. The applicability of test conditions can be further justified in the model documentation, so the Stress Corrosion Cracking Slip Dissolution/Film Rupture Model is assigned to Bin 2. In addition, further laboratory tests are needed to provide more complete data for model validation.

\subsubsection{Manufacturing Defects Abstraction Model (J.12-1)}


Description: This is a probabilistic abstraction intended to predict the distribution and size of fabrication flaws that are expected to occur in waste package closure welds. Most of the inputs for this abstraction are derived from the calculation report entitled: Abstraction of Models of Stress Corrosion Cracking of Drip Shield and Waste Package Outer Barrier and Hydrogen Induced Corrosion of Drip Shield (CRWMS M\&O 2000c). Abstraction is primarily restricted to assigning uncertainty to the flaw detection distribution and fraction (based on location in the weld cross-section) of flaws to be considered for WAPDEG.

Use of the Model: Information on the number and size of surface-breaking and near-surface flaws per length of waste package closure welds is input to WAPDEG Analysis of Waste Package and Drip Shield Degradation (CRWMS M\&O 2000br) for use in TSPA-SR.

Review Findings: In the absence of deterministic values, input parameters have been distributed over specified ranges. Distributions for the parameters used in the probabilistic calculations have not been validated by sensitivity analyses. It is stated that the method of validation is to review the model parameters for reasonableness, or consistency of all relevant data. However, no substantial argument for the bases of the parameters is presented, therefore this model is assigned to Bin 2.

\subsubsection{Stress and Stress Intensity Factor Profile Abstraction Model (J.12-2)}

Description: This abstraction is intended to calculate the overall stress state and stress intensity profile for waste package closure welds as a function of depth from the outer surface. The information is used to assess the conditions that contributed to stress corrosion cracking (SCC). The model is documented in the AMR entitled: Abstraction of Models of Stress Corrosion Cracking of Drip Shield and Waste Package Outer Barrier and Hydrogen Induced Corrosion of Drip Shield (CRWMS M\&O 2000c). Two methods for addressing uncertainty are described; these are based on the stress improvement techniques being investigated for use on closure weld regions: 1) laser-peening, and 2) induction-heating solution annealing. Only the hoop stress induced by the fabrication process is considered in the abstraction.

Use of the Model: A description of the tensile stress through the weld, as a function of depth from the outside surface, is input to WAPDEG Analysis of Waste Package and Drip Shield Degradation (CRWMS M\&O 2000br) for use in TSPA-SR.

Review Findings: Stress profiles and stress intensity values are based only on the hoop stress. This hoop stress appears to be the tensile component of the welding residual stress aligned normal to the expected weld heat affected zone. Clarification is necessary to further define the stress condition of the as-welded component. Also, it appears that an assumption is being made that most welding residual stresses are mitigated during the fabrication process by laser-peening and induction heat stress annealing. This assumption requires further validation. Recognizing these needs for additional justification, the Stress and Stress Intensity Factor Profile Abstraction Model is assigned to Bin 2.

\subsubsection{Slip Dissolution Abstraction Model (J.12-3)}

Description: This abstraction is intended to provide values for the parameters of crack growth pre-exponent $(A)$ and repassivation slope $(n)$ for use in the Stress Corrosion Cracking Slip Dissolution/Film Rupture Model (J.11-2). The model is documented in the AMR entitled: Abstraction of Models of Stress Corrosion Cracking of Drip Shield and Waste Package Outer Barrier and Hydrogen Induced Corrosion of Drip Shield (CRWMS M\&O 2000c). The model 
uses statistical sampling of these parameters within their expected ranges to capture effects of the corrosion processes that drive stress corrosion cracking (SCC). Uncertainty and variability in these values are also addressed.

Use of the Model: Parameters for estimating the SCC growth rate, as a function of crack tip strain and expected weld defects present, are input to WAPDEG Analysis of Waste Package and Drip Shield Degradation (CRWMS M\&O 2000br) for use in TSPA-SR.

Review Findings: The main purpose of the abstraction is to assess uncertainty and variability associated with the parameters in the SCC Slip Dissolution/Film Rupture Model. Bounding analyses are implemented to examine model response for time-to-failure, given the variability in repassivation slope $(n)$ and stress intensity factor $\left(K_{I}\right)$ for an iterative crack growth process. However, the bases for the limits in the bounding analyses are not fully described; it is suggested that they are products of the original model presented in Stress-Corrosion Cracking of the Drip Shield, the Waste Package Outer Barrier and the Stainless Steel Structural Material (CRWMS M\&O 2000bi). If so, the values for the model parameters are questionable as no welded specimens were tested in the laboratory. Further justification is needed for the stress levels used in this model as discussed previously in (Section 6.10.14 of this report). Accordingly, the Slip Dissolution Abstraction Model is assigned to Bin 2.

\subsubsection{Threshold Stress Intensity Factor Abstraction Model (J.12-4)}

Description: A cumulative distribution function is introduced to address uncertainty in the range of input parameters for the Stress Corrosion Cracking Threshold Model (J.11-1). The model is documented in the AMR entitled: Abstraction of Models of Stress Corrosion Cracking of Drip Shield and Waste Package Outer Barrier and Hydrogen Induced Corrosion of Drip Shield (CRWMS M\&O 2000c).

Use of the Model: The model is used in this AMR, but is not used directly in TSPA-SR.

Review Findings: This is an abstraction used to provide a distribution function for the original Stress Corrosion Cracking Threshold Model (J.11-1). The abstraction appears to be consistent with the original model. With additional documentation the Threshold Stress Intensity Factor Abstraction Model can be validated, and accordingly, the model is assigned to Bin 2.

\subsection{Model Area K: Waste Form Degradation}

\subsubsection{Inventory Abstraction (K.1)}

Description: A conceptual model is presented wherein different scenarios for EBS failure and isotope release mechanisms are considered. For time periods of 100 to $10,000 \mathrm{yr}$ and 20,000 to $1,000,000 \mathrm{yr}$ a set of isotopes was determined to account for $95 \%$ of the inhalation and ingestion dose for average waste forms. Based on the various possible times and rates associated with these scenarios a list of isotopes that need to be considered for each scenario is recommended. This model is documented in the AMR entitled: Inventory Abstraction (BSC 2001i).

Use of the Model: Initial values of radionuclide inventories used directly in TSPA-SR.

Review Findings: The assumptions used for the release modes appear to be excessively conservative, i.e. the calculations are bounding in that they assume that all of the inventory could be released at one time and all of the isotopes that are present in the repository at that time could contribute to dose. The calculations used to determine these recommended values appear to be 
referenced but it is not apparent upon what assumptions the calculations themselves are based. The fact that ${ }^{236} \mathrm{U}$ is cited in many of the tables, but is stated as only applicable to BWR fuel, for example, is questionable given that the enrichment and neutron spectrum for BWR and PWR are quite similar. The Inventory Abstraction Model needs additional clarification and is assigned to Bin 2.

\subsubsection{CSNF Waste Form Summary Degradation Abstraction (K.2)}

Description: A current summary of data and models for dissolution of commercial spent nuclear fuel (CSNF) is provided. These are bounding models, valid within a range of qualified experimental data: $\mathrm{pH}$ of 3 to $10, \mathrm{pO}_{2}$ of .002 to $0.2 \mathrm{Atm}$, and at $\mathrm{pH}<7, \mathrm{pCO}_{2}$ of $.001 \mathrm{Atm}$. The model is documented in the AMR entitled: CSNF Waste Form Degradation: Summary Abstraction (CRWMS M\&O 2000r).

Use of the Model: CSNF fuel dissolution rates over a range of water chemistry and gas composition are input to TSPA-SR.

Review Findings: The model is based on test results on CSNF over a range of flow rates and water chemistry deemed representative of repository conditions. The results are conservative (but not excessively so) insofar as fresh spent fuel ( $<30 \mathrm{yr}$ in this study) is probably more reactive than aged fuel (due to decrease in activity) and the majority of the fuel is unlikely to be exposed to groundwater until well after the regulatory period of 10,000 yr. The CSNF Waste Form Summary Degradation Abstraction Model is assigned to Bin 1.

\subsubsection{DHLW Glass Degradation (K.3)}

Description: This is a model designed to conservatively estimate the rates for radionuclide releases from glass, being directly dependent on glass dissolution rate. Measured glass dissolution rates in aqueous media are used to bound dissolution rate behavior at unsaturated conditions. The model is supported by measurements on a variety of glasses including some SRL glass, and is also supported by data from peer reviewed journals. This model is documented in the AMR entitled: Defense High Level Waste Glass Degradation (CRWMS M\&O 2001f).

The starting point is Equation 1 of the AMR, a mechanistic equation for the dissolution rate that is taken from previous work on dissolution of aluminosilicate minerals (see the AMR for details). A full rate equation is derived; parameters for it are derived and to the extent possible, are based on measured data.

The important result is found in Equations 7 and 8, giving dissolution rate per surface area exposed with appropriate estimates and bounds for the parameters. The conservatism of the model can be inferred from Table 3 and Figure 4 of the AMR.

Use of the Model: The parameterized Defense High Level Waste (DHLW) glass dissolution expression model is input to the waste form degradation abstraction for TSPA-SR.

Review Findings: The validation argument for this model is based on model-data comparison and complies with AP-3.10Q Section 5.3.b. However, there are several important points in the model development that should be clarified or justified:

- The assumption of congruent release of radionuclides, given the potential for radiation damage to the glass waste form. 
- The form of the glass dissolution rate expression is valid, but the dissolution rate will depend on the surface area, which will vary with the aggregation state of the glass. Because measured dissolution rates are used to estimate model parameters, consideration of inclusions (e.g., amorphous and crystalline forms in glass produced from the vitrification process) as separate model parameters may be unnecessary. The same may be true of compositional variation in different kinds of borosilicate glass (e.g., the $\mathrm{Al}, \mathrm{Ca}$, and $\mathrm{Na}$ constituents).

- The bounding rates seem overly conservative.

On balance, the assumptions and methods used in this model are defensible and supported by available data and technical literature. A more careful validation argument that incorporates more extensive model-data comparison and treatment of the literature on this subject can produce a compliant validation argument. Accordingly, the DHLW Glass Degradation Model is assigned to Bin 2 .

\subsubsection{Waste Form Degradation Abstraction - Upper Limit Model (K.4-1)}

Description: This abstraction model incorporates a review of the available data for degradation of 11 types of DOE spent nuclear fuel (DSNF) and recommends appropriate types of limiting or bounding case models to be used in TSPA for degradation of these types. Although 11 submodels are incorporated in the document, the output takes the form of what are termed: upper limit, conservative, and best estimates for the degradation of these types of fuel. This model review applies to the "upper limit" type of model. In this case, the DSNF is assumed to be instantly released upon failure of the waste package. This is the model that is eventually recommended for all of the DSNF. This model is documented in the AMR entitled: DSNF and Other Waste Form Degradation Abstraction (BSC 2001n).

Use of the Model: Release rates for all of the DSNF types other than LWR and naval reactor fuel are available for use in TSPA-SR.

Review Findings: Although some of the data pertaining to degradation of the DSNF fuels considered in this AMR are preliminary or incomplete, the limited quantities of these waste forms that are involved (and an assessment that the contribution of this small fraction of the waste would be negligible to the overall dose), combined with the fact that these models are simplified to a limiting case (e.g., the fuel either dissolves instantly upon exposure to water or, in the case of naval DSNF, acts identically to LWR fuel) renders this model an upper bound estimate on release rates, as intended. Conservatism is not a basis for model validation in accordance with AP-3.10Q, however, the test data and other empirical information used in development of this model can be used to satisfy the need for confidence building commensurate with the intended use. A compliant argument (not based on conservatism) does not presently exist, but can be developed with the available information. Accordingly, the Waste Form Degradation Abstraction - Upper Limit Model is assigned to Bin 2.

\subsubsection{Waste Form Degradation Abstraction - Conservative Model (K.4-2)}

Description: As noted for the previous Upper Limit Model (Section 6.11 .4 of this report) this abstraction model incorporates a review of the available data for degradation of 11 types of DOE spent nuclear fuel (DSNF) and recommends appropriate types of limiting or bounding case models to be used in TSPA for degradation of these types. Although 11 submodels are incorporated in the document, the output takes the form of what are termed: upper limit, 
conservative, and best estimates for the degradation of these types of fuel. This model review applies to the "conservative" type of model. In this case rates are assumed as conservative bounds (not instantaneous degradation as assumed in the foregoing model) based on available data (for most types there are no such data) or data for materials assumed to be analogous. These analogous materials are ceramics, uranium dioxide spent nuclear fuel (SNF), and uranium metal SNF for which there are more data available. The "conservative" model that is recommended by the AMR to represent all DSNF is based on the Hanford N-reactor SNF model because more qualified data are available for this fuel type. This model is documented in the AMR entitled: DSNF and Other Waste Form Degradation Abstraction (BSC 2001n).

Use of the Model: The "conservative" model for all DSNF is not recommended for use in TSPA-SR.

Review Findings: Although some of the data for degradation of the DSNF fuels considered in this AMR are preliminary or incomplete, the empirical information used in development of this model could probably be used as the basis for a compliant validation argument. Accordingly, the Waste Form Degradation Abstraction - Conservative Model is assigned to Bin 2.

\subsubsection{Waste Form Degradation Abstraction - Best Estimate Model (K.4-3)}

Description: This abstraction model incorporates a review of the available data for degradation of 11 types of DSNF and recommends appropriate types of limiting case models to be used in TSPA for the degradation of these types. Although 11 submodels are incorporated in the document, the output takes the form of what are termed upper limit, conservative and best estimates for the degradation of these types of fuel. This model review applies to the "best estimate" type of model, in which the rates are based on experimental data appropriate to the materials of interest. The materials for which data are available are not necessarily identical to the DSNF waste forms but are considered representative. The "best estimate" model that is recommended by the AMR to represent all DSNF is based on the Hanford N-reactor SNF model because more qualified data are available for this fuel type. This model is documented in the AMR entitled: DSNF and Other Waste Form Degradation Abstraction (BSC 2001n).

Use of the Model: A best-estimate model is recommended for Pu-ceramic waste forms (based on published data) and for Naval SNF based on LWR fuel and N-reactor spent fuel. The model is available but was not used for TSPA-SR.

Review Findings: It is the judgment of the reviewer that the empirical information used in development of this model would probably not be sufficient as the basis for a compliant validation argument for a "best estimate" model. Accordingly, the Waste Form Degradation Abstraction - Best Estimate is assigned to Bin 3 (impact review is provided in Appendix IV).

Impact Review Summary: The model is based on preliminary or approximate information and has limitations as discussed in the AMR. Based on these limitations, the "conservative" approach was used for TSPA-SR in lieu of this model. Thus, this model was not used in TSPA-SR and did not serve as a basis for screening out FEPs. Consequently, the model validation review findings associated with this model have no impact on the conclusions of TSPA-SR (CRWMS M\&O 2000bm, Section 6.1).

\subsubsection{Waste Form Degradation Abstraction - Immobilized Pu Model (K.4-4)}

Description: Like the foregoing models, this abstraction model incorporates a review of the available data for degradation of 11 types of DSNF and recommends appropriate types of 
limiting case models to be used in TSPA. This review applies to the model developed to describe degradation of immobilized $\mathrm{Pu}$. In this case, rates are recommended based on published data from two other reports wherein a titanate based ceramic was used as a surrogate for the immobilized $\mathrm{Pu}$ waste form. This model is documented in the AMR entitled: DSNF and Other Waste Form Degradation Abstraction (BSC 2001n).

Use of the Model: Estimated rates for dissolution of immobilized $\mathrm{Pu}$ are available but were not used in TSPA-SR.

Review Findings: There is some possibility that a compliant validation argument could be constructed, focusing on the intended use of the model and its impact on TSPA. However, it is the judgment of the reviewer that the empirical information used in development of this model would probably not be sufficient as the basis for a compliant validation argument. Accordingly, the Waste Form Degradation Abstraction - Immobilized Pu Model is assigned to Bin 3 .

Impact Review Summary: This model was not used to represent waste form degradation for TSPA-SR. The immobilized $\mathrm{Pu}$ inventory was averaged into the $\mathrm{HLW}$ glass radionuclide inventory, and the immobilized $\mathrm{Pu}$ waste form was treated as HLW glass for TSPA-SR. This approach is the "conservative" model developed in the subject AMR (BSC 2001n). Consequently, the model validation review findings associated with this model have no impact on the conclusions of TSPA-SR (CRWMS M\&O 2000bm, Section 6.1).

\subsubsection{In-Package Source Term Abstraction (K.5)}

Description: The model incorporated in this AMR is a conceptual model wherein the waste form is assumed to degrade according to the time since a particular canister failed, rather than the time since a single, global zero-time point. This model is documented in the AMR entitled: InPackage Source Term Abstraction (CRWMS M\&O 2000ap).

Use of the Model: Modification of TSPA-VA approach for use in TSPA-SR.

Review Findings: The slight change from "absolute time" (actually a fixed zero-time) to real time (time since the waste package failed) is reasonable, (although trivial) based upon the validation information given in the AMR. Accordingly, the In-Package Source Term Abstraction Model is assigned to Bin 1.

\subsubsection{In-Package Chemistry for Waste Forms (K.6)}

Description: The AMR identifies the equilibrium chemistry from in-package fluids contacting materials such as steel alloys (used as the basket material), and the breached waste forms consisting of the commercial spent nuclear fuel (CSNF), DOE spent nuclear fuel (DSNF), and Defense High Level Waste (DHLW). A single form of DSNF is used for all cases. Other components available for chemical reaction include ambient $\mathrm{CO}_{2}$ and $\mathrm{O}_{2}$. This model attempts to calculate the major-element composition of in-package fluids as a result of exposure of over 10,000 to $50,000 \mathrm{yr}$ in the repository. The model consists of adding SCFT (solid-centered flowthrough mode) to the much-used EQ3/6 software package. EQ6 models the consequences of aqueous medium reacting with a set of reactants in a time frame. The results of the model calculations are not definitive, mostly as a result of limited input data and the simplification assumptions inherent to this type of model. 
The water to which the breached packages are exposed is assumed to be J-13 well water. Only the $\mathrm{J}-13$ composition at $25^{\circ} \mathrm{C}$ is used. Microbially mediated processes are not considered. Major uncertainties exist in the drip rate and degradation rates, which are therefore represented by ranges of values. The various outputs (solute concentrations and $\mathrm{pH}$ as functions of time) depend strongly on estimates of the cladding coverage, degradation rate, and drip rate. Large ionic strengths are predicted to be outside the range of support by the EQ3/6 software and thermodynamic database.

This model is documented in the AMR entitled: Summary of In-Package Chemistry for Waste Forms (CRWMS M\&O 2000bj).

Use of the Model: Input to the AMR: In-Package Chemistry Abstraction (BSC 2001h, which generates the in-package chemistry information used for TSPA-SR.

Review Findings: The model development is based on conservatism, and compliant validation will require additional work. A validation argument can probably be constructed to comply with AP-3.10Q Section 5.3.b, and, accordingly, the In-Package Chemistry for Waste Forms Model is assigned to Bin 2. On examining the output there are: 1) great excursions of elemental concentrations and $\mathrm{pH}$ with reaction extent, and 2) very different output conditions for the different cases. The results require explanation. The surface areas, waste package component dissolution rates, and thermodynamic database used in the EQ3/6 SCFT calculation need justification. The narrative suggests that only small changes in model output will result because of possible changes to input data, but this is not justified.

\subsubsection{In-Package Chemistry Abstraction (K.7)}

Description: This abstraction model is based on Summary of In-Package Chemistry for Waste Forms (CRWMS M\&O 2000bj). As in that report, ranges of values for water flux, fuel exposure, and WP corrosion rates are used as a device for treating uncertainties in these inputs to the calculation. The abstraction considers $\mathrm{pH}$ to be a "master variable" controlling the concentrations for chemical species of interest. The abstraction is reported as a "response surface" where the $\mathrm{pH}$ is shown as a function of fuel exposure and water flux for two cases of base- and low-corrosion rates for the waste package.

The $\mathrm{pH}$ is stated to control the solution potential Eh in a linear manner, so Eh is abstracted into the $\mathrm{pH}$. The ionic strength histories are calculated for the various input data sets and they vary widely for the cases considered. A further abstraction is to represent the ionic strength history by a triangular distribution whose shape is determined by extreme and average values of the ionic strength during the time history. Separate $\mathrm{pH}$ response surfaces are generated for the period from 0 to $200 \mathrm{yr}$ after breach and the period from 200 to $10,000 \mathrm{yr}$ after breach.

The abstraction is simplified by the assumption that the fugacities of oxygen and carbon dioxide are constant and known. The model provides $\mathrm{pH}$ histories, which in turn control Eh and elemental concentrations for in-package fluids for breached waste packages. The model output includes $\mathrm{pH}$ response surfaces using fuel exposure and water flux as independent variables for two cases of corrosion rate and for four time intervals $(0-200 \mathrm{yr}$; $200-10,000 \mathrm{yr}$.; $10,000-$ $300,000 \mathrm{yr}$; and $300,000-10^{6} \mathrm{yr}$.). The dependence of $\mathrm{Eh}$ on $\mathrm{pH}$ is reported. Ionic strength histories are presented for the various cases and abstracted by a triangular distribution.

Use of the Model: The model output is input to TSPA-SR. 
Review Findings: Like the In-Package Chemistry for Waste Forms Model (Section 6.11 .9 of this report) development of this model is based partly on conservatism, and compliant validation will require additional work. A validation argument can probably be constructed to comply with AP-3.10Q Section 5.3.b, and, accordingly, the In-Package Chemistry Abstraction Model is assigned to Bin 2. The technical defensibility of the abstraction depends on the process-level information from which it is derived (CRWMS M\&O 2000bj). Uncertainties in the independent variable inputs (degradation rates, water drip rate) propagate into uncertainty as to which part of the response surface to use in TSPA.

\subsubsection{Pure-Phase Solubility Limits (K.8)}

Description: This model seeks to calculate solubility limits for $\mathrm{Np}$ and $\mathrm{Pu}$, and other radionuclides of secondary importance to dose, in J-13 groundwater. The EQ3/6 software code was used for the calculations. Equilibrium constants for precipitation reactions presumed to be operative are taken from various sources. Data for $\mathrm{Np}, \mathrm{Pu}$ and $\mathrm{Tc}$ are presented and discussed. The model is documented in the AMR entitled: Pure Phase Solubility Limits - LANL (CRWMS M\&O 2001n).

The equilibrium solubilities for various $\mathrm{Np}$-containing compounds (candidates for controlling solid) are shown graphically as a function of $\mathrm{pH}$. At $\mathrm{pH} 6$ and 7 and low \& high $\mathrm{CO}_{2}$ content $\mathrm{NpO}_{2}+$ is the predominant species in solution, and $\mathrm{NpO}_{2} \cdot \mathrm{xH}_{2} \mathrm{O}$ is identified as the stable solid phase in contact with the solution. The role of $\mathrm{Eh}$ (redox potential) on the $\mathrm{pH}$ dependence of $\mathrm{Np}$ solubility is also discussed.

The analysis for $\mathrm{Pu}$ is presented as a comparison of total $\mathrm{Pu}$ at three temperatures $\left(25^{\circ} \mathrm{C}, 60^{\circ} \mathrm{C}\right.$, $90^{\circ} \mathrm{C}$ ) and $\mathrm{pH}$ values of 6,7 , and 8 with a comparison to values from the literature. Plutonium solubility determination is based on experimental thermodynamic data.

No other plots similar to those presented for $\mathrm{Np}$ are shown for any other nuclide and the $\mathrm{pH}$ dependence is addressed only briefly for $\mathrm{Ni}, \mathrm{Ra}, \mathrm{Sn}$ and $\mathrm{Zr}$.

Use of the Model: The output from this model is used as input to: Summary of Dissolved Concentration Limits (CRWMS M\&O 2001q). Solubility modeling for radionuclides is used in TSPA-SR.

Review Findings: A compliant validation argument based on use of laboratory test data can probably be constructed, hence the Pure Phase Solubility Limits Model is assigned to Bin 2. The scientific basis is somewhat incomplete. For example, the conclusions regarding $\mathrm{Np}$ are noted to have changed significantly between documentation of models K.8 and K.9. Also, the results for other nuclides are very limited.

\subsubsection{Dissolved Concentration Limits (K.9)}

Description: This analysis and model is based on the Pure-Phase Solubility Limits Model LANL (CRWMS M\&O 2001n) with extensive changes. The goal is to establish revised solubility response surfaces for $\mathrm{Np}, \mathrm{Pu}$, and $\mathrm{Ni}$. The solubility of some other nuclides considered in the foregoing AMR are not treated here. The key improvement of the model is the use of the newly qualified database (data 0.YMP.R0) with EQ3/6. One feature of the model is that the occurrence of chemical species is controlled by kinetic relationships rather than equilibrium. The process of precipitation is modeled using the Ostwald step rule whereby metastable species are followed by stable species. 
With the improved database and a more robust view of the chemistry, the authors found very different solubility for $\mathrm{Np}$. The oxide phase $\mathrm{Np}_{2} \mathrm{O}_{5}$ is identified as the controlling solid. Equations representing total nuclide solubility as functions of $\mathrm{pH}$ and $\mathrm{CO}_{2}$ fugacity are given for $\mathrm{U}, \mathrm{Np}, \mathrm{Pu}, \mathrm{Am}$, and Ac. A brief discussion is presented for other nuclides not treated quantitatively.

Use of the Model: The output of this model is used for TSPA-SR.

Review Findings: This model might be considered validated, except for areas of concern with the important radionuclide $\mathrm{Np}$. The changes in input data and modeling approach between models K.8 and K.9 produced a large change in Np solubility for repository-relevant conditions. There is a need for explanation of why the newer results should be considered reliable and the model is therefore assigned to Bin 2.

Part of the output is a comparison of $\mathrm{Np}$ model predictions with drip test results from Argonne National Laboratory. If the drip test results are accepted as reasonable and accurate, then the model must be extremely conservative. In all cases the model prediction for $\mathrm{Np}$ activity is much larger than that observed. In one case the discrepancy is more than 6 orders of magnitude.

\subsubsection{Secondary Uranium-Phase Paragenesis and Incorporation of Radionuclides into Secondary Phases (K.10)}

Description: This conceptual model assesses the potential for uranium(VI) compounds formed during the oxidative corrosion of spent uranium-oxide $\left(\mathrm{UO}_{2}\right)$ fuels to sequester certain radionuclides and, thereby, limit their release. The approach uses analysis of limited laboratory data along with thermodynamic arguments and the behavior of uranium in nature. The "unsaturated drip tests" conducted at Argonne National Laboratory (ANL) provide the basis for this model. The ANL drip tests on spent fuel are the only experiments on fuel corrosion from which solids have been analyzed for trace levels of radionuclides. Brief summaries are provided of the results from other selected corrosion and dissolution experiments on spent $\mathrm{UO}_{2}$ fuels, specifically those conducted under nominally oxidizing conditions. Quantitative data, although not supportive of the sequestration hypothesis, are presented. Discussion of the current understanding of thermodynamic and kinetic properties of uranyl compounds is provided in order to outline the scientific basis for modeling precipitation and dissolution of potential radionuclide-bearing phases under repository-relevant conditions. Attachment I of the AMR provides additional information on corrosion mechanisms and behaviors of radionuclides from tests at ANL. Attachment II reviews occurrence, formation, and alteration (collectively known as paragenesis) of naturally occurring uranyl minerals, because natural mineral occurrences can be used to assess the possible long-term behaviors of uranyl compounds formed in short-term laboratory experiments and to extrapolate experimental results to repository-relevant time scales. The model is documented in the AMR entitled: Secondary Uranium Phase Paragenesis and Incorporation of Radionuclides into Secondary Phases (CRWMS M\&O 2000bd).

The output is a set of general conclusions (e.g., whether corrosion products and uranium paragenesis products can or cannot be proven to sequester or retard radionuclides). The model concludes that Pu-239, Am 241, Tc-99, and I-129 are not sequestered or retarded by corrosion products and uranium paragenesis products. For C-14 and Sc-79 behavior there are no definite conclusions pertaining to retardation by corrosion products and uranium paragenesis products. The AMR suggests (p. 40), but does not prove that Np-237 may behave congruently with uranium paragenesis products. The AMR results are not quantitative in nature. 
Use of the Model: This supporting AMR concludes (p. 43) that more work is needed to develop a quantitative model for retardation of radionuclides by corrosion and uranium paragenesis products in order to reduce overall uncertainties in the TSPA. Since this model does not provide uncertainty-reducing data or conclusions that support its sequestration hypothesis, it is only used by reference in the TSPA-SR.

Review Findings: The conclusions of this model are based on the analysis of limited laboratory data, thermodynamic arguments and the behavior of uranium in nature. The validation argument complies with AP-3.10Q Section 5.3.b, i.e., it is based on model-data comparison supported by other relevant information. No benefit to calculated performance was proven and the results have not been incorporated in TSPA. The argument could be revised (see below) so that it is adequate for the intended use of the model, i.e. not in TSPA, therefore the Secondary Uranium-Phase Paragenesis and Incorporation of Radionuclides into Secondary Phases Model is assigned to Bin 2. If new studies indicate that credit can be taken in TSPA for retardation by corrosion products and uranium paragenesis products, a more rigorous validation process for the model will be required.

It is recommended that more use be made of observations from the Oklo natural reactors and from underground nuclear weapon tests at the NTS to see if any relevant information (e.g., radioelement ratios vs. distance and time) can be used to support this model. Also, coprecipitation and sorption relationships that support the sequestration hypothesis may be found in data available from other natural analogs (e.g., Pena Blanca) and from contaminated sites (e.g., transport data from INEEL). Data that support this model's sequestration hypothesis may be relevant to transport phenomena in the UZ and SZ, so there should be integration with those technology areas to assure that maximum value is achieved from any future work.

\subsubsection{Initial Oxide Thickness (K.11-1)}

Description: The model is documented in the AMR entitled: Initial Cladding Condition (CRWMS M\&O 2000ao). Equation 6.4-1 in the AMR is an abstracted model that determines the initial oxide layer thickness based on final assembly burnup. Surface oxidation of cladding occurs as a fuel is irradiated in the reactor. Cladding thins as the oxide layer grows. Maximum oxide thickness occurs near the upper end of the fuel rod, where there are both high coolant temperatures and high linear power. A linear relationship was suggested by Van Swam, et al. (1997a, 1997b). Plots of peak oxide thickness as a function of rod average burnup (MWd/kgU), Figures 11 and 12 in this AMR, were fitted to linear relationships, with an inflection point at approximately $37 \mathrm{MWd} / \mathrm{kgU}$. Based on data from commercial PWR cladding, two linear equations were chosen: one for burnup $\leq 37 \mathrm{MWd} / \mathrm{kgU}$ and one for values $>37 \mathrm{MWd} / \mathrm{kgU}$. Oxide thickness is stated to be less than or equal to 120 microns.

Use of the Model: The output of this model is cladding oxide thickness vs. rod average burnup. Oxide thickness is not directly used for TSPA. In the AMR entitled: Hydride-Related Degradation of SNF Cladding Under Repository Conditions (CRWMS M\&O 2001k) oxide thickness is used to calculate residual stress in CSNF cladding. In the AMR: Clad Degradation Dry Unzipping (CRWMS M\&O 2000n) oxide thickness is discussed in relation to stress corrosion cracking (SCC).

Review Findings: This model was not identified as a model in the AMR. It should be identified as a model and a validation section should be added. There is sufficient information provided in 
the AMR to validate the equations over the range of burnup. Accordingly, the Initial Oxide Thickness Model is assigned to Bin 2.

\subsubsection{Rod Internal Pressure (K.11-2)}

Description: Rod internal pressure is equal to the sum of the initial fill pressure, fission gas pressure, and, for an extended time duration, helium gas pressure from alpha decay. Fission gas pressure is determined by combining the fission gas production rate in the fuel rod, fission gas release rate from fuel pellets, free volume for the gas to occupy, and temperature. The model is documented in the AMR entitled: Initial Cladding Condition (CRWMS M\&O 2000ao). Equation 6.3-2 in this AMR defines fission gas pressure. The fission gas pressure is limited to above reactor coolant pressure to prevent cladding from creeping away from fuel pellets during reactor operation. The internal pressure of the rod influences possible rod failure from cladding creep, hydride reorientation, delayed hydride cracking, and stress corrosion cracking. This model is used in the statistical analysis to estimate probability distribution functions for fuel gas pressure in a fuel rod. Fission gas pressure is the product of the burnup times the mass of $U$ in one rod times temperature in Kelvin at time of measurement times standard temperature $\left(273^{\circ} \mathrm{K}\right)$. Equation 6.3-4 defines helium gas pressure. Helium gas pressure will increase over time by the production of helium from alpha decay. This equation, equates helium pressure to the product of burnup rate, temperature, helium release fraction and reactor discharge of fuel time raised to an exponent, depending on time.

Use of the Model: The rod internal pressure is not used directly in the TSPA. However, it is used in the cladding stress hoop calculation, which is an input to the stress corrosion cracking (SCC) model for the cladding. SCC of the cladding is included in the TSPA.

Review Findings: This model was not identified as a model in the AMR. The abstracted model should be listed as such and a model validation section should be added that discusses how the abstracted model is an appropriate representation of the data. This will probably result in a compliant validation argument, hence the Rod Internal Pressure Model is assigned to Bin 2.

\subsubsection{Cladding Crack Depth (K.11-3)}

Description: Equation 6.6-2 of the AMR is an abstraction model, which estimates the depth of a crack in cladding based on several studies of commercial fuel. The model is documented in the AMR entitled: Initial Cladding Condition (CRWMS M\&O 2000ao). The crack depth estimate is in the form of a probability distribution function. It is assumed that the crack size distribution is exponentially shaped. The shape and depth of the crack determine the stress intensity factor at the crack tip. Cladding with larger initial cracks would be expected to fail during reactor operation as the cracks propagate through the cladding.

Use of the Model: Although the cladding crack depth is not a direct input to the TSPA-SR, it is an input to the cladding stress corrosion cracking (SCC) calculation. SCC is included in the overall TSPA-SR model.

Review Findings: This model was not identified as a model in the AMR. The abstracted model should be listed as such and a model validation section should be added that discusses how the abstracted model is an appropriate representation of the data. There appears to be sufficient information in the AMR for a compliant validation argument. Accordingly, the Cladding Crack Depth Model is assigned to Bin 2. 


\subsubsection{Overall Cladding Stress (K.11-4)}

Description: This model uses a thin-wall approximation for hoop stress to determine overall cladding stress. Cladding stress is total rod gas pressure multiplied by the cladding inside diameter, divided by twice the difference of initial cladding thickness minus metal loss from oxide layer minus crack depth. This function calculates a distribution for the hoop stress in the cladding. This thin wall approximation underestimates the stress at the inside of the cladding by approximately 7 percent. The stress-distribution in the cladding includes only hoop stresses caused by the differential pressure between the interior and the exterior of the rod. The model is documented in the AMR entitled: Initial Cladding Condition (CRWMS M\&O 2000ao).

Use of the Model: Cladding stress is not used directly for TSPA. Cladding stress is used as an input to calculate cladding stress corrosion cracking, which is included in the overall TSPA-SR model.

Review Findings: The information in the Rothman (1984) and Pescatore et al. (1990) is probably sufficient for a compliant validation strategy. The abstracted model should be listed as a model and a validation section should be added. Accordingly, the Overall Cladding Stress Model is assigned to Bin 2.

\subsubsection{Initial Rod Failure (K.11-5)}

Description: This model provides distribution of physical rod failures based on fuel conditions from reactor operations, storage, and transportation. Failures during reactor operation assume that no blending occurs and fuel is loaded into the waste package in the chronological order that it was discharged from the reactor. Rods failed in the PWR waste package varied from $0 \%$ to approximately $0.127 \%$ with a median of $0.032 \%$ and a mean of $0.030 \%$. Rod failure during dry storage and transportation has been characterized by a creep correlation developed by Matsuo (1987) and a delayed hydride cracking correlation. Delayed hydride cracking is considered unlikely and is not included in the abstraction for the TSPA-SR analysis. During normal shipping of fuel, no failures have been identified in the literature. Testing has given a normal transport failure probability of $2 \times 10^{-7}$ per rod, which is conservatively assumed to be $1 \times 10^{-4}$ per rod to account for other accident conditions. The model is documented in the AMR entitled: Initial Cladding Condition (CRWMS M\&O 2000ao).

Use of the Model: The output of this model is rod failure probability. The rod failure probability is directly used in the TSPA.

Review Findings: This model was not identified as a model in the AMR, however the AMR contains sufficient data to validate the model. Accordingly, the Initial Rod Failure Model is assigned to Bin 2.

\subsubsection{Zircaloy Corrosion Rate Model (K.12)}

Description: This model calculates the corrosion performance of the Zircaloy clad under various environmental conditions. The environmental conditions were divided into four regions: 1) $\mathrm{pH}>$ 3.18 and fluorides less than $5 \mathrm{ppm}$; 2) $3.18<\mathrm{pH}<5$, fluorides greater than $5 \mathrm{ppm}$ and temperature less than $55^{\circ} \mathrm{C}$; 3) $3.18<\mathrm{pH}<5$, fluorides up to $100 \mathrm{ppm}$ and temperatures between $55^{\circ} \mathrm{C}$ and $100^{\circ} \mathrm{C}$; and (4) $\mathrm{pH}<3.18$ and fluorides greater than $5 \mathrm{ppm}$. The authors state 
that there is no need for corrosion rates at temperatures above $55^{\circ} \mathrm{C}$, while fluorides are greater than $100 \mathrm{ppm}$, as there will be instantaneous corrosion.

A technical comment noted that the model does not provide a corrosion correlation for $\mathrm{pH}<3.18$ and fluoride $<5 \mathrm{ppm}$. In the first specified region, the Hillner, et al. equation is used to calculate the general corrosion rate. In the second and third regions, the corrosion rate is assumed to be bounded an equation and $.007 \mathrm{~mm} / \mathrm{yr}$. However, in this region, it is stated that there are no data to support the recommendation for $\mathrm{pH}$ values between 3.18 and 5 . In the fourth region, it is assumed that the cladding will be instantaneously penetrated.

The model is documented in the AMR entitled: Clad Degradation-Local Corrosion of Zirconium and Its Alloys Under Repository Conditions (CRWMS M\&O 20000).

Use of the Model: The corrosion rate is used in the TSPA-SR model to determine localized corrosion. The results from the second, third, and fourth regions are used to calculate the amount of localized corrosion in the TSPA. The results from the first region are used to exclude general corrosion as a FEP.

Review Findings: This model was not identified as a model in the AMR. Sufficient information exists for region (1) to properly validate the correlation. Although there is a claim that there is no data for the second and third regions, there are references in the open literature to validate this model or potentially a more accurate model. The information on the fourth region is not

validated. Rather a very conservative and bounding approach is used. This approach is not valid or accurate for all $\mathrm{pH}$ and fluoride combinations in that region. However, it should be noted that it is a bounding approach. With additional work using available information from the open literature, the models (which may need to be revised) for the second, third, and fourth regions can be validated. Accordingly, the Zircaloy Corrosion Rate Model is assigned to Bin 2.

Additional research is needed into the interaction of $\mathrm{pH}$, fluorides, and temperature, starting with more complete use of the available open literature. In addition, the work to develop the corrosion correlations for low $\mathrm{pH}$ conditions (though ultimately not used) is flawed in several ways. The reviewer agrees that the correlations should not be used, but does not agree that the bounding approach is appropriate. The flaws in the correlations include an inverse relationship with both temperature and chlorides (i.e., as temperature goes up, the model has corrosion rate going down and as chlorides go up, the model has corrosion rate going down). It is recommended that the correlations from the low $\mathrm{pH}$ regions be deleted.

\subsubsection{Residual Stress in CSNF Cladding Material (K.13)}

Description: This abstraction model describes the first approximation of the calculation of oxide induced residual stress. This residual stress is produced by expansion of $\mathrm{Zr}$-oxide volume in the waterside oxide layer in contact with the metallic layer in CSNF metal. The oxide layer affects the microstructure of CSNF cladding, which in turn affects hydride related degradation. Results of these conservative calculations indicate that the stress-intensity factor on a flaw in the metallic layer (in contact with the coolant-side layer) is a strong function of the thickness of the adherent uncracked oxide that contains a high volume-fraction of tetragonal $\mathrm{ZrO}_{2}$. During hot-cell burst tests of PWR SNF cladding in inert gas environment at $290^{\circ}$ to $325^{\circ} \mathrm{C}$, cracks initiated on the outer surface of the cladding metal covered with an oxide layer. This indicated oxide-induced residual stress. The abstraction equation is used to calculate these stresses. This abstraction is considered conservative, and is a function of the thickness of the oxide layer. The degree of 
thickness of the oxide layer influences the degree of hydrogen uptake. Oxide thickness in PWR cladding is expected to be 15 to 80 microns. Figure 2 of this AMR shows oxidation-induced residual stress in CSNF cladding material along with characteristic oxide cracking and hydrogen concentration beneath the cracked oxide. The oxygen morphology helps explain the enhanced hydrogen uptake and hydriding. This model assumes negligible elastic strain, Young's modulus of $200 \mathrm{GPa}$, and a metal layer thickness of 560 microns.

The model is documented in the AMR entitled: Hydride-Related Degradation of SNF Cladding Under Repository Conditions (CRWMS M\&O 2001k).

Use of the Model: This model is used to calculate residual cladding stress. Results of calculations are shown in Table 1 of the AMR. The output is used to screening features, events and processes (FEPs) for hydride related metallurgical processes, such as delayed hydride cracking, hydride reorientation and hydride embrittlement.

Review Findings: Although the AMR indicates that models were not used, Figure 3 in the AMR is entitled: "Summary of Model Designed to Calculate Residual Stress in CSNF Cladding Metal as a result of Oxide Volume Expansion and Circumferential Cracking of Oxide." Also, page 15 of the AMR discusses work by Chung and Yaggee (1984) that supports models of Figure 3. This model should be listed as such and a model validation section should be added that discusses how the model provides an accurate representation of the residual stress in cladding. The information in Chung and Yaggee (1984) should be expanded upon to provide a compliant validation. Therefore, the Residual Stress in CSNF Cladding Material Model is assigned to Bin 2.

\subsubsection{Alternative Wet Clad Unzipping Model (K.14-1)}

Description: The analysis and model applies to the rate of breach extension ("unzipping") of a spent nuclear fuel rod over the long term, where the cladding has been breached and water is present. The purpose of the model appears to be to support recommendations for simplified/bounding models of this process for use in TSPA, but this is not fully explained. The model is documented in the AMR entitled: Clad Degradation - Wet Unzipping (CRWMS M\&O 2000 ).

The model applies to unzipping of CSNF when the temperature of the nuclear fuel rod is below $100{ }^{\circ} \mathrm{C}$, water or moist air is present, and there is a pre-existing breach in the fuel rod. (If any of these conditions are not met the model is not applicable). An alternative evaluation is also presented in Section 6.7.2 of the AMR, based on experimentally observed shape of partially "unzipped" fuel rods at long times (on the order of years). In this approach the degraded fuel is modeled as a cylinder with the greatest extent of alteration furthest from the crack tip. This is similar to a model developed in AMR: Clad Degradation - Summary and Abstraction (CRWMS M\&O 2001d). The observations are based on photographs of failed rods. The rate of unzipping calculated using this approach is $10^{5}$ to $10^{6} \mathrm{yr}$.

Use of the Model: The model output consists of rates for clad "unzipping" under condensingwater conditions, which are input to the cladding abstraction (CRWMS M\&O 2001d), and are used in TSPA-SR.

Review Findings: The data support for this model is inadequate for validation. It may be possible to qualify this approach, but much work would be required. Accordingly, the Alternative Wet Clad Unzipping Model is assigned to Bin 3. The times required for unzipping 
are so long $(10,000$ to $>1,000,000 \mathrm{yr})$ that this seems unlikely to be an important failure mode for the cladding.

It is recommended that this model be combined with another model, incorporating the rates of general and pitting corrosion of the cladding, such that when individual rods are breached the failure of the overall rod can be treated using this model. Other clad degradation models found in: Clad Degradation - Dry Unzipping (CRWMS M\&O 2000n) and Clad Degradation Summary and Abstraction (CRWMS M\&O 2001d) should be reconciled with this model using the appropriate irradiated clad properties.

Impact Review Summary: This model estimates the range of the unzipping rate multiplier and thus the effectiveness of cladding in limiting CSNF release. The model is used in TSPA-SR but did not serve as the basis for screening out FEPs. Because the contribution of cladding to total system performance is minor, there is no significant impact from uncertainty in the cladding degradation model, on the conclusions of TSPA-SR (CRWMS M\&O 2000bm, Section 6.1). Based on this information and the additional detail provided in Appendix IV, the model validation review findings associated with this model have no significant impact on the conclusions TSPA-SR.

\subsubsection{Bounding Model for Clad Unzipping Velocity (K.14-2)}

Description: This model applies to the rate of breach extension ("unzipping") of a spent nuclear fuel rod over the long term where the cladding has been breached and water is present. This model is intended to be abstracted into the system model found in Section 6.11.24 of this report (K.16). The Bounding Model for Clad Unzipping Velocity only applies when the temperature of the nuclear fuel rod is below $100^{\circ} \mathrm{C}$ and water or moist air is present and there is a pre-existing breach in the fuel rod.

The spent fuel alteration rate is first calculated using the kinetics modeled in a separate AMR: CSNF Waste Form Degradation: Summary Abstraction (CRWMS M\&O 2000r). Diffusion of water in the gap and the properties (density and volume) of the probable alteration species are coupled to a fracture mechanics model of the crack propagation. Unzipping velocities calculated from this approach predict times on the order of $10^{4}$ to $10^{5} \mathrm{yr}$ (shorter than the "alternative model" discussed in Section 6.11.21 of this report).

The model is documented in the AMR entitled: Clad Degradation - Wet Unzipping (CRWMS $\mathrm{M} \& \mathrm{O} 2000 \mathrm{p})$.

Use of the Model: The rate of clad unzipping failure under conditions where the SNF is exposed to moisture is used for TSPA-SR.

Review Findings: The data used for this model, i.e., clad/fuel gap size, fuel alteration kinetics, and material properties of irradiated cladding, appear to be based on reliable sources. The model is appropriate for the conditions for which it is intended, i.e., when the waste package has been breached and the fuel is exposed to condensing water. The predicted rates of failure for individual rods with pre-existing cracks are reasonable. Since the predicted unzipping times are so long, the sensitivity of the TSPA-SR is limited and the model is clearly valid for its intended use. Accordingly, the Bounding Model for Clad Unzipping Velocity is assigned to Bin 1.

It is recommended that this model be combined with another model, incorporating the rates of general and pitting corrosion of the cladding, such that when individual rods are breached the failure of the overall rod can be treated using this model. Other clad degradation models found 
in: Clad Degradation - Dry Unzipping (CRWMS M\&O 2000n) and Clad Degradation Summary and Abstraction (CRWMS M\&O 2001d) should be reconciled with this model using the appropriate irradiated clad properties.

\subsubsection{Clad Dry Unzipping Model (K.15)}

Description: An analysis and a semi-empirical model are presented for the unzipping of cladding due to dry oxidation of the uranium oxide matrix spent fuel. The oxidation of the fuel and consequent phase transformations are modeled as a function of time and temperature in an oxygen environment. The swelling of the fuel results in strain of the cladding and propagation of existing defects into splits along the axis of the clad. The oxidation of the clad is modeled using a fit to qualified and accepted experimental data. The pellet/clad gap is assumed to be zero. The existing defects are assumed to expand at zero (additional) strain. The properties of the cladding (yield strength, Poisson ratio, etc.) are assumed to be those of un-irradiated Zircaloy; this assumption is justified in the AMR entitled: Clad Degradation - Wet Unzipping (CRWMS M\&O 2000p), but not in this AMR. The unzipping velocity is controlled by the oxidation rate of the fuel and the elastic properties of the cladding. The simplified form of the crack opening velocity, derived in this model, is:

$$
\mathrm{V}=\mathrm{G} / \sigma_{\mathrm{y}} \cdot(\mathrm{dr} / \mathrm{dt})
$$

where $\mathrm{dr} / \mathrm{dt}$ is the time rate of change of the radial expansion of the fuel at the crack tip. Based on comparison with literature values the model overestimates crack velocities at lower temperatures by up to 3 orders of magnitude, although in some cases the match is nearly perfect.

This model is documented in AMR: Clad Degradation - Dry Unzipping (CRWMS M\&O 2000n).

Use of the Model: The output of this model (oxidation rate of $\mathrm{UO}_{2}$ and cladding failure under hot and dry oxidation conditions) is not used in TSPA-SR because the waste packages remain intact long after the fuel has cooled below the temperature range of interest.

Review Findings: The information used in the model on oxidation of $\mathrm{UO}_{2}$ and CSNF is valid because it is based on test data and information reported in published literature. However, the data for properties of Zircaloy are not supported and are the properties of un-irradiated Zircaloy taken from a table in an undergraduate textbook. The assertion is made that this is conservative based on the expectation that irradiated Zircaloy would exhibit a higher yield stress. The approach taken in AMR: Clad Degradation - Wet Unzipping (CRWMS M\&O 2000p) for wet unzipping is preferable, in which both qualified test data and literature data are used to develop a data set for properties of irradiated Zircaloy. This model should be combined with the cited model (CRWMS M\&O 2000p) and the abstraction (CRWMS M\&O 2001d), and all the models should use the same set of data. Accordingly, the Clad Dry Unzipping Model is assigned to Bin 2.

The models used for clad failure would be significantly improved by incorporating a model of early clad failure when no pre-existing defects are present (i.e., defect nucleation and growth.)

\subsubsection{Summary and Abstraction - Clad Unzipping and Fuel Dissolution (K.16)}

Description: An empirical model is presented for wet unzipping of CSNF. The model is intended to be an abstraction of the unzipping models described in Clad Degradation - Wet Unzipping (CRWMS M\&O 2000p), however it principally uses the geometric and observational 
concepts from the Alternative Wet Clad Unzipping Model (K.14-1; see Section 6.11.21 of this report). The results are then put into a "computationally efficient" form. The predicted unzipping times are presented in Figure 19 of the subject AMR (CRWMS M\&O 2001d) and are on the order of $10^{5} \mathrm{yr}$.

This model is documented in the AMR entitled: Clad Degradation - Summary and Abstraction (CRWMS M\&O 2001d).

Use of the Model: The rate of clad unzipping under wet conditions and the distribution of failed rods in multiple waste packages are used in TSPA-SR.

Review Findings: This model differs from the three models previously considered under the headings of wet and dry unzipping (Sections 6.11.21 through 6.11.23 of this report). The model development is based on reasonable engineering and mathematical constructs, but the data support is inadequate. This model is similar to the others in the sense that they predict clad unzipping times significantly greater than $10^{4} \mathrm{yr}$. However, the times to failure are different, and the model development limited, such that model validation will require additional work to understand the differences. This model should be reconciled with those reviewed previously. Accordingly, the Clad Unzipping and Fuel Dissolution Model is assigned to Bin 3 (impact review is provided in Appendix IV).

The models used for clad failure would be significantly improved by incorporating a model of early clad failure when no pre-existing defects are present (i.e., defect nucleation and growth.)

Impact Review Summary: This model estimates the range of the unzipping rate multiplier and thus the effectiveness of cladding in limiting CSNF release. This model is used in TSPA-SR but did not serve as the basis for screening out FEPs. Because the contribution of cladding to total system performance is minor, there is no significant impact from uncertainty in the cladding degradation model, on the conclusions of TSPA-SR (CRWMS M\&O 2000bm, Section 6.1). Based on this information and the additional detail provided in Appendix IV, the model validation review findings associated with this model have no significant impact on the conclusions TSPA-SR. Additional work performed to address the DOE-NRC agreements on Key Technical Issue related to cladding degradation, will provide additional confidence in the cladding abstraction model.

\subsubsection{Stainless Steel in WPs for TSPA-SR (K.17)}

Description: This model includes a calculation and justification for the average number of stainless steel clad assemblies in the waste packages and the expected distribution thereof. This AMR is not marked as a model, but the assumptions on which it is based express a model for the loading of waste containers whereby they are loaded in a way that fuel assemblies are distributed in batches as available. The model is documented in the calculation report: Stainless Steel in Waste Packages for TSPA-SR (CRWMS M\&O 2000bh).

Use of the Model: The average number of stainless steel assemblies in the waste packages is input to the cladding degradation abstraction.

Review Findings: The model is documented as a calculation report, but should be a model. The approach is straightforward and a compliant validation argument can probably be developed. Accordingly the Stainless Steel in Waste Packages for TSPA-SR Model is assigned to Bin 2. 


\subsubsection{Thermal Evaluation of Breached 21-PWR Waste Packages (K.18)}

Description: This model is documented as a "calculation" that determines the radial temperature distribution in a breached waste package as a function of time. The output is a temperature distribution for the waste package resulting from using a conceptual model that provides conservative (higher) temperatures. The conceptual model is based on proven analytical heat transfer modeling methods and many simplifying assumptions, such as the geometry and thermal properties of the waste package, environmental conditions, and engineering judgement about dominant heat transfer mechanisms.

Temperature histories are calculated for a 21-pressurized water reactor spent nuclear fuel waste package loaded with: 1) average-heat output fuel assemblies, and 2) design-basis-heat output fuel assemblies. Different repository design configurations were investigated. The results of this calculation support evaluations of credit for cladding integrity in performance assessment analyses. The model is documented in calculation report: Thermal Evaluation of Breached 21PWR Waste Packages (CRWMS M\&O 1999f).

Use of the Model: The model output consists of tables of calculated surface and internal temperature histories for different repository designs. The AMR: Clad Degradation - Summary and Abstraction (CRWMS M\&O 2001d) uses peak internal temperatures from Tables 6-2 and 6-7 from the documentation of this model (CRWMS M\&O 1999f) to evaluate uncertainty. The differences between rod gas plenum and peak clad temperatures (Table 6-4) are used to estimate strain correlation. The model was used for TSPA-SR as described, and was also used as the basis for FEP screening. Note that output from this model is not used in Model Area I, EBS Moisture Distribution and TH (Section 6.9 of this report).

Review Findings: The model is documented as a calculation report, but should be a model. The author chose a conceptual model whereby heat transfer within the waste packages is limited to thermal radiation. Other heat transfer mechanisms could have been selected, and the model has not been validated. The choice to ignore axial conduction and buoyant convection as heat transfer mechanisms is not at all obvious and should be validated by documenting sample calculations that illustrate the relative importance of those mechanisms compared to thermal radiation with particular attention to prediction of temperatures in different locations within the waste packages. Reservations about the reliability of the calculated output are expressed in Section 6, of (CRWMS M\&O 1999d), and must be addressed to improve confidence. Assumptions claimed to be conservative may not produce conservative system performance, depending on how the output from this report is used. In accordance with these comments, the Thermal Evaluation of Breached 21-PWR Waste Packages Model is assigned to Bin 3 (impact review is provided in Appendix IV). One possible method of validation proposed by the reviewer is to directly simulate heat transfer using a set of rod heaters to simulate spent fuel rods.

Impact Review Summary: This model calculates the difference in temperature between the cladding and the waste package surface to support evaluation of cladding degradation. The model is used in TSPA-SR and did serve as a basis for screening out FEPs. Because the contribution of cladding to total system performance is minor, there is no significant impact from uncertainty with respect to cladding degradation, on the conclusions of TSPA-SR (CRWMS $\mathrm{M} \& \mathrm{O} 2000 \mathrm{bm}$, Section 6.1). Based on this information and the additional detail provided in 
Appendix IV, the model validation review findings associated with this model have no significant impact on the conclusions TSPA-SR.

\subsubsection{Breakage of CSNF Clad by Seismic Loading (K.19-1)}

Description: In this model the expected rate for breakage of commercial spent nuclear fuel cladding caused by seismic mechanical loading is estimated. The model that is used was developed on well-founded, rigorous engineering principals and conservative assumptions. For seismic loading, the equations of motion for a fuel rod in an assembly are solved for impact of the assembly on an unyielding surface. The solution to the equations of motion is used to determine the fragility, that is, the probability that the fuel rod will break under the specified loading. The fragility of the fuel rod is convolved with the expected seismic hazard to determine the rate of rod breakage. The model is documented in calculation report: Breakage of Commercial Spent Nuclear Fuel Cladding by Mechanical Loading (CRWMS M\&O 1999a).

Use of the Model: The output from this model consists of a table summarizing seismic risk to cladding integrity (Table 2 of the AMR) that is input to: Clad Degradation - Summary and Abstraction (CRWMS M\&O 2001d).

Review Findings: This model is not a simple calculation, and although it is based on wellfounded principles and assumptions, it has not been validated using test data or information from the literature. The overly conservative conclusion of this model goes against the fact that nuclear fuel elements have been designed not to fail in nuclear power plants that are licensed to be operated in seismic (or battle-shock) environments. Nuclear reactor fuel seismic design and testing information should be reviewed for information relevant to this modeling activity. Also, a test using a mock-up waste package could be performed at modest cost in an existing seismic test facility. It is the opinion of the reviewer that a study of nuclear power plant seismic design reports and/or realistic tests would reverse (not validate) the conclusion of this model and demonstrate that failure of CSNF cladding is highly unlikely from any credible seismic loading scenario. Accordingly, the Breakage of CSNF Clad by Seismic Loading Model is assigned to Bin 3 (impact review is provided in Appendix IV).

Impact Review Summary: This model was used to determine the likelihood of a seismic event causing breakage of the cladding. This model is abstracted for use in TSPA-SR but did not serve as a basis for screening out FEPs. Because the contribution of cladding to total system performance is minor, there is no significant impact from uncertainty in the cladding degradation model, on the conclusions of TSPA-SR (CRWMS M\&O 2000bm, Section 6.1). Based on this information and the additional detail provided in Appendix IV, the model validation review findings associated with this model have no significant impact on the conclusions TSPA-SR. Additional work performed to address the DOE-NRC agreements on Key Technical Issues related to cladding degradation, will provide additional confidence in the cladding abstraction model.

\subsubsection{Breakage of CSNF Clad by Static Loading (K.19-2)}

Description: This model describes the calculated, expected rate for breakage of commercial spent nuclear fuel cladding caused by static mechanical loading. The model that is used was developed on well-founded and rigorous engineering principals. For static loading by backfill or rubble, the amount of material is used to determine the gravitational load on a fuel rod. That static load is compared with the calculated load a rod can support to determine whether the rods 
can support the load. The model is documented in the calculation report: Breakage of CSNF Cladding by Mechanical Loading (CRWMS M\&O 1999a).

Use of the Model: The output from this model includes a conclusion that static loads could fail the cladding. However, damage to the waste package and its contents during the 10,000-yr regulatory performance period is screened out because the drip shield and waste package are robust with respect to rockfall (CRWMS M\&O 2000cd; Section 6.2.1.5). After this period, cladding failure by other modes would occur before failure from rockfall loading. As a result, this model is not used for TSPA-SR.

Review Findings: This model is not a simple calculation, and although based on well-founded principles and assumptions, it has not been validated using test data or information from the literature. The model appears to be overly conservative, and not realistic, and is therefore assigned to Bin 3 (impact review is provided in Appendix IV). A test using rubble, of the type expected to result from rockfall, to load a mock-up waste package (with the drip shield) may reverse the static load failure conclusion. It is the opinion of the reviewer that a realistic test would reverse (not validate) the static load failure conclusion of this model and demonstrate that failure of the Waste Package and its fuel elements is highly unlikely from credible roof-fall or backfill-loading scenario.

Impact Review Summary: This model was not used in TSPA-SR and did not serve as a basis for screening out FEPs. The cladding will be protected from static loading by the waste package and drip shield, throughout the 10,000-yr performance period. Based on this information and the additional detail provided in Appendix IV, the model validation review findings associated with this model have no significant impact on the conclusions TSPA-SR (CRWMS M\&O 2000bm, Section 6.1).

\subsubsection{Colloid-Associated Radionuclide Concentration Limits (K.21)}

Description: This model provides an upper-bound correlation of available colloid data from waste form corrosion tests at Argonne National Laboratory (ANL). The model develops a useful description of the following waste-form colloid characteristics: 1) composition, 2) size distribution, and 3) quantification of the rate of waste form colloid generation. The composition and size distribution information support analysis of the potential transport of the sparingly soluble radionuclides associated with waste-form colloids. The rate of colloid generation supports analysis of the waste form colloid-associated radionuclide concentrations. In addressing the above characteristics, available data are interpreted to address mechanisms for colloid formation and stability. The model is documented in the AMR: Colloid-Associated Radionuclide Concentration Limits (CRWMS M\&O 2001e).

Use of the Model: The model output is a conservative upper-bound of experimental data, which includes maximum colloid concentrations and absorption values for $\mathrm{Pu}$ and $\mathrm{Am}$ on colloids. The model provides part of the input used by: Waste Form Colloid-Associated Concentration Limits: Abstraction and Summary (CRWMS M\&O 2001s).

Review Findings: The supporting AMR describes a model not an analysis. The conservative upper bound for Pu colloidal releases was validated using the best-available experimental data from "drip tests" conducted on waste forms at chemical conditions that are relevant to the potential repository. A validation argument can be developed to comply with AP-3.10Q Section 
5.3.b, based on model-data comparison supported by other relevant information. Accordingly, the Colloid-Associated Radionuclide Concentration Limits Model is assigned to Bin 2.

If water chemistry scenarios other than those used to gather the experimental data have significantly high probability in the TSPA, additional modeling and validating test data will be needed to assure that the model covers the entire range of expected conditions.

\subsubsection{Waste Form Colloid-Associated Concentration Limits: Abstraction and Summary (K.22)}

Description: This abstraction model is based on data developed primarily by Argonne National Laboratory (ANL) and Los Alamos National Laboratory (LANL). The process models invoked in this abstraction describe the observed types, formation, and stability of radionuclide-bearing colloids that result from degradation and decomposition of the waste forms contained in failed waste packages. In addition, contaminant-colloid attachment/detachment mechanisms and transport characteristics anticipated in the repository are discussed for colloids formed from degradation of the waste forms, colloids formed from man-made materials introduced into the repository, and naturally occurring colloids. The abstraction of the process models captures the most important characteristics of radionuclide-colloid behavior for use in predicting the potential impact on repository performance of colloid-facilitated radionuclide transport. The model is documented in the AMR entitled: Waste Form Colloid-Associated Concentration Limits: Abstraction and Summary (CRWMS M\&O 2001s).

Output of the model consists of colloid concentration as a function of ionic strength, assuming that colloids are stable. This information is suitable for estimating the stability of smectite and iron-(hydr)oxide colloids as functions of ionic strength and $\mathrm{pH}$. The abstraction uses bounding relationships that are closely tied to the colloid generation and characterization experimental programs conducted at ANL and LANL. The abstraction is conservative and incorporates as much realism as is considered defensible.

Use of the Model: The concentrations of colloids and colloid-associated radionuclides are output for use directly in TSPA-SR.

Review Findings: The model description of colloidal releases was validated using experimental data from ANL and LANL conducted on YMP-type waste forms, and data from natural analog systems with chemical conditions relevant to the potential repository. A validation argument can be developed to comply with AP-3.10Q Section 5.3.b, based on model-data comparison supported by other relevant information. Accordingly, the Waste Form Colloid-Associated Concentration Limits: Abstraction and Summary Model is assigned to Bin 2.

If water chemistry scenarios other than those used to gather the experimental data abstracted by this model have significantly high probability in the TSPA, additional modeling and validating experimental data will be required to assure that the colloid abstraction model covers the entire range of expected conditions. 


\subsection{Model Area L: EBS Degradation}

\subsubsection{DRKBA Rockfall Model (L.1)}

Description: The DRKBA Rockfall Model is used to examine the time-dependent degradation of unsupported drifts under static (as-excavated), thermal, and seismic loading conditions. The model is documented in the AMR entitled: Drift Degradation Analysis (CRWMS M\&O 2000x). The model considers progressive block failure along preexisting joint sets, such that when an initial block falls and is removed, then additional failure can continue until the crown becomes kinematically and mechanically stable and no additional blocks can fall. Distributions for the number and sizes of fallen rock blocks as a function of excavation length and lithostratigraphic unit are determined based on key block analyses and probabilistic representations of the joint sets in the units.

Static and quasi-static key block analysis results are presented for four lithostratigraphic units of the Topopah Spring welded tuff. These are identified as: 1) crystal poor upper lithophysal zone, 2) crystal poor middle nonlithophysal zone, 3) crystal poor lower lithophysal zone, and 4) crystal poor lower nonlithophysal zone. The quasi-static analyses account for seismic and thermal effects on key blocks.

For the static key block analyses, results include the cumulative frequency of occurrence vs. block volume for drift orientations between $0^{\circ}$ and $180^{\circ}$ at $15^{\circ}$ intervals. For the seismic analyses, drift orientations with azimuths of $105^{\circ}$ and $75^{\circ}$ were analyzed. Cumulative frequency of occurrence vs. block volume are provided for three levels of earthquakes representing 1,000-, 5,000-, and 10,000-year events. Rock falls related to thermal effects are presented at 0, 200, 2,000 , and $10,000 \mathrm{yr}$ for drift orientations with azimuths of $105^{\circ}$ and $75^{\circ}$ without backfill and for the $105^{\circ}$ drift orientation with backfill. In addition to cumulative frequencies of occurrence vs. block volume, the predicted average volume of rock fall per unit length of drift is tabulated for the four lithostratigraphic units.

Use of the Model: Worst-case drift profiles for each of the lithostratigraphic units are provided for various loading scenarios and configurations. These are provided for consideration in seepage modeling. Block size information is also used for performance analyses of engineered barriers subject to rockfall.

Review Findings: Validation of the DRKBA Rockfall Model was addressed in the subject AMR (CRWMS M\&O 2000x). Methods documented for validating the model include: 1) comparing the results of the model against relevant field observations at Yucca Mountain; 2) comparison of model results with an alternative model; and 3) comparison of the model results with natural analogs. Whereas these methods are appropriate to validate a model according to AP-3.10Q, the validation effort did not sufficiently address the performance of the model for its intended use (i.e., thermal- and seismic-related postclosure drift degradation). Accordingly, the DRKBA Rockfall Model is assigned to Bin 3 (impact review is provided in Appendix IV). Thermal effects were accounted for in the model by reducing the joint cohesion as a function of time, whereas the seismic effects were accounted for by reducing both the joint cohesion and the friction angle. Simulating the effects of additional loads (thermal or seismic) by reductions in strength is not a conventional approach in rock mechanics, and the validation of this approach presented in the AMR was not sufficient to develop confidence in the methodology. The use of natural analogs does increase the level of confidence that the response to seismic effects would 
be minor. Comparison of model predictions for block size and distribution under static (asexcavated) conditions with the rock falls observed in the ESF and ECRB cross-drift does serve as an excellent example to validate the static analyses. However, the intended use of the model is not limited to the static condition.

An alternative distinct-element simulation technique (UDEC) was used to calibrate joint property parameters in response to seismic loads based on a single realization of the joint spacing and orientation. It is the opinion of the reviewers that the comparison of the DRKBA Rockfall Model results with the UDEC results was a calibration effort and does not constitute model validation.

The reviewers agree with the report of a panel of tunneling, mining, and rock mechanics experts regarding postclosure performance of the emplacement drifts, entitled: Panel Report on the Drift Stability Workshop, Las Vegas, Nevada, December 9-11, 1998. (Brekke et al., 1999). This report concludes that, "A variety of rock fracture-degrading mechanisms will be driven by the steep $\Delta \mathrm{T}$, $\Delta \mathrm{P}$, and $\Delta \sigma$ gradients for a long period of time. This is likely to lead inevitably to the collapse of some of the drifts." The DRKBA Rockfall Model considers only rock falls associated with preexisting joint sets and does not account for: 1) brittle failure or crushing of intact rock caused by excavation and thermally induced stresses; 2) ravelling of rock within intensely fractured zones; and 3) stress concentrations and rock failure caused when the drift profile changes after a rock fall. Deterministic analyses of these processes were included in the aforementioned panel report, and the results were used in conjunction with the DRKBA Rockfall Model to develop the abstraction of drift degradation used in the Seepage Model for PA Including Drift Collapse (Model E.2), Section 6.5.2 of this report, (CRWMS M\&O 2000be).

The AMR entitled: Ground Control for Emplacement Drifts for SR (BSC 2001f) is a design analysis that includes stability analysis for unsupported drifts. Potentially, this AMR could be merged with the DRKBA Rockfall Model to provide a more comprehensive analysis of drift degradation.

Impact Review Summary: The DRKBA Rock Fall Model is used in TSPA-SR and did serve as a basis for screening out features, events, and processes. The DRKBA model is reasonably conservative for predicting the occurrence of large rockfall blocks; these blocks are then used for structural analysis of the drip shield design. Although the DOE recognizes the need for, and has agreed to make, model improvements and evaluate alternative modeling approaches, the current model and its supporting and related documentation are considered to provide adequate confidence that the effects of rockfall on integrity of the waste package can be limited for 10,000 years by use of the drip shield. This is the basis for the rockfall screening decision for TSPA-SR. Based on the results of additional evaluations, described in Appendix IV and including activities that will addresses DOE-NRC agreements on Key Technical Issues, DOE will reconsider the screening decision for inclusion or exclusion of rockfall in performance assessment analyses. Based on the above information and the additional detail provided in Appendix IV, the model validation review findings associated with the DRKBA Rock Fall Model have no significant impact on the conclusions of TSPA-SR (i.e. dose calculations; CRWMS M\&O 2000bm, Section 6.1).

\subsubsection{Rockfall on Drip Shield Model (L.3)}

Description: This product was documented as an engineering calculation to determine the effects on the drip shield from falling rock blocks with masses of $2,4,6,8$, or 52 metric tons. The calculation showed that the drip shield design considered would not fail even if impacted by 
a 52-metric ton rock falling form directly above. It is a model according to the criteria in Section 3.4.2, and it is documented in Rockfall on Drip Shield (CRWMS M\&O 2000bw).

Use of the Model: The output of the model includes: 1) the conclusion that rock falls of 2, 4, 6, 8 , and 52 MT will not cause the DS to fail (rupture); 2) a tabulation of the potential number and sizes (lengths) of stress corrosion cracks (SCCs) associated with the rockfalls; and 3) figures showing the magnitudes of the residual tensile stresses caused by the rock falls.

The information on the number and sizes of SCCs, and the residual tensile stresses, is used in several other models that represent SCCs in the drip shield and liquid flow through them. The specific reports include: Stress Corrosion Cracking of the Drip Shield, the Waste Package Outer Barrier, and the Stainless Steel Structural Material (CRWMS M\&O 2000bi); Flow of Water and Pooling in a Waste Package (BSC 2001c); and EBS Radionuclide Transport Abstraction (CRWMS M\&O 2000z).

Review Findings: The Rockfall on Drip Shields calculation should be documented as a model because of the potential impact on drip shield performance. The information needed for validation is readily available, hence the model is assigned to Bin 2 . The primary objective of the activity was to determine whether the drip shield would fail (rupture) if impacted by rock blocks with a mass of $2,4,6,8$, or 52 metric tons. Had the calculation report been limited to this objective, it would have been classified correctly as an engineering calculation. However, the activity went beyond the primary objective and also determined the number of potential stress corrosion cracks (SCCs) and their sizes in areas where plastic deformation caused by the rock fall yielded residual tensile stresses. The SCC and residual tensile stress calculations are being used in higher-level models addressing seepage through the DS. Since SCCs are potential pathways for seepage during early times (patch penetrations by general corrosion require much longer time periods), the calculations of the number and sizes of SCCs and of the residual tensile stresses may be critical to evaluating DS performance. Consequently, the Rockfall on Drip Shields Model should be documented as a model and validated per AP-3.10Q criteria.

The Rockfall on Drip Shields Model can be validated using data from other AMRs and analogs reported in the open literature. Specifically, the distributions of rock block sizes and rock fall locations provided in the Drift Degradation Analysis (CRWMS M\&O 2000x; changes are recommended to this model also, see above) can be used to validate the conservatism of the model with respect to the rock block sizes. The technical literature is expected to contain analogous applications of transient-dynamic analyses to validate the approach for modeling the impact of falling bodies on metal structures (e.g., pipelines).

The Rock Fall on Drip Shields Model should also consider the interaction between the drip shield and the invert. The current modeling approach maximizes deformation of the drip shield and the potential for rupture by placing the drip shield on a rigid foundation. However, it seems likely that a large rock fall would cause the thin base of the drip shield to punch into the crushedtuff invert ballast material. This would cushion the loading of the drip shield, reducing plastic deformation of the metal. However, this process could yield more vertical displacement of the drip shield, thereby potentially affecting the integrity of the joint between adjacent drip shield segments. 


\subsubsection{Flow into Waste Packages Through Small Lid Openings (FLO) Model (L.6)}

Description: The FLO Model provides a conceptual and mathematical basis for predicting the quantity of liquid water leakage through different types of breaches in the waste package and drip shield. It was documented in the AMR entitled: Flow of Water and Pooling in a Waste Package (BSC 2001c). The model assumes that water contacting a breach may originate from a source directly above, or may have flowed laterally from another source location. Lateral flow in the model is controlled by a spreading factor that depends on the surface roughness. All the water contacting breaches in the drip shield will flow through. If the opening in the WP is large enough (e.g., large openings formed by igneous disruption) all the water that contacts the opening will flow through. For stress corrosion cracks, comparison of the water film thickness with the crack width determines the fraction of the incident flow that flows through. An SCC can become saturated and bridged by water, whereupon through-flow of liquid is impeded. The model consists of two main parts: 1) the fraction of the drift seepage that contacts breaches, and 2) the fraction of contacting water that flows through breaches.

Use of the Model: Development of the FLO Model is relatively recent (2001) and the output has not been incorporated in other models or reports.

Review Findings: The FLO Model is assigned to Bin 3 (impact review is provided in Appendix IV). In Section 6.6.7, this AMR claims the model is appropriate for its intended use, in compliance with AP-3.10Q because: “...1) model inputs and assumptions are reasonable and produce reasonable results for the intended use; 2) model results are within bounds provided by conservative derivations." However, neither statement complies with the validation options provided in Section 5.3 of AP-3.10Q.

Although theoretical and experimental work published in the open literature is incorporated in the development of the FLO Model, the reviewer is not aware of results in other AMRs or publications that could be readily used to validate the results of the FLO Model. Consequently, experiments on mockups of the EBS components need to be conducted to provide flow data for comparison with the results of the model.

Impact Review Summary: This model was developed after TSPA-SR. The purpose of the model was to improve understanding of processes controlling water flow into waste packages. This model was not used in TSPA-SR and did not serve as a basis for FEP screening. Based on this information and the additional detail provided in Appendix IV, the model validation review findings associated with this model have not impact on the conclusions of TSPA-SR.

\subsection{Model Area M: EBS Radionuclide Transport}

\subsubsection{In-Drift Colloids and Concentrations Model (M.3)}

Description: This model is a conceptual model for: 1) colloid transport through the EBS, and 2) transport of actinides that can either sorb to colloids (pseudocolloids) or form pure-phase colloids. The abstraction is conceptualized as a sequence of individual chemical/physical processes that are designed to be coded and incorporated into GoldSim (CRWMS M\&O 2000bl; Sections 6.2.2 and 6.3.4.6). The sequence includes:

- Source terms: Mass balance for colloids produced in the waste package, mass balance for actinides that are dissolved or associated with colloids, and calculation of ionic strength and $\mathrm{pH}$ based on multiple inputs from the waste package. 
- Determination of colloid stability, for 3 classes of colloids, as a function of calculated ionic strength and $\mathrm{pH}$. Modification of colloid mass fractions between mobile and immobile.

- Redistribution of adsorbed radionuclides based on $\mathrm{Kd}$ 's and an assumption of thermodynamic equilibrium.

Implicit in the conceptual model is the assumption that the invert can be treated as a completely mixed reactor. It is the only way to deal with fluid mixing, colloid stability, and radionuclide adsorption processes in the manner they are presented in this report. This model is documented in the AMR entitled: In-Drift Colloids and Concentration (CRWMS M\&O 2000al).

Use of the Model: Colloidal transport of radionuclides in the engineered barrier system, and also in the unsaturated zone downgradient, are described by this model. Certain algorithms developed in this model are implemented directly in TSPA-SR.

Review Findings: This model is placed into Bin 3 (impact review is provided in Appendix IV) primarily because it is a conceptual model that cannot be readily tested against experimental data, yet the model provides direct input to TSPA-SR representing an important mode of radionuclide transport. The parameters and most assumptions of the model are well described and referenced. However, the general conceptual approach and some of the assumptions should be addressed by peer review. Although colloidal transport can be very complex, the objective of the model is to provide a conservative estimate while maintaining some basis for validation (which cannot be based on conservatism in accordance with AP-3.10Q). Although the model is well constructed and would be an excellent basis for research hypotheses, it is possible that there is simply not enough published information to support, even by peer review, the sequential elements of the model.

The conceptual model is not validated against experimental data and such data are probably not available. Therefore, validation rests on the validity of the assumptions and basic concepts. Many of the assumptions (such as ignoring colloid filtration) are probably reasonable for generating conservative estimates of transport (i.e., the most rapid transport).

The conceptual approach is limited in terms of explaining how individual processes will be calculated or how they are incorporated. Ignoring the particular values used for input parameters, here are conceptual questions that should be addressed:

- Can the invert be accurately treated as a completely mixed reactor? By including colloid stability in the model there is an assumption that different classes of colloids will be spatially separated depending on the $\mathrm{pH}$ and ionic strength. Even if $\mathrm{pH}$ and ionic strength are not gradients along the transport path, there will be a separation of colloidal materials.

- Is a constant $\mathrm{Kd}$ appropriate? Although the effects of $\mathrm{pH}$ and ionic strength are included for determining colloid stability, there is no such functionality for radionuclide adsorption. The choice of using constant Kd's rather than sorption parameters that are sensitive to $\mathrm{pH}$ or ionic strength needs justification.

- Although filtration and settling of colloids is ignored in the name of conservatism, the mechanism for colloid removal by instability is not discussed. Destabilized colloids appear as a mass-loss expression. Since transport is vertical, and the invert is a dual 
porosity material, it is possible that gravitational settling of coagulated particles and more rapid transport would be a more conservative approach.

- Along these same lines, and given the mixed reactor nature of the model, the destabilized colloids appear to be removed from playing a role in solute re-partitioning.

- Given the large mass of colloidal material available from the waste package, the portion that is de-stabilized could impact the porosity and conductivity of the invert over an extended period of time.

- Given the potential for physical separation of classes of colloids, which is implied by the use of stability diagrams, it may not be appropriate to apply a uniform set of Kd's to the re-partitioning of solutes. Downstream particles should not communicate with upstream particles in this way.

- One of the assumptions touches on the issue of adsorption/desorption kinetics for pseudocolloids, however the model adopts only a local equilibrium assumption for adsorption.

- There is some question concerning the partitioning in the waste package (presumably part of another model and not this one) and re-partitioning in the invert. What solid phase is the dissolved concentration equilibrated with the colloids or the degrading materials?

- Americium may not be a good analog for Pu depending on the oxidation state. This assumption should be refined. The likely Pu oxidation state(s) should be stated and the use of Am as an analog should be justified (this can be a contentious issue). The reviewer believes that $\mathrm{Np}(\mathrm{V})$ and $\mathrm{Am}(\mathrm{V})$ are considered to be good analogs for $\mathrm{Pu}(\mathrm{V})$, but not necessarily for other oxidation states of $\mathrm{Pu}$.

Plutonium can exist in several oxidation states simultaneously which can complicate interpretation of the behavior of $\mathrm{Pu}$ in separation, processing, and environmental systems. To assist in obtaining data on the behavior of the separate oxidation states, it is useful to use data for analogous elements that form redox-stable cations with similar chemical behavior to $\mathrm{Pu}$ in the same oxidation state. For example, such analogs are $\mathrm{Eu}(\mathrm{III})$ and $\mathrm{Am}(\mathrm{III})$ for $\mathrm{Pu}(\mathrm{III})$, Th(IV) for $\mathrm{Pu}(\mathrm{IV}), \mathrm{Np}(\mathrm{V}) \mathrm{O}_{2}{ }^{+}$for $\mathrm{Pu}(\mathrm{V}) \mathrm{O}_{2}{ }^{+}$, and $\mathrm{U}(\mathrm{VI}) \mathrm{O}_{2}{ }^{2+}$ for $\mathrm{Pu}(\mathrm{VI}) \mathrm{O}_{2}{ }^{2+}$. The agreement in chemical properties is very good for the trivalent and pentavalent analogs, and acceptable for the hexavalent analogs. The tetravalent analogs require adjustments be made to the analog data to represent $\mathrm{Pu}(\mathrm{IV})$.

The important elements of colloid facilitated transport have been summarized and appear to be adequate in this model. However, the step-wise construction of the conceptual model and the absence of relevant processes from the conceptual model need further justification. This is a good example of where experimental data (column experiments) would play a critical role if they were available. Use of a mixed-reactor model rather than at least a 1-D transport model needs to be justified, but such refinement is difficult in the absence of experimental data.

Impact Review Summary: This model is a conceptual model that cannot be readily tested against experimental data, yet the model provides direct input to TSPA-SR representing a potentially important mode of radionuclide transport. The In-Drift Colloids and Concentrations Model is used in TSPA-SR and served as a basis for FEP screening. Colloid-associated radionuclide releases calculated by TSPA-SR are not significantly impacted by model validation review findings associated with this model because the TSPA-SR colloid abstraction approach 
uses conservative and/or bounding values for parameters that are not well supported by data. Use of a similar conservative and/or bounding approach for FEP screening is likewise not impacted. Based on this information and the additional detail provided in Appendix IV, there is no impact on the conclusions of TSPA-SR (CRWMS M\&O 2000bm, Section 6.1) from model validation review findings associated with this model.

\subsubsection{Invert Diffusion Properties Model (M.1)}

Description: This model describes a relationship between the moisture content of a porous matrix and the diffusivity of soluble ions, and is described in the AMR entitled: Invert Diffusion Properties Model (CRWMS M\&O 2000as). Motivation for developing the model is based on the difficulty in directly measuring ion diffusion in a porous matrix because practical experimental issues and the long times required. The model relies on earlier work by Conca and Wright (1992) that established a defensible correlation between ion mobility, conductivity, and moisture content of porous media. The premise, supported by experimental evidence, is that the aqueous phase and dissolved solutes in a porous media alone control conductivity. Electrical conductivity of a homogeneous solution of electrolytes is a function of free ion mobility and is described by the well-established Nernst-Einstein thermodynamic relationship. The assumption is that the NernstEinstein relationship applies to thin films of water that follow tortuous paths in unsaturated media. The relationship is semi-empirical and includes at least one empirical coefficient. Finally, the model is based on a power-law relationship (Archie's Law saturation exponent) between conductivity and moisture content. The data from Conca and Wright (1992) are plotted to derive an empirical but practical relationship that includes translation of the conductivity data using the Nernst-Einstein equation.

Use of the Model: The diffusion coefficient for crushed tuff invert ballast material, as a function of temperature and saturation, is used directly in TSPA.

Review Findings: Validation of this model does not use direct experimental data, i.e., measurement of ion diffusion in a porous matrix as a function of moisture content. Rather, the relationship is indirect. Validation draws on: 1) experimental data for the conductivity of a variety of granular geologic materials with variable liquid saturation, 2) acceptability of the Nernst-Einstein equation, and 3) an assumption that pore water in saturated or unsaturated media behaves in a manner that is fundamentally similar to a homogeneous solution of electrolytes. Given the absence of direct experimental data, these indirect relationships need to be well justified, and any limitations of the model should be carefully specified. The general model development appears to be sound in terms of the principles that apply. However, there are potential weakness in the model validation approach that should be readily resolved, and thus the Invert Diffusion Properties Model is assigned to Bin 2.

The experimental data set (Conca and Wright, 1992) appears to be reasonable for the purpose of establishing or verifying Archie's Law. The expected functional relationship is confirmed within limits. Figure 1 of the AMR tends to be misleading because this is just a plot of calculated values using Archie's Law, and not (as the inclusion of data symbols would imply) a plot of experimental data overlying theoretical data. It appears that Archie's law is a reasonable model as displayed in Figure 2. However, nonlinearity of Archie's Law at low water content has been pointed out in the literature, and using it down to $1 \%$ volumetric moisture content may be challenged, and should be better justified. 
The validation also rests on the assumption that the Nernst-Einstein equation holds for liquids in porous media. This has not been confirmed by direct observation (which may be impracticable) and the approach could be challenged, particularly at greater ionic strength. The mobility of ions in an electric field in the presence of charged surfaces with electrical double layers is complex. Highest ion mobility is thought to occur near the solid-solution interfaces. In addition, electroosmosis and counterion flow create complex advective currents. Hydrolysis reactions at mineral surfaces will generate surface charge, the double layer, and mobile ions. Therefore, the extension of the Nernst-Equation to these systems has not yet been defended although the relatively tight clustering of data for different materials and moisture contents suggests that the assumption is a good one. In the absence of being able to test the model directly, the boundaries for concentration should be noted. Usefulness at low ionic strengths where ions are more affected by the structure of a double layer could be a problem, but might not be an issue for the conditions expected in a repository.

It is recommended that the justification for validation of the Nernst-Einstein equation for thin films be expanded, to demonstrate that the mobility of the ions of interest can account for measured conductivity with the expected relationship. This could be done by eliminating alternative explanations, which is partly done in this report, and is also covered by Conca and Wright (1992).

Although not stated in the model development, it should also be noted that this model does not apply to solutes that sorb to the solid matrix. This is implicit in the use of the Nernst-Einstein equation, which applies only to free ions in solution. Although not explicitly discussed in this model summary, the title of the document and intended use of the model implies that this model will be used to estimate radioisotope migration. Since most radioelements that are relevant to repository performance sorb to mineral surfaces (some very strongly), this model is presumably conservative. Another concern is the assumption that a "corrected concentration" (i.e., ion activity) can be calculated using the extended Debye-Huckel equation (or similar activity model) for use in Fick's $1^{\text {st }}$ Law. Although temperature is a parameter in this type of activity-model equation, it is not valid to use the equation to compare ion activities at different temperatures. These relationships say nothing about changes in standard state conditions, only changes in free energy as a function of ionic strength.

\subsubsection{In-Drift Transport of Radionuclides Model (M.2)}

Description: This is a simple, 1-D advection/diffusion/dispersion model for vertical transport of radionuclides through a matrix with properties of the invert, or of a sand. The model is documented in the AMR entitled: EBS Radionuclide Transport Model (CRWMS M\&O 2000aa). Fluid that is deflected from the waste package by the drip shield, enters the invert and flows laterally as well as vertically. The extent of lateral flow is not specified in the model. The model is not intended for use in the TSPA, and in fact, seems inconsistent with other models where the invert is treated as a completely mixed batch reactor. This model is used for a sensitivity study that shows how breakthrough curves change for different dispersion coefficients and Peclet numbers. Accordingly, it could possibly be classified as an analysis. However, in this discussion it is treated as a model because of the uncertainty with respect to the flow field in the invert.

Use of the Model: Used for sensitivity testing; not used for TSPA.

Review Findings: This model is primarily a sensitivity analysis and there is no significant attempt at validation. It is not validated against experimental data although it seems that either 
published data from column studies could have been used, or it should have been possible to cite references that have already demonstrated that this 1-D approach is appropriate in analogous systems. In the latter case, a suitable comparison between the conditions in the invert and the reported studies would be provided, and any bounding conditions would be summarized. Other comments on the model are:

- The bulk of the model development seems to be focused on estimating a diffusion/dispersion coefficient in the absence of experimental data. The constancy of the coefficient is an assumption that is not justified. Experimental data testing the range of conditions for which this coefficient is constant would have been helpful.

- Another assumption is that the pore water velocity is the Darcy flux divided by the volumetric moisture context. This depends on all water present in the material constituting a connected phase. This assumption is questionable at low saturation.

- It is not clear whether lateral flux of water is incorporated into the model. Including the plots from NUFT calculations (CRWMS M\&O 2000aa, Figures 2, 3, 4, 5, 7, 8, 10, and 11) would seem to imply that lateral flux is included. This requires clarification.

- There are conflicting statements regarding whether the solutes are sorbing. One assumption says they are not (equivalent to conservative tracers?) while another models sorption as a Kd. However, a retardation factor is not part of the model so sorption may not be included. This also requires clarification.

- The range of moisture contents used in this model appears to extend beyond the bounds established in the referenced report entitled: Invert Diffusion Properties Model (CRWMS M\&O 2000as).

In recognition of these questions and limitations, the Model for In-Drift Transport of Radionuclides is assigned to Bin 3 (impact review is provided in Appendix IV).

Impact Review Summary: This model is primarily a sensitivity analysis and there is no significant attempt at validation. It is not validated against experimental data although published data from column studies could have been used, or the technical literature could have been used to demonstrate that this 1-D approach is appropriate in analogous systems. The EBS Radionuclide Transport Model was not used in TSPA-SR and did not serve as the basis for FEP screening. Deviation of invert transport behavior from the average response represented by this model would have no impact on the TSPA-SR except for waste package early failure scenarios, and the effect of invert transport on dose consequences for such scenarios is small. Based on this information and the additional detail provided in Appendix IV, there is no impact on the conclusions of TSPA-SR (CRWMS M\&O 2000bm, Section 6.1) from model validation review findings associated with this model.

\subsubsection{EBS Radionuclide Transport Abstraction Model (M.5)}

Description: The EBS Radionuclide Transport Abstraction Model is a conceptual and mathematical model that is used to determine the rate of release of radionuclides from the EBS to the UZ in the TSPA-SR model. It is documented in the AMR entitled: EBS Radionuclide Transport Abstraction (CRWMS M\&O 2000z). Specifically, this model is used to quantify the time-dependent radionuclide releases from a failed waste package and the subsequent transport through the drift to the drift wall. In effect, the model provides algorithms for transporting 
radionuclides using the flow geometry and radionuclide concentrations determined by other elements of the TSPA model (e.g., waste form degradation, radionuclide solubility/concentration limits, and near-field thermodynamic environment and percolation flux). The emphasis is on a reasonable approach that conservatively bounds the response of the EBS.

The model can be divided into three components: 1) a flow abstraction for various flowpaths within the EBS; 2) an abstraction for radionuclide transport from the waste package through the invert to the UZ; and 3) an abstraction for the thermal and mechanical response of the drip shield. The baseline flow abstraction is a quasi-steady "flow-through" model with no long-term build-up and retention of liquid within any of the EBS components. The transport abstraction includes advective and diffusive transport from the waste package (which may not be active simultaneously depending on the types of penetrations and the local seepage conditions) and one-dimensional advection and diffusion through the porous medium in the invert. Both dissolved and colloidal species are considered; dispersion and adsorption are neglected for conservatism. The potential for displacing and separating adjacent drip shields by thermal expansion, floor heave, rock fall, seismic events, and emplacement pallet failure is considered in the drip shield response abstraction, and each of these five mechanisms are screened out of the TSPA.

The EBS Radionuclide Transport Abstraction Model also considers an alternate model for flow through the WP. The alternative model, called the bathtub model, allows fluid to collect in the WP before being released. The bathtub model is screened out because results demonstrate that the flow-through model is generally more conservative with respect to repository performance than the bathtub model.

Overall, this model summarizes the parameters and processes that are likely to be important in the EBS. It is thorough, well organized, and well conceived. It is a conceptual model and cannot be validated against experimental data. It will rely on validation of component models that it uses (other AMRs) and acceptability of the assumptions.

Use of the Model: The model provides a mathematical linkage between other elements of the TSPA model. Its output (time-dependent radionuclide releases to the emplacement drift wall/UZ interface) is used directly in the EBS transport module of the TSPA-SR model. However, the supporting AMR does not provide any quantitative results

Review Findings: This AMR (Section 7.2) makes a strong case that "...the [model] is valid and appropriate for its intended use because it is designed to represent fundamental flow and transport processes in a bounding or conservative framework." The AMR makes very transparent and logical arguments regarding model features and assumptions that provide confidence in the model. However, these arguments do not satisfy any of the specific validation criteria in AP$3.10 \mathrm{Q}$, and there are questions that may require laboratory testing, hence the EBS Radionuclide Transport Abstraction Model is assigned to Bin 3 (impact review is provided in Appendix IV).

Portions of the model are strongly supported by data and probably could be deemed "valid" by the AP-3.10Q criteria. Specifically, the use of the self-diffusion coefficient of water as an upper bound for diffusion of all species is supported by comparison to experimental data, and the mathematical relationship for the diffusion coefficient is developed from Archie's law (Invert Diffusion Properties Model, CRWMS M\&O 2000as). However, the validity of portions of the model does not constitute validity of the model as a whole. 
As discussed in Section 7.2 of this AMR, the EBS Radionuclide Transport Abstraction Model is based on "typical flow processes" and on transport processes that are "commonly considered for contaminant transport calculations." Their implementation in a "bounding or conservative framework" is fairly transparent and probably could be validated by peer review, review by international collaborations, or technical review through publication in the open literature. However, the AMR makes no mention of these types of reviews, and the reviewer is not aware that these methods were used.

Although the conceptualization of flow and transport through stress corrosion cracks (SCCs) is logically developed and physically appealing, no experimental observations or studies of natural or man-made analogs are cited by the supporting and related AMRs for corroboration of the concepts. It seems likely to the reviewer that flow and transport through fine cracks in smooth surfaces under various seepage conditions has been addressed in other areas and that models and experimental data may be available in the open literature. This would presumably be done in conjunction with validation for the Water Distribution and Removal Model (CRWMS M\&O 2001t).

Impact Review Summary: The AMR makes transparent and logical arguments regarding model features and assumptions that provide confidence in the representation of EBS performance in the TSPA-SR. However, these arguments do not satisfy any of the specific validation criteria in $\mathrm{AP}-3.10 \mathrm{Q}$, and there are questions that may require laboratory testing. The EBS Transport Abstraction was used in TSPA-SR but did not serve as a basis for screening out FEPs. The abstraction provides conservative and/or bounding representation of processes controlling radionuclide transport from the waste package to the drift wall. However, conservatism is not considered as sufficient basis for compliant model validation. Based on this information and the additional detail provided in Appendix IV, there is no impact on the conclusions of TSPA-SR (CRWMS M\&O 2000bm, Section 6.1) from model validation review findings associated with this model.

\subsubsection{Seepage/Invert Interactions Model (M.4)}

Description: The Seepage/Invert Interactions Model is a conceptual model for physical/chemical interactions between seepage and the invert materials (crushed tuff, steel, and a small amount of copper). It is documented in the AMR entitled: Seepage/Invert Interactions (CRWMS M\&O $2000 \mathrm{bg}$ ). The model is strictly conceptual, describing the potential chemical processes and reactions along various pathways to the invert, for thermal and ambient conditions. The AMR outlines how the seepage/invert interactions may be quantified, but does not provide any mathematical models that yield quantitative results. The Seepage/Invert Interactions Model specifically excludes detailed discussion of precipitation of salts in the invert because modeling and analysis of precipitates is addressed separately in the AMR entitled: In-Drift Precipitates/Salts Analysis (CRWMS M\&O 2001l).

Use of the Model: The model's intended use is input to another model/analysis and input to other technical products. A stated function of the model is to determine transport under both low and high temperature conditions.

The Physical and Chemical Environmental Abstraction Model (CRWMS M\&O 2000az) incorporates the concepts from the Seepage/Invert Interactions Model by reference. However, this other report also presents only a conceptualization of the environment in the emplacement drifts and states (Section 6.0) that “... it does not document an analysis or model in the strict 
sense of those terms." Consequently, it appears that mathematical models, which give the quantitative description used in TSPA-SR for the processes and reactions described in the Seepage/Invert Interactions Model (CRWMS M\&O 2000bg), and the Physical and Chemical Environment Abstraction Model AMR (CRWMS M\&O 2000az), have not been documented.

It is also noted that the AMR entitled: EBS Features, Events, and Processes (CRWMS M\&O 2001h; FEP 2.1.09.01.00, Section 6.4.48) states that the Seepage/Invert Interactions Model, as incorporated in the Physical and Chemical Environment Abstraction Model (CRWMS M\&O 2000az), is included in the TSPA-SR (as part of the TSPA disposition for the FEP). However, the Total System Performance Assessment (TSPA) Model for the Site Recommendation (CRWMS M\&O 2000bl) does not cite either of these reports, and Total System Performance for the Site Recommendation (CRWMS M\&O 2000bm, Section 3.3.4.5.7) includes only qualitative discussion of the Seepage/Invert Interactions Model.

Review Findings: The subject AMR does not attempt to validate the Seepage/Invert Interactions Model. In Section 7.4, the AMR states that "...it is not possible to validate a discussion of a conceptual model with further discussion..." and "...the only possible method that could be used in validation of the seepage/invert interactions model would be implementation of this model." Documentation of a full or partial mathematical implementation of the conceptual model was not found here or in other documents. The Seepage/Invert model cannot be validated by any of the criteria in AP-3.10Q without documentation of the mathematical model that is actually used to describe these interactions in TSPA-SR (such documentation was not found). It is recommended that when the mathematical model is documented, that the Seepage/Invert Interaction Model be "rolled into" the same document. The subject AMR is thus a "white paper" that could be useful in this way, but it may be too broad to be implemented without further simplifying assumptions, bounding calculations, and screening of processes. In recognition of these concerns, the Seepage/Invert Interaction Model is assigned to Bin 3 (impact review is provided in Appendix IV).

Impact Review Summary: The Seepage/Invert model cannot be validated by any of the criteria in AP-3.10Q without documentation of the mathematical model that is actually used to describe these interactions in TSPA-SR. The Seepage-Invert Interaction was not used in TSPA-SR but did serve as a basis for FEP screening. Impacts on the conclusions of TSPA-SR from model model validation review findings are minimal because the invert has small benefit for performance, and invert materials will not exert significant influence on water composition. Based on this information and the additional detail provided in Appendix IV, there is no impact on the conclusions of TSPA-SR (CRWMS M\&O 2000bm, Section 6.1) from model validation review findings associated with this model.

\subsection{Model Area N: Unsaturated Zone Transport}

\subsubsection{Equilibrium Matrix Sorption Basis (N.1-1)}

Description: Development and test of a conceptual model and mathematical implementation for the key transport process of chemical adsorption, documented in the AMR entitled: Unsaturated Zone and Saturated Zone Transport Properties (CRWMS M\&O 2001r). In particular, the development addresses whether partition coefficients determined during column tests need to be used explicitly for modeling site performance, or whether values derived from simpler batch tests will suffice. Batch tests on crushed tuff, column tests on crushed tuff, and column tests on intact 
tuff cores were conducted. This document contains the conceptual and mathematical basis for estimating the parameters used in TSPA-SR.

Use of the Model: The model output consists primarily of the justification for using batch-Kds in the transport models that directly support TSPA-SR.

Review Findings: For purposes of validating the conclusion of this model, better justification is needed for use of qualitative agreement between the model used to interpret laboratory tests, and the laboratory data for transport of $\mathrm{Np}$ and $\mathrm{Se}$.

For example, "good agreement" is claimed between batch and column results for Np transport, but this criterion is not defined. Comparison of reported values in Table 11 of the AMR shows that the agreement ranges from perfect to very different. This is the basis for using partition coefficients determined from batch tests to model $\mathrm{Np}$ transport in the Yucca Mountain tuff matrix. Consequently, validation of the modeling approach per AP-3.10Q Section 5.3.b is not achieved.

For $\mathrm{Pu}$ experiments, no agreement between batch and column test results is claimed, yet there appears to be an inconsistency between this result and what is used in Table $2 \mathrm{a}$ of the AMR for minimum Pu sorption coefficients. The column experiments discussed in Section 6.5.1.2.2 on pp. 98-99 indicate that for devitrified tuff at some flow velocities, up to $60 \%$ of the Pu breaks through early and is not retarded. For this reason, the minimum value in Table $2 \mathrm{a}$ and $2 \mathrm{~b}$ should be zero for plutonium, yet non-zero values are entered.

For Se experiments the partition coefficient values for zeolitic tuff determined in batch and intact column tests were determined to be 0.08 and $0.26 \mathrm{~mL} / \mathrm{g}$ (CRWMS M\&O 2001r, Section 6.5.2.2.2). The column tests were performed using ultracentrifugation (UFA method) to overcome difficulties with long test times associated with natural-gradient methods. The batch and column test results are claimed to "indicate agreement" without justification even though some values differ by $225 \%$. There is discussion later about numerical scatter in the batch tests results caused by subtraction of one measurement from another. For this example, some additional discussion is needed to show how the $0.26 \mathrm{~mL} / \mathrm{g}$ value was derived from the results presented. The conceptual model presented earlier in the section seemed to indicate that batch tests would yield larger $\mathrm{Kds}$ due to transient interference effects in intact cores. Some explanation of the opposite finding, of lower $\mathrm{Kds}$ in intact cores vs. batch tests on crushed rock, needs to be added.

In accordance with this discussion, the Equilibrium Matrix Sorption Basis Model is assigned to Bin 2. This is appropriate because the information exists in the document by which to validate this conceptual model, for species with relatively low partition coefficient values, if the issues described above are addressed.

It is recommended that a rigorous criterion be established as the basis for a conclusion that batchderived partition coefficients are adequate for use in simulating transport through the tuff matrix. Alternatively, some other line of reasoning could be used to explain why the agreement is sufficient to support the conclusion. It is the position of the reviewer that the approach of using batch-derived sorption on crushed tuff to represent transport within the matrix is fundamentally valid especially if supported by test results. Concerns about exposure of fresh mineral surfaces in crushed tuff are mostly mollified since mineral exposure is extensive within the rock matrix. It is noted that sorption onto fracture surfaces is addressed in other documents. 


\subsubsection{Fracture Sorption (N.1-2)}

Description: This model contains the development and testing for a conceptual model and mathematical representation for radionuclide sorption onto mineral alteration products on fracture surfaces. The model is documented in the AMR entitled: Unsaturated Zone and Saturated Zone Transport Properties (CRWMS M\&O 2001r). The hypothesis that significant sorptive retardation occurs during transport through fractures is tested using the FEHM transport simulator, applied to elution results from a fractured-rock column to fit $\mathrm{Np}$ sorption.

Use of the Model: The model results are not used because TSPA-SR conservatively assumes no sorption of radionuclides onto fracture surfaces.

Review Findings: The Fracture Sorption Model is assigned to Bin 2 because of reliance on earlier documentation (e.g., Robinson et al. 1995; pp. 63-70). Also, no plots are shown that directly compare the model predictions with column test results. Rather, they are referenced to Robinson et al. (1995, pp. 63-70). A conclusion is presented that significant sorption does occur on fracture surfaces, and that fracture flow does not necessarily result in fast pathways for actinide transport. This conclusion corroborates the assertion that ignoring fracture sorption in the TSPA-SR is conservative. It is recommended that the test data and model-data comparison be incorporated explicitly in the validation argument, and that the report provide more thorough discussion of why the model matches the observed column elution results.

\subsubsection{Matrix Diffusion (N.1-3)}

Description: The TRACRN transport simulator is used to obtain model fits to rock-beaker diffusion experiments, to evaluate diffusion coefficients in the matrix of devitrified, welded tuff. The model is documented in the AMR entitled: Unsaturated Zone and Saturated Zone Transport Properties (CRWMS M\&O 2001r).

Use of the Model: The average results from fitting of diffusion coefficients using model-data comparison, from Table 16 (CRWMS M\&O 2001r, Section 6.6.1.3), are used directly in the TSPA-SR model.

Review Findings: Although an example of the TRACRN model fit to measured data is shown in Figure 28 and the fit appears to be very good, no criteria are identified to establish whether this fit is adequate. As a result of the lack of discussion on adequacy of the fitted matrix diffusion parameter, this model is assigned to Bin 2. It is recommended that the AMR be revised to include discussion of the adequacy of this model fit in order to satisfy the criteria of AP-3.10Q 5.3.b, commensurate with the importance of the matrix diffusion parameter to site performance calculated in TSPA. This discussion could be qualitative, although the fit seems adequate to withstand quantitative scrutiny if appropriately documented. A quantitative approach would also allow validation of the other fitted values shown in Table 16, on page 119 of the AMR.

\subsubsection{Colloidal Transport (N.2-1)}

Description: This model describes modification to the FEHM 2.0 transport simulator to allow simulation of colloidal transport in variably saturated porous media. The conceptual development for colloidal transport is based on conservative assumptions. The model is documented in the AMR entitled: UZ Colloid Transport Model (CRWMS M\&O 2000bp). The mathematical representation is used to demonstrate model implementation, but model-data comparison is not included. 
Use of the Model: The colloidal transport functionality of FEHM is implemented directly in the TSPA-SR (CRWMS M\&O 2000bl, Section 6.3.6.1).

Review Findings: Validation of the colloidal transport model using site-specific data is not possible because such data are not generally available from the Yucca Mountain site or any other. Rather, validation is achieved using a conservative development approach based on understanding of the system and comparison of model results with alternative conceptual models. This approach meets the criterion of AP-3.10Q Section 5.3.c.4. Although conservatism is not generally accepted as the basis for model validation, in this instance the model validation argument does effectively provide "... supporting information to establish basis for confidence in selected model." Accordingly, the Colloidal Transport Model is assigned to Bin 1.

The conceptual model and mathematical representation for colloidal transport were developed and tested in this AMR. However, this information is apparently not cited or used in other AMRs that describe UZ transport, specifically Particle Tracking Model and Abstraction of Transport Processes (CRWMS M\&O 2000ay) and the Total System Performance Assessment (TSPA) Model for Site Recommendation (CRWMS M\&O 2000bl).

It is recommended that the conceptual and mathematical development information be extracted from this AMR and incorporated into Particle Tracking Model and Abstraction of Transport Processes (CRWMS M\&O 2000ay). It is further recommended that model-data comparison be included using results from the Busted Butte colloid test results. This change could result in pertinent information regarding validation of $\mathrm{UZ}$ transport models located in a single report. Then the subject AMR: UZ Colloid Transport Model (CRWMS M\&O 2000bp) could be eliminated.

Supplementary Response from Project Staff: This model was not effectively validated because data were not available. Validation of the UZ Colloidal Transport Model needs to be carried out using available data from the Busted Butte Tests, and additional data from fractured, welded tuff that are expected to result from ongoing testing. Further testing and validation of this model are not expected to impact the conclusions of the TSPA-SR because the model is not used directly.

\subsubsection{Pu Sorption on Colloids (N.2-2)}

Description: Transport simulator TRACRN is applied to lab data to fit forward and backward rates for $\mathrm{Pu}$ sorption onto clay colloids. A Levenberg-Marquardt optimization algorithm is used for minimizing a sum of squared differences between lab and simulated values. The model is documented in the AMR entitled: UZ Colloid Transport Model (CRWMS M\&O 2000bp).

Use of the Model: Forward/backward rates for $\mathrm{Pu}$ sorption/desorption onto clay colloids are provided, however these results are apparently not used directly. Forward and reverse rates are not used in the TSPA-SR, instead reversible sorption is determined by multiplying the colloid $\mathrm{Kd}$ by the colloid concentration (CRWMS M\&O 2000b1, Section 6.3.6.1, p. 386).

Review Findings: The optimized model-data fit is adopted without establishing whether the fit is adequate. Perhaps the author intended the chi-square statistics for this purpose. The chi-square statistic is introduced and used to qualify 1 of 4 fits as good and 1 of 4 as bad, leaving the other two indeterminate. The appropriate conclusion from this statistical approach is not obvious. Accordingly, the model is assigned to Bin 2 because although test data are used in the validation strategy, the documentation is incomplete. The model validation arguments can satisfy AP-3.10Q 
Section 5.3.b if revised. Further, it is recommended that there be clarification as whether the chisquare statistics are used to validate the model fits to the measured data. Other approaches may be more useful.

\subsubsection{FRACL Calibration to Borehole Chloride (N.3-1)}

Description: The FRACL transport model is tested by comparison to field measurements of chloride in water samples extracted from rock core obtained from borehole UE-25 UZ\#16. This model is documented in the AMR entitled: Radionuclide Transport Models Under Ambient Conditions (CRWMS M\&O 2000ba). A 1-dimensional model is abstracted from the 3-D UZ transport model domain for simulation with FRACL. Model-data comparison is based on modeling of chemical effects from changes in net infiltration associated with climate transition from pre-Holocene to modern conditions. Model results are compared directly with the measured borehole chloride "profile." Also a claim is presented for benchmarking/validation/calibration of the T2R3D model against the same set of FRACL and field-measured results.

Use of the Model: This model nominally supports TSPA-SR because it is one calibration for the UZ transport model. This would actually be the case if the calibration were successful.

Review Findings: "Reasonable agreement" is claimed for comparison of the FRACL results and the measured chloride profile presented in Figure 6.4.4 (page 73 of the AMR). This figure does not indicate reasonable agreement. There is too great a spread of the measured chloride values at depth for the model comparison to be meaningful. The text of the AMR states that all the chloride data originated from a single borehole, but given the spread on the values, the veracity of the data is questionable.

Another problem is that the description of the model, especially the upper boundary conditions for transport, is inadequate. The model results appear to be totally constrained by the unexplained upper boundary condition. From the AMR entitled: UZ Flow Models and Submodels (CRWMS M\&O 2000bq, Section 6.4.1.2) it seems that the upper boundary should have been prescribed as a first-type boundary with a concentration of $0.62 \mathrm{mg} / \mathrm{L}$. Yet the discussion in the subject AMR (CRWMS M\&O 2000ba; p. 73) describes a constantconcentration upper boundary condition of $\sim 38 \mathrm{mg} / 1$. This discrepancy needs to be explained.

Additionally, the comparison to $\mathrm{T} 2 \mathrm{R} 3 \mathrm{D}$ model results is meaningless. The only $\mathrm{T} 2 \mathrm{R} 3 \mathrm{D}$ results presented are for a steady-state model, and are apparently all equal to the prescribed upper boundary condition. The text refers to T2R3D results for other time simulations that are not presented in the AMR, thus there appears to be no basis to the claim on page 70 of the AMR that the "positive comparison supports the validation and calibration of the Radionuclide Transport Model."

In recognition of these issues, the FRACL Calibration to Borehole Chloride Model is assigned to Bin 3 (impact review is provided in Appendix IV). If the authors still believe that this FRACL model is calibrated to the borehole $\mathrm{Cl}$ profile, then it is recommended that this same modeling exercise be performed using the FEHM 2.1 particle transport simulator since that is the code being used for simulating transport in the TSPA-SR (CRWMS M\&O 2000bl, Sections 3.1.2 and 6.3.6).

Impact Review Summary: This model is used in TSPA-SR but did not serve as a basis for screening out FEPs. However, the FRACL model itself was not used to simulate radionuclide transport in the TSPA-SR. Instead, UZ radionuclide transport was simulated for TSPA-SR using 
the FEHM code with the residence time transfer function particle-tracking technique (CRWMS M\&O 2000ay). The FRACL model-data comparison results for chloride do support the UZ radionuclide transport model. The results are consistent with other methods used to assess the reasonableness of the UZ radionuclide transport model, and would not significantly affect the overall TSPA-SR results even if they were excluded. The overall validation of the transport calculations is also addressed in DOE-NRC agreements on Key Technical Issues. Future revisions of this AMR to address these agreements will incorporate additional information (such as porewater chloride concentrations) that will provide further confidence in the validation of the FRACL model. Based on this information and the additional detail provided in Appendix IV, there is no impact on the conclusions of TSPA-SR (CRWMS M\&O 2000bm, Section 6.1) from model validation review findings associated with this model.

\subsubsection{EOS9nT Calibration to ESF Chloride Profile (N.3-2)}

Description: The EOS9nT transport model is tested by comparison to field measurements of chloride in water samples extracted from rock core obtained from the Exploratory Studies Facility (ESF). The application is conceptually similar to that described above for the FRACL Calibration to Borehole Chloride Model, except that the model-data comparison is better. This model is documented in the AMR entitled: Radionuclide Transport Models Under Ambient Conditions (CRWMS M\&O 2000ba).

Use of the Model: Model output consists of predicted chloride concentrations along the ESF profile. These results are apparently not used directly in any other model, however, the model demonstrates the capability to create a site-scale transport model calibrated to site-specific data and thus provides important support to model validation arguments.

Review Findings: A claim of "satisfactory agreement" between the EOS9nT results and the ESF chloride profile data is made without substantiating discussion. A comparison plot showing the simulated and observed chloride profiles is shown in Figure 6.4.5 of the AMR, but it is difficult to agree that the model-data comparison is adequate unless some criterion is established. This may not be simple because there is much spread on the measured values. One approach that could be taken to improve the model validation argument is to expand the qualitative discussion to meet the requirements of AP-3.10Q Section 5.3.b.

Another AMR entitled: Unsaturated Zone Flow Model and Submodels (CRWMS M\&O 2000bq; Section 6.4) addresses this same topic of modeling chloride concentrations for comparison to the ESF chloride profiles. There is a discrepancy between the results for T2R3D presented in this other AMR (Section 6.4 and Figure 6-28 on page 83), and in the subject AMR (CRWMS M\&O $2000 \mathrm{ba}$; Figure 6.4 .5 on page 75 ). Both figures portray T2R3D results using the "calibrated infiltration," yet the plotted results are different. The results in the AMR (CRWMS M\&O $2000 \mathrm{bq}$ ) exhibit a better match to the observed data. This apparent discrepancy should be resolved

In accordance with these review comments, the EOS9nT Calibration to ESF Chloride Profile Model is assigned to Bin 2. It is recommended that the chloride simulations in the two AMRs discussed above be consolidated. Most important, it is recommended that an equivalent FEHM 2.1 particle transport simulation be developed to demonstrate that the transport code being used for the TSPA-SR can show the same degree of calibration. 


\subsubsection{Fracture-to-Matrix Colloid Filtration (N.4)}

Description: This model consists of a conceptual development and mathematical representation for estimating the fraction of colloids that can advect from the fractures into the tuff matrix, based on the distribution of saturated matrix pore openings. The approach uses moisture retention curves and the Laplace equation. This model is documented in the AMR entitled: Particle Tracking Model and Abstraction of Transport Processes (CRWMS M\&O 2000ay).

Use of the Model: Output from this model consists of the fraction of 100-nm colloids that can enter the matrix domain from the fracture domain. Forward and reverse filtration rates for estimating colloid retardation in fractures were fitted, but were not used in TSPA-SR because the effect of colloid retardation on UZ transport is conservatively neglected (i.e., retardation factor for colloids in fractures is unity).

Review Findings: The model is a relatively simple application of the Laplace equation with sitespecific moisture retention data for each formation. However, no validation arguments are offered and thus AP-3.10Q, Section 5.3.c, is not satisfied. Accordingly, the Fracture-to-Matrix Colloid Filtration Model is assigned to Bin 2. It is recommended that the AMR be revised to include justification for model validation, commensurate with the use of the model (not used in TSPA-SR).

\subsection{Model Area O: Saturated Zone Flow}

\subsubsection{Site-Scale Saturated Zone Flow Model (O.5)}

Description: This model provides the groundwater flow fields to be used for calculating the saturated zone (SZ) transport of radionuclides. The model was developed using site-specific hydrogeological data such as hydrostratigraphy, permeability and the groundwater water levels. In addition, output from the regional scale saturated zone model and the vadose zone model are used in the model. Numerical codes were used to simulate the flow field. This model is documented in the AMR entitled: Calibration of the Site-Scale SZ Flow Model (CRWMS M\&O $2000 \mathrm{j}$ ), supported by various other AMRs in the SZ Flow model area (see Table 1 of this report).

Use of the Model: The model output is the hydraulic flow field of the domain, which is used in the SZ transport model for TSPA.

Review Findings: Insufficient information is provided in the AMR to support some critical details and assumptions. This includes explanation of permeability and temperature profiles, justification for use of water levels and head data, use of the hydrogeologic framework model, and justification for the distribution of hydrogeologic features including faults. It is also noted that output from an unvalidated regional scale model was used to validate the site-scale saturated zone model. A compliant validation argument can likely be developed from the information available, so the Site-Scale Saturated Zone Flow Model is assigned to Bin 2.

\subsection{Model Area P: Saturated Zone Transport}

\subsubsection{Sub-Gridblock Scale Dispersion in 3-D Heterogeneous Fractured Media (P.6)}

Description: This model provides estimates of dispersion (transverse and longitudinal) that could be in effect at the sub-gridblock scale within the saturated zone (SZ), in the fractured Tertiary volcanic rocks downgradient from the potential repository. The model development includes the use of grid blocks containing both the background fracture characteristics, and 
enhanced permeability. Point and distributed planar sources for tracer or contaminant were considered. The model is documented in the AMR entitled: Modeling Sub Gridblock Scale Dispersion in Three-Dimensional Heterogeneous Fractured Media (S0015) (CRWMS M\&O 2000au).

An indicator-geostatistical simulation routine was used to create background permeability and background fracture permeability models. Enhanced permeability models were created using a similar approach. The FEHM transport simulator was used to solve for a steady state flow solution on the heterogeneous fields, and to determine particle tracks using the steady state solution (CRWMS M\&O 2000au, Section 6.2.2). A separate routine was used to calculate longitudinal and transverse dispersivities from the output of FEHM.

Use of the Model: The transverse and longitudinal dispersivity distributions output from this model were used to justify the values used in SZ flow and transport modeling for TSPA-SR (CRWMS M\&O 2000cp, Section 5.4).

Review Findings: The distribution of measured permeability was checked against permeability models by determining the value of the cumulative distribution function at each threshold for each of the 50 realizations for both the background and enhanced conceptual models of fracture permeability. Differences are within $10 \%$ or less of the input values. The distribution of transect sampling was compared to the spacing of known flowing features. The model was shown to produce average feature spacings that are within the distribution of measured spacings.

The background fracture permeability model was validated by calculating the variogram on each realization in the three principal directions $(\mathrm{X}, \mathrm{Y}, \mathrm{Z})$ and comparing the results to the input variogram model. The authors concluded that the input variogram was maintained but at a lower variance. The realizations are considered valid. In accordance with these findings the SubGridblock Scale Dispersion in 3-D Heterogeneous Fractured Media Model is assigned to Bin 1.

\subsubsection{Saturated Zone Colloid Facilitated Transport (P.2)}

Description: This model estimates plausible ranges of retardation factors for colloids with irreversibly attached radionuclides, in both saturated fractured tuff and saturated alluvium, for use in the TSPA-SR. Field data and its interpretation from the C-wells tracer test are compared with Reactive Transport Application (RTA) model predictions for colloids in the tuff. The time scales associated with colloid filtration and detachment in the saturated tuff are assumed to be small relative to fluid residence time, therefore allowing the assumption of local equilibrium. The values used in the RTA model for the flow velocity in fractures and the dispersion coefficient are from interpretation of the non-sorbing tracers in the C-wells tests. Attachment and detachment rates, used to estimate retardation factors, are adjusted in the RTA model to improve comparison with field data. A distribution of retardation factors describing the multiple field tests is developed for use in predictive transport models. The assumption of local equilibrium is evaluated by calculation of Damkohler numbers (rate constant times the residence time).

Due to absence of field data, a theoretical analysis based upon field tests at other sites is used to predict retardation factors for colloidal transport in alluvial material. Key input variables to the standard colloid filtration theory equation are treated as uncertain parameters. The GoldSim model (Golder Associates 2000) is used to develop a probability distribution for retardation factors, representing the range of uncertainty associated with input variables. The model is 
documented in the AMR entitled: Saturated Zone Colloid Facilitated Transport (CRWMS M\&O 2000bc).

Use of the Model: Model output is used in the AMR: Uncertainty Distribution for Stochastic Parameters (CRWMS M\&O 2000bo), which is used in developing: Input and Results of the Base-Case SZ Flow and Transport Model for TSPA (CRWMS M\&O 2000aq).

Review Findings: A validation section for colloidal transport in fractured tuff is included in this AMR. Validation of the assumption of local equilibrium is achieved by the use of well accepted Damkohler equations comparing rate constants with residence time. Validation must include the choice of the dual porosity model for fitting the experimental data. A similar exercise is needed to validate the interpretation of the $\mathrm{C}$-wells test data.

Validation of the model for colloidal transport in alluvium is also included. The model is a standard equation from colloid filtration theory that has been validated in the open literature. The appropriateness of this equation has been demonstrated by comparison with field data. The necessary assumption of local equilibrium was validated by evaluating the ratio of attachment and detachment rate constants to residence times that are a function of porewater velocities. Existing validation would be strengthened by checking the parameters required to calibrate the model for reasonableness in accordance with AP-3.10Q, Section 5.3.c. Model validation can be readily achieved using available information, so the Saturated Zone Colloid Facilitated Transport Model is assigned to Bin 2.

\subsubsection{Pipe Model for Daughter Radionuclides (P.4-1)}

Description: A process model for 1-D transport with radioactive decay and in-growth is developed for simulated transport of four decay chains (see CRWMS M\&O 2000bl, Table 6-28). The model is implemented for the saturated zone (SZ), directly in the simulator used for TSPASR as a series of "pipes" or streamtubes (CRWMS M\&O 2000bl, Section 6.3.7). A series of five streamtubes cover the transport pathway from the repository to $80 \mathrm{~km}$ downgradient (CRWMS M\&O 2000aq, Section 6.1). The results of the 1-D transport model are only used for the daughter radionuclides; transport of the parent nuclides is simulated using FEHM. The "pipe" model simulates advection, longitudinal dispersion, retardation, decay and in-growth, and the effects of matrix diffusion. Retardation includes sorption and colloid-facilitated transport. Average groundwater flow and transport properties from the SZ Site-Scale Flow Model are used within streamtube segments. Average specific discharge for each segment is estimated using the 3-D SZ Site-Scale Flow Model. The solution for matrix diffusion in fractured media is verified by comparison with an analytical solution developed by Sudicky and Frind (1982). The model is documented in the AMR entitled: Input and Results of the Base-Case SZ Flow and Transport Model for TSPA (CRWMS M\&O 2000aq).

Use of the Model: Output of the model consists of a numerical file describing 1-D transport of daughter products from decay and in-growth in four decay chains, which is used directly in the TSPA-SR model (CRWMS M\&O 2000bl, Section 6.3.7.1).

Review Findings: Validation of the 1-D "pipe" transport model is attempted by comparison of the results from the 3-D FEHM model of flow and transport (CRWMS M\&O 2000aq, Section 6.5.2 and Figures 25 and 26). The conclusion that the average breakthrough times are generally comparable between the two models is not well supported. One example shows that the streamtube model significantly underestimates the contaminant mass flux. Validation should be 
much more rigorous. Quantitative acceptance criteria for such comparisons should be established. Accordingly, the Pipe Model for Daughter Radionuclides is assigned to Bin 2.

\subsubsection{Abstraction of FEHM and Coupling with UZ Mass Flux (P.4-2)}

Description: This abstraction model uses the convolution integral method in GoldSim (Golder Associates 2000) to couple the 3-D Saturated Zone (SZ) Site-Scale Flow and Transport Model (FEHM) with the "pipe" model for daughter radionuclides (CRWMS M\&O 2000aq, Sections 6.2 and 6.5), to represent the radionuclide mass flux at the SZ/Biosphere interface $20 \mathrm{~km}$ downgradient of the repository as a function of the transient radionuclide mass flux at the water table beneath the repository. The input functions are the saturated-zone mass breakthrough curve as simulated by FEHM, the time-dependent radionuclide mass flux at the water table simulated by the UZ transport model, and the transport of daughter radionuclides calculated by the "pipe" model. The effects of climate change on radionuclide transport in the SZ are incorporated into the convolution integral analysis by assuming instantaneous change from one steady-state flow condition to another steady-state flow condition in the SZ. The changes in flow condition are multiples of the groundwater flux of the base case. Radioactive decay is applied to transport as loss of mass by first order decay through the interval of travel time. The model is documented in the AMR entitled: Input and Results of the Base-Case SZ Flow and Transport Model for TSPA (CRWMS M\&O 2000aq).

Use of the Model: The convolution integral method and associated parameters of radionuclide transport, and scaling factors to represent the effect of climate change on the SZ, are input directly to TSPA-SR (CRWMS M\&O 2000bl, Section 6.3.7).

Review Findings: The description of this model is not clear in this AMR. It needs more complete explanation that might be easier done in a different document, such as the Total System Performance Assessment (TSPA) Model for Site Recommendation (CRWMS M\&O 2000bl). Validation of this model is critical to building confidence in the overall modeling approach to evaluate the repository performance. The FEHM transport code has been verified, stochastic input parameters have been developed, and a library of breakthrough curves has been generated, but validation of these models has not been done. Validation at this point must include the appropriateness of using the FEHM code and its results, the coupling of the FEHM output with the UZ mass flux and the output of the "pipe" model for daughter radionuclides, and the representation of climate change. Confidence must be established as to the representativeness of the predicted results. This might be done by a combination of approaches, e.g., review of calibration data, comparison of model predictions with test results, and comparison of the modeling approach with similar applications published in the open literature. This has already been started (CRWMS M\&O 2000bl; Section 6.3.7) but the scope of validation activities needed is significant, so the Abstraction of FEHM and Coupling with UZ Mass Flux Model is assigned to Bin 3 (impact review is provided in Appendix IV).

Impact Review Summary: This model is used in TSPA-SR but did not serve as a basis for screening out FEPs. It is a simple calculation procedure that uses other validated models with appropriate qualified data, and uses verified software to perform the calculations. The conceptual approach for representing SZ transport is straightforward and is commonly used in groundwater contaminant transport investigations. Accordingly, the model is considered to be appropriate for its intended use. Additional model validation activities are in process that will include verification of the model by comparison to other model results and experimental results. 
Additional model validation documentation will be provided in a revision of this AMR. Based on this information and the additional detail provided in Appendix IV, there is no impact on the conclusions of TSPA-SR (CRWMS M\&O 2000bm, Section 6.1) from model validation review findings associated with this model.

\subsubsection{Transport Parameters from C-Wells and Laboratory Studies (P.4-3)}

Description: This process model includes analysis of laboratory tests conducted in parallel with field tracer tests in the lower Bullfrog Tuff and the lower Prow Pass Tuff at the C-wells. Field data are fitted to breakthrough curves generated by the dual porosity transport models RELAP and RETRAN. Non-sorbing tracer data were used to estimate dispersivity, matrix diffusion, and porosity. Lithium tracer data were used to estimate sorption parameters, and colloid tracer data was used to estimate filtration rate constants. Laboratory studies included batch tests to characterize lithium sorption, diffusion cell tests to measure matrix diffusion coefficients, and column tests to evaluate transport in fractured and crushed tuff. Comparison of results from batch and column tests of lithium sorption with the field responses is used to show that laboratory-derived radionuclide sorption parameters can be used defensibly in field-scale predictive calculations. Comparison of the laboratory derived matrix diffusion and mass-transfer coefficients with the field responses shows that those estimated from the field tests are smaller than from the lab results. The model is documented in the AMRs entitled: Unsaturated Zone and Saturated Zone Transport Properties (CRWMS M\&O 2001r) and: Input and Results of the BaseCase SZ Flow and Transport Model for TSPA (CRWMS M\&O 2000aq). The latter AMR is assigned as the principal supporting document because of the important connection between the base-case model and the input data used.

Use of the Model: Sorption parameters and matrix diffusion coefficients developed in this model are used in: Saturated Zone Colloid Facilitated Transport (CRWMS M\&O 2000bc) and Uncertainty Distributions for Stochastic Parameters (CRWMS M\&O 2000bo). In addition, the use of an equilibrium linear sorption model for TSPA-SR is based on this AMR (CRWMS M\&O 2000aq, Section 5.1). The model is also used for FEP screening (CRWMS M\&O 2001ac).

Review Findings: There is no validation section in the part of the AMR that addresses the Cwell field and laboratory tests, and such a section must be added. Confidence-building is needed for the assumption of dual-porosity flow and transport in the saturated zone, and the implementation of RELAP and RETRAN (CRWMS M\&O 2001r, Sections 6.9.2 and 6.9.3). In addition, validation should address the output data (dispersivity and matrix diffusion coefficients) and the use of the lithium tracer test to justify the use of laboratory-measured $\mathrm{Kd}$ values. Perhaps alternative models could be used for curve fitting, with comparison of the models, and review of the calibration parameters in accordance with AP-3.10Q 5.3.c. Again, the scope of validation activities needed is significant, so the Transport Parameters from C-Wells and Laboratory Studies Model is assigned to Bin 3 (impact review is provided in Appendix IV).

Impact Review Summary: This model is used in TSPA-SR and did serve as a basis for screening out FEPs. Comparison of results from laboratory and field responses was used to show that laboratory-derived sorption parameters could be used defensibly in field-scale predictive calculations. The use of overlapping laboratory and field testing, and complementary analyses of laboratory and field test results, provide confidence in the parameterization of the dual-porosity model used for TSPA-SR. Additional validation activities, including laboratory testing, will provide further confidence in the selection of the dual-porosity model, the use of RELAP and 
RETRAN to simulate field test results, and the use of laboratory-measured $K_{d}$ values as well as other specific parameters to model SZ transport. Based on this information and the additional detail provided in Appendix IV, there is no impact on the conclusions of TSPA-SR from model validation review findings associated with this model.

\subsection{Model Area Q: Biosphere}

\subsubsection{Crop Interception Fraction Submodel (Q.2-1)}

Description: This empirical model is used to estimate the fraction of material deposited on plant surfaces via wet or dry deposition processes that is retained by the plant. Output from the model is used as the basis for an input parameter to the plant uptake model. This model is documented in the AMR entitled: Identification of Ingestion Exposure Parameters (CRWMS M\&O 2000ai).

Use of the Model: Model output is used within the GENII-S code to determine the biosphere dose conversion factors that are used for TSPA-SR (CRWMS M\&O $2001 \mathrm{~m}$, Sections 3 and 4.1).

Review Findings: The basic equation is based on field data and the review in the AMR also draws on additional studies and data in the open literature. Information necessary for a compliant validation argument exists in the AMR, so the Crop Interception Fraction Submodel is assigned to Bin 1.

\subsubsection{Irrigation Rate Submodel (Q.2-2)}

Description: The irrigation rate parameter is used as an input to the leaching model and is a function of evapotranspiration, precipitation, and the deep percolation needed to remove salts that could damage crop yield. This model is documented in the AMR entitled: Identification of Ingestion Exposure Parameters (CRWMS M\&O 2000ai).

Use of the Model: Model output is used in the formulation of inputs to the GENII-S code to determine the biosphere dose conversion factors that are used for TSPA-SR (CRWMS M\&O $2001 \mathrm{~m}$, Sections 3 and 4.1).

Review Findings: Validation is established by comparing predicted evapotranspiration values with those of another model as well as measurements. Deep percolation is calculated using sitespecific conductivity data for well waters as input to an established approach given by Donahue et al. (1997). Information necessary for a compliant validation argument therefore exists in the AMR, so the Irrigation Rate Submodel is assigned to Bin 1.

\subsubsection{Dose Conversion for Ingestion (Q.4-1)}

Description: GENII-S (CRWMS M\&O 1999b, Section 3) includes an implementation of the ICRP-30 (ICRP 1979) dosimetric models in order to calculate the 50-yr effective dose equivalent of a given radionuclide following ingestion. Each biokinetic model consists of a series of linked ordinary differential equations that trace the metabolism of a radionuclide in selected organs of a reference human. The resulting dose conversion factors (DCFs) are given as Sieverts per Becquerel of radiation ingested. This model is documented in the AMR entitled: Dose Conversion Factor Analysis: Evaluation of GENII-S Dose Assessment Methods (CRWMS M\&O 1999b).

Use of the Model: Model output is used within the GENII-S code to determine the biosphere dose conversion factors that are used for TSPA-SR (CRWMS M\&O 1999b, Section 4.1). 
Review Findings: The GENII-S DCFs for a set of radionuclides are compared with those published in the Environmental Protection Agency's (EPA) Federal Guidance Report (FGR) No. 11 (Eckerman et al. 1988), and it is found that DCFs are in reasonable agreement with the FGR values, except for Tc-99, which GENII-S overestimated by 50\%. Accordingly, sufficient information is provided in the AMR to support a conclusion that the model is valid for its intended use, and the Dose Conversion for Ingestion Model is assigned to Bin 1.

\subsubsection{Dose Conversion for Inhalation (Q.4-2)}

Description: GENII-S (CRWMS M\&O 1999b, Section 3) includes an implementation of the ICRP 30 (ICRP 1979) dosimetric models in order to calculate the 50-yr effective dose equivalent of a given radionuclide following inhalation. Each biokinetic model consists of a series of linked ordinary differential equations that trace the metabolism of a radionuclide in selected organs of a reference human. A key component of these models is the ICRP-30 lung model. The resulting dose conversion factors (DCFs) are given as Sieverts per Becquerel of radiation inhaled. This model is documented in the AMR entitled: Dose Conversion Factor Analysis: Evaluation of GENII-S Dose Assessment Methods (CRWMS M\&O 1999b). Additional input to this model is provided in: Input Parameter Values for External \& Inhalation Radiation Exposure Analysis (CRWMS M\&O 2000ar).

Use of the Model: Model output is used within the GENII-S code to determine the biosphere dose conversion factors that are used for TSPA-SR (CRWMS M\&O 1999b, Section 4.1).

Review Findings: The GENII-S DCFs for a set of radionuclides are compared with those published in the EPA's Federal Guidance Report (FGR) No. 11 (Eckerman et al. 1988), and it is found that the DCFs are in reasonable agreement with the FGR values, specifically, that all values are within 15\% of the FGR values (CRWMS M\&O 1999b, Section 6.4). Accordingly, sufficient information is provided in the AMR to support a conclusion that the model is valid for its intended use, and the Dose Conversion for Inhalation Model is assigned to Bin 1.

\subsubsection{Dose Conversion for External Exposure (Q.4-3)}

Description: This model is documented in the AMR entitled: Dose Conversion Factor Analysis: Evaluation of GENII-S Dose Assessment Methods (CRWMS M\&O 1999b). Section 6.5 of the AMR notes that GENII-S allows for the introduction of dose conversion factors (DCFs) for external exposures to be introduced from another source. Consequently, the AMR adopts the DCFs from the EPA's Federal Guidance Report (FGR) No. 12 (Eckerman and Ryman 1993) for external exposures to radionuclide uniformly mixed to a depth of $15 \mathrm{~cm}$, which is the default depth of soil contamination. GENII-S, however, may not include an adjustment for radiation shielding while indoors. Additional input to this model is provided in: Input Parameter Values for External \& Inhalation Radiation Exposure Analysis (CRWMS M\&O 2000ar).

Use of the Model: Model output is used within the GENII-S code to determine the biosphere dose conversion factors that are used for TSPA-SR (CRWMS M\&O 1999b, Sections 3 and 4.1).

Review Findings: An accurate estimate of external exposures also requires the use of a radiation shielding factor-see NCRP 129 (NCRP 1999) for appropriate factors and justification. If the areal extent of surface contamination due to irrigation of lawns/gardens is limited, then external radiation dose will be over estimated by the EPA's FGR 12 (Eckerman and Ryman 1993). Comparisons with other models for determining external radiation would be beneficial, especially if the distribution of a soil radionuclide is inconsistent with the assumptions implicit in 
FGR 12 (Eckerman and Ryman 1993). Some revision of the model documentation is needed based on available information, so the Dose Conversion for External Exposure Model is assigned to $\operatorname{Bin} 2$.

\subsubsection{Resuspension Model (Q.9-2)}

Description: GENII-S uses a resuspension factor approach to relate the concentration of a radionuclide in soil to the concentration of the radionuclide in air above soil (CRWMS M\&O $2001 \mathrm{~m}$, Appendix I). The resuspension factor can be estimated from mass loading information as well as by resuspension models. Resuspension is also a process that can impact the deposition of radionuclides onto crop surfaces. This model is primarily documented in the AMR entitled: Nominal Performance Biosphere Dose Conversion Factor Analysis (CRWMS M\&O 2001m). Additional input to this model is provided in: Input Parameter Values for External \& Inhalation Radiation Exposure Analysis (CRWMS M\&O 2000ar).

Use of the Model: Model output is used within the GENII-S code (CRWMS M\&O 2001m, Sections 3 and 6.5) to determine the biosphere dose conversion factors that are used for TSPASR.

Review Findings: The transfer of soil contaminants to air in GENII-S (CRWMS M\&O 2001m, Section 3) can be estimated using an empirically-based, time-dependent model (Anspaugh et al., 1975) or a mass loading approach. A natural-analogue approach was adopted to estimate the resuspension factor using particulate mass loading data (CRWMS M\&O 2001m, Appendix I). A compliant validation argument can be developed from available information, so the Resuspension Model is assigned to Bin 2. The following are some additional recommendations:

- Although the use of the mass loading data is a reasonable way to estimate the resuspension factor, especially when no site-specific resuspension studies have been conducted, this approach does not necessarily lead to conservative estimates.

- NCRP Report No. 129 (NCRP 1999) provides a review of resuspension models and default values for selected cases (e.g., disturbed and undisturbed soils) along with uncertainty estimates. Data are also provided on the median diameters of resuspended particles based on field studies and literature reviews.

- GENII-S supposedly allows the use of a shielding factor, as implied on p. 20 of AMR: Input Parameter Values for External and Inhalation Radiation Exposure Analysis (CRWMS M\&O 2000ar, Section 5.2.1) when indoors to protect against the inhalation of outdoor particles, however, various studies show that a building shell may not really provide significant protection.

- GENII-S uses the same breathing rate indoors as outdoors. This is incorrect, as the breathing rate indoors includes many hours at rest/sleep and low intensity activities. Also, the breathing rate used in GENII-S should be consistent with the energy intake of the diet supporting the given lifestyle (Layton 1993).

\subsubsection{Plant Uptake Model (Q.9-3)}

Description: This model, which is represented in its basic form as Equation I-8 and supporting inputs (CRWMS M\&O 2001m) simulates the accumulation of soil-derived radionuclides in plants/crops as a function of deposition processes (i.e., deposition of airborne particles resuspended from contaminated soil and deposition of sprinkler irrigation derived from 
contaminated well water) and root uptake from contaminated soil. This model is primarily documented in the AMR entitled: Nominal Performance Biosphere Dose Conversion Factor Analysis (CRWMS M\&O 2001m).

Use of the Model: Model output is used within the GENII-S code to determine the biosphere dose conversion factors that are used for TSPA-SR (CRWMS M\&O 2001m, Sections 3 and 6.5).

Review Findings: The model consists of about 10 different parameters, and although the GENII-S documentation states that it "has been shown to be reasonable by direct comparison of modeled and measured values" (Jaquish and Napier 1987) no data or comparisons are provided. Its applicability to irrigated arid land is unknown. An analysis of some of the input parameters is provided in AMR: Transfer Coefficient Analysis (CRWMS M\&O 2000bn), but no integrated evaluation of model performance is provided. Also, the model does not include rinse-off of particles by consumers. A compliant validation argument can be developed from the available information, so the Plant Uptake Model is assigned to Bin 2.

One way of providing additional validation of the performance of the soil-plant system represented by GENII-S is to collect measurements or data on elements in plants grown on arid soils as a natural analogue to the processes being simulated.

\subsubsection{Surface Soil Model in GENII-S (Q.9-1)}

Description: This model is defined by Equation I-1 (CRWMS M\&O 2001m) and is used to predict the concentration of a radionuclide in surficial soil in the Amargosa Valley after the introduction of irrigation water obtained from a well that contains radionuclides derived from the potential repository. The calculation is performed within the GENII-S code. A key assumption of this model is that a radionuclide dissolved in irrigation water is instantaneously mixed into the soil to a fixed depth, which is assumed to be $15 \mathrm{~cm}$. The radionuclide concentration in soil is a pivotal component in estimating human exposures to radionuclides derived from well water because soil-based exposures include the incidental ingestion of soil, inhalation of soil resuspended into air, external exposure to radiation, and consumption of produce grown on contaminated soil. The model does not include surface erosion either by water or aeolian removal processes. Moreover, it does not implicitly deal with elevated soil salinity that could affect the $\mathrm{Kd}$ values used to determine the soil leaching half-life. This model is primarily documented in the AMR entitled: Nominal Performance Biosphere Dose Conversion Factor Analysis (CRWMS M\&O 2001m).

Use of the Model: Model output is used within the GENII-S code to determine the biosphere dose conversion factors that are used for TSPA-SR (CRWMS M\&O 2001m, Sections 3 and 6.5).

Review Findings: The originating journal article (Baes and Sharp 1983) provides limited validation data, and cautions that the lack of long-term solute migration data "precludes verification of the model." Combined effects of soil parameters and processes on radionuclide soil concentrations are not validated in the model (e.g., surface erosion, soil salinity impact on $\mathrm{Kd}$ ). No validation is provided regarding concentrations in soil following irrigation applications in an arid environment. In addition, the basic assumption of a uniformly contaminated soil zone to $15 \mathrm{~cm}$ may not be realistic, as there could be a depth-dependent concentration profile. If so, the model could contribute to underestimates of resuspension of radionuclides in the top $5 \mathrm{~cm}$, as an example. Considerable revision is needed to provide a compliant validation argument, so the Surface Soil Model in GENII-S is assigned to Bin 3 (impact review is provided in Appendix IV 
of this report). The following recommendations are also provided for consideration:

- Perform laboratory $\mathrm{Kd}$ measurements for selected radionuclides/elements using representative soils from the Amargosa Valley.

- Develop or adapt alternative surficial soil models that incorporate relevant soil properties and processes and compare the results with those of the GENII-S model.

- Compare model predictions with field data at current agricultural sites in the Amargosa Valley or elsewhere to demonstrate either uniform mixing in tilled soils or depthdependent changes in selected ions.

- Complete an AMR that synthesizes all of the various inputs to the surface soil model, including predicted irrigation applications for alternative crops, expected soil salinity, erosion rates, etc.

Impact Review Summary: The current soil model is a simplified evaluation of the processes that affect the buildup of radionuclides in the soil. This model is used in TSPA-SR but did not serve as a basis for screening out FEPs. The relatively simple approach used in this model has several advantages that are appropriate for analysis of long-term performance, although it produces demonstrably conservative results. The approach (CRWMS M\&O 2001m) is the same basic model used in other Biosphere models developed internationally, and is both accepted and well documented. Additional development activities will address DOE-NRC agreements on Key Technical Issues, related to the selection of $\mathrm{Kd}$ values, the assessment of the realistic vs. conservative nature of the Kd values, and other element-specific biosphere parameters important in the BDCF calculations. These activities are expected to increase confidence in the model, and to provide a quantitative demonstration that the model adequately represents uncertainty and variability. The current approach is simplified and conservative, and therefore defensible for its intended use. Potential future revisions of the model are unlikely to significantly impact dose assessments. Based on this information and the additional detail provided in Appendix IV, there is no impact on the conclusions of TSPA-SR (CRWMS M\&O 2000bm, Section 6.1) from model validation review findings associated with this model.

\subsubsection{Radionuclide Transfer to Animals (Q.9-4)}

Description: This empirical model is used to estimate the accumulation of radionuclides in animal tissue; the calculation is performed within the GENII-S code (CRWMS M\&O 2001m, Section 3). The two intake pathways are ingestion of contaminated water and the consumption of feed/forage. A radionuclide transfer factor is used to relate intakes to accumulation of a radionuclide in the tissue of an animal. This model is primarily documented in the AMR entitled: Nominal Performance Biosphere Dose Conversion Factor Analysis (CRWMS M\&O $2001 \mathrm{~m}$ ).

Use of the Model: Model output is used within the GENII-S code to determine the biosphere dose conversion factors that are used for TSPA-SR (CRWMS M\&O 2001m, Section 6.5) .

Review Findings: No model-specific validation information was provided. Moreover, the model does not include incidental ingestion of contaminated soil while grazing or inhalation of resuspended particles. Model should be revised to reflect the additional pathways. Accordingly, the Radionuclide Transfer to Animals Models is assigned to Bin 3 (impact review is provided in Appendix IV). 
Impact Review Summary: No model-specific validation information was provided, and the model does not include incidental ingestion of contaminated soil while grazing, or inhalation of resuspended particles. The Radionuclide Transfer to Animals Model was used in TSPA-SR but did not serve as a basis for screening out FEPs. The GENII-S based approach used for TSPA-SR dose calculations includes the primary pathways that contribute to dose. Contributions from additional pathways are much smaller and not likely to be significant to dose calculations. Based on this information and the additional detail provided in Appendix IV, there is no significant impact on the conclusions of TSPA-SR (CRWMS M\&O 2000bm, Section 6.1) from the model validation review findings associated with this model.

\subsubsection{Radionuclide Transfer to Aquatic Food (Q.9-5)}

Description: This empirical model is used to estimate the accumulation of radionuclides in aquatic food; the calculation is performed within the GENII-S code. This model is primarily documented in the AMR entitled: Nominal Performance Biosphere Dose Conversion Factor Analysis (CRWMS M\&O $2001 \mathrm{~m}$ ). The model is given as the product of the concentration of a radionuclide in water and an estimated bioaccumulation factor. The fish are presumably grown in a pond fed by well water, as shown in Figure 2 of the AMR (radionuclide concentrations controlled by well water, not pond water).

Use of the Model: Model output is used within the GENII-S code (CRWMS M\&O 2001m, Sections 3 and 6.5) to determine the biosphere dose conversion factors that are used for TSPASR.

Review Findings: The most significant finding is the absence of a model to predict the concentration of a radionuclide in pond water. Inclusion of a simple fate model for radionuclides in a lake is probably the best approach for dealing with this particular exposure pathway. Model should be revised to reflect the additional pathway. Accordingly, the Radionuclide Transfer to Aquatic Food Model is assigned to Bin 3 (impact review is provided in Appendix IV).

Impact Review Summary: The Radionuclide Transfer to Aquatic Foods was used in TSPA-SR but did not serve as a basis for screening out FEPs. The need for the aquatic food pathway in Amargosa Valley arose because a small commercial catfish farm was in operation. For all radionuclides except ${ }^{14} \mathrm{C}$, the dose contribution from aquatic foods is insignificant. For ${ }^{14} \mathrm{C}$ in groundwater (if any should be present from the repository) the dose was overestimated by an order of magnitude. Because these results are demonstrably conservative, there is no detrimental impact to the conclusions of TSPA-SR from the use of this model. Furthermore, the catfish farm no longer operates. Based on this information and the additional information provided in Appendix IV, there is no impact on the conclusions of TSPA-SR (CRWMS M\&O 2000bm, Section 6.1) from the model validation review findings associated with this model.

\subsection{Model Area R: Disruptive Events/Igneous Disruption Consequences}

\subsubsection{Conditional Distribution for Number of Eruptive Centers Model (R.1)}

Description: Mathematical formulation to assess the conditional distribution for the number of eruptive centers within the footprint of the potential repository based on the concept that eruptive centers will occur at uncertain locations along the length of the dike associated with a volcanic event. The number of eruptive centers per volcanic event and spatial distribution of eruptive centers along the length of the dike were not defined in the Probabilistic Volcanic Hazards Assessment for Yucca Mountain, Nevada (PVHA) (CRWMS M\&O 1996) expert elicitation 
process. The authors propose a model of eruptive center distributions along the length of a dike length based on two assumptions and use information from the PVHA experts and mapped volcano locations. The model is documented in AMR: Characterize Framework for Igneous Activity at Yucca Mountain, Nevada (CRWMS M\&O 20001).

Use of the Model: The output is conditional probability distributions for dike length, dike azimuth, and number of eruptive centers appropriate for the mean frequency of intersection of the primary block and contingency block. The output of this model is used in calculation report Number of Waste Packages Hit by Intrusions (CRWMS M\&O 2000ax) to calculate the cumulative distribution function (CDF) for the number of waste packages hit by igneous intrusion. The CDF is used as input to AMR: Igneous Consequence Modeling for TSPA-SR (CRWMS M\&O 2000aj) to generate output that is then used in the TSPA-SR.

Review Findings: This AMR needs additional documentation to develop model validation arguments that comply with AP-3.10Q Section 5.3.b and 5.3.c. Documentation of the selection of input from the PVHA relative to new information/data/studies since 1996 is adequate. Additional documentation is needed to validate the model of how eruptive centers are associated with dike length and why the five random location approaches to assess eruptive centers (statistical formulations) are reasonable approaches for dike intrusion processes at or near Yucca Mountain. Documentation should also be added concerning what the statistically derived spatial distributions represent in models of eruptive centers and dike orientations relative to the potential repository. For example, the justification for the statistical spatial distributions should account for these scenarios:

- The eruptive center is outside the repository footprint closer to the critical group and is fed by a dike flowing through the repository.

- The dike and eruptive center models used in AMR: Characterize Eruptive Processes at Yucca Mountain, Nevada (CRWMS M\&O 2000k)

Also, additional documentation is needed to support the approach that considers how the potential repository induces an eruptive center. Some documentation of the validation methodology used in the expert elicitation PVHA would also increase confidence in the use of PVHA input to this model. In accord with the need for revision using available information, the Conditional Distribution for Number of Eruptive Centers Model is assigned to Bin 2.

\subsubsection{Geometry of Volcanic Feeder System Model (R.2)}

Description: The Geometry of Volcanic Feeder System Model is documented in the AMR entitled: Characterize Eruptive Processes at Yucca Mountain, Nevada (CRWMS M\&O 2000k). This AMR combines a set of input data and assumptions to formulate a conceptual model that characterizes the eruptive processes at Yucca Mountain. Most of the outputs are parameters or parameter distributions gleaned from the literature and from studies of analogous sites, which do not by themselves constitute a model. In some cases, the analyzed events and their associated parameters could be supported more strongly by a wider review of literature (see below). A more serious concern is the development of an implicit model that restricts the number of waste packages available for eruption to those within the circular cross-sectional area of the volcanic conduit. The authors point out early in Section 6 that: 
"Because of the effect of the (1) conduit diameter and (2) depth (to which a conduit extends before merging into a simple feeder dike) on the number of waste packages disrupted... it is important to constrain both these variables".

Discussions follow dealing with conduit diameter, dike thickness, and dike swarms, but depth at which the conduit merges with a dike is given very little further discussion. Instead, there seems to be an implicit model assumed throughout this report and in the other AMRs that use the output that either: 1) the conduit merges with its feeder dike at depths greater than that of the repository; or 2) once initiated near the surface, the conduit bores its way downward through the repository quickly as reduced pressure allows the fragmentation depth to increase. In either case, only the cross-sectional area of the conduit specifies those waste packages that will contribute to the radionuclide release scenarios.

Use of the Model: Parameters, probability distributions, and the model that is implicit in this AMR are used in TSPA-SR (CRWMS M\&O 2000bl, Section 6.3.9) indirectly through other reports such as Igneous Consequence Modeling for the TSPA-SR (CRWMS M\&O 2000aj).

Review Findings: The model chosen for the volcanic eruptive process should be clearly described, and confidence building should be approached by providing descriptions of alternative models that were considered and rejected and reasons for their rejection. The Geometry of Volcanic Feeder System Model was assigned to Bin 3 (impact review is provided in Appendix IV) because both the model and its validation are unclear.

It is recommended that other models for eruption geometry be considered, specifically cases in which the depth of merging is above the repository and a fissure eruption from a dike that cuts the entire repository, allowing the possibility that waste from all packages damaged by the dike could contribute inventory to the eruption. Although there is no indication of it in this AMR, such models may have been considered and rejected because they can be shown to be less conservative than the implicit model (i.e., low flow velocities and lack of fragmentation in relatively thin dikes cannot effectively entrain waste and carry it to the conduit or to the fissure eruption). If so, increased confidence could be gained here by showing that alternative eruption models have been considered and that the one chosen for use in the TSPA-SR is the one that contributes the most waste inventory to the eruption or the highest exposure to the critical group.

Even though many of the parameters estimated in this AMR for use in TSPA-SR are truly analyses and not models, higher confidence can be gained in the parameter distributions by amassing more supportive information from published literature. For instance, a couple of simple searches of a well-known catalogue of geoscience references (using "intrusion temperature" and "contact temperature" as keywords) found 36 papers which estimate temperatures of mafic intrusions using a variety of methods including mineral paleothermometers, metamorphic assemblages, modifications of vitrinite reflectance in wall rocks, and generation of coke in contact zones.

Impact Review Summary: The Geometry of Volcanic Feeder System Model was used in TSPA-SR but did not serve as a basis for screening out FEPs. For TSPA-SR only eruptions fed by conduits were considered, and the inference was made that any waste package that was wholly or partly intersected by a conduit would be damaged to the extent that it would provide no further protection for waste. This model is conservative in several respects. Although additional validation activities are underway which will address the DOE-NRC agreements on Key Technical Issues, it is unlikely that the conclusions of the TSPA-SR will be affected by 
these activities. Based on this information and the additional detail provided in Appendix IV, there is no significant impact on the conclusions of TSPA-SR (CRWMS M\&O 2000bm, Section 6.1) from the model validation review findings associated with this model.

\subsubsection{Volcanic Eruption Release Model (R.3-1)}

Description: This model is documented in the AMR: Igneous Consequence Modeling for $T S P A-S R$ (CRWMS M\&O 2000aj). A volcanic eruption release model is discussed in Section 6 of the AMR, which forms the basis for selecting the ASHPLUME computer code (Jarzemba et al. 1997) and associated parameters. The model includes the subsurface physics of the erupting column, atmospheric transport and surface deposition of erupted material, and incorporation of the radionuclides with the biosphere leading to potential doses within humans. Quantitative distributions are developed for parameters of two model components, subsurface physics and atmospheric transport.

The model uses input from the AMR: Characterize Framework for Igneous Activity at Yucca Mountain, Nevada (CRWMS M\&O 20001) to produce an estimate of eruptive volume, expressed as a cumulative distribution function (CDF). It also quantifies the initial eruptive velocity and ash particle sizes, as CDFs based on information from AMR: Characterize Eruptive Processes at Yucca Mountain, Nevada (CRWMS M\&O 2000k). The model output also includes a dispersion controlling constant. The model also calculates wind rose diagrams of direction and velocity based on wind measurements in the area.

Use of the Model: Model is used primarily as input to the ASHPLUME code (Jarzemba et al. 1997).

Review Findings: This AMR needs additional documentation to constitute model validation arguments that comply with AP-3.10Q Section 5.3.b and 5.3.c. No justification is presented for redefinition of the eruptive volume CDF. General acceptance of the Suzuki (1983) model does not demonstrate its applicability for use at YM. No physical basis is used to justify the incorporation ratio $(0.3)$ or the concepts that waste particles must be attached to ash particles for transport and that waste particle sizes, greater than half the ash particle size, will not be transported. Such basis may be presented in Jarzemba et al. (1997) but should be discussed here. Revision of the model documentation is needed, based on available information, so the Volcanic Eruption Release Model is assigned to Bin 2. The following additional comments are provided for consideration:

- Confidence would be gained by adding discussion of model testing or comparison of models to real volcanic events published in the open literature.

- Statements that justify an assumption or use of model based on "no data available" or "results in conservative estimates" should be avoided.

- Discuss or make reference to discussion of alternatives, and clearly show the reasons for the chosen or calculated parameter values or distributions.

- Whenever possible, compare parameter distributions to data from other regions, or previous studies for the Yucca Mountain region. Examples are: 1) compare the calculated wind rose diagram to other published results; and 2) the assumption that modern wind characteristics are the same as those for other climatological conditions in the past and 
future should be justified by comparison to published studies of Pleistocene aeolian processes in the region.

\subsubsection{In-drift Damage Due to Dike Intersection Model (R.3-2)}

Description: Despite the speculation and discussion in AMR: Dike Propagation Near Drifts (CRWMS M\&O 2000u) about the possible effects of a dike intersecting a repository drift, this model simply assumes a model in which the 3 to 4 waste packages on either side of the dike are completely destroyed (and the contents become available for groundwater transport) and that the remaining packages receive a specified damage (loss of all drip shields, loss of cladding, and penetrating damage to welds). The model is documented in the AMR: Igneous Consequence Modeling for TSPA-SR (CRWMS M\&O 2000aj).

Use of the Model: Model output to TSPA-SR consists of the waste inventories immediately available for groundwater or magmatic transport, and the inventories in damaged packages that would contribute to transport in groundwater.

Review Findings: This AMR needs additional documentation to develop model validation arguments that comply with AP-3.10Q Section 5.3.b and 5.3.c. Specifically, the validation argument needs to compare results with data acquired from natural or man-made analogs and other releyant observations, and with results from implementation of alternative conceptual models.

There is no justification in any of the AMRs reviewed for this model area, for the model used to estimate damage to waste packages. This applies to both the model for complete failure of 3 to 4 containers on each side of the dike, and to the model specifying a 15-meter zone on each side of the dike in which all containers experience complete failure. Where the two are discussed together, as in Number of Waste Packages Hit by Intrusions (CRWMS M\&O 2000ax), the reasoning seems circular.

Considerable insight could be gained by discussions of damage to metallic objects that are engulfed by magma or pyroclastic debris in recent eruptions. Also, a 1978 eruption in Iceland could provide valuable insight. Basalt magma erupted from a $\sim 1100 \mathrm{~m}$ deep geothermal well after it was intersected by an intruding basalt dike (Larsen et al. 1979; Brandsdottir and Einarsson 1979). Perhaps this analog could provide some justification for the damage model proposed. The damage model is purported to be conservative, but that is not well supported or documented.

Many of the speculations in Dike Propagation Near Drifts (CRWMS M\&O 2000u) could be screened, i.e., eliminated because more speculative modeling is avoided by adopting a model of prescribed damage in each intersected drift. These screened effects include complete filling of drifts by magma, filling of drifts by pyroclastic material, shock wave propagation in the drifts, effects from temperatures and pressures attained in the drifts, blocking of the drift by EBS debris and magmatic material, and the effects of these processes on waste containers.

A significant effort is needed to develop model validation arguments, but the information is probably available, so the In-drift Damage Due to Dike Intersection Model is assigned to Bin 2.

\subsubsection{Mass Loading Decay Model Following Deposition of Volcanic Ash (R.5-1)}

Description: The decline in the annual average mass loading following the deposition of volcanic ash on the ground surface was calculated using an exponential decay model. This model 
is documented in AMR: Disruptive Event Biosphere Dose Conversion Factor Analysis (CRWMS M\&O 2001ah) and also: Input Parameter Values for External and Inhalation Radiation Exposure Analysis (CRWMS M\&O 2000ar).

Use of the Model: Development of disruptive-event biosphere dose conversion factors for the TSPA-SR (CRWMS M\&O 2000bl, Section 6.3.9).

Review Findings: The AMR reviewed alternative resuspension models and selected the exponential decay model. This model may be reasonable, given the inherent uncertainties in such a simulation; however, the rationale for determining the decay rate constant is not well supported. Accordingly, the Mass Loading Decay Model Following Deposition of Volcanic Ash is assigned to Bin 2. It is recommended that relevant data collected before and after the eruption of Mount St. Helens, and the subsequent drop off in concentrations, could be used to bracket the decay rate in particulate mass loading derived from resuspended ash.

\subsubsection{Dose Conversion Factor Model for Inhalation - Igneous Disruption (R.5-2)}

Description: Inhalation of ash particles following a volcanic eruption will result in a smaller amount of particle deposition in the lungs compared with the nominal case. The inhalation dose conversion factors (DCFs) calculated using $1 \mu \mathrm{m}$ diameter particles were adjusted to account for the decreased amount of deposition.

This model is documented in AMR: Disruptive Event Biosphere Dose Conversion Factor Analysis (CRWMS M\&O 2001ah) and also: Dose Conversion Factor Analysis: Evaluation of GENII-S Dose Assessment Methods (CRWMS M\&O 1999b).

Use of the Model: Development of disruptive-event biosphere dose conversion factors for the TSPA-SR (CRWMS M\&O 2000bl, Section 6.3.9).

Review Findings: This model uses a recognized formula for converting the ICRP-30 (ICRP 1979) DCFs based on $1 \mu \mathrm{m}$ diameter particles to other particle sizes. As a another validation, a more direct comparison of DCFs can be completed using the newer ICRP lung model. Accordingly, the Dose Conversion Factor Model for Inhalation - Igneous Disruption is assigned to Bin 2.

\subsection{Model Area S: Seismic Hazards}

\subsubsection{Vibratory Ground Motion Hazard Model (S.1-1)}

Description: This model is implicit in the report entitled: Characterize Framework for Seismicity \& Structural Deformation at Yucca Mountain, Nevada (CRWMS M\&O 2000m). It is a summary of information developed and presented in Probabilistic Seismic Hazard Analyses for Fault Displacement and Vibratory Ground Motion at Yucca Mountain, Nevada (Wong and Stepp 1998).

This model provides annual exceedance probabilities for accelerations ranging from 0.01 to $3.0 \mathrm{~g}$ for structural frequencies from $100 \mathrm{~Hz}$ to $1 \mathrm{~Hz}$, from 0.002 to $1.0 \mathrm{~g}$ for structural frequencies of $0.5 \mathrm{~Hz}$ and $0.3 \mathrm{~Hz}$, and from 0.2 to $500 \mathrm{~cm} / \mathrm{s}$ for peak ground velocity. Response spectrum accelerations for $10^{-3}$ and $10^{-4}$ annual exceedance probabilities are also given for six additional structural periods. The model results were developed using a formal elicitation process from six teams of experts who provided alternative characterizations of the tectonic setting, Quaternary faults, and earthquake source parameters, and another seven experts who provided evaluations of 
ground motion attenuation. The FRISK88 (CRWMS M\&O 2000m, Attachment II) software package was used to compute the vibratory ground motion results. Both random and model uncertainties are incorporated into the results. Sensitivity analyses of the PSHA results are also included.

Use of the Model: This model is not used directly for TSPA-SR because degradation of the engineered and natural barriers by vibratory ground motion has generally been "screened out" in the FEPs screening process. It is, however, used indirectly as follows. In the AMR: Breakage of CSNF Clad by Seismic Loading (CRWMS M\&O 1999a), the calculated fragility of a fuel rod is convolved with the expected seismic hazard predicted by this model to determine the rate of rod breakage. The output from this model is a table summarizing seismic risk to cladding integrity (Table 2 of CRWMS M\&O 1999a) that is input to the AMR: Clad Degradation Summary and Abstraction (CRWMS M\&O 2001d). These results are simplified for use directly in the TSPA-SR (CRWMS M\&O 2000bl, Section 6.3.4.3, p. 283). The model may also be used indirectly now or in the future because design analyses for the engineered components, including those components designed to function for $10,000 \mathrm{yr}$, may include response to ground motion.

Review Findings: The Vibratory Ground Motion Hazard Model appears not to have been formally validated, and is assigned to Bin 2 . However, the model and its output have undergone peer review and received technical review through publication in the open literature. What is needed is to identify it as a model, and cite the achievement of its validation under AP-3.10Q criteria.

\subsubsection{Fault Rupture Hazard Model (S.1-2)}

Description: This model is implicit in the report entitled: Characterize Framework for Seismicity \& Structural Deformation at Yucca Mountain, Nevada (CRWMS M\&O 2000m). It is a summary of information developed and presented in Probabilistic Seismic Hazard Analyses for Fault Displacement and Vibratory Ground Motion at Yucca Mountain, Nevada (Wong and Stepp 1998).

This model forecasts fault displacements at nine demonstration points near the Yucca Mountain repository site for annual exceedance probabilities from about $10^{-4}$ to $10^{-8}$. The points selected represent the expected range of fault displacement hazard conditions within the site area in terms of the types of features that may be encountered. The model results were developed using a formal elicitation process from six teams of experts who provided alternative characterizations of the tectonic setting, Quaternary faults, and fault source parameters.

Use of the Model: This model is not used directly for TSPA-SR because degradation of the engineered and natural barriers by fault rupture has been "screened out" in the FEPs screening process (CRWMS M\&O 2000bm, Appendix B). It is, however, used indirectly as follows. UZ flow along existing faults has been evaluated in support of the TSPA-SR (CRWMS M\&O $2000 \mathrm{ch}$ ). A bounding value of $10 \mathrm{~m}$ is used for fault offset with annual probability of $10^{-8}$. This bounding fault offset value is supported in Features, Events, and Processes: Disruptive Events (CRWMS M\&O 2000ad) by reference to the Fault Rupture Hazard Model supporting documentation. This model may also be used indirectly now or in the future because design analyses for the engineered components, including those components designed to function for $10,000 \mathrm{yr}$, may include response to fault rupture. 
Review Findings: The Fault Rupture Hazard Model appears not to have been formally validated, and is assigned to Bin 2 . However, the model and its output have undergone peer review and received technical review through publication in the open literature. What is needed is to identify it as a model, and cite the achievement of its validation under AP-3.10Q criteria.

\subsection{Model Area T: Integrated Site Model}

No models were identified in this model area. The three AMRs reviewed contain compilations and statistical analyses of site characterization data; Geologic Framework Model Analysis Model Report (BSC 2001e); Mineralogical Model (MM3.0) (CRWMS M\&O 2000at); and Rock Properties Model Analysis Model Report (CRWMS M\&O 2000bb). The uses of this information related to TSPA-SR (CRWMS M\&O 2000bl) were reviewed, and found not to require documentation of the analyses as models. This result may change depending on future use of the information.

Any decision to document technical work as an analysis or model is ultimately based on judgment as to the confidence needed for the intended use (see Section 3.4.2). These are recommendations only, and not final determinations as to the future status of these AMRs.

\subsection{Model Area U: Performance Assessment Model}

\subsubsection{Total System Performance Assessment-Site Recommendation Model (U.1-1)}

Description: The TSPA-SR is a computer model used to evaluate the performance of a potential repository system at Yucca Mountain, Nevada in isolating waste for future times after the closure of the repository. The model is documented in the AMR entitled: Total System Performance Assessment Model for Site Recommendation (CRWMS M\&O 2000bl). Additional documentation is provided in a companion technical report: Total System Performance for the Site Recommendation (CRWMS M\&O 2000bm).

The model integrates various submodels representing nine process submodel areas: Unsaturated Zone Flow, Thermal Hydrology, Waste Package and Drip Shield Degradation, Waste Form Degradation, Engineered Barrier System Transport, Unsaturated Zone Transport, Saturated Zone Flow and Transport, Biosphere, and Disruptive Events. Information about the configuration and performance of the engineered and natural barriers, and characteristics of the biosphere, is integrated in the TSPA-SR Model, and documented in the nine Process Model Reports (PMRs) corresponding to these submodel areas, and the associated Analysis Model Reports (AMRs).

The ultimate purpose of the TSPA-SR Model is to provide an estimate of the dose that could be received by a future member of public (MOP) located $20 \mathrm{~km}$ south of the repository in the Amargosa Valley, because of potential releases of radionuclides from the repository. (The 20-km distance has since changed when 40 CFR 197 [2001] was finalized.) The maximum dose to the MOP within $10,000 \mathrm{yr}$ (for compliance) and the peak dose within one million years, are estimated for undisturbed (nominal) and disturbed (igneous activity and human intrusion) performance of the repository system.

The model is implemented using the GoldSim probabilistic/dynamic-modeling platform (Golder Associates 2000). A single GoldSim file includes the input, the code, and the output for each facility performance scenario or case simulated. 
Use of the Model: Model output consists of time-series for total and radionuclide-specific annual dose at the receptor location, groundwater concentrations, and other intermediate results for the base-case and three disruptive-events scenarios. Additional simulations (sensitivity studies) are performed by modifying key parameters or models.

Review Findings: The TSPA-SR Model is assigned to Bin 3 (impact review is provided in Appendix IV), because additional confidence-building effort is needed to achieve model validation commensurate with the intended use.

The binning decision is based on the following:

- The validation criteria presented in Section 6.5 of the AMR do not address the intent of model validation stated in AP-3.10Q, Section 5.3. The validation discussion in Section 6.0 is more appropriate as documentation of integrated code verification. The AMR lacks a compliant validation argument, and the effort needed to achieve this is not limited to documentation of available information (the definition for Bin 2).

- Output of the integrated TSPA model is not tested against real data where feasible and/or the TSPA-SR has not been formally peer reviewed, in accordance with AP-3.10Q Section 5.3.b or 5.3.c.

Some aspects of model validation are either not clearly described or are lacking: 1) approach to uncertainty analysis; 2) implementation criteria for the system model; 3) integrated treatment of parameter uncertainty in the model; 4) Stage-3 verification of the integrated TSPA model in Section 6.5 of the AMR; 5) comparison of model predictions with best available sets of relevant field data involving YMP analogs; and 6) justification of the Monte Carlo sample size. For further details of the model validation review see Appendix II of this report.

The report: Total System Performance Assessment for the Site Recommendation (CRWMS M\&O $2000 \mathrm{bm}$ ) documents activities that are appropriate for validation of the TSPA-SR Model. These include discussions of integrated mathematical models (numerical methods, spatial and temporal variability) supporting the conceptual models, parameterization, input and output distributions in Sections 2 through 6; sensitivity analysis performed for the integrated model as well as the component models in Sections 5 and 6; and uncertainty analysis discussed in Section 6 and Appendix F. The Repository Safety Strategy (CRWMS M\&O 20010) also provides documentation of contributing model validation activity. It is concluded that these documents combined contain incomplete model validation as required by AP-3.10Q.

The following additional comments and recommendations are offered for consideration:

- It is assumed that submodels called by GoldSim (CRWMS M\&O 2000bl, Sections 6.2 and 6.3) are validated and documented in their respective AMRs. However, specific conceptual models that are implemented using GoldSim transport elements need better documentation of their validation. These include: cladding degradation, waste form degradation, EBS transport, 1-D SZ transport, indirect volcanism, and human intrusion.

- Develop criteria to perform the model validation, responsive to the validation requirements stated in AP-3.10Q. Document all validation activities and analyses performed following these criteria.

- Reorganize the TSPA-SR Model AMR and the TSPA-SR technical report to achieve greater transparency and tractability for model validation arguments and analyses. 
- The criteria and specific activities to demonstrate the reasonableness of the TSPA-SR Model output should address the following (the related parts of AP-3.10Q are indicated parenthetically):

- Acceptability of the mathematical models used to implement the conceptual model (mathematical models include physically-based, empirical, and compartmental models) (5.3.a)

- Reasonableness of the numerical schemes employed (5.3.a)

- Reasonable treatment of spatial and temporal variability (5.3.a)

- Reasonableness of the input parameters that are held constant (bounding conservative assumptions) (5.3.b, 5.3.c)

- Reasonableness of the input parameter distributions (uncertainty well quantified) (5.3.b, 5.3.c)

- Reasonableness and consistency of the integrated model output distributions, given the inputs to component models and the time periods (5.3.b, 5.3.c)

- Sensitivity analyses are performed for the integrated model as well as the component models (5.3.a)

- Uncertainty analyses are performed for the integrated model as well as the component models (5.3.a)

- Unquantified uncertainties are investigated (5.3.a)

- Robustness analysis (5.3.a)

Specific recommendations for report reorganization are included in Appendix III of this report. Briefly, the material supporting the integrated code verification (Section 6 of the AMR) should be moved to an appendix, and most of the material currently presented in the TSPA-SR technical report should be moved to the TSPA-SR Model AMR.

Impact Review Summary: The TSPA-SR incorporates the appropriate degree of confidence needed to demonstrate that a Yucca Mountain repository would comply with the draft regulatory postclosure performance standards. The evolution of TSPA has undergone independent peer review (Budnitz et al. 1999) with favorable conclusions regarding the basic framework and the use of abstractions and component models. In addition, a summary is available from the recently completed Joint NEA-IAEA International Peer Review (Riotte 2001). The conclusions expressed include findings that the overall structure of the TSPA-SR, and the FEP screening methodology, conform to international best practices. In response to a request from DOE to provide a statement regarding the adequacy of the overall TSPA-SR approach for supporting the site recommendation decision, the peer review summary included statements that the TSPA-SR method is soundly based and implemented in a competent manner, and that overall, the peer review team considers that the TSPA-SR approach provides an adequate basis for supporting an evaluation of regulatory compliance within the regulatory period of 10,000 years, and accordingly, for the site recommendation. Additional details of this peer review summary are provided in the impact reviews of Appendix IV. These statements show that there is consensus among an important part of the international technical community that the TSPA-SR methodology is adequate for its intended use. 
Sensitivity studies in the form of uncertainty importance analyses, subsystem sensitivity analyses, and robustness analyses clearly represent the level of uncertainty, the influence of conservatism, the limitations of the models, and the impacts on individual-dose associated with various time periods and hazards. These analyses indicate that the TSPA-SR model is sufficiently robust that even given the uncertainties that may exist in the subsystem models, the system model provides an adequate representation for comparison against the relevant draft performance standards. Additional sensitivity analyses (BSC 2001d, 2001k) have been performed to provide insight into potential conservatism and optimism in the TSPA-SR, to express a wider representation of uncertainty, and to provide updated and more realistic models for processes. Based on this information and the additional detail provided in Appendix IV, there is no significant impact on the conclusions of TSPA-SR (CRWMS M\&O 2000bm, Section 6.1) from the review findings identified for the TSPA-SR system model by this review.

\subsubsection{Soil Removal Model for Volcanic Disruption (U.1-2)}

Description: The soil removal model is associated with volcanic releases. The model computes a soil removal factor that represents the loss of soil caused by normal erosion processes.

The model is contained in GoldSim subroutine SOILEXP (CRWMS M\&O 2000b1, Sections 3.1.10 and Attachment III) which calculates the cumulative soil removal factor used to determine radionuclide concentration at deposition points over the life of the repository.

The model is documented in the AMR entitled: Total System Performance Assessment (TSPA) Model for Site Recommendation (CRWMS M\&O 2000bl). Additional supporting documentation is found in: Evaluate Soil/Radionuclide Removal by Erosion and Leaching (CRWMS M\&O 2001i).

Use of the Model: This result from the SOILEXP subroutine is used in conjunction with biosphere dose conversion factors (BDCFs) and event probability to calculate the dose to the critical group from the volcanic release event for TSPA-SR (CRWMS M\&O 2000bl, Section 6.3.9.1).

Review Findings: No theoretical basis, verification, or validation of the soil removal model is given. The AMR: Evaluate Soil/Radionuclide Removal by Erosion and Leaching (CRWMS $\mathrm{M} \& \mathrm{O} 2001 \mathrm{i})$ is referenced for detailed discussion of soil erosion. However, it does not explicitly include the functional form for computing the soil removal factor or any validation information for soil erosion models. In the judgment of the reviewers, model development and/or data collection may be needed in addition to revision of the documentation, to achieve a validation argument that complies with AP-3.10Q. Accordingly, the Soil Removal Model for Volcanic Disruption is assigned to Bin 3 (impact review is provided in Appendix IV).

Impact Review Summary: The Soil Removal Model for Volcanic Disruption was used in TSPA-SR but did not serve as a basis for screening out FEPs. Instead of explicitly including aeolian and fluvial processes that could transport sediment from the area of ashfall to the location of the receptor, TSPA-SR analyses used a conservative approach in which the wind direction was fixed toward the receptor for all eruptive events. This and other features of the model resulted in overestimating radiation exposure from volcanic disruption. The overall representation of ash redistribution processes in the model for TSPA-SR is conservative. Based on this information and the additional detail provided in Appendix IV, there is no significant 
impact on the conclusions of TSPA-SR (CRWMS M\&O 2000bm, Section 6.1) from the model validation review findings associated with this model.

\subsubsection{Pu-Ceramic Degradation Model for TSPA-SR (U.4)}

Description: This model provides the aqueous dissolution rate for plutonium ceramic. The Synroc-C model is proposed as representative of the ceramic, based on the argument that the ceramic waste form is inherently more stable and degrades more slowly than the glass. This AMR constitutes the justification for using high-level waste glass as a surrogate for plutonium disposal in can-in-canister ceramic in the TSPA-SR (CRWMS M\&O 2000bl). The model is documented in AMR: Performance Assessment and Sensitivity Analysis of Disposal of Plutonium as Can-in-Canister Ceramic (CRWMS M\&O 2001u).

Use of the Model: Three waste form categories are represented in TSPA-SR (CRWMS M\&O 2000bl, Section 6.3.4.1): 1) commercial spent nuclear fuel (CSNF), 2) DOE-owned spent nuclear fuel (DSNF), and 3) high-level radioactive waste (HLW) glass. These are contained and disposed of in two types of waste packages - the CSNF waste package and the co-disposal waste package, with the latter containing both DSNF and HLW glass. As such, the immobilized plutonium waste form is not explicitly represented in TSPA-SR, but is represented by $\mathrm{HLW}$ glass. The subject AMR is a sensitivity study that is not used for TSPA-SR.

Review Findings: No validating information is provided for this model in the AMR. Validation of this model is required only if plutonium-disposition ceramic waste forms are included in TSPA-SR (CRWMS M\&O 2000bl). In the judgment of the reviewers, model development and/or data collection may be needed in addition to revision of the documentation, to achieve a validation argument that complies with AP-3.10Q. Accordingly, the Pu-Ceramic Degradation Model for TSPA-SR is assigned to Bin 3 (impact review is provided in Appendix IV).

Impact Review Summary: This model was used in a sensitivity analysis of the potential effects from using HLW as a surrogate for plutonium in canister-in-canister ceramic form. The model was not used in TSPA-SR and was not used for screening out FEPs. Based on this information and the additional detail provided in Appendix IV, there is no significant impact on the conclusions of TSPA-SR (CRWMS M\&O 2000bm, Section 6.1) from the model validation review findings associated with this model. 


\section{INTENTIONALLY LEFT BLANK}




\section{Summary and Conclusions}

This Model Validation Status Review (MVSR) evaluated the AMRs and other technical documents that support Total System Performance Assessment - Site Recommendation (TSPASR; CRWMS M\&O $2000 \mathrm{bl}$ and $2000 \mathrm{bm}$ ). Models were identified, and compliance with the procedural criteria for model validation (AP-3.10Q Section 5.3) was assessed using a binning strategy.

A total of 128 models were identified, of which 34 were assigned to Bin 3 (not in compliance and not readily validated). Impact reviews were performed for these models, and are included as Appendix IV to this report. For all 34 Bin-3 models, the impact reviews found no significant impact from the model validation review findings, on the conclusions of the TSPA-SR (i.e. calculated annual dose; CRWMS M\&O 2000bm, Section 6.1).

The reviews were performed by 32 independent reviewers, called technical specialists, under the direction of the BSC Chief Science Office. They reviewed 125 AMRs, plus other documents and data, in 21 designated model areas (Table 1). The focus of the review was to identify and describe the models, and bin them according to the extent to which model validation was achieved in compliance with AP-3.10Q (Analyses and Models). A series of workshops and teleconferences was held in July and August, 2001, to discuss and integrate the review findings. Description of the model areas is provided in Section 3, along with the review methodology. The documents reviewed are described in Section 4. Biographical information for the 32 technical specialists is provided in Section 5. The identification and description of individual models, description of how the models were used, basis for binning, and recommendations for improved documentation or further model development are detailed in Section 6.

Identification and binning of the 128 models is shown in Table 6 . In this report the models are assigned a unique alphanumeric code X.N-M, where $X$ is the letter corresponding to the model area (as in Table 1), $\mathrm{N}$ is the document number from the list in the second column of Table 1, and $\mathrm{M}$ is a serial number assigned when more than one model was found in a particular document. For example, model Q.9-1 belongs to Model Area Q (Biosphere), and the principal AMR is number Q.9 from Table 1 (Nominal Performance Biosphere Dose Conversion Factor Analysis, CRWMS M\&O $2001 \mathrm{~m}$ ). Model Q.9-1 is the first of five models identified in this AMR, hence the serial number "-1" is appended.

The MVSR was a one-time evaluation of model validation associated with the AMRs supporting TSPA-SR (CRWMS M\&O 2000bl and 2000bm). This review was performed in response to Corrective Action Request BSC-01-C-001 (Clark 2001, Krisha 2001) pursuant to Quality Assurance review findings of an adverse trend in model validation deficiency.

\subsection{Summary of Review Findings by Model Area}

Models were identified from the documentation assigned to each of the 21 model areas (Table 1). For each model a short description was developed (Section 6), and the model was assigned to one of three bins defined as follows:

- Bin 1 - Validation (as defined in AP-3.10Q Section 5.3) was achieved in a single, principal AMR. The entire content of the AMR was considered, not just the model validation subsections. 
- Bin 2 - Validation was not achieved in a principal AMR, however, existing documentation (possibly including other AMRs, other reports, data, publications, etc.) provides adequate confidence to support compliant model validation, commensurate with the intended use of the model.

- Bin 3 - Validation was not achieved in a principal AMR, and cannot be readily achieved using available information. Additional work (e.g., model development, testing, data collection) is needed to provide adequate confidence to support compliant validation.

Identification and review of each model were the responsibilities of the lead technical specialist assigned to each model area (Table 4). The lead technical specialists were assisted by other reviewers assigned as support. Overlap between the model areas was established to promote consistency in the reviews. Further integration was achieved using teleconferences, and in a final workshop in Las Vegas that was also attended by Project staff responsible for the models. Review findings including model descriptions were submitted by the technical specialists on signed forms. Most of the detailed information from these forms is contained in Section 6, and Appendices I, II, and III.

The following is a brief summary of the review findings organized by model area. The reader is referred to Section 6 for details of the reviews, and to Appendix IV for details of the impact reviews for the Bin-3 models:

- Model Area A: Climate - One model was identified in this area (Section 6.1): the Precession-Based Orbital Clock Model (A.1), which was assigned to Bin 1. The review determined that the data used for this model are all published in peer-reviewed literature.

- Model Area B: Infiltration - One model was identified in this area (Section 6.2): the Net Infiltration Model (B.1). It is assigned to Bin 2 because additional documentation is needed to justify the treatment of plant rooting depth, and the use of neutron moisture $\operatorname{logs}$.

- Model Area C: Unsaturated Zone Flow - Nine models were identified in this area (Section 6.3) and assigned to Bins 1 and 2:

Unsaturated Zone Flow - Bin 1:

- UZ Flow Model (C.1-1)

- Geothermal Model (C.1-2)

- Conceptual Model of Perched Water (C.1-3)

- 3-D Perched Water Calibration Model (C.1-4)

Unsaturated Zone Flow - Bin 2:

- Conceptual Model of UZ Flow (C.6-1)

- Numerical Grids Model (C.4)

- Numerical Model of UZ Flow (C.6-2)

- Active Fracture Model (C.6-3)

- Calibrated Properties Model (C.2)

It is recommended that conceptual and numerical model development not be documented as separate AMRs. Rather, they should be documented in other ways (e.g., technical 
reports or analyses), or combined incorporated into the predictive-model AMRs, so that model-data comparison can be used in validating the ensemble.

- Model Area D: Mountain-Scale Thermal-Hydrology - One model was identified in this area (Section 6.4): the Mountain-Scale Coupled Processes (TH) Model. It was assigned to Bin 2. Recommended improvements to the documentation include: update model comparisons with field data, justify neglect of THC and THM coupled processes, and provide further justification of model inputs, numerical gridding schemes, and differences among the mountain-scale models.

- Model Area E: Ambient/Thermal Drift Seepage - Four models were identified in this area (Section 6.5) and assigned to Bin 2:

Ambient/Thermal Drift Seepage - Bin 2:

- Seepage Calibration Model (E.1)

- Seepage Model for PA Including Drift Collapse (E.2)

- Abstraction of Drift Seepage (E.3-1)

- Abstraction of Thermal Seepage Effects (E.3-2).

Recommended improvements to the documentation include: update model comparisons with field-test data, justify treatment of evaporation in the interpretation of field tests, justify neglect of THM effects in the seepage model, and develop an alternative approach for validating the thermal seepage model.

- Model Area F: Mountain-Scale/Near-Field Thermal-Hydrologic-Chemical Model Two models were identified in this area (Section 6.6): the DST THC Calibration Model (F.1-1), and a suite of models collectively referred to as the THC Seepage Model (F.1-2). Both are assigned to Bin 2. Recommended improvements to the documentation include: clear definition of the conceptual basis, justification of assumptions, and establishment of model validation criteria that define the relationship between the DST and validation of models describing the post-closure repository. Additional detailed recommendations are provided in Appendix I.

- Model Area G: Mountain-Scale/Near-Field Thermal-Hydrologic-Mechanical Model - One model was identified in this area: the THM Model (G.1), which was assigned to Bin 3. Review findings indicate that the model is too simplistic, and that further model development is needed (Section 6.7.1). Impact review for this model (see Section 6.7.1 and Appendix IV) shows that the model validation review findings associated with this model have no significant impact on the conclusions of TSPA-SR (CRWMS M\&O $2000 \mathrm{bm}$, Section 6.1) because the magnitude of the potential THM effect on permeability is limited. Additional work has already been performed, and other work is ongoing, to support further assessment of THM processes.

- Model Area H: In-Drift Chemistry - Ten models were identified in this model area (Section 6.8) and assigned to Bins 2 and 3:

In-Drift Chemistry - Bin 2:

- High-Relative Humidity Salts Model (H.1)

- In-Drift Microbial Communities (H.6) 
- Gas Flux and Fugacity Model (H.3-1)

- In-Drift Gas Flux and Composition Model (H.5)

- Normative Precipitates/Salts Model (H.3-6)

Recommended improvements to the documentation for the above models include: examine the intended use for these models, justify extrapolation of $\mathrm{pH}$ predictions to higher ionic strength, establish the context of a "reaction cell" in the repository environment, establish the relevant microbial processes, and integrate model inputs and results with other activities in the Project.

In-Drift Chemistry - Bin 3:

- Cement Grout Model (H.3-2)

- Seepage/Cement Interaction Model (H.7)

- Corrosion of Steel Used in the Ex-Container EBS (H.3-3)

- Effect of Evaporation in the Invert (H.3-4)

- EBS Colloids Model (H.3-5)

Recommended changes to the development and documentation of these Bin-3 models include: compare model results to experimental data, model cement evolution over time, use more realistic cement mineral phases, justify steel corrosion modeling approach, model THC processes using fully coupled reactive transport, and conduct direct measurement of porosity-permeability behavior for the invert ballast material.

Impact reviews for these Bin-3 models (see Section 6.8 and Appendix IV) show that the model validation review findings associated with this model have no significant impact on the conclusions of TSPA-SR (CRWMS M\&O 2000bm, Section 6.1). None of these Bin-3 models were used for TSPA-SR; some were used for FEP screening and arguments are provided as why these has no impact on the conclusions of TSPA-SR.

- Model Area I: EBS Moisture Distribution and Thermal-Hydrology - Eight models were identified in this model area (Section 6.9) and assigned to Bins 1, 2, and 3:

EBS Moisture Distribution and Thermal-Hydrology - Bin 1:

- Water Diversion (I.2-2)

EBS Moisture Distribution and Thermal-Hydrology - Bin 2:

- Multiscale TH Model (I.1)

- Water Drainage (I.2-1)

- Thermohydrologic Model (1.2-3)

- DS Condensation Model (I.2-4)

Recommended improvements to the documentation for the above models include: justify the assumptions and approach used, justify neglect of THC and THM coupled processes in TH models, justify neglect of lateral flow for drainage models, justify representation of plugging of the host rock below the drifts, explain how spatial heterogeneity of rock properties affects the results, and incorporate more results from thermal testing. 
EBS Moisture Distribution and Thermal-Hydrology - Bin 3:

- In-Drift THC Model (I.7)

- Effective Thermal Conductivity Model (I.8)

- Ventilation Model (I.5)

Recommended changes to the development and documentation of these Bin-3 models include: reformulate the models used for "clear-fluid" simulations so they implement Navier-Stokes equations, and develop a more mechanistic physical basis for representing in-drift heat and mass transfer during both the preclosure and post-closure periods.

Impact reviews for these Bin-3 models (see Section 6.9 and Appendix IV) show that the model validation review findings associated with this model have no significant impact on the conclusions of TSPA-SR (CRWMS M\&O 2000bm, Section 6.1). None of these Bin-3 models were used for screening out FEPs from TSPA-SR. The Ventilation Model was used only to establish the feasibility of preclosure ventilation, and the Effective Thermal Conductivity Model was used as a simplification for TSPA-SR that has since been evaluated using an approach that explicitly models thermal radiation.

- Model Area J: Waste Package/Drip Shield Degradation - Eighteen models were identified in this model area (Section 6.10) and assigned to Bins 1, 2, and 3:

Waste Package/Drip Shield Degradation - Bin 1:

- Alloy-22 Potential-Based Localized Corrosion Initiation Threshold and Rate Abstraction Model (J.5-1)

- Ti Grade 7 Potential-Based Localized Corrosion Initiation Threshold and Rate Abstraction Model (J.5-2)

Waste Package/Drip Shield Degradation - Bin 2:

- General \& Localized Corrosion of the WPOB (J.1)

- Aging and Phase Stability: Precipitation Model (J.2-1)

- Aging and Phase Stability: Long Range Ordering Model (J.2-2)

- General and Localized Corrosion of the Drip Shield (J.3)

- Degradation of Stainless Steel Structural Materials (J.4)

- Calculation of General Corrosion Rate of DS and WPOB to Support WAPDEG Analysis (J.7)

- DS Passive Corrosion (J.10-1)

- DS Galvanic Coupling (J.10-2)

- SCC Threshold Model (J.11-1)

- SCC Slip Dissolution/Film Rupture Model (J.11-2)

- Manufacturing Defects Abstraction Model (J.12-1)

- Stress and Stress Intensity Factor Profile Abstraction (J.12-2)

- Slip Dissolution Abstraction Model (J.12-3)

- Threshold Stress Intensity Factor Abstraction Model (J.12-4)

Recommended improvements to the documentation for the above models include: rely more on probabilistic methodology, provide better documentation of causal relationships, use alternative methods for residual stress analysis, incorporate recent data, make more 
use of analogous information on stainless steels, perform more complete interpretation of corrosion test data, and make more use of the technical literature.

Waste Package/Drip Shield Degradation - Bin 3:

- WAPDEG Analysis of WP and DS Degradation (J.6)

- Incorporation of Uncertainty and Variability of DS and WP Degradation in WAPDEG (J.8)

Recommended changes to the development and documentation of these Bin-3 models include: enhance the model for localized corrosion and SCC particularly at heat-affected zones, use stress analysis as an alternative to finite element modeling, and probabilistically combine mechanisms for localized corrosion and SCC in TSPA.

Impact reviews for these Bin-3 models (see Section 6.10 and Appendix IV) show that the model validation review findings associated with these models have no significant impact on the conclusions of TSPA-SR (CRWMS M\&O 2000bm, Section 6.1). Neither of these Bin-3 models was used for FEP screening. The WAPDEG model (J.6) was used for TSPA-SR, but is founded on measured data.

- Model Area K: Waste Form Degradation - Thirty models were identified in this model area (Section 6.11) and assigned to Bins 1, 2, and 3:

Waste Form Degradation - Bin 1:

- CSNF Waste Form Summary Degradation Abstraction (K.2)

- In-Package Source Term Abstraction (K.5)

- Bounding Model for Clad Unzipping Velocity (K.14-2)

Waste Form Degradation - Bin 2:

- Inventory Abstraction (K.1)

- DHLW Glass Degradation (K.3)

- Waste Form Degradation Abstract. - Upper Limit Model (K.4-1)

- Waste Form Degradation Abstraction - Conservative Model (K.4-2)

- In-Package Chemistry for Waste Forms (K.6)

- In-Package Chemistry Abstraction (K.7)

- Pure-Phase Solubility Limits (K.8)

- Dissolved Concentration Limits (K.9)

- Secondary Uranium-Phase Paragenesis and Incorporation of Radionuclides into Secondary Phases (K.10)

- Initial Oxide Thickness (K.11-1)

- Rod Internal Pressure (K.11-2)

- Cladding Crack Depth (K.11-3)

- Overall Cladding Stress (K.11-4)

- Initial Rod Failure (K.11-5)

- Zircaloy Corrosion Rate (K.12)

- Residual Stress in CSNF Cladding Material (K.13)

- Clad Dry Unzipping Model (K.15)

- Stainless Steel in WPs for TSPA-SR (K.17) 
- Colloid-Associated Radionuclide Concentration Limits (K.21)

- WF Colloid-Associated Concentration Limits: Abstraction and Summary (K.22)

Recommended improvements to the documentation for the above models include: eliminate excessive conservatism in waste-form degradation models, consider inclusions in glass, use more extensive model-data comparison, make more complete use of data from the literature, provide more careful explanation of chemical model output, propagate uncertainties on inputs into model output, update $\mathrm{Np}$ solubility models, and revise the documentation for cladding degradation models so they are not calculations or analyses (i.e. provide validation).

Waste Form Degradation - Bin 3:

- Waste Form Degradation Abstraction - Best Estimate Model (K.4-3)

- Waste Form Degradation Abstraction - Immobilized Pu Model (K.4-4)

- Alternative Wet Clad Unzipping Model (K.14-1)

- Summary and Abstraction - Clad Unzipping and Fuel Dissolution (K.16)

- Thermal Evaluation of Breached 21-PWR WPs (K.18)

- Breakage of CSNF Clad by Seismic Loading (K.19-1)

- Breakage of CSNF Clad by Static Loading (K.19-2)

Recommended changes to the development and documentation of these Bin- 3 models include: develop better data support for waste form degradation models, consolidate cladding degradation models to reconcile them and decrease the effort of documentation, provide more realistic in-package heat transfer analysis, use nuclear-plant seismic design approaches to evaluate seismic effects on cladding, and provide more realistic static loading analysis.

Impact reviews for these Bin-3 models (see Section 6.11 and Appendix IV) show that the model validation review findings associated with these models have no significant impact on the conclusions of TSPA-SR (CRWMS M\&O 2000bm, Section 6.1). Of the Bin-3 models listed above, only the Thermal Evaluation of Breached 21-PWR WPs (K.18) was used for FEP screening. Models K.4-3, K.4-4, and K.19-2 were not used for TSPA-SR. The model validation review findings associated with those which were used for TSPASR, have no significant impact on the conclusions of TSPA-SR because the contribution of cladding to system performance has been shown to be minor.

- Model Area L: EBS Degradation - Three models were identified in this model area (Section 6.12) and assigned to Bins 2 and Bin 3 :

EBS Degradation - Bin 2:

- Rockfall on DS Model (L.3)

Recommended improvements to the documentation for the above model include: use examples of transient-dynamic analysis from the literature to validate the approach for modeling rockfall on the drip shield, and explicitly represent drip shield interaction with the invert. 
EBS Degradation - Bin 3:

- DRKBA Rockfall Model (L.1)

- Flow into WPs Through Small Lid Openings Model (L.6)

Recommended changes to the development and documentation of these Bin-3 models include: improve the approach for estimating thermal and seismic effects on rockfall, consider breakage of intact rock and ravelling, consider stress concentration around profiles altered by rockfall, and compare rockfall model results with alternative, mechanistic models.

Impact reviews for these Bin-3 models (see Section 6.12 and Appendix IV) show that the model validation review findings associated with these models have no significant impact on the conclusions of TSPA-SR (CRWMS M\&O 2000bm, Section 6.1). The DRKBA Rockfall Model (L.1) is reasonably conservative for predicting the occurrence of large rockfall blocks; these blocks are then used for structural analysis of the drip shield design. Although the DOE has agreed to make model improvements and evaluate alternative modeling approaches, the current model and its supporting and related documentation are considered to provide adequate confidence. The Flow into WPS Through Small Lid Openings Model (L.6) was not used for TSPA-SR or for FEP screening.

- Model Area M: EBS Radionuclide Transport - Five models were identified in this model area (Section 6.13) and assigned to Bins 2 and 3:

EBS Radionuclide Transport - Bin 2:

- Invert Diffusion Properties Model (M.1)

Recommended improvements to the documentation for the above model include: expand the empirical justification for the diffusion-electrical analog, estimate the lower limit of water content for application of Archie's Law, develop a basis for neglecting electrical conduction and diffusion in thin moisture films, develop a theoretically based approach for representing ion activity at different temperatures, and include sorption in the model.

EBS Radionuclide Transport - Bin 3:

- In-Drift Colloids and Concentrations (M.3)

- In-Drift Transport of Radionuclides (M.2)

- EBS Radionuclide Transport Abstraction Model (M.5)

- Seepage/Invert Interactions Model (M.4)

Recommended changes to the development and documentation of these Bin-3 models include: conduct a peer review of the In-drift Colloids and Concentrations Model (M.3), justify representing the invert as a reaction cell, provide better justification of the constant-Kd approach, consider the effects of colloid settling, evaluate changes in invert properties from accumulation of de-stabilized colloids, combine conceptual and mathematical models to facilitate validation, and use testing and the open literature to support models for water movement in the EBS.

Impact reviews for these Bin-3 models (see Section 6.13 and Appendix IV) show that the model validation review findings associated with these models have no significant impact 
on the conclusions of TSPA-SR (CRWMS M\&O 2000bm, Section 6.1). Only the In-Drift Colloids and Concentrations Model (M.3) and the EBS Radionuclide Transport Abstraction Model (M.5) were used for TSPA-SR. Both of these models are bounding and/or conservative, and therefore tend to overestimate the potential for transport of dissolved and colloidal species from breached waste packages. Impacts on the conclusions of TSPA-SR from these model validation review findings are also minimal because the invert has small benefit for performance, and invert materials will not exert significant influence on water composition.

- Model Area N: Unsaturated Zone Transport - Eight models were identified in this model area (Section 6.14) and assigned to Bins 1, 2, and 3:

Unsaturated Zone Transport - Bin 1:

- Colloid Transport (N.2-1)

Unsaturated Zone Transport - Bin 2:

- Equilibrium Matrix Sorption Basis (N.1-1)

- Fracture Sorption (N.1-2)

- Matrix Diffusion (N.1-3)

- Pu Sorption on Colloids (N.2-2)

- EOS9nT Calibration to ESF Chloride Profile (N.3-2)

- Fracture-to-Matrix Colloid Filtration (N.4)

Recommended improvements to the documentation for the above models include: develop rigorous criteria for model-data comparisons and apply them consistently to different chemical species, include the measured data in AMRs where validation is discussed, consolidate conceptual and mathematical model development to facilitate validation, and ensure that the different transport models used by the Project yield comparable results when applied to model-data comparisons.

Unsaturated Zone Transport - Bin 3:

- FRACL Calibration to Borehole Chloride (N.3-1)

Recommended changes to the development and documentation of this Bin-3 model include: develop an objective criterion for model-data comparison, and incorporate additional measured data in the comparison.

Impact review for this Bin-3 model (see Section 6.14 and Appendix IV) shows that the model validation review findings are not significant to the conclusions of TSPA-SR (CRWMS M\&O 2000bm, Section 6.1). The FRACL Calibration to Borehole Chloride Model (N.3-1) is only one line of reasoning by which the representation of UZ flow and transport for TSPA-SR is validated.

- Model Area O: Saturated Zone Flow - One model was identified in this area (Section 6.15): the SZ Flow Model (O.1). It is assigned to Bin 2 because justification is needed for permeability and temperature profiles, the use of water levels and head data, the use of the hydrogeologic framework model, and the distribution of hydrogeologic features including faults. It is also noted that output from an unvalidated regional-scale model was used to validate the site-scale $\mathrm{SZ}$ flow model. 
- Model Area P: Saturated Zone Transport - Five models were identified in this model area (Section 6.16) and assigned to Bins 1,2, and 3:

Saturated Zone Transport - Bin 1:

- Sub-Gridblock Scale Dispersion in 3-Dimensional Heterogeneous Fractured Media (P.6)

Saturated Zone Transport - Bin 2:

- Saturated Zone Colloid Facilitated Transport (P.2)

- Pipe Model for Daughter Radionuclides (P.4-1)

Recommended improvements to the documentation for the above models include: check model calibration parameters for reasonableness in accordance with AP-3.10Q, and develop a more rigorous basis for model-model comparison used to validate the "pipe" model.

Saturated Zone Transport - Bin 3:

- Abstraction of FEHM and Coupling with UZ Mass Flux (P.4-2)

- Transport Parameters from C-Wells and Laboratory Studies (P.4-3)

Recommended changes to the development and documentation of these Bin-3 models include: clearly describe the integration of the SZ model used in TSPA-SR and coupling with the UZ model, make more use of model-data comparison or alternative validation methods, and justify selection of the dual-permeability model by comparison to alternatives.

Impact reviews for these Bin-3 models (see Section 6.16 and Appendix IV) show that the model validation review findings are not significant to the conclusions of TSPA-SR (CRWMS M\&O 2000bm, Section 6.1). Coupling of the UZ transport model with the "pipe" model is a calculational procedure that was checked against an analytical solution. Additional validation activities are planned and will provide additional confidence in the representation of SZ transport, but are unlikely to change the conclusions of TSPA-SR.

- Model Area Q: Biosphere - Ten models were identified in this model area (Section 6.17) and were assigned to Bins 1, 2, and 3:

Biosphere-Bin 1:

- Crop Interception Fraction Submodel (Q.2-1)

- Irrigation Rate Submodel (Q.2-2)

- Dose Conversion for Ingestion (Q.4-1)

- Dose Conversion for Inhalation (Q.4-2)

Biosphere - Bin 2:

- Dose Conversion for External Exposure (Q.4-3)

- Resuspension Model (Q.9-2)

- Plant Uptake Model (Q.9-3)

Recommended improvements to the documentation for the above models include: 
document GENII-S components as models instead of analyses, develop a site-specific external exposure model with an appropriate shielding factor, justify application of the Resuspension Model (Q.9-2) as implemented in GENII-S, and expand the empirical basis for the Plant Uptake Model (Q.9-3).

Biosphere - Bin 3:

- Surface Soil Model in GENII-S (Q.9-1)

- Radionuclide Transfer to Animals (Q.9-4)

- Radionuclide Transfer to Aquatic Food (Q.9-5)

Recommended changes to the development and documentation of these Bin-3 models include: address the cautions in the original journal article on the Surface Soil Model (Q.9-1) approach, develop site-specific aspects of radionuclide accumulation in soil including laboratory measurements and analogs, and revise the representation of radionuclides in pond water to include a simple fate model.

Impact reviews for these Bin-3 models (see Section 6.17 and Appendix IV) show that the model validation review findings are not significant to the conclusions of TSPA-SR (CRWMS M\&O 2000bm, Section 6.1). The primary pathways that lead to dose are included in the implementation of GENII-S for TSPA-SR; the approach is the same basic model that is used in other Biosphere models developed internationally, and is widely accepted. Incorporation or modification of minor pathway models is not likely to change the conclusions of TSPA-SR.

- Model Area R: Disruptive Events/Igneous Disruption Consequences - Six models were identified in this model area (Section 6.18) and assigned to Bins 2 and 3:

Disruptive Events/Igneous Disruption Consequences - Bin 2:

- Conditional Distribution for Number of Eruptive Centers Model (R. 1)

- Volcanic Eruption Release Model (R.3-1)

- In-drift Damage Due to Dike Intersection Model (R.3-2)

- Mass Loading Decay Model Following Deposition of Volcanic Ash (R.5-1)

- Dose Conversion Factor Model for Inhalation - Igneous Disruption (R.5-2)

Recommended improvements to the documentation for the above models include: provide additional documentation of the basis for the Conditional Distribution for Number of Eruptive Centers Model (R.1), make greater use of relevant technical literature and data from Yucca Mountain and elsewhere, use empirical data for metallic objects engulfed in magma or pyroclastic debris, use data from Mt. St. Helens to bracket the decay rate in particulate loading derived from resuspended ash, and update the Dose Conversion Factor Model for Inhalation - Igneous Disruption (R.5-2).

Disruptive Events/Igneous Disruption Consequences - Bin 3:

- Geometry of Volcanic Feeder System Model (R.2)

Recommended changes to the development and documentation of this Bin-3 model include: show that alternative eruption models have been considered, and make greater use of technical literature to support model parameterization.

Impact review for this Bin-3 model shows that the model validation review findings are 
not significant to the conclusions of TSPA-SR (CRWMS M\&O 2000bm, Section 6.1). The model used for TSPA-SR is conservative in several respects (see Section 6.18 and Appendix IV). Although additional validation activities are underway, it is unlikely that the conclusions of the TSPA-SR will be affected by these activities.

- Model Area S: Seismic Hazards - Two models were identified in this model area (Section 6.19) and assigned to Bin 2:

Seismic Hazards - Bin 2:

- Vibratory Ground Motion Hazard (S.1-1)

- Fault Rupture Hazard (S.1-2)

The present documentation includes enough information to develop validation arguments, and it is recommended that these be documented as models in accordance with AP$3.10 \mathrm{Q}$.

- Model Area T: Integrated Site Model - No models were identified in this area (Section 6.20).

- Model Area U: Performance Assessment Model - Three models were identified in this model area (Section 6.21) and assigned to Bin 3:

Performance Assessment - Bin 3:

- TSPA-SR Model (U.1-1)

- Soil Removal Model for Volcanic Disruption (U.1-2)

- Pu-Ceramic Degradation Model for TSPA-SR (U.4)

Recommended changes to the development and documentation of the TSPA-SR Model (U.1-1) include: develop the approach to uncertainty analysis, perform model-data comparison involving natural analogs, provide fully documented Stage-3 documentation (explained in CRWMS M\&O 2000bl, pp. 548-550), conduct a peer review, justify the Monte Carlo sample size, document models implemented directly in GoldSim, and reorganize the TSPA model documentation. Recommendations for the Soil Removal Model for Volcanic Disruption (U.1-2) include documenting and validating the model explicitly. Recommendations for the Pu-Ceramic Degradation Model for TSPA-SR (U.4) include providing model validation, if the $\mathrm{Pu}$-disposition ceramic waste form is to be incorporated in a future TSPA.

Impact review for these Bin-3 models shows that the model validation review findings are not significant to the conclusions of TSPA-SR. The approach used for TSPA-SR is basically the same as that used by other waste management programs internationally. The recent TSPA for the Yucca Mountain Viability Assessment was peer reviewed, with favorable conclusions regarding the approach. In addition, preliminary findings from the recently completed Joint NEA-IAEA International Peer Review of the TSPA-SR include statements that the overall structure of the TSPA-SR, and the FEP screening methodology, conform to international best practices. In response to a request from DOE to provide a statement regarding the adequacy of the overall TSPA-SR approach for supporting the site recommendation decision, the findings include statements that the TSPA-SR method is soundly based and implemented in a competent manner, and that overall, the peer review team considers that the TSPA-SR approach provides an adequate 
basis for supporting an evaluation of regulatory compliance within the regulatory period of 10,000 years, and accordingly, for the site recommendation. Additional support for the TSPA-SR model comes from sensitivity, uncertainty, and "robustness" analyses (CRWMS M\&O 2000bm, Section 5.3). The results show that the TSPA-SR model is sufficiently robust that even given uncertainties in the subsystem models, the relevant draft performance standards will likely be met. Additional sensitivity analyses (BSC $2001 \mathrm{~d}, 2001 \mathrm{k}$ ) show that relevant draft performance standards can be met for a range of thermal operating modes, by a margin of several orders of magnitude.

The Soil Removal Model for Volcanic Disruption was used in TSPA-SR, but uses a conservative approach that overestimates radiation exposure from volcanic disruption. The Pu-Ceramic Degradation Model for TSPA-SR is a sensitivity study that was not used for TSPA-SR or FEP screening. Based on this information and the additional detail provided in Section 6 and Appendix IV, there is no significant impact on the conclusions of TSPA-SR (CRWMS M\&O 2000bm, Section 6.1) from the model validation review findings associated with these models.

\subsection{Impact Reviews for Bin-3 Models}

Of the 34 Bin-3 models, the impact reviews for 24 can be summarized as follows:

- Sixteen were found not to support TSPA-SR, i.e., the output from these models was not used as input to the system model (Table 7). All 16 of these models are developmental, and some have been superseded by other products. Of these 16 models, only six were used for screening out of features, events, and processes (FEPs) from the TSPA-SR. Inspection of the FEPs AMRs shows that this usage was generally qualitative in nature.

- Five additional Bin-3 models (not among the 16 discussed above) were originally intended by the authors to be analyses or calculations (i.e., not requiring validation; these are models I.8, K.16, K.18, K.19-1, and R.2 in Table 7). At the time this work was documented, the authors determined that model validation was not required based on consideration of details such as the analysis methods used and the contribution to TSPA$\mathrm{SR}$. The review team identified these as models requiring validation, using the criteria from Section 3.4.2 of this report.

- Three additional Bin-3 models in the Biosphere area were embedded within the GENII-S dose-assessment code and were not previously recognized as discrete models (see Sections $6.17 .8,6.17 .9$, and 6.17 .10 of this report). The GENII-S code is a widely used and accepted approach, but application to a potential Yucca Mountain geologic repository is not yet fully documented.

The remaining 10 of the Bin-3 models were used for TSPA-SR, and were originally identified as models requiring validation:

- WAPDEG Analysis of Waste Package and Drip Shield Degradation (J.6)

- Alternative Wet Clad Unzipping Model (K. 14-1)

- DRKBA Rockfall Model (L.1)

- In-Drift Colloids and Concentrations (M.3)

- EBS Radionuclide Transport Abstraction Model (M.5)

- FRACL Calibration to Borehole Chloride (N.3-1)

- Abstraction of FEHM and Coupling with UZ Mass Flux (P.4-2) 
- Transport Parameters from C-Wells and Laboratory Studies (P.4-3)

- TSPA-SR Model (U.1-1)

- Soil Removal Model for Volcanic Disruption (U.1-2)

Impact reviews for all $34 \mathrm{Bin}-3$ models are provided in Appendix IV to this report. In each case these impact reviews find that there is no impact of the model validation review findings, on the conclusions of the TSPA-SR (i.e. calculated annual dose; CRWMS M\&O 2000bm, Section 6.1).

The most frequent explanation for no impact is that these models are bounding, or conservative with respect to the effects of model uncertainty on the calculated annual dose in TSPA-SR. (Conservatism is not a compliant grounds for model validation according to AP-3.10Q Section 5.3.) Another type of explanation is based on the potential impact of the model on TSPA-SR dose calculations. For example, impact reviews for the cladding models (Appendix IV) point out that a sensitivity study for TSPA-SR minimized the performance of cladding, and showed that the impact of uncertainty in cladding models on calculated annual dose is minor.

Yet another type of explanation is based on differing technical opinions. For example, for the TSPA-SR Model (Section 6.21.1) and WAPDEG Model (Section 6.10.8) there are disagreements between reviewers and the responsible Project staff as to whether the available validating information provides sufficient confidence for the intended uses of the models (i.e., Bin 2 or Bin 3 ). The impact reviews for these models reiterate how the available information does support sufficient confidence in the model results. It is anticipated that the potential for such disagreements in the future will decrease through revision and enhancement of the documentation.

In summary, for all 34 models assigned to Bin 3, however, the impact reviews provide a sound, documented basis for a determination that the conclusions of TSPA-SR (i.e. calculated annual dose; CRWMS M\&O 2000bm, Section 6.1) are not significantly impacted by the findings of this review.

\subsection{Summary of Binning Results}

The 125 AMRs and supplementary materials reviewed for this activity (as discussed in Section 4) yielded 128 models (Table 6). Of these 128 models, 17 models (13\%) were assigned to Bin 1 signifying that model validation was achieved in compliance with AP-3.10Q (Table 6). An additional 77 models $(60 \%)$ were assigned to Bin 2, meaning that sufficient information exists in all available sources to achieve compliant model validation (i.e. these are documentation problems). The remaining 34 models $(27 \%)$ were assigned to Bin 3 indicating that additional work (i.e. model development, testing, data collection) is needed to achieve model validation in compliance with the procedure, in support of licensing activities. The Bin-3 models are listed separately in Table 7.

The review findings combined assessments of procedural compliance, with expert opinions concerning the suitability of the validation arguments and the availability of additional supporting information. Procedural compliance was relied upon to discern Bin-1 models from noncompliant ones. The reviewers often expressed the need for better documentation of models for which they were in basic agreement, and for which validating information is available. This helps to explain the relatively large population of models in Bin 2 . The need for additional documentation is attributable to the need for better communication to an external audience, and 
limitations on the technical information available and known to the authors when the reports were prepared.

The basis for assignment to Bins 2 and 3 varied somewhat among the reviewers according to their individual technical understanding of the models and the intended uses. Neither Bin 2 or Bin 3 are in compliance with $\mathrm{AP}-3.10 \mathrm{Q}$, so the binning decision is based mostly on expert opinion as to the suitability of available information from all sources, to support the models for their intended uses. This situation is expected where expert opinion is used to represent confidence in technical work reviewed in detail.

In the process of identifying models, this MVSR identified 33 analyses and calculations defined according to AP-3.10Q and AP-3.12Q, respectively, which were not documented as models but should be, with model validation appropriate for the intended use. These products are listed in Table 8. They are products which are indicated in Table 1 as analyses, calculations, or technical reports, but which constitute the principal documentation for models listed in Table 6. The criteria used by the MVSR reviewers to judge when model documentation is required are discussed in Section 3.4.

In addition, 13 AMRs were identified which had been designated as models, but which are used in such a way that validation is not required. These products are listed in Table 9. They are products which are indicated in Table 1 as models, but which are not the basis for models listed in Table 6. For example, the three reports that support the Integrated Site Model (Table 1, Model Area T) were designated by the authors as models, but considered to be analyses by this review. The MVSR technical specialists recommend that these products be reconsidered as analyses or calculations which have no need for model validation. 
INTENTIONALLY LEFT BLANK 


\section{References}

\subsection{Documents Cited}

Ahlers, C.F.; Finsterle, S.; and Bodvarsson, G.S. 1999. "Characterization and Prediction of Subsurface Pneumatic Response at Yucca Mountain, Nevada." Journal of Contaminant Hydrology, 38, (1-3), 47-68. New York, New York: Elsevier. TIC: 244160.

Andresen, P.L. 1999. Interim Report to TRW, "Stress Corrosion Crack Growth Measurements in Environments Relevant to High Level Nuclear Waste Packages", September 1999. [Schenectady, New York]: General Electric Corporate Research \& Development Center. ACC: MOL.20000316.0182.

Ang A.H-S. and Tang W.H. 1984. Probability Concepts in Engineering Planning and Design, Volume II: Design, Risk and Probability. New York, New York: John Wiley \& Sons. Copyright Requested Library Tracking Number-250800.

Anspaugh, L.R.; Shinn, J.H.; Phelps, P.L.; and Kennedy, N.C. 1975. "Resuspension and Redistribution of Plutonium in Soils." Health Physics, 29, (4), 571-582. New York, New York: Pergamon Press. TIC: 248619.

Baes, C.F., III and Sharp, R.D. 1983. "A Proposal for Estimation of Soil Leaching and Leaching Constants for Use in Assessment Models." Journal of Environmental Quality, 12, (1), 17-28. Madison, Wisconsin: American Society of Agronomy. TIC: 245676.

Bandurraga, T.M. and Bodvarsson, G.S. 1999. "Calibrating Hydrogeologic Parameters for the 3D Site-Scale Unsaturated Zone Model of Yucca Mountain, Nevada.." Journal of Contaminant Hydrology, 38, (1-3), 25-46. New York, New York: Elsevier. TIC: 244160.

Bogen, K.T. and Spear, R.C. 1987. "Integrating Uncertainty and Interindividual Variability in Environmental Risk Assessment." Risk Analysis, 7, (4), 427-436. New York, New York: Plenum Press. TIC: 245810.

Bogen, K.T. 1995. "Methods to Approximate Joint Uncertainty and Variability in Risk." Risk Analysis, 15, (3), 411-419. [New York, New York: Plenum Press]. TIC: 250653.

Bogen, K.T.; Conrado, C.L.; and Robison, W.L. 1997. "Uncertainty and Variability in Updated Estimates of Potential Dose and Risk at a U.S. Nuclear Test Site-Bikini Atoll." Health Physics, 73, (1), 115-126. [New York, New York: Pergamon Press]. TIC: 250654.

Brandsdottir, B. and Einarsson, P. 1979. "Seismic Activity Associated with the September 1977 Deflation of the Krafla Central Volcano in North-Eastern Iceland." Journal of Volcanology and Geothermal Research, 6, 197-212. Amsterdam, The Netherlands: Elsevier. TIC: 250651.

Brekke, T.L.; Cording, E.J.; Daemen, J.; Hart, R.D.; Hudson, J.A.; Kaiser, P.K.; and Pelizza, S. 1999. Panel Report on the Drift Stability Workshop, Las Vegas, Nevada, December 9-11, 1998. Las Vegas, Nevada: Management and Technical Support Services. ACC: MOL.19990331.0102.

BSC (Bechtel SAIC Company) 2001a. Drift-Scale Coupled Processes (DST and THC Seepage) Models. MDL-NBS-HS-000001 REV 01 ICN 01. Las Vegas, Nevada: Bechtel SAIC Company. ACC: MOL.20010418.0010. 
BSC (Bechtel SAIC Company) 2001b. Features, Events, and Processes in UZ Flow and Transport. ANL-NBS-MD-000001 REV 01. Las Vegas, Nevada: Bechtel SAIC Company. ACC: MOL.20010423.0321.

BSC (Bechtel SAIC Company) 2001c. Flow of Water and Pooling in a Waste Package. ANLEBS-MD-000055 REV 00. Las Vegas, Nevada: Bechtel SAIC Company. ACC: MOL.20010604.0218.

BSC (Bechtel SAIC Company) 2001d. FY01 Supplemental Science and Performance Analyses, Volume 1: Scientific Bases and Analyses. TDR-MGR-MD-000007 REV 00. Las Vegas, Nevada: Bechtel SAIC Company. ACC: MOL.20010712.0062.

BSC (Bechtel SAIC Company) 2001e. Geologic Framework Model Analysis Model Report. MDL-NBS-GS-000002 REV 00 ICN 02. Las Vegas, Nevada: Bechtel SAIC Company. ACC: MOL.20010313.0505.

BSC (Bechtel SAIC Company) 2001f. Ground Control for Emplacement Drifts for SR. ANLEBS-GE-000002 REV 00 ICN 01. Las Vegas, Nevada: Bechtel SAIC Company. ACC: MOL. 20010627.0028.

BSC (Bechtel SAIC Company) 2001g. Coupled Thermal-Hydrologic-Mechanical Effects on Permeability Analysis and Models Report. ANL-NBS-HS-000037 REV 00. Las Vegas, Nevada: Bechtel SAIC Company. ACC: MOL.20010822.0092.

BSC (Bechtel SAIC Company) 2001h. In-Package Chemistry Abstraction. ANL-EBS-MD000037 REV 01. Las Vegas, Nevada: Bechtel SAIC Company. ACC: MOL.20010315.0053.

BSC (Bechtel SAIC Company) 2001i. Inventory Abstraction. ANL-WIS-MD-000006 REV 00 ICN 02. Las Vegas, Nevada: Bechtel SAIC Company. ACC: MOL.20010416.0088.

BSC (Bechtel SAIC Company) 2001j. Technical Work Plan for Model Validation Status Review. TWP-WIS-MD-000006 REV 00. Las Vegas, Nevada: CRWMS M\&O. ACC: MOL.20010718.0226.

BSC (Bechtel SAIC Company) 2001k. FY01 Supplemental Science and Performance Analyses, Volume 2: Performance Analyses. TDR-MGR-PA-000001 REV 00. Las Vegas, Nevada: Bechtel SAIC Company. ACC: MOL.20010724.0110.

BSC (Bechtel SAIC Company) 2001l. Training Lesson Plan, Introduction to Quality Assurance, Course Number LPGET00-013, Revision 1, Dated 3/9/01. Las Vegas, Nevada: Bechtel SAIC Company. ACC: MOL.20011002.0001.

BSC (Bechtel SAIC Company) 2001m. Training Lesson Plan, Technical Specialist/Reviewer Briefing, Course Number BRETEC01-030, Revision 0, Dated 6/20/01. Las Vegas, Nevada: Bechtel SAIC Company. ACC: MOL.20011002.0002.

BSC (Bechtel SAIC Company) 2001n. DSNF and Other Waste Form Degradation Abstraction. ANL-WIS-MD-000004 REV 01 ICN 01. Las Vegas, Nevada: Bechtel SAIC Company. ACC: MOL.20010316.0002.

BSC (Bechtel SAIC Company) 20010. Analysis of Geochemical Data for the Unsaturated Zone. ANL-NBS-HS-000017 REV 00 ICN 01. Las Vegas, Nevada: Bechtel SAIC Company. ACC: MOL.20010405.0013. 
BSC (Bechtel SAIC Company) 2001p. Committed Materials in Repository Drifts. CAL-GCSGE-000002 REV 00. Las Vegas, Nevada: Bechtel SAIC Company. ACC: MOL.20010412.0157.

BSC (Bechtel SAIC Company) 2001q. Evaluation of the Applicability of Biosphere-Related Features, Events, and Processes (FEP). ANL-MGR-MD-000011 REV 01. Las Vegas, Nevada: Bechtel SAIC Company. ACC: MOL.20010226.0003.

BSC (Bechtel SAIC Company) 2001r. In-Drift Thermal-Hydrological-Chemical Model. ANLEBS-MD-000026 REV 00 ICN 02. Las Vegas, Nevada: Bechtel SAIC Company. ACC: MOL.20010406.0012.

Budnitz, B.; Ewing, R.C.; Moeller, D.W.; Payer, J.; Whipple, C.; and Witherspoon, P.A. 1999. Peer Review of the Total System Performance Assessment-Viability Assessment Final Report. Las Vegas, Nevada: Total System Performance Assessment Peer Review Panel. ACC: MOL.19990317.0328.

Chandler, N.; Davison, C.C.; Gee, G.; LaPointe, P.; and Neuman, S. 1999. Yucca Mountain Project, A Consensus Peer Review of Predictions of Seepage into the Drifts of a Proposed Repository at Yucca Mountain. Las Vegas, Nevada: Drift Seepage Peer Review Panel. ACC: MOL. 20000303.1378.

Chung, H.M. and Yaggee, F.L. 1984. Characteristics of Brittle-Type Fracture of High-Burnup Fuel Cladding Under Internal Gas-Pressurization Loading. Volume 1 of Materials Science and Technology Division Light-Water-Reactor Safety Research Program: Quarterly Progress Report January-March 1983. NUREG/CR-3689. Washington, D.C.: U.S. Nuclear Regulatory Commission. TIC: 246236.

Clark, R.W. 2001. "Issuance of Corrective Action Request (CAR) BSC-01-C-001 Resulting from the Office of Quality Assurance (OQA) Suspect Trend Investigation Report (STIR) BSC-01004." Letter from R.W. Clark (DOE) to K.G. Hess (BSC), May 3, 2001, OQA:JB-1125, with enclosure. ACC: MOL.20010620.0253.

Conca, J.L. and Wright, J. 1992. "Diffusion and Flow in Gravel, Soil, and Whole Rock." Applied Hydrogeology, 1, 5-24. Hanover, Germany: Verlag Heinz Heise GmbH. TIC: 224081.

Cornell, V. 2001. "Meeting Summary - NRC/DOE TSPAI Technical Exchange, August 6-10, 2001." E-mail from V. Cornell to A. Gil, August 15, 2001, with attachment. ACC: MOL.20010921.0120; MOL.20010921.0121.

Crouch, S.L. and Starfield, A.M. 1983. Boundary Element Methods in Solid Mechanics, with Applications in Rock Mechanics and Geological Engineering. Boston, Massachusetts: Allen \& Unwin. TIC: 4370 .

CRWMS M\&O 1996. Probabilistic Volcanic Hazard Analysis for Yucca Mountain, Nevada. BA0000000-01717-2200-00082 REV 0. Las Vegas, Nevada: CRWMS M\&O. ACC: MOL. 19971201.0221.

CRWMS M\&O 1999a. Breakage of Commercial Spent Nuclear Fuel Cladding by Mechanical Loading. CAL-EBS-MD-000001 REV 00. Las Vegas, Nevada: CRWMS M\&O. ACC: MOL.19991213.0237. 
CRWMS M\&O 1999b. Dose Conversion Factor Analysis: Evaluation of GENII-S Dose Assessment Methods. ANL-MGR-MD-000002 REV 00. Las Vegas, Nevada: CRWMS M\&O. ACC: MOL.19991207.0215.

CRWMS M\&O 1999c. Not Used.

CRWMS M\&O 1999d. Seismic Ground Motion Hazard Inputs. Input Transmittal WP-NEP99309. Ta. Las Vegas, Nevada: CRWMS M\&O. ACC: MOL. 19991019.0379.

CRWMS M\&O 1999e. Seismic Ground Motion Hazard Inputs. Input Transmittal WP-NEP99309.T. Las Vegas, Nevada: CRWMS M\&O. ACC: MOL.19991005.0147.

CRWMS M\&O 1999f. Thermal Evaluation of Breached 21-PWR Waste Packages. CAL-UDCME-000002 REV 00. Las Vegas, Nevada: CRWMS M\&O. ACC: MOL.20000120.0447.

CRWMS M\&O 1999g. In Drift Corrosion Products. ANL-EBS-MD-000041 REV 00. Las Vegas, Nevada: CRWMS M\&O. ACC: MOL.20000106.0438.

CRWMS M\&O 1999h. Recharge and Lateral Groundwater Flow Boundary Conditions for the Saturated Zone Site-Scale Flow and Transport Model. ANL-NBS-MD-000010 REV 00. Las Vegas, Nevada: CRWMS M\&O. ACC: MOL.19991118.0188.

CRWMS M\&O 2000a. Abstraction of Drift-Scale Coupled Processes. ANL-NBS-HS-000029 REV 00. Las Vegas, Nevada: CRWMS M\&O. ACC: MOL.20000525.0371.

CRWMS M\&O 2000b. Abstraction of Models for Pitting and Crevice Corrosion of Drip Shield and Waste Package Outer Barrier. ANL-EBS-PA-000003 REV 00. Las Vegas, Nevada: CRWMS M\&O. ACC: MOL.20000526.0327.

CRWMS M\&O 2000c. Abstraction of Models of Stress Corrosion Cracking of Drip Shield and Waste Package Outer Barrier and Hydrogen Induced Corrosion of Drip Shield. ANL-EBS-PA000004 REV 00 ICN 01. Las Vegas, Nevada: CRWMS M\&O. ACC: MOL.20001213.0065.

CRWMS M\&O 2000d. Aging and Phase Stability of Waste Package Outer Barrier. ANL-EBSMD-000002 REV 00. Las Vegas, Nevada: CRWMS M\&O. ACC: MOL.20000410.0407.

CRWMS M\&O 2000e. Analysis of Infiltration Uncertainty. ANL-NBS-HS-000027 REV 00. Las Vegas, Nevada: CRWMS M\&O. ACC: MOL.20000525.0377.

CRWMS M\&O 2000f. Calculation of General Corrosion Rate of Drip Shield and Waste Package Outer Barrier to Support WAPDEG Analysis. CAL-EBS-PA-000002 REV 01. Las Vegas, Nevada: CRWMS M\&O. ACC: MOL.20001024.0075.

CRWMS M\&O 2000g. Calculation of Permeability Change Due to Coupled ThermalHydrological-Mechanical Effects. CAL-NBS-MD-000002 REV 00. Las Vegas, Nevada: CRWMS M\&O. ACC: MOL.20000711.0192.

CRWMS M\&O 2000h. Calculation of Probability and Size of Defect Flaws in Waste Package Closure Welds to Support WAPDEG Analysis. CAL-EBS-PA-000003 REV 00. Las Vegas, Nevada: CRWMS M\&O. ACC: MOL.20000424.0676.

CRWMS M\&O 2000i. Calibrated Properties Model. MDL-NBS-HS-000003 REV 00. Las Vegas, Nevada: CRWMS M\&O. ACC: MOL.19990721.0520.

CRWMS M\&O 2000j. Calibration of the Site-Scale Saturated Zone Flow Model. MDL-NBSHS-000011 REV 00. Las Vegas, Nevada: CRWMS M\&O. ACC: MOL.20000825.0122. 
CRWMS M\&O 2000k. Characterize Eruptive Processes at Yucca Mountain, Nevada. ANLMGR-GS-000002 REV 00. Las Vegas, Nevada: CRWMS M\&O. ACC: MOL.20000517.0259.

CRWMS M\&O 20001. Characterize Framework for Igneous Activity at Yucca Mountain, Nevada. ANL-MGR-GS-000001 REV 00 ICN 01. Las Vegas, Nevada: CRWMS M\&O. ACC: MOL.20001221.0001.

CRWMS M\&O 2000m. Characterize Framework for Seismicity and Structural Deformation at Yucca Mountain, Nevada. ANL-CRW-GS-000003 REV 00. Las Vegas, Nevada: CRWMS M\&O. ACC: MOL.20000510.0175.

CRWMS M\&O 2000n. Clad Degradation - Dry Unzipping. ANL-EBS-MD-000013 REV 00. Las Vegas, Nevada: CRWMS M\&O. ACC: MOL.20000503.0200.

CRWMS M\&O 2000o. Clad Degradation-Local Corrosion of Zirconium and Its Alloys Under Repository Conditions. ANL-EBS-MD-000012 REV 00. Las Vegas, Nevada: CRWMS M\&O. ACC: MOL.20000405.0479.

CRWMS M\&O 2000p. Clad Degradation-Wet Unzipping. ANL-EBS-MD-000014 REV 00. Las Vegas, Nevada: CRWMS M\&O. ACC: MOL.20000502.0398.

CRWMS M\&O 2000q. Conceptual and Numerical Models for UZ Flow and Transport. MDLNBS-HS-000005 REV 00. Las Vegas, Nevada: CRWMS M\&O. ACC: MOL.19990721.0526.

CRWMS M\&O 2000r. CSNF Waste Form Degradation: Summary Abstraction. ANL-EBS-MD000015 REV 00. Las Vegas, Nevada: CRWMS M\&O. ACC: MOL.20000121.0161.

CRWMS M\&O 2000s. Degradation of Stainless Steel Structural Material. ANL-EBS-MD000007 REV 00. Las Vegas, Nevada: CRWMS M\&O. ACC: MOL.20000329.1188.

CRWMS M\&O 2000t. Development of Numerical Grids for UZ Flow and Transport Modeling. ANL-NBS-HS-000015 REV 00. Las Vegas, Nevada: CRWMS M\&O. ACC: MOL. 19990721.0517.

CRWMS M\&O 2000u. Dike Propagation Near Drifts. ANL-WIS-MD-000015 REV 00 ICN 1. Las Vegas, Nevada: CRWMS M\&O. ACC: MOL.20001213.0061.

CRWMS M\&O 2000v. Not Used.

CRWMS M\&O 2000w. Disruptive Events Process Model Report. TDR-NBS-MD-000002 REV 00 ICN 02. Las Vegas, Nevada: CRWMS M\&O. ACC: MOL.20001220.0047.

CRWMS M\&O 2000x. Drift Degradation Analysis. ANL-EBS-MD-000027 REV 01. Las Vegas, Nevada: CRWMS M\&O. ACC: MOL.20001206.0006.

CRWMS M\&O 2000y. Not Used.

CRWMS M\&O 2000z. EBS Radionuclide Transport Abstraction. ANL-WIS-PA-000001 REV 00 ICN 02. Las Vegas, Nevada: CRWMS M\&O. ACC: MOL.20001204.0029.

CRWMS M\&O 2000aa. EBS Radionuclide Transport Model. ANL-EBS-MD-000034 REV 00 ICN 01. Las Vegas, Nevada: CRWMS M\&O. ACC: MOL.20000727.0091.

CRWMS M\&O 2000ab. Engineered Barrier System: Physical and Chemical Environment Model. ANL-EBS-MD-000033 REV 01. Las Vegas, Nevada: CRWMS M\&O. ACC: MOL.20001228.0081. 
CRWMS M\&O 2000ac. Environment on the Surfaces of the Drip Shield and Waste Package Outer Barrier. ANL-EBS-MD-000001 REV 00 ICN 01. Las Vegas, Nevada: CRWMS M\&O. ACC: MOL.20001219.0080.

CRWMS M\&O 2000ad. Features, Events, and Processes: Disruptive Events. ANL-WIS-MD000005 REV 00 ICN 1. Las Vegas, Nevada: CRWMS M\&O. ACC: MOL.20001218.0007.

CRWMS M\&O 2000ae. Fracture Geometry Analysis for the Stratigraphic Units of the Repository Host Horizon. ANL-EBS-GE-000006 REV 00. Las Vegas, Nevada: CRWMS M\&O. ACC: MOL.20000918.0286.

CRWMS M\&O 2000af. General Corrosion and Localized Corrosion of the Drip Shield. ANLEBS-MD-000004 REV 00. Las Vegas, Nevada: CRWMS M\&O. ACC: MOL.20000329.1185.

CRWMS M\&O 2000ag. General Corrosion and Localized Corrosion of Waste Package Outer Barrier. ANL-EBS-MD-000003 REV 00. Las Vegas, Nevada: CRWMS M\&O. ACC: MOL.20000202.0172.

CRWMS M\&O 2000ah. Hydrogen Induced Cracking of Drip Shield. ANL-EBS-MD-000006 REV 00 ICN 01. Las Vegas, Nevada: CRWMS M\&O. ACC: MOL.20001025.0100.

CRWMS M\&O 2000ai. Identification of Ingestion Exposure Parameters. ANL-MGR-MD000006 REV 00. Las Vegas, Nevada: CRWMS M\&O. ACC: MOL.20000216.0104.

CRWMS M\&O 2000aj. Igneous Consequence Modeling for the TSPA-SR. ANL-WIS-MD000017 REV 00 ICN 01. Las Vegas, Nevada: CRWMS M\&O. ACC: MOL.20001204.0022.

CRWMS M\&O 2000ak. Incorporation of Uncertainty and Variability of Drip Shield and Waste Package Degradation in WAPDEG Analysis. ANL-EBS-MD-000036 REV 00. Las Vegas, Nevada: CRWMS M\&O. ACC: MOL.20000526.0333.

CRWMS M\&O 2000al. In-Drift Colloids and Concentration. ANL-EBS-MD-000042 REV 00. Las Vegas, Nevada: CRWMS M\&O. ACC: MOL.20000509.0242.

CRWMS M\&O 2000am. In-Drift Gas Flux and Composition. ANL-EBS-MD-000040 REV 00. Las Vegas, Nevada: CRWMS M\&O. ACC: MOL.20000523.0154.

CRWMS M\&O 2000an. In-Drift Microbial Communities. ANL-EBS-MD-000038 REV 00 ICN 01. Las Vegas, Nevada: CRWMS M\&O. ACC: MOL.20001213.0066.

CRWMS M\&O 2000ao. Initial Cladding Condition. ANL-EBS-MD-000048 REV 00 ICN 01. Las Vegas, Nevada: CRWMS M\&O. ACC: MOL.20001002.0145.

CRWMS M\&O 2000ap. In-Package Source Term Abstraction. ANL-WIS-MD-000018 REV 00. Las Vegas, Nevada: CRWMS M\&O. ACC: MOL.20001219.0106.

CRWMS M\&O 2000aq. Input and Results of the Base Case Saturated Zone Flow and Transport Model for TSPA. ANL-NBS-HS-000030 REV 00. Las Vegas, Nevada: CRWMS M\&O. ACC: MOL.20000526.0330.

CRWMS M\&O 2000ar. Input Parameter Values for External and Inhalation Radiation Exposure Analysis. ANL-MGR-MD-000001 REV 01 ICN 00. Las Vegas, Nevada: CRWMS M\&O. ACC: MOL. 20001122.0005.

CRWMS M\&O 2000as. Invert Diffusion Properties Model. ANL-EBS-MD-000031 REV 01. Las Vegas, Nevada: CRWMS M\&O. ACC: MOL.20000912.0208. 
CRWMS M\&O 2000at. Mineralogical Model (MM3.0). MDL-NBS-GS-000003 REV 00 ICN 01. Las Vegas, Nevada: CRWMS M\&O. ACC: MOL.20000120.0477.

CRWMS M\&O 2000au. Modeling Sub Gridblock Scale Dispersion in Three-Dimensional Heterogeneous Fractured Media (S0015). ANL-NBS-HS-000022 REV 00 ICN 01. Las Vegas, Nevada: CRWMS M\&O. ACC: MOL.20001107.0376.

CRWMS M\&O 2000av. Mountain-Scale Coupled Processes (TH) Models. MDL-NBS-HS000007 REV 00. Las Vegas, Nevada: CRWMS M\&O. ACC: MOL.19990721.0528.

CRWMS M\&O 2000aw. Multiscale Thermohydrologic Model. ANL-EBS-MD-000049 REV 00 ICN 01. Las Vegas, Nevada: CRWMS M\&O. ACC: MOL.20001208.0062.

CRWMS M\&O 2000ax. Number of Waste Packages Hit by Igneous Intrusion. CAL-WIS-PA000001 REV 01. Las Vegas, Nevada: CRWMS M\&O. ACC: MOL.20001220.0041.

CRWMS M\&O 2000ay. Particle Tracking Model and Abstraction of Transport Processes. ANL-NBS-HS-000026 REV 00. Las Vegas, Nevada: CRWMS M\&O. ACC: MOL.20000502.0237.

CRWMS M\&O 2000az. Physical and Chemical Environmental Abstraction Model. ANL-EBSMD-000046 REV 00 ICN 01. Las Vegas, Nevada: CRWMS M\&O. ACC: MOL.20001204.0023.

CRWMS M\&O 2000ba. Radionuclide Transport Models Under Ambient Conditions. MDLNBS-HS-000008 REV 00. Las Vegas, Nevada: CRWMS M\&O. ACC: MOL.19990721.0529.

CRWMS M\&O 2000bb. Rock Properties Model Analysis Model Report. MDL-NBS-GS-000004 REV 00 ICN 02. Las Vegas, Nevada: CRWMS M\&O. ACC: MOL.20010216.0001.

CRWMS M\&O 2000bc. Saturated Zone Colloid-Facilitated Transport. ANL-NBS-HS-000031 REV 00. Las Vegas, Nevada: CRWMS M\&O. ACC: $\underline{\text { MOL.20000609.0266. }}$

CRWMS M\&O 2000bd. Secondary Uranium-Phase Paragenesis and Incorporation of Radionuclides into Secondary Phases. ANL-EBS-MD-000019 REV 00. Las Vegas, Nevada: CRWMS M\&O. ACC: MOL.20000414.0644.

CRWMS M\&O 2000be. Seepage Model for PA Including Drift Collapse. MDL-NBS-HS000002 REV 01. Las Vegas, Nevada: CRWMS M\&O. ACC: MOL.20010221.0147.

CRWMS M\&O 2000bf. Seepage/Cement Interactions. ANL-EBS-MD-000043 REV 00. Las Vegas, Nevada: CRWMS M\&O. ACC: MOL.20000317.0262.

CRWMS M\&O 2000bg. Seepage/Invert Interactions. ANL-EBS-MD-000044 REV 00. Las Vegas, Nevada: CRWMS M\&O. ACC: MOL.20000523.0156.

CRWMS M\&O 2000bh. Stainless Steel in Waste Packages for TSPA-SR. CAL-WIS-MD-000010 REV 00. Las Vegas, Nevada: CRWMS M\&O. ACC: MOL.20000630.0249.

CRWMS M\&O 2000bi. Stress Corrosion Cracking of the Drip Shield, the Waste Package Outer Barrier, and the Stainless Steel Structural Material. ANL-EBS-MD-000005 REV 00 ICN 01. Las Vegas, Nevada: CRWMS M\&O. ACC: MOL.20001102.0340.

CRWMS M\&O 2000bj. Summary of In-Package Chemistry for Waste Forms. ANL-EBS-MD000050 REV 00. Las Vegas, Nevada: CRWMS M\&O. ACC: MOL.20000217.0217. 
CRWMS M\&O 2000bk. Thermal Tests Thermal-Hydrological Analyses/Model Report. ANLNBS-TH-000001 REV 00 ICN 01. Las Vegas, Nevada: CRWMS M\&O. ACC: MOL.20010109.0004.

CRWMS M\&O 2000bl. Total System Performance Assessment (TSPA) Model for Site Recommendation. MDL-WIS-PA-000002 REV 00. Las Vegas, Nevada: CRWMS M\&O. ACC: MOL.20001226.0003.

CRWMS M\&O 2000bm. Total System Performance Assessment for the Site Recommendation. TDR-WIS-PA-000001 REV 00 ICN 01. Las Vegas, Nevada: CRWMS M\&O. ACC: MOL. 20001220.0045.

CRWMS M\&O 2000bn. Transfer Coefficient Analysis. ANL-MGR-MD-000008 REV 00 ICN 02. Las Vegas, Nevada: CRWMS M\&O. ACC: MOL.20001016.0005.

CRWMS M\&O 2000bo. Uncertainty Distribution for Stochastic Parameters. ANL-NBS-MD000011 REV 00. Las Vegas, Nevada: CRWMS M\&O. ACC: MOL.20000526.0328.

CRWMS M\&O 2000bp. UZ Colloid Transport Model. ANL-NBS-HS-000028 REV 00. Las Vegas, Nevada: CRWMS M\&O. ACC: MOL.20000822.0005.

CRWMS M\&O 2000bq. UZ Flow Models and Submodels. MDL-NBS-HS-000006 REV 00. Las Vegas, Nevada: CRWMS M\&O. ACC: MOL.19990721.0527.

CRWMS M\&O 2000br. WAPDEG Analysis of Waste Package and Drip Shield Degradation. ANL-EBS-PA-000001 REV 00 ICN 01. Las Vegas, Nevada: CRWMS M\&O. ACC: MOL. 20001208.0063.

CRWMS M\&O 2000bs. Waste Package Degradation Process Model Report. TDR-WIS-MD000002 REV 00 ICN 02. Las Vegas, Nevada: CRWMS M\&O. ACC: MOL.20001228.0229.

CRWMS M\&O 2000bt. Waste Package Operations Fabrication Process Report. TDR-EBS-ND000003 REV 01. Las Vegas, Nevada: CRWMS M\&O. ACC: MOL.20000927.0002.

CRWMS M\&O 2000bu. Water Diversion Model. ANL-EBS-MD-000028 REV 00. Las Vegas, Nevada: CRWMS M\&O. ACC: MOL.20000107.0329.

CRWMS M\&O 2000bv. Water Drainage Model. ANL-EBS-MD-000029 REV 00 ICN 01. Las Vegas, Nevada: CRWMS M\&O. ACC: MOL.20000705.0095.

CRWMS M\&O 2000bw. Rock Fall on Drip Shield. CAL-EDS-ME-000001 REV 00. Las Vegas, Nevada: CRWMS M\&O. ACC: MOL.20000509.0276.

CRWMS M\&O 2000bx. Ventilation Model. ANL-EBS-MD-000030 REV 00. Las Vegas, Nevada: CRWMS M\&O. ACC: MOL.20000107.0330.

CRWMS M\&O 2000by. Analysis of Hydrologic Properties Data. ANL-NBS-HS-000002 REV 00. Las Vegas, Nevada: CRWMS M\&O. ACC: MOL.19990721.0519.

CRWMS M\&O 2000bz. Natural Analogs for the Unsaturated Zone. ANL-NBS-HS-000007 REV 00. Las Vegas, Nevada: CRWMS M\&O. ACC: MOL.19990721.0524.

CRWMS M\&O 2000ca. In Situ Field Testing of Processes. ANL-NBS-HS-000005 REV 00. Las Vegas, Nevada: CRWMS M\&O. ACC: MOL.20000504.0304. 
CRWMS M\&O 2000cb. Seepage/Backfill Interactions. ANL-EBS-MD-000039 REV 00. Las Vegas, Nevada: CRWMS M\&O. ACC: MOL.20000509.0243.

CRWMS M\&O 2000cc. Analysis of Mechanisms for Early Waste Package Failure. ANL-EBSMD-000023 REV 02. Las Vegas, Nevada: CRWMS M\&O. ACC: MOL.20001011.0196.

CRWMS M\&O 2000cd. Clad Degradation - FEPs Screening Arguments. ANL-WIS-MD000008 REV 00 ICN 01. Las Vegas, Nevada: CRWMS M\&O. ACC: MOL.20001208.0061.

CRWMS M\&O 2000ce. Analysis of Base-Case Particle Tracking Results of the Base-Case Flow Fields (ID: U0160). ANL-NBS-HS-000024 REV 00. Las Vegas, Nevada: CRWMS M\&O. ACC: MOL.20000207.0690.

CRWMS M\&O 2000cf. Analysis Comparing Advective-Dispersive Transport Solution to Particle Tracking. ANL-NBS-HS-000001 REV 00. Las Vegas, Nevada: CRWMS M\&O. ACC: MOL.19990721.0518.

CRWMS M\&O 2000cg. Abstraction of Flow Fields for TSPA. ANL-NBS-HS-000023 REV 00 ICN 01. Las Vegas, Nevada: CRWMS M\&O. ACC: MOL.20001208.0060.

CRWMS M\&O 2000ch. Fault Displacement Effects on Transport in the Unsaturated Zone. ANL-NBS-HS-000020 REV 01. Las Vegas, Nevada: CRWMS M\&O. ACC: MOL.20001002.0154.

CRWMS M\&O 2000ci. Not Used.

CRWMS M\&O 2000cj. Probability Distribution for Flowing Interval Spacing. ANL-NBS-MD000003 REV 00 ICN 01. Las Vegas, Nevada: CRWMS M\&O. ACC: MOL.20001204.0034.

CRWMS M\&O 2000ck. Saturated Zone Transport Methodology and Transport Component Integration. MDL-NBS-HS-000010 REV 00. Las Vegas, Nevada: CRWMS M\&O. ACC: MOL. 20000824.0513.

CRWMS M\&O 2000cl. Groundwater Usage by the Proposed Farming Community. ANL-NBSMD-000006 REV 00. Las Vegas, Nevada: CRWMS M\&O. ACC: MOL 20000407.0785.

CRWMS M\&O $2000 \mathrm{~cm}$. Non-Disruptive Event Biosphere Dose Conversion Factor Sensitivity Analysis. ANL-MGR-MD-000010 REV 00. Las Vegas, Nevada: CRWMS M\&O. ACC: MOL. 20000420.0074 .

CRWMS M\&O 2000cn. Disruptive Event Biosphere Dose Conversion Factor Sensitivity Analysis. ANL-MGR-MD-000004 REV 00. Las Vegas, Nevada: CRWMS M\&O. ACC: MOL.20000418.0826.

CRWMS M\&O 2000co. Effects of Fault Displacement on Emplacement Drifts. ANL-EBS-GE000004 REV 00 ICN 01. Las Vegas, Nevada: CRWMS M\&O. ACC: MOL.20000504.0297.

CRWMS M\&O 2000cp. Saturated Zone Flow and Transport Process Model Report. TDR-NBSHS-000001 REV 00 ICN 02. Las Vegas, Nevada: CRWMS M\&O. ACC: MOL.20001102.0067.

CRWMS M\&O 2000cq. Features, Events, and Processes: System-Level and Criticality. ANLWIS-MD-000019 REV 00. Las Vegas, Nevada: CRWMS M\&O. ACC: MOL.20010108.0012.

CRWMS M\&O 2001a. Abstraction of BDCF Distributions for Irrigation Periods. ANL-NBSMD-000007 REV 00 ICN 01. Las Vegas, Nevada: CRWMS M\&O. ACC: MOL 20010201.0027. 
CRWMS M\&O 2001b. Abstraction of Drift Seepage. ANL-NBS-MD-000005 REV 01. Las Vegas, Nevada: CRWMS M\&O. ACC: MOL.20010309.0019.

CRWMS M\&O 2001c. Abstraction of NFE Drift Thermodynamic Environment and Percolation Flux. ANL-EBS-HS-000003 REV 00 ICN 02. Las Vegas, Nevada: CRWMS M\&O. ACC: MOL.20010221.0160.

CRWMS M\&O 2001d. Clad Degradation - Summary and Abstraction. ANL-WIS-MD-000007 REV 00 ICN 01. Las Vegas, Nevada: CRWMS M\&O. ACC: MOL.20010214.0229.

CRWMS M\&O 2001e. Colloid-Associated Radionuclide Concentration Limits: ANL. ANLEBS-MD-000020 REV 00 ICN 01. Las Vegas, Nevada: CRWMS M\&O. ACC: MOL.20010216.0003.

CRWMS M\&O 2001f. Defense High Level Waste Glass Degradation. ANL-EBS-MD-000016 REV 00 ICN 01. Las Vegas, Nevada: CRWMS M\&O. ACC: MOL.20010130.0004.

CRWMS M\&O 2001g. Effective Thermal Conductivity for Drift-Scale Models Used in TSPA-SR. CAL-EBS-HS-000001 REV 00. Las Vegas, Nevada: CRWMS M\&O. ACC: MOL.20010301.0252.

CRWMS M\&O 2001h. Engineered Barrier System Features, Events, and Processes. ANL-WISPA-000002 REV 01. Las Vegas, Nevada: CRWMS M\&O. ACC: MOL 20010312.0024.

CRWMS M\&O 2001i. Evaluate Soil/Radionuclide Removal by Erosion and Leaching. ANLNBS-MD-000009 REV 00 ICN 01. Las Vegas, Nevada: CRWMS M\&O. ACC: MOL.20010214.0032.

CRWMS M\&O 2001j. Features, Events, and Processes in Thermal Hydrology and Coupled Processes. ANL-NBS-MD-000004 REV 00 ICN 01. Las Vegas, Nevada: CRWMS M\&O. ACC: MOL.20010220.0007.

CRWMS M\&O 2001k. Hydride-Related Degradation of SNF Cladding Under Repository Conditions. ANL-EBS-MD-000011 REV 00 ICN 01. Las Vegas, Nevada: CRWMS M\&O. ACC: MOL.20010117.0019.

CRWMS M\&O 2001l. In-Drift Precipitates/Salts Analysis. ANL-EBS-MD-000045 REV 00 ICN 02. Las Vegas, Nevada: CRWMS M\&O. ACC: MOL.20010220.0008.

CRWMS M\&O $2001 \mathrm{~m}$. Nominal Performance Biosphere Dose Conversion Factor Analysis. ANL-MGR-MD-000009 REV 01. Las Vegas, Nevada: CRWMS M\&O. ACC: MOL.20010123.0123.

CRWMS M\&O 2001n. Pure Phase Solubility Limits - LANL. ANL-EBS-MD-000017 REV 00 ICN 01. Las Vegas, Nevada: CRWMS M\&O. ACC: MOL.20010126.0005.

CRWMS M\&O 20010. Repository Safety Strategy: Plan to Prepare the Safety Case to Support Yucca Mountain Site Recommendation and Licensing Considerations. TDR-WIS-RL-000001 REV 04 ICN 01. Two volumes. Las Vegas, Nevada: CRWMS M\&O. ACC: MOL.20010329.0825.

CRWMS M\&O 2001p. Seepage Calibration Model and Seepage Testing Data. MDL-NBS-HS000004 REV 01. Las Vegas, Nevada: CRWMS M\&O. ACC: MOL.20010122.0093. 
CRWMS M\&O 2001q. Summary of Dissolved Concentration Limits. ANL-WIS-MD-000010 REV 01. Las Vegas, Nevada: CRWMS M\&O. ACC: MOL.20010223.0061.

CRWMS M\&O 2001r. Unsaturated Zone and Saturated Zone Transport Properties (U0100). ANL-NBS-HS-000019 REV 00 ICN 1. Las Vegas, Nevada: CRWMS M\&O. ACC: MOL.20010201.0026.

CRWMS M\&O 2001s. Waste Form Colloid-Associated Concentrations Limits: Abstraction and Summary. ANL-WIS-MD-000012 REV 00 ICN 01. Las Vegas, Nevada: CRWMS M\&O. ACC: MOL.20010130.0002.

CRWMS M\&O 2001t. Water Distribution and Removal Model. ANL-EBS-MD-000032 REV 01. Las Vegas, Nevada: CRWMS M\&O. ACC: MOL.20010214.0031.

CRWMS M\&O 2001u. Performance Assessment and Sensitivity Analysis of Disposal of Plutonium as Can-in-Canister Ceramic. ANL-WIS-PA-000003 REV 00. Las Vegas, Nevada: CRWMS M\&O. ACC: MOL.20010205.0015.

CRWMS M\&O 2001v. Not Used.

CRWMS M\&O 2001w. Precipitates/Salts Model Results for THC Abstraction. CAL-EBS-PA000008 REV 00 ICN 01. Las Vegas, Nevada: CRWMS M\&O. ACC: MOL.20010125.0231.

CRWMS M\&O 2001x. FEPs Screening of Processes and Issues in Drip Shield and Waste Package Degradation. ANL-EBS-PA-000002 REV 01. Las Vegas, Nevada: CRWMS M\&O. ACC: MOL.20010216.0004.

CRWMS M\&O 2001y. Not Used.

CRWMS M\&O 2001z. Hydrogeologic Framework Model for the Saturated-Zone Site-Scale Flow and Transport Model. ANL-NBS-HS-000033 REV 00 ICN 01. Las Vegas, Nevada: CRWMS M\&O. ACC: MOL.20010403.0147.

CRWMS M\&O 2001aa. Not Used.

CRWMS M\&O 2001ab. Geochemical and Isotopic Constraints on Groundwater Flow Directions, Mixing, and Recharge at Yucca Mountain, Nevada. ANL-NBS-HS-000021 REV 00 ICN 1. Las Vegas, Nevada: CRWMS M\&O. ACC: MOL. 20010123.0120.

CRWMS M\&O 2001ac. Features, Events, and Processes in SZ Flow and Transport. ANL-NBSMD-000002 REV 01. Las Vegas, Nevada: CRWMS M\&O. ACC: MOL.20010214.0230.

CRWMS M\&O 2001ad. Identification of the Critical Group (Consumption of Locally Produced Food and Tap Water). ANL-MGR-MD-000005 REV 01 ICN 00. Las Vegas, Nevada: CRWMS M\&O. ACC: MOL. 20010119.0109.

CRWMS M\&O 2001ae. Environmental Transport Parameter Analysis. ANL-MGR-MD-000007 REV 00 ICN 01. Las Vegas, Nevada: CRWMS M\&O. ACC: MOL.20010208.0001.

CRWMS M\&O 2001af. Distribution Fitting to the Stochastic BDCF Data. ANL-NBS-MD000008 REV 00 ICN 01. Las Vegas, Nevada: CRWMS M\&O. ACC: MOL.20010221.0148.

CRWMS M\&O 2001ag. Not Used.

CRWMS M\&O 2001ah. Disruptive Event Biosphere Dose Conversion Factor Analysis. ANLMGR-MD-000003 REV 01. Las Vegas, Nevada: CRWMS M\&O. ACC: MOL.20010125.0233. 
CRWMS M\&O 2001ai. Miscellaneous Waste-Form FEPS. ANL-WIS-MD-000009 REV 00 ICN 01. Las Vegas, Nevada: CRWMS M\&O. ACC: MOL.20010216.0006.

DOE (U.S. Department of Energy) 1998. Total System Performance Assessment. Volume 3 of Viability Assessment of a Repository at Yucca Mountain. DOE/RW-0508. Washington, D.C.: U.S. Department of Energy, Office of Civilian Radioactive Waste Management. ACC: MOL.19981007.0030.

Donahue, R.L.; Miller, R.W.; and Shickluna, J.C. 1977. Soils: An Introduction to Soils and Plant Growth. 4th Edition. Englewood Cliffs, New Jersey: Prentice Hall. TIC: 242506.

Fabryka-Martin, J.T.; Wolfsberg, A.V.; Dixon, P.R.; Levy, S.S.; Musgrave, J.A.; and Turin, H.J. 1997. Summary Report of Chlorine-36 Studies: Sampling, Analysis, and Simulation of Chlorine36 in the Exploratory Studies Facility. LA-13352-MS. Los Alamos, New Mexico: Los Alamos National Laboratory. ACC: MOL.19980812.0254.

Farmer, J.C.; McCright, R.D.; Roy, A.K.; Gdowski, G.E.; Wang, F.T.; Estill, J.C.; King, K.J.; Gordon, S.R.; Fleming, D.L.; and Lum, B.Y. 1998. "Development of Corrosion Models for High-Level Waste Containers." ICONE-6: Proceedings of the 6th International Conference on Nuclear Engineering, San Diego, California, May 10-15, 1998. 15 Pages. New York, New York: American Society of Mechanical Engineers.. TIC: $\underline{248740 .}$

Gardner, D. 2001. "Meeting Summary, NRC/DOE Technical Exchange on Repository Design and Thermal-Mechanical Effects KTI, Feburary 6-8, 2001." E-mail from D. Gardner to C. Hanlon, February 21, 2001, with attachment. ACC: MOL.20010307.0510; MOL.20010307.0511.

Golder Associates 2000. User's Guide, GoldSim, Graphical Simulation Environment. Version 6.02. Draft \#3. Redmond, Washington: Golder Associates. TIC: 247324.

GoldSim Consulting Group 2001. "Simulation-Based Decision Support." Accessed September 18, 2001. http://www.goldsim.com/software/modules2.asp\#problems.

Grisak, G.E. and Pickens, J.F. 1980. "Solute Transport Through Fractured Media, 1. The Effect of Matrix Diffusion." Water Resources Research, 16, (4), 719-730. Washington, D.C.: American Geophysical Union. TIC: 222056.

Hardin, E.L. 1998. Near-Field/Altered-Zone Models Report. UCRL-ID-129179. Livermore, California: Lawrence Livermore National Laboratory. ACC: MOL. 19980630.0560.

Hardin, E.L. 2001a. "Guidance for the Model Validation Status Review." Memorandum from E.L. Hardin (BSC) to Distribution, July 24, 2001, CSO.07/01.004. ACC: MOL.20010816.0114

Hardin, E.L. 2001. "Model Validation Status Review - Reviewer Selection, Training, Qualifications, and Instructions." Memorandum from E.L. Hardin (BSC) to M. Voegele, September 14, 2001, CSO.09/01.002, with attachment. ACC: MOL.20011026.0199.

Hardin, E.L. 2001. "Model Validation Status Review - Final Workshop Status Memo." Memo from E.L. Hardin (BSC), and G. Mann (BSC) to M. Voegele, September 24, 2001, CSO.09/01.003, with enclosure. ACC: MOL.20011026.0200. 
IAEA (International Atomic Energy Agency) 2001. An International Peer Review of the Biosphere Modelling Programme of the US Department of Energy's Yucca Mountain Site Characterization Project, Report of the IAEA International Review Team. Vienna, Austria: International Atomic Energy Agency. TIC: 250092 .

Jarzemba, M.S.; LaPlante, P.A.; and Poor, K.J. 1997. ASHPLUME Version 1.0-A Code for Contaminated Ash Dispersal and Deposition, Technical Description and User's Guide. CNWRA 97-004, Rev. 1. San Antonio, Texas: Center for Nuclear Waste Regulatory Analyses. TIC: 239303.

Jaquish, R.E. and Napier, B.A. 1987. A Comparison of Environmental Radionuclide Concentrations Calculated by a Mathematical Model with Measured Concentration. PNL-SA14720. Richland, Washington: Pacific Northwest Laboratory. TIC: 234513.

Kelkar, S. and Travis, B. 1999. Independent Test Case Report for TRACRN Version 1.0. [Los Alamos, New Mexico]: Los Alamos National Laboratory. ACC: MOL.19991221.0361.

Kelmenson, R. 2000. "Meeting Summary, NRC/DOE Technical Exchange Meeting on Container Life and Source Term (CLST), September 12-13, 2000." E-mail from R. Kelmenson to C. Hanlon (DOE/YMSCO), T. Gunter (DOE/YMSCO), A. Gil (DOE/YMP), P. Russell (DOE/YMSCO), September 27, 2000, with attachment. ACC: MOL.20001127.0049.

Krisha, D.T. 2001. "Contract No. DE-AC08-01NV12101 - Corrective Action Request (CAR) BSC-01-C-001." Letter from D.T. Krisha (BSC) to R.W. Clark (DOE/YMSCO), June 5, 2001, QA.06/01.001, with enclosures. ACC: MOL.20010705.0194.

Larsen, G.; Grönvold, K.; and Thorarinsson, S. 1979. "Volcanic Eruption Through a Geothermal Borehole at Namafjall, Iceland." Nature, 278, (5706), 707-710. [London, England]: Macmillan Journals. TIC: 250655 .

Layton, D.W. 1993. "Metabolically Consistent Breathing Rates for use in Dose Assessments." Health Physics, 64, (1), 23-36. [New York, New York: Pergamon Press]. TIC: 250652.

Maloszewski, P. and Zuber, A. 1985. "On the Theory of Tracer Experiments in Fissured Rocks with a Porous Matrix." Journal of Hydrology, 79, 333-358. Amsterdam, The Netherlands: Elsevier Science Publishers B.V.. TIC: 222390.

Maloszewski, P. and Zuber, A. 1991. "Influence of Matrix Diffusion and Exchange Reactions on Radiocarbon Ages in Fissured Carbonate Aquifers." Water Resources Research, 27, (8), 1937 1945. Washington, D.C.: American Geophysical Union. TIC: 247494.

Matsuo, Y. 1987. "Thermal Creep of Zircaloy-4 Cladding Under Internal Pressure." Journal of Nuclear Science and Technology, 24, (2), 111-119. Tokyo, Japan: Atomic Energy Society of Japan. TIC: 237137.

McCullough, B.D. and Wilson, B. 1999. "On the Accuracy of Statistical Procedures in Microsoft Excel 97." Computational Statistics \& Data Analysis, 31, ([1]), 27-37. [Amsterdam, The Netherlands: North-Holland]. TIC: 250737.

National Research Council 1994. Science and Judgment in Risk Assessment. Washington, D.C.: National Academy Press. TIC: 215158. 
Neretnieks, I. 1980. "Diffusion in the Rock Matrix: An Important Factor in Radionuclide Retardation." Journal of Geophysical Research, 85, (B8), 4379-4397. Washington, D.C.: American Geophysical Union. TIC: 221345.

NRC (U.S. Nuclear Regulatory Commission) 2000. Summary of the Resolution of the Key Technical Issue on Igneous Activity. [Presented at the DOE/NRC Technical Exchange and Management Meeting on Igneous Activity, August 29-31, 2000, Las Vegas, Nevada. Washington, D.C.: U.S. Nuclear Regulatory Commission]. ACC: MOL.20001101.0107.

Pescatore, C.; Cowgill, M.G.; and Sullivan, T.M. 1990. Zircaloy Cladding Performance Under Spent Fuel Disposal Conditions, Progress Report May 1-October 31, 1989. BNL 52235. Upton, New York: Brookhaven National Laboratory. ACC: NNA.19900710.0055.

Reamer, C.W. 2001. "Unsaturated and Saturated Flow Under Isothermal Conditions (USFIC) Key Technical Issue Agreements." Letter from C.W. Reamer (NRC) to S. Brocoum (DOE/YMSCO), June 20, 2001, with enclosure. ACC: MOL.20010913.0149.

Reamer, C.W. and Williams, D.R. 2000. Summary Highlights of NRC/DOE Technical Exchange and Management Meeting on Radionuclide Transport. Meeting held December 5-7, 2000, Berkeley, California. Washington, D.C.: U.S. Nuclear Regulatory Commission. ACC: MOL.20010117.0063.

Reamer, C.W. and Williams, D.R. 2001. Summary Highlights of NRC/DOE Technical Exchange and Management Meeting on Thermal Effects on Flow. Meeting held January 8-9, 2001, Pleasanton, California. [Washington, D.C.]: U.S. Nuclear Regulatory Commission. ACC: MOL.20010202.0095; MOL.20010202.0096; MOL.20010202.0097; MOL.20010202.0098; MOL.20010202.0099; MOL.20010202.0100; MOL.20010202.0102; MOL.2 0010202.0103; MOL.20010202.0104; MOL.20010202.0105; MOL.20010202.0106; MOL.20010202.0107; MOL. 20010202.0108 .

Reardon, E.J. 1990. "An Ion Interaction Model for the Determination of Chemical Equilibria in Cement Water Systems." Cement and Concrete Research, 20, 175-192. Elmsford, New York: Pergamon Press. TIC: 239801.

Reimus, P.W.; Adams, A.; Haga, M.J.; Humphrey, A.; Callahan, T.; Anghel, I.; and Counce, D. 1999. Results and Interpretation of Hydraulic and Tracer Testing in the Prow Pass Tuff at the CHoles. Milestone SP32E7M4. Los Alamos, New Mexico: Los Alamos National Laboratory. TIC: 246377.

Riotte, H. 2001. OECD/NEA - IAEA Joint International Peer Review of the Yucca Mountain Site Characterization Project's Total System Performance Assessment Supporting the Site Recommendation Process Letter to Lake Barrett ACC: MOL.20011113.0240.

Robinson, B.A. 1994. "A Strategy for Validating a Conceptual Model for Radionuclide Migration in the Saturated Zone Beneath Yucca Mountain." Radioactive Waste Management and Environmental Restoration, 19, (1-3), 73-96. Yverdon, Switzerland: Harwood Academic Publishers. TIC: 222513.

Robinson, B.A.; Wolfsberg, A.V.; Zyvoloski, G.A.; and Gable, C.W. 1995. An Unsaturated Zone Flow and Transport Model of Yucca Mountain. Milestone 3468. Draft. Los Alamos, New Mexico: Los Alamos National Laboratory. ACC: MOL.19960415.0218. 
Rothman, A.J. 1984. Potential Corrosion and Degradation Mechanisms of Zircaloy Cladding on Spent Nuclear Fuel in a Tuff Repository. UCDD-20172. Livermore, California: Lawrence Livermore National Laboratory. ACC: NNA.19870903.0039.

Sudicky, E.A. and Frind, E.O. 1981. "Carbon 14 Dating of Groundwater in Confined Aquifers: Implications of Aquitard Diffusion." Water Resources Research, 17, (4), 1060-1064. Washington, D.C.: American Geophysical Union. TIC: 247712.

Sudicky, E.A. and Frind, E.O. 1982. "Contaminant Transport in Fractured Porous Media: Analytical Solutions for a System of Parallel Fractures." Water Resources Research, 18, (6), 1634-1642. Washington, D.C.: American Geophysical Union. TIC: 217475.

Suzuki, T. 1983. "A Theoretical Model for Dispersion of Tephra." Arc Volcanism: Physics and Tectonics, Proceedings of a 1981 IAVCEI Symposium, August-September, 1981, Tokyo and Hakone. Shimozuru, D. and Yokoyama, I., eds. Pages 95-113. Tokyo, Japan: Terra Scientific Publishing Company. TIC: 238307.

Turcotte, D.L. and Schubert, G. 1982. Geodynamics, Applications of Continuum Physics to Geological Problems. New York, New York: John Wiley \& Sons. TIC: 235924.

USGS (U.S. Geological Survey) 2000. Future Climate Analysis. ANL-NBS-GS-000008 REV 00. Denver, Colorado: U.S. Geological Survey. ACC: MOL.20000629.0907.

USGS (U.S. Geological Survey) 2001a. Simulation of Net Infiltration for Modern and Potential Future Climates. ANL-NBS-HS-000032 REV 00 ICN 01. Denver, Colorado: U.S. Geological Survey. ACC: MOL.20010405.0002.

USGS (U.S. Geological Survey) 2001b. Water-Level Data Analysis for the Saturated Zone SiteScale Flow and Transport Model. ANL-NBS-HS-000034 REV 00 ICN 01. Denver, Colorado: U.S. Geological Survey. ACC: MOL.20010405.0211.

Van Swam, L.F.; Strasser, A.A.; Cook, J.D.; and Burger, J.M. 1997a. "Behavior of Zircaloy-4 and Zirconium Liner Zircaloy-4 Cladding at High Burnup." Proceedings of the 1997 International Topical Meeting on LWR Fuel Performance, Portland, Oregon, March 2-6, 1997. Pages 421-431. La Grange Park, Illinois: American Nuclear Society. TIC: 232556.

Van Swam, L.F.; Bain, G.M.; Dey, W.C.; Davis, D.D; and Heckermann, H. 1997b. "BWR and PWR Fuel Performance at High Burnup." Proceedings of the 1997 International Topical Meeting on LWR Fuel Performance, Portland, Oregon, March 2-6, 1997. 455-462. La Grange Park, Illinois: American Nuclear Society. TIC: 232556.

Voegele, M.D. 2001. "Assignment of Team Lead for the Model Validation Status Review." Memorandum from M.D. Voegele (BSC) to E.L. Hardin, July 16, 2001, CSO.07/01.003. ACC: MOL.20010813.0427.

Wilkins, D.R. and Heath, C.A. 1999. "Direction to Transition to Enhanced Design Alternative II." Letter from D.R. Wilkins (CRWMS M\&O) and C.A. Heath (CRWMS M\&O) to Distribution, June 15, 1999, LV.NS.JLY.06/99-026, with enclosures, "Strategy for Baselining EDA II Requirements" and "Guidelines for Implementation of EDA II." ACC: MOL.19990622.0126; MOL.19990622.0127; MOL.19990622.0128.

Williams, D. 2001. "TEF/ENFE Meeting Summary and Agreement Matrix." E-mail from D. Williams to G. Hellstrom, January 16, 2001, with attachments. ACC: MOL.20010306.0262. 
Wolfram, S. 1996. The Mathematica Book. 3rd Edition. Champaign, Illinois: Wolfram. TIC: $\underline{250735}$.

Wong, I.G. and Stepp, C. 1998. Probabilistic Seismic Hazard Analyses for Fault Displacement and Vibratory Ground Motion at Yucca Mountain, Nevada. Milestone SP32IM3, September 23, 1998. Three volumes. Oakland, California: U.S. Geological Survey. ACC: MOL.19981207.0393.

YMP (Yucca Mountain Site Characterization Project) 2001. Evaluation of Uncertainty Treatment in the Technical Documents Supporting TSPA-SR. Las Vegas, Nevada: Yucca Mountain Site Characterization Office. ACC: $\underline{\text { MOL.20010502.0084. }}$

\subsection{Codes, Standards, Regulations, and Procedures}

40 CFR 197. 2001. Protection of Environment: Public Health and Environmental Radiation Protection Standards for Yucca Mountain, Nevada. Readily available. 40CFR197.html.

64 FR 67054. Office of Civilian Radioactive Waste Management; General Guidelines for the Recommendation of Sites for Nuclear Waste Repositories; Yucca Mountain Site Suitability Guidelines. Proposed rule 10 CFR Part 963. Readily available.

66 FR 55732. Disposal of High-Level Radioactive Wastes in a Proposed Geologic Repository at Yucca Mountain, NV. Final Rule 10 CFR Part 63. Readily available.

AP-2.1Q, Rev. 2. Indoctrination and Training of Personnel. Washington, D.C.: U.S. Department of Energy, Office of Civilian Radioactive Waste Management. ACC: MOL.20010917.0186.

AP-2.2Q, Rev. 1. Establishment and Verification of Required Education and Experience of Personnel. Washington, D.C.: U.S. Department of Energy, Office of Civilian Radioactive Waste Management. ACC: MOL.20010724.0108.

AP-2.12Q, Rev. 0, ICN 3. Peer Review. [Washington, D.C.]: U.S. Department of Energy, Office of Civilian Radioactive Waste Management. ACC: MOL.20010904.0452.

AP-2.14Q, REV 2, ICN 0. Review of Technical Products and Data. [Washington, D.C.]: U.S. Department of Energy, Office of Civilian Radioactive Waste Management. ACC: MOL.20010801.0316.

AP-2.21Q, Rev. 1, ICN 0, BSCN 1. Quality Determinations and Planning for Scientific, Engineering, and Regulatory Compliance Activities. Washington, D.C.: U.S. Department of Energy, Office of Civilian Radioactive Waste Management. ACC: MOL.20010212.0018.

AP-3.10Q Rev. 02, ICN 4 ECN 1. Analyses and Models. Washington, D.C.: U.S. Department of Energy, Office of Civilian Radioactive Waste Management. ACC: MOL.20010827.0114.

AP-3.11Q, Rev. 2, ICN 0. Technical Reports. Washington, D.C.: U.S. Department of Energy, Office of Civilian Radioactive Waste Management. ACC: MOL.20010405.0010.

AP-3.12Q, Rev. 0, ICN 4. Calculations. Washington, D.C.: U.S. Department of Energy, Office of Civilian Radioactive Waste Management. ACC: MOL.20010404.0008.

AP-SV.1Q, Rev. 0, ICN 2. Control of the Electronic Management of Information. Washington, D.C.: U.S. Department of Energy, Office of Civilian Radioactive Waste Management. ACC: MOL. 20000831.0065 . 
DOE (U.S. Department of Energy) 2000. Quality Assurance Requirements and Description. DOE/RW-0333P, Rev. 10. Washington, D.C.: U.S. Department of Energy, Office of Civilian Radioactive Waste Management. ACC: MOL.20000427.0422.

Eckerman, K.F.; Wolbarst, A.B.; and Richardson, A.C.B. 1988. Limiting Values of Radionuclide Intake and Air Concentration and Dose Conversion Factors for Inhalation, Submersion, and Ingestion. EPA 520/1-88-020. Federal Guidance Report No. 11. Washington, D.C.: U.S. Environmental Protection Agency. TIC: 203350.

Eckerman, K.F. and Ryman, J.C. 1993. External Exposure to Radionuclides in Air, Water, and Soil, Exposure-to-Dose Coefficients for General Application, Based on the 1987 Federal Radiation Protection Guidance. EPA 402-R-93-081. Federal Guidance Report No. 12. Washington, D.C.: U.S. Environmental Protection Agency, Office of Radiation and Indoor Air. TIC: 225472 .

Eisenberg, N.A.; Lee, M.P.; Federline, M.V.; Wingefors, S.; Andersson, J.; Norrby, S.; Sagar, B.; and Wittmeyer, G.W. 1999. Regulatory Perspectives on Model Validation in High-Level Radioactive Waste Management Programs: A Joint NRC/SKI White Paper. NUREG-1636. Washington, D.C.: U.S. Nuclear Regulatory Commission. TIC: 246310.

ICRP (International Commission on Radiological Protection) 1979. Limits for Intakes of Radionuclides by Workers. Volume 2, No. 3/4 of Annals of the ICRP. Sowby, F.D., ed. ICRP Publication 30 Part 1. New York, New York: Pergamon Press. TIC: 4939.

NCRP (National Council on Radiation Protection and Measurements) 1999. Recommended Screening Limits for Contaminated Surface Soil and Review of Factors Relevant to Site-Specific Studies. NCRP Report No. 129. Bethesda, Maryland: National Council on Radiation Protection and Measurements. TIC: 250396.

\subsection{Source Data, Listed by Data Tracking Numbers}

GS000508312332.001. Water-Level Data Analysis for the Saturated Zone Site-Scale Flow and Transport Model. Submittal date: 06/01/2000.

LA0003AM831341.001. Probability Distributions for Sorption Coefficients (Kd's). Submittal date: $03 / 29 / 2000$.

MO0003SEPSDARS.002. Preliminary Seismic Design Acceleration Response Spectra for the Repository Level (Point B). Submittal date: 03/30/2000. Submit to RPC URN-0203

MO0006SPAPVE03.001. Preliminary Volcanic Eruption Biosphere Dose Conversion Factors. Submittal date: 06/15/2000.

SN9908T0581999.001. Recharge and Lateral Groundwater Flow Boundary Conditions for the Saturated Zone (SZ) Site-Scale Flow and Transport Model. Submittal date: 08/19/1999. 
INTENTIONALLY LEFT BLANK 
Table 1. List of Model Areas and Associated AMRs and Other Reports for Status Review

\begin{tabular}{|c|c|c|c|}
\hline $\begin{array}{l}\text { 1. Model } \\
\text { Area }\end{array}$ & 2. AMRs and Other Documents Reviewed ${ }^{A}$ & $\begin{array}{l}\text { 3. Model or } \\
\text { Analysis? }\end{array}$ & $\begin{array}{l}\text { 4. \# Unique } \\
\text { Reports }\end{array}$ \\
\hline A. Climate & A.1 Future Climate Analysis (ANL-NBS-GS-000008 Rev. 00; USGS 2000) & A & 1 Unique AMR \\
\hline B. Infiltration & $\begin{array}{l}\text { B.1 Simulation Net Infiltration for Modern and Potential Future Climates (ANL-NBS-HS-000032 Rev. } 00 \text { ICN 1; } \\
\text { USGS 2001a) } \\
\text { B.2 Analysis of Infiltration Uncertainty (ANL-NBS-HS-000027 Rev. 00; CRWMS M\&O 2000e) }\end{array}$ & $A+M$ & 2 Unique AMRs \\
\hline C. UZ Flow & $\begin{array}{l}\text { C. } 1 \text { UZ Flow Models and Submodels (MDL-NBS-HS-000006 Rev. 00; CRWMS M\&O 2000bq) } \\
\text { C. } 2 \text { Calibrated Properties Model (MDL-NBS-HS-000003 Rev. 00; CRWMS M\&O 2000i) } \\
\text { C.3 Analysis of Hydrologic Properties Data (ANL-NBS-HS-000002 Rev. 00; CRWMS M\&O 2000by) } \\
\text { C. } 4 \text { Development of Numerical Grids for UZ Flow and Transport Modeling (ANL-NBS-HS-000015 Rev. 00; } \\
\quad \text { CRWMS M\&O 2000t) } \\
\text { C.5 Analysis of Geochemical Data for the UZ (ANL-NBS-HS-000017 Rev. 00 ICN 1; BSC 20010) } \\
\text { C.6 Conceptual and Numerical Models of UZ Flow and Transport (MDL-NBS-HS-000005 Rev. 00; CRWMS M\&O 2000q) } \\
\text { C.7 Natural Analogs for the UZ (ANL-NBS-HS-000007 Rev. 00; CRWMS M\&O 2000bz) } \\
\text { C. } 8 \text { Features, Events, and Processes in UZ Flow and Transport (ANL-NBS-MD-000001 Rev. 01; BSC 2001b) }\end{array}$ & $\begin{array}{l}A+M \\
A+M \\
A \\
A+M \\
A \\
M \\
A \\
A\end{array}$ & 8 Unique AMRs \\
\hline $\begin{array}{l}\text { D. Mountain- } \\
\text { Scale TH }\end{array}$ & $\begin{array}{l}\text { D. } 1 \text { Mountain-Scale Coupled Processes (TH) Models (MDL-NBS-HS-000007 Rev. 00; CRWMS M\&O 2000av) } \\
\text { C. } 8 \text { Features, Events, and Processes in UZ Flow and Transport (ANL-NBS-MD-000001 Rev. 01; BSC 2001b) }\end{array}$ & $\begin{array}{c}A+M \\
A\end{array}$ & $\begin{array}{l}1 \text { Unique AMR } \\
\text { (C. } 8 \text { is tallied } \\
\text { in Area } C \text { ) }\end{array}$ \\
\hline $\begin{array}{l}\text { E. Ambient/ } \\
\text { Thermal } \\
\text { Drift } \\
\text { Seepage }\end{array}$ & $\begin{array}{l}\text { E.1 Seepage Calibration Model and Seepage Testing Data (MDL-NBS-HS-000004 Rev. 01; CRWMS M\&O 2001p) } \\
\text { E.2 Seepage Model for PA Including Drift Collapse (MDL-NBS-HS-000002 Rev. 01; CRWMS M\&O 2000be) } \\
\text { E.3 Abstraction of Drift Seepage (ANL-NBS-MD-000005 Rev. 01; CRWMS M\&O 2001b) } \\
\text { E.4 In Situ Field Testing of Processes (ANL-NBS-HS-000005 Rev. 00; CRWMS M\&O 2000ca) } \\
\text { C.8 Features, Events, and Processes in UZ Flow and Transport (ANL-NBS-MD-000001 Rev. 01; BSC 2001b) } \\
\text { I.6 Abstraction of NFE Drift Thermodynamic Environment and Percolation Flux (ANL-EBS-HS-000003 Rev. 00 ICN2; } \\
\text { CRWMS M\&O 2001C) } \\
\text { L.1 Drift Degradation Analysis (ANL-EBS-MD-000027 Rev. 01; CRWMS M\&O 2000x) }\end{array}$ & $\begin{array}{l}M \\
M \\
A+M \\
A \\
A \\
A \\
A+M\end{array}$ & $\begin{array}{l}4 \text { Unique AMRs } \\
(C .8,1.6, \text { and } \\
L .1 \text { are tallied } \\
\text { in Areas } C, I \\
\text { and } L \\
\text { respectively) }\end{array}$ \\
\hline $\begin{array}{l}\text { F. Mountain- } \\
\text { Scale/Ne } \\
\text { ar-Field } \\
\text { THC }\end{array}$ & $\begin{array}{l}\text { F.1 Drift-Scale Coupled Processes (DST and THC Seepage) Models (MDL-NBS-HS-000001 Rev. } 01 \text { ICN 1; BSC 2001a) } \\
\text { F.2 Abstraction of Drift-Scale Coupled Processes (ANL-NBS-HS-000029 Rev. 00; CRWMS M\&O 2000a) } \\
\text { F.3 Thermal Tests Thermal/Hydrological Analyses/Model Report (ANL-NBS-TH-000001 Rev. } 00 \text { ICN 1; } \\
\text { CRWMS M\&O 2000bk) } \\
\text { I.1 Multiscale Thermohydrologic Model (ANL-EBS-MD-000049 Rev. 00 ICN 1; CRWMS M\&O 2000aW) } \\
\text { I.9 FEPs in Thermal Hydrology and Coupled Processes (ANL-NBS-MD-000004 Rev. 00 ICN 1; CRWMS M\&O 2001j) }\end{array}$ & $\begin{array}{c}A+M \\
A+M \\
M \\
A\end{array}$ & $\begin{array}{c}3 \text { Unique AMRs } \\
\text { (I.1 \& } 1.9 \\
\text { are tallied in } \\
\text { Area I) }\end{array}$ \\
\hline
\end{tabular}


Table 1. List of Model Areas and Associated AMRs and Other Reports for Status Review (continued)

\begin{tabular}{|c|c|c|c|}
\hline $\begin{array}{l}\text { 1. Model } \\
\text { Area }\end{array}$ & 2. AMRs and Other Documents Reviewed ${ }^{A}$ & \begin{tabular}{|l|} 
3. Model or \\
Analysis?
\end{tabular} & $\begin{array}{l}\text { 4. \# Unique } \\
\text { Reports }\end{array}$ \\
\hline $\begin{array}{l}\text { G. Mountain- } \\
\text { Scale/ } \\
\text { Near-Field } \\
\text { THM }\end{array}$ & $\begin{array}{l}\text { G.1 Calculation of Permeability Change Due to Coupled THM Effects (CAL-NBS-MD-000002 Rev. 00; } \\
\text { CRWMS M\&O 2000g) } \\
\text { 1.9 FEPs in Thermal Hydrology and Coupled Processes (ANL-NBS-MD-000004 Rev. } 00 \text { ICN 1; CRWMS M\&O 2001j) }\end{array}$ & \begin{tabular}{c|c} 
(calculation) \\
$A$
\end{tabular} & $\begin{array}{c}\text { No Unique } \\
\text { AMRs } \\
\text { (G.1 is a Calc. } \\
\text { Report; } 1.9 \text { is } \\
\text { tallied in Area I) }\end{array}$ \\
\hline $\begin{array}{l}\text { H. In-Drift } \\
\text { Chemistry }\end{array}$ & $\begin{array}{l}\text { H.1 In-Drift Precipitates/Salts Analysis (ANL-EBS-MD-000045 Rev. } 00 \text { ICN 2; CRWMS M\&O 2001/) } \\
\text { H. } 2 \text { Environment on the Surfaces of the Drip Shield and Waste Package Outer Barrier (ANL-EBS-MD-000001 } \\
\text { Rev. } 00 \text { ICN 1; CRWMS M\&O 2000ac) } \\
\text { H.3 EBS Physical and Chemical Environment Model (ANL-EBS-MD-000033 Rev. 01; CRWMS M\&O 2000ab) } \\
\text { H.4 In-Drift Corrosion Products (ANL-EBS-MD-000041 Rev. 00; CRWMS M\&O 1999g) } \\
\text { H.5 In-Drift Gas Flux and Composition (ANL-EBS-MD-000040 Rev. 00; CRWMS M\&O 2000am) } \\
\text { H.6 In-Drift Microbial Communities (ANL-EBS-MD-000038 Rev. 00 ICN 1; CRWMS M\&O 2000an) } \\
\text { H.7 Seepage/Cement Interactions (ANL-EBS-MD-000043 Rev. 00; CRWMS M\&O 2000bf) } \\
\text { H.8 Physical and Chemical Environmental Abstraction Model (ANL-EBS-MD-000046 Rev. } 00 \text { ICN 1; CRWMS M\&O } \\
2000 \text { az) } \\
\text { H.9 Precipitates/Salts Model Results for THC Abstraction (CAL-EBS-PA-000008 Rev. } 00 \text { ICN 1; CRWMS M\&O 2001w) } \\
\text { H.10 Seepage/Backfill Interaction (ANL-EBS-MD-000039 Rev. 00; CRWMS M\&O 2000cb) } \\
\text { L.5 EBS Features, Events, and Processes (ANL-WIS-PA-000002 Rev. 01; CRWMS M\&O 2001h) }\end{array}$ & \begin{tabular}{c|}
$A+M$ \\
$A$ \\
$M$ \\
$M$ \\
$A$ \\
$A+M$ \\
$A$ \\
$M$ \\
(calculation) \\
$A+M$ \\
$A$
\end{tabular} & $\begin{array}{l}9 \text { Unique AMRs } \\
\text { (H.9 is a Calc. } \\
\text { Report; L.5 is } \\
\text { tallied in } \\
\text { Area L) }\end{array}$ \\
\hline
\end{tabular}


Table 1. List of Model Areas and Associated AMRs and Other Reports for Status Review (continued)

\begin{tabular}{|c|c|c|c|}
\hline $\begin{array}{l}\text { 1. Model } \\
\text { Area }\end{array}$ & 2. AMRs and Other Documents Reviewed ${ }^{A}$ & $\begin{array}{l}\text { 3. Model or } \\
\text { Analysis? }\end{array}$ & $\begin{array}{l}\text { 4. \# Unique } \\
\text { Reports }\end{array}$ \\
\hline $\begin{array}{l}\text { I. EBS } \\
\text { Moisture } \\
\text { Distribu- } \\
\text { tion and } \\
\text { TH }\end{array}$ & $\begin{array}{l}\text { I.1 Multiscale Thermohydrologic Model (ANL-EBS-MD-000049 Rev. } 00 \text { ICN 1; CRWMS M\&O 2000aw) } \\
\text { I.2 Water Distribution and Removal Model (ANL-EBS-MD-000032 Rev. 01; CRWMS M\&O 2001t) } \\
\text { I.3 Water Diversion Model (ANL-EBS-MD-000028 Rev. 00; CRWMS M\&O 2000bu) } \\
\text { I.4 Water Drainage Model (ANL-EBS-MD-000029 Rev. 00 ICN 1; CRWMS M\&O 2000bv) } \\
\text { I.5 Ventilation Model (ANL-EBS-MD-000030 Rev. 00; CRWMS M\&O 2000bx) } \\
\text { I.6 Abstraction of NFE Drift Thermodynamic Environment and Percolation Flux (ANL-EBS-HS-000003 Rev. } 00 \text { ICN2; } \\
\text { CRWMS M\&O 2001c) } \\
\text { I.7 In-Drift THC Model (ANL-EBS-MD-000026 Rev. 00 ICN 2; BSC 2001r) } \\
\text { I.8 Effective Thermal Conductivity for Drift-Scale Models Used in TSPA-SR (CAL-EBS-HS-000001 Rev. 00; } \\
\text { CRWMS M\&O 2001g) } \\
\text { I.9 FEPs in Thermal Hydrology and Coupled Processes (ANL-NBS-MD-000004 Rev. 00 ICN 1; CRWMS M\&O 2001j) } \\
\text { F.3 Thermal Tests Thermal/Hydrological Analyses/Model Report (ANL-NBS-TH-000001 Rev. 00 ICN 1; } \\
\text { CRWMS M\&O 2000bk) } \\
\text { L.5 EBS Features, Events, and Processes (ANL-WIS-PA-000002 Rev. 01; CRWMS M\&O 2001h) } \\
\text { M.5 EBS Radionuclide Transport Abstraction (ANL-WIS-PA-000001 Rev. 00 ICN 2; CRWMS M\&O 2000z) }\end{array}$ & \begin{tabular}{|c|}
$M$ \\
$A+M$ \\
$M$ \\
$M$ \\
$M$ \\
$A$ \\
$A+M$ \\
(calculation) \\
$A$ \\
$A$ \\
$A$ \\
$M$
\end{tabular} & $\begin{array}{l}8 \text { Unique AMRs } \\
\text { ( } 1.8 \text { is a Calc. } \\
\text { Report; F.3, } \\
\text { L.5, and M.5 } \\
\text { are tallied in } \\
\text { Areas F, L, } \\
\text { and M, } \\
\text { respectively.) }\end{array}$ \\
\hline $\begin{array}{l}\text { J. Waste } \\
\text { Package/ } \\
\text { Drip Shield } \\
\text { Degra- } \\
\text { dation: } \\
\text { General } \\
\text { and } \\
\text { Localized } \\
\text { Corrosion }\end{array}$ & $\begin{array}{l}\text { J.1 General and Localized Corrosion of the Waste Package Outer Barrier (ANL-EBS-MD-000003 Rev. 00; } \\
\text { CRWMS M\&O 2000ag) } \\
\text { J.2 Aging and Phase Stability of the Waste Package Outer Barrier (ANL-EBS-MD-000002 Rev. 00; CRWMS M\&O 2000d) } \\
\text { J.3 General and Localized Corrosion of the Drip Shield (ANL-EBS-MD-000004 Rev. 00; CRWMS M\&O 2000af) } \\
\text { J.4 Degradation of Stainless Steel Structural Material (ANL-EBS-MD-000007 Rev. 00; CRWMS M\&O 2000s) } \\
\text { J.5 Abstraction of Models for Pitting and Crevice Corrosion of Drip Shield and Waste Package Outer Barrier } \\
\text { (ANL-EBS-PA-000003 Rev. 00; CRWMS M\&O 2000b) } \\
\text { J.6 WAPDEG Analysis of Waste Package and Drip Shield Degradation (ANL-EBS-PA-000001 Rev. 00 ICN 1; } \\
\text { CRWMS M\&O 2000br) } \\
\text { J.7 Calculation of General Corrosion Rate of Drip Shield and Waste Package Outer Barrier to Support WAPDEG Analysis } \\
\text { (CAL-EBS-PA-000002 Rev. 01; CRWMS M\&O 2000f) } \\
\text { J.8 Incorporation of Uncertainty and Variability of Drip Shield and Waste Package Degradation in WAPDEG Analysis } \\
\text { (ANL-EBS-MD-000036 Rev. 00; CRWMS M\&O 2000ak) } \\
\text { H.2 Environment on the Surfaces of the Drip Shie/d and Waste Package Outer Barrier (ANL-EBS-MD-000001 } \\
\text { Rev. OO ICN 1; CRWMS M\&O 2000ac) }\end{array}$ & $\begin{array}{c} \\
M \\
M \\
M \\
A+M \\
A+M \\
\text { (calculation) } \\
A \\
A\end{array}$ & $\begin{array}{l}12 \text { Unique AMRs } \\
\text { (J.7 and } \mathrm{J} .13 \\
\text { are Calc. } \\
\text { Reports; } \mathrm{H} .2 \text { is } \\
\text { tallied in } \\
\text { Area } \mathrm{H} \text { ). }\end{array}$ \\
\hline
\end{tabular}


Table 1. List of Model Areas and Associated AMRs and Other Reports for Status Review (continued)

\begin{tabular}{|c|c|c|c|}
\hline $\begin{array}{l}\text { 1. Model } \\
\text { Area }\end{array}$ & 2. AMRs and Other Documents Reviewed ${ }^{A}$ & $\begin{array}{l}\text { 3. Model or } \\
\text { Analysis? }\end{array}$ & $\begin{array}{l}\text { 4. \# Unique } \\
\text { Reports }\end{array}$ \\
\hline $\begin{array}{l}\text { J. Waste } \\
\text { Package/ } \\
\text { Drip Shield } \\
\text { Degra- } \\
\text { dation: } \\
\text { Other } \\
\text { Corrosion } \\
\text { Modes }\end{array}$ & $\begin{array}{l}\text { J.9 Analysis of Mechanisms for Early Waste Package Failure (ANL-EBS-MD-000023 Rev. 02; CRWMS M\&O 2000cC) } \\
\text { J.10 Hydrogen Induced Cracking of Drip Shield (ANL-EBS-MD-000006 Rev. 00 ICN 1; CRWMS M\&O 2000ah) } \\
\text { J.11 Stress-Corrosion Cracking of the Drip Shield, the Waste Package Outer Barrier, and the Stainless Steel Structural } \\
\text { Material (ANL-EBS-MD-000005 Rev. 00 ICN 1; CRWMS M\&O 2000bi) } \\
\text { J.12 Abstraction of Models of Stress-Corrosion Cracking of Drip Shield and Waste Package Outer Barrier, and Hydrogen- } \\
\text { Induced Corrosion of the Drip Shield (ANL-EBS-PA-000004 Rev. O0 ICN 1; CRWMS M\&O 2000c) } \\
\text { J.13 Calculation of Probability and Size of Defect Flaws in Waste Package Closure Welds to Support WAPDEG Analysis } \\
\text { (CAL-EBS-PA-000003 Rev. 00; CRWMS M\&O 2000h) } \\
\text { J.14 FEPs Screening of Processes and Issues in Drip Shield and Waste Package Degradation } \\
\text { (ANL-EBS-PA-000002 Rev. 01; CRWMS M\&O 2001x) }\end{array}$ & $\begin{array}{c}A \\
A+M \\
M \\
A+M \\
\text { (calculation) } \\
\text { A }\end{array}$ & \\
\hline $\begin{array}{l}\text { K. Waste } \\
\text { Form } \\
\text { Degra- } \\
\text { dation: } \\
\text { General } \\
\text { Infor- } \\
\text { mation } \\
\end{array}$ & $\begin{array}{l}\text { K.1 Inventory Abstraction (ANL-WIS-MD-000006 Rev. } 00 \text { ICN 2; BSC 2001i) } \\
\text { K.2 CSNF Waste Form Degradation Summary Abstraction (ANL-EBS-MD-000015 Rev. 00; CRWMS M\&O 2000r) } \\
\text { K.3 DHLW Glass Degradation (ANL-EBS-MD-000016 Rev. } 00 \text { ICN 1; CRWMS M\&O 2001f) } \\
\text { K.4 DSNF and Other Waste Form Degradation Abstraction (ANL-WIS-MD-000004 Rev. 01 ICN 1; BSC 2001n) } \\
\text { K.5 In-Package Source Term Abstraction (ANL-WIS-MD-000018 Rev. 00; CRWMS M\&O 2000ap) }\end{array}$ & $\begin{array}{c}A \\
A+M \\
M \\
M \\
A+M\end{array}$ & $\begin{array}{l}19 \text { Unique AMRs } \\
\text { (K.17, K.18, } \\
\text { and K. } 19 \text { are } \\
\text { Calc. Reports) }\end{array}$ \\
\hline $\begin{array}{l}\text { K. Waste } \\
\text { Form } \\
\text { Degra- } \\
\text { dation: } \\
\text { In-Package } \\
\text { Chemistry }\end{array}$ & $\begin{array}{l}\text { K.6 Summary of In-Package Chemistry for Waste Forms (ANL-EBS-MD-000050 Rev. 00; CRWMS M\&O 2000bj) } \\
\text { K.7 In-Package Chemistry Abstraction (ANL-EBS-MD-000037 Rev. 01; BSC 2001h) }\end{array}$ & $\begin{array}{c}A+M \\
M\end{array}$ & \\
\hline $\begin{array}{l}\text { K. Waste } \\
\text { Form } \\
\text { Degra- } \\
\text { dation: } \\
\text { Solubility } \\
\text { Constraints }\end{array}$ & $\begin{array}{l}\text { K.8 Pure-Phase Solubility Limits - LANL (ANL-EBS-MD-000017 Rev. } 00 \text { ICN 1; CRWMS M\&O 2001n) } \\
\text { K.9 Summary of Dissolved Concentration Limits (ANL-WIS-MD-000010 Rev. 01; CRWMS M\&O 2001q) } \\
\text { K.10 Secondary Uranium Phase Paragenesis and Incorporation of Radionuclides Into Secondary Phases } \\
\text { (ANL-EBS-MD-000019 Rev. 00; CRWMS M\&O 2000bd) }\end{array}$ & $\begin{array}{c}A \\
A+M \\
A\end{array}$ & \\
\hline
\end{tabular}


Table 1. List of Model Areas and Associated AMRs and Other Reports for Status Review (continued)

\begin{tabular}{|c|c|c|c|}
\hline $\begin{array}{l}\text { 1. Model } \\
\text { Area }\end{array}$ & 2. AMRs and Other Documents Reviewed ${ }^{A}$ & $\begin{array}{l}\text { 3. Model or } \\
\text { Analysis? }\end{array}$ & $\begin{array}{l}\text { 4. \# Unique } \\
\text { Reports }\end{array}$ \\
\hline $\begin{array}{l}\text { K. Waste } \\
\text { Form } \\
\text { Degra- } \\
\text { dation: } \\
\text { Cladding } \\
\text { Degra- } \\
\text { dation }\end{array}$ & $\begin{array}{l}\text { K.11 Initial Cladding Condition (ANL-EBS-MD-000048 Rev. } 00 \text { ICN 1; CRWMS M\&O 2000ao) } \\
\text { K.12 Clad Degradation - Localized Corrosion of Zirconium and Its Alloys Under Repository Conditions } \\
\text { (ANL-EBS-MD-000012 Rev. 00; CRWMS M\&O 2000o) } \\
\text { K.13 Hydride-Related Degradation of SNF Cladding Under Repository Conditions (ANL-EBS-MD-000011 Rev. } 00 \text { ICN } 1 \text {; } \\
\text { CRWMS M\&O 2001k) } \\
\text { K.14 Clad Degradation - Wet Unzipping (ANL-EBS-MD-000014 Rev. 00; CRWMS M\&O 2000p) } \\
\text { K.15 Clad Degradation - Dry Unzipping (ANL-EBS-MD-000013 Rev. 00; CRWMS M\&O 2000n) } \\
\text { K.16 Clad Degradation - Summary and Abstraction (ANL-WIS-MD-000007 Rev. 00 ICN 1; CRWMS M\&O 2001d) } \\
\text { K.17 Stainless Steel in Waste Packages for TSPA-SR (CAL-WIS-MD-000010 Rev. 00; CRWMS M\&O 2000bh) } \\
\text { K.18 Thermal Evaluation of Breached 21-PWR Waste Packages (CAL-UDC-ME-000002 Rev. 00; CRWMS M\&O 1999f) } \\
\text { K.19 Breakage of CSNF Cladding by Mechanical Loading (CAL-EBS-MD-000001 Rev. 00; CRWMS M\&O 1999a) } \\
\text { K.20 Clad Degradation - FEPs Screening Arguments (ANL-WIS-MD-000008 Rev. 00 ICN 1; CRWMS M\&O 2000cd) }\end{array}$ & $\begin{array}{c}A \\
A+M \\
A \\
M \\
A+M \\
A \\
\text { (calculation) } \\
\text { (calculation) } \\
\text { (calculation) } \\
\text { A }\end{array}$ & \\
\hline $\begin{array}{l}\text { K. Waste } \\
\text { Form } \\
\text { Degra- } \\
\text { dation: } \\
\text { Colloid } \\
\text { Release }\end{array}$ & $\begin{array}{l}\text { K.21 Colloid-Associated Radionuclide Concentration Limits: ANL (ANL-EBS-MD-000020 ReV. } 00 \text { ICN 1; } \\
\text { CRWMS M\&O 2001e) } \\
\text { K.22 Waste Form Colloid-Associated Concentration Limits: Abstraction and Summary (ANL-WIS-MD-000012 } \\
\text { Rev. 00 ICN 1; CRWMS M\&O 2001s) }\end{array}$ & $\begin{array}{l}A \\
M\end{array}$ & \\
\hline $\begin{array}{l}\text { L. EBS } \\
\text { Degra- } \\
\text { dation }\end{array}$ & $\begin{array}{l}\text { L.1 Drift Degradation Analysis (ANL-EBS-MD-000027 Rev. 01; CRWMS M\&O 2000x) } \\
\text { L.2 Fracture Geometry Analysis for the Stratigraphic Units of the Repository Host Horizon (ANL-EBS-GE-000006 Rev. 00; } \\
\text { CRWMS M\&O 2000ae) } \\
\text { L.3 Rockfall on Drip Shield (CAL-EDS-ME-000001 Rev. 00; CRWMS M\&O 2000bw) } \\
\text { L.4 Committed Materials in Repository Drifts (CAL-GCS-GE-000002 Rev. 00; BSC 2001p) } \\
\text { L.5 EBS Features, Events, and Processes (ANL-WIS-PA-000002 Rev. 01; CRWMS M\&O 2001h) } \\
\text { L.6 Flow of Water and Pooling in a Waste Package (ANL-EBS-MD-000055 Rev. 00; BSC 2001c) } \\
\text { H.3 EBS Physical and Chemical Environment Mode/ (ANL-EBS-MD-000033 Rev. 01; CRWMS M\&O 2000ab) } \\
\text { H.7 Seepage/Cement Interaction (ANL-EBS-MD-000043 Rev. 00; CRWMS M\&O 2000bf) } \\
\text { H.10 Seepage/Backfill Interaction (ANL-EBS-MD-000039 Rev. 00; CRWMS M\&O 2000cb) } \\
\text { M.5 EBS Radionuclide Transport Abstraction (ANL-WIS-PA-000001 Rev. 00 ICN 2; CRWMS M\&O 2000z) }\end{array}$ & $\begin{array}{c}A+M \\
A+M \\
\text { (calculation) } \\
\text { (calculation) } \\
A \\
A+M \\
M \\
A \\
A+M \\
M\end{array}$ & $\begin{array}{l}4 \text { Unique AMRs } \\
\text { (L. } 3 \text { and } L .4 \\
\text { are Calc. } \\
\text { Reports; H.3, } \\
\text { H.7, and H. } 10 \\
\text { are tallied in } \\
\text { Area } H \text {, and } \\
\text { M.5 is tallied in } \\
\text { Area } M)\end{array}$ \\
\hline
\end{tabular}


Table 1. List of Model Areas and Associated AMRs and Other Reports for Status Review (continued)

\begin{tabular}{|c|c|c|c|}
\hline $\begin{array}{l}\text { 1. Model } \\
\text { Area }\end{array}$ & 2. AMRs and Other Documents Reviewed ${ }^{A}$ & $\begin{array}{l}\text { 3. Model or } \\
\text { Analysis? }^{B}\end{array}$ & $\begin{array}{l}\text { 4. \# Unique } \\
\text { Reports }\end{array}$ \\
\hline $\begin{array}{l}\text { M. EBS } \\
\text { Radio- } \\
\text { nuclide } \\
\text { Transport }\end{array}$ & $\begin{array}{l}\text { M. } 1 \text { Invert Diffusion Properties Model (ANL-EBS-MD-000031 Rev. 01; CRWMS M\&O 2000as) } \\
\text { M.2 EBS Radionuclide Transport Model (ANL-EBS-MD-000034 Rev. } 00 \text { ICN 1; CRWMS M\&O 2000aa) } \\
\text { M.3 In-Drift Colloids and Concentration (ANL-EBS-MD-000042 Rev. 00; CRWMS M\&O 2000al) } \\
\text { M.4 Seepage/Invert Interactions (ANL-EBS-MD-000044 Rev. 00; CRWMS M\&O 2000bg) } \\
\text { M.5 EBS Radionuclide Transport Abstraction (ANL-WIS-PA-000001 Rev. 00 ICN 2; CRWMS M\&O 2000z) } \\
\text { L.5 EBS Features, Events, and Processes (ANL-WIS-PA-000002 Rev. 01; CRWMS M\&O 2001h) }\end{array}$ & $\begin{array}{l}M \\
A+M \\
M \\
M \\
M \\
A\end{array}$ & $\begin{array}{c}5 \text { Unique AMRs } \\
\text { (L.5 is tallied in } \\
\text { Area } L \text { ) }\end{array}$ \\
\hline $\begin{array}{l}\text { N. UZ } \\
\text { Transport }\end{array}$ & $\begin{array}{l}\text { N.1 UZ and SZ Transport Properties (ANL-NBS-HS-000019 Rev. } 00 \text { ICN 1; CRWMS M\&O 2001r) } \\
\text { N.2 UZ Colloid Transport Model (ANL-NBS-HS-000028 Rev. 00; CRWMS M\&O 2000bp) } \\
\text { N.3 Radionuclide Transport Models Under Ambient Conditions (MDL-NBS-HS-000008 Rev. 00; CRWMS M\&O 2000ba) } \\
\text { N.4 Particle Tracking Model and Abstraction of Transport Processes (ANL-NBS-HS-000026 Rev. 00; } \\
\quad \text { CRWMS M\&O 2000ay) } \\
\text { N.5 Analysis of Base-Case Particle Tracking Results of the Base-Case Flow Fields (ID: U0160) (ANL-NBS-HS-000024 } \\
\quad \text { Rev. 00; CRWMS M\&O 2000ce) } \\
\text { N.6 Analysis Comparing Advective-Dispersive Transport Solution to Particle Tracking (ANL-NBS-HS-000001 Rev. 00; } \\
\quad \text { CRWMS M\&O 2000cf) } \\
\text { N.7 Abstraction of Flow Fields for TSPA (ANL-NBS-HS-000023 Rev. 00 ICN 1; CRWMS M\&O 2000cg) } \\
\text { N.8 Fault Displacement Effects on Transport in the UZ (ANL-NBS-HS-000020 Rev. 01; CRWMS M\&O 2000ch) } \\
\text { C.8 Features, Events, and Processes in UZ Flow and Transport (ANL-NBS-MD-000001 Rev. 01; BSC 2001b) }\end{array}$ & $\begin{array}{l}A+M \\
A+M \\
A+M \\
A+M \\
A \\
A \\
A \\
A \\
A\end{array}$ & $\begin{array}{c}8 \text { Unique AMRs } \\
\text { (C.8 is tallied in } \\
\text { Area } C)\end{array}$ \\
\hline O. SZ Flow & $\begin{array}{l}\text { O.1 Hydrogeologic Framework Model for the SZ Site-Scale Flow and Transport Model (ANL-NBS-HS-000033 } \\
\text { Rev. } 00 \text { ICN 1; CRWMS M\&O 2001z) } \\
\text { O.2 Water Level Data Analysis for the SZ Site-Scale Flow and Transport Model (ANL-NBS-HS-000034 Rev. } 00 \text { ICN 1; } \\
\text { USGS 2001b) } \\
\text { O.3 Recharge and Lateral Groundwater Flow Boundary Conditions for the SZ Site-Scale Flow and Transport Model } \\
\text { (ANL-NBS-MD-000010 Rev. 00; CRWMS M\&O 1999h) } \\
\text { O.4 Geochemical and Isotopic Constraints on Groundwater Flow Directions, Mixing, and Recharge (ANL-NBS-HS-000021 } \\
\text { Rev. } 00 \text { ICN 1; CRWMS M\&O 2001ab) } \\
\text { O.5 Calibration of the Site-Scale SZ Flow Model (MDL-NBS-HS-000011 Rev. 00; CRWMS M\&O 2000j) } \\
\text { O.6 FEPs in Saturated Zone Flow and Transport (ANL-NBS-MD-000002 Rev. 01; CRWMS M\&O 2001ac) }\end{array}$ & $\begin{array}{l}A+M \\
A \\
A \\
A+M \\
A\end{array}$ & 6 Unique AMRs \\
\hline
\end{tabular}


Table 1. List of Model Areas and Associated AMRs and Other Reports for Status Review (continued)

\begin{tabular}{|c|c|c|c|}
\hline $\begin{array}{l}\text { 1. Model } \\
\text { Area }\end{array}$ & 2. AMRs and Other Documents Reviewed ${ }^{A}$ & $\begin{array}{l}\text { 3. Model or } \\
\text { Analysis? }\end{array}$ & $\begin{array}{l}\text { 4. \# Unique } \\
\text { Reports }\end{array}$ \\
\hline $\begin{array}{l}\text { P. SZ } \\
\text { Transport }\end{array}$ & $\begin{array}{l}\text { P.1 Probability Distribution for Flowing Interval Spacing (ANL-NBS-MD-000003 Rev. 00 ICN 1; CRWMS M\&O 2000cj) } \\
\text { P.2 SZ Colloid Facilitated Transport (ANL-NBS-HS-000031 Rev. 00; CRWMS M\&O 2000bc) } \\
\text { P.3 Uncertainty Distribution for Stochastic Parameters (ANL-NBS-MD-000011 Rev. 00; CRWMS M\&O 2000bo) } \\
\text { P.4 Input and Results of the Base-Case SZ Flow and Transport Model for TSPA (ANL-NBS-HS-000030 Rev. 00; } \\
\text { CRWMS M\&O 2000aq) } \\
\text { P.5 SZ Transport Methodology and Transport Component Integration (MDL-NBS-HS-000010 Rev. 00; } \\
\quad \text { CRWMS M\&O 2000ck) } \\
\text { P.6 Modeling Sub-Gridblock Scale Dispersion in 3-D Heterogeneous Fractured Media (ANL-NBS-HS-000022 } \\
\quad \text { Rev. 00 ICN 1; CRWMS M\&O 2000au) } \\
\text { N.1 UZ and SZ Transport Properties (ANL-NBS-HS-000019 Rev. 00 ICN 1; CRWMS M\&O 2001r) } \\
\text { O.6 FEPs in Saturated Zone Flow and Transport (ANL-NBS-MD-000002 ReV. 01; CRWMS M\&O 2001ac) }\end{array}$ & $\begin{array}{c}A \\
A+M \\
A \\
A+M \\
M \\
A+M \\
A+M \\
A \\
\end{array}$ & $\begin{array}{l}6 \text { Unique AMRs } \\
\text { (N.1 and } 0.6 \\
\text { are tallied in } \\
\text { Areas } N \text { and } O \text {, } \\
\text { respectively) }\end{array}$ \\
\hline Q. Biosphere & $\begin{array}{l}\text { Q.1 Groundwater Usage by the Proposed Farming Community (ANL-NBS-MD-000006 Rev. 00; CRWMS M\&O 2000cl) } \\
\text { Q.2 Identification of Ingestion Exposure Parameters (ANL-MGR-MD-000006 Rev. 00; CRWMS M\&O 2000ai) } \\
\text { Q.3 Input Parameter Values for External and Inhalation Radiation Exposure Analysis (ANL-MGR-MD-000001 Rev. 01; } \\
\text { CRWMS M\&O 2000ar) } \\
\text { Q.4 Dose Conversion Factor Analysis: Evaluation of GENII-S Dose Assessment Methods (ANL-MGR-MD-000002 Rev. 00; } \\
\text { CRWMS M\&O 1999b) } \\
\text { Q.5 Identification of the Critical Group (Consumption of Locally Produced Food and Tap Water) (ANL-MGR-MD-000005 } \\
\text { Rev. 01; CRWMS M\&O 2001ad) } \\
\text { Q.6 Environmental Transport Parameter Analysis (ANL-MGR-MD-000007 Rev. 00 ICN 1; CRWMS M\&O 2001ae) } \\
\text { Q.7 Transfer Coefficient Analysis (ANL-MGR-MD-000008 Rev. 00 ICN 2; CRWMS M\&O 2000bn) } \\
\text { Q.8 Evaluate Soil/Radionuclide Removal by Erosion and Leaching (ANL-NBS-MD-000009 Rev. 00 ICN 1; } \\
\quad \text { CRWMS M\&O 2001i) } \\
\text { Q.9 Nominal Performance Biosphere Dose Conversion Factor Analysis (ANL-MGR-MD-000009 Rev. 01; } \\
\text { CRWMS M\&O 2001m) } \\
\text { Q.10 Non-Disruptive Event Biosphere Dose Conversion Factor Sensitivity Analysis (ANL-MGR-MD-000010 Rev. 00; } \\
\text { CRWMS M\&O 2000cm) } \\
\text { Q.11 Distribution Fitting to the Stochastic BDCF Data (ANL-NBS-MD-000008 Rev. 00 ICN 1; CRWMS M\&O 2001af) } \\
\text { Q.12 Abstraction of BDCF Distributions for Irrigation Periods (ANL-NBS-MD-000007 Rev. 00 ICN 1; CRWMS M\&O 2001a) } \\
\text { Q.13 Evaluation of the Applicability of Biosphere-Related FEPs (ANL-MGR-MD-000011 Rev. 01; BSC 2001q) }\end{array}$ & $\begin{array}{l}\text { A } \\
A \\
A \\
A \\
A \\
A \\
A \\
A \\
A+M \\
A \\
A \\
A \\
A\end{array}$ & 13 Unique AMRs \\
\hline
\end{tabular}


Table 1. List of Model Areas and Associated AMRs and Other Reports for Status Review (continued)

\begin{tabular}{|c|c|c|c|}
\hline $\begin{array}{l}\text { 1. Model } \\
\text { Area }\end{array}$ & 2. AMRs and Other Documents Reviewed ${ }^{A}$ & $\begin{array}{l}\text { 3. Model or } \\
\text { Analysis? }\end{array}$ & $\begin{array}{l}\text { 4. \# Unique } \\
\text { Reports }\end{array}$ \\
\hline $\begin{array}{l}\text { R. Disruptive } \\
\text { Events: } \\
\text { Igneous } \\
\text { Disruption } \\
\text { Conse- } \\
\text { quences }\end{array}$ & $\begin{array}{l}\text { R.1 Characterize Framework for Igneous Activity at Yucca Mountain, Nevada (ANL-MGR-GS-000001 Rev. } 00 \text { ICN 1; } \\
\text { CRWMS M\&O 2000I) } \\
\text { R.2 Characterize Eruptive Processes at Yucca Mountain, Nevada (ANL-MGR-GS-000002 Rev. 00; CRWMS M\&O 2000k) } \\
\text { R.3 Igneous Consequence Modeling for the TSPA-SR (ANL-WIS-MD-000017 Rev. } 00 \text { ICN 1; CRWMS M\&O 2000aj) } \\
\text { R.4 Dike Propagation Near Drifts (ANL-WIS-MD-000015 Rev. 00 ICN 1; CRWMS M\&O 2000u) } \\
\text { R.5 Disruptive Event Biosphere Dose Conversion Factor Analysis (ANL-MGR-MD-000003 Rev. 01; } \\
\text { CRWMS M\&O 2001ah) } \\
\text { R.6 Disruptive Event Biosphere Dose Conversion Factor Sensitivity Analysis (ANL-MGR-MD-000004 Rev. 00; } \\
\text { CRWMS M\&O 2000cn) } \\
\text { R.7 DTN: MO0006SPAPVE03.001 (Documentation of BCDF input provided for TSPA-SR) } \\
\text { R.8 Miscellaneous Waste-Form FEPs (ANL-WIS-MD-000009 Rev. 00 ICN 1; CRWMS M\&O 2001ai) } \\
\text { R.9 Number of Waste Packages Hit by Igneous Intrusion (CAL-WIS-PA-000001 Rev. 01; CRWMS M\&O 2000ax) } \\
\text { R.10 Features, Events, and Processes: Disruptive Events (ANL-WIS-MD-000005 Rev. 00 ICN 1; CRWMS M\&O 2000ad) }\end{array}$ & \begin{tabular}{|c|} 
\\
\\
$M$ \\
$A$ \\
$A+M$ \\
$A$ \\
(data) \\
A \\
(calculation) \\
A
\end{tabular} & $\begin{array}{l}8 \text { Unique AMRs } \\
\text { (R.6 is data; R.7 } \\
\text { is a Calc. } \\
\text { Report) }\end{array}$ \\
\hline $\begin{array}{l}\text { S. Seismic } \\
\text { Hazard }\end{array}$ & $\begin{array}{l}\text { S.1 Characterize Framework for Seismicity and Structural Deformation at YM (ANL-CRW-GS-000003 Rev. 00; } \\
\text { CRWMS M\&O 2000m) } \\
\text { S.2 Effects of Fault Displacement on Emplacement Drifts (ANL-EBS-GE-000004 Rev. } 00 \text { ICN 1; CRWMS M\&O 2000co) } \\
\text { N.8 Fault Displacement Effects on Transport in the UZ (ANL-NBS-HS-000020 Rev. 01; CRWMS M\&O 2000ch) }\end{array}$ & $\begin{array}{l}\text { A } \\
\text { A } \\
\text { A }\end{array}$ & $\begin{array}{l}2 \text { Unique AMRs } \\
\text { (N.8 is tallied in } \\
\quad \text { Area } N)\end{array}$ \\
\hline $\begin{array}{l}\text { T. Integrated } \\
\text { Site } \\
\text { Model }\end{array}$ & $\begin{array}{l}\text { T.1 Geologic Framework Model (MDL-NBS-GS-000002 Rev. } 00 \text { ICN 2; BSC 2001e) } \\
\text { T.2 Mineralogical Model (MDL-NBS-GS-000003 Rev. } 00 \text { ICN 1; CRWMS M\&O 2000at) } \\
\text { T.3 Rock Properties Model (MDL-NBS-GS-000004 Rev. } 00 \text { ICN 2; CRWMS M\&O 2000bb) }\end{array}$ & $\begin{array}{l}M \\
M \\
M\end{array}$ & 3 Unique AMRs \\
\hline $\begin{array}{l}\text { U. PA } \\
\text { Modeling }\end{array}$ & $\begin{array}{l}\text { U.1 TSPA Model for SR (MDL-WIS-PA-000002 Rev. 00; CRWMS M\&O 2000bl) } \\
\text { U.2 Total System Performance for Site Recommendation (TDR-WIS-PA-000001 Rev. } 00 \text { ICN 1; CRWMS M\&O 2000bm) } \\
\text { U.3 Features, Events, and Processes: System Level and Criticality (ANL-WIS-MD-000019 Rev. 00; } \\
\quad \text { CRWMS M\&O 2000cq) } \\
\text { U.4 Performance Assessment and Sensitivity Analysis of Disposal of Plutonium as Can-in-Canister Ceramic (ANL-WIS- } \\
\text { PA-000003 Rev. 00; CRWMS M\&O 2001u) }\end{array}$ & $\begin{array}{c}\text { M } \\
\text { (tech. report) } \\
\text { A } \\
\text { A }\end{array}$ & $\begin{array}{c}3 \text { Unique AMRs } \\
\text { (U.2 is a } \\
\text { Technical } \\
\text { Report) }\end{array}$ \\
\hline
\end{tabular}


Table 1. List of Model Areas and Associated AMRs and Other Reports for Status Review (continued)

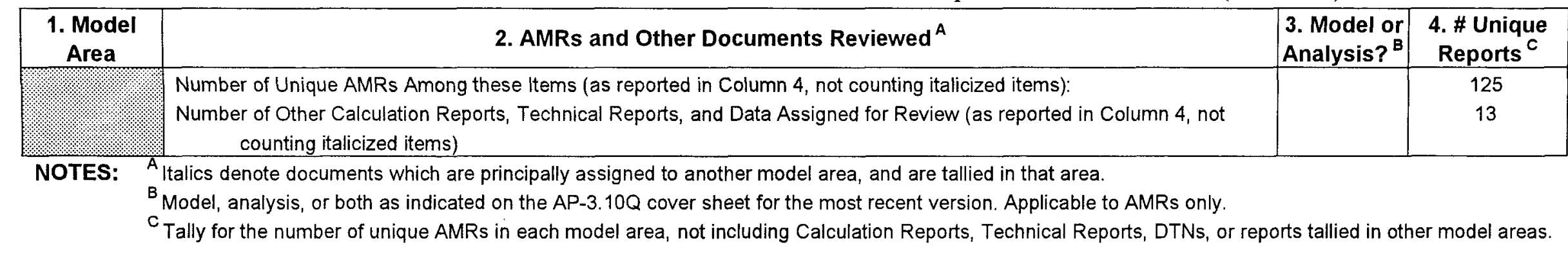


Table 2. Original Planned Schedule of Tasks Performed for Model Validation Status Review

\begin{tabular}{|c|c|}
\hline Task & $\begin{array}{c}\text { Date } \\
\text { Completed }\end{array}$ \\
\hline 1. Approve Technical Work Plan & $7 / 11 / 01$ \\
\hline 2. Identify technical specialists and model-area leads & $6 / 27 / 01$ \\
\hline 3. Conduct training workshops. & $\begin{array}{c}6 / 21 / 01 \\
6 / 28 / 01 \\
7 / 9-10 / 01\end{array}$ \\
\hline 4. Complete electronic distribution of review materials & $7 / 10 / 01$ \\
\hline 5. Receive reviews from lead technical specialists. & $8 / 3 / 01$ \\
\hline $\begin{array}{l}\text { 6. Conduct final workshop to report findings and } \\
\text { formulate recommendations. }\end{array}$ & $8 / 6-8 / 01$ \\
\hline $\begin{array}{l}\text { 7. Receive additional input from model-area leads } \\
\text { (Science \& Analysis Project) on recommendations }\end{array}$ & $8 / 14 / 01$ \\
\hline $\begin{array}{l}\text { 8. Draft report sent to review team members for } \\
\text { comments. }\end{array}$ & $8 / 17 / 01$ \\
\hline $\begin{array}{l}\text { 9. Consolidated draft submitted for checking and } \\
\text { review. }\end{array}$ & $8 / 27 / 01$ \\
\hline 10. Final approval & $9 / 20 / 01$ \\
\hline 11. Distribute approved report to affected managers. & $9 / 20 / 01$ \\
\hline 12. Submit Records Package. & $9 / 30 / 01$ \\
\hline 13. Follow-up activities. & TBD \\
\hline
\end{tabular}


Table 3. List of Supplementary Materials Distributed to the Technical Specialists

\begin{tabular}{|c|c|}
\hline Source & Comment \\
\hline \multicolumn{2}{|l|}{ Documents } \\
\hline $\begin{array}{l}\text { Andresen, P.L. 1999. Interim Report to TRW, "Stress Corrosion } \\
\text { Crack Growth Measurements in Environments Relevant to High } \\
\text { Level Nuclear Waste Packages", September } 1999 .\end{array}$ & $\begin{array}{l}\text { Obtained from Records Information } \\
\text { System }\end{array}$ \\
\hline $\begin{array}{l}\text { BSC (Bechtel SAIC Company) 2001f. Ground Control for } \\
\text { Emplacement Drifts for SR. ANL-EBS-GE-000002 REV } 00 \text { ICN } \\
01 .\end{array}$ & $\begin{array}{l}\text { Obtained from Records Information } \\
\text { System (unavailable from Document } \\
\text { Control) }\end{array}$ \\
\hline $\begin{array}{l}\text { Brekke, T.L.; Cording, E.J.; Daemen, J.; Hart, R.D.; Hudson, J.A.; } \\
\text { Kaiser, P.K.; and Pelizza, S. 1999. Panel Report on the Drift } \\
\text { Stability Workshop, Las Vegas, Nevada, December 9-11, } 1998 .\end{array}$ & $\begin{array}{l}\text { Obtained from Records Information } \\
\text { System }\end{array}$ \\
\hline $\begin{array}{l}\text { Budnitz, B.; Ewing, R.C.; Moeller, D.W.; Payer, J.; Whipple, C.; } \\
\text { and Witherspoon, P.A. 1999. Peer Review of the Total System } \\
\text { Performance Assessment-Viability Assessment Final Report. }\end{array}$ & $\begin{array}{l}\text { Obtained from Records Information } \\
\text { System }\end{array}$ \\
\hline $\begin{array}{l}\text { Chandler, N.; Davison, C.C.; Gee, G.; LaPointe, P.; and Neuman, } \\
\text { S. 1999. Yucca Mountain Project, A Consensus Peer Review of } \\
\text { Predictions of Seepage into the Drifts of a Proposed Repository } \\
\text { at Yucca Mountain. }\end{array}$ & $\begin{array}{l}\text { Obtained from Records Information } \\
\text { System }\end{array}$ \\
\hline $\begin{array}{l}\text { Crouch, S.L. and Starfield, A.M. 1983. Boundary Element } \\
\text { Methods in Solid Mechanics, with Applications in Rock Mechanics } \\
\text { and Geological Engineering. }\end{array}$ & Textbook \\
\hline $\begin{array}{l}\text { CRWMS M\&O 1999d. Seismic Ground Motion Hazard Inputs. } \\
\text { Input Transmittal WP-NEP-99309.Ta. }\end{array}$ & $\begin{array}{l}\text { Obtained from Records Information } \\
\text { System }\end{array}$ \\
\hline $\begin{array}{l}\text { CRWMS M\&O 1999e. Seismic Ground Motion Hazard Inputs. } \\
\text { Input Transmittal WP-NEP-99309.T. }\end{array}$ & $\begin{array}{l}\text { Obtained from Records Information } \\
\text { System }\end{array}$ \\
\hline $\begin{array}{l}\text { CRWMS M\&O 2000bt. Waste Package Operations Fabrication } \\
\text { Process Report. TDR-EBS-ND-000003 REV } 01 .\end{array}$ & Obtained from Document Control. \\
\hline $\begin{array}{l}\text { CRWMS M\&O 2000w. Disruptive Events Process Model Report. } \\
\text { TDR-NBS-MD-000002 REV } 00 \text { ICN } 02 .\end{array}$ & Obtained from Document Control. \\
\hline $\begin{array}{l}\text { CRWMS M\&O 2000bs. Waste Package Degradation Process } \\
\text { Model Report. TDR-WIS-MD-000002 REV 00 ICN } 02 .\end{array}$ & Obtained from Document Control. \\
\hline $\begin{array}{l}40 \text { CFR 197. 2001. Protection of Environment: Public Health and } \\
\text { Environmental Radiation Protection Standards for Yucca } \\
\text { Mountain, Nevada. 40CFR197.html. }\end{array}$ & $\begin{array}{l}\text { Cited the Electronic Code of Federal } \\
\text { Regulations for latest version. }\end{array}$ \\
\hline $\begin{array}{l}\text { Fabryka-Martin, J.T.; Wolfsberg, A.V.; Dixon, P.R.; Levy, S.S.; } \\
\text { Musgrave, J.A.; and Turin, H.J. 1997. Summary Report of } \\
\text { Chlorine-36 Studies: Sampling, Analysis, and Simulation of } \\
\text { Chlorine-36 in the Exploratory Studies Facility. }\end{array}$ & $\begin{array}{l}\text { Obtained from Records Information } \\
\text { System }\end{array}$ \\
\hline $\begin{array}{l}\text { Kelkar, S. and Travis, B. 1999. Independent Test Case Report for } \\
\text { TRACRN Version 1.0 }\end{array}$ & $\begin{array}{l}\text { Obtained from Records Information } \\
\text { System }\end{array}$ \\
\hline
\end{tabular}


Table 3. List of Supplementary Materials Distributed to the Technical Specialists (continued)

\begin{tabular}{|c|c|}
\hline Source & Comment \\
\hline $\begin{array}{l}\text { Reimus, P.W.; Adams, A.; Haga, M.J.; Humphrey, A.; Callahan, } \\
\text { T.; Anghel, I.; and Counce, D. 1999. Results and Interpretation of } \\
\text { Hydraulic and Tracer Testing in the Prow Pass Tuff at the C- } \\
\text { Holes. }\end{array}$ & $\begin{array}{l}\text { Obtained from Records Information } \\
\text { System }\end{array}$ \\
\hline $\begin{array}{l}\text { Robinson, B.A.; Wolfsberg, A.V.; Zyvoloski, G.A.; and Gable, } \\
\text { C.W. 1995. An Unsaturated Zone Flow and Transport Model of } \\
\text { Yucca Mountain. }\end{array}$ & $\begin{array}{l}\text { Obtained from Records Information } \\
\text { System }\end{array}$ \\
\hline $\begin{array}{l}\text { Turcotte, D.L. and Schubert, G. 1982. Geodynamics, Applications } \\
\text { of Continuum Physics to Geological Problems. }\end{array}$ & Textbook \\
\hline \multicolumn{2}{|l|}{ Data } \\
\hline $\begin{array}{l}\text { GS000508312332.001. Water-Level Data Analysis for the } \\
\text { Saturated Zone Site-Scale Flow and Transport Model. }\end{array}$ & $\begin{array}{l}\text { All data in this record were transmitted } \\
\text { as supplementary review material. }\end{array}$ \\
\hline $\begin{array}{l}\text { LA0003AM831341.001. Probability Distributions for Sorption } \\
\text { Coefficients (Kd's). }\end{array}$ & $\begin{array}{l}\text { All data in this record were transmitted } \\
\text { as supplementary review material. }\end{array}$ \\
\hline $\begin{array}{l}\text { MO0003SEPSDARS.002. Preliminary Seismic Design } \\
\text { Acceleration Response Spectra for the Repository Level (Point } \\
\text { B). }\end{array}$ & $\begin{array}{l}\text { All data in this record were transmitted } \\
\text { as supplementary review material. }\end{array}$ \\
\hline $\begin{array}{l}\text { SN9908T0581999.001. Recharge and Lateral Groundwater } \\
\text { Flow Boundary Conditions for the Saturated Zone (SZ) Site- } \\
\text { Scale Flow and Transport Model. }\end{array}$ & $\begin{array}{l}\text { All data in this record were transmitted } \\
\text { as supplementary review material. }\end{array}$ \\
\hline \multicolumn{2}{|c|}{ Document Input Reference System (DIRS) Reports } \\
\hline $\begin{array}{l}\text { DIRS report listing all documents and data cited by the TSPA- } \\
\text { SR Model AMR (CRWMS M\&O 2000bl) }\end{array}$ & Report for DIRS reference 148384 . \\
\hline $\begin{array}{l}\text { Edited compilation of DIRS reports listing Project reports cited by } \\
\text { various documents assigned to Model Areas J and M in Table } 1 \\
\text { (cited by: CRWMS M\&O 2000b, 2000c, 2000d, 2000f, 2000h, } \\
2000 \text { s, 2000ac, 2000af, 2000ag, 2000ah, 2000ak, 2000bi, } \\
2000 \mathrm{z} \text {, and 2000cc; and CRWMS M\&O 2001x). }\end{array}$ & $\begin{array}{l}\text { Compiled reports for DIRS references: } \\
\text { 147648, 151549, 147639, 152542, } \\
144551,110182,151568,144971 \\
144229,151559,146546,151564, \\
150792,152097 \text {, and } 153937 .\end{array}$ \\
\hline
\end{tabular}


Table 4. Assignment of Technical Specialists to Model Areas (X denotes supporting role)

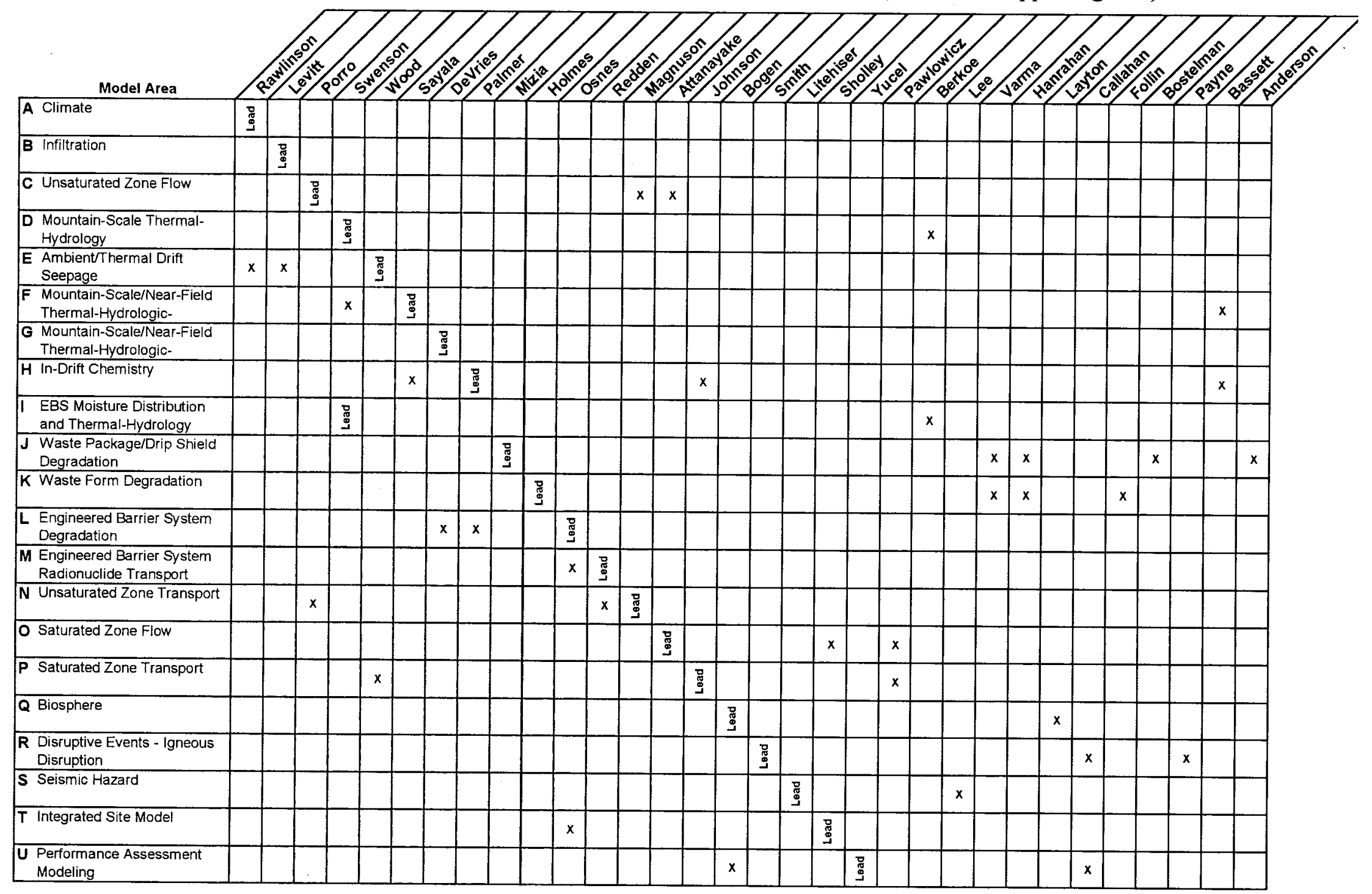


Table 5. Model Area Leads, Representing Responsible Project Staff

\begin{tabular}{|c|c|}
\hline Model Area & Assigned Staff (see note) \\
\hline A Climate & Ming Zhu/Bo Bodvarsson \\
\hline B Infiltration & Ming Zhu/Bo Badvarsson \\
\hline C UZ Flow & Ming Zhu/Bo Bodvarsson \\
\hline D Mountain-Scale TH & Ming Zhu/Bo Bodvarsson \\
\hline E Ambient/Thermal Drift Seepage & Ming Zhu/Bo Bodvarsson \\
\hline F Mountain-Scale/Near-Field THC & Dave Dobson/Yvonne Tsang \\
\hline G Mountain-Scale/Near-Field THM & Dave Dobson/Yvonne Tsang \\
\hline H In-Drift Chemistry & Bob MacKinnon \\
\hline I EBS Moisture Distribution and TH & Bob Mackinnon \\
\hline J Waste Package/Drip Shield Degradation & Tammy Summers \\
\hline K Waste Form Degradation & Christine Stockman \\
\hline L EBS Degradation & Bob MacKinnon \\
\hline M EBS Radionuclide Transport & Bob MacKinnon \\
\hline N UZ Transport & Ming Zhu/Bo Bodvarsson \\
\hline O SZ Flow & Al Eddebbarh \\
\hline P SZ Transport & Al Eddebbarh \\
\hline Q Biosphere & Tony Smith \\
\hline R Disruptive Events - Igneous Disruption & Richard Quittmeyer \\
\hline S Seismic Hazard & Richard Quittmeyer \\
\hline $\mathrm{T}$ Integrated Site Model & Clinton Lum \\
\hline U PA Modeling & Jerry McNeish \\
\hline
\end{tabular}

Note: Selection and participation of Model Area Leads is discussed in Section 5.2. 
Table 6. Summary of Binning Results from Model Validation Status Review, for Each Model Area

\begin{tabular}{|c|c|c|c|}
\hline $\begin{array}{l}\text { 1. Model } \\
\text { Area }\end{array}$ & 2. AMRs and Other Documents (see note) & 3. Models Identified & 4. Bin \\
\hline A. Climate & A.1 Future Climate Analysis (ANL-NBS-GS-000008 Rev. 00) & $\begin{array}{l}\text { Precession-Based Orbital Clock (A.1) } \\
\text { Bin Subtotals: Bin 1: } 1 \text { Bin 2:0 Bin 3:0 }\end{array}$ & 1 \\
\hline B. Infiltration & $\begin{array}{l}\text { B.1 Simulation Net Infiltration for Modern and Potential Future Climates } \\
\text { (ANL-NBS-HS-000032 Rev. } 00 \text { ICN 1) } \\
\text { B.2 Analysis of Infiltration Uncertainty (ANL-NBS-HS-000027 Rev. 00) }\end{array}$ & $\begin{array}{l}\text { Net Infiltration Model (B.1) } \\
\text { Bin Subtotals: Bin 1:0 Bin 2: } 1 \text { Bin 3: } 0\end{array}$ & 2 \\
\hline C. UZ Flow & $\begin{array}{l}\text { C. } 1 \text { UZ Flow Models and Submodels (MDL-NBS-HS-000006 Rev. 00) } \\
\text { C. } 2 \text { Calibrated Properties Model (MDL-NBS-HS-000003 Rev. 00) } \\
\text { C. } 3 \text { Analysis of Hydrologic Properties Data (ANL-NBS-HS-000002 Rev. 00) } \\
\text { C. } 4 \text { Development of Numerical Grids for UZ Flow and Transport Modeling } \\
\text { (ANL-NBS-HS-000015 Rev. 00) } \\
\text { C. } 5 \text { Analysis of Geochemical Data for the UZ (ANL-NBS-HS-000017 Rev. 00 ICN 1) } \\
\text { C. } 6 \text { Conceptual and Numerical Models of UZ Flow and Transport (MDL-NBS-HS-000005 } \\
\text { Rev. 00) } \\
\text { C.7 Natural Analogs for the UZ (ANL-NBS-HS-000007 Rev. 00) } \\
\text { C.8 Features, Events, and Processes in UZ Flow and Transport (ANL-NBS-MD-000001 } \\
\quad \text { Rev. 01) }\end{array}$ & $\begin{array}{l}\text { Conceptual Model of UZ Flow (C.6-1) } \\
\text { Numerical Grids Model (C.4) } \\
\text { Numerical Model of UZ Flow (C.6-2) } \\
\text { Active Fracture Model (C.6-3) } \\
\text { Calibrated Properties Model (C.2) } \\
\text { UZ Flow Model (C.1-1) } \\
\text { Geothermal Model (C.1-2) } \\
\text { Conceptual Model of Perched Water (C.1-3) } \\
\text { 3-D Perched Water Calibration Model (C.1-4) } \\
\text { Bin Subtotals: Bin 1: } 4 \text { Bin 2: } 5 \text { Bin 3:0 }\end{array}$ & $\begin{array}{l}2 \\
2 \\
2 \\
2 \\
2 \\
1 \\
1 \\
1 \\
1\end{array}$ \\
\hline $\begin{array}{l}\text { D. Mountain- } \\
\text { Scale TH }\end{array}$ & $\begin{array}{l}\text { D.1 Mountain-Scale Coupled Processes (TH) Models (MDL-NBS-HS-000007 Rev. 00) } \\
\text { C.8 Features, Events, and Processes in UZ Flow and Transport (ANL-NBS-MD-000001 } \\
\text { Rev. 01) }\end{array}$ & $\begin{array}{l}\text { Mountain-Scale Coupled Processes (TH) } \\
\text { Model (D.1) } \\
\text { Bin Subtotals: Bin 1: } 0 \text { Bin 2: } 1 \text { Bin 3: } 0\end{array}$ & 2 \\
\hline $\begin{array}{l}\text { E. Ambient/ } \\
\text { Thermal } \\
\text { Drift } \\
\text { Seepage }\end{array}$ & $\begin{array}{l}\text { E.1 Seepage Calibration Model and Seepage Testing Data (MDL-NBS-HS-000004 } \\
\text { Rev. 01) } \\
\text { E.2 Seepage Model for PA Including Drift Collapse (MDL-NBS-HS-000002 Rev. 01) } \\
\text { E.3 Abstraction of Drift Seepage (ANL-NBS-MD-000005 Rev. 01) } \\
\text { E.4 In Situ Field Testing of Processes (ANL-NBS-HS-000005 Rev. 00) } \\
\text { C.8 Features, Events, and Processes in UZ Flow and Transport } \\
\text { (ANL-NBS-MD-000001 Rev. 01) } \\
\text { I.6 Abstraction of NFE Drift Thermodynamic Environment and Percolation Flux } \\
\text { (ANL-EBS-HS-000003 Rev. O0 ICN2) } \\
\text { L.1 Drift Degradation Analysis (ANL-EBS-MD-000027 Rev. 01) }\end{array}$ & $\begin{array}{l}\text { Seepage Calibration Model (E.1) } \\
\text { Seepage Model for PA Including Drift } \\
\quad \text { Collapse (E.2) } \\
\text { Abstraction of Drift Seepage (E. 3-1) } \\
\text { Abstraction of Thermal Seepage Effects (E.3-2) } \\
\text { Bin Subtotals: Bin 1: } 0 \text { Bin 2: } 4 \text { Bin 3: } 0\end{array}$ & $\begin{array}{l}2 \\
2 \\
2 \\
2\end{array}$ \\
\hline
\end{tabular}


Table 6. Summary of Binning Results from Model Validation Status Review, for Each Model Area (continued)

\begin{tabular}{|c|c|c|c|}
\hline $\begin{array}{l}\text { 1. Model } \\
\text { Area }\end{array}$ & 2. AMRs and Other Documents (see note) & 3. Models Identified & 4. Bin \\
\hline $\begin{array}{l}\text { F. Mountain- } \\
\text { Scalel } \\
\text { Near-Field } \\
\text { THC }\end{array}$ & $\begin{array}{l}\text { F.1 Drift-Scale Coupled Processes (DST and THC Seepage) Models } \\
\text { (MDL-NBS-HS-000001 Rev. } 01 \text { ICN 1) } \\
\text { F.2 Abstraction of Drift-Scale Coupled Processes (ANL-NBS-HS-000029 Rev. 00) } \\
\text { F.3 Thermal Tests Thermal/Hydrological Analyses/Model Report (ANL-NBS-TH-000001 } \\
\text { Rev. 00 ICN 1) } \\
\text { I.1 Multiscale Thermohydrologic Model (ANL-EBS-MD-000049 Rev. 00 ICN 1) } \\
\text { I.9 FEPs in Thermal Hydrology and Coupled Processes (ANL-NBS-MD-000004 } \\
\text { Rev. 00 ICN 1) }\end{array}$ & $\begin{array}{l}\text { DST THC Calibration Model (F.1-1) } \\
\text { THC Seepage Model (F.1-2) } \\
\text { Bin Subtotals: Bin 1:0 Bin 2: } 2 \text { Bin 3:0 }\end{array}$ & $\begin{array}{l}2 \\
2\end{array}$ \\
\hline $\begin{array}{l}\text { G. Mountain- } \\
\text { Scale/ } \\
\text { Near-Field } \\
\text { THM }\end{array}$ & $\begin{array}{l}\text { G.1 Calculation of Permeability Change Due to Coupled THM Effects } \\
\text { (CAL-NBS-MD-000002 Rev. O0) } \\
\text { 1.9 FEPs in Thermal Hydrology and Coupled Processes (ANL-NBS-MD-000004 } \\
\text { Rev. } 00 / C N 1 \text { ) }\end{array}$ & $\begin{array}{l}\text { THM Model (G.1) } \\
\text { Bin Subtotals: Bin 1:0 Bin 2: } 0 \text { Bin 3: } 1\end{array}$ & 3 \\
\hline $\begin{array}{l}\text { H. In-Drift } \\
\text { Chemistry }\end{array}$ & $\begin{array}{l}\text { H.1 In-Drift Precipitates/Salts Analysis (ANL-EBS-MD-000045 Rev. 00 ICN 2) } \\
\text { H.2 Environment on the Surfaces of the DS and WP Outer Barrier (ANL-EBS-MD-000001 } \\
\quad \text { Rev. 00 ICN 1) } \\
\text { H.3 EBS Physical and Chemical Environment Model (ANL-EBS-MD-000033 Rev. 01) } \\
\text { H.4 In-Drift Corrosion Products (ANL-EBS-MD-000041 Rev. 00) } \\
\text { H.5 In-Drift Gas Flux and Composition (ANL-EBS-MD-000040 Rev. 00) } \\
\text { H.6 In-Drift Microbial Communities (ANL-EBS-MD-000038 Rev. 00 ICN 1) } \\
\text { H.7 Seepage/Cement Interactions (ANL-EBS-MD-000043 Rev. 00) } \\
\text { H.8 Physical and Chemical Environmental Abstraction Model (ANL-EBS-MD-000046 } \\
\quad \text { Rev. 00 ICN 1) } \\
\text { H.9 Precipitates/Salts Model Results for THC Abstraction (CAL-EBS-PA-000008 } \\
\quad \text { Rev. 00 ICN 1) } \\
\text { H.10 Seepage/Backfill Interaction (ANL-EBS-MD-000039 Rev. 00) } \\
\text { L.5 EBS Features, Events, and Processes (ANL-WIS-PA-000002 Rev. 01) }\end{array}$ & 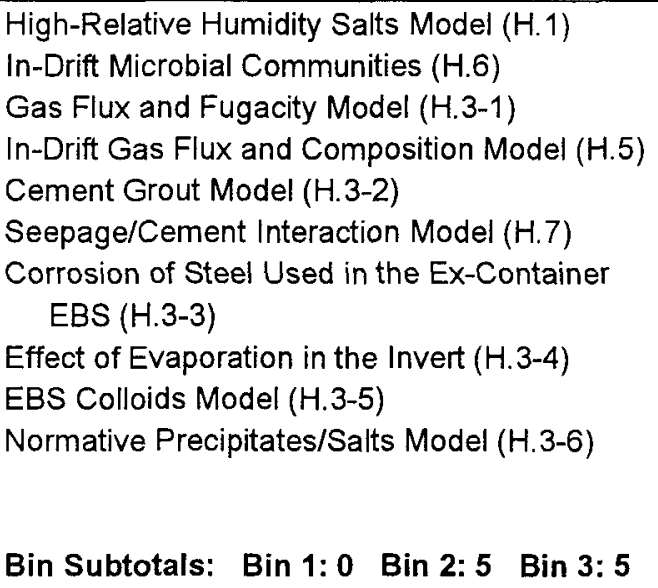 & $\begin{array}{l}2 \\
2 \\
2 \\
2 \\
3 \\
3 \\
3 \\
3 \\
3 \\
2\end{array}$ \\
\hline
\end{tabular}


Table 6. Summary of Binning Results from Model Validation Status Review, for Each Model Area (continued)

\begin{tabular}{|c|c|c|c|}
\hline $\begin{array}{l}\text { 1. Model } \\
\text { Area }\end{array}$ & 2. AMRs and Other Documents (see note) & 3. Models Identified & 4. Bin \\
\hline
\end{tabular}


Table 6. Summary of Binning Results from Model Validation Status Review, for Each Model Area (continued)

\begin{tabular}{|c|c|c|c|}
\hline $\begin{array}{l}\text { 1. Model } \\
\text { Area }\end{array}$ & 2. AMRs and Other Documents (see note) & 3. Models Identified & 4. Bin \\
\hline \begin{tabular}{|l|} 
J. Waste \\
Packagel \\
Drip Shield \\
Degra- \\
dation: \\
General \\
and \\
Localized \\
Corrosion
\end{tabular} & $\begin{array}{l}\text { J.1 General and Localized Corrosion of the WP Outer Barrier (ANL-EBS-MD-000003 } \\
\text { Rev. 00) } \\
\text { J.2 Aging and Phase Stability of the WP Outer Barrier (ANL-EBS-MD-000002 Rev. 00) } \\
\text { J.3 General and Localized Corrosion of the DS (ANL-EBS-MD-000004 Rev. 00) } \\
\text { J.4 Degradation of Stainless Steel Structural Material (ANL-EBS-MD-000007 Rev. 00) } \\
\text { J.5 Abstraction of Models for Pitting and Crevice Corrosion of DS and WP Outer Barrier } \\
\text { (ANL-EBS-PA-000003 Rev. 00) } \\
\text { J.6 WAPDEG Analysis of WP and DS Degradation (ANL-EBS-PA-000001 Rev. 00 ICN 1) } \\
\text { J.7 Calculation of General Corrosion Rate of DS and WP Outer Barrier to Support } \\
\text { WAPDEG Analysis (CAL-EBS-PA-000002 Rev. 01) } \\
\text { J.8 Incorporation of Uncertainty and Variability of DS and WP Degradation in WAPDEG } \\
\text { Analysis (ANL-EBS-MD-000036 Rev. 00) } \\
\text { H.2 Environment on the Surfaces of the DS and WP Outer Barrier } \\
\text { (ANL-EBS-MD-000001Rev. O0 ICN 1) }\end{array}$ & $\begin{array}{l}\text { General \& Localized Corrosion of the } \\
\text { WPOB (J.1) } \\
\text { Aging and Phase Stability: Precipitation } \\
\text { Model (J.2-1) } \\
\text { Aging and Phase Stability: Long Range } \\
\text { Ordering Model (J.2-2) } \\
\text { General and Localized Corrosion of the Drip } \\
\text { Shield (J.3) } \\
\text { Degradation of Stainless Steel Structural } \\
\text { Materials (J.4) } \\
\text { Alloy-22 Potential-Based Localized Corrosion } \\
\text { Initiation Threshold and Rate Abstraction } \\
\text { Model (J.5-1) } \\
\text { Ti Grade } 7 \text { Potential-Based Localized } \\
\text { Corrosion Initiation Threshold and Rate } \\
\text { Abstraction Model (J.5-2) } \\
\text { WAPDEG Analysis of WP and DS } \\
\text { Degradation (J.6) } \\
\text { Calculation of General Corrosion Rate of DS } \\
\text { and WPOB to Support WAPDEG } \\
\text { Analysis (J.7) } \\
\text { Incorporation of Uncertainty and Variability of } \\
\text { DS and WP Degradation in WAPDEG (J.8) } \\
\text { Bin Subtotals: Bin 1: } 2 \text { Bin 2: } 6 \text { Bin } 3: 2\end{array}$ & $\begin{array}{l}2 \\
2 \\
2 \\
2 \\
2 \\
1 \\
1 \\
3 \\
2\end{array}$ \\
\hline
\end{tabular}


Table 6. Summary of Binning Results from Model Validation Status Review, for Each Model Area (continued)

\begin{tabular}{|c|c|c|c|}
\hline $\begin{array}{l}\text { 1. Model } \\
\text { Area }\end{array}$ & 2. AMRs and Other Documents (see note) & 3. Models Identified & 4. Bin \\
\hline \begin{tabular}{|l|} 
J. Waste \\
Package/ \\
Drip Shield \\
Degra- \\
dation: \\
Other \\
Corrosion \\
Modes
\end{tabular} & $\begin{array}{l}\text { J.9 Analysis of Mechanisms for Early WP Failure (ANL-EBS-MD-000023 ReV. 02) } \\
\text { J.10 Hydrogen Induced Cracking of DS (ANL-EBS-MD-000006 Rev. 00 ICN 1) } \\
\text { J.11 Stress-Corrosion Cracking of the DS, the WP Outer Barrier, and the Stainless Steel } \\
\text { Structural Material (ANL-EBS-MD-000005 Rev. 00 ICN 1) } \\
\text { J.12 Abstraction of Models of Stress-Corrosion Cracking of Drip Shield and WP Outer } \\
\text { Barrier, and Hydrogen-Induced Corrosion of the DS (ANL-EBS-PA-000004 } \\
\text { Rev. 00 ICN 1) } \\
\text { J.13 Calculation of Probability and Size of Defect Flaws in WP Closure Welds to Support } \\
\text { WAPDEG Analysis (CAL-EBS-PA-000003 Rev. 00) } \\
\text { J.14 FEPs Screening of Processes and Issues in DS and WP Degradation } \\
\text { (ANL-EBS-PA-000002 Rev. 01) }\end{array}$ & $\begin{array}{l}\text { DS Passive Corrosion (J.10-1) } \\
\text { DS Galvanic Coupling (J.10-2) } \\
\text { SCC Threshold Model (J.11-1) } \\
\text { SCC Slip Dissolution/Film Rupture } \\
\text { Model (J.11-2) } \\
\text { Manufacturing Defects Abstraction } \\
\text { Model (J.12-1) } \\
\text { Stress and Stress Intensity Factor Profile } \\
\quad \text { Abstraction (J.12-2) } \\
\text { Slip Dissolution Abstraction Model (J.12-3) } \\
\text { Threshold Stress Intensity Factor Abstraction } \\
\quad \text { Model (J.12-4) } \\
\text { Bin Subtotals: Bin 1: } 0 \text { Bin 2: } 8 \text { Bin 3: } 0\end{array}$ & $\begin{array}{l}2 \\
2 \\
2 \\
2 \\
2 \\
2 \\
2 \\
2 \\
2\end{array}$ \\
\hline $\begin{array}{l}\text { K. Waste } \\
\text { Form } \\
\text { Degra- } \\
\text { dation: } \\
\text { General } \\
\text { Infor- } \\
\text { mation }\end{array}$ & $\begin{array}{l}\text { K.1 Inventory Abstraction (ANL-WIS-MD-000006 Rev. 00 ICN 2) } \\
\text { K.2 CSNF Waste Form Degradation Summary Abstraction (ANL-EBS-MD-000015 Rev. 00) } \\
\text { K.3 DHLW Glass Degradation (ANL-EBS-MD-000016 Rev. 00 ICN 1) } \\
\text { K.4 DSNF and Other Waste Form Degradation Abstraction (ANL-WIS-MD-000004 } \\
\quad \text { Rev. } 01 \text { ICN 1) } \\
\text { K.5 In-Package Source Term Abstraction (ANL-WIS-MD-000018 Rev. 00) }\end{array}$ & $\begin{array}{l}\text { Inventory Abstraction (K.1) } \\
\text { CSNF Waste Form Summary Degradation } \\
\text { Abstraction (K.2) } \\
\text { DHLW Glass Degradation (K.3) } \\
\text { Waste Form Degradation Abstract. - Upper } \\
\text { Limit Model (K.4-1) } \\
\text { Waste Form Degradation Abstraction - } \\
\text { Conservative Model (K.4-2) } \\
\text { Waste Form Degradation Abstraction - Best } \\
\text { Estimate Model (K.4-3) } \\
\text { Waste Form Degradation Abstraction - } \\
\text { Immobilized Pu Model (K.4-4) } \\
\text { Bin Subtotals: Bin 1: 1 Bin 2: } 4 \text { Bin 3: } 2\end{array}$ & $\begin{array}{l}2 \\
1 \\
2 \\
2 \\
2 \\
3 \\
3\end{array}$ \\
\hline \begin{tabular}{l|} 
Kaste \\
Form \\
Degra- \\
dation: \\
In-Package \\
Chemistry
\end{tabular} & $\begin{array}{l}\text { K.6 Summary of In-Package Chemistry for Waste Forms (ANL-EBS-MD-000050 Rev. 00) } \\
\text { K.7 In-Package Chemistry Abstraction (ANL-EBS-MD-000037 Rev. 01) }\end{array}$ & $\begin{array}{l}\text { In-Package Source Term Abstraction (K.5) } \\
\text { In-Package Chemistry for Waste Forms (K.6) } \\
\text { In-Package Chemistry Abstraction (K.7) } \\
\text { Bin Subtotals: Bin 1: } 1 \text { Bin 2: } 2 \text { Bin 3:0 }\end{array}$ & $\begin{array}{l}1 \\
2 \\
2\end{array}$ \\
\hline
\end{tabular}


Table 6. Summary of Binning Results from Model Validation Status Review, for Each Model Area (continued)

\begin{tabular}{|c|c|c|c|}
\hline $\begin{array}{l}\text { 1. Model } \\
\text { Area }\end{array}$ & 2. AMRs and Other Documents (see note) & 3. Models Identified & 4. Bin \\
\hline $\begin{array}{l}\text { K. Waste } \\
\text { Form } \\
\text { Degra- } \\
\text { dation: } \\
\text { Solubility } \\
\text { Con- } \\
\text { straints } \\
\end{array}$ & $\begin{array}{l}\text { K.8 Pure-Phase Solubility Limits - LANL (ANL-EBS-MD-000017 Rev. 00 ICN 1) } \\
\text { K.9 Summary of Dissolved Concentration Limits (ANL-WIS-MD-000010 Rev. 01) } \\
\text { K.10 Secondary Uranium Phase Paragenesis and Incorporation of Radionuclides Into } \\
\text { Secondary Phases (ANL-EBS-MD-000019 Rev. 00) }\end{array}$ & $\begin{array}{l}\text { Pure-Phase Solubility Limits (K. 8) } \\
\text { Dissolved Concentration Limits (K.9) } \\
\text { Secondary Uranium-Phase Paragenesis and } \\
\text { Incorporation of Radionuclides into } \\
\text { Secondary Phases (K.10) } \\
\text { Bin Subtotals: Bin 1: } 0 \text { Bin 2: } 3 \text { Bin 3: } 0\end{array}$ & $\begin{array}{l}2 \\
2 \\
2\end{array}$ \\
\hline $\begin{array}{l}\text { K. Waste } \\
\text { Form } \\
\text { Degra- } \\
\text { dation: } \\
\text { Cladding } \\
\text { Degra- } \\
\text { dation }\end{array}$ & $\begin{array}{l}\text { K.11 Initial Cladding Condition (ANL-EBS-MD-000048 Rev. 00 ICN 1) } \\
\text { K.12 Clad Degradation - Localized Corrosion of Zirconium and Its Alloys Under Repository } \\
\text { Conditions (ANL-EBS-MD-000012 Rev. 00) } \\
\text { K.13 Hydride-Related Degradation of SNF Cladding Under Repository Conditions } \\
\text { (ANL-EBS-MD-000011 Rev. 00 ICN 1) } \\
\text { K.14 Clad Degradation - Wet Unzipping (ANL-EBS-MD-000014 Rev. 00) } \\
\text { K.15 Clad Degradation - Dry Unzipping (ANL-EBS-MD-000013 Rev. 00) } \\
\text { K.16 Clad Degradation - Summary and Abstraction (ANL-WIS-MD-000007 Rev. 00 ICN 1) } \\
\text { K.17 Stainless Steel in WPs for TSPA-SR (CAL-WIS-MD-000010 Rev. 00) } \\
\text { K.18 Thermal Evaluation of Breached 21-PWR WPS (CAL-UDC-ME-000002 Rev. 00) } \\
\text { K.19 Breakage of CSNF Cladding by Mechanical Loading (CAL-EBS-MD-000001 Rev. 00) } \\
\text { K.20 Clad Degradation - FEPs Screening Arguments (ANL-WIS-MD-000008 Rev. 00 ICN 1) }\end{array}$ & $\begin{array}{l}\text { Initial Oxide Thickness (K.11-1) } \\
\text { Rod Internal Pressure (K.11-2) } \\
\text { Cladding Crack Depth (K.11-3) } \\
\text { Overall Cladding Stress (K.11-4) } \\
\text { Initial Rod Failure (K.11-5) } \\
\text { Zircaloy Corrosion Rate (K.12) } \\
\text { Residual Stress in CSNF Cladding } \\
\quad \text { Material (K.13) } \\
\text { Alternative Wet Clad Unzipping Model (K.14-1) } \\
\text { Bounding Model for Clad Unzipping } \\
\quad \text { Velocity (K.14-2) } \\
\text { Clad Dry Unzipping Model (K.15) } \\
\text { Summary and Abstraction - Clad Unzipping and } \\
\quad \text { Fuel Dissolution (K.16) } \\
\text { Stainless Steel in WPs for TSPA-SR (K.17) } \\
\text { Thermal Evaluation of Breached 21-PWR } \\
\text { WPs (K.18) } \\
\text { Breakage of CSNF Cladding by Seismic } \\
\text { Loading (K.19-1) } \\
\text { Breakage of CSNF Cladding by Static } \\
\text { Loading (K.19-2) } \\
\text { Bin Subtotals: Bin 1: } 1 \text { Bin 2: } 9 \text { Bin 3: } 5\end{array}$ & $\begin{array}{l}2 \\
2 \\
2 \\
2 \\
2 \\
2 \\
2 \\
3 \\
1 \\
2 \\
3 \\
2 \\
3 \\
3 \\
3\end{array}$ \\
\hline
\end{tabular}


Table 6. Summary of Binning Results from Model Validation Status Review, for Each Model Area (continued)

\begin{tabular}{|c|c|c|c|}
\hline $\begin{array}{l}\text { 1. Model } \\
\text { Area } \\
\end{array}$ & 2. AMRs and Other Documents (see note) & 3. Models Identified & 4. Bin \\
\hline $\begin{array}{l}\text { W. Waste } \\
\text { Form } \\
\text { Degra- } \\
\text { dation: } \\
\text { Colloid } \\
\text { Release }\end{array}$ & $\begin{array}{l}\text { K.21 Colloid-Associated Radionuclide Concentration Limits: ANL (ANL-EBS-MD-000020 } \\
\text { Rev. } 00 \text { ICN 1) } \\
\text { K.22 Waste Form Colloid-Associated Concentration Limits: Abstraction and Summary } \\
\text { (ANL-WIS-MD-000012 Rev. 00 ICN 1) }\end{array}$ & $\begin{array}{l}\text { Colloid-Associated Radionuclide Concentration } \\
\quad \text { Limits (K.21) } \\
\text { WF Colloid-Associated Concentration Limits: } \\
\quad \text { Abstraction and Summary (K.22) } \\
\text { Bin Subtotals: Bin 1: } 0 \text { Bin 2: } 2 \text { Bin 3:0 }\end{array}$ & 2 \\
\hline $\begin{array}{l}\text { L. EBS } \\
\text { Degra- } \\
\text { dation }\end{array}$ & $\begin{array}{l}\text { L.1 Drift Degradation Analysis (ANL-EBS-MD-000027 Rev. 01) } \\
\text { L.2 Fracture Geometry Analysis for the Stratigraphic Units of the Repository Host Horizon } \\
\text { (ANL-EBS-GE-000006 Rev. 00;) } \\
\text { L.3 Rockfall on DS (CAL-EDS-ME-000001 Rev. 00) } \\
\text { L.4 Committed Materials in Repository Drifts (CAL-GCS-GE-000002 Rev. 00) } \\
\text { L.5 EBS Features, Events, and Processes (ANL-WIS-PA-000002 Rev. 01) } \\
\text { L.6 Flow of Water and Pooling in a WP (ANL-EBS-MD-000055 Rev. 00) } \\
\text { H.3 EBS Physical and Chemical Environment Model (ANL-EBS-MD-000033 Rev. 01) } \\
\text { H.7 Seepage/Cement Interaction (ANL-EBS-MD-000043 Rev. 00) } \\
\text { H.10 Seepage/Backfill Interaction (ANL-EBS-MD-000039 Rev. 00) } \\
\text { M.5 EBS Radionuclide Transport Abstraction (ANL-WIS-PA-000001 Rev. 00 ICN 2) }\end{array}$ & $\begin{array}{l}\text { DRKBA Rockfall Model (L. } 1) \\
\text { Rockfall on DS Model (L. } 3) \\
\text { Flow into WPs Through Small Lid Openings } \\
\text { Model (L.6) }\end{array}$ & $\begin{array}{l}3 \\
2 \\
3\end{array}$ \\
\hline $\begin{array}{l}\text { M. EBS } \\
\text { Radio- } \\
\text { nuclide } \\
\text { Transport }\end{array}$ & $\begin{array}{l}\text { M.1 Invert Diffusion Properties Model (ANL-EBS-MD-000031 Rev. 01) } \\
\text { M.2 EBS Radionuclide Transport Model (ANL-EBS-MD-000034 Rev. 00 ICN 1) } \\
\text { M.3 In-Drift Colloids and Concentration (ANL-EBS-MD-000042 Rev. 00) } \\
\text { M.4 Seepage/Invert Interactions (ANL-EBS-MD-000044 Rev. 00) } \\
\text { M.5 EBS Radionuclide Transport Abstraction (ANL-WIS-PA-000001 Rev. 00 ICN 2) } \\
\text { L.5 EBS Features, Events, and Processes (ANL-WIS-PA-000002 Rev. 01) }\end{array}$ & $\begin{array}{l}\text { In-Drift Colloids and Concentrations (M.3) } \\
\text { Invert Diffusion Properties Model (M.1) } \\
\text { In-Drift Transport of Radionuclides (M.2) } \\
\text { EBS Radionuclide Transport Abstraction } \\
\text { Model (M.5) } \\
\text { Seepage/Invert Interactions Model (M.4) } \\
\text { Bin Subtotals: Bin 1: } 0 \text { Bin 2: } 1 \text { Bin 3: } 4\end{array}$ & $\begin{array}{l}3 \\
2 \\
3 \\
3\end{array}$ \\
\hline $\begin{array}{l}\text { N. UZ } \\
\text { Transport }\end{array}$ & $\begin{array}{l}\text { N.1 UZ and SZ Transport Properties (ANL-NBS-HS-000019 Rev. O0 ICN 1) } \\
\text { N.2 UZ Colloid Transport Model (ANL-NBS-HS-000028 Rev. 00) } \\
\text { N.3 Radionuclide Transport Models Under Ambient Conditions (MDL-NBS-HS-000008 } \\
\text { Rev. 00) } \\
\text { N.4 Particle Tracking Model and Abstraction of Transport Processes } \\
\text { (ANL-NBS-HS-000026 Rev. 00) } \\
\text { N.5 Analysis of Base-Case Particle Tracking Results of the Base-Case Flow Fields } \\
\text { (ID: U0160) (ANL-NBS-HS-000024 Rev. 00) } \\
\text { N.6 Analysis Comparing Advective-Dispersive Transport Solution to Particle Tracking } \\
\text { (ANL-NBS-HS-000001 Rev. 00) } \\
\text { N.7 Abstraction of Flow Fields for TSPA (ANL-NBS-HS-000023 Rev. 00 ICN 1) }\end{array}$ & $\begin{array}{l}\text { Equilibrium Matrix Sorption Basis (N.1-1) } \\
\text { Fracture Sorption (N.1-2) } \\
\text { Matrix Diffusion (N.1-3) } \\
\text { Colloid Transport (N.2-1) } \\
\text { Pu Sorption on Colloids (N.2-2) } \\
\text { FRACL Calibration to Borehole Chloride (N.3-1) } \\
\text { EOS9nT Calibration to ESF Chloride } \\
\quad \text { Profile (N.3-2) } \\
\text { Fracture-to-Matrix Colloid Filtration (N.4) }\end{array}$ & $\begin{array}{l}2 \\
2 \\
2 \\
1 \\
2 \\
3 \\
2 \\
2\end{array}$ \\
\hline
\end{tabular}


Table 6. Summary of Binning Results from Model Validation Status Review, for Each Model Area (continued)

\begin{tabular}{|c|c|c|c|}
\hline $\begin{array}{c}\text { 1. Model } \\
\text { Area }\end{array}$ & 2. AMRs and Other Documents (see note) & 3. Models Identified & 4. Bin \\
\hline $\begin{array}{l}\text { N. UZ } \\
\text { Transport } \\
\text { (continued) }\end{array}$ & $\begin{array}{l}\text { N. } 8 \text { Fault Displacement Effects on Transport in the UZ (ANL-NBS-HS-000020 Rev. 01) } \\
\text { C. } 8 \text { Features, Events, and Processes in UZ Flow and Transport (ANL-NBS-MD-000001 } \\
\text { Rev. } 01\end{array}$ & Bin Subtotals: Bin 1: 1 Bin 2: 6 Bin 3: 1 & \\
\hline O. SZ Flow & $\begin{array}{l}\text { O.1 Hydrogeologic Framework Model for the SZ Site-Scale Flow and Transport Model } \\
\text { (ANL-NBS-HS-000033 Rev. 00 ICN 1) } \\
\text { O.2 Water Level Data Analysis for the SZ Site-Scale Flow and Transport Model } \\
\text { (ANL-NBS-HS-000034 Rev. 00 ICN 1) } \\
\text { O.3 Recharge and Lateral Groundwater Flow Boundary Conditions for the SZ Site-Scale } \\
\text { Flow and Transport Model (ANL-NBS-MD-000010 Rev. 00) } \\
\text { O.4 Geochemical and Isotopic Constraints on Groundwater Flow Directions, Mixing, and } \\
\text { Recharge (ANL-NBS-HS-000021 Rev. 00 ICN 1) } \\
\text { O.5 Calibration of the Site-Scale SZ Flow Model (MDL-NBS-HS-000011 Rev. 00) } \\
\text { O.6 FEPs in Saturated Zone Flow and Transport (ANL-NBS-MD-000002 Rev. 01) }\end{array}$ & Site-Scale Saturated Zone Flow Model (O.5) & 2 \\
\hline $\begin{array}{l}\text { P. SZ } \\
\text { Transport }\end{array}$ & $\begin{array}{l}\text { P.1 Probability Distribution for Flowing Interval Spacing (ANL-NBS-MD-000003 } \\
\text { Rev. 00 ICN 1) } \\
\text { P.2 SZ Colloid Facilitated Transport (ANL-NBS-HS-000031 Rev. 00) } \\
\text { P.3 Uncertainty Distribution for Stochastic Parameters (ANL-NBS-MD-000011 Rev. 00) } \\
\text { P.4 Input and Results of the Base-Case SZ Flow and Transport Model for TSPA } \\
\text { (ANL-NBS-HS-000030 Rev. 00) } \\
\text { P.5 SZ Transport Methodology and Transport Component Integration } \\
\text { (MDL-NBS-HS-000010 Rev. 00) } \\
\text { P.6 Modeling Sub-Gridblock Scale Dispersion in 3-D Heterogeneous Fractured Media } \\
\text { (ANL-NBS-HS-000022 Rev. 00 ICN 1) } \\
\text { N.1 UZ and SZ Transport Properties (ANL-NBS-HS-000019 Rev. 00 ICN 1) } \\
\text { O.6 FEPs in Saturated Zone Flow and Transport (ANL-NBS-MD-000002 Rev. 01) }\end{array}$ & $\begin{array}{l}\text { Sub-Gridblock Scale Dispersion in 3- } \\
\quad \text { Dimensional Heterogeneous Fractured } \\
\text { Media (P.6) } \\
\text { Saturated Zone Colloid Facilitated } \\
\text { Transport (P.2) } \\
\text { Pipe Model for Daughter Radionuclides (P.4-1) } \\
\text { Abstraction of FEHM and Coupling with UZ } \\
\text { Mass Flux (P.4-2) } \\
\text { Transport Parameters from C-Wells and } \\
\text { Laboratory Studies (P.4-3) } \\
\text { Bin Subtotals: Bin 1: } 1 \text { Bin 2: } 2 \text { Bin 3: } 2\end{array}$ & $\begin{array}{l}2 \\
2 \\
3 \\
3\end{array}$ \\
\hline Q. Biosphere & $\begin{array}{l}\text { Q.1 Groundwater Usage by the Proposed Farming Community (ANL-NBS-MD-000006 } \\
\text { Rev. 00) } \\
\text { Q.2 Identification of Ingestion Exposure Parameters (ANL-MGR-MD-000006 Rev. 00) } \\
\text { Q.3 Input Parameter Values for External and Inhalation Radiation Exposure Analysis } \\
\text { (ANL-MGR-MD-000001 Rev. 01) } \\
\text { Q.4 Dose Conversion Factor Analysis: Evaluation of GENII-S Dose Assessment Methods } \\
\text { (ANL-MGR-MD-000002 Rev. 00) } \\
\text { Q.5 Identification of the Critical Group (Consumption of Locally Produced Food and Tap } \\
\text { Water) (ANL-MGR-MD-000005 Rev. 01) } \\
\text { Q.6 Environmental Transport Parameter Analysis (ANL-MGR-MD-000007 Rev. 00 ICN 1) }\end{array}$ & $\begin{array}{l}\text { Crop Interception Fraction Submodel (Q.2-1) } \\
\text { Irrigation Rate Submodel (Q.2-2) } \\
\text { Dose Conversion for Ingestion (Q.4-1) } \\
\text { Dose Conversion for Inhalation (Q.4-2) } \\
\text { Dose Conversion for External Exposure (Q.4-3) } \\
\text { Resuspension Model (Q.9-2) } \\
\text { Plant Uptake Model (Q.9-3) } \\
\text { Surface Soil Model in GENII-S (Q.9-1) } \\
\text { Radionuclide Transfer to Animals (Q.9-4) } \\
\text { Radionuclide Transfer to Aquatic Food (Q.9-5) }\end{array}$ & $\begin{array}{l}1 \\
1 \\
1 \\
1 \\
2 \\
2 \\
2 \\
3 \\
3 \\
3\end{array}$ \\
\hline
\end{tabular}


Table 6. Summary of Binning Results from Model Validation Status Review, for Each Model Area (continued)

\begin{tabular}{|c|c|c|c|}
\hline $\begin{array}{l}\text { 1. Model } \\
\text { Area }\end{array}$ & 2. AMRs and Other Documents (see note) & 3. Models Identified & 4. Bin \\
\hline $\begin{array}{l}\text { Q. Biosphere, } \\
\text { continued }\end{array}$ & $\begin{array}{l}\text { Q.7 Transfer Coefficient Analysis (ANL-MGR-MD-000008 Rev. 00 ICN 2) } \\
\text { Q.8 Evaluate Soil/Radionuclide Removal by Erosion and Leaching (ANL-NBS-MD-000009 } \\
\text { Rev. 00 ICN 1) } \\
\text { Q.9 Nominal Performance Biosphere Dose Conversion Factor Analysis } \\
\text { (ANL-MGR-MD-000009 Rev. 01) } \\
\text { Q.10 Non-Disruptive Event Biosphere Dose Conversion Factor Sensitivity Analysis } \\
\text { (ANL-MGR-MD-000010 Rev. 00) } \\
\text { Q.11 Distribution Fitting to the Stochastic BDCF Data (ANL-NBS-MD-000008 } \\
\text { Rev. 00 ICN 1) } \\
\text { Q.12 Abstraction of BDCF Distributions for Irrigation Periods (ANL-NBS-MD-000007 } \\
\text { Rev. 00 ICN 1) } \\
\text { Q.13 Evaluation of the Applicability of Biosphere-Related FEPs (ANL-MGR-MD-000011 } \\
\text { Rev. 01) }\end{array}$ & Bin Subtotals: Bin 1: 4 Bin 2: 3 Bin $3: 3$ & \\
\hline \begin{tabular}{|l} 
R. Disruptive \\
Events: \\
Igneous \\
Disruption \\
Conse- \\
quences
\end{tabular} & $\begin{array}{l}\text { R.1 Characterize Framework for Igneous Activity at Yucca Mountain, Nevada } \\
\text { (ANL-MGR-GS-000001 Rev. } 00 \text { ICN 1) } \\
\text { R.2 Characterize Eruptive Processes at Yucca Mountain, Nevada (ANL-MGR-GS-000002 } \\
\text { Rev. 00) } \\
\text { R.3 Igneous Consequence Modeling for the TSPA-SR (ANL-WIS-MD-000017 } \\
\text { Rev. 00 ICN 1) } \\
\text { R.4 Dike Propagation Near Drifts (ANL-WIS-MD-000015 Rev. 00 ICN 1) } \\
\text { R.5 Disruptive Event Biosphere Dose Conversion Factor Analysis (ANL-MGR-MD-000003 } \\
\text { Rev. 01) } \\
\text { R.6 Disruptive Event Biosphere Dose Conversion Factor Sensitivity Analysis } \\
\text { (ANL-MGR-MD-000004 Rev. 00) } \\
\text { R.7 DTN: MO0006PAPVE03.001 (Documentation of BCDF input provided for TSPA-SR) } \\
\text { R.8 Miscellaneous Waste-Form FEPs (ANL-WIS-MD-000009 Rev. 00 ICN 1) } \\
\text { R.9 Number of WPs Hit by Igneous Intrusion (CAL-WIS-PA-000001 Rev. 01) } \\
\text { R.10 Features, Events, and Processes: Disruptive Events (ANL-WIS-MD-000005 } \\
\text { Rev. 00 ICN 1) }\end{array}$ & $\begin{array}{l}\text { Conditional Distribution for Number of Eruptive } \\
\text { Centers Model (R.1) } \\
\text { Geometry of Volcanic Feeder System } \\
\text { Model (R.2) } \\
\text { Volcanic Eruption Release Model (R.3-1) } \\
\text { In-drift Damage Due to Dike Intersection } \\
\text { Model (R.3-2) } \\
\text { Mass Loading Decay Model Following } \\
\text { Deposition of Volcanic Ash (R.5-1) } \\
\text { Dose Conversion Factor Model for Inhalation - } \\
\text { Igneous Disruption (R.5-2) }\end{array}$ & $\begin{array}{l}2 \\
3 \\
2 \\
2 \\
2 \\
2\end{array}$ \\
\hline
\end{tabular}


Table 6. Summary of Binning Results from Model Validation Status Review, for Each Model Area (continued)

\begin{tabular}{|c|c|c|c|}
\hline $\begin{array}{c}\text { 1. Model } \\
\text { Area }\end{array}$ & 2. AMRs and Other Documents (see note) & 3. Models Identified & 4. Bin \\
\hline $\begin{array}{c}\text { S. Seismic } \\
\text { Hazard }\end{array}$ & $\begin{array}{l}\text { S.1 Characterize Framework for Seismicity and Structural Deformation at YM } \\
\text { (ANL-CRW-GS-000003 Rev. 00) } \\
\text { S.2 Effects of Fault Displacement on Emplacement Drifts (ANL-EBS-GE-000004 } \\
\text { Rev. 00 ICN 1) } \\
\text { N.8 Fault Displacement Effects on Transport in the UZ (ANL-NBS-HS-000020 Rev. 01) }\end{array}$ & $\begin{array}{l}\text { Vibratory Ground Motion Hazard (S.1-1) } \\
\text { Fault Rupture Hazard (S.1-2) } \\
\text { Bin Subtotals: Bin 1:0 Bin 2: } 2 \text { Bin 3: } 0\end{array}$ & $\begin{array}{l}2 \\
2\end{array}$ \\
\hline $\begin{array}{l}\text { T. Integrated } \\
\text { Site Model }\end{array}$ & $\begin{array}{l}\text { T.1 Geologic Framework Model (MDL-NBS-GS-000002 Rev. } 00 \text { ICN 2) } \\
\text { T.2 Mineralogical Model (MDL-NBS-GS-000003 Rev. } 00 \text { ICN 1) } \\
\text { T.3 Rock Properties Model (MDL-NBS-GS-000004 Rev. } 00 \text { ICN 2) }\end{array}$ & $\begin{array}{l}\text { Only analyses were identified in this model } \\
\text { area. } \\
\text { Bin Subtotals: Bin 1:0 Bin 2: } 0 \text { Bin 3: } 0\end{array}$ & N/A \\
\hline $\begin{array}{l}\text { U. PA } \\
\text { Modeling }\end{array}$ & $\begin{array}{l}\text { U.1 TSPA Model for SR (MDL-WIS-PA-000002 Rev. 00) } \\
\text { U.2 Total System Performance for Site Recommendation (TDR-WIS-PA-000001 } \\
\quad \text { Rev. 00 ICN 1) } \\
\text { U.3 Features, Events, and Processes: System Level and Criticality (ANL-WIS-MD-000019 } \\
\quad \text { Rev. 00) } \\
\text { U.4 Performance Assessment and Sensitivity Analysis of Disposal of Plutonium as Can-in- } \\
\quad \text { Canister Ceramic (ANL-WIS-PA-000003 Rev. 00) }\end{array}$ & $\begin{array}{l}\text { TSPA-SR Model (U.1-1) } \\
\text { Soil Removal Model for Volcanic } \\
\quad \text { Disruption (U.1-2) } \\
\text { Pu-Ceramic Degradation Model for } \\
\quad \text { TSPA-SR (U.4) } \\
\text { Bin Subtotals: Bin 1:0 Bin 2: } 0 \text { Bin 3: } 3\end{array}$ & $\begin{array}{l}3 \\
3 \\
3\end{array}$ \\
\hline & & $\begin{array}{l}\text { Bin Grand Totals: } \\
\text { Bin 1: } 17 \text { Bin 2: } 77 \text { Bin 3: } 34 \\
\text { Total Number of Models Identified: } 128\end{array}$ & \\
\hline
\end{tabular}

NOTE: For bibliographic citations associated with the documents listed in Column 2, please see Column 2 of Table 1. Italics denote documents which are principally assigned to another model area, but were reviewed in multiple model areas for completeness. 
Table 7. Summary of Bin-3 Models, Showing How They are Used, and the Model Designation in the Principal Supporting Document

\begin{tabular}{|c|c|c|c|c|c|c|}
\hline & Model Area & Models Identified & Bin & $\begin{array}{l}\text { Used for } \\
\text { TSPA? }\end{array}$ & $\begin{array}{l}\text { Used for } \\
\text { FEPs? }\end{array}$ & $\begin{array}{l}\text { Called a Model } \\
\text { or Other? }^{A}\end{array}$ \\
\hline 1 & G. Mountain-Scale/Near-Field THM & THM Model (G.1) & 3 & $\mathrm{~N}$ & $\bar{Y}$ & $\mathrm{C}$ \\
\hline $\begin{array}{l}2 \\
3 \\
4 \\
5 \\
6 \\
\end{array}$ & H. In-Drift Chemistry & $\begin{array}{l}\text { Cement Grout Model (H.3-2) } \\
\text { Seepage/Cement Interaction Model (H.7) } \\
\text { Corrosion of Steel Used in the Ex-Container EBS (H.3-3) } \\
\text { Effect of Evaporation in the Invert (H.3-4) } \\
\text { EBS Colloids Model }(\mathrm{H} .3-5)\end{array}$ & $\begin{array}{l}3 \\
3 \\
3 \\
3 \\
3\end{array}$ & $\begin{array}{l}N \\
N \\
N \\
N \\
N\end{array}$ & $\begin{array}{l}Y \\
N \\
Y \\
Y \\
N\end{array}$ & $\begin{array}{l}M \\
A \\
M \\
M \\
M\end{array}$ \\
\hline $\begin{array}{l}7 \\
8 \\
9 \\
\end{array}$ & $\begin{array}{l}\text { I. EBS Moisture Distribution and } \\
\text { TH }\end{array}$ & $\begin{array}{l}\text { In-Drift THC Model (1.7) } \\
\text { Effective Thermal Conductivity Model (1.8) } \\
\text { Ventilation Model (1.5) }\end{array}$ & $\begin{array}{l}3 \\
3 \\
3\end{array}$ & $\begin{array}{l}N \\
Y \\
N\end{array}$ & $\begin{array}{l}N \\
N \\
N\end{array}$ & $\begin{array}{c}A+M \\
C \\
M\end{array}$ \\
\hline $\begin{array}{l}10 \\
11\end{array}$ & $\begin{array}{l}\text { J. Waste Package/Drip Shield } \\
\text { Degradation: General and } \\
\text { Localized Corrosion }\end{array}$ & $\begin{array}{l}\text { WAPDEG Analysis of WP and DS Degradation (J.6) } \\
\text { Incorp. of Uncert. \& Variability of DS \& WP } \\
\text { Degradation in WAPDEG (J.8) }\end{array}$ & $\begin{array}{l}3 \\
3\end{array}$ & $\begin{array}{l}\mathrm{Y} \\
\mathrm{N}\end{array}$ & N & $\begin{array}{c}A+M \\
A\end{array}$ \\
\hline $\begin{array}{l}12 \\
13 \\
\end{array}$ & $\begin{array}{l}\text { K. Waste Form Degradation: } \\
\text { General Information }\end{array}$ & $\begin{array}{l}\text { Waste Form Degradation Abstract. - Best Estimate Model (K.4-3) } \\
\text { Waste Form Degradation Abstract. - Immobilized Pu Model (K.4-4) }\end{array}$ & $\begin{array}{l}3 \\
3 \\
\end{array}$ & $\begin{array}{l}N \\
N\end{array}$ & $\begin{array}{l}N \\
Y\end{array}$ & $\begin{array}{l}M \\
M\end{array}$ \\
\hline $\begin{array}{l}14 \\
15 \\
16 \\
17 \\
18 \\
\end{array}$ & $\begin{array}{l}\text { K. Waste Form Degradation: } \\
\text { Cladding Degradation }\end{array}$ & $\begin{array}{l}\text { Alternative Wet Clad Unzipping Model (K.14-1) } \\
\text { Summary and Abstract. - Clad Unzipping \& Fuel Dissolution (K.16) } \\
\text { Thermal Evaluation of Breached 21-PWR WPS (K.18) } \\
\text { Breakage of CSNF Clad by Seismic Loading (K.19-1) } \\
\text { Breakage of CSNF Clad by Static Loading (K.19-2) } \\
\end{array}$ & $\begin{array}{l}3 \\
3 \\
3 \\
3 \\
3\end{array}$ & $\begin{array}{l}\mathrm{Y} \\
\mathrm{Y} \\
\mathrm{Y} \\
\mathrm{Y} \\
\mathrm{N}\end{array}$ & $\begin{array}{l}N \\
N \\
Y \\
N \\
N\end{array}$ & $\begin{array}{l}\text { M } \\
\text { A } \\
\text { C } \\
\text { C }\end{array}$ \\
\hline $\begin{array}{l}19 \\
20 \\
\end{array}$ & L. EBS Degradation & $\begin{array}{l}\text { DRKBA Rockfall Model (L.1) } \\
\text { Flow into WPs Through Small Lid Openings Model (L.6) }\end{array}$ & $\begin{array}{l}3 \\
3\end{array}$ & $\begin{array}{l}\mathrm{Y} \\
\mathrm{N}\end{array}$ & $\begin{array}{l}\mathrm{Y} \\
\mathrm{N}\end{array}$ & $\begin{array}{l}A+M \\
A+M\end{array}$ \\
\hline $\begin{array}{l}21 \\
22 \\
23 \\
24\end{array}$ & M. EBS Radionuclide Transport & $\begin{array}{l}\text { In-Drift Colloids and Concentrations (M.3) } \\
\text { In-Drift Transport of Radionuclides (M.2) } \\
\text { EBS Radionuclide Transport Abstraction Model (M.5) } \\
\text { Seepage/Invert Interaction Model (M.4) }\end{array}$ & $\begin{array}{l}3 \\
3 \\
3 \\
3\end{array}$ & $\begin{array}{l}Y \\
N \\
Y \\
N\end{array}$ & $\begin{array}{l}\mathrm{Y} \\
\mathrm{N} \\
\mathrm{N} \\
\mathrm{Y}\end{array}$ & $\begin{array}{c}M \\
A+M \\
M \\
M\end{array}$ \\
\hline 25 & N. UZ Transport & FRACL Calibration to Borehole Chloride (N.3-1) & 3 & $Y$ & $\mathrm{~N}$ & $A+M$ \\
\hline $\begin{array}{l}26 \\
27 \\
\end{array}$ & P. SZ Transport & $\begin{array}{l}\text { Abstraction of FEHM and Coupling with UZ Mass Flux (P.4-2) } \\
\text { Transport Parameters from C-Wells and Laboratory Studies (P.4-3) }\end{array}$ & $\begin{array}{l}3 \\
3\end{array}$ & $\begin{array}{l}Y \\
Y \\
\end{array}$ & $\begin{array}{l}N \\
Y\end{array}$ & $\begin{array}{l}A+M \\
A+M\end{array}$ \\
\hline $\begin{array}{l}28 \\
29 \\
30\end{array}$ & Q. Biosphere & $\begin{array}{l}\text { Surface Soil Model in GENII-S (Q.9-1) } \\
\text { Radionuclide Transfer to Animals (Q.9-4) } \\
\text { Radionuclide Transfer to Aquatic Food (Q.9-5) }\end{array}$ & $\begin{array}{l}3 \\
3 \\
3\end{array}$ & $\begin{array}{l}Y \\
Y \\
Y\end{array}$ & $\begin{array}{l}N \\
N \\
N\end{array}$ & $\begin{array}{l}A+M \\
A+M \\
A+M\end{array}$ \\
\hline 31 & R. Igneous Disruption & Geometry of Volcanic Feeder System Model (R.2) & 3 & $Y$ & $\mathrm{~N}$ & A \\
\hline $\begin{array}{l}32 \\
33 \\
34\end{array}$ & U. PA Modeling & $\begin{array}{l}\text { TSPA Model (U.1-1) } \\
\text { Soil Removal Model for Volcanic Disruption (U. 1-2) } \\
\text { Pu-Ceramic Degradation Model for TSPA-SR (U.4) }\end{array}$ & $\begin{array}{l}3 \\
3 \\
3\end{array}$ & $\begin{array}{l}\mathrm{Y} \\
\mathrm{Y} \\
\mathrm{N}\end{array}$ & $\begin{array}{l}Y \\
N \\
N\end{array}$ & $\begin{array}{l}M \\
M \\
A\end{array}$ \\
\hline
\end{tabular}

Notes: A. Based on designation in original document ( $M$ " = model, " $A$ " = analysis, and " $C$ " = calculation). For $A M R s$ that contain model( $(s)$ and analysis, " $A+M$ " is used. 
Table 8. Models which are Documented as Analyses or Calculations

\begin{tabular}{|c|c|c|c|}
\hline & Model Area & Models Documented as Analyses or Calculations & $\begin{array}{c}\text { Documents Recommended to } \\
\text { Become Models }\end{array}$ \\
\hline 1 & A. Climate & Precession-Based Orbital Clock (A.1) & USGS (2000) \\
\hline 2 & G. Mountain-Scale/Near-Field THM & THM Model (G.1) & CRWMS M\&O (2000g) \\
\hline $\begin{array}{l}3 \\
4\end{array}$ & H. In-Drift Chemistry & $\begin{array}{l}\text { In-Drift Gas Flux and Composition Model }(\mathrm{H} .5) \\
\text { Seepage/Cement Interaction Model }(\mathrm{H} .7)\end{array}$ & $\begin{array}{l}\text { CRWMS M\&O (2000am) } \\
\text { CRWMS M\&O (2000bf) }\end{array}$ \\
\hline 5 & 1. EBS Moisture Distribution and TH & Effective Thermal Conductivity Model (1.8) & CRWMS M\&O (2001g) \\
\hline 6 & $\begin{array}{l}\text { J. Waste Package/Drip Shield } \\
\text { Degradation: General and } \\
\text { Localized Corrosion }\end{array}$ & $\begin{array}{l}\text { Calculation of General Corrosion Rate of DS and WPOB to Support } \\
\text { WAPDEG Analysis (J.7) } \\
\text { Incorporation of Uncertainty and Variability of DS and WP Degradation } \\
\text { in WAPDEG (J.8) }\end{array}$ & $\begin{array}{l}\text { CRWMS M\&O (2000f) } \\
\text { CRWMS M\&O (2000ak) }\end{array}$ \\
\hline 8 & $\begin{array}{l}\text { K. Waste Form Degradation: General } \\
\text { Information }\end{array}$ & Inventory Abstraction (K.1) & BSC (2001i) \\
\hline $\begin{array}{l}9 \\
10\end{array}$ & $\begin{array}{l}\text { K. Waste Form Degradation: Solubility } \\
\text { Constraints }\end{array}$ & $\begin{array}{l}\text { Pure-Phase Solubility Limits (K.8) } \\
\text { Secondary Uranium-Phase Paragenesis and Incorporation of } \\
\text { Radionuclides into Secondary Phases (K.10) }\end{array}$ & $\begin{array}{l}\text { CRWMS M\&O (2001n) } \\
\text { CRWMS M\&O (2000bd) }\end{array}$ \\
\hline $\begin{array}{l}11 \\
12 \\
13 \\
14 \\
15 \\
16 \\
17 \\
18 \\
19 \\
20 \\
21\end{array}$ & $\begin{array}{l}\text { K. Waste Form Degradation: Cladding } \\
\text { Degradation }\end{array}$ & $\begin{array}{l}\text { Initial Oxide Thickness (K.11-1) } \\
\text { Rod Internal Pressure (K.11-2) } \\
\text { Cladding Crack Depth (K.11-3) } \\
\text { Overall Cladding Stress (K.11-4) } \\
\text { Initial Rod Failure (K.11-5) } \\
\text { Residual Stress in CSNF Cladding Material (K.13) } \\
\text { Summary and Abstract. - Clad Unzipping \& Fuel Dissolution (K.16) } \\
\text { Stainless Steel in WPs for TSPA-SR (K.17) } \\
\text { Thermal Evaluation of Breached 21-PWR WPs (K.18) } \\
\text { Breakage of CSNF Clad by Seismic Loading (K.19-1) } \\
\text { Breakage of CSNF Clad by Static Loading (K.19-2) }\end{array}$ & $\begin{array}{l}\text { CRWMS M\&O (2000ao) } \\
\text { CRWMS M\&O (2000ao) } \\
\text { CRWMS M\&O (2000ao) } \\
\text { CRWMS M\&O (2000ao) } \\
\text { CRWMS M\&O (2000ao) } \\
\text { CRWMS M\&O (2001k) } \\
\text { CRWMS M\&O (2001d) } \\
\text { CRWMS M\&O (2000bh) } \\
\text { CRWMS M\&O (1999f) } \\
\text { CRWMS M\&O (1999a) } \\
\text { CRWMS M\&O (1999a) } \\
\end{array}$ \\
\hline 22 & $\begin{array}{l}\text { K. Waste Form Degradation: Colloid } \\
\text { Release }\end{array}$ & Colloid-Associated Radionuclide Concentration Limits (K.21) & CRWMS M\&O (2001e) \\
\hline 23 & L. EBS Degradation & Rockfall on Drip Shield Model (L.3) & CRWMS M\&O (2000bw) \\
\hline $\begin{array}{l}24 \\
25 \\
26 \\
27 \\
28\end{array}$ & Q. Biosphere & $\begin{array}{l}\text { Crop Interception Fraction Submodel (Q.2-1) } \\
\text { Irrigation Rate Submodel (Q.2-2) } \\
\text { Dose Conversion for Ingestion (Q.4-1) } \\
\text { Dose Conversion for Inhalation (Q.4-2) } \\
\text { Dose Conversion for External Exposure (Q.4-3) }\end{array}$ & $\begin{array}{l}\text { CRWMS M\&O (2000ai) } \\
\text { CRWMS M\&O (2000ai) } \\
\text { CRWMS M\&O (1999b) } \\
\text { CRWMS M\&O (1999b) } \\
\text { CRWMS M\&O (1999b) }\end{array}$ \\
\hline
\end{tabular}


Table 8. Models which are Documented as Analyses or Calculations (continued)

\begin{tabular}{|l|l|l|l|}
\hline \multicolumn{1}{|c|}{ Model Area } & \multicolumn{1}{|c|}{ Models Documented as Analyses or Calculations } & \multicolumn{1}{c|}{$\begin{array}{c}\text { Documents Recommended to } \\
\text { Become Models }\end{array}$} \\
\hline 29 & R. Igneous Disruption & $\begin{array}{l}\text { Conditional Distribution for Number of Eruptive Centers Model (R.1) } \\
\text { Geometry of Volcanic Feeder System Model (R.2) }\end{array}$ & $\begin{array}{l}\text { CRWMS M\&O (2000I) } \\
\text { CRWMS M\&O (2000k) }\end{array}$ \\
\hline 30 & & $\begin{array}{l}\text { Vibratory Ground Motion Hazard (S.1-1) } \\
\text { Fault Rupture Hazard (S.1-2) }\end{array}$ & $\begin{array}{l}\text { CRWMS M\&O (2000m) } \\
\text { CRWMS M\&O (2000m) }\end{array}$ \\
\hline 32 & S. Seismic Hazard & Pu-Ceramic Degradation Model for TSPA-SR (U.4) & CRWMS M\&O (2001u) \\
\hline 33 & U. PA Modeling &
\end{tabular}


Table 9. Recommended Analyses Identified in This Review which are Documented as Models

\begin{tabular}{|c|c|c|}
\hline & Model Area & Analyses Which Are Documented as Models \\
\hline 1 & F. Mountain-Scale/Near-Field THC & $\begin{array}{l}\text { F.3 Thermal Tests Thermal/Hydrological Analyses/Model Report (ANL-NBS-TH-000001 } \\
\text { Rev. 00 ICN 1; CRWMS M\&O 2000bk) }\end{array}$ \\
\hline $\begin{array}{l}2 \\
3\end{array}$ & H. In-Drift Chemistry & $\begin{array}{l}\text { H.4 In-Drift Corrosion Products (ANL-EBS-MD-000041 Rev. 00; CRWMS M\&O 1999g) } \\
\text { H.8 Physical and Chemical Environmental Abstraction Model (ANL-EBS-MD-000046 } \\
\text { Rev. } 00 \text { ICN 1; CRWMS M\&O 2000az) } \\
\text { H.10 Seepage/Backfill Interaction (ANL-EBS-MD-000039 Rev. 00; CRWMS M\&O 2000cb) }\end{array}$ \\
\hline $\begin{array}{l}5 \\
6 \\
\end{array}$ & 1. EBS Moisture Distribution and TH & $\begin{array}{l}\text { I.3 Water Diversion Model (ANL-EBS-MD-000028 Rev. 00; CRWMS M\&O 2000bu) } \\
\text { 1.4 Water Drainage Model (ANL-EBS-MD-000029 Rev. 00 ICN 1; CRWMS M\&O 2000bv) }\end{array}$ \\
\hline $\begin{array}{l}7 \\
8\end{array}$ & L. EBS Degradation & $\begin{array}{l}\text { L.2 Fracture Geometry Analysis for the Stratigraphic Units of the Repository Host Horizon } \\
\text { (ANL-EBS-GE-000006 Rev. 00; CRWMS M\&O 2000ae) }\end{array}$ \\
\hline 9 & O. Saturated Zone Flow & $\begin{array}{l}\text { O.1 Hydrogeologic Framework Model for the SZ Site-Scale Flow and Transport Model } \\
\text { (ANL-NBS-HS-000033 Rev. 00 ICN 1; CRWMS M\&O 2001Z) }\end{array}$ \\
\hline 10 & P. Saturated Zone Transport & $\begin{array}{l}\text { P.5 SZ Transport Methodology and Transport Component Integration (MDL-NBS-HS-000010 } \\
\text { Rev. 00; CRWMS M\&O 2000ck) }\end{array}$ \\
\hline $\begin{array}{l}11 \\
12 \\
13\end{array}$ & T. Integrated Site Model & $\begin{array}{l}\text { T.1 Geologic Framework Model (MDL-NBS-GS-000002 Rev. } 00 \text { ICN 2; BSC 2001e) } \\
\text { T.2 Mineralogical Model (MDL-NBS-GS-000003 Rev. } 00 \text { ICN 1; CRWMS M\&O 2000at) } \\
\text { T.3 Rock Properties Model (MDL-NBS-GS-000004 Rev. 00 ICN 2; CRWMS M\&O 2000bb) }\end{array}$ \\
\hline
\end{tabular}




\title{
Appendix I
}

\author{
(8 pages)
}

\section{Additional Comments and Recommendations on the}

\section{Drift-Scale Test Thermal-Hydrologic-Chemical Calibration Model (F.1-1) and the Thermal-Hydrologic-Chemical Seepage Model (F.1-2) \\ by Dash Sayala}

The following additional recommendations are made concerning the thermal-hydrologicalchemical modeling (THC) approach documented in AMR: Drift-Scale Coupled Processes (DST and THC Seepage) Models (BSC 2001a):

\section{Comments on Input Data}

Justify the use of input data that are different than known repository conditions, and assess the impact of those choices on the validation.

Section 4.0 Inputs - Data and Parameters: Input data appropriateness and qualification are not adequately met for parameter calibration, and for reasonableness and consistency. It appears some input data are not consistent between the DST THC and the THC Seepage Models. The choices for input data and references used are not explained in some important ways, depicted by the examples in the following paragraphs.

On p. 31, Section 4.1, it is mentioned that additional thermodynamic data, not specific to Yucca Mountain, are required for all models. The thermodynamic data used are taken from different sources, and some values may be inconsistent. The text should explain what kind of uncertainty the choice of thermodynamic data engenders, and how it could impact the model predictions. The discussion should address whether thermodynamic data can dictate unrealistic dissolution and precipitation reaction trends regardless of other data inputs. Further, the thermodynamic and kinetic data used for calcite is not well developed. From the references, it is not obvious whether the currently used thermodynamic data conform to applicable international ICSU/CODATA and NIST data standards. Conduct comparisons to establish confidence in the data used, especially for calcite.

Molar volumes of alteration minerals are typically greater than for the reactant minerals. Explain whether the selection of minerals, and the chemical data that described their behavior in the model, could significantly impact the porosity reduction predictions.

On p. 31, Section 4.1.1.1, it is stated that the data sets include calibrated and uncalibrated properties (porosity, temperatures and thermal conductivities). What does this say about model validity when more-representative data are mixed with non-representative data? Explain what uncertainty is introduced by this practice.

On p. 34 and Table $3, \mathrm{CO}_{2}$ partial pressures and $\mathrm{pH}$ are calculated by equilibrating with calculated concentrations of bicarbonate, hematite, and secondary silicate minerals at $17^{\circ} \mathrm{C}$ and $25^{\circ} \mathrm{C}$. These types of data do not represent the expected repository conditions, especially for the pre-closure period with ventilation exhaust and low RH. First, are such data appropriately extrapolated to higher temperatures? Secondly, although this is a validation test, the AMR should 
justify how these results apply to expected higher repository temperatures. The measured 400 ppmv $\mathrm{CO}_{2}$ concentration may be due to equilibration with atmospheric $\mathrm{CO}_{2}$; explain why this is not so.

Simulations using all data from the revised EQ3/6 V7.2b databases (Section 4.1.4.1) may not yield the same agreement between simulations and field test data, as was obtained using the modifications described in Attachment V. Testing of the sensitivity of model results to thermodynamic input data is needed for confidence in the model predictions.

Under Section 4.1.6 on Transport Parameters (p. 42), diffusion coefficients are apparently used for aqueous and gaseous species. However, the same values are not suitable for both aqueous and gaseous species, and dispersion may be important in either case. As such a classical equation of this form may be appropriate:

$$
\mathrm{D}=\mathrm{D}_{\mathbf{0}} \tau+\alpha \mathrm{V}(\theta)
$$

where $\quad \mathrm{D}=$ Dispersion coefficient (units of length ${ }^{2} / \mathrm{time}$ )

$D_{0}=$ Free-phase diffusion coefficient (length ${ }^{2} /$ time )

$\tau=$ Tortuosity (length/length, i.e., dimensionless)

$\alpha=$ Dispersivity (length)

$\mathrm{V}=$ Solute velocity (length/time), as a function of volumetric water content $\theta$ (volume/volume, i.e., dimensionless)

Further, the equation used on p. 42 considers the gas constant, which is indicative of an ideal state function that does not apply to liquids, and temperature and pressure which may not be representative of initial and elevated temperature conditions in the repository. Justify use of these parameters in the model, including all assumptions.

On p. 32, Section 4.1.2 on Mineralogical Data, there are no criteria for selecting the set of primary and secondary minerals, and it is not clear what is the total mineralogical content of the rocks under consideration. For AMR: In-Drift Microbial Communities (CRWMS M\&O 2000an) biotite was used as one of the important minerals in the analysis, and this mineral is not considered in the THC Seepage Models.

On p. 34, Section 4.1.3 on Water and Gas Chemistry, it is stated that full characterization of Tptpll pore-waters is unavailable because of insufficient data, yet various additional species are included for model input. Concentrations of iron, aluminum and total carbonate were not measured, but are calculated. Justify this approach, and consider the effect on model results and uncertainty.

Applicable codes and standards are not listed under Section 4.3. With regard to thermodynamic data, international and NIST standards should be addressed to establish more confidence in the data and consequential simulations.

\section{Comments on Assumptions}

Some assumptions are not justified by defensible arguments, and some assumptions are confusing. Examples of these are given in the following: 
In the case of the DST THC, Assumption 17 on p. 52 is problematic because it is restrictive; limiting reaction due to ionic strength greater than 2 , or liquid saturations less than $10^{-4}$, is an artifact and not realistic. If the code cannot be modified to avoid this problem, then additional justification is needed, including sensitivity testing to determine the effect on model results. This problem with the modeling approach does impact reliability of model results and may require impact review.

In the assumptions listed for the DST model (p. 53) there is no discussion of the effect of cement used in the DST on the $\mathrm{CO}_{2}$ mass-balance. Concrete used in construction will be a significant sink for $\mathrm{CO}_{2}$.

It is stated that the dual-permeability approach is validated by comparison of measured geochemical data to results of simulations presented, and no further justification is necessary ( $p$. 49, Section 5.A). This statement seems to be without justification. Also, $\mathrm{CO}_{2}$ partial pressures resulting from heating calcite and gas transport are mentioned. However, the rationale is not given for not using other gases such as $\mathrm{Cl}, \mathrm{F}, \mathrm{HF}$ and water vapor (which are found) in combination with $\mathrm{CO}_{2}$ for model simulations.

In point A.7 (p. 50), a constant thickness product layer of $10 \mu \mathrm{m}$ thick on the glass surface was assumed for calculating the rate constant at $25{ }^{\circ} \mathrm{C}$. However, no rationale was given for considering this temperature, which is not representative of postclosure repository conditions. In addition, it is nearly impossible to dissolve silica glass at that temperature unless there are concentrated reactants such as HF in contact with the glass.

Explanation given (see point A.8 on p. 50) for the assumption that $\mathrm{pH}$ effects are not important for the THC processes is not justified because the stability of silicate and non-silicate minerals generally depends on $\mathrm{Eh}$ and $\mathrm{pH}$, and under conditions of low water activity (i.e. dryout) the given assumption may not be applicable.

In point $A .10$ on p. 51, mineral dissolution and precipitation are assumed to be uniform over the fracture walls. This is not realistic because, mineral dissolution depends on grain size and degree of grain contact with dissolving solution. Further, dissolution rates of silicate minerals are different from non-silicate minerals. Similarly, precipitation trends for silicate and non-silicate minerals are different and are governed by temperature, pressure and saturation indices at a given area. What are the consequences of these assumptions?

\section{Comments on Model Development}

Section 6.0 on p. 57 lacks integrating discussion. In some instances the discussion leads to questionable justification or selection of parameters or data. Uncertainty and sensitivity analyses are not treated statistically, and much improvement is needed for model validation. Some examples are given in the following paragraphs:

Explain why the models are run discontinuously, for the pre-closure and postclosure periods, as opposed to running continuously.

Some model simulations are run with the TOUGHREACT V.2.2 and others by TOUGHREACT V2.3 (see p. 59), and the later version of the code was not qualified for the most recent revision. Explain whether this would have any impact on the model predictions. 
In a general sense, for a multi-component system, both major and minor mineral constituents may influence the kinetic reactions of mineral dissolution and precipitation that could affect the porosity and permeability. Provide justification for the constituents included, considering those that may be present in the natural system, and the potential reactions that could affect hydrologic properties.

In the THC Seepage Models the dissolution of primary silicates occurs at repository temperatures. To help validate the simulation results, compare and corroborate with observations of dissolution of those minerals as reported in the literature.

In estimating the porosity reduction, consider the possible effects of pore occlusion due to colloids or suspended clays.

\section{Comments on Validation Strategy}

Clearly define the validation criteria, especially in regard to the aqueous and gas-phase modeldata comparisons. Although aqueous and gaseous processes are certainly coupled, the data collection methods, sampling points, and transport phenomena are different and discrete validation requirements are needed. Apply specific observations from natural analogues to provide greater confidence in the validation strategy.

Comparisons between simulations and field data (taken from two boreholes only) are clearly more defensible for the $\mathrm{CO}_{2}$ behavior than for aqueous chemical transport. Validation criteria are currently liberally defined trends rather than firm, objective confidence-building requirements. Tests of a reactive transport model should include components directly involved in the reactions as well as conservative components, and must have clearly defined spatial and temporal validation criteria. It is assumed that many more data are forthcoming in the near future, and that the authors are awaiting laboratory analysis of samples collected during the DST. With these new data, the validation should focus on key processes. For example, do trends in aqueous concentrations simply represent dilution from condensate coupled with precipitation of calcite, or do they permit a defensible test of the model capability to predict silicate mineral dissolution?

Are the DST data actually sufficient to test the reactive chemical transport (THC) model? Before this question can be answered, the use of the test data for fully testing thermal-hydrologic (TH) models must be demonstrated. This is especially important if the DST is to be the defining experiment for building confidence in simulations of long-term repository near-field processes. Justify whether model-data comparisons using the limited available field data are sufficient for model validation, given the implications of modeling uncertainties for long-term repository predictions. The validation strategy for complex THC simulations cannot have been optimized, so justification of the validation approach actually used requires a demonstration that we understand what improvements to the test and/or the validation strategy could increase confidence.

The $\mathrm{CO}_{2}$ gas phase composition and isotopic content were monitored. Model validation should address the isotopic signatures of the $\mathrm{CO}_{2}$-this is important. This type of comparison has the potential to confirm $\mathrm{CO}_{2}$ sources and transport pathways, and the nature of $\mathrm{CO}_{2}$ partitioning to the liquid phase. These pathways are simulated in the model and a validation procedure would benefit from approach. Further the ${ }^{14} \mathrm{C}$ data could be used to evaluate $\mathrm{CO}_{2}$ introduced from the 
observation and access drifts. This question is important to a mass balance for $\mathrm{CO}_{2}$ in the driftrock-water system.

Much of effort to simulate the DST is focused on calcite dissolution and precipitation kinetics. In this regard, the validation strategy should address the use of pre-test and post-test observations of calcite in the matrix and on fracture surfaces.

The sensitivity studies report modifications of input parameters in order to demonstrate the effects on the simulations. Where these sensitivity changes improve the simulations and are then subsequently used in seepage models, it should be noted that this was a calibration step, not just a sensitivity test. When implementing model-data comparisons, use a statistical approach to quantify improvement in simulation accuracy (and confidence), so that the relative effects of different changes to the model inputs can be compared.

The validation effort should also include minerals. Some discussion is needed concerning what minerals are predicted by the model to be present after the DST, and where they can be found. The value of any post-test coring, borehole video surveys, or other sampling will be enhanced if pre-test model predictions are made. This type of validation is crucial to building confidence in the predictive capabilities. All subsequent seepage models are predictive and their validation depends on the confidence established in this comparison of the DST model with experimental data. If this validation is weak, so will be the validation for the seepage models.

DST THC model simulations of porosity change due to calcite precipitation were on the order of $0.1 \%$ (Section 6.2 .7 .5 on p. 94) but in the THC Seepage Model simulations, using a base-case and extended cases with silicate and non-silicate minerals, it was on the order of $1 \%$ to $3 \%$ for $20,000 \mathrm{yr}$ and 100,000 yr respectively (Section 6.4.5.2 on p. 168, and Section 6.6.5.2 on p. 207). For validation purposes use the same approach for the DST and the THC Seepage Model simulations, including the same sets of minerals.

To improve the documentation, it would be useful to include tables with all relevant input data, the modeled output data, and the corresponding statistics of model-data agreement. Such presentation should be used for the DST THC Model, the THC Seepage Model, the Plug-Flow test simulations, and natural analogs or any other type of comparison that is included in the model validation strategy.

To test and validate the geochemical models developed for the DST THC, and the Tptpmn and Tptpll THC Seepage Models, kinetic simulations of tuff dissolution rates, under isothermal conditions with initial rock and water compositions, were accomplished using a Crushed-Tuff Experiment and a Plug-Flow Reactor Experiment with conditions that are different from those in the repository. (See pp. 57 and 212, Sections 6 and 6.7). It should be made clear that this is primarily a verification case study for testing the geochemical code, and the conceptual basis, and is not intended as direct validation of the THC Seepage Models. Crushed tuff samples are highly reactive and provide dissolution rates and compositions that may be significantly different from uncrushed samples.

It is suggested that the sensitivity analysis section of the plug-flow test comparison is really a calibration of the model, because the data were not matched to a high degree of confidence using only the base case. Numerous simulations were performed with different inputs such as variable surface area, mineral compositions, and corrections to experimental data before a satisfactory 
match was achieved. Validation would require a simulation that met validation criteria using a calibrated model, or using input parameters that were pre-selected and justified. It is not clear that the calibration procedure has improved the predictive capability of the THC modeling methods. In our opinion this is inverse modeling and not predictive. Additional confidence could be provided if $\mathrm{pH}$, and bicarbonate as well as $\mathrm{Mg}, \mathrm{Fe}, \mathrm{SO}_{4}, \mathrm{Al}$, etc. and other minerals, were predicted as well. Nevertheless, since this work is not extrapolated to the DST, it does not directly impact validation of the field-scale models.

Under Section 6.2.7 on Simulation Results and Model Validation by Comparison to Measured Data, it is mentioned that "There are no experimental data by which the THC Seepage Models can be validated ...validation of the DST model effectively validates them as well" (see paragraph 3, p. 73). Published hydrothermal seepage experimental and natural analog data are available, and by comparing with these, confidence in validation can be augmented. For example, published descriptions of experiments on granite cores, and natural analogs such as the Salton Sea trough hydrothermal system are available in the open literature.

\section{Recommendation for Systematic Model Development}

Consider a Systems Engineering approach to describing the model inputs and components, and exhibit the following through schematic diagrams:

- Show the model prediction domain in the context of the overall repository system. Establish the relevant regulatory compliance requirements and the corresponding information needs for the drift-system.

- Schematically establish a hierarchy of submodels. Develop a conceptual model, outlining the relevant processes and the scenario-based implications on repository system integrity. Based on the processes, implications and information needs, establish the needs of type of mathematical modeling (including the codes and standards) and Validation/ corroborative studies/experiments needed. Finally, lead into how you would accomplish the model simulations and their validation. Also show, how these simulations are fed to other related studies.

\section{Comments on Integration and Documentation}

In validating the models, the results of other pertinent models and investigations, such as performed in the areas of UZ Flow (Model Area C, Section 6.3), Mountain-Scale TH (Model Area D, Section 6.4), Ambient/Thermal Drift Seepage (Model Area E, Section 6.5), MountainScale/Near-field THM (Model Area G, Section 6.7), and In Drift Chemistry (Model Area H, Section 6.8), should be integrated to build confidence. Integration with other activities needs to be extensively addressed in the documentation.

Among the THC Seepage Models described, it is not clear which one best represents the actual expected repository conditions and is most appropriate.

It is not clear how the model output is used for predicting the hydrologic flow and chemical transport in the repository environment, and the implications for integrity of engineered barriers. 
In many sections, authors refer to previous work without defining the relevant information; thus it is difficult to establish the relevance to model validation. This AMR should be a stand-alone report; therefore, more complete description of previous work is needed.

\section{Other Comments and Recommendations}

Under the section on Purpose (p. 23) too much unnecessary information is given and the scope was included.

It would be useful to have an introductory section with background, purpose/objective and scope as subheadings. Establish the relevant regulatory compliance requirements and the corresponding information needs for the drift-system. 
INTENTIONALLY LEFT BLANK 


\title{
Appendix II
}

\author{
(10 pages)
}

\section{Additional Comments and Recommendations on the}

\section{Total System Performance Assessment - Site Recommendation Model (U.1): \\ Basis for Assessment of Validation for the TSPA-SR Model \\ by Ken Bogen}

The TSPA-SR analysis/model report (AMR) is intended to "...describe the integration of information that represents different aspects of the repository, into one comprehensive model. ... This AMR provides detail as to how these component models are implemented in the TSPASR model" (Total System Performance Assessment (TSPA) Model for Site Recommendation, CRWMS M\&O 2000bl, p. 25). A corresponding technical report (Total System Performance Assessment for the Site Recommendation, CRWMS M\&O 2000bm) which describes modeling results obtained using the integrated TSPA-SR model and its application to support SR, contains some background as well as technical material that also is presented in the TSPA-SR model report (CRWMS M\&O 2000bl). These two reports should be merged into a single document that presents the basis and validation of an integrated TSPA model, or some material currently in the technical report (CRWMS M\&O 2000bm) but not in the model report (CRWMS M\&O $2000 \mathrm{bl}$ ) needs to be reproduced in a revised model report as explained below. In either case, model validation could be improved for the specific reasons listed below.

\section{Some Aspects of the Integrated Model Are Not Clearly Described}

The integrated TSPA-SR model is described in Section 6 of the AMR (CRWMS M\&O 2000bl, p. 80), which dwells primarily on the architecture of the system model in relation to its component (sub)models, and on the mechanics of operating the integrated stochastic model under different assumption, together with a concluding section (Section 6.5, p. 542-559) entitled "Model Validation." However, validation of the integrated TSPA-SR model as a system model, must refer to more than a listing of model components, data-transfer pathways, and modeloperation mechanics; it must also refer to the design and operation of the system model as a whole as one that can produce output that is meaningfully related to the intended purpose of the model. The intended purpose of the model is stated in Section 1 of the AMR (CRWMS M\&O $2000 \mathrm{bl}$, p. 25) to be "to assist the Performance Assessment Operations (PAO) and its Engineered Barrier Performance Section in analyzing the performance of the repository system in isolating waste for long periods of time." For the purposes of this review, it seems useful to infer that an additional purpose of the model is to support SR in its evaluations of the potential repository with respect to criteria specified in DOE's proposed regulation 10 CFR 963 (64 FR 67054).

As indicated in proposed 10 CFR 963.16, one of the bases for the evaluation of site suitability will be "a total system performance assessment to evaluate the ability of the geologic repository to meet the applicable radiation protection standard...." Applicable radiation protection standards are the NRC's proposed 10 CFR 63 (66 FR 55732) and the EPA's final 40 CFR 197 (2001). Final guidance on the methods for compliance with the NRC and EPA standards is not yet available, and is expected to be provided by the NRC prior to any submittal of a license application. Preliminary comments to the DOE from the NRC on the adequacy of the TSPA-SR 
were provided in a technical exchange in August 2001 (Cornell 2001). This technical exchange resulted in a set of agreements between the $\mathrm{NRC}$ and DOE regarding additional information about the TSPA that will be needed prior to submittal of any License Application. These agreements, plus any subsequent guidance provided by the NRC, are an appropriate basis for determining the adequacy of the TSPA model for the purpose of evaluating performance with respect to the NRC and EPA regulations.

For the purposes of this review, it is appropriate to note that regulatory criteria in both proposed 10 CFR 63 and final 40 CFR 197 involve consideration of uncertainty in model predictions. With respect to the treatment of uncertainty, the technical documentation should define (1) the underlying approach to uncertainty analysis; and, (2) specific criteria with which output from the model can be used to demonstrate compliance, and what specific model output(s) are intended to address these specified criteria. Each of these topics is discussed below.

\subsection{Approach to Uncertainty Analysis}

The treatment of uncertainty analysis, and the use of sensitivity analysis, in analyses involving the TSPA-SR model are topics covered explicitly in Sections 2.2.4 through 2.2.5 of the TSPASR technical report (CRWMS M\&O 2000bm, p. 2-34 through 2-44). It is recommended that this material should also be incorporated within the TSPA-SR model report (CRWMS M\&O $2000 \mathrm{bl}$ ). The incorporation of this information needs to clarify what is meant by the distinction between "uncertainty" and "variability" within the integrated probabilistic TSPA framework (CRWMS M\&O 2000bm, Section 2.2.4, p. 2-34 through 2-35):

"The parameters of the model used to predict the performance of the disposal system are also subject to uncertainty and/or variability. Uncertainty in model parameters arises because of imperfect knowledge or limited data and, in principle, can be reduced with additional measurements. ... Variability refers to the randomness or heterogeneity in physical and/or behavioral characteristics. It is an intrinsic property of the system and cannot be reduced by additional information. ... Often, variability and uncertainty in a parameter are commingled because of imprecise knowledge. ... This leads to a situation where the inputs of the TSPA model (i.e., scenarios, mathematical and conceptual models, and parameters) are uncertain and/or variable, which will therefore result in the output of the model being uncertain as well. As described in the following sections, a probabilistic framework has been adopted in TSPA-SR for translating uncertainties in model inputs to corresponding uncertainties in model predictions."

The definition of "variability," in particular, given above is not quite the same as that for "variability" that generally is used in the context of uncertainty/variability analysis undertaken for the purpose of environmental risk assessment (Bogen and Spear, 1987; National Research Council, 1994; Bogen 1995). Specifically, the distinction being made above for the purpose of TSPA-SR analysis is questioned by this reviewer because: (1) the distinction appears to have no practical impact on the method of analysis undertaken, and (2) it is only "uncertainty" (as correctly defined above, and not "variability" as defined above) that causes and accounts for all "uncertainty" (as defined above) in TSPA-model output. In other words, no TSPA-model variate 
that strictly reflects a spatially and/or temporally variable/heterogeneous quantity (as defined above) - by itself - produces any uncertainty in model output. This is expected to be the case if the impact of any and all sources of variability and heterogeneity (independent of the uncertainties) averages out to zero over the relevant spatial and temporal dimensions, as the TSPA is implemented for a substantial duration such as 10,000 yr. On the other hand, if the extent or character of a specific source of variability or heterogeneity is itself uncertain, then this uncertainty may contribute to uncertainty in TSPA model output.

If the conclusion just stated is incorrect, the AMR needs to explain clearly why this is so, using one or more specific examples. If not, then references to modeled "variability" need to be explained to clarify the relevance of this information to model performance and to the interpretation of model output.

\subsection{Implementation Criteria for the System Model}

As noted above, validation of the integrated TSPA-SR model, as a system model, must refer to more than an enumeration of model components, data-transfer pathways, and mechanics of operating a computerized implementation of the TSPA model. It must also refer to (1) implementation criteria for the integrated system model that meaningfully relate model output to the intended purpose of the model, and (2) other issues bearing on the validation of the integrated model. Topic (1) will be discussed below in this part of the appendix, and topic (2) is discussed in parts 2 through 4 that follow.

As inferred for the purpose of this review, the intended purpose of the TSPA-SR model is to support SR in a way that relates to specific licensing criteria. As discussed in Section 1.3 of the TSPA-SR technical report (CRWMS M\&O 2000bm, p. 1-8) key regulatory criteria are contained in proposed NRC regulation 10 CFR 63 (66 FR 55732) and in EPA regulation 40 CFR 197 (2001; proposed at the time the TSPA-SR documents were prepared, but now available in final form). The discussion in Section 1.3 of that report does not appear in the corresponding TSPASR model report (CRWMS M\&O 2000bl), nor are key regulatory criteria interpreted in either document in an explicit manner.

A key requirement in proposed 10 CFR 63 (66 FR 55732, Part 63 Section 31) is that DOE must provide reasonable assurance that the expected annual dose to the average member of the critical group does not exceed the postclosure performance objective (as provided in 40 CFR 197) within $10,000 \mathrm{yr}$ of site closure. This requirement unambiguously refers to reasonable assurance that must be provided concerning the quantitative value of a specific estimator of (i.e., statistic estimating) predicted dose-namely, the expected value of predicted dose-to a specified receptor (i.e., the average member of the critical group within $10,000 \mathrm{yr}$ of site closure). In contrast, the corresponding key requirement in 40 CFR 197 (2001) found in $\$ 197.13$ and $\S 197.14$ quoted below, is:

\footnotetext{
" $\$ 197.13$ How is subpart B implemented?

"The NRC implements this subpart B. The DOE must demonstrate to NRC that there is a reasonable expectation of compliance with this subpart before NRC may issue a license. In the case of the specific numerical requirements in $\$ 197.20$ of this subpart, and if performance assessment is used to demonstrate compliance with the specific numerical requirements in $\S \S 197.25$ and 197.30 of this subpart, NRC will determine compliance
} 
based upon the mean of the distribution of projected doses of DOE's performance assessments which project the performance of the Yucca Mountain disposal system for 10,000 years after disposal.

\section{" $\$ 197.14$ What is a reasonable expectation?}

Reasonable expectation means that NRC is satisfied that compliance will be achieved based upon the full record before it. Characteristics of reasonable expectation include that it:

"(a) Requires less than absolute proof because absolute proof is impossible to attain for disposal due to the uncertainty of projecting long-term performance;

"(b) Accounts for the inherently greater uncertainties in making long-term projections of the performance of the Yucca Mountain disposal system;

“(c) Does not exclude important parameters from assessments and analyses simply because they are difficult to precisely quantify to a high degree of confidence; and

"(d) Focuses performance assessments and analyses upon the full range of defensible and reasonable parameter distributions rather than only upon extreme physical situations and parameter values."

The relevant numerical criterion in the EPA regulation cited here $(15 \mathrm{mrem} / \mathrm{yr})$ is somewhat less than that specified in the NRC regulation $(<25 \mathrm{mrem} / \mathrm{yr})$. Otherwise, the EPA criterion is conceptually similar to that of NRC in that the former requirement (at \$197.13) refers to a "reasonable expectation" that must be provided concerning the quantitative value of a specific estimator of (i.e., statistic estimating) predicted dose-namely, in this case, the mean value of dose predicted using stochastic modeling methods. Because $\$ 197.13$ clearly states that "NRC will determine compliance based upon the mean of the distribution of projected doses," and because "mean of the distribution of projected [annual] doses" and "expected annual dose" are synonymous phrases in this context, this line of reasoning would assert that the EPA and NRC criteria stated above are operationally identical ( $\$ 197.14$ notwithstanding). Before discussing an alternative reasonable interpretation of 40 CFR 197, implications of the interpretations given above for determining corresponding TSPA model-performance criteria will be described.

The interpretation above implies that the EPA and NRC regulatory criteria discussed can be satisfied jointly only if DOE demonstrates a reasonable expectation/assurance that the expected value of predicted "critical-target" dose (CTD) will not exceed $15 \mathrm{mrem} / \mathrm{yr}$ as maximum annual committed effective groundwater dose equivalent to the specified representative critical person within $10,000 \mathrm{yr}$ after site closure. The "expected value" in the context of a "distribution of projected doses" can only refer to the mathematical expectation of that distribution, which in turn consists of the "true population mean" of an infinite number of hypothetical integrated-TSPAmodel realizations. Notationally, the joint regulatory criterion (under the presently assumed interpretation) can thus be expressed as $\mathrm{E}(\mathrm{CTD})<15 \mathrm{mrem} / \mathrm{yr}$, where $\mathrm{E}$ here denotes the mathematical expectation operator. Notably, under this interpretation, the relevant criterion does not refer directly to uncertainty (e.g., that might be reflected in a shape statistic) per se associated with the predicted CTD distribution, but rather refers directly only to the E(CTD) (a location parameter of the predicted distribution). Therefore, this criterion is not very sensitive to the predicted likelihood, e.g., that CTD might exceed $15 \mathrm{mrem} / \mathrm{yr}$. Indeed, in the limiting case in which uncertainty in CTD is modeled approximately as a 2-point probability mass function with one point at $\mathrm{CTD}=0 \mathrm{mrem} / \mathrm{yr}$, the criterion implies that a "reasonable expectation" of 
compliance means the acceptability of any likelihood $p$ whatsoever that CTD will exceed (15/p) $\mathrm{mrem} / \mathrm{yr}$, where $0<p<1$.

Because only a finite number of TSPA model realizations can be simulated, a "reasonable expectation" concerning the value of E(CTD) can only be made if E(CTD) can be calculated either: 1) exactly (e.g., analytically, conditional on all distributed inputs); or 2) with respect to some "reasonable" upper bound with respect to uncertainty in some feasibly obtained estimator ( $\overline{\mathrm{CTD}}$ ) of E(CTD) (e.g., traditionally, a 2-tailed upper $95 \%$ confidence limit, or $97.5^{\text {th }}$ percentile). Conditional on a specified modeling scenario, such as the "base case" considered in the TSPA, the TSPA model described in the TSPA-SR technical report (CRWMS M\&O $2000 \mathrm{bm}$ ) calculates $\overline{\mathrm{CTD}}$ as the arithmetic mean of some number $n$ Monte-Carlo-simulated CTD realizations, i.e., as $\overline{\mathrm{CTD}}=\operatorname{Sum}\left[\mathrm{CTD}_{i}\right] / n$, where the value of $n$ used has been 100 to $500-$ see CRWMS M\&O 2000bm, p. 5-9). Nowhere in the TSPA-SR technical report is any reference made to uncertainty in $\mathrm{CTD}$, to a reasonable upper bound on that uncertainty (i.e. a suitable definition of $\overline{\mathrm{CTD}}^{*}$ ), or to a TSPA-implementation procedure intended to demonstrate that $\overline{\mathrm{CTD}}^{*}<15 \mathrm{mrem} / \mathrm{yr}$. Such a procedure needs to account for the fact that there is sampling error associated with any Monte Carlo estimate such as $\overline{\mathrm{CTD}}$, which can be addressed approximately by analytic methods (see Ang and Tang, 1984, p. 291-292) or by using the information obtained directly via the Monte Carlo calculation performed (see Bogen et al., 1997). Therefore, under the interpretation stated above and given the reasonable inference that evaluations of compliance with final NRC and EPA regulations will be one of the intended uses of the TSPA, the AMR for the integrated TSPA model needs to include additional discussion to adhere to the YMP QA requirement that "criteria used to evaluate the appropriateness and adequacy of the model for its intended use ... must be justified in the model documentation" (AP-3.10Q §5.3b).

The following paragraphs present an alternative approach to interpreting the requirements for compliance with 40 CFR 197. This interpretation approach has not been reviewed for consistency with available guidance from the NRC regarding the use of the mean for evaluations of compliance (such as the documentation of the recent Technical Exchange meeting on the Total System Performance Assessment Integration Key Technical Issue (Cornell 2001). Insofar as $\$ 197.13$ requires DOE to provide a "reasonable expectation" that, e.g., CTD $<15 \mathrm{mrem} / \mathrm{yr}$, but the outcome being modeled is the true CTD value that will actually occur within (and hence, can possibly only be known after) a period of $10,000 \mathrm{yr}$, standard engineering practice used in the context of analogous scenarios involving risk of future failure of durable structures dictates that consideration be given to some "reasonable" upper limit on the estimated likelihood that the specified CTD limit will be exceeded. This alternative interpretation is consistent with established engineering judgment that "consistent levels of safety and reliability may be achieved only if the criteria for design are based on ... probabilistic measures of reliability" (Ang and Tang, 1984, p. 4).

The first approach discussed above based only on E(CTD) collapses all information considered in relation to TSPA modeling for a Yucca Mountain repository to a single output measure of the type most useful for making decisions on the basis of "expected utility." This approach to decision making is reasonably guaranteed to be acceptable "in the long run" (Ang and Tang, 1984 , p. 16 and p. 68) for conditions under which similar decisions and corresponding outcome evaluations can be repeated many times. In the case of the Yucca Mountain repository, long- 
term outcome can only be determined in the remote future. Consequently, the rational basis of risk acceptability criteria based only on E(CTD) is unclear, in view of which it would be prudent to consider an alternative interpretation described below.

The alternative interpretation would involve showing that some "reasonable" (e.g., 1-tailed $100(1-p)^{\text {th }}$ percentile) upper confidence bound $\left(\mathrm{CTD}^{*}\right)$ on simulated CTD satisfies the required dose limitation, e.g., that CTD* $<15 \mathrm{mrem} / \mathrm{yr}$ for $p=0.1,0.05$, or 0.01 . Note that such a criterion is not necessarily more conservative than one based solely on $\mathrm{E}(\mathrm{CTD})$, because $\mathrm{E}(\mathrm{CTD})$ may exceed CTD* if the estimated CTD distribution is sufficiently positively skewed conditional on any value of $p<1$. For example, if CTD is approximately log-normally distributed with a specified geometric standard deviation (GSD), then E(CTD) $>\mathrm{CTD}^{*}$ if it is the case that GSD $>\exp [2 \Phi(1-p)]$, where $\Phi$ here denotes the standard normal cumulative distribution function. An alternative CTD-estimator, CTD ${ }^{* *}$, may be defined as the expected value of CTD conditional on Prob(dose $>$ CTD) $=1-p$. That is, $\mathrm{CTD}^{* *}$ is the mean value of the upper tail of the CTD distribution, or the conditional mean value of CTD ignoring all potential CTD realizations less than its unconditional $100(1-p)$ percentile value, for some "reasonable" value(s) of $p$ (such as $0.01,0.05,0.1$, or even 0.5 ) consistent with the $\$ 197.14(\mathrm{~d})$ requirement that reliance not be made "only upon extreme physical situations and parameter values." Note that CTD** > $\mathrm{E}$ (CTD) always unless CTD is a constant (i.e., is not uncertain). Thus defined, the CTD** estimator has the advantage that it satisfies 40 CFR 197 requirements stated both in $\$ 197.13$ (insofar as it is a "reasonable" conditional expectation) and in $\$ 197.14(\mathrm{~d})$ (insofar as it explicitly addresses CTD uncertainty, but does so in a "reasonable" way). CTD** can be estimated via Monte Carlo simulation by Sum[CTD $\left.{ }_{(i)}\right] /(p n)$, where (i) denotes the ordered $i^{\text {th }}$-largest among $n$ simulated CTD-realization values, where $i=(1-p) n, 1+(1-p) n, \ldots, n$.

Again, nowhere in the TSPA-SR technical report (CRWMS M\&O 2000bm) is any reference made to a suitable definition of CTD* (and/or CTD**) or to a TSPA-implementation procedure (as discussed above) intended to demonstrate that CTD* (and/or CTD**) $<15 \mathrm{mrem} / \mathrm{yr}$.

\section{Integrated Treatment of Parameter Uncertainty in the TSPA Model is Defective}

Validation of the integrated TSPA model as a system model, means that parameter uncertainty pertaining to each input distribution that is used to characterize uncertainty is characterized using appropriate information. In some cases, this may imply that one or more of the input distributions have the form of a compound distribution (e.g., a normal distribution for which the location and scale parameters are themselves represented by distributions reflecting uncertainty in these parameters). This is especially true for any input distribution that is estimated from a relatively small data set. For example, if uncertainty in a model input $\mathrm{X}$ is assumed to be normally distributed with mean $\mathrm{M}$ and variance $\mathrm{V}$ based on $n$ empirical measures or observations (e.g., for estimated values of a quantity reported in the literature, which a particular TSPA-model parameter is intended to model), and these observations have a corresponding sample mean and variance equal to $\mathrm{m}$ and $\mathrm{v}$, respectively, then integrated uncertainty in $\mathrm{M}$ should be modeled as $\mathrm{t}^{*} \mathrm{v} / \mathrm{Sqrt}[n]$ where $\mathrm{t}$ is Student $\mathrm{t}$-distributed with $n$ - 1 degrees of freedom, and relative uncertainty in $\mathrm{V}$ should also be modeled as $\mathrm{c} /(n-1)$ where $\mathrm{c}$ is chi-square-distributed with $n$ - 1 degrees of freedom and where $\mathrm{c}$ and $\mathrm{t}$ are independent. Thus, $\mathrm{X}$ should in this case be modeled using a dually compound normal distribution. For relatively large $n$ (e.g., $n>500$ ), uncertainties in $\mathrm{M}$ and $\mathrm{V}$ can reasonably be considered negligible and therefore can be ignored. 
Although final determinations of the adequacy of the approach with respect to regulatory requirements will be made by the $\mathrm{NRC}$, this review maintains that the above approach was not adopted, e.g., in the treatment of Biosphere dose-conversion factors (CRWMS M\&O 2000bl, p. 438-444). This section of the AMR (p. 439-440) refers to the use of Microsoft Excel-based minimum-chi-square fits of statistical distributions that were obtained to fairly small $(n=130)$ sets of simulated parameter values. In the case of each TSPA-model-parameter distribution for which an apparently reliable fit was obtained, corresponding uncertainty pertaining to the values of the fitting parameters was not included in the TSPA-SR uncertainty analysis (e.g., a compound distribution). Note that the accuracy of some statistical procedures in Microsoft Excel 97 has recently been called into question (McCullough and Wilson 1999). Furthermore, it is not made clear in the AMR why such distribution-modeling techniques were used, rather than relying directly on original assumptions regarding the underlying plausible distributions of individual TSPA-model parameters. It may expedite subsequent modeling to consolidate several (say, $k$ ) stochastic TSPA inputs into a single stochastic input parameter where feasible, so that one input rather than $k$ inputs must be sampled during each Monte Carlo evaluation. But loss in accuracy need not occur by taking this expedited approach, in contrast to the accuracy loss implied by empirical distribution functions that were used in the TSPA model to model inputs for which adequate parametric fits could not be obtained to model corresponding composite distributions (e.g., BDCF values for ${ }^{210} \mathrm{~Pb},{ }^{242} \mathrm{Pu},{ }^{226} \mathrm{Ra}$, and ${ }^{230} \mathrm{Th}$; see CRWMS M\&O 2000bl, p. 440-441).

\section{Stage-3 Verification of the Integrated TSPA Model is Deficient}

Validation of the integrated TSPA-SR model is discussed in Section 6.5 of the AMR (CRWMS $\mathrm{M} \& \mathrm{O}$ 2000bl, p. 542-557). In this section the explanation of Stage-3 model verification (Integrated Model Output Testing, p. 548-550) performed for TSPA-SR states:

\footnotetext{
"Integrated model output testing can be accomplished by careful evaluation of the model results, in this case dose, in response to the upstream feeds. For the TSPA model the general measure of performance is dose. ... The total system integrated model is the sum of the subsystem models coupled together using common input data and propagating changes in a logical order through the system, during a simulation. It can be demonstrated through a series of plots...that the integrated total system model is performing as expected. ...The TSPA-SR model has been carefully scrutinized to establish its agreement with the conceptual models developed in the relevant AMRs. This included verifying all of the data fields in the TSPA-SR model. This verification ensures that the input digital model is in accord with the conceptual model... The internal computations performed within the GoldSim code have been verified to be correct when the integrated model is implemented... All the external dynamically linked library routines (DLLs) have been verified under the GoldSim code command. The data transfers to and from the DLLs in the GoldSim code have been verified when the integrated model is implemented... The integrated total system model behaves as expected, and results from each subsystem model component are consistent with the entire total system model."
}

This summary of criteria for Stage-3 verification does not appear to include any test of whether or not the specific model output of interest (say, the maximum annual dose from the dominant radionuclide over $10,000 \mathrm{yr}$ ) actually equals the corresponding value calculated independently 
using alternative methods (e.g., analytic methods where feasible) linked to Monte Carlo calculations for remaining model inputs or steps that can only be solved numerically. To perform this test for numerically intensive TSPA model components, it may be sufficient for each component or scenario, to run the numerical model only once. It is likely that the result for a particular radionuclide could be related in a simple manner (e.g., linearly with time $t$ ) to the magnitude of the source function used (e.g., the rate of release from a waste package beginning at time $t_{0}$ ). Such simplification and verification have not been done for TSPA-SR.

This exercise could be undertaken using expected values for all stochastic inputs, after solving for predicted dose as some analytic and/or interpolated function $G$ of a linear function $L(\mathbf{x})$ of a reduced set of input model parameters $\mathbf{x}$, where $\mathbf{x}$ is a reduced set of intermediate quantities that reflect the output of previous, simplifying analytic calculations. An independent calculation of the expected values $\mathrm{E}(G(L(\mathbf{x}))) \mid(\mathbf{x}=\mathrm{E}(\mathbf{x}))$ will be facilitated by the ability to integrate over any (numerically derived, but symbolically specified) arbitrarily nonlinear function $G$ that is interpolated with great accuracy, as might be achieved using the commercially available symbolic software Mathematica ${ }^{\otimes}$ (Wolfram 1999). The value of going through this exercise would be to reveal any absolute or relative error that might be introduced by the GoldSim integration of the TSPA-SR system model (CRWMS M\&O 2000bl, Sections 6.2 and 6.3), in contrast to the component, dynamically linked submodels contained in the integrated TSPA-SR model. Such absolute or relative error may not be revealed by the analysis of model-outputbehavior figures as described above for the current approach to Step-3 model verification.

\section{Validation of Integrated TSPA Model Using AP-3.10Q \$5.3.b Requires Comparison of Model Predictions With Best-Available Sets of Relevant Field Data Involving YMP Analogs}

If validation of the TSPA model is to be achieved using the approach described in AP-3.10Q 5.3.b (in lieu of, or in addition to 5.3.c) then at least some completed examples are needed in which TSPA model predictions are compared systematically to relevant field data involving analogs. This approach would require careful consideration of the uncertainty distributions selected for relevant TSPA input parameters. Some comparisons along these lines are included in Total System Performance Assessment for the Site Recommendation (CRWMS M\&O 2000bm, Appendix C which is entitled: Natural-Analogue Investigations in Support of Performance Assessment of the Potential Yucca Mountain Radioactive-Waste Repository, p. C-13 and C-16). This source states that:

"...there are no rock-analysis data from beneath the [Nopal I uranium] ore body, and the fate of uranium in downward percolating water, such as modeled by the Repository Integration Program, cannot be corroborated by field data at this time. ... The analysis indicates that a groundwater-sampling program could provide data with which to estimate realistic transport parameters for the Peña Blanca site. By analogy, these parameters may be a useful tool in estimating the performance assessment of the Yucca Mountain site. ... Because of the paucity of well data and other hydrogeologic data for the area, the estimated direction and gradient of groundwater flow is highly uncertain. ... The tentative conclusions developed as a result of the modeling previously described could be enhanced or modified with the implementation of the drilling program described ... [and recommended]. These recommendations were designed to help provide data with which 
to more accurately define the magnitude and direction of the groundwater gradient in the vicinity of the Nopal I mine. The proposed additional monitor wells are also needed to provide water-sampling locations that will be used to calibrate future performanceassessment modeling in the Peña Blanca area."

Completion of activities such as the Nopal I study and comparative analysis recommended in the report quoted above, is needed for the TSPA system model to comply with AP-3.10Q $\S 5.3 b$. Such validation would be helpful in addition to the model verification and peer review approaches that have already been implemented.

\section{Monte Carlo Sample Size}

The TSPA-SR model (CRWMS M\&O 2000bl) is considered by this reviewer to require evaluation of the Monte Carlo sample sizes used in all applications of the system model to explore the parameter hyperspace involved and justify the sample-size. The set of all distributed inputs and their relative contributions to uncertainty in predicted critical dose should be summarized more clearly and concisely (including the use of a single comparative plot to convey this information). The AMR indicates that there are a relatively large number (perhaps more than 100) distributed inputs involved in the TSPA-SR model, and yet only 100 to 300 realizations were generated using Latin-Hypercube sampling. The bases for this sample size should be accompanied by supplementary analyses, and corresponding clear discussion of resulting evidence, demonstrating that nearly all parameters involved have negligible individual effects as well as negligible interactive (e.g., synergistic) effects. Because of the small sample size used, it is possible that interactive effects may not have been fully explored. To see why, suppose, for simplicity, that each of a hypothetical set of 5 independent input parameters $\left(x_{i}, i=\right.$ $1,2, \ldots, 5)$ is monotonically proportional to predicted dose $D$ as a function $f\left(x_{i}\right)$ of the vector $x_{i}$. Again for simplicity, approximate each $i^{\text {th }}$ corresponding input probability distribution function (pdf) by a corresponding 2-point approximating probability mass function (apmf), where in each $i^{\text {th }}$ case apmf $=\left\{\left\{\mathrm{x}_{\mathrm{i} 1}, 1-\mathrm{p}\right\},\left\{\mathrm{x}_{\mathrm{i} 2}, \mathrm{p}\right\}\right\}$ where $\mathrm{x}_{\mathrm{i} 2}$ is the mean of the upper tail (with probability mass $p$ ) of the $i^{\text {th }}$ pdf and $x_{i 1}$ is the corresponding mean of the complementary lower tail (with probability mass 1-p) of this pdf, for some constant $0<p<1$ (e.g., $p=25 \%$ ) (see, e.g., Bogen 1995). Now, unless unexpected synergy can be ruled out a priori, for example based on analysis of model structure, a complete exploration of the potential for substantial (and first order) synergistic interaction among these 5 stochastic variates requires that dose be simulated using all possible $\left(2^{5}\right.$ or 512$)$ combinations of the apmf-approximated $\mathrm{x}_{\mathrm{i}}$-values, after which a corresponding approximate pdf for dose can be constructed using discrete probability calculus or "DPC" (see Bogen 1995). If 30 variates were involved, this complete exploration would require more than $10^{9}$ realizations. In summary, even if only 5 independent input variates are involved, and if only 100 realizations were used, it is substantially likely that the simulations could fail to reveal the possibility that a large dose could arise when just two of the inputs are only moderately large (e.g., $\mathrm{x}_{12}$ and $\mathrm{x}_{22}$, each with $\mathrm{p}=0.25$ and thus with a joint likelihood $>5 \%$ ). The likelihood of such a large dose may affect the expected dose value, but any such affect will be missed (resulting in a biased underestimate of expected dose) if Monte Carlo simulation fails to identify this likelihood because the number $\left(n_{\text {sim }}\right)$ of realizations is too low. This argument applies to the estimation of upper-bounds on dose as well as to the estimation of expected dose. 
The magnitude of potential bias due to $\mathrm{n}_{\text {sim }}$ being too low depends of the degree of nonlinearity of the problem and on the variance of the relative contributions from uncertainty in each of the $\mathrm{x}_{\mathrm{i}}$ to predicted uncertainty in $\mathrm{D}$. For instance, let $\mathrm{D}=A\left(B^{C}+D^{E}\right)$ involving only five stochastic variates $\{A, B, C, D, E\}$ each distributed as log-normal with a median of 1 and a geometric standard deviation (GSD) of 2 . Contrast the mean value of $\mathrm{D}(\overline{\mathrm{D}})$ estimated using a single simulation of $D$ via Latin-Hypercube sampling with $n_{\text {sim }}=100$, vs. the distribution of $\bar{D}$ estimates obtained when such a simulation is repeated (randomly) 100 times. In this example, this reviewer calculates that the coefficient of variation of $\bar{D}$ (i.e., the standard deviation divided by the expected value of any single estimate of expected dose) is $>100 \%$. Therefore, a single estimate of the expected value of $D$ based on $n_{\text {sim }}=100$ is unreliable in this highly non-linear example, even though Latin-Hypercube sampling was used.

In practice, the investigation of potentially substantial synergistic interactions in the YMP simulation problem may be simplified. It is reasonable to focus this investigation only on, e.g., the 5 to 9 input variates that yield the greatest relative univariate contribution (RC) to uncertainty in predicted critical 10,000-yr annual maximum dose D. (Reasonable bases for assuming that substantial synergistic interactions involving the remaining variates would not be expected-e.g., based on arguments involving the TSPA-SR model structure-must be discussed in the AMR.) The apmf-DPC approach described above could then be used for a complete exploration of potential $1^{\text {st }}$-order synergy among the selected input variates conditional, e.g., on the mean value of all remaining input variates, and to generate a corresponding apmf for predicted dose, using only 512 or fewer simulations. (The AMR must state that the potential for any higher-order synergy among input variates was not investigated systematically, or state how any such investigation was done.) An alternative approach would be to use an RC-based importancesampling approach rather than Latin-Hypercube sampling for Monte Carlo simulations (or if this approach was in fact used in the TSPA-SR analysis, this fact must be clarified and explained). If neither approach is used, the AMR for the TSPA model must reasonably defend the assumption (which in this case should be stated explicitly in the AMR) that interactions among (or at least between pairs of) uncertain input variates have no substantial synergistic effect on predicted uncertain output. In this case, the Monte Carlo sample size (i.e., number of simulated TSPA model realizations) must always be demonstrated quantitatively to be adequate in relation to specified risk acceptability criteria adopted for a probabilistic risk analysis (Ang and Tang, 1984).

The sample-size issue discussed above is not addressed in the AMR. It is discussed, however in the TSPA-SR technical report (CRWMS M\&O 2000bm) in Sections 4.1.4 (for nominal performance) and 4.2 .3 (for igneous disruption), where results of analyses using larger numbers of realizations are presented to allow visual confirmation of the adequacy of the sample size. As noted by the NRC in their comments on the TSPA-SR (Cornell 2001, p. 24) additional information regarding sample size and the stability of the model results will be needed to support a potential License Application. 


\title{
Appendix III
}

\section{(2 pages)}

\section{Additional Comments on Document Content Reorganization for}

\author{
the TSPA Model Report, and the TSPA Technical Report \\ (by Gary Callahan)
}

The following reorganization of content is recommended based on the assumption that the purpose of the AMR: Total System Performance Assessment (TSPA) Model for the Site Recommendation (CRWMS M\&O 2000bl, hereinafter called the U.1 document) is to describe the TSPA model, and the purpose of the technical report: Total System Performance Assessment for the Site Recommendation (CRWMS M\&O 2000bm, hereinafter called the U.2 document) is to present the TSPA analysis based on the TSPA model presented in the U.1 document. An alternative approach would be to prepare three documents: 1) the philosophy and approach to TSPA, 2) description of the TSPA model (i.e., pretty much what U.1 is presently-a description of the GoldSim (Golder Associates 2000) implementation and its component models, and 3) documentation of the TSPA with results and conclusions.

The following discussion pertains to the organization of the U.2 document (CRWMS M\&O 2000bm):

1. Chapter 2, especially Section 2.2 (Methodology) includes model philosophy and details that should be included in the U.1 document. A short summary presenting the methodology of the TSPA model, with reference to the U.1 document, would be better in the U.2 document Chapter 2. The addition of this information to U.1 would be an improvement to the overall presentation of the model development. The U.1 document presently starts out more or less with a bang-presenting software immediately.

2. Portions of Chapter 3 would fit better into the TSPA model report (U.1 document). In particular, discussion of the AMRs supporting the TSPA model and discussion of model conceptualizations are better suited to the U.1 document.

3. Appendix B (Summary of Screening Decision and Basis Information Contained in Revision 00 of the Yucca Mountain Project and Features, Events, and Processes Database) in the U.2 document would be more appropriate for the U.1 document under this recommended reorganization. This would provide the much needed supporting information for those FEPs that are not presently included in the TSPA model.

4. Appendix C (Natural-Analogue Investigations in Support of Performance Assessment of the Potential Yucca Mountain Radioactive-Waste Repository) in the U.2 document provides information on natural analogues that provide confidence in the TSPA model. Thus, Appendix C could be moved to the U.1 document. Comment: The differences between ASHPLUME Version 2.0 and Version 1.4LV are not described in sufficient detail to determine whether or not the simulation results are meaningful. One would expect the two versions of the code to produce similar results. Since the parameters were adjusted (calibrated) to obtain a reasonable fit to the observed data, one would expect the comparison between the calculated and observed data to be reasonable. 
5. Appendix F (Synthesis of Major Assumptions and Conservatisms Included in Total System Performance Assessment-Site Recommendation) in the U.2 document summarizes major assumptions used in the individual component models and the degree of conservatism that has been incorporated in these models. Therefore, Appendix F should be included in Chapter 5 of the U.1 document.

6. Process model-area names should be consistent throughout the U.1 and U.2 documents. For example, compare the naming convention used in the U.2 document (p. 3-1, Table 31) with terminology used in the U.1 document (Purpose section on p. 25, Figure 6-1 on p. 83, and Section 6-3 on p. 112). 


\section{Appendix IV}

\section{(72 pages)}

Impact Reviews for Bin-3 Models as Submitted by Responsible Project Staff

This appendix contains 34 impact reviews prepared by Project staff (model area leads) responsible for model development. The impact reviews correspond to the Bin-3 models identified in Section 6 and listed in Table 7 of this report.

The impact reviews clearly indicate whether each model was used for TSPA-SR, and whether it was used for FEP screening. Use for TSPA-SR means that quantitative output from the model was used as input to the TSPA-SR system model. This does not necessarily mean that all output from the model was used. Use for FEP screening means that the model is cited in a FEP screening analysis AMR, so the use may be qualitative, and may be limited to certain aspects of the model. 
MODEL VALIDATION - IMPACT REVIEW

A. Model: Thermal-Hydrologic-Mechanical (THM) Model (G.1)
B. AMR: Calculation of Permeability Change Due to Coupled Thermal-Hydrological-Mechanical Effects. (CAL-NBS-MD-000002 Rev. 00) (CRWMS M\&O 2000g)

C. Category (Check appropriate case)

1) Model NOT USED in TSPA and DID NOT SERVE as a basis for screening FEPS.

Result: No impact on TSPA-SR

$\triangle$ 2) Model NOT USED in TSPA but DID SERVE as a basis for screening FEPs.

Result: Assess impact of validation findings on FEP screening - Complete Section $D$ below

3) Model IS USED in TSPA but DID NOT SERVE as a basis for screening FEPs.

Result: Assess impact of validation findings on TSPA-SR results - Complete Section D below

4) Model IS USED in TSPA and DID SERVE as a basis for screening FEPS.

Result: Assess impact of validation findings on TSPA-SR results and FEP screening Complete Section D below

D. Path Forward and Impact on TSPA (Describe in detail)

See continuation sheet.

E. Responsible Individual: 


\section{Continuation of item D (Model G.1):}

The reviewer's comments concerning this calculation are acknowledged. These issues of model validation have been recognized for approximately one year, after an audit of the near-field environment program. Steps have already been taken to address them; additional model development and documentation have been completed, and further activities are planned. These findings of the model validation review do not affect the conclusions of the TSPA-SR because the arguments discussed below indicate that "screening-out" of THM effects from the TSPA was reasonable and defensible.

This original calculation report (CRWMS M\&O 2000g) has been revised and converted to a new AMR (BSC 2001g) effective in August, 2001. The new AMR includes more extensive information on model validation based primarily on measured field data (including rock displacement measurements) from the Drift Scale Test (DST), Large Block Test, and Single Heater Test (SHT) (Section 4.3.7). This information is also summarized briefly in the SSPA (BSC 2001d; Section 4.3.7).

Alternative modeling approaches have also been compared, to support validation for THM models for Yucca Mountain, as permitted by AP-3.10Q Section 5.3.c.4. A continuum model has been used along with the discrete fracture model in the new AMR (BSC 2001ag). In addition, the THM effects have been independently studied using another continuum model (TOUGH-FLAC) (BSC 2001d, Section 3.2.7). In that effort, the model is calibrated against data from field air permeability measurements in the DST that provide bounds for magnitude of residual apertures as a result of THM processes.

All analyses completed to date indicate that the THM effects on permeability are relatively small (within an order of magnitude change in permeability, based on measurements from both the SHT and DST) compared to the range of permeability (three to four orders of magnitude) arising from natural spatial heterogeneity. Recent results confirm the screening decision not to incorporate THM effects on permeability into the TSPA-SR. THM model validation is the subject of a KTI agreement, for the Repository Design and Thermal-Mechanical Effects (RDTME) Key Technical Issue (Gardner 2001) which is summarized as follows:

- Provide additional validation analysis of field tests related to the thermal-mechanical effects on fracture permeability (RDTME 3.21)

Resolution of this agreement item will fully address the model validation review finding. This will involve alternative representations of fracture geometry and constitutive relationships. It is noted that resolution of other agreement items from KTI technical exchange meetings may also contribute to model validation.

THM effects on permeability will be further investigated in ongoing and planned underground testing at Yucca Mountain, analyzed using both the discrete fracture modeling approach and continuum modeling, and the results will be documented in revisions to the AMRs that would support a potential License Application. 
MODEL VALIDATION - IMPACT REVIEW

\begin{tabular}{|c|c|}
\hline A. Model: Cement Grout Model (H.3-2) & $\begin{array}{l}\text { B. AMR: Engineered Barrier System: Physical } \\
\text { and Chemical Environment Model. (ANL-EBS- } \\
\text { MD-000033 Rev. 01) (CRWMS M\&O 2000ab) }\end{array}$ \\
\hline
\end{tabular}

C. Category (Check appropriate case)

1) Model NOT USED in TSPA and DID NOT SERVE as a basis for screening FEPS.

Result: No impact on TSPA-SR

$\triangle$ 2) Model NOT USED in TSPA but DID SERVE as a basis for screening FEPs.

Result: Assess impact of validation findings on FEP screening - Complete Section D below

3) Model IS USED in TSPA but DID NOT SERVE as a basis for screening FEPs.

Result: Assess impact of validation findings on TSPA-SR results - Complete Section D below

4) Model IS USED in TSPA and DID SERVE as a basis for screening FEPS.

Result: Assess impact of validation findings on TSPA-SR results and FEP screening Complete Section D below

D. Path Forward and Impact on TSPA (Describe in detail)

See continuation sheets.

E. Responsible Individual: $\frac{\text { Robert MacKinnon/ Robet Mocthon }}{\text { Type Name/Signature }} \frac{11 / 15 / 01}{\text { Date: }}$ 
Continuation of item D (Model H.3-2):

Model results were used to screen out the effects of cement leachate on the composition of the water in the EBS bulk environment. Cement leachate may affect the concentrations of soluble and colloidal radionuclides and waste package and drip shield degradation. The impact on TSPA-SR is not significant because the predicted cement leachate composition is conservatively alkaline as discussed below, and because current models for waste package and drip shield corrosion rates indicate that water compositions similar to cement leachate will have no significant effect. See CRWMS M\&O (2000af; 2000ag) for description of the corrosion models, and Section 3.4 of CRWMS M\&O (2000bm) for a summary of the implementation of $\mathrm{pH}$ dependence for waste package corrosion, in TSPA-SR. The $\mathrm{pH}$ of alkaline cement leachate is expected to be no greater than $\mathrm{pH} 13$ on equilibration with portlandite in a silica-rich environment, and substantially lower than this after reaction with $\mathrm{CO}_{2}$ in the environment.

It is noted that corrosion data for high-pH conditions that are used in models that support TSPASR, are limited to results from cyclic polarization testing of thermally aged Alloy-22 samples in BSW-13 solution at pH 13 (CRWMS M\&O 2000ag). As stated in this AMR (Section 6.7.2) more quantitative testing (which would include long-term corrosion testing in strongly alkaline conditions) is needed to support definitive statements on the effects of high-pH (or thermal aging) on corrosion rates.

There are several KTI agreements related to the issue of cement/seepage interactions and their potential effects on performance, for the Evolution of the Near-Field Environment (ENFE) Key Technical Issue (Williams 2001) and the Total System Performance Assessment Integration Issue (Cornell 2001):

- Address the effects of cementitious materials on hydrologic properties of the host rock (ENFE 1.4)

- Evaluate data and model uncertainties for specific in-drift geochemical environment submodels used in TSPA calculations and propagate those uncertainties through the submodels in a systematic approach (ENFE 2.5)

- Evaluate the impact of the range of local chemistry (e.g., dripping of equilibrated evaporated cement leachate and corrosion products) conditions at the drip shield and waste package considering the chemical divide phenomena that may propagate small uncertainties into large effects (ENFE 2.6)

- Provide additional information about the range of composition of waters that could contact the drip shield or waste package, including whether such waters are of the bicarbonate or chloride-sulfate type (ENFE 2.10)

- Evaluate the possibility of preferential dripping from engineered materials including rockbolts, and give appropriate consideration to the uncertainties of the water sources, as well as their potential impact on other models (TSPAI 3.7)

- Provide documentation of the integrated analyses and comprehensive uncertainty analyses related to the EBS physical and chemical environment in documentation associated with TSPA for any potential License Application (TSPAI 3.10)

These agreements will address many of the points raised in the model validation review. It is noted that resolution of other agreement items from KTI technical exchange meetings may also contribute to model validation. The additional consideration of alternative cement phase 
minerals, recommended by the model validation reviewer, will provide further confidence in predicted leachate composition. Substitution of more stable phases will tend to decrease the concentrations of key chemical components in cement leachate. Incorporation of multiple invariant points in leachate composition will limit the duration of the most alkaline compositions. Integrated analyses and uncertainty analyses will include evaluation of the effects of cement leachate on other models, i.e. other barriers such as the drip shield and waste package.

The path forward includes revising the cement/seepage model and conducting cement/seepage testing to provide data for model validation. The model revision will revisit the treatment of mineral phases (e.g., ettringite, calcium-silicate-hydrate, and tobermorite) used to represent the cement assemblage (CRWMS M\&O 2000ab; Section 6.3), and justify the selections on conservative or other grounds. The current model is conservative in the sense that it assumes highly alkaline portlandite is present, and does not allow for in-place evolution of cement minerals to more stable, less soluble and less alkaline phases with time. Therefore the evolution of cement leachate to less alkaline compositions as cement mineral constituents are exhausted, is not considered with respect to leachate composition. This latter aspect of the cement behavior may be incorporated in future work if it can be adequately validated, otherwise a more conservative approach will be used. It is expected that the Cement Model will be further developed and documented in a new AMR for analysis of introduced materials in the EBS.

Capillary properties of the cement grout will control its water content, and the tendency for flow to converge toward and through the grout. The grout permeability is expected to be small, on the order of $10^{-19} \mathrm{~m}^{2}$, which limits the amount of leachate that could potentially reach the drift opening. The current model is based on the concept that capillary affinity for water is inversely related to permeability, so that if the grout absorbs water from the rock then its permeability will be low. If the grout cracks or its fabric is altered by mineral evolution, then flow in the grout will become channelized and the extent of interaction with highly alkaline cementitious phases will decrease. This possibility is addressed in the current model by use of flux scaling, i.e. a geometrical argument (rather than based on permeability) for limiting the amount of cement leachate that could reach the drift opening.

In summary, the current model for cement leachate composition is believed to be reasonably conservative, and the possibility for interaction of high-pH waters with the waste package outer barrier has been addressed in the corrosion modeling. The need for improvement of the cement model is acknowledged, and will be addressed by planned work if cementitious materials continue to be used in the design. This topic has been discussed previously with the NRC staff and is the subject of KTI agreement items. While planned work will address the model validation review findings noted in this review, the conclusions of TSPA-SR with respect to the effects of cement on waste package performance are very unlikely to change as a result of new information. 
MODEL VALIDATION - IMPACT REVIEW

A. Model: Corrosion of Steel Used in the ExContainer EBS (H.3-3)
B. AMR: Engineered Barrier System: Physical and Chemical Environment Model. (ANL-EBS-MD000033 Rev. 01) (CRWMS M\&O 2000ab)

C. Category (Check appropriate case)

1) Model NOT USED in TSPA and DID NOT SERVE as a basis for screening FEPs.

Result: No impact on TSPA-SR

$\triangle$ 2) Model NOT USED in TSPA but DID SERVE as a basis for screening FEPs.

Result: Assess impact of validation findings on FEP screening - Complete Section D below

3) Model IS USED in TSPA but DID NOT SERVE as a basis for screening FEPS.

Result: Assess impact of validation findings on TSPA-SR results - Complete Section D below

4) Model IS USED in TSPA and DID SERVE as a basis for screening FEPs.

Result: Assess impact of validation findings on TSPA-SR results and FEP screening Complete Section D below

D. Path Forward and Impact on TSPA (Describe in detail)

See continuation sheets.

E. Responsible Individual: $\frac{\text { Robert MacKinnon / Robut Mackmon }}{\text { Type Name/Signature }} 11 / 5 / 01$ 
Continuation of item D (Model H.3-3):

This model is referenced in the screening argument for FEPs related to biological activity in the waste and EBS (CRWMS M\&O 2001h; Section 6.4.65).

This model estimates a range of effects from steel corrosion, on the oxygen content of the gas phase in the emplacement drifts. The model is preliminary in that an important assumption is made, that the steel corrosion rate measured for atmospheric conditions decreases in proportion to the oxygen fugacity. This assumption is identified to-be-verified (TBV-4931). Based on this assumption, which is a first-order approximation, a range of oxygen fugacities was calculated (CRWMS M\&O 2000ab, Section 6.3 and 6.7) and used to evaluate possible changes in the equilibrium redox chemistry for water and mineral compositions representing the EBS. The effects of microbial activity were included as a multiplier on the steel corrosion rate, based on preliminary data. The result of the model indicated that excursions in oxygen fugacity are possible, but the calculated magnitude is not significant.

Apart from the assumption, the approach is conservative in that for purposes of modeling oxygen consumption, the most consumptive reaction (producing $\mathrm{Fe}_{2} \mathrm{O}_{3}$ ) is used. Hydrolysis with production of hydrogen would tend to consume less oxygen from the gas phase. Hydrogen species from hydrolysis reactions are known to cause embrittlement in titanium, but this possibility was considered based on direct contact between carbon steel and titanium (CRWMS $\mathrm{M} \& \mathrm{O} 2000 \mathrm{ah}$ ). Also, in the application of the steel corrosion model to gas-phase oxygen calculations, a 2-D approach was used, ignoring mixing of the gas phase along the third axis (parallel to the drift axis).

Other sources of uncertainty with respect to the rate of oxygen consumption were identified by the reviewers, and are acknowledged. These include microbial activity which is represented using preliminary data, represented by a simple multiplication factor. However, the calculated range of oxygen fugacities was shown to be far less (in a logarithmic sense) than that which would be needed to significantly affect the redox potential of the aqueous phase (CRWMS M\&O 2000ab; Section 6.7). Consequently, order-of-magnitude changes in the rate of oxygenconsuming processes such as steel corrosion would not affect the calculated result of this model. Accordingly, the use of this model in FEP screening is justified. Further model development, testing, and comparison to natural or man-made analogs may be undertaken if steel remains part of the emplacement-drift design, but would not be expected to change this conclusion.

Some of the concerns raised by the model validation review are similar to previous KTI agreement items for the Evolution of the Near-Field Environment (ENFE) Key Technical Issue (Williams 2001) and the Total System Performance Assessment Integration (TSPAI) Key Technical Issue (Cornell 2001):

- Resolution of preliminary and to-be-verified information, will include TBV-4931 which relates to the form of the predictive expression for reasonable-bound rates of oxygen consumption by steel corrosion (TSPAI 2.1 Item 58)

- Evaluate the impact of the range of local chemistry (e.g., dripping of equilibrated evaporated cement leachate and corrosion products) conditions at the drip shield and waste package considering the chemical divide phenomena that may propagate small uncertainties into large effects (ENFE 2.6)

It is noted that resolution of other agreement items from KTI technical exchange meetings may 
also contribute to model validation. It is expected that the Steel Corrosion Model will be further developed and documented in a new AMR for analysis of introduced materials in the EBS. 


\section{MODEL VALIDATION - IMPACT REVIEW}

\begin{tabular}{|c|c|}
\hline $\begin{array}{l}\text { A. Model: Effect of Evaporation in the Invert } \\
(H .3-4)\end{array}$ & $\begin{array}{l}\text { B. AMR: Engineered Barrier System: Physical and } \\
\text { Chemical Environment Model. (ANL-EBS-MD- } \\
000033 \text { Rev. 01) (CRWMS M\&O 2000ab) }\end{array}$ \\
\hline
\end{tabular}

C. Category (Check appropriate case)

1) Model NOT USED in TSPA and DID NOT SERVE as a basis for screening FEPS.

Result: No impact on TSPA-SR

$\triangle 2$ ) Model NOT USED in TSPA but DID SERVE as a basis for screening FEPs.

Result: Assess impact of validation findings on FEP screening - Complete Section D below

3) Model IS USED in TSPA but DID NOT SERVE as a basis for screening FEPs.

Result: Assess impact of validation findings on TSPA-SR results - Complete Section D below

4) Model IS USED in TSPA and DID SERVE as a basis for screening FEPS.

Result: Assess impact of validation findings on TSPA-SR results and FEP screening Complete Section $\mathrm{D}$ below

D. Path Forward and Impact on TSPA (Describe in detail)

See continuation sheet.

E. Responsible Individual: $\frac{\text { Robert MacKinnon / RobatMrathe }}{\text { Type Name/Signature }} \frac{11 / 15 / 01}{\text { Date: }}$ 
Continuation of item D (Model H.3-4):

No impact. This model was not used for TSPA-SR and will not be carried forward to License Application (LA).

Physical degradation of the invert and invert materials has been screened out of the TSPA-SR based on low consequence. The screening argument recognizes that the invert is a minor barrier to flow in comparison to the drip shield, waste package, and unsaturated zone beneath the drift Changes in porosity of the invert would have little effect on radionuclide transport. The typical depth dimension of the invert, about one meter, is much less than the transport distance through the unsaturated zone, and the effect on radionuclide transport is therefore much less.

The path forward includes analyses of coupled processes in the EBS per KTI agreement ENFE 2.7 (Williams 2001). At this time, however, no experiments are planned as the final invert design and material selection has not been made. Tests involving crushed tuff have low priority because the invert offers little waste isolation performance. It is noted that resolution of other agreement items from KTI technical exchange meetings may also contribute to model validation. 
A. Model: EBS Colloids Model (H.3-5)
B. AMR: Engineered Barrier System: Physical and Chemical Environment Model. (ANL-EBS-MD000033 Rev. 01) (CRWMS M\&O 2000ab)

C. Category (Check appropriate case)

$\triangle 1$ ) Model NOT USED in TSPA and DID NOT SERVE as a basis for screening FEPs.

Result: No impact on TSPA-SR

2) Model NOT USED in TSPA but DID SERVE as a basis for screening FEPs.

Result: Assess impact of validation findings on FEP screening - Complete Section D below

3) Model IS USED in TSPA but DID NOT SERVE as a basis for screening FEPs.

Result: Assess impact of validation findings on TSPA-SR results - Complete Section D below

4) Model IS USED in TSPA and DID SERVE as a basis for screening FEPs.

Result: Assess impact of validation findings on TSPA-SR results and FEP screening Complete Section D below

D. Path Forward and Impact on TSPA (Describe in detail)

No impact. This bounding model was not used for TSPA-SR, and will not be carried forward to LA. This model was used in preliminary process model screening considerations only (see Table I1, CRWMS M\&O, 2001h) and was not used as a basis for screening FEPs for TSPA-SR.

The reviewer's concerns with the need for test data, the use of hematite to represent iron oxide, and the use of constant Kd's, are acknowledged. However, this model is not used, and colloidal transport in the waste package and the ex-container engineered barrier system (EBS) is included in the TSPA-SR based on other models (CRWMS M\&O 2000al; 2001s). These other models are reviewed elsewhere in this report (Sections 6.11.29, 6.11.30, and 6.13.1) with findings and recommendations that are not related to the subject model.

The specific details associated with colloids comprised of iron oxides are not addressed by a KTI agreement. However, there are existing KTI agreements for the Evolution of the Near-Field Environment (ENFE) Key Technical Issue (Williams 2001) and the Total System Performance Assessment Integration (TSPAI) Key Technical Issue (Cornell 2001) that pertain to evaluation of colloid properties that control sorption (ENFE 4.6), particles larger than colloids (TSPAI 2.1), and changes in colloid concentrations due to changes in $\mathrm{pH}$ and ionic strength (TSPAI 3.42). Resolution of these agreements will help to reduce uncertainty in modeling of colloidal processes. It is noted that resolution of other agreement items from KTI technical exchange meetings may also contribute to model validation.

E. Responsible Individual: $\frac{\text { Robert MacKinnon / Robat Mocknown }}{\text { Type Name/Signature }} / 1 / 15 / 01$ 


\section{MODEL VALIDATION - IMPACT REVIEW}

\begin{tabular}{|l|l}
\hline A. Seepage/Cement Interaction Model (H.7) & B. $\begin{array}{l}\text { AMR: Seepage/Cement Interactions (ANL-EBS- } \\
\text { MD-000043 ReV. 00) (CRWMS M\&O 2000bf) }\end{array}$ \\
\hline
\end{tabular}

C. Category (Check appropriate case)

$\triangle 1$ 1) Model NOT USED in TSPA and DID NOT SERVE as a basis for screening FEPS.

Result: No impact on TSPA-SR

2) Model NOT USED in TSPA but DID SERVE as a basis for screening FEPs.

Result: Assess impact of validation findings on FEP screening - Complete Section $D$ below

3) Model IS USED in TSPA but DID NOT SERVE as a basis for screening FEPS.

Result: Assess impact of validation findings on TSPA-SR results - Complete Section D below

4) Model IS USED in TSPA and DID SERVE as a basis for screening FEPs.

Result: Assess impact of validation findings on TSPA-SR results and FEP screening Complete Section D below

D. Path Forward and Impact on TSPA (Describe in detail)

See continuation sheet.

E. Responsible Individual: $\frac{\text { Robert MacKinnon / Robat Mochan }}{\text { Type Name/Signature }} \frac{11 / 15 / 0}{\text { Date: }}$ 
Continuation of item D (Model H.7):

No impact. This bounding model was not used for TSPA-SR, and will not be carried forward to LA in its present form. The reviewer's concerns with appropriate boundary conditions and diffusion parameter values, and the need for comparison to test data, are acknowledged for this developmental model.

There are several existing KTI agreements related to the issue of cement/seepage interactions and their potential effects on performance, for the Evolution of the Near-Field Environment (ENFE) Key Technical Issue (Williams 2001):

- Address the effects of cementitious materials on hydrologic properties of the host rock (ENFE 1.4)

- Evaluate data and model uncertainties for specific in-drift geochemical environment submodels used in TSPA calculations and propagate those uncertainties through the submodels in a systematic approach (ENFE 2.5)

- Evaluate the impact of the range of local chemistry (e.g., dripping of equilibrated evaporated cement leachate and corrosion products) conditions at the drip shield and waste package considering the chemical divide phenomena that may propagate small uncertainties into large effects (ENFE 2.6)

- Provide additional information about the range of composition of waters that could contact the drip shield or waste package, including whether such waters are of the bicarbonate or chloride-sulfate type (ENFE 2.10)

It is noted that resolution of other agreement items from KTI technical exchange meetings may also contribute to model validation. This model overlaps with the Cement Model (H.3-2) which was addressed in a previous impact review. Both models will be consolidated, developed, and documented in a new AMR that evaluates the effects of introduced materials in the EBS. 
A. Model: Ventilation Model (1.5)

B. AMR: Ventilation Model. (ANL-EBS-MD-000030 Rev. 00) (CRWMS M\&O 2000bx)

C. Category (Check appropriate case) ,

$\bigotimes$ 1) Model NOT USED in TSPA and DID NOT SERVE as a basis for screening FEPs.

Result: No impact on TSPA-SR

2) Model NOT USED in TSPA but DID SERVE as a basis for screening FEPs.

Result: Assess impact of validation findings on FEP screening - Complete Section D below

3) Model IS USED in TSPA but DID NOT SERVE as a basis for screening FEPs.

Result: Assess impact of validation findings on TSPA-SR results - Complete Section D below

4) Model IS USED in TSPA and DID SERVE as a basis for screening FEPS.

Result: Assess impact of validation findings on TSPA-SR results and FEP screening Complete Section $\mathrm{D}$ below

D. Path Forward and Impact on TSPA (Describe in detail)

See continuation sheets.

E. Responsible Individual: $\frac{\text { Robert MacKinnon/ Pheut }}{\text { Type Name/Signature }}$ 
Continuation of item D (Model I.5):

The model validation findings have no significant impact on the TSPA-SR model (CRWMS M\&O 2000bl) primarily because output from the Ventilation Model was not used directly for TSPA-SR. The Ventilation Model was not used for FEP exclusion, because the preclosure ventilation FEP was included in TSPA-SR. (The effects of preclosure ventilation on system performance were represented by models other than the Ventilation Model.)

The Ventilation Model is used to verify that forced ventilation can remove a prescribed fraction $(70 \%)$ of waste-generated heat during the 50 -yr preclosure period. The thermal analyses and thermal-hydrologic models that use this information, do so by decreasing the waste heat output by $70 \%$. All other information produced by the Ventilation Model is not used. Only the feasibility of $70 \%$ heat removal resulted from this model, and this result is not questioned in the model validation review. The representation of heat removal by preclosure ventilation in other models may be questioned, but that is not part of the Ventilation Model.

The lumped-parameter averaging approach for temperature and air velocity, the representation of buoyancy, and the representation of drift-wall temperature, are adequate to establish ventilation feasibility as intended. The effects of these model representations on ventilation efficiency are likely to be minor, i.e., readily compensated by minor adjustments in ventilation parameters such as the air flow rate. Evaporation of water from the near-field rock would tend to increase ventilation efficiency if considered in the model. Additional confidence in the predictive model is not needed until detailed design of the ventilation system, which will be done at a later time.

The following actions planned in response to KTI agreement items for the Repository Design and Thermal-Mechanical Effects (RDTME) Key Technical Issue (Gardner 2001) and the Thermal Effects on Flow (TEF) Key Technical Issue (Reamer and Williams 2001) will address some of the findings identified by the model validation review:

- Provide the technical basis for the range of relative humidities, and the possible presence of liquid-phase water, that could affect ground support longevity (RDTME 3.1)

- Provide the results of ventilation testing in an update to the Ventilation Model, which will include the technical basis for discretization, and the basis for application to repository simulation (RDTME 3.14, TEF 2.7)

It is noted that resolution of other agreement items from KTI technical exchange meetings may also contribute to model validation.

The following activities have been undertaken, or are ongoing, to further develop confidence in ventilation models:

- A revision to this AMR (revision to CRWMS M\&O 2000bx) is in preparation. It compares the original model (considered for the model validation review) to another model that includes more explicit representation of heat and mass transfer.

- The Supplemental Science and Performance Analyses (BSC 2001d, Section 5.3.2) investigated the sensitivity of temperatures to ventilation efficiency and to the temporal evolution of ventilation efficiency. For the high-temperature and low-temperature cases considered, the peak waste package temperature changes about 0.7 and $0.35^{\circ} \mathrm{C}$ for each $1 \%$ change in ventilation efficiency. Peak preclosure temperatures were very sensitive to the temporal evolution of the efficiency, which is helpful for guiding future predictive modeling activities. 
Based on this discussion it is concluded that the current Ventilation Model adequately establishes the feasibility of preclosure ventilation to remove $70 \%$ of waste-generated heat in a $50-y r$ preclosure period. The need for additional model validation information in the Rev. 00 AMR is acknowledged, and this need is being addressed by additional modeling and testing activities. 


\section{MODEL VALIDATION - IMPACT REVIEW}

A. Model: In-Drift THC Model (1.7)

B. AMR: In-Drift THC Model. (ANL-EBS-MD000026 Rev. 01) (BSC 2001r)

C. Category (Check appropriate case)

$\bigotimes$ 1) Model NOT USED in TSPA and DID NOT SERVE as a basis for screening FEPs.

Result: No impact on TSPA-SR

2) Model NOT USED in TSPA but DID SERVE as a basis for screening FEPs.

Result: Assess impact of validation findings on FEP screening - Complete Section $D$ below

3) Model IS USED in TSPA but DID NOT SERVE as a basis for screening FEPs.

Result: Assess impact of validation findings on TSPA-SR results - Complete Section D below

4) Model IS USED in TSPA and DID SERVE as a basis for screening FEPs.

Result: Assess impact of validation findings on TSPA-SR results and FEP screening Complete Section D below

D. Path Forward and Impact on TSPA (Describe in detail)

No impact. This model was not used for TSPA-SR, and will not be carried forward to LA. The reviewer's concerns with this model are acknowledged, including model representation of the buoyant flow physics, spatial resolution, and other aspects. This model is preliminary in nature and was developed to represent the original EDA-II backfill design case (Wilkins and Heath 1999). Coupled thermal-hydrologic-chemical processes were to be incorporated in a revision). This backfill case is similar to the current conceptual design, but includes crushed-tuff backfill, slightly different thermal loading, and different thermal management objectives. Although documentation changes to the AMR have been implemented, the original model has not been changed or updated.

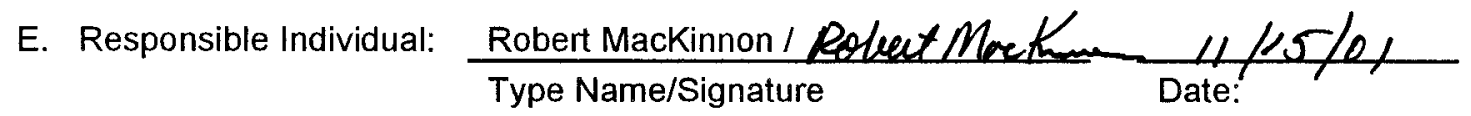


MODEL VALIDATION - IMPACT REVIEW

\begin{tabular}{l|l}
\hline $\begin{array}{l}\text { A. Model: Effective Thermal Conductivity Model } \\
\text { (1.8) }\end{array}$ & B. $\begin{array}{l}\text { AMR: Effective Thermal Conductivity for Drift- } \\
\text { Scale Models Used in TSPA-SR. (CAL-EBS-HS- } \\
000001 \text { Rev. 00.; a calculation report) (CRWMS } \\
\end{array}$ \\
\hline
\end{tabular}

C. Category (Check appropriate case)

$\square$ 1) Model NOT USED in TSPA and DID NOT SERVE as a basis for screening FEPS.

Result: No impact on TSPA-SR

2) Model NOT USED in TSPA but DID SERVE as a basis for screening FEPs.

Result: Assess impact of validation findings on FEP screening - Complete Section $D$ below

$\triangle$ 3) Model IS USED in TSPA but DID NOT SERVE as a basis for screening FEPS.

Result: Assess impact of validation findings on TSPA-SR results - Complete Section D below

4) Model IS USED in TSPA and DID SERVE as a basis for screening FEPS.

Result: Assess impact of validation findings on TSPA-SR results and FEP screening Complete Section $D$ below

D. Path Fonward and Impact on TSPA (Describe in detail)

See continuation sheet.

E. Responsible Individual: $\frac{\text { Robert MacKinnon/Ge fuet Mathm }}{\text { Type Name/Signature }} \frac{11 / 15 / 01}{\text { Date: }}$ 


\section{Continuation of item D (Model I.8):}

The effective thermal conductivity parameter is applied directly in two process-level models (Multiscale Thermohydrologic Model and the Thermal-Hydrologic-Chemical Seepage Model) that supply information to TSPA-SR. The effective thermal conductivity parameter controls the rate at which heat is transported from the waste packages to the drift wall during the preclosure period, and from the drip shield to the drift wall in the postclosure period. This influences the calculated temperatures and relative humidity values at the waste package, drip shield, and drift wall.

The impact of validation issues associated with this model, on the conclusions of TSPA-SR (i.e. calculated annual dose; see CRWMS M\&O 2000bm, Section 6.1) is insignificant because: 1) the uncertainty in repository temperature predictions is small, on the order of a few degrees Celsius as shown by predictions from field thermal tests (CRWMS M\&O 2000bk); and 2) the sensitivity of system performance to repository temperature is not important to calculated dose (CRWMS M\&O 2000bm, Section 5).

The reviewer's concerns are acknowledged; the greatest known potential for error in the model is the suppression of local variations in temperature. Temperatures computed using this approach are spatially smoothed such that local "hot" or "cold" spots along the surfaces of the waste package, drip shield, or drift wall are not predicted although small differences in temperature (for example, comparable in magnitude to the predictive uncertainty discussed above) could occur in the repository. The overall impact of this approximation on the annual dose calculated by TSPASR is not important because temperature changes have been shown, in general, not to be important to calculated dose as discussed above. It is noted that the TSPA-SR component models (e.g., WAPDEG; CRWMS M\&O 2000br) do not have the spatial resolution that would be needed to simulate corrosion and other processes at "hot" or "cold" spots in the engineered barrier system.

The DOE-NRC agreements concerning the Thermal Effects on Flow (TEF) Key Technical Issue (Reamer and Williams 2001) include items which when resolved, will contribute to validation of this model or alternative approaches that may be used in the future:

- Represent the "cold-trap" effect in thermal-hydrologic simulations, and provide technical justification for inclusion or exclusion of the effect in the various scale models that support TSPA. The analysis will consider thermal effects on flow, and the in-drift geochemical environment (TEF 2.5).

It is noted that resolution of other agreement items from KTI technical exchange meetings may also contribute to model validation.

Simulating the "cold-trap" effect is similar to the prediction of small-scale variation of environmental conditions discussed above. It involves the representation of heat and mass transport processes within the emplacement drifts, with sufficient fidelity to predict variations caused by buoyant convection, condensation, and other processes. 


\section{MODEL VALIDATION - IMPACT REVIEW}

A. Model: WAPDEG Analysis of Waste Package and Drip Shield Degradation (J.6)
B. AMR: WAPDEG Analysis of Waste Package and Drip Shield Degradation (ANL-EBS-PA-000001 Rev. 00 ICN 01) (CRWMS M\&O 2000br)

C. Category (Check appropriate case)

1) Model NOT USED in TSPA and DID NOT SERVE as a basis for screening FEPS.

Result: No impact on TSPA-SR

2) Model NOT USED in TSPA but DID SERVE as a basis for screening FEPs.

Result: Assess impact of validation findings on FEP screening - Complete Section $D$ below

3) Model IS USED in TSPA but DID NOT SERVE as a basis for screening FEPs.

Result: Assess impact of validation findings on TSPA-SR results - Complete Section D below

4) Model IS USED in TSPA and DID SERVE as a basis for screening FEPS.

Result: Assess impact of validation findings on TSPA-SR results and FEP screening Complete Section D below

D. Path Forward and Impact on TSPA (Describe in detail).

See continuation sheets.

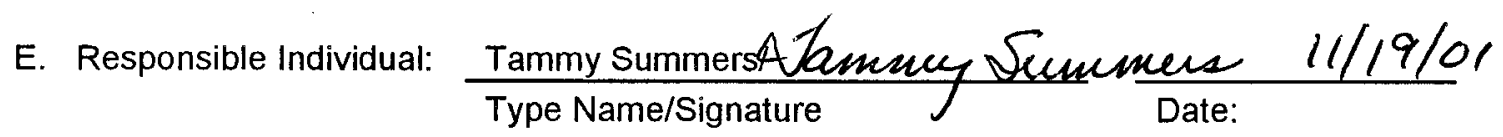




\section{Continuation of Item D (Model J.6):}

There is no impact from the model validation review findings from Section 6.10.8 of this report, on the conclusions of TSPA-SR (CRWMS M\&O 2000bm, Section 6.1). The models used to describe waste package and drip shield performance for TSPA-SR are adequate because they are closely based on the process models (also reviewed for model validation) which in turn are based on Project-generated data relevant to repository conditions.

The technical basis for the process models is the focus of KTI agreement items for the Container Life and Source Term (CLST) Key Technical Issue (Kelmenson 2001) which include:

- Provide data that characterizes passive film stability, including welded and thermally aged specimens (CLST 1.9)

- Provide documentation for Alloy-22 and titanium performance, including the following: measured potentials in the long-term corrosion tests, critical potentials on welded samples, separate effects of water composition on damage/buffering behavior, and critical potentials in environments containing heavy metal concentrations (CLST 1.10)

- Provide documentation for Alloy-22 and titanium performance, including the following: qualify and optimize mitigation processes, generate SCC data over a range of conditions, continue slow strain-rate testing, determine repassivation constants for film rupture model, continue direct current potential drip crack propagation rate measurements extended to additional environments, evaluate SCC resistance of welded/stress mitigated vs. unwelded samples, and evaluate SCC of full-thickness welded material (CLST 1.12)

- Provide documentation for Alloy-22 and titanium performance, including the following: install specimens from mock-up in long-term corrosion tests, evaluate scaling and weld process factors related to actual containers, and provide welded samples for MIC, aging, and localized corrosion testing (CLST 1.15)

- Provide documentation for Alloy-22 performance, including the following: evaluate data input to current models, continue ongoing aging and evaluation of Alloy-22 samples, use theoretical modeling to enhance confidence in kinetic modeling, use welded and nonwelded samples for SCC compact tension tests, expand test program to welded and cold-worked materials, evaluate effects of stress mitigation on phase stability, and expand aging tests to include lower temperatures (CLST 2.5)

- Provide documentation for path-forward items including the following: expand rockfall effect calculations to include weld embrittlement/aging, drip shield thinning, hydrogen embrittlement of drip shield, and effects of multiple rock blocks; and calculate effects from static loading by fallen rock blocks during ground motion events (CLST 2.8)

The data being collected to address the KTI agreement items will be incorporated into the process-model AMRs supporting the WAPDEG AMR and will fully address the model validation review findings identified by the review team. It is noted that resolution of other agreement items from KTI technical exchange meetings may also contribute to model validation.

The following comments apply to the overall conclusions from the model validation review concerning the WAPDEG model:

- The WAPDEG model is an integration model for waste package degradation analysis and based on the supporting process models and abstraction models. Validation of the process models in the Waste Package/Drip Shield Degradation model area is discussed in Section 
6.10 of this report (specifically, Sections 6.10.1 through 6.10.5, and also 6.10.11, 6.10.12, and 6.10.14).

- The WAPDEG model is based on the set of abstraction models identified in the subject AMR (CRWMS M\&O 2000br, Section 6.4.18). The validation for each of these contributing abstractions has determined that they are consistent with the parent processlevel models.

- The software for WAPDEG has been qualified. The qualification efforts included execution of approximately 100 test cases involving verifying the operation of various segments of the code.

Specific comments from the review team (in italics) and responses are provided below.

Review comment: The WAPDEG Analysis of Waste Package and Drip Shield Degradation Model is assigned to Bin 3 because there is a missing model that should be incorporated. This model will bring together the effects of the weld, weld heat-affected zone, and base plate microstructure/residual stress profile to predict the resistance of the waste package outer barrier (WPOB) to localized corrosion and stress corrosion cracking. The model will incorporate weld process variation within the number of passes needed to fill the joint and the effects of repair welding. The model will also incorporate the effect of chemistry variation in different heats of Alloy 22 weld wire and base plate.

This model will develop the characterization of the microstructure and residual stress for Alloy 22 under the following conditions:

- Welds and weld heat affected zone of the WPOB in the as-welded and as-welded/solution annealed condition (aged and un-aged)

- The weld and weld heat affected zone of the inner lid of the outer barrier in the WPOB in the as-welded and as-welded/laser peened condition (aged and un-aged)

- The weld and weld heat affected zone of the outer lid of the WPOB in the as-welded and as-welded/induction annealed condition (aged and un-aged)

Analysis: The effects of the weld and weld heat-affected zone on corrosion of WPOB are modeled with a general corrosion enhancement factor due to aging (assumed uniform distribution between 1 and 2.5). The enhancement factor is based on the cyclic polarization data for fully aged and un-aged Alloy 22 base-metal samples in a highly aggressive condition relevant to the potential repository, and this is documented in: General Corrosion and Localized Corrosion of Waste Package Outer Barrier (CRWMS M\&O 2000ag). No localized corrosion initiation was shown for the expected open circuit potential range. The cyclic polarization data are also supported by the various weld samples being tested in the Long-Term Corrosion Testing Facility (LTCTF). The weld samples that have been exposed for up to $2 \mathrm{yr}$ do not show any noticeable differences in the corrosion behaviors (general corrosion, localized corrosion and SCC) and rates from non-welded samples in the LTCTF. The weld process effects on the corrosion resistance are part of the NRC CLST KTI agreements. The lower-tier AMRs (CRWMS M\&O 2000ag; 2000bi) supporting the WAPDEG AMR will be updated to incorporate additional data and analyses for the weld process effects, which are being generated to address the NRC agreements. 
It is agreed that the effects of potential weld process and materials variations are not addressed in the supporting AMRs (CRWMS M\&O 2000ag; 2000bi). These effects are part of the ongoing and planned testing programs in response to NRC CLST KTI agreements. The supporting AMRs will be updated to incorporate additional data and analyses for the weld process and materials variation effects, which are being generated to address the NRC agreements. Updated models and data that will be incorporated into WAPDEG, will resolve the model validation review findings identified by the review team.

Review comment: The finite element model analysis in the AMR: Stress Corrosion Cracking of the Drip Shield, the Waste Package Outer Barrier, and the Stainless Steel Structural Material (CRWMS M\&O 2000bi) is presented but not validated with measurements of residual stress for the as-welded condition before laser peening or induction annealing.

Analysis: The use of finite element (FEM) analysis (ANSYS analysis; see CRWMS M\&O $2000 \mathrm{bi}$ ) for the stress state in the closure weld regions after the local induction heating treatment is justified. This is based on the fact that the resulting stress state is due to the imposed cool down from annealing temperatures and not related to the as-welded stress state. The ANSYS analysis adequately represents the effect of quenching operations. In addition, preliminary unpublished data from testing by McDermott Technology Inc. of Alloy 22 welds and base metal, show that induction annealing produces compressive residual stresses to a significant depth, thus mitigating the potential for SCC. These measurements represent the combined effects of welding and induction annealing. Although not yet fully documented, it is expected that these data will confirm the FEM analysis.

Review comment: There are assumptions made for the inner lid induction annealed case but no validating data are presented.

Analysis: The induction annealing process does not apply to the inner lid, but has been proposed for the outer lid. Validation of the residual stresses in the outer lid closure weld following induction annealing has recently been achieved. Confirmatory data were generated as part of the ongoing production and study of prototypical mockups, and show that the residual stresses are compressive to greater than $5 \mathrm{~mm}$ in depth.

Review comment: The residual stress data presented for the laser-peened WPOB inner lid closure weld are based on other nickel-based materials and weld joint designs that may not reflect accurately the relevant closure weld design.

Analysis: The stress state for the laser peened WPOB inner lid is based on the measured residual stress before and after peening. While the data are obtained on welded Alloy-22 plate and not on the closure weld configuration, they represent the magnitude (if not the exact configuration) of weld-induced residual stresses and are therefore valid for the intended use, until more representative data become available. The residual stress data for shot-peening of Incoloy 908 were used only to define the stress uncertainty range and were not used for the absolute stress values (CRWMS M\&O 2000bi, Section 6.2.2.5).

Review comment: The residual stress measurement data presented do not account for changes due to the welding process (weld fit-up variations, variations in heat input, travel speed) and possible subsequent repair processes. More data are also required on the equivalence of shot peening and laser peening.

TDR-WIS-MD-000005 REV 00

Page IV-24

November 2001 
Analysis: It is agreed that residual stress measurements on mock ups with the closure lid design configurations would provide more reliable supporting data for the model. However, as mentioned in the previous response, the residual stress data are representative of the magnitude of weld-induced stresses (at yield strength or above) and it is the post-mitigated stress state that is important for the model. The shot-peening data are analogous, and are used where more directly relevant are not yet available.

Review comment: The use of the term "hoop stress" to define residual stress needs clarification.

Analysis: The term "hoop stress" is used for the z-component (circumferential direction) of the three principal components of the residual stresses in the outer and inner closure lids, as discussed in Sections 6.2.2.1 through 6.2.2.5 of the supporting AMR (CRWMS M\&O 2000bi). Accordingly, the same term is used in the subject AMR: WAPDEG Analysis of Waste Package and Drip Shield Degradation (CRWMS M\&O 2000br) and in the abstraction AMR: Abstraction of Models of Stress Corrosion Cracking of Drip Shield and Waste Package Outer Barrier and Hydrogen Induced Corrosion of Drip Shield. (CRWMS M\&O 2000c).

Review comment: The output of the WAPDEG model concludes that localized corrosion is not possible within the 10,000-year evaluation period as represented in the TSPA-SR. However, it is the strongly held opinion of the reviewers that the materials used in the waste package should not be judged on the low rate of general corrosion but localized corrosion or stress corrosion cracking (SCC). This is not adequately expressed in WAPDEG despite the uncertainty that surrounds the technical issues. An example of this is the lack of expression of uncertainty related to the efficacy of the stress mitigation procedures for the WPOB welds. More data are needed (microstructure and residual stress) from representative weld mockups fabricated using the weld process, joint design, and stress mitigation techniques selected for the WPOB.

Analysis: Localized corrosion is represented in the TSPA. However, it is not triggered because the required threshold environmental conditions do not occur in the system model (CRWMS M\&O $2000 \mathrm{bm}$, Section 5.2.3). In addition, thicker welds that are prototypical of the welding process currently planned have been tested for crevice corrosion in a Basic Saturated Water (BSW-12; see CRWMS M\&O 2000ac, Table 27). Preliminary, unpublished data from testing by McDermott Technology Inc. show no evidence of localized corrosion in either the weld or heat affected zone, and there was no difference in corrosion behavior observed for the Alloy 22 weld and base metal. Residual stress measurements from samples that were either laser peened or induction annealed have shown that it is possible to produce compressive residual stresses to a significant depth by these stress mitigation techniques, thus mitigating the potential for SCC.

Review Comment: The multiple mechanisms for localized corrosion and cracking, as described in the abstraction models, should be combined probabilistically and then included in the WAPDEG abstraction model. This is needed to avoid oversimplification and to satisfy stakeholders.

Analysis: Stochastic models have been developed for the occurrence (initiation and propagation) of crevice corrosion and SCC and implemented in the WAPDEG model for the TSPA-SR (CRWMS M\&O 2000br). Localized corrosion of the SCC cracks (or at the SCC crack tips) is not considered in the TSPA-SR WAPDEG analysis because the WPOB is not subject to localized corrosion under expected repository exposure conditions as indicated by the relevant project cyclic polarization data (CRWMS M\&O 2000ag). 
For the TSPA-SR base case WAPDEG analysis (CRWMS M\&O 2000br, Section 6.5.1) the initial breach of waste packages is in fact by SCC in the closure weld regions, and the SCC failure is estimated to begin at about $11,000 \mathrm{yr}$ after the repository closure. Penetration of the waste packages by general corrosion is estimated to begin at about $30,000 \mathrm{yr}$.

The efficacy of the stress mitigation processes is incorporated in the model as part of the uncertainty bands on the stress profiles used in the model. In addition, more recent analyses have included probabilistic estimates of potential improper heat treatment conditions and the effects on waste package performance. These analyses are documented in the SSPA Volume 1 (BSC 2001d). It is agreed that more data are needed and additional sample testing from the mockups subjected to stress mitigation are planned as part of the ongoing activities to address KTI agreements. 


\section{MODEL VALIDATION - IMPACT REVIEW}
A. Model: Incorporation of Uncertainty and Variability of Drip Shield and Waste Package Degradation in WAPDEG (J.8)

B. AMR: Incorporation of Uncertainty and Variability of Drip Shield and Waste Package Degradation in WAPDEG (ANL-EBS-MD-000036 Rev. 00) (CRWMS M\&O 2000ak)

C. Category (Check appropriate case)

$\triangle 1$ 1) Model NOT USED in TSPA and DID NOT SERVE as a basis for screening FEPs.

Result: No impact on TSPA-SR

2) Model NOT USED in TSPA but DID SERVE as a basis for screening FEPs.

Result: Assess impact of validation findings on FEP screening - Complete Section $D$ below

3) Model IS USED in TSPA but DID NOT SERVE as a basis for screening FEPS.

Result: Assess impact of validation findings on TSPA-SR results - Complete Section D below

4) Model IS USED in TSPA and DID SERVE as a basis for screening FEPs.

Result: Assess impact of validation findings on TSPA-SR results and FEP screening Complete Section D below

D. Path Forward and Impact on TSPA (Describe in detail)

See continuation sheet. E. Responsible Individual: $\frac{\text { Tammy Summers/tammy }}{\text { Type Name/Signature }}$ Sumees $1 / 19 / 01$ 
Continuation of item D (Model J.8):

The model validation review findings have no impact on the conclusions from TSPA-SR (i.e calculated annual dose; see CRWMS M\&O 2000bm, Section 6.1) for the following reasons:

- This AMR was not used in the TSPA-SR, i.e. not referenced by the TSPA-SR reports (CRWMS M\&O 2000bl; 2000bm) or the principal supporting WAPDEG report (CRWMS $\mathrm{M} \& \mathrm{O} 2000 \mathrm{br}$ ).

- This AMR was intended to address the effects of uncertainty and variability in various data sets which are used as input to WAPDEG. However, the analysis was not fully developed and so was not used for TSPA-SR.

- The AMR designated as Model J.8 in the model validation review (Table 6 of this report) was originally identified by the author as an analysis and not a model. Further, the AMR was prepared with the intention that it would provide supplemental information only, and would not be used for the TSPA-SR model (CRWMS M\&O 2000bl). The subject matter is developmental, and it was originally intended that the AMR would be revised before use in TSPA.

- Representation of uncertainty and variability with respect to waste package and drip shield corrosion processes has been incorporated into the process model and abstraction AMRs that support TSPA-SR (e.g., CRWMS M\&O 2000ag; 2000br).

- Revised input from the technical specialist reviewers assigned to this model area indicates that this model should be considered as an analysis instead, in which case there is no need for validation. (This revised input was received recently, after the model-binning and impact reviews were conducted, and could not be incorporated in the findings of the review.)

The analysis may be updated for use for TSPA-LA and if so, will include additional new information generated from ongoing and future testing and model development activities. 


\section{MODEL VALIDATION - IMPACT REVIEW} A. Model: Waste Form Degradation Abstraction -
Best Estimate Model (K.4-3)
B. AMR: DSNF and Other Waste Form Degradation Abstraction (ANL-WIS-MD-000004 Rev. 01 ICN 1) (BSC 2001n)

C. Category (Check appropriate case)

$\triangle 1$ 1) Model NOT USED in TSPA and DID NOT SERVE as a basis for screening FEPS.

Result: No impact on TSPA-SR

2) Model NOT USED in TSPA but DID SERVE as a basis for screening FEPs.

Result: Assess impact of validation findings on FEP screening - Complete Section $D$ below

3) Model IS USED in TSPA but DID NOT SERVE as a basis for screening FEPs.

Result: Assess impact of validation findings on TSPA-SR results - Complete Section D below

4) Model IS USED in TSPA and DID SERVE as a basis for screening FEPs.

Result: Assess impact of validation findings on TSPA-SR results and FEP screening Complete Section D below

D. Path Forward and Impact on TSPA (Describe in detail)

This model was not used to represent waste form degradation for TSPA-SR (CRWMS M\&O $2000 \mathrm{bl}$ ) which used the "conservative" representation. The subject AMR (BSC 2001n) was cited in the waste form FEPs screening analysis (CRWMS M\&O 2000ai) but the screening arguments do not depend on this model. Therefore the model validation review findings have no impact on the conclusions of the TSPA-SR (i.e. calculated annual dose; see CRWMS M\&O 2000bm, Section 6.1). The model is based at least in part on preliminary or approximate information, and the limitations of the model are discussed in the AMR, including the need for additional validation. In accordance with those limitations, the conservative approach (as defined in the subject AMR) is recommended and used for TSPA.

E. Responsible Individual:

Christine Stockman/

Type Name/Signature

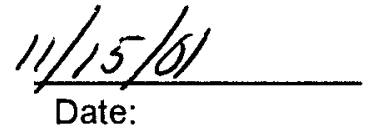

Date: 
MODEL VALIDATION - IMPACT REVIEW

\begin{tabular}{l|l}
\hline $\begin{array}{l}\text { A. Waste Form Degradation Abstraction - } \\
\text { Immobilized Pu Model (K.4-4) }\end{array}$ & $\begin{array}{l}\text { B. AMR: DSNF and Other Waste Form } \\
\text { Degradation Abstraction (ANL-WIS-MD-000004 } \\
\text { Rev. 01 ICN 1) (BSC 2001n) }\end{array}$ \\
\hline
\end{tabular}

C. Category (Check appropriate case)

1) Model NOT USED in TSPA and DID NOT SERVE as a basis for screening FEPs.

Result: No impact on TSPA-SR

$\bigotimes$ 2) Model NOT USED in TSPA but DID SERVE as a basis for screening FEPs.

Result: Assess impact of validation findings on FEP screening - Complete Section D below

3) Model IS USED in TSPA but DID NOT SERVE as a basis for screening FEPs.

Result: Assess impact of validation findings on TSPA-SR results - Complete Section D below

4) Model IS USED in TSPA and DID SERVE as a basis for screening FEPs.

Result: Assess impact of validation findings on TSPA-SR results and FEP screening Complete Section $D$ below

D. Path Forward and Impact on TSPA (Describe in detail)

This model was not used to represent waste form degradation for TSPA-SR (CRWMS M\&O 2000bl). The immobilized Pu inventory was averaged into the HLW glass radionuclide inventory, and the immobilized Pu waste form was treated as HLW glass for TSPA-SR. This approach is the "conservative" model developed in the subject AMR (BSC 2001n). The subject AMR was cited in the waste form FEPs screening analysis (CRWMS M\&O 2000ai) but the screening arguments do not depend on this model. Accordingly, the model validation review findings have no impact on the conclusions of the TSPA-SR (CRWMS M\&O 2000bm, Section 6.1). The model is based at least in part on preliminary or approximate information, and the limitations of the model are discussed in the AMR, including the need for additional validation.

E. Responsible Individual: $\frac{\text { christine Stockman }}{\text { Type NamelSignature }}$ 


\section{MODEL VALIDATION - IMPACT REVIEW}

\begin{tabular}{l|l}
\hline $\begin{array}{l}\text { A. Model: Alternate Wet Clad Unzipping Model } \\
\text { (K.14-1) }\end{array}$ & $\begin{array}{l}\text { B. AMR: Clad Degradation - Wet Unzipping (ANL- } \\
\text { EBS-MD-000014 Rev. 00) (CRWMS M\&O } \\
2000 p)\end{array}$ \\
\hline
\end{tabular}

C. Category (Check appropriate case)

1) Model NOT USED in TSPA and DID NOT SERVE as a basis for screening FEPS.

Result: No impact on TSPA-SR

2) Model NOT USED in TSPA but DID SERVE as a basis for screening FEPs.

Result: Assess impact of validation findings on FEP screening - Complete Section $D$ below

3) Model IS USED in TSPA but DID NOT SERVE as a basis for screening FEPs.

Result: Assess impact of validation findings on TSPA-SR results - Complete Section D below

4) Model IS USED in TSPA and DID SERVE as a basis for screening FEPS.

Result: Assess impact of validation findings on TSPA-SR results and FEP screening Complete Section D below

D. Path Forward and Impact on TSPA (Describe in detail)

This model was used in estimating the range of the unzipping multiplier used in TSPA-SR (CRWMS M\&O 2000bm, Section 3.5.4.2) and thus influences the time of unzipping and the effectiveness of the cladding in isolating the CSNF inventory. Although the review findings are not specific, the use of limited data support is acknowledged, and future work will address the need for more data to the extent practicable. There is no significant impact of this finding on the conclusions of TSPA-SR (i.e. calculated annual dose; see CRWMS M\&O 2000bm, Section 6.1) because the contribution of cladding, as represented in TSPA-SR, is minor for the 10,000-yr compliance period. It is noted that the unzipping model provides relatively little benefit to system performance, and that future work on this model would be greatly reduced if unzipping models were eliminated from a future TSPA model.

The contribution of cladding to total system performance was evaluated in a sensitivity study, documented in the "robustness analysis" for the TSPA-SR (CRWMS M\&O 2000bm, Sections 5.3 and 5.3.4.1). This is a case in which cladding credit is substantially diminished by setting four of the five cladding parameters used in TSPA-SR, at their $95 \%$ distribution points signifying greater release rates. The result was a minor increase in the calculated average annual dose over the first $100,000 \mathrm{yr}$ for 100 realizations of the TSPA-SR model (a factor of 1.5), compared to the nominal case in which these parameters were sampled over their full ranges. Thus the cladding model, while based on limited data with attendant model validation questions, does not impact the conclusions of the TSPA-SR (CRWMS M\&O 2000bm, Section 6.1).

E. Responsible Individual:

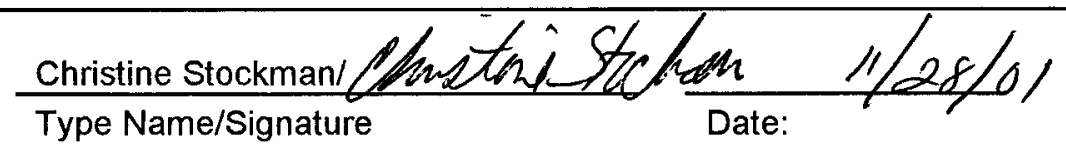

Type Name/Signature

Date: 


\section{MODEL VALIDATION - IMPACT REVIEW}

\begin{tabular}{l|l}
\hline A. Model: Summary and Abstraction - Clad & B. AMR: Clad Degradation - Summary and \\
Unzipping and Fuel Dissolution (K.16) & $\begin{array}{l}\text { Abstraction. (ANL-WIS-MD-000007 Rev. 00 ICN } \\
\text { 1) (CRWMS M\&O 2001d) }\end{array}$
\end{tabular}

C. Category (Check appropriate case)

1) Model NOT USED in TSPA and DID NOT SERVE as a basis for screening FEPs.

Result: No impact on TSPA-SR

2) Model NOT USED in TSPA but DID SERVE as a basis for screening FEPs.

Result: Assess impact of validation findings on FEP screening - Complete Section $D$ below

$\triangle 3$ ) Model IS USED in TSPA but DID NOT SERVE as a basis for screening FEPs.

Result: Assess impact of validation findings on TSPA-SR results - Complete Section D below

4) Model IS USED in TSPA and DID SERVE as a basis for screening FEPS.

Result: Assess impact of validation findings on TSPA-SR results and FEP screening Complete Section D below

D. Path Forward and Impact on TSPA (Describe in detail)

See continuation sheet.

E. Responsible Individual: Christine Stockman/

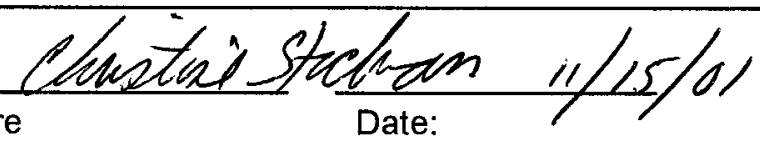




\section{Continuation of item D (Model K.16):}

This is the cladding degradation summary process model compiled from the submodels documented in the calculation report: Thermal Evaluation of Breached 21-PWR Waste Packages (CRWMS M\&O 1999f) and in the AMR: Clad Degradation-Wet Unzipping (CRWMS M\&O 2000p). This model also includes development of the abstraction used for TSPA-SR (CRWMS $\mathrm{M} \& \mathrm{O}$ 2000bl, Section 6.3.4.3). Although the review findings are not specific, the use of limited data support is acknowledged, and future work will address the need for more data to the extent practicable. Some aspects of the review findings are addressed by the KTI agreements discussed below. There is no significant impact of this finding on the conclusions of TSPA-SR (i.e. calculated annual dose; see CRWMS M\&O 2000bm, Section 6.1) because the contribution of cladding, as represented in TSPA-SR, is minor for the 10,000-yr compliance period.

The contribution of cladding to total system performance was evaluated in a sensitivity study, documented in the "robustness analysis" for the TSPA-SR (CRWMS M\&O 2000bm, Sections 5.3 and 5.3.4.1). This is a case in which cladding credit is substantially diminished by setting four of the five cladding parameters used in TSPA-SR, at their 95\% distribution points signifying greater release rates. The result was a minor increase in the calculated average annual dose over the first $100,000 \mathrm{yr}$ for 100 realizations of the TSPA-SR model (a factor of 1.5), compared to the nominal case in which these parameters were sampled over their full ranges. Thus the cladding model, while based on limited data with attendant model validation questions, does not impact the conclusions of the TSPA-SR (CRWMS M\&O 2000bm, Section 6.1).

The following KTI agreements are related to the Cladding Summary and Abstraction Model, for the Container Life and Source Term (CLST) Key Technical Issue (Kelmenson 2001):

- Perform tests for cladding SCC critical stress, under more aggressive conditions (CLST 3.9)

- Update rockfall model and determine if mechanical breakage should be incorporated in the cladding degradation abstraction (CLST 3.10)

Changes resulting form these agreements are expected to provide additional confidence in the cladding abstraction model. It is noted that resolution of other agreement items from KTI technical exchange meetings may also contribute to model validation. 


\section{MODEL VALIDATION - IMPACT REVIEW}

A. Model: Thermal Evaluation of Breached 21PWR Waste Packages (K.18)

B. AMR: Thermal Evaluation of Breached 21-PWR Waste Packages. (CAL-UDC-ME-000002 Rev. 00, a calculation report) (CRWMS M\&O 1999f)

C. Category (Check appropriate case)

1) Model NOT USED in TSPA and DID NOT SERVE as a basis for screening FEPS.

Result: No impact on TSPA-SR

2) Model NOT USED in TSPA but DID SERVE as a basis for screening FEPS.

Result: Assess impact of validation findings on FEP screening - Complete Section $D$ below

3) Model IS USED in TSPA but DID NOT SERVE as a basis for screening FEPS.

Result: Assess impact of validation findings on TSPA-SR results - Complete Section D below

$\triangle 4$ ) Model IS USED in TSPA and DID SERVE as a basis for screening FEPS.

Result: Assess impact of validation findings on TSPA-SR results and FEP screening Complete Section D below

D. Path Forward and Impact on TSPA (Describe in detail)

This model provides the difference in temperature between the cladding and the waste package surface. Although this work is documented as a calculation, reviewer comments on the need for validation are acknowledged. The model is used to determine the likelihood of dry oxidation, and as input to the creep model which depends on peak cladding temperature. In the model, 3-D heat transfer, conduction, and convective heat transfer are neglected. The model calculates the peak cladding temperature and the associated uncertainty. The model results are not used for other purposes for which conservatism may not be assured. Accordingly, there is no impact of the model validation review findings on the conclusions of TSPA-SR (i.e. calculated annual dose; see CRWMS M\&O 2000bm, Section 6.1).

In addition, the contribution of cladding to total system performance was evaluated in a sensitivity study, documented in the "robustness analysis" for the TSPA-SR (CRWMS M\&O 2000bm, Sections 5.3 and 5.3.4.1). Cladding credit is substantially diminished by setting four of the five cladding parameters used in TSPA-SR at their $95 \%$ distribution points signifying greater release rates. The result was a minor increase in the calculated average annual dose over the first 100,000 $\mathrm{yr}$ for 100 realizations of the TSPA-SR model (a factor of 1.5 increase), compared to the nominal case in which these parameters were sampled over their full ranges. Thus the contribution of cladding, as represented in TSPA-SR, is minor for the 10,000-yr compliance period. This further supports the position that the validation review findings pertaining to this model do not impact the conclusion of the TSPA-SR (CRWMS M\&O 2000bm, Section 6.1).

E. Responsible Individual:

Christine Stockman/

Type Name/Signature

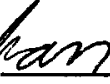

Date:

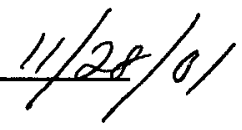


MODEL VALIDATION - IMPACT REVIEW

A. Model Breakage of CSNF Clad by Seismic Loading (K.19-1)
B. AMR: Breakage of CSNF Cladding by Mechanical Loading (CAL-EBS-MD-000001 Rev. 00) (CRWMS M\&O 1999a)

C. Category (Check appropriate case)

1) Model NOT USED in TSPA and DID NOT SERVE as a basis for screening FEPs.

Result: No impact on TSPA-SR

2) Model NOT USED in TSPA but DID SERVE as a basis for screening FEPs.

Result: Assess impact of validation findings on FEP screening - Complete Section $D$ below

$\triangle 3$ ) Model IS USED in TSPA but DID NOT SERVE as a basis for screening FEPs.

Result: Assess impact of validation findings on TSPA-SR results - Complete Section D below

4) Model IS USED in TSPA and DID SERVE as a basis for screening FEPS.

Result: Assess impact of validation findings on TSPA-SR results and FEP screening Complete Section D below

D. Path Forward and Impact on TSPA (Describe in detail)

This model (documented as a calculation) was used to determine the likelihood of a seismic event causing breakage of the cladding. The reviewer's comments on the robustness of cladding are acknowledged. The model is thus conservative, so there is no impact from the validation review findings on the conclusions of TSPA-SR (i.e. calculated annual dose; see CRWMS M\&O 2000bm, Section 6.1).

The KTI agreements for the Repository Design and Thermal-Mechanical Effects (RDTME) Key Technical Issue (Gardner 2001) include a commitment to further develop and test rockfall models, and to reevaluate the application of rockfall model output to other models including the Model of Breakage of CSNF Clad by Seismic Loading (RDTME 3.19). It is noted that resolution of other agreement items from KTI technical exchange meetings may also contribute to model validation.

In addition, the contribution of cladding to total system performance was evaluated in a sensitivity study, documented in the "robustness analysis" for the TSPA-SR (CRWMS M\&O 2000bm, Sections 5.3 and 5.3.4.1). Cladding credit is substantially diminished by setting four of the five cladding parameters used in TSPA-SR at their $95 \%$ distribution points signifying greater release rates. The result was a minor increase in the calculated average annual dose over the first 100,000 yr for 100 realizations of the TSPA-SR model (a factor of 1.5 increase), compared to the nominal case in which these parameters were sampled over their full ranges. Thus the contribution of cladding, as represented in TSPA-SR, is minor for the $10,000-y r$ compliance period. This further supports the position that the validation review findings pertaining to this model do not impact the conclusion of the TSPA-SR (i.e. calculated annual dose; see CRWMS M\&O 2000bm, Section 6.1).

E. Responsible Individual:

Christine Stockman/

Type Name/Signature

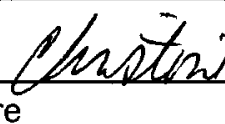

stin

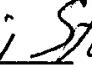

Sted

Date:

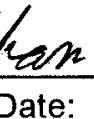

\section{$n$}

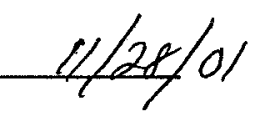


MODEL VALIDATION - IMPACT REVIEW

A. Model: Breakage of CSNF Clad by Static Loading (K.19-2)
B. AMR: Breakage of CSNF Cladding by Mechanical Loading (CAL-EBS-MD-000001 Rev. 00) (CRWMS M\&O 1999a)

C. Category (Check appropriate case)

$\triangle$ 1) Model NOT USED in TSPA and DID NOT SERVE as a basis for screening FEPS.

Result: No impact on TSPA-SR

2) Model NOT USED in TSPA but DID SERVE as a basis for screening FEPs.

Result: Assess impact of validation findings on FEP screening - Complete Section D below

3) Model IS USED in TSPA but DID NOT SERVE as a basis for screening FEPs.

Result: Assess impact of validation findings on TSPA-SR results - Complete Section D below

4) Model IS USED in TSPA and DID SERVE as a basis for screening FEPS.

Result: Assess impact of validation findings on TSPA-SR results and FEP screening Complete Section D below

D. Path Forward and Impact on TSPA (Describe in detail)

This model (documented as a calculation) was used to evaluate breakage of the cladding caused by loading from backfill or debris from rockfall. The reviewer's comments on the robustness of cladding are acknowledged. The model is conservative, so there is no impact from the validation review findings on the conclusions of TSPA-SR (i.e. calculated annual dose; see CRWMS M\&O $2000 \mathrm{bm}$, Section 6.1).

The KTI agreements for the Repository Design and Thermal-Mechanical Effects (RDTME) Key Technical Issue (Gardner 2001) include a commitment to further develop and test rockfall models, and to reevaluate the application of rockfall model output to other models including the Model of Breakage of CSNF Clad by Static Loading (RDTME 3.19). It is noted that resolution of other agreement items from KTI technical exchange meetings may also contribute to model validation.

In addition, the contribution of cladding to total system performance was evaluated in a sensitivity study, documented in the "robustness analysis" for the TSPA-SR (CRWMS M\&O 2000bm, Sections 5.3 and 5.3.4.1). Cladding credit is substantially diminished by setting four of the five cladding parameters used in TSPA-SR at their 95\% distribution points signifying greater release rates. The result was a minor increase in the calculated average annual dose over the first 100,000 yr for 100 realizations of the TSPA-SR model (a factor of 1.5 increase), compared to the nominal case in which these parameters were sampled over their full ranges. Thus the contribution of cladding, as represented in TSPA-SR, is minor for the 10,000-yr compliance period. This further supports the position that the validation review findings pertaining to this model do not impact the conclusion of the TSPA-SR (i.e. calculated annual dose; see CRWMS M\&O 2000bm, Section 6.1).

E. Responsible Individual:

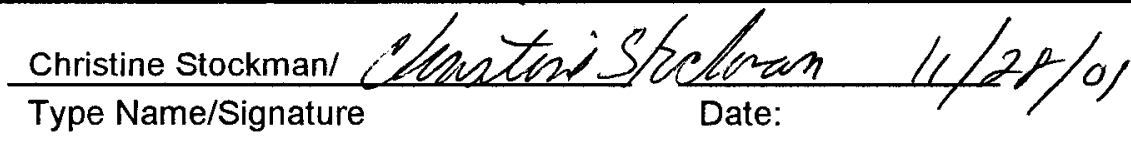




\section{MODEL VALIDATION - IMPACT REVIEW}
A. Model: DRKBA Rockfall Model (L.1)
B. AMR: Drift Degradation Analysis (ANL-EBS- MD-000027 Rev. 01) (CRWMS M\&O 2000x)

C. Category (Check appropriate case)

1) Model NOT USED in TSPA and DID NOT SERVE as a basis for screening FEPs.

Result: No impact on TSPA-SR

2) Model NOT USED in TSPA but DID SERVE as a basis for screening FEPs.

Result: Assess impact of validation findings on FEP screening - Complete Section D below

3) Model IS USED in TSPA but DID NOT SERVE as a basis for screening FEPS.

Result: Assess impact of validation findings on TSPA-SR results - Complete Section D below

$\triangle 4$ ) Model IS USED in TSPA and DID SERVE as a basis for screening FEPS.

Result: Assess impact of validation findings on TSPA-SR results and FEP screening Complete Section D below

D. Path Forward and Impact on TSPA (Describe in detail)

See continuation sheets.

E. Responsible Individual: $\frac{\text { Robert MacKinnon / Roleat Mor Kum }}{\text { Type Name/Signature }} \frac{11 / 15 / 01}{\text { Date: }}$ 
As stated by DOE in the summary from the Technical Exchange on the Repository Design and Thermal-Mechanical Effects (RDTME) Key Technical Issue (February 6-8, 2001, Las Vegas, NV) "... the Drift Degradation Analysis is consistent with current understanding of the Yucca Mountain site and the present level of detail for the design." Elaborating on this point, the DRKBA model is reasonably conservative for predicting the occurrence of large rockfall blocks, which are then used as the basis for structural analysis of the drip shield design in the calculation report: Rock Fall on Drip Shield (CRWMS M\&O 2000bw). The DOE has committed to model improvements and alternative modeling approaches as discussed below, but the current model and its supporting and related documentation are considered to provide adequate confidence that the effects of rockfall on integrity of the waste package can be limited for $10,000 \mathrm{yr}$ by the presence of the drip shield, and can therefore be "screened out" for TSPA-SR.

The path forward for model validation is consistent with the KTI agreements for the Repository Design and Thermal-Mechanical Effects (RDTME) Key Technical Issue (Gardner 2001). The relevant agreement items are:

- Provide field data and analysis of rock bridges between rock joints that are treated as cohesion in DRKBA modeling together with a technical basis for how a reduction in cohesion adequately accounts for thermal effects (RDTME 3.15)

- Provide a technical basis for the DOE position that the method used to model joint planes as circular discs does not under-represent the smaller trace-length fractures (RDTME 3.16)

- Provide the technical basis for effective maximum rock size including consideration of the effect of variation of the joint dip angle (RDTME 3.17)

- The acceptability of the process models (Drift Degradation) that determine whether rock fall can be screened out from performance assessment abstractions needs to be substantiated by the DOE (RDTME 3.19)

Considered in more detail, the DOE has committed to: 1) provide revised DRKBA analyses using appropriate ranges of strength properties for rock joints taken from a design parameters analysis report (or other document), accounting for their long-term degradation; 2) provide an analysis of block sizes based on the full distribution of joint trace length data from Fracture Geometry Analysis for the Stratigraphic Units of the Repository Host Horizon (CRWMS M\&O 2000ae), supplemented by available small joint trace length data; and 3) verify the results of the revised DRKBA analyses using:

- Appropriate boundary conditions for thermal and seismic loading

- Critical fracture patterns from the DRKBA Monte Carlo simulations (at least two patterns for each rock unit)

- Thermal and mechanical properties for rock blocks and joints from a design parameters analysis report (or other document)

- Long-term degradation of joint strength parameters

- Site-specific ground motion time histories appropriate for post-closure period.

This additional verification work will address the performance of the rock fall model for its intended use (i.e., thermal- and seismic-related postclosure drift degradation). It will improve 
upon the current UDEC validation results (CRWMS M\&O 2000x, Attachment V) by using multiple realizations of fracture patterns modeled in three-dimensional space, with seismic and thermal loads directly applied. This work will extend the validation approach presented in the Drift Degradation Analysis (CRWMS M\&O 2000x, Section 6.5) and improve confidence in the methodology.

Based on the results of the analyses above and subsequent revision to drip shield structural calculations, the DOE will reconsider the screening decision for inclusion or exclusion of rockfall in performance assessment analysis. Any changes to screening decisions will be documented in analyses prior to a potential License Application. Note that verification of the results from the revised DRKBA will be developed using a distinct-element modeling approach that can represent both seismic and thermal loads explicitly. It is noted that resolution of other agreement items from KTI technical exchange meetings may also contribute to model validation. 
A. Model: Flow into Waste Packages Through Small Lid Openings (FLO) Model (L.6)
B. AMR: Flow of Water and Pooling in a Waste Package (ANL-EBS-MD-000055 Rev. 00) (BSC 2001c)

C. Category (Check appropriate case)

$\triangle 1$ ) Model NOT USED in TSPA and DID NOT SERVE as a basis for screening FEPS.

Result: No impact on TSPA-SR

2) Model NOT USED in TSPA but DID SERVE as a basis for screening FEPs.

Result: Assess impact of validation findings on FEP screening - Complete Section D below

3) Model IS USED in TSPA but DID NOT SERVE as a basis for screening FEPs.

Result: Assess impact of validation findings on TSPA-SR results - Complete Section D below

4) Model IS USED in TSPA and DID SERVE as a basis for screening FEPs.

Result: Assess impact of validation findings on TSPA-SR results and FEP screening Complete Section D below

D. Path Forward and Impact on TSPA (Describe in detail)

No impact. This model was developed after TSPA-SR (CRWMS M\&O 2000bl). The purpose of the model was to improve understanding of processes controlling water flow into waste packages.

As currently documented, the model is not planned for direct application in any future TSPA. As stated by the review, this model may find use as an alternative model for comparison to the EBS Radionuclide Transport Model (CRWMS M\&O 2000aa).

It is also noted that this model describes water movement through cracks in the drip shield or waste package, which is a topic that is also addressed by an AMR entitled: Water Diversion Model (CRWMS M\&O 2000bu), and another entitled: Water Distribution and Removal Model (CRWMS M\&O 2001t), for which model validation was found to be better developed and documented.

E. Responsible Individual:
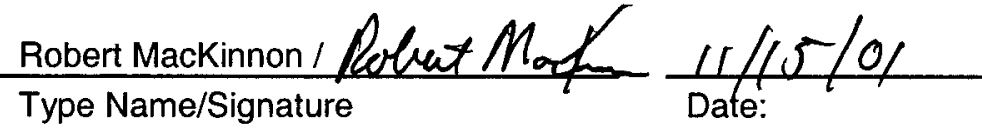
MODEL VALIDATION - IMPACT REVIEW

\begin{tabular}{|c|c|}
\hline $\begin{array}{l}\text { A. Model: In-Drift Transport of Radionuclides } \\
\text { Model (M.2) }\end{array}$ & $\begin{array}{l}\text { B. AMR: EBS Radionuclide Transport Model (ANL- } \\
\text { EBS-MD-000034 Rev. } 00 \text { ICN 1) (CRWMS M\&O } \\
\text { 2000aa) }\end{array}$ \\
\hline
\end{tabular}

C. Category (Check appropriate case)

$\triangle 1$ 1) Model NOT USED in TSPA and DID NOT SERVE as a basis for screening FEPs.

Result: No impact on TSPA-SR

2) Model NOT USED in TSPA but DID SERVE as a basis for screening FEPS.

Result: Assess impact of validation findings on FEP screening - Complete Section $D$ below

3) Model IS USED in TSPA but DID NOT SERVE as a basis for screening FEPS.

Result: Assess impact of validation findings on TSPA-SR results - Complete Section D below

4) Model IS USED in TSPA and DID SERVE as a basis for screening FEPS.

Result: Assess impact of validation findings on TSPA-SR results and FEP screening Complete Section D below

D. Path Forward and Impact on TSPA (Describe in detail)

See continuation sheet.

E. Responsible Individual: $\frac{\text { Robert MacKinnon/ Robent Mor/Ln }}{\text { Type Name/Signature }} \frac{11 / 15 / 01}{\text { Date: }}$ 
Continuation of item D (Model M.2):

This model validation review finding has no impact on the conclusions of TSPA-SR (i.e. calculated annual dose; see CRWMS M\&O 2000bm, Section 6.1) because:

- The EBS Radionuclide Transport Model (CRWMS M\&O 2000aa) is primarily a sensitivity study that was not used for TSPA-SR.

- The model will not be used directly for the TSPA or other products that support the License Application.

To the extent that the conceptual model is the same as that used in the EBS Radionuclide Transport Abstraction Model (CRWMS M\&O 2000z) which was used for TSPA-SR, the following comments apply:

Lateral flux in the invert is not represented in the 1-D model, but if so then the flow path would be longer, because the 1-D flowpath represented by the model is the shortest possible given the invert geometry. The flow in the invert will be unsaturated, so the vertical (shortest path) velocity is controlled by the water content and the gravitational potential.

The use of a continuum approximation to represent pore water velocity (calculated from volumetric water content and liquid flux) may be nonconservative if liquid flow occurs in channels where the velocity is faster than the average. For TSPA-SR (CRWMS M\&O 2000bm, Section 5.2.5.1) invert diffusion affects system performance only during early time (diminishing a few thousands years after repository closure) when water content in the invert ballast material, and thus the advective flow velocity, is decreased. Waste package failures are not predicted during early time, so deviation of invert transport behavior from the average response would have no impact on system performance except for waste package early failure scenarios, moreover, the effect of invert transport on dose consequences for such scenarios is small.

The 1-D approach is analogous to column studies, for which abundant experimental data are available. Whereas such analogous data were not included in the AMR, this does not impact the conceptual model for invert transport used for TSPA-SR. Sorption is conservatively ignored in the TSPA-SR, so the treatment of sorption in this AMR has no impact. 
MODEL VALIDATION - IMPACT REVIEW

\begin{tabular}{l|l}
\hline $\begin{array}{l}\text { A. Model: In-Drift Colloids and Concentrations } \\
\text { Model (M.3) }\end{array}$ & $\begin{array}{l}\text { B. AMR: In-Drift Colloids and Concentration (ANL- } \\
\text { EBS-MD-000042 Rev. 00) (CRWMS M\&O } \\
2000 \text { al) }\end{array}$ \\
\hline
\end{tabular}

C. Category (Check appropriate case)

1) Model NOT USED in TSPA and DID NOT SERVE as a basis for screening FEPS.

Result: No impact on TSPA-SR

2) Model NOT USED in TSPA but DID SERVE as a basis for screening FEPs.

Result: Assess impact of validation findings on FEP screening - Complete Section $D$ below

3) Model IS USED in TSPA but DID NOT SERVE as a basis for screening FEPs.

Result: Assess impact of validation findings on TSPA-SR results - Complete Section D below

$\triangle 4$ ) Model IS USED in TSPA and DID SERVE as a basis for screening FEPs.

Result: Assess impact of validation findings on TSPA-SR results and FEP screening Complete Section $D$ below

D. Path Forward and Impact on TSPA (Describe in detail)

The representation of in-drift colloid-associated radionuclide transport for TSPA-SR, is not significantly impacted by the validation review findings, because the TSPA-SR colloidabstraction approach documented in the subject AMR uses conservative and/or bounding values for parameters where data are insufficient. Use of the approach to support FEP screening is likewise not impacted. In addition, sensitivity studies have shown that the contribution of colloidal transport in the waste package and the invert is small (CRWMS M\&O 2000bm, Section 5.3.4.2). Therefore, the impact of the model validation review findings for this model on the conclusions of TSPA-SR (i.e. calculated annual dose; see CRWMS M\&O 2000bm, Section 6.1) is insignificant.

E. Responsible Individual: $\frac{\text { Robert MacKinnon/ Roluet Mor Kan }}{\text { Type Name/Signature }} \frac{11 / 15 / 01}{\text { Date: }}$ 


\section{MODEL VALIDATION - IMPACT REVIEW}

\begin{tabular}{l|l}
\hline $\begin{array}{l}\text { A. Model: Seepage/Invert Interactions Model } \\
\text { (M.4) }\end{array}$ & B. $\begin{array}{l}\text { AMR: Seepage/Invert Interactions (ANL-EBS- } \\
\text { MD-000044 Rev. 00) (CRWMS M\&O 2000bg) }\end{array}$ \\
\hline
\end{tabular}

C. Category (Check appropriate case)

1) Model NOT USED in TSPA and DID NOT SERVE as a basis for screening FEPs.

Result: No impact on TSPA-SR

$\bigotimes$ 2) Model NOT USED in TSPA but DID SERVE as a basis for screening FEPs.

Result: Assess impact of validation findings on FEP screening - Complete Section $D$ below

3) Model IS USED in TSPA but DID NOT SERVE as a basis for screening FEPs.

Result: Assess impact of validation findings on TSPA-SR results - Complete Section D below

4) Model IS USED in TSPA and DID SERVE as a basis for screening FEPs.

Result: Assess impact of validation findings on TSPA-SR results and FEP screening Complete Section D below

D. Path Forward and Impact on TSPA (Describe in detail)

The model validation review findings have no impact on TSPA-SR because the invert has small benefit to performance (CRWMS M\&O 2000bm, Sections 5.2.5.1 and 5.3.4.2). The primary conclusion from this model is that invert materials will not be present in sufficient quantities, relative to the host rock and other EBS materials, to exert significant influence on water composition. For example, the crushed tuff to be used as invert ballast material would be derived from the host rock, so that waters which had already been conditioned to the host rock would exhibit limited changes in composition on contact with the invert. Also, the structural steel that used in the invert would not affect water composition because substantial quantities of similar steel would be located elsewhere in the EBS including inside the waste package, and because the steel corrosion products will be insoluble in the oxidizing environment of the invert.

In addition, potential changes in hydrologic and transport properties of the invert would have little impact on transport of radionuclides in the EBS or elsewhere, because the invert contribution to performance is not significant. The typical depth dimension of the invert, about one meter, is much less than the transport distance through the unsaturated zone, and the effect on radionuclide transport is therefore much less.

The path forward includes analyses of coupled processes in the EBS per KTI agreement ENFE 2.7 (Williams 2001). It is noted that resolution of other agreement items from KTI technical exchange meetings may also contribute to model validation. At this time, however, no experiments are planned as the final invert design and material selection has not been made. Tests involving crushed tuff have low priority because the invert offers little waste isolation performance.

\footnotetext{
E. Responsible Individual: $\frac{\text { Robert MacKinnon / Povent Mock }}{\text { Type Name/Signature }} \frac{11 / 15 / 01}{\text { Date: }}$
} 
A. Model: EBS Radionuclide Transport Abstraction Model (M.5)
B. AMR: EBS Radionuclide Transport Abstraction (ANL-WIS-PA-000001 Rev. 00 ICN 2) (CRWMS M\&O 2000z)

C. Category (Check appropriate case)

1) Model NOT USED in TSPA and DID NOT SERVE as a basis for screening FEPs.

Result: No impact on TSPA-SR

2) Model NOT USED in TSPA but DID SERVE as a basis for screening FEPs.

Result: Assess impact of validation findings on FEP screening - Complete Section $D$ below

\3) Model IS USED in TSPA but DID NOT SERVE as a basis for screening FEPS.

Result: Assess impact of validation findings on TSPA-SR results - Complete Section D below

4) Model IS USED in TSPA and DID SERVE as a basis for screening FEPS.

Result: Assess impact of validation findings on TSPA-SR results and FEP screening Complete Section $D$ below

D. Path Forward and Impact on TSPA (Describe in detail)

There is no impact from the findings of the model validation review on the conclusions of the TSPA-SR, because this model provides bounding treatment of processes controlling radionuclide transport from the waste package to the drift wall. This position is supported by the part of the review which states that Section 7.2 of the AMR makes a strong case that the model is valid and appropriate for its intended use, to represent fundamental flow and transport processes in a bounding or conservative framework. The review goes on to state that the AMR makes transparent and logical arguments that provide confidence in the model. However, conservatism is not considered grounds for compliant model validation. Hence the Bin-3 designation is justified, although there is no resulting impact on TSPA-SR that would cause calculated dose consequences to increase.

The path forward includes an experiment to validate the model's representation of flow entering and leaving breached waste packages and drip shields. The path forward also includes developing a representation of the performance consequences from drip shield displacement by thermal expansion of the titanium, floor heave, pallet failure, and seismic ground motion. Ongoing process modeling activities will provide additional support. Based on the outcome of model development, the representation of drip shield performance would be included in TSPA rather than screening out these effects. These two items will address what the authors believe to be the major needs for confidence building in the abstraction model.

E. Responsible Individual: $\frac{\text { Robert Mackinnon / Ledeet MerK }}{\text { Type Name/Signature }} \frac{11 / 15 / 01}{\text { Date: }}$ 


\section{MODEL VALIDATION - IMPACT REVIEW}

A. Model: FRACL Calibration to Borehole Chloride (N.3-1)
B. AMR: Radionuclide Transport Models Under Ambient Conditions (MDL-NBS-HS-000008 Rev. 00) (CRWMS M\&O 2000ba)

C. Category (Check appropriate case)

1) Model NOT USED in TSPA and DID NOT SERVE as a basis for screening FEPS.

Result: No impact on TSPA-SR

2) Model NOT USED in TSPA but DID SERVE as a basis for screening FEPS.

Result: Assess impact of validation findings on FEP screening - Complete Section D below

$\triangle 3$ ) Model IS USED in TSPA but DID NOT SERVE as a basis for screening FEPS.

Result: Assess impact of validation findings on TSPA-SR results - Complete Section D below

4) Model IS USED in TSPA and DID SERVE as a basis for screening FEPS.

Result: Assess impact of validation findings on TSPA-SR results and FEP screening Complete Section $D$ below

D. Path Forward and Impact on TSPA (Describe in detail)

See continuation sheets.

E. Responsible Individual: $\frac{\text { Bo Bodvarsson } / 100}{\text { Type Name/Signature }}(1 / 15 / \phi)$

TDR-WIS-MD-000005 REV 00E $\left.\mathcal{q}_{1 / 1 S^{A}}\right|^{01}$ 


\section{Continuation of item D (Model N.3-1).}

The reviewer has correctly pointed out that there is significant variability in the measured chloride data used to assess the FRACL model, and disputes that the correlation presented represents "reasonable agreement." For several reasons, the AMR authors believe that the FRACL model-chloride data comparison results do support the UZ radionuclide transport model, and are appropriate for use. Also, the results are consistent with other methods used to assess the reasonableness of the $\mathrm{UZ}$ radionuclide transport model, and would not significantly affect the overall TSPA-SR results even if they were excluded.

Validation of the model flow field in fractured rock below the repository is the subject of a KTI agreement, for the Radionuclide Transport (RT) Key Technical Issue (Reamer and Williams 2000). DOE has agreed to provide the analysis of geochemical data used for support of the flow field below the repository (RT 3.2). It is noted that resolution of other agreement items from KTI technical exchange meetings may also contribute to model validation.

Resolution of this agreement item will partially address the model validation review findings pertaining to the use of chloride data for model-data comparison. The FRACL model-chloride data comparisons are appropriate for use in supporting the radionuclide transport model for the following reasons:

Firstly, concerning the validation of FRACL against measured chloride data from borehole UE25 UZ\#16 described in this AMR, it should be noted that all the measurements in Figure 6.4 .4 of the AMR came from the UE-25 UZ\#16 well. A better measure of the agreement is also provided by Figure 6-27 in the AMR: UZ Flow Models and Submodels (CRWMS M\&O 2000bq), which shows the very large deviation of predictions based on uncalibrated infiltration, and supports the claim of reasonable agreement made in this AMR. Regarding the boundary condition, it should be noted that the $0.62 \mathrm{mg} / \mathrm{L}$ concentration was not used for consideration of this model in either AMR; the value used was $38 \mathrm{mg} / \mathrm{L}$ in both cases. The reviewer is correct that the measured chloride concentrations are widely variable, and there is also some uncertainty associated with the chloride concentration in the infiltrating water. Additional data have since been collected on porewater chloride, and it is anticipated that the FRACL model would be reevaluated using the new data during the next revision of the AMR to support a potential License Application.

Secondly, it should be pointed out that models and results from this AMR are not directly used in the TSPA-SR. The FRACL code was used to simulate the chloride profile for borehole UE-25 UZ\#16 in an attempt to gain confidence in its use within the context of the Radionuclide Transport Model described in this AMR, which is then abstracted in the TSPA-SR. The FRACL model itself was not used to simulate radionuclide transport in the TSPA. Instead, UZ radionuclide transport was simulated for TSPA-SR using the FEHM code with the residence time transfer function particle-tracking technique, which is described in the AMR: Particle Tracking Model and Abstraction of Transport Processes (CRWMS M\&O 2000ay). For this reason the validation status of the FRACL code is not likely to have a significant impact on, or change the conclusions of, the TSPA-SR.

In summary, due to its limited use in the comparison to the chloride data of UE-25 UZ\#16, which is used to corroborate the transport calculations in the TSPA-SR, it is appropriate to consider and use the FRACL results. The overall validation of the transport calculations is addressed in other AMRs, and is partially addressed by NRC KTIs related to transport. Also, future revisions of this AMR for the LA will incorporate additional data (such as porewater 
chloride concentrations) that will provide further confidence in the calibration and validation of the FRACL model. 


\section{MODEL VALIDATION - IMPACT REVIEW}

A. Model: Abstraction of FEHM and Coupling with UZ Mass Flux (P.4-2)
B. AMR: Input and Results of the Base-Case SZ Flow and Transport Model for TSPA (ANL-NBSHS-000030 Rev. 00) (CRWMS M\&O 2000aq)

C. Category (Check appropriate case)

1) Model NOT USED in TSPA and DID NOT SERVE as a basis for screening FEPs.

Result: No impact on TSPA-SR

2) Model NOT USED in TSPA but DID SERVE as a basis for screening FEPs.

Result: Assess impact of validation findings on FEP screening - Complete Section D below

$\triangle$ 3) Model IS USED in TSPA but DID NOT SERVE as a basis for screening FEPs.

Result: Assess impact of validation findings on TSPA-SR results - Complete Section D below

4) Model IS USED in TSPA and DID SERVE as a basis for screening FEPS.

Result: Assess impact of validation findings on TSPA-SR results and FEP screening Complete Section D below

D. Path Forward and Impact on TSPA (Describe in detail)

See continuation sheets.

E. Responsible Individual:

Bruce Robinson 1 Type Name/Signature

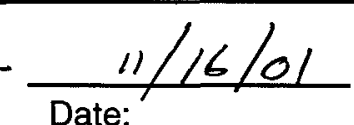

Date: 
Continuation of item D (Model P.4-2):

This model uses a convolution integral method implemented as an external routine called by GoldSim (Golder Associates 2000) to couple breakthrough curves generated using the 3-D SZ site-scale flow and transport model (based on the FEHM code), with the breakthrough curves from the "pipe" model used to represent transport of daughter radionuclides, and the radionuclide mass releases from the UZ, in the TSPA calculations. In this way the radionuclide mass flux at the biosphere downgradient of the repository is estimated as a function of the transient mass flux at the water table beneath a repository. The effects of climate change are incorporated into the convolution integral analysis by assuming instantaneous change from one steady-state flow condition to another in the SZ. This is believed to be conservative because climate changes in the TSPA-SR model tend to increase flow in the SZ (i.e., only from drier to wetter conditions) and the associated increases in storage of contaminants in the SZ is neglected in the model. The changes in flow condition are approximated as multiples of the groundwater flux for the base case. Radioactive decay is applied to transport as loss of mass by first-order decay through the interval of travel time. Visual inspection is used to check the breakthrough curves; other techniques are also being considered to check these intermediate results.

The UZ mass coupling is performed using a convolution subroutine that simply executes mathematical manipulations. The approach is based on a conceptual model of the saturated zone as a linear system that can be represented by breakthrough curves calculated from linear sorption behavior, for unit input fluxes of different radionuclides. The linear sorption approach is approximate, but it is mathematically tractable and widely used in groundwater contaminant transport modeling. Essentially, every Performance Assessment analysis performed in the Yucca Mountain and the Waste Isolation Pilot Plant (WIPP) has used the linear sorption "Kd" approach. Although it is recognized that the process of sorption is complex and that $\mathrm{Kd}$ values are specific to the chemical conditions and mineralogy, as a practical matter, linear sorption is assumed, and the uncertainties associated with this assumption are incorporated into the parameter uncertainty distribution used in the TSPA analysis. The sorption parameters (Kds) are based on consistent application of laboratory and field data as detailed for the Transport Parameters from C-Wells and Laboratory Studies Model (P.4-3). The flow fields are calculated by the SZ Flow Model (O.5). The implementing software was verified and documented in the subject AMR.

In summary, this model integrates the following component models in a simple computation scheme:

- The SZ flow model is validated elsewhere (see Model O.5)

- The "pipe" model is considered separately (see Model P.4-1)

- Mass flux from the UZ is calculated upstream in the TSPA-SR model

Documentation of the mathematical steps in this integration model, and testing to fully demonstrate appropriate representation of chemical transport, radioactive decay, climate change, and fracture heterogeneity, is not yet complete. The reviewer's comments are acknowledged, and the path forward will include improved documentation, justification for the approach, and verification as discussed below. However, the conceptual model is straightforward, and the implementing calculation has been checked, so future activities are unlikely to change the SZ transport model in a way that would significantly affect the conclusions of TSPA-SR. 
The description of this model will be clarified, and more complete explanation of the calculation procedure will be provided, in future revisions of the reports that support a potential License Application. Validation and verification activities will include evaluation of the appropriateness of the FEHM modeling, coupling of FEHM output with UZ mass flux, incorporation of FEHM output with that from the "pipe" model, and the representation of climate change. Confidence will also be improved by review of model input parameters, comparison with testing results where practicable, and verification of model output following the approach begun in Total System Performance Assessment (TSPA) Model for Site Recommendation (CRWMS M\&O 2000bl; Section 6.3.7). 
MODEL VALIDATION - IMPACT REVIEW

A. Model: Transport Parameters from C-Wells and Laboratory Studies (P.4-3)
B. AMR: Input and Results of the Base-Case SZ Flow and Transport Model for TSPA (ANL-NBSHS-000030 Rev. 00) (CRWMS M\&O 2000ag)

C. Category (Check appropriate case)

1) Model NOT USED in TSPA and DID NOT SERVE as a basis for screening FEPs.

Result: No impact on TSPA-SR

2) Model NOT USED in TSPA but DID SERVE as a basis for screening FEPs.

Result: Assess impact of validation findings on FEP screening - Complete Section D below

3) Model IS USED in TSPA but DID NOT SERVE as a basis for screening FEPs.

Result: Assess impact of validation findings on TSPA-SR results - Complete Section D below

\4) Model IS USED in TSPA and DID SERVE as a basis for screening FEPs.

Result: Assess impact of validation findings on TSPA-SR results and FEP screening Complete Section $D$ below

D. Path Forward and Impact on TSPA (Describe in detail)

See continuation sheet.

E. Responsible Individual:

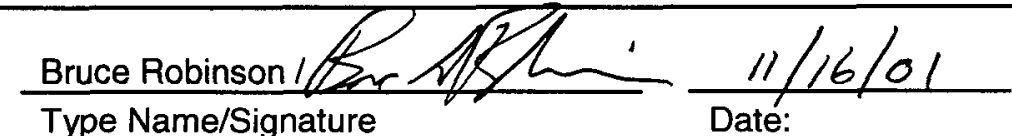

Type Name/Signature

Date: 
Continuation of item D (Model P.4-3):

This model consists of results from laboratory transport tests and field tracer tests, and fitting of field breakthrough curves generated using dual-porosity transport models (RELAP and RETRAN, CRWMS M\&O 2001r). Fitting of breakthrough curves for non-sorbing tracers was used to estimate dispersivity, matrix diffusion, and porosity. Fitting of the lithium tracer data was used to estimate sorption parameters. Fitting of colloid tracer data was used to estimate filtration rate constants. Laboratory studies included batch tests to characterize lithium sorption, matrix diffusion measurements, and column tests to evaluate transport in fractured and crushed tuff. Comparison of results from laboratory and field responses was used to show that laboratoryderived sorption parameters could be used defensibly in field-scale predictive calculations. Comparison of laboratory-derived matrix diffusion and mass-transfer coefficients with field responses showed that parameter values estimated from the field tests were smaller (less matrix diffusion) than from the lab results.

The reviewer's comments on lack of validation documentation are acknowledged, but this finding does not impact the conclusions of the TSPA-SR. This is because the model is based on overlapping laboratory and field testing, and complementary analyses of laboratory and field test results, which provide confidence in the parameterization of the dual-porosity model used for TSPA-SR. Evidence for dual-porosity behavior is provided by both field tracer testing, and laboratory diffusion testing. Field testing has been performed in transmissive rock units in the saturated zone downgradient from the Yucca Mountain site, that represent likely transport pathways for radionuclides. The simultaneous use of tracers with distinct transport properties (diffusion coefficients and $\mathrm{Kd}$ values) provided very strong evidence that the dual-porosity approach is valid for the fractured volcanic tuffs. Use of dual-porosity models to represent flow and transport in fractured rock has been reported extensively in the technical literature. For example, transport for comparable hydrogeologic conditions was evaluated by Grisak and Pickens (1980), Neretnicks (1980), Sudicky and Frind (1981), Maloszewski and Zuber (1985, 1991 ) and found to be appropriate for interpreting transport tests in fractured rock. Robinson (1994) documented an approach for validating this model for use in the Yucca Mountain SZ far in advance of the actual C-Wells experiments. Therefore, the validation of matrix diffusion in a dual-porosity system was the fundamental design basis for the test, and results subsequently showed that the model is appropriate for the volcanic tuffs.

Further validation effort will be applied to the selection of the dual-porosity model, and the use of RELAP and RETRAN (CRWMS M\&O 2001r) to simulate field test results. In addition, additional documentation is needed to address the validity of the output data (dispersivity and matrix diffusion coefficients) and the use of the lithium tracer tests to justify the use of laboratory-measured $\mathrm{Kd}$ values to model $\mathrm{SZ}$ transport. Where possible, alternative models will be used for curve fitting to provide additional justification for the approach selected as the basis for $\mathrm{SZ}$ parameter estimation. The additional validation will be documented in the revision of this AMR for a future License Application. 


\begin{tabular}{l|l}
\hline $\begin{array}{l}\text { A. Model: Surface Soil Model in GENII-S } \\
(Q .9-1)\end{array}$ & B. AMRs: \\
& Evaluate Soil/Radionuclide Removal by Erosion and \\
Leaching (ANL-NBS-MD-000009 Rev. 00, ICN 01) \\
(CRWMS M\&O 2001i) \\
Nominal Performance Biosphere Dose Conversion Factor \\
Analysis (ANL-MGR-MD-000009 Rev. 01) (CRWMS M\&O \\
$2001 \mathrm{~m})$
\end{tabular}

C. Category (Check appropriate case)

1) Model NOT USED in TSPA and DID NOT SERVE as a basis for screening FEPS.

Result: No impact on TSPA-SR

2) Model NOT USED in TSPA but DID SERVE as a basis for screening FEPS.

Result: Assess impact of validation findings on FEP screening - Complete Section $D$ below

$\triangle 3$ ) Model IS USED in TSPA but DID NOT SERVE as a basis for screening FEPs.

Result: Assess impact of validation findings on TSPA-SR results - Complete Section D below

4) Model IS USED in TSPA and DID SERVE as a basis for screening FEPS.

Result: Assess impact of validation findings on TSPA-SR results and FEP screening Complete Section D below

D. Path Forward and Impact on TSPA (Describe in detail)

See continuation sheets.

E. Responsible Individual:

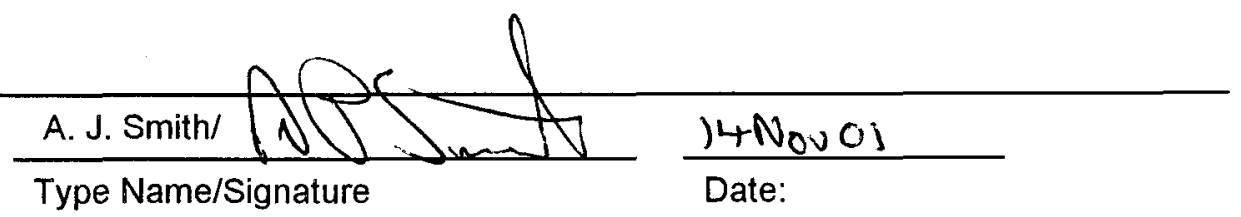




\section{Continuation of item D (Model Q.9-1):}

The reviewer's comment is correct that the current soil model does not explicitly simulate the details of several processes that may affect radionuclide concentrations in soils, and that there are several methods (including those recommended by the reviewer) that could be used to increase the level of detail of the analysis. However, it should also be noted that the relatively simple approach used in this model has several advantages which are appropriate for analysis of longterm performance. The current model is reasonable and technically justifiable, although it produces demonstrably conservative results.

Whether or not the assumption of a uniformly contaminated soil zone to $15 \mathrm{~cm}$ is realistic is not specifically addressed by a KTI agreement. However, the KTI agreements for the Total System Performance Assessment Integration Key Technical Issue (Cornell 2001) partially address the model validation reviewer's comments. In particular, the following agreement items address Kd values used for radionuclides in soil and sampling methodology used in GENII-S (CRWMS M\&O 2001m):

- Provide justification that the Kd values used for radionuclides in the soil in Amargosa Valley are realistic or conservative for actual conditions at the receptor location (KTS 3.33)

- Provide the technical basis for selection of radionuclide or element specific biosphere parameters (except for Kds which are addressed in KTS 3.33) that are important in the BDCF calculations (e.g., soil to plant transfer factors) (KTS 3.34)

- Provide a quantitative analysis that the sampling method including the correlations between BDCFs utilized by the TSPA code to abstract the GENII-S process model data adequately represent the uncertainty and variability and correlations for the biosphere process model (KTS 3.37)

The current approach is defensible for its intended use, for several reasons, and potential future revisions of the model are unlikely to result in dose assessments that differ significantly from TSPA-SR. Firstly, the current approach (CRWMS M\&O 2001m) is the same basic model that is used in other Biosphere models developed both in the USA and by international radiation protection programs (IAEA 2001, Section 5.3).

Secondly, it is true that the current model does not capture details of the variability of radionuclide concentrations in soils due to processes such as non-uniform mixing, erosion or aeolian transport, and also does not explicitly incorporate temporal variation (generally building up over time). However, these simplifications will generally result in the overprediction of radionuclides available for uptake (with some exceptions such as the possible concentration in the upper $5 \mathrm{~cm}$ of soil noted by the reviewer). Similarly, the data feed to TSPA-SR (via downstream AMRs (CRWMS M\&O 2001a; 2001af) does not rely upon the predicted time evolution of the radionuclide build-up in soils. The biosphere dose conversion factor (BDCF) data feed to the TSPA-SR model depends only on the asymptotic (i.e., steady state) value of radionuclide build-up in soil, and the simplicity of this approach is considered not only adequate but also a distinct advantage. This approach is believed to be reasonable, since the purpose of the TSPA is to evaluate the long term behavior of the system.

Future revisions of the AMR will consider the uncertainties identified by the reviewer, including the effects of spatially variable radionuclide concentrations and soil salinity on Kds, to determine whether additional data collection, modeling or sensitivity studies are needed. The revised AMR 
will address the effects of uncertainties related to each of the model inputs. As the AMR authors continue to evaluate the uncertainties inherent in the process models, they will incorporate additional detail as necessary, or perform sensitivity studies as appropriate, to provide additional information necessary for validation. 


\section{MODEL VALIDATION - IMPACT REVIEW}

\begin{tabular}{l|l}
\hline A. Model: Radionuclide & B. AMRs: \\
Transfer to Animals (Q.9-4) & $\begin{array}{l}\text { Transfer Coefficient Analysis (ANL-MGR-MD-000008 Rev. 00 ICN 2) } \\
\text { (CRWMS M\&O 2000bn) } \\
\text { Nominal Performance Biosphere Dose Conversion Factor Analysis (ANL- } \\
\text { MGR-MD-000009 Rev. 01) (CRWMS M\&O 2001m) }\end{array}$ \\
\hline
\end{tabular}

C. Category (Check appropriate case)

1) Model NOT USED in TSPA and DID NOT SERVE as a basis for screening FEPS.

Result: No impact on TSPA-SR

2) Model NOT USED in TSPA but DID SERVE as a basis for screening FEPs. below

Result: Assess impact of validation findings on FEP screening - Complete Section $D$

$\triangle$ 3) Model IS USED in TSPA but DID NOT SERVE as a basis for screening FEPS. below

Result: Assess impact of validation findings on TSPA-SR results - Complete Section D

4) Model IS USED in TSPA and DID SERVE as a basis for screening FEPS.

Result: Assess impact of validation findings on TSPA-SR results and FEP screening Complete Section $D$ below

D. Path Forward and Impact on TSPA (Describe in detail)

The model validation reviewer has noted that several specific pathways are not included in the Radionuclide Transfer to Animals model, and that model-specific validation information has not been provided. The GENII-S based approach used for TSPA-SR dose calculations (CRWMS $\mathrm{M} \& \mathrm{O} 2001 \mathrm{~m}$ ) is believed to contain the primary pathways contributing to dose, and the contributions from additional pathways would be small and not significantly affect the conclusions of the TSPA-SR.

In the TSPA-SR dose calculations, the primary pathways for delivering dose to the receptor are drinking water and leafy vegetables. This model as implemented in GENII-S is similar to the equivalent model developed and in use by the International Atomic Energy Agency (see CRWMS M\&O 2000bn, Section 4.1 for a list of corroborating literature sources). Consequently, the dose calculated by TSPA-SR is only weakly dependent on the details of the model for transfer of radionuclides to animals, and any impact on dose would be small in comparison to the primary pathways.

The radionuclide transfer to animals model used in GENII-S is in the process of being validated, and will be documented in a revision of the AMR for the potential License Application. If the validation effort indicates that the current model is lacking, a more comprehensive model incorporating additional pathways may be developed.

The omission of incidental ingestion of contaminated soil while grazing, and inhalation of resuspended particles, is not specifically addressed by a KTI agreement.

E. Responsible Individual:

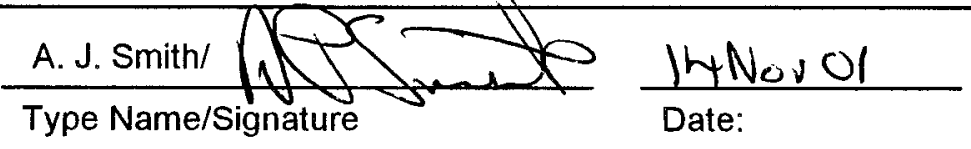


A. Model: Radionuclide Transfer to Aquatic Food (Q.9-5)
B. AMRs:

Transfer Coefficient Analysis (ANL-MGR-MD-000008 Rev, 00 ICN 2) (CRWMS M\&O 2000bn)

Nominal Performance Biosphere Dose Conversion Factor Analysis (ANLMGR-MD-000009 Rev. 01) (CRWMS M\&O 2001m)

C. Category (Check appropriate case)

1) Model NOT USED in TSPA and DID NOT SERVE as a basis for screening FEPs.

Result: No impact on TSPA-SR

2) Model NOT USED in TSPA but DID SERVE as a basis for screening FEPS.

Result: Assess impact of validation findings on FEP screening - Complete Section D below

$\triangle$ 3) Model IS USED in TSPA but DID NOT SERVE as a basis for screening FEPs.

Result: Assess impact of validation findings on TSPA-SR results - Complete Section D below

4) Model IS USED in TSPA and DID SERVE as a basis for screening FEPS.

Result: Assess impact of validation findings on TSPA-SR results and FEP screening Complete Section D below

D. Path Forward and Impact on TSPA (Describe in detail)

See continuation sheet.

E. Responsible Individual:

A. J. Smith/

Type Name/Signature

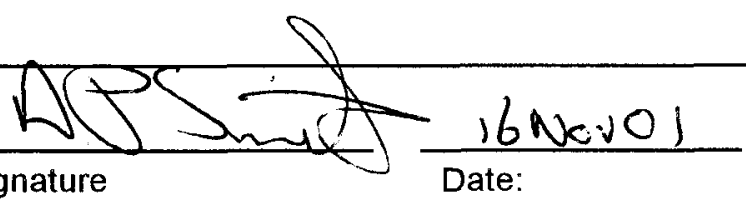




\section{Continuation of item D (Model Q.9-5):}

For the reasons summarized below, the issues raised by the model validation review concerning validation of this model do not impact the conclusions of the TSPA-SR (i.e. calculated annual dose; CRWMS M\&O 2000bm, Section 6.1). Further, due to a change in the potential pathways available, further assessment of dose consequences from aquatic pathways may be unnecessary.

The aquatic food pathway which uses the radionuclide transfer to aquatic food model is based on an infinite (large) source of water with a uniform concentration of radionuclides. In this large volume, all aquatic life including plants in the food chain are contaminated and the availability of radionuclides not constrained by a finite mass. The need for the aquatic food pathway in Amargosa Valley arose because, when the eating habits survey was conducted (CRWMS M\&O 2000 bn, Section 4.1.1), there was a small commercial catfish farm in operation. The farm used a limited sized tank to raise the fish, which represents a limited supply of radionuclides. Thus, doses estimated with this model were overly conservative and provided a higher dose than would actually be expected from this pathway. If necessary, a correction to account for the limited water supply, and associated improvement to the model are relatively simple and could be incorporated in the code update.

However, for all radionuclides except ${ }^{14} \mathrm{C}$, the dose contribution from aquatic foods is insignificant. For ${ }^{14} \mathrm{C}$ in the groundwater (if indeed there should be any present) the dose was overestimated by a factor of about ten. Because the TSPA-SR results are demonstrably conservative, there is no detrimental impact to the conclusions from a compliance perspective. Also, the catfish farm is no longer in operation so there are now no existing aquatic pathways.

The need (or lack thereof) for a model to represent aquaculture as an aquatic pathway for radionuclide uptake by humans in the Amargosa Valley is not specifically addressed by a KTI agreement. 


\section{MODEL VALIDATION - IMPACT REVIEW}

A. Model: Geometry of Volcanic Feeder System Model (R.2)
B. AMR: Characterize Eruptive Processes at Yucca Mountain, Nevada (ANL-MGR-GS000002 Rev. 00) (CRWMS M\&O 2000k)

C. Category (Check appropriate case)

1) Model NOT USED in TSPA and DID NOT SERVE as a basis for screening FEPs.

Result: No impact on TSPA-SR

2) Model NOT USED in TSPA but DID SERVE as a basis for screening FEPs.

Result: Assess impact of validation findings on FEP screening - Complete Section $D$ below

$\triangle 3$ ) Model IS USED in TSPA but DID NOT SERVE as a basis for screening FEPs.

Result: Assess impact of validation findings on TSPA-SR results - Complete Section D below

4) Model IS USED in TSPA and DID SERVE as a basis for screening FEPs.

Result: Assess impact of validation findings on TSPA-SR results and FEP screening Complete Section $\mathrm{D}$ below

D. Path Forward and Impact on TSPA (Describe in detail)

See continuation sheet.

E. Responsible Individual:

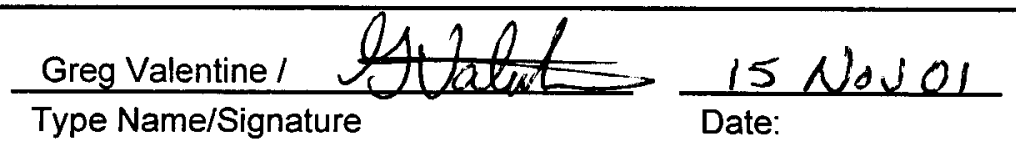




\section{Continuation of item D (Model R.2):}

This is a conceptual model that is used as a framework to determine how much contaminated volcanic ash could reach a control group. Briefly, the model assumes that magma propagates from depth through dikes. Initially these dikes may intersect the Earth's surface and erupt along a fissure (the trace of the dike). With time, upward flow of magma at shallow depths (within a few hundred meters of the Earth's surface) tends to focus into one or a few vents that are fed by conduits. These conduits represent places where thermal and mechanical erosion of the wall rocks have locally widened the dike. Each of these conduits may feed a growing scoria cone with attendant lava flows. Relatively explosive eruptions that are capable of spreading contamination as far as a control population are generally only fed from conduits (rather than the initial fissureeruption phases). Therefore for TSPA-SR, only eruptions fed by conduits were considered in the subject AMR. It was also assumed that any waste package that is wholly or partly intersected by a conduit would be disintegrated and available for dispersal in a volcanic plume. Although this conceptual model is used as the basis for calculation of eruptive doses in TSPA-SR, it is not completely and explicitly described by any individual document in the current suite of AMRs.

Because there is no practical way to directly test the conceptual model for the effect of eruptive processes on potential radionuclide releases associated with volcanic activity, there will inherently be significant uncertainty associated with this model. However, the present model is conservative in several respects, so it is considered unlikely that the conclusions of the TSPA-SR would be affected by additional validation activities. In the present model, all waste packages within the cross section of the conduit are considered to be entrained in an eruption, and the entire contents of each package are dispersed in the volcanic plume. If future work to further validate the conceptual model identifies modifications to the existing model, or credible alternative conceptual models, it is possible that more or fewer waste packages would be involved in the volcanic eruption scenario examined as part of the TSPA or that a larger or smaller radionuclide source term may need to be used for groundwater release scenarios.

There are several KTI agreements related to the issue of repository disruption by volcanic, for the Igneous Activity (IA) Key Technical Issue (NRC 2000):

- Document the approach for estimating the number of waste packages incorporated into the volcanic conduit, and the possible consequences of conduit elongation parallel to drifts (IA 2.5)

- Document the calculation of the number of waste packages hit by the intrusion (IA 2.10)

The additional documentation will involve revisions to several AMRs (CRWMS M\&O 2000l; 2000u; 2000aj; 2000ax) as well as the subject AMR noted above. These agreement items will address many of the points raised in the model validation review. It is noted that resolution of other agreement items from KTI technical exchange meetings may also contribute to model validation.

The current conceptual model and the basis for numerical estimates of the number of waste packages breached, will be explicitly described in revisions to the referenced AMRs. In addition, further analog investigations, modeling and testing studies are planned that will strengthen the technical bases for the conceptual model. Sensitivity studies will be performed to assess the extent to which uncertainties in eruptive processes could affect total system results. 


\section{MODEL VALIDATION - IMPACT REVIEW}

\begin{tabular}{l|l}
\hline A. Model: Total System Performance & B. $\begin{array}{l}\text { AMR: Total System Performance Assessment } \\
\text { (TSPA) Model for Site Recommendation (MDL- } \\
\text { Assessment-Site Recommendation Model } \\
\text { W.1-1) }\end{array}$ \\
& \begin{tabular}{l} 
WIS-PA-000002 Rev. 00) (CRWMS M\&O \\
\hline
\end{tabular} \\
\hline
\end{tabular}

C. Category (Check appropriate case) N/A, Model is the TSPA Model

1) Model NOT USED in TSPA and DID NOT SERVE as a basis for screening FEPS.

Result: No impact on TSPA-SR

2) Model NOT USED in TSPA but DID SERVE as a basis for screening FEPS.

Result: Assess impact of validation findings on FEP screening - Complete Section D below

3) Model IS USED in TSPA but DID NOT SERVE as a basis for screening FEPS.

Result: Assess impact of validation findings on TSPA-SR results - Complete Section D below

$\triangle 4)$ Model IS USED in TSPA and DID SERVE as a basis for screening FEPs.

Result: Assess impact of validation findings on TSPA-SR results and FEP screening Complete Section D below

D. Path Forward and Impact on TSPA (Describe in detail)

See continuation sheets.

E. Responsible Individual: $\frac{\text { Jerry McNeish / Jeng Q hineinh }}{\text { Type Name/Signalure }} \frac{11.14 .01}{\text { Date: }}$ 
Continuation of item D (Model U.1-1).

\section{Background}

Development of the TSPA-SR model (CRWMS M\&O 2000bl) was based on supporting abstraction and process-level models that represent different aspects of the repository. These abstraction and process-level models were specifically developed for use in the TSPA-SR model for Yucca Mountain.

The hierarchical aspect of total system performance assessment modeling in support of a potential License Application is based on a sequence of modeling activities that starts with the development of process level models that capture the key aspects of the natural and engineered systems. In turn these process models are frequently simplified into abstraction models. These simplified models are compared to the process models on which they are based to build confidence and to ensure that the key aspects of the system are being captured. Once confidence in these processes and abstractions is demonstrated, they become key components in determining the validity-ofestablishing confidence in the total system model, where the total system model is probabilistic and stochastic in nature and is intended to capture the behavior of the entire system. These modeling activities are intended to build upon each other sequentially so that when the total system model is finalized, one is confident that the total system is adequately represented.

Currently, model validation is defined as "a process to determine and document the adequacy of the scientific basis (i.e., confidence) for a model and to demonstrate that the model is appropriate and adequate for its intended use" (AP-3.10Q Rev. 2 ICN 4). Thus, model validation of the total system model depends upon the confidence-building activities that are conducted for the key underlying process and abstraction models. The scientific process established on the Yucca Mountain project to accomplish model validation includes comparing analyses or modeling results to data acquired from the laboratory, field experiments, natural and man-made analog studies or other relevant observations such as classical case histories from the literature. In addition to these technical confidence-building activities, the documentation process ensures the traceability, transparency and quality assurance of key modeling inputs such as data, assumptions, and computer software. The component models of the TSPA-SR model undergo verification and validation independently within the source AMRs. Then it is demonstrated that the integrated model is validated, with emphasis on integrated data and results, and the flow of data from each sub-component to the next. Criteria used to demonstrate this integrated model validation consist of: 1) comparison of the final results (in this case dose) to intermediate sub-system results; 2) verification of the implementation of AMR abstractions within the TSPASR model, including appropriate use of associated dynamically linked libraries (DLLs); and 3) ensuring correct data are passed between each DLL and the GoldSim code (Golder Associates 2000). When verification of the subsystem models and review of the integrated model are completed, confidence in the model is demonstrated.

For each of the process-level or abstraction analyses or models used as direct inputs to or component models of the TSPA-SR model, a "Results and Verification" subsection is included in Sections 6.3.1 through 6.3.9 of the subject AMR (CRWMS M\&O 2000bl). These subsections show the results from a median-value simulation (i.e., median values for all input parameters), and show that the process-level or abstraction models from the supporting AMRs have been implemented appropriately into the TSPA-SR model. Sections 6.5.1 through Sections 6.5.4 of the subject AMR discusses an "integrated testing" approach to the validation of the TSPA-SR model. 
There are several KTI agreements related to validation of the TSPA model, for the Total System Performance Assessment Integration (TSPAI) Key Technical Issue (Cornell 2001). In particular, agreements items 3.1 through 3.42 include many actions that will enhance the submodels for TSPA, and their documentation. In addition, the following agreement items pertain more directly to the TSPA system model:

- DOE will document the incorporation of alternative conceptual models into the TSPA model, and document the guidance given to process-level modeling experts, overall ensuring that incorporation of alternative models does not cause risk to be underestimated (TSPAI 4.01).

- DOE will document the methods that will be used to determine that the overall TSPA results are stable, and that the contributing submodels are numerically stable. The method will address the number of realizations, and describe the statistical treatment that will be used to evaluate stability (TSPAI 4.03).

- DOE will conduct appropriate analyses to demonstrate that the results of the TSPA are stable with respect to the effects of temporal and spatial discretization (TSPAI 4.04).

- DOE will document the process, and the implementation of the process, used to develop confidence in the TSPA models. The documentation will demonstrate compliance with model confidence-building criteria in accordance with applicable procedures (TSPAI 4.05 and 4.06).

The additional documentation will be provided in the form of revised procedures, and the analysis/model reports (AMRs) and other technical documents that will support the TSPA for a future License Application. It is noted that resolution of other agreement items from KTI technical exchange meetings may also contribute to validation of the TSPA system model.

\section{Model Validation Review}

Two major reasons why the TSPA-SR model was classified as Bin 3 are: 1) sufficiency of the approach used to demonstrate adequacy of the Monte Carlo approach, and 2) the TSPA-SR is of such high importance to the Yucca Mountain Project that additional validation activities such as an independent Peer Review must be conducted. In addition, the review includes findings and recommendations related to the completeness of documentation and testing of the system model, and the approach to validation in compliance with AP-3.10Q. The major points are discussed further in the following paragraphs.

\section{Statistical Analysis}

One review finding is that the Monte Carlo sample size used was so low that very little exploration actually was made of the parameter hyperspace involved, coupled with the fact that no discussion and/or justification of this very fundamental aspect of the sample-size issue appears anywhere in the AMR (Appendix II, Part 5). Although the focus on the mean dose is driven by regulatory guidelines (40 CFR 197 [2001] Section 197.13 specifies use of the mean) the 5th, 50th and 95th percentile results are presented to provide an indication of the expected range of model outcomes. The analysis of the sensitivity of these quantities to the number of realizations for the nominal scenario is documented in Section 4.1.4 (Precision of Probabilistic Results) of the TSPA-SR technical report (CRWMS M\&O 2000bm). Figure 4.1-22 of that report shows a comparison of mean, median, 5th percentile and 95th percentile dose histories for 100 , 300 and 500 realizations. Very little difference can be seen between all three cases over the simulated time period of 100,000 yr. Based on these visual comparisons, 300 realizations have 
been selected for analyzing the reference case and carrying out the uncertainty importance analyses (Section 5.1). The 100-realization sample is considered to be adequate for comparing the trends in predictions of mean annual dose for the various sensitivity cases.

Clearly, the stability of the results for all statistical measures shown in Figure 4.1-22 of the TSPA-SR technical report (CRWMS M\&O 2000bm) refutes the idea that the sample size used in the Latin-Hypercube sampling scheme produced results which do not fully and reasonably represent the uncertainty on calculated annual dose. Based on these observations, the likelihood that the TSPA systematically missed a combination of parameter values that could have produced extremely high doses appears to be very small for all three sample sizes.

During the ongoing International Peer Review (see following discussion), an issue involving confidence intervals on the expected dose was raised. Further confirmation of the stability of the results was provided in the YMP response on confidence intervals on the 300-sample probabilistic results. The standard error in estimates of the expected dose range from 0.019 (mean $=0.112$ ) at $40,000 \mathrm{yr}$ to 7.494 (mean=66.112) at $100,000 \mathrm{yr}$. The $95 \%$ confidence intervals on the 95th percentile values are $0.64-0.76$ at $40,000 \mathrm{yr}$ and $274-374$ at $100,000 \mathrm{yr}$.

\section{Peer Reviews}

Although the reviewers concluded that the TSPA-SR should be assigned Bin 3 due to the lack of additional validating activities such as a peer review, it should be noted that a peer review was conducted on the previous TSPA model (Budnitz et al. 1999) and an international peer review is currently underway on the TSPA-SR.

The TSPA-SR integrated model (CRWMS M\&O 2000bl) is very similar conceptually to the preceding model (DOE 1998) used for performance assessment of a Yucca Mountain repository: the TSPA model for the Viability Assessment (TSPA-VA). The TSPA-VA model underwent extensive peer review and the TSPA-VA Peer Review Panel concluded, in part (Budnitz et al. 1999, p. 43):

"The Panel believes that the basic framework or architecture of the TSPA-VA is sound, as is the use of abstractions of component models for purposes of computational efficiency. Where the Panel has concerns, it is more often due to the specific methods applied and the details of the component models, rather than with how the models were linked."

Because the TSPA-SR model (CRWMS M\&O 2000bl) is quite similar in architecture to the TSPA-VA model, this conclusion adds confidence to the validity of the integrated TSPA-SR model.

In addition, the GoldSim risk-based methodology and software has been used by nuclear waste management programs in other countries. For example, the URL http://www.goldsim.com/software/modules2.asp\#problems (GoldSim 2001) provides the following documentation:

"The GoldSim Contaminant Transport Module has been used to address complex contaminant transport problems in North and South America, Europe, Asia, and Australia. A few of the more high-profile applications of the software (specifically, in the area of radioactive waste management) are listed below:

- "Spanish Radioactive Waste Disposal Research. ENRESA, the Spanish radioactive waste management agency, has been using GoldSim (and RIP) since 1992 to evaluate potential host rocks as 
part of a program to select a disposal site for the nation's spent nuclear fuel.

- "Evaluation of Waste Disposal Sites, Los Alamos, New Mexico. Los Alamos National Laboratory is using GoldSim to aid in characterizing risks and to help identify monitoring requirements for low-level radioactive waste disposal areas.

- "Remediation and Closure of Mine Workings and Facilities. GoldSim has been used in Germany to evaluate alternative remediation and closure options for abandoned mine workings and tailings facilities associated with former uranium mining operations."

Additionally, the Yucca Mountain Project has initiated the Joint NEA-IAEA International Peer Review of the TSPA-SR. The objective of this peer review is to provide, consulting the bases of available international standards and guidance as appropriate, an independent assessment of the methodology developed by the DOE Yucca Mountain Site Characterization Project and reported in: Total System Performance Assessment for the Site Recommendation (CRWMS M\&O $2000 \mathrm{bm})$.

The peer review is a review and critical analysis of the performance assessment methodology and rationale being used in support of the current site recommendation decision process. It is being conducted taking account of the international experience in preparing for and conducting system-level post-closure performance assessments. In addition, the relevant international standards and practices, and specifically the requirements proposed by the U.S. Environmental Protection Agency and the U.S. Nuclear Regulatory Commission, are being considered as bases. One aspect of the review is to identify consistencies and inconsistencies between methods being used at YMP and those being considered or developed in international recommendations, standards or practices.

Preliminary findings from the Joint NEA-IAEA International Peer Review (Riotte 2001) with regard to the methodology used for TSPA-SR include the following statements:

- The overall structure of the TSPA-SR methodology, and the approach of building on previous performance assessments, conforms to international best practice.

- The FEP screening methodology used for TSPA-SR also conforms to international best practice.

- The TSPA-SR places far greater emphasis on probabilistic assessment than equivalent programs in other countries. The limitations and strengths of the probabilistic method need to be addressed to ensure a defensible analysis.

- The TSPA-SR does not emphasize natural analogs as much as in some other international studies, and more such effort is recommended.

In response to a request from DOE to provide a statement regarding the adequacy of the overall TSPA-SR approach for supporting the site recommendation decision, the peer review team states:

"While presenting room for improvement, the TSPA-SR methodology is soundly based and has been implemented in a competent manner. Moreover, the modelling incorporates many conservatisms, including the extent to which water is able to contact the waste packages, the performance of engineered barriers and 
retardation provided by the geosphere.

"Overall, the [International Review Team, IRT] considers that the implemented performance assessment approach provides an adequate basis for supporting a statement on likely compliance within the regulatory period of 10,000 years and, accordingly, for the site recommendation decision.

"On the basis of a growing international consensus, the IRT stresses that understanding of the repository system and its performance and how it provides for safety should be emphasised more in future iterations, both during and beyond the regulatory period. Also, further work is required to increase confidence in the robustness of the TSPA."

These statements show that, notwithstanding the findings of the model validation status review, there is consensus among an important part of the international technical community that the TSPA-SR methodology is adequate for its intended use.

\section{Model Testing and Documentation}

Planned activities include more complete documentation of submodels that are implemented in the TSPA system model. Also, as recommended, more cemplete - sensitivity and uncertainty analyses will be applied to the system model. For additional confidence building or validation, specific criteria from the applicable implementing procedure (e.g., AP-3.10Q) will be considered. Finally, the documentation for TSPA will be improved to provide additional transparency, consistent at least with the intent of the recommendations in Section 6 and the additional comments in Appendix III.

\section{Conclusion}

As stated above, model validation of the total system model depends upon the confidence building activities that are conducted for the key underlying process and abstraction models Confidence building is an iterative and ongoing process, and the Yucca Mountain Project continues to develop confidence building activities as part of the model validation for process models and abstractions that are used as the underlying bases for the TSPA. In order to provide an appropriate level of confidence in the models used to demonstrate compliance with the draft regulations considered in the site recommendation documents, it is necessary to:

- Confirm that the relevant draft numerical performance standards have been met

- Confirm that the analyses are realistic with reasonable conservatism for uncertainties, that limitations in the analyses are well understood, and appropriate allowances have been made for time period, hazards and uncertainties.

The level of confidence required should be consistent with the importance to performance. With respect to the first bullet, the results of the TSPA-SR indicate that relevant draft numerical performance standards can be met by several orders of magnitude. It should be noted that there are differences between the draft 10 CFR 63 (66 FR 55732) and the final 40 CFR 197 (2001) numerical performance standards and the points of compliance. With respect to the second bullet, sensitivity studies have been documented in the TSPA-SR in the form of uncertainty importance analyses, subsystem sensitivity analyses, and robustness analyses (CRWMS M\&O $2000 \mathrm{bm}$; Sections 5.1, 5.2, and 5.3) that indicate the level of uncertainties and conservatisms, the limitations of the models, and the impacts and individual contributions associated with various time periods and likely and unlikely hazards. These sensitivity analyses indicate that the TSPA 
model is sufficiently robust that even given the uncertainties that may exist in the confidence of the subsystem models, the relevant draft numerical performance standards will likely be met. Additional sensitivity analyses have been documented in Volumes 1 and 2 of the FY01 Supplemental Science and Performance Analyses (BSC 2001d and 2001k, respectively) specifically to provide additional insights into the potential conservatisms and optimisms in the TSPA, to capture a wider range of uncertainties, and to provide updated and more realistic representations of processes. The results of these additional sensitivity analyses also indicate that the relevant draft numerical performance standards can be met for a range of thermal operating modes by several orders of magnitude.

The model validation work performed to date on the TSPA Model including all of the uncertainty and sensitivity analyses coupled with the past and ongoing peer reviews result in a TSPA Model that is sufficiently robust and provides adequate confidence that the model is suitable for its intended use (a site recommendation) and that the relevant draft numerical performance standards will be met in a potential License Application (LA). As model validation exercises continue for subsystem models, confidence will increase in the appropriateness of the models for incorporation into the TSPA iteration required for a potential LA submittal. The TSPA iteration required for a potential LA will be used to confirm that the relevant final numerical performance standards have been met. 
MODEL VALIDATION - IMPACT REVIEW

A. Model: Soil Removal Model for Volcanic Disruption (U.1-2)
B. AMR: Total System Performance Assessment (TSPA) Model for Site Recommendation (MDLWIS-PA-000002 Rev. 00) (CRWMS M\&O 2000bl)

C. Category (Check appropriate case)

1) Model NOT USED in TSPA and DID NOT SERVE as a basis for screening FEPS.

Result: No impact on TSPA-SR

2) Model NOT USED in TSPA but DID SERVE as a basis for screening FEPS.

Result: Assess impact of validation findings on FEP screening - Complete Section D below

囚 3) Model IS USED in TSPA but DID NOT SERVE as a basis for screening FEPs.

Result: Assess impact of validation findings on TSPA-SR results - Complete Section D below

4) Model IS USED in TSPA and DID SERVE as a basis for screening FEPs.

Result: Assess impact of validation findings on TSPA-SR results and FEP screening Complete Section $\mathrm{D}$ below

D. Path Forward and Impact on TSPA (Describe in detail)

See continuation sheet.

E. Responsible Individual: $\frac{\text { Jerry McNeish/ !emya hicheinh }}{\text { Type Name/Signature }} \frac{11.14 .01}{\text { Date: }}$ 
Continuation of item D (Model U.1-2):

As discussed in the FY01 Supplemental Science and Performance Analyses (BSC 2001d, Section 3.3.1.2.5) the approach taken in TSPA-SR (CRWMS M\&O 2000bm, Sections 3.10.2 through 3.10.4) does not explicitly include the effects of possible surface redistribution of contaminated ash following deposition. Specifically, aeolian and fluvial processes may result in transport of sediment from other regions within the area of the ashfall to the location of the receptor. Instead of explicitly including these processes, TSPA-SR analyses used a conservative approach (CRWMS M\&O 2000bm, Section 3.10.4) in which the wind direction was fixed toward the receptor for all eruptive events, overestimating the amount of ash initially deposited at the location receptor. Furthermore, the transition-phase biosphere dose conversion factors (BDCFs) used for calculating eruptive annual dose at all times following ash deposition used high air-mass loading values applicable for fresh, unconsolidated ash, rather than the more appropriate longterm BDCFs calculated for stabilized soils. This overestimate of long-term air-mass loading, combined with the assumption for the purpose of calculating the inhalation dose that all radionuclides would be concentrated in the upper $1 \mathrm{~cm}$ of the ash layer regardless of its thickness, form the basis for the assertion in the TSPA-SR technical report that the overall treatment is conservative with respect to ash redistribution processes (CRWMS M\&O 2000bm, Section 3.10.4).

Also, as described in the FY01 Supplemental Science and Performance Analyses (BSC 2001d, Sections 3.3.1.2.4 and 3.3.1.2.5, and Figure 3.3.1.2.4-3) the no-soil removal case provides an upper bound on conditional annual doses that might result if surficial redistribution processes cause deposition of contaminated sediment at the location of the receptor, as long as concentrations of radionuclides in the redeposited sediments are equal to or less than concentrations in the initial ash layer.

The soil redistribution model will be updated to reflect processes that both add to and remove soil from the receptor site. Rates for soil addition and removal will be based on field studies of soil movement in the Yucca Mountain vicinity. Redistribution of ash from the Lathrop Wells eruption will also be examined. It is planned that these studies will be documented in a revision to the report: Characterize Eruptive Processes at Yucca Mountain, Nevada (CRWMS M\&O $2000 \mathrm{k}$ ) and results will be intended for use in TSPA-LA. 


\section{MODEL VALIDATION - IMPACT REVIEW}

A. Model: Pu-Ceramic Degradation Model for TSPA-SR (U.4)
B. AMR: Performance Assessment and Sensitivity Analysis of Disposal of $\mathrm{Pu}$ as Can-in-Canister Ceramic. (ANL-WIS-PA-000003 Rev. 00) (CRWMS M\&O 2001u)

C. Category (Check appropriate case)

$\triangle 1$ 1) Model NOT USED in TSPA and DID NOT SERVE as a basis for screening FEPS.

Result: No impact on TSPA-SR

2) Model NOT USED in TSPA but DID SERVE as a basis for screening FEPS.

Result: Assess impact of validation findings on FEP screening - Complete Section D below

3) Model IS USED in TSPA but DID NOT SERVE as a basis for screening FEPs.

Result: Assess impact of validation findings on TSPA-SR results - Complete Section D below

4) Model IS USED in TSPA and DID SERVE as a basis for screening FEPs.

Result: Assess impact of validation findings on TSPA-SR results and FEP screening Complete Section $D$ below

D. Path Forward and Impact on TSPA (Describe in detail)

The validation review findings on this model do not impact the conclusions of the TSPA-SR, because the model is used only for a sensitivity study, and not for dose calculations that can be compared to regulatory standards. The subject AMR is not cited in the TSPA-SR model or technical report documents (CRWMS M\&O 2000bl; 2000bm).

In the subject AMR several models for aqueous dissolution of the ceramic are compared by examining the annual dose results (dose history) for the median value nominal case (median values for distributed inputs). These models include two different ceramic models, plus the HLW glass model, and also an instantaneous dissolution model. The dose results for the LLNL ceramic model, the HLW glass model, and instantaneous dissolution model showed virtually equivalent calculated annual dose on the million-year dose history plot (Figures 6.5-5 and 6.5-6 of the subject AMR). The other ceramic model (Synroc ceramic) showed somewhat lower annual dose at later times (after about 40,000 yr; Figure 6.5-6) and is therefore regarded as less conservative than the other models.

The LLNL ceramic model is selected for use in the sensitivity study because it is mechanistic and thus physically meaningful, but produces results that are equivalent to the bounding instantaneous dissolution model. The fourth model (Synroc ceramic) would require additional justification. The reason that all four models produce such similar results is that release from the failed waste package depends more on actinide solubility than the waste form dissolution rate. The Synroc ceramic model imposes greater kinetic limitation on dissolution, and after 40,000 yr begins to limit the amounts of actinides available for transport out of a failed package, which is why it produces somewhat lower annual dose at later times.

E. Responsible Individual: $\frac{\text { Jerry McNeish/ Jeng A nitheioh }}{\text { Type Name/Signature }} \frac{i 1.14 .01}{\text { Date: }}$ 
INTENTIONALLY LEFT BLANK 